\title{
Message from our President
}

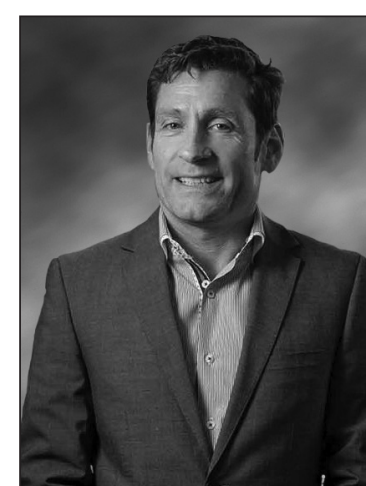

Dr. Andrew MacNeily, CUA President

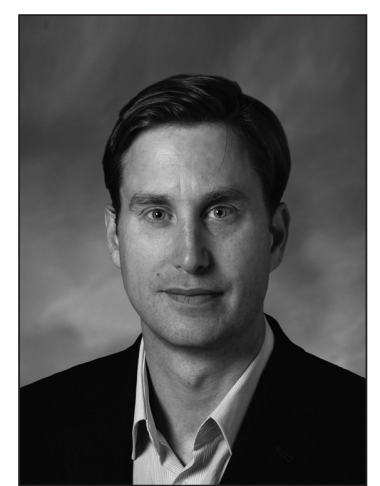

Dr. Keith Rourke, CUA 2020 Scientific Committee Co-Chair $\mathrm{n}$ an abstract sort of way, it has been quite the ride.

I suppose I will go down in the annals of the CUA as the COVID President. Not to be outdone, your 2020 CUA Scientific Committee Chairs (the tall boys) will most likely be remembered as the COVID Chairs.

Keith Rourke (tallest) and Peter Black (wisest) really stepped up and delivered a great scientific program for CUA 2020. Unfortunately, it was not meant to be.

Instead of wasting all that effort for naught, your CUAJ has published the accepted abstracts for CUA 2020. These should have, could have, and would have been debated in Victoria. Please take the time to study the abstracts, which represent the hard work of all the would-be presenters. Keith, Peter, and their Scientific Committee put a tremendous amount of work into creating the 2020 CUA program and it should not go unnoticed. In fact, with the support of the Scientific Planning Committee, the CUA is proud to be offering a week-long, not-to-be missed, virtual "CUA Night School" every night from 8:00-10:15 pm from June 22-26, reflecting what would have been some of the program highlights of the CUA 2020 annual meeting. I encourage you to participate in this Section 1-accredited event at nightschool.cua.events.

Of course, all of this is just an abstraction...

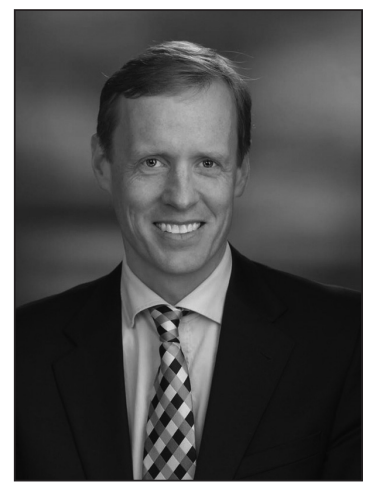

Dr. Peter Black, CUA 2020

Scientific Committee Co-Chair 


\section{TABLE OF CONTENTS}

\section{Podium Sessions:}

1: Endourology, Nephrolithiasis S23

2: Oncology - Prostate ..S28

3: Urinary Incontinence, Voiding Dysfunction ..S33

4: Oncology - Other

5: Training, Reconstruction

\section{Unmoderated Poster Sessions:}

1: Prostate Cancer, Endourology, BPH S44

2: Uro-Oncology, Pediatric Urology, Sexual Dysfunction, Transplant

3: Prostate Cancer, Functional Urology, Other Urology Topics

\section{Moderated Poster Sessions:}

1: Endourology I S79

2: Trauma, Reconstruction, Pelvic Pain S86

3: Uro-Oncology S91

4: Prostate Cancer I S97

5: Technical Advances in Urology, Improving Patient Relations $\mathrm{S} 104$

6: Training, Evaluation S109

7: Benign Prostatic Hyperplasia...

8: Bladder Cancer S127

9: Endourology II

10: Prostate Cancer II S138

11: Urinary Incontinence, Voiding Dysfunction, Sexual Dysfunction, Transplant

12: Pediatric Urology S149

Author Index S160 


\section{Podium Session 1: Endourology, Nephrolithiasis}

\section{POD-1.1}

Three-year outcomes after Aquablation compared to TURP: Efficacy and ejaculatory improvements sustained

Kevin C. Zorn' ${ }^{1}$, Naeem Bhojani ${ }^{1}$, Dean S. Elterman², S. Larry Goldenberg', Alan I. So , Ryan F. Paterson ${ }^{3}$, Mihir Desai ${ }^{4}$, Roehrborn Roehrborn ${ }^{5}$, Steven Kaplan ${ }^{5}$, Peter Gilling ${ }^{6}$

${ }^{1}$ Urology, University of Montreal Hospital Center (CHUM), Montreal, QC, Canada; ${ }^{2}$ Urology, University of Toronto, Toronto, ON, Canada; ${ }^{3}$ Urology, University of British Columbia, Vancouver, BC, Canada; ${ }^{4}$ Urology, UTSW, Dallas, TX, United States; ${ }^{5}$ Urology, USC, Los Angeles, CA, United States; ${ }^{6}$ Urology, University of Auckland, Auckland, New Zealand; ATER2/ WATER Study Group

Introduction: We aimed to compare three-year (last followup of the proto$\mathrm{col})$ safety and efficacy outcomes after Aquablation or transurethral resection of the prostate (TURP) for the treatment of lower urinary tract symptoms (LUTS) related to benign prostate hyperplasia (BPH).

Methods: A total of 181 patients with $\mathrm{BPH}$ were assigned at random (2:1 ratio) to either Aquablation or TURP. Patients and followup assessors at each site were blinded to treatment. Assessments included International Prostate Symptom Score (IPSS), Male Sexual Health Questionnaire (MSHQ), International Index of Erectile Function (IIEF), and uroflow.

Results: At three years, IPSS scores improved by 14.2 points in the Aquablation group and 15 points in TURP $(p=0.7050,95 \%$ confidence interval $[\mathrm{Cl}]$ for difference -3.6 to 5.3 points). Three-year improvements in maximum flow rate (Qmax) were large in both groups at 10 and $9.7 \mathrm{cc} / \mathrm{sec}$ for Aquablation and

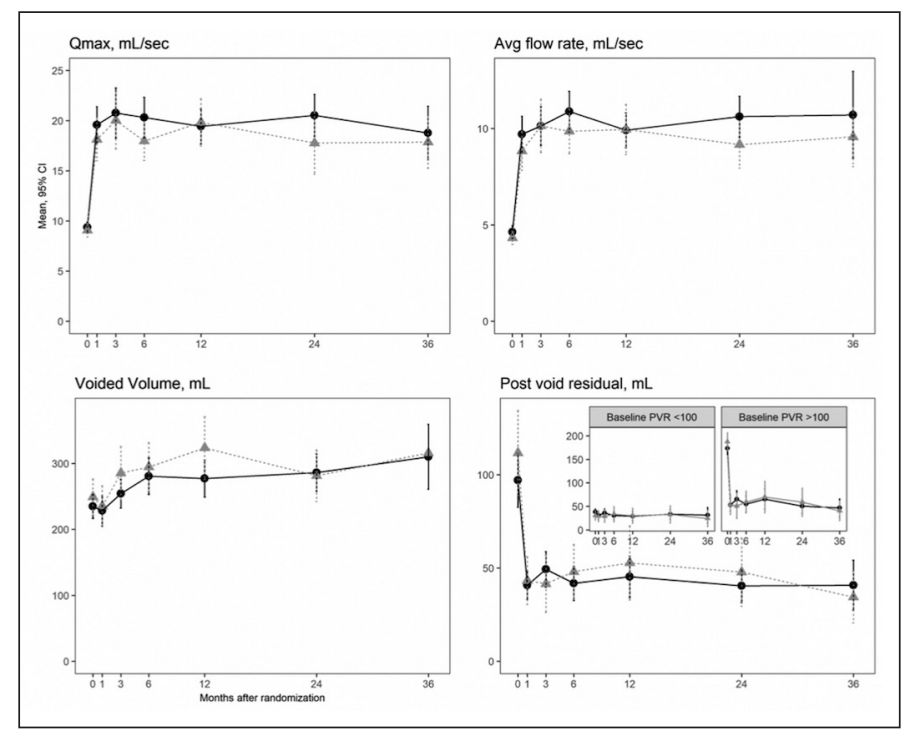

POD-1.1. Fig. 1. Uroflow measures by treatment and time. For PVR, insert graph shows analysis of those with elevated (>100 cc) and not elevated (<100 cc) baseline PVR. Black circles=aquablation; gray triangles=TURP. PVR: post-void residual; TURP: transurethral resection of the prostate.

\begin{tabular}{|c|c|c|}
\hline Characteristic & $\begin{array}{c}\text { Aquablation } \\
\mathrm{n}=117\end{array}$ & $\begin{array}{l}\text { TURP } \\
\mathrm{n}=67\end{array}$ \\
\hline Age, years, mean (SD) & $66.0(7.3)$ & $65.8(7.2)$ \\
\hline Body mass index, mean (SD) & $28.4(4.1)$ & $28.2(4.5)$ \\
\hline Prostate size (TRUS)* ${ }^{*}$ gm; mean (SD) & $54.1(16.2)$ & $51.8(13.8)$ \\
\hline $\begin{array}{l}\text { Prostate specific antigen, } \mathrm{g} / \mathrm{dL} \text {; mean } \\
\text { (SD) }\end{array}$ & $3.7(3.0)$ & $3.3(2.3)$ \\
\hline \multicolumn{3}{|l|}{ Cystoscopy findings } \\
\hline $\begin{array}{l}\text { Lobes present } \\
\text { Lateral lobe only } \\
\text { Middle lobe only } \\
\text { Both lateral and middle }\end{array}$ & $\begin{array}{c}50(42.7 \%) \\
9(7.7 \%) \\
55(47.0 \%)\end{array}$ & $\begin{aligned} 31 & (46.3 \%) \\
3 & (4.5 \%) \\
88 & (47.8 \%)\end{aligned}$ \\
\hline $\begin{array}{l}\text { Degree of middle lobe obstruction } \\
\text { None } \\
\text { Mild } \\
\text { Moderate } \\
\text { Severe }\end{array}$ & $\begin{array}{l}23(19.7 \%) \\
25(21.4 \%) \\
35(29.9 \%) \\
14(12.0 \%)\end{array}$ & $\begin{array}{l}15922.4 \%) \\
15(22.4 \%) \\
22(32.8 \%) \\
7(10.4 \%)\end{array}$ \\
\hline Bladder neck obstruction & $30(25.6 \%)$ & $24(35.8 \%)$ \\
\hline \multicolumn{3}{|l|}{ Baseline questionnaires } \\
\hline IPSS score, mean (SD) & $22.9(6.0)$ & $22.2(6.1)$ \\
\hline IPSS QOL, mean (SD) & $4.8(1.1)$ & $4.8(1.0)$ \\
\hline Sexually active, N (\%) [MSHQ-EjD] & $93(80.2 \%)$ & $54(83.1 \%)$ \\
\hline MSHQ-EjD mean (SD) ${ }^{* *}$ & $8.1(3.7)$ & $8.8(3.6)$ \\
\hline IIEF-5, mean $(S D)^{* *}$ & $17.2(6.5)$ & $18.2(7.0)$ \\
\hline
\end{tabular}

TURP, respectively ( $p=0.9078,95 \% \mathrm{CI}$ for difference -2.9 to 6.4 ). Sexual function as assessed by MSHQ-EjD was stable in the Aquablation group and decreased slightly in the TURP group; these changes persisted at year three. At three years, prostate-specific antigen was reduced significantly in both groups but showed no difference between groups $(p=0.4074$ for difference). The average annual retreatment occurrence was less than $2 \%$ in both treatment groups.

Conclusion: Three-year efficacy outcomes after TURP and Aquablation were similar and the rate of surgical retreatment was low and similar to TURP. Efficacy results have maintained their improvement since the 90-day followup for both groups that are consistent with all other resective surgical techniques.

Trial registration: ClinicalTrials.gov number, NCT02505919 
POD-1.1. Table 1B. Number of subjects with urologic events by year 1 (adjudicated by CEC)

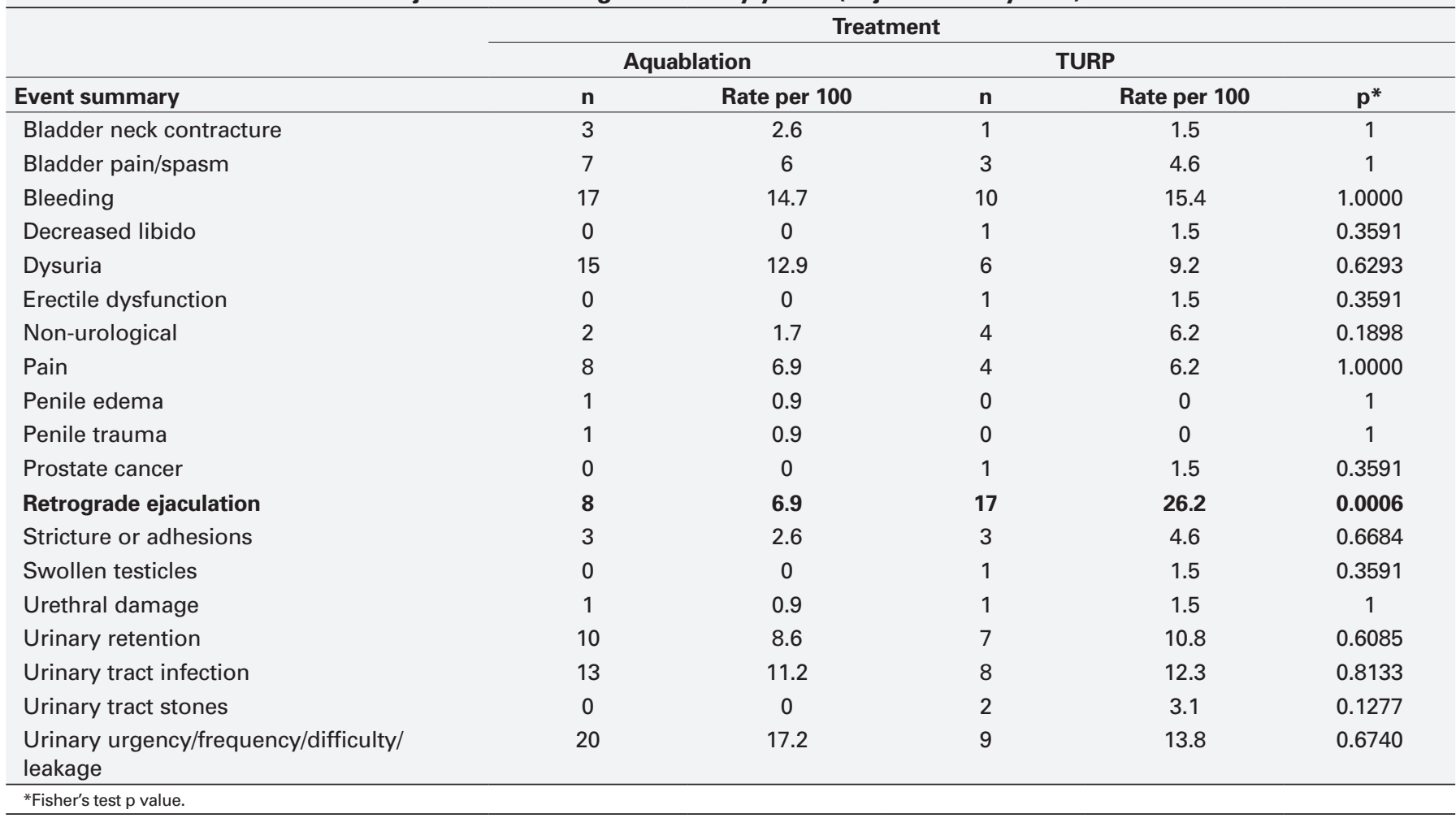

\section{POD-1.2}

Surgical trends in pediatric urolithiasis management in Ontario from 2002-2016

Fernanda Gabrigna Berto ${ }^{1}$, Zhan Tao (Peter) Wang ${ }^{1}$, Jennifer Bjazevic ${ }^{1}$, Guido Filler' ${ }^{2}$, Blayne K. Welk', Hassan Razvi', Sumit Dave ${ }^{1}$

${ }^{1}$ Urology, University of Western Ontario, London, ON, Canada; ${ }^{2}$ Pediatric Nephrology, University of Western Ontario, London, ON, Canada

Introduction: A global increase in pediatric urolithiasis (PUL) incidence has been reported. ${ }^{1-5}$ As a potentially recurrent condition, PUL has implications on morbidity ${ }^{5,6}$ and healthcare costs. ${ }^{7}$ This study evaluates surgical trends and outcomes of PUL in Ontario over a 15 year-period (2002-2016).

Methods: This retrospective cohort study used administrative databases held at the Institute of Clinical Evaluative Sciences to identify all children ( $<18$ years) who underwent their first surgical treatment for PUL using the Canadian Institute for Health Information-Discharge Abstract Database and ICD10th revision. Stone location and treatment modality were simultaneously identified. Descriptive statistics were used to summarize patient demographics and surgical trends. Logistic regression analysis was performed to identify independent risk factors for repeat surgical intervention within six months of index procedure.

Results: A total of 706 patients undergoing surgical procedure for PUL were identified (mean age $12.6 \pm 4.8$ years); $68.5 \%$ of children were older than 12 years and $55.2 \%$ presented with ureteric stones. There was no statistical increase in the number of procedures performed, gender predominance, or age difference at surgery. Ureteroscopy (URS) was the most commonly performed procedure $(63.7 \%)$ and its application increased over time ( $p<0.0001)$ (Fig. 1). Conversely, extracorporeal shockwave lithotripsy (ESWL) use decreased. Percutaneous nephrolithotripsy rates $(9.6 \%)$ were stable. Renal stone location (odds ratio [OR] 2.79; $p=0.0002$ ) and ESWL (OR 1.66; $p=0.0025)$ were independent risk factors for repeat surgical procedure within six months. Stone recurrence requiring additional surgery after five years was $33 \%$.
Conclusions: The incidence of surgically treated PUL is stable over 15 years in Ontario, Canada. URS has become the most common surgical treatment modality for PUL and ESWL rates have dropped, possibly secondary to the higher rates of re-do surgical intervention.

\section{References}

1. Alfandary $\mathrm{H}$, Haskin $\mathrm{O}$, Davidovits $\mathrm{M}$, et al. Increasing prevalence of nephrolithiasis in association with increased body mass index in children: A population-based study. J Urol 2018;199:1044-9. https:// doi.org/10.1016/j.juro.2017.10.023

2. Park HK, Kim JH, Min GE, et al. Change of trends in the treatment modality for pediatric nephrolithiasis: Retrospective analysis of a U.S.-based insurance claims database. J Endourol 2019;33:614-8. https://doi.org/10.1089/end.2019.0154

3. Sas DJ, Hulsey TC, Shatat IF, et al. Increasing incidence of kidney stones in children evaluated in the emergency department. J Pediatr 2010;157:132-7. https://doi.org/10.1016/j.jpeds.2010.02.004

4. Matlaga BR, Schaeffer AJ, Novak TE, et al. Epidemiologic insights into pediatric kidney stone disease. Urol Res 2010;38:453-7. https:// doi.org/10.1007/s00240-010-0327-

5. Routh JC, Graham DA, Nelson CP. Epidemiological trends in pediatric urolithiasis at United States freestanding pediatric hospitals. J Urol 2010;184:1100-4. https://doi.org/10.1016/j.juro.2010.05.018

6. Schaeffer AJ, Feng Z, Trock BJ, et al. Medical comorbidities associated with pediatric kidney stone disease. Urology 2011;77:195-9. https://doi.org/10.1016/j.urology.2010.06.062

7. Kusumi K, Becknell B, Schwaderer A. Trends in pediatric urolithiasis: Patient characteristics, associated diagnoses, and financial burden. Pediatr Nephrol 2015;30:805-10. https://doi.org/10.1007/s00467014-3012-3 


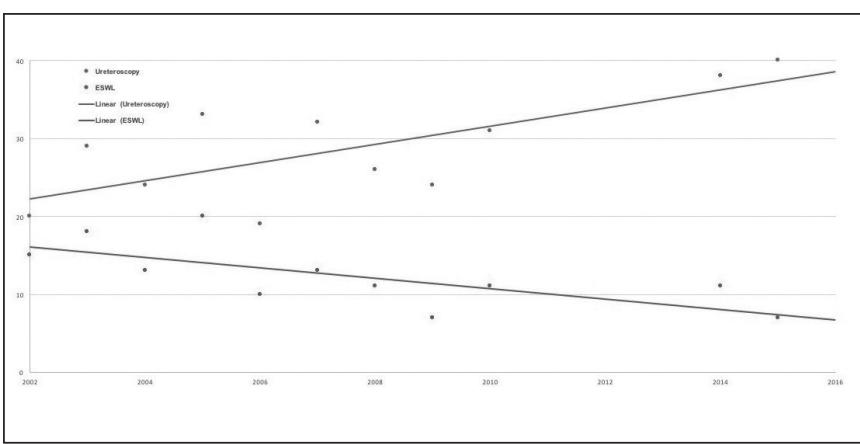

POD-1.2. Fig. 1. Number of PUL surgical stone procedures performed in Ontario (2002-2016).

\section{POD-1.3}

Increased risk of new persistent opioid use in pediatric and young adult urolithiasis patients prescribed opioids at presentation

Gregory Hosier ${ }^{1}$, Thomas McGregor ${ }^{1}$, Darren T. Beiko', Gregory E. Tasian², Michael A. Di Lena' ${ }^{1}$, Christopher M. Booth ${ }^{3}$, Marlo Whitehead ${ }^{1}$, D. Robert Siemens

${ }^{1}$ Urology, Queen's University, Kingston, ON, Canada; ${ }^{2}$ Pediatric Urology, Children's Hospital of Philadelphia, Philadelphia, PA, United States; ${ }^{3}$ Medical Oncology, Queen's University, Kingston, ON, Canada Support: PSI Foundation

Introduction: The incidence of pediatric urolithiasis has been steadily increasing for the past several decades. Pediatric and young adults are a vulnerable patient population for development of addiction after opioid exposure. However, the long-term impact of opioid prescribing in pediatric and young adult patients with urolithiasis is not known. Our objective was to describe rates of opioid prescription and identify risk factors for persistent opioid use in patients age 25 years or younger with urolithiasis.

\begin{tabular}{|c|c|c|c|c|c|}
\hline Characteristic & Rate & $\begin{array}{l}\text { Univariate analysis } \\
\text { OR }(95 \% \mathrm{Cl})\end{array}$ & p & $\begin{array}{l}\text { Multivariable analysis } \\
\text { OR (95\% Cl) }\end{array}$ & $\mathbf{p}$ \\
\hline \multicolumn{6}{|l|}{ Patient-related } \\
\hline \multicolumn{6}{|l|}{ Age, years } \\
\hline $0-11$ & 2 & $0.21(0.03-1.51)$ & 0.18 & $0.16(0.02-1.22)$ & 0.22 \\
\hline $12-18$ & 9 & $1.24(0.87-1.75)$ & & $1.20(0.83-1.70)$ & \\
\hline 19-21 & 7 & Ref & & Ref & \\
\hline $22-25$ & 9 & $1.20(0.91-1.6)$ & & $1.06(0.8-1.4)$ & \\
\hline \multicolumn{6}{|l|}{ Sex } \\
\hline Female & 9 & $1.22(0.97-1.5)$ & 0.09 & $1.15(0.90-1.48)$ & 0.26 \\
\hline Male & 7 & Ref & & Ref & \\
\hline \multicolumn{6}{|c|}{ Mental health history } \\
\hline No & 7 & Ref & 0.0025 & Ref & 0.0338 \\
\hline Yes & 10 & $1.43(1.13-1.80)$ & & $1.32(1.02-1.71)$ & \\
\hline \multicolumn{6}{|l|}{ Care-related } \\
\hline \multicolumn{6}{|c|}{ Duration urolithiasis episode } \\
\hline$<60$ days & 6 & $0.45(0.35-0.57)$ & $<0.0001$ & $0.62(0.47-0.82)$ & 0.0008 \\
\hline$>60$ days & 13 & Ref & & Ref & \\
\hline \multicolumn{6}{|c|}{$\begin{array}{l}\text { \# ED visits for urolithiasis after index } \\
\text { visit }\end{array}$} \\
\hline 0 & 6 & $0.24(0.17-0.35)$ & $<0.0001$ & $0.49(0.32-0.74)$ & 0.0059 \\
\hline 1 & 8 & $0.32(0.21-0.47)$ & & $0.5(0.33-0.79)$ & \\
\hline 2 & 13 & $0.57(0.36-0.90)$ & & $0.68(0.42-1.09)$ & \\
\hline$>2$ & 21 & Ref & & Ref & \\
\hline \multicolumn{6}{|l|}{ Had surgery } \\
\hline No & 7 & Ref & $<0.0001$ & Ref & 0.0009 \\
\hline Yes & 14 & $2.30(1.77-2.99)$ & & $1.7(1.24-2.34)$ & \\
\hline \multicolumn{6}{|l|}{ Opioid-related } \\
\hline \multicolumn{6}{|c|}{$\begin{array}{l}\text { Total oral morphine equivalents } \\
\text { during acute stone episode }\end{array}$} \\
\hline $1-99$ & 5 & Ref & $<0.0001$ & Ref & $<0.0001$ \\
\hline 100-149 & 6 & $1.07(0.71-1.62)$ & & $1.0(0.65-1.52)$ & \\
\hline 150-199 & 7 & $1.29(0.85-1.95)$ & & $1.18(0.77-1.80)$ & \\
\hline 200-299 & 8 & $1.62(1.09-2.42)$ & & $1.35(0.90-2.04)$ & \\
\hline$>300$ & 17 & $3.79(2.64-5.44)$ & & $2.21(1.49-3.29)$ & \\
\hline
\end{tabular}


Methods: Using previously validated linked administrative databases, we performed a population-based retrospective study of all opioid-naive patients age 25 years or younger with a diagnosis of urolithiasis between July 1, 2013 and September 30, 2017 in Ontario, Canada. Our primary outcome was persistent opioid use defined as filling a prescription for an opioid between 91 and 180 days after initial urolithiasis visit. Secondary outcomes were opioid addiction and opioid overdose.

Results: Of the 6962 patients identified, 56\% were prescribed an opioid at presentation and $34 \%$ of those were dispensed more than 200 oral morphine equivalents. There was persistent opioid use in $313(8 \%)$ patients who filled an initial opioid prescription. Those prescribed an opioid initially had a significantly higher risk of persistent opioid use (odds ratio $[\mathrm{OR}] 1.8 ; 95 \%$ confidence interval $[\mathrm{Cl}] 1.5-2.3)$, opioid addiction (OR 3.6; 1.2-10.6), and opioid overdose (OR 2.8; 0.9-8.6) compared to those without initial opioid exposure. In adjusted analysis, initial exposure of $>300$ oral morphine equivalents (OR $2.2 ; 1.5-3.3$ ), history of mental illness (OR 1.3; 1.0-1.7), and need for surgery (OR 1.7; 1.2-2.3) were all risk factors for persistent opioid use (Table 1).

Conclusions: Among urolithiasis patients age 25 years or younger, filling an opioid prescription after presentation is associated with an increased risk of persistent opioid use 3-6 months later and a higher risk of serious long-term complications, such as opioid addiction and overdose.

\section{POD-1.4}

Metabolic syndrome negatively impacts stone specific quality of life: Results from the North American Stone Quality of Life Consortium

Lonathan Lim ', Kymora Scotland', Seth K. Bechis², Roger L. Sur'², Stephen Y. Nakada ${ }^{3}$, Sri Sivalingam ${ }^{4}$, Timothy D. Averch ${ }^{5}$, Jaime Landman ${ }^{6}$, Thomas Chi $^{7}$, Sero Andonian ${ }^{8}$, Naeem Bhojani ${ }^{9}$, Noah E. Canvasser ${ }^{10}$, Jonathan D. Harper $^{11}$, Kristina L. Penniston ${ }^{3}$, Ben H. Chew ${ }^{1}$

'Department of Urologic Sciences, University of British Columbia, Vancouver, BC, Canada; ${ }^{2}$ School of Medicine, University of San Diego, San Diego, CA, United States; ${ }^{3}$ School of Medicine and Public Health, University of Wisconsin, Madison, WI, United States; ${ }^{4}$ Glickman Urological and Kidney Institute, Cleveland Clinic, Cleveland, $\mathrm{OH}$, United States; ${ }^{5}$ Palmetto Health USC Medical Group, Columbia, SC, United States; ${ }^{6}$ School of Medicine, University of California Irvine, Orange, CA, United States; ${ }^{7}$ School of Medicine, University of California San Francisco, San Francisco, CA, United States; ${ }^{8}$ McGill University Health Centre, Montreal, QC, Canada; ${ }^{9}$ Université de Montréal, Montreal, QC, Canada; ${ }^{10}$ School of Medicine, University of California Davis, Sacramento, CA, United States; ${ }^{11}$ University of Washington, Seattle, WA, United States Introduction: Metabolic syndrome (MetS) is a collection of metabolic comorbidities that is linked to the formation of atherosclerotic cardiovascular disease. ${ }^{1}$ Recently, MetS has been connected to an increased risk of nephrolithiasis. ${ }^{2}$ In light of this association, this study aimed to assess the hypothesis that the presence of MetS is linked to a decreased health-related quality of life (HRQOL) in active stone-forming patients. ${ }^{3}$ Methods: This is a multi-institutional prospective study by the North American Stone Quality of Life Consortium. All sites used the Wisconsin stone quality of life questionnaire (WISQOL) - a validated survey - to evaluate patient stone specific HRQOL. ${ }^{4}$ In addition to WISQOL, sites also collected medical history. This was used to distinguish MetS patients from non-MetS patients. Patients who were active stone-formers were identified. Among these active stone-formers, MetS patients were compared to non-MetS patients using a Wilcoxon rank sum test.

Results: In active stone-formers, there was a significant difference in stone specific HRQOL between MetS (median 102/140) and non-MetS patients (median 106/140; $\mathrm{p}<0.05)$. Patients with body mass index $(\mathrm{BMI})>30$ or diabetes mellitus (part of criteria for MetS) also had a significantly lower HRQOL compared to non-BMI $>30$ and non-diabetes mellitus patients, respectively. In this study, all patients had stones, but those with MetS or associated criterion had lower HRQOL.

Conclusions: In the management of stones, asymptomatic or tolerably symptomatic stones may not require surgical treatment. Thus, factors that are associated with symptoms need to be identified. These factors can then be treated to decrease symptomaticity. Observed through a lower
HRQOL score, this study indicates an association between MetS and increased nephrolithiasis symptoms. Specific criterion of MetS (BMI >30 and diabetes mellitus) are linked to lower HRQOL scores as well. This lends support to holistic, personalized stone patient care.

\section{References}

1. Grundy SM, Cleeman JI, Daniels SR, et al. Diagnosis and management of the metabolic syndrome: An American Heart Association/National Heart, Lung, and Blood Institute Scientific Statement. Circulation 2005;112:2735-52. https://doi.org/10.1161/ CIRCULATIONAHA.105.169404

2. DiBianco JM, Jarrett TW, Mufarrij P. Metabolic syndrome and nephrolithiasis risk: Should the medical management of nephrolithiasis include the treatment of metabolic syndrome? Rev Urol 2015;17:117-28.

3. Karimi M, Brazier J. Health, health-related quality of life, and quality of life: What is the difference? Pharmacoeconomics 2016;34:645-9. https://doi.org/10.1007/s40273-016-0389-9

4. Penniston KL, Antonelli J, Viprakasit D, et al. Validation and reliability of the Wisconsin stone quality of life questionnaire. J Urol 2017;197:1280-8. https://doi.org/10.1016/j.juro.2016.11.097

\section{POD-1.5}

Wisconsin Quality of Life Machine Learning Algorithm for predicting the quality of life in kidney stone patients

David-Dan Nguyen 1,2 , Jack W. Luo ${ }^{1}$, Jonathan R.Z Lim³ ${ }^{3}$ Kymora Scotland ${ }^{3}$, Seth K. Bechis ${ }^{4}$ Roger L. Sur ${ }^{4}$, Stephen Y. Nakada ${ }^{5}$, Jody A. Antonelli, Necole M. Streepe ${ }^{7}$, Sri Sivalingam ${ }^{8}$, Davis P. Viprakasit ${ }^{9}$, Timothy D. Averch $^{10}$, Jaime Landman ${ }^{11}$, Thomas Chi ${ }^{12}$, Vernon M. Pais, Jr. ${ }^{13}$, Vincent G. Bird ${ }^{14}$, Sero Andonian ${ }^{15}$, Noah E. Canvasser ${ }^{16}$, Jonathan D. Harper ${ }^{17}$ Ben H. Chew ${ }^{3}$, Kristina L Penniston ${ }^{5}$, Naeem Bhojani ${ }^{18}$

${ }^{1}$ Faculty of Medicine, McGill University, Montreal, QC, Canada; ${ }^{2}$ Health Policy and Management, Harvard T.H. Chan School of Public Health, Boston, MA, United States; ${ }^{3}$ Urologic Sciences, University of British Columbia, Vancouver, BC, Canada; ${ }^{4}$ Urology, University of California San Diego School of Medicine, San Diego, CA, United States; ${ }^{5}$ Urology, University of Wisconsin School of Medicine and Public Health, Madison, WI, United States; ${ }^{6}$ Urology, University of Texas Southwestern Medical Center, Dallas, TX, United States; ${ }^{7}$ Urology, Pennsylvania State University College of Medicine, Hershey, PA, United States; ${ }^{8}$ Glickman Urological

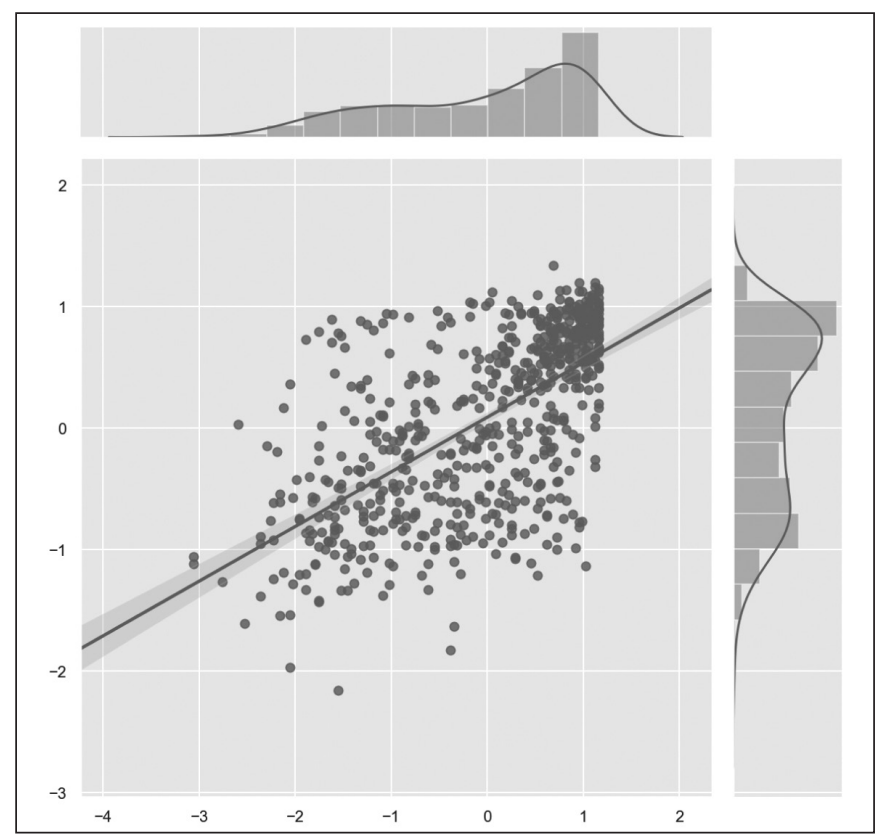

POD-1.5. Fig. 1. Out-of-sample correlation between quality of life estimates and WISQOL total score $(\mathrm{r}=0.622)$ 


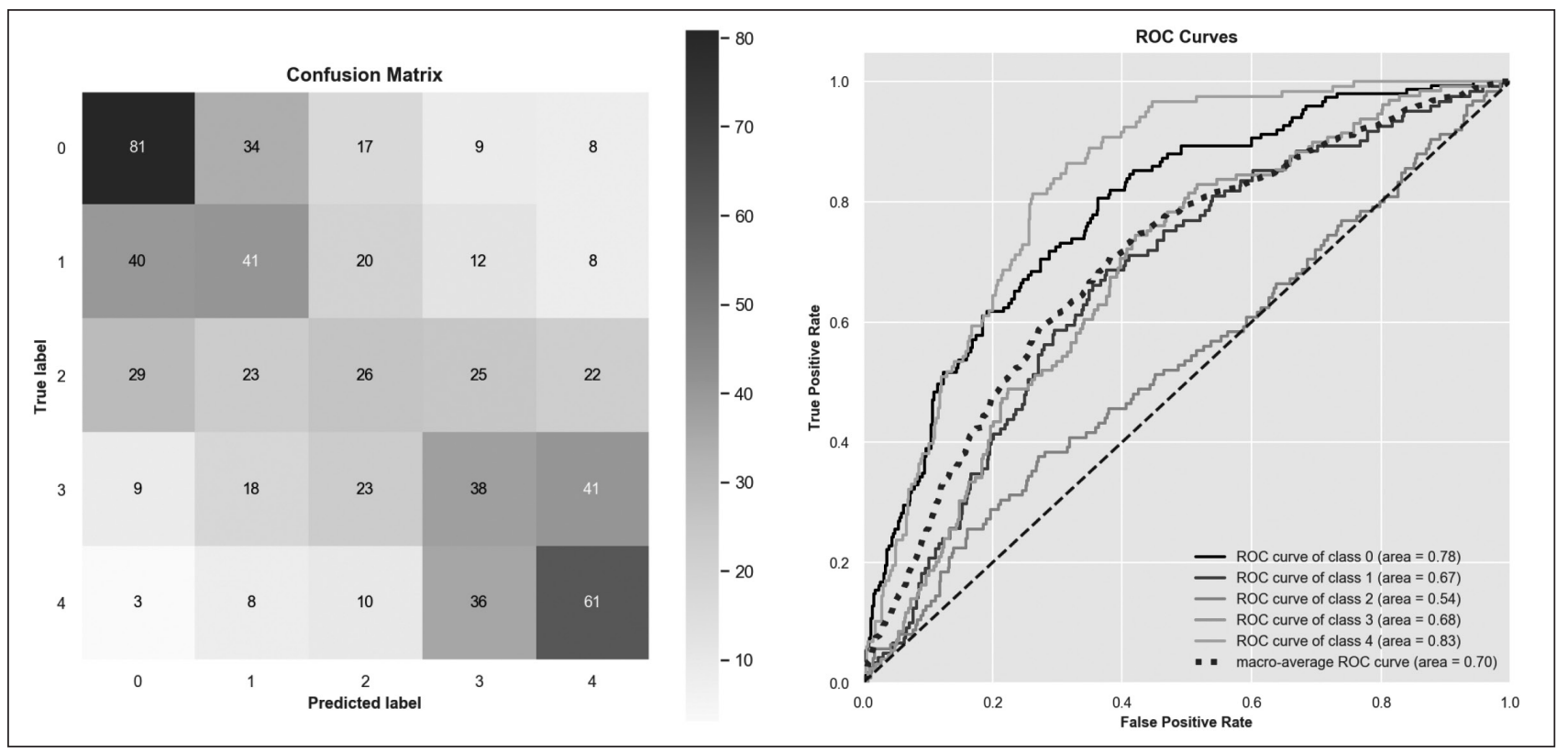

POD-1.5. Fig. 2. Out-of-sample ROC curve for quintile stratification $(A U C=0.70)$ and corresponding confusion matrix.

and Kidney Institute, Cleveland Clinic, Cleveland, $\mathrm{OH}$, United States; ${ }^{9}$ Urology, University of North Carolina School of Medicine, Chapel Hill, NC, United States; ${ }^{10}$ Urology, Palmetto Health USC Medical Group, Columbia, SC, United States; ${ }^{11}$ Urology, University of California Irvine School of Medicine, Orange, CA, United States; ${ }^{12}$ Urology, University of California San Francisco School of Medicine, San Francisco, CA, United States: ${ }^{13}$ Urology, Dartmouth Hitchcock Medical Center, Lebanon, $\mathrm{NH}$, United States; ${ }^{14}$ Urology, University of Florida College of Medicine, Gainesville, FL, United States; ${ }^{5}$ Urology, McGill University Health Centre, Montreal, QC, Canada; ${ }^{16}$ Urology, University of California Davis School of Medicine, Sacramento, CA, United States; ${ }^{17}$ Urology, University of Washington, Seattle, WA, United States; ${ }^{18}$ Urology, Université de Montréal, Montreal, QC, Canada

Introduction: The Wisconsin Stone Quality of Life (WISQOL) questionnaire, a quality of life (QOL) measurement tool designed specifically for kidney stone patients, was recently validated. Using the WISQOL score as the gold standard of the model, we built the WISQOL Machine Learning Algorithm (WISQOL-MLA) to predict patients' QOL based on demographic, symptomatic, and clinical data collected for the validation of the WISQOL. Methods: Using gradient boosting and deep learning models implemented in Python, QOL scores for all 3206 patients were predicted. We also stratified QOL scores by quintiles. The dataset was split using a standard 70/10/20\% training/validation/testing ratio. Per usual machine learning practice, categorical variables were numerically discretized. Variables were then standardized to mean of 0 and standard deviation of 1 to aid model convergence. Regression performance was evaluated using Pearson's correlation (r). Classification was evaluated with area under the ROC curve (AUC). Results: Gradient boosting obtained a test correlation of 0.622 (Fig. 1). Deep learning obtained a correlation of 0.592 . Multivariate regression only achieved a correlation of 0.4375 . Quintile stratification on all WISQOL patients obtained an average test AUC of 0.70 for the five classes. The model performed best in distinguishing between lowest $(0.79)$ and highest quintile (0.83) (Fig. 2). Feature importance analysis showed that the model correctly weights in symptomatic status, body mass index, and age, as well as other medical and demographic features to estimate QOL.

Conclusions: Harnessing the power of the WISQOL questionnaire, WISQOL-MLA can accurately predict a stone patient's QOL from readily available clinical information and outperforms linear models. Future endeavors include scaling the tool as an aid to urologists that don't have the resources to collect precise QOL scores via questionnaire.

\section{POD-1.6}

The effect of a bacterial urinary tract infection isolate and antibiotics on calcium urolithiasis: A novel role for zinc? Jennifer Bjazevic ${ }^{1}$, Kaitlin Al${ }^{2}$, Hassan Razvi', Jeremy Burton ${ }^{2}$

${ }^{1}$ Surgery, Western University, London, ON, Canada; ${ }^{2}$ Microbiology \& Immunology, Western University, London, ON, Canada

Support: Canadian Urological Association Scholarship Foundation Introduction: The formation of calcium-based stone disease may be impacted by both urinary bacteria and antibiotics. Zinc (Zn), which is involved as an early nidus for the mineralization process of urinary stones and is known to be involved in both immune system function and bacterial pathogenesis, may play an integral role in this process. Our study aims to further clarify the role of urinary bacteria and antibiotics on the pathogenesis of urolithiasis, potentially through modulating Zn transporters as part of the immune response in a Drosophila melanogaster (DM) urolithiasis model.

Methods: Wild-type DM were reared under standard conditions at $25^{\circ} \mathrm{C}$, $40 \%$ humidity and a 12-12-hour light/dark cycle with either a standard or lithogenic diet supplemented with $0.1 \%$ sodium oxalate. DM flies were either treated with a combination of a non-urease-producing strain $\mathrm{E}$. coli (UTI89), ciprofloxacin $(0.2 \mu \mathrm{g} / \mathrm{mL})$, or TMP-SMX $(30 / 10 \mu \mathrm{g} / \mathrm{mL})$ for seven or 14 days. Stone burden was assessed through measurement of pixel intensity of $\mathrm{CaOx}$ crystals in dissected Malpighian tubules. DM flies were anesthetized with $\mathrm{CO}_{2}$ and homogenized; total RNA was isolated and converted to CDNA; quantitative PCR was undertaken for each DM Zn transporter gene (CG3994, CG17723, CG11163), using ${ }^{\circledR}-$ tubulin as an internal control.

Results: Treatment with UTI89 ( $p=0.005)$, ciprofloxacin $(p<0.001)$, and TMP-SMX ( $p=0.003$ ) increased CaOx stone formation in DM flies at day 7. Preliminary results demonstrate a trend towards increased expression of Zn transporter gene ZnT41F (CG1163) with UTI89 treatment. Co-treatment of the DM flies with both E. coli UTI89 and either antibiotic reduced the expression of ZnT41F to baseline levels.

Conclusions: These findings suggest that non-urease-producing E. coli, and the antibiotics ciprofloxacin and TMP-SMX impact CaOx stone formation. Zn may be involved in this process through the modulation of its transport proteins. Further investigation is required to confirm these results and delineate the exact mechanism involved. 


\section{Podium Session 2: Oncology - Prostate}

\section{POD-2.1}

Transrectal vs. transperineal prostate biopsy under local anesthesia: Prospective comparative analysis of cancer detection, safety, and tolerability at a single center

Pui Long Wilson Hung ${ }^{1}$, Stacia Chun ${ }^{1}$, Chiu Fung Tsang ${ }^{1}$, Brian $\mathrm{Ho}^{1}$, Ada $\mathrm{Ng}^{1}$, Hok Leung Tsu', Wayne Lam ${ }^{7}$

${ }^{1}$ Urology, Surgery, Queen Mary Hospital, The University of Hong Kong, HK, Hong Kong

Introduction: Transrectal ultrasound-guided prostate biopsy under local anesthesia (LA-TRUSB) has been the standard for prostate cancer detection for decades. Transperineal prostate biopsy under local anesthesia (LA-TPB) has emerged as an alternative office-based procedure to seek improvement in cancer detection and safety. This study aimed to compare the cancer detection rates, complications, and patient tolerability using patientreported outcome measure (PROM) questionnaires.

Methods: The patients included in our study underwent systematic LA-TPB using a transperineal access device or standard LA-TRUSB from October 2018 to November 2019. Inclusion criteria were: serum prostatespecific antigen (PSA) $4-20 \mathrm{ng} / \mathrm{mL}$, age 55-80 years, and biopsy-naive. Exclusion criteria were: suspected tumor $\geq \mathrm{cT} 3$ on digital rectal examination or medically unfit for treatment. Cancer detection and complications were recorded. A validated PROM was used to compare overall patient tolerability and safety.

Results: A total of 146 of 215 patients who underwent biopsy were eligible. Median age was 68 years. Mean presenting PSA was $7.3 \mathrm{ng} / \mathrm{mL}$. One hundred and four $(48.4 \%)$ patients underwent LA-TRUSB and $111(51.6 \%)$ had LA-TPB. More prostate cancers $(p=0.209)$ and clinically significant cancer $(p=0.534)$ were detected with LA-TPB; $46 \%$ of LA-TPB patients had cancer detected in anterior prostate. None of the LA-TPB patients was complicated with sepsis (vs. $5.8 \%$ in TRUSB; $p=0.012$ ) or urinary tract infection with bacteriuria (vs. $10.6 \%$ in TRUSB; $p<0.001$ ). Mean International Index of Erectile Function (IIEF-5) change immediately after biopsy was 2.60 in LA-TRUSB and 6.81 in LA-TPB ( $p=0.004$ ). There was no statistically significant difference in pain scores, urinary retention rate, IPSS change, sexual satisfaction, or bowel and psychological functions.

Conclusions: Office-based LA-TPB was a well-tolerated procedure compared to standard LA-TRUSB, with comparable cancer detection rates and the ability to prevent post-biopsy sepsis. However, erectile function could be affected in the initial period following LA-TPB, and patients should be counselled carefully before the procedure.

\section{POD-2.2}

Prospective, phase $2 / 3$, multicenter study of prostate-specific membrane antigen-targeted positron emission tomography imaging in prostate cancer patients (OSPREY): Detection rates of regional and distant metastases at initial staging by 18F-DCFPyL $\mathrm{PET} / \mathrm{CT}$

Frédéric Pouliot ${ }^{1}$, Peter Carroll2, Stephan Probst ${ }^{3}$, Kenneth J. Pienta ${ }^{4}$, Stephen P. Rowe ${ }^{5}$, Lawrence Saperstein ${ }^{6}$, Barry Siegel $^{7}$, Akash Patnaik ${ }^{8}$, Mark A. Preston ${ }^{9}$, Ajjai Shivaram Alva ${ }^{10}$, Michael A. Gorin ${ }^{11}$, Michael J. Morris ${ }^{12}$

${ }^{1}$ Surgery, CHU de Québec-Université Laval, Quebec City, QC, Canada; ${ }^{2}$ Urology, University of California San Francisco, San Francisco, CA, United States; ${ }^{3}$ Medicine, Jewish General Hospital, Montreal, QC, Canada; ${ }^{4}$ Urology, Johns Hopkins University School of Medicine, Baltimore, MD, United States; ${ }^{5}$ Radiology, Johns Hopkins University School of Medicine, Baltimore, MD, United States; ${ }^{6}$ Medicine, Yale School of Medicine, New
Haven, CT, United States; ${ }^{7}$ Medicine, Washington University, Saint Louis, MO, United States; ${ }^{8}$ Medicine, Beth Israel Deaconess Medical Center/ Dana-Farber Cancer Institute, Boston, MA, United States; ${ }^{9}$ Medicine, Brigham and Women's Hospital/Dana-Farber Cancer Institute, Boston, MA, United States; ${ }^{10}$ Medicine, University of Michigan, Ann Harbor, MI, United States; ${ }^{11}$ Urology, Johns Hopkins University School of Medicine, Baltimore, MD, United States; ${ }^{12}$ Medicine, Memorial Sloan Kettering Cancer Center, New York, NY, United States

Introduction: Current imaging modalities are suboptimal for the initial staging of men at risk of harboring occult metastatic prostate cancer (PCa). Prostate-specific membrane antigen (PSMA)-based imaging is considered highly promising for PCa detection. ${ }^{18} \mathrm{~F}-\mathrm{DCFPyL}$ is a novel PSMA-targeted radiopharmaceutical for positron emission tomography (PET) that may be useful in staging of patients with high-risk PCa. The diagnostic performance of ${ }^{18} \mathrm{~F}$-DCFPyL regarding regional and distant metastases has been previously reported. Here, we report on detection rates and the resulting impact ${ }^{18} \mathrm{~F}$-DCFPyL may have on staging of patients with high-risk PCa. Methods: ${ }^{18} \mathrm{~F}$-DCFPyL PET/CT was evaluated in 252 men with high-risk PCa who were planned for radical prostatectomy with lymphadenectomy (RP-PLND). Based on TNM staging, ${ }^{18} \mathrm{~F}$-DCFPyL PET/CT detection rates, including lesion counts, were systematically analyzed: prostatic (T), pelvic LN (N), extra-pelvic LN (M1a), bone (M1b), and other visceral organs/soft tissue (M1c). Three central, blinded, and independent readers evaluated the ${ }^{18} \mathrm{~F}$-DCFPyL scans.

Results: At study entry, $97 \%$ and $99 \%$ of all evaluable patients had no known nodal or metastatic disease, respectively, based on standard crosssectional imaging. Of these, ${ }^{18} \mathrm{~F}-\mathrm{DCFPyL}$ PET/CT staged $37(14.7 \%)$ patients with $\mathrm{N} 1$ disease and $27(10.7 \%)$ patients with $\mathrm{M} 1$ disease (one [0.4\%] M1a, 23 [9.1\%] M1b, and three [1.2\%] M1c). In total, 56 (22\%) patients were upstaged to $\mathrm{N} 1$ or $\mathrm{M} 1$ disease by ${ }^{18} \mathrm{~F}$-DCFPyL. The positive predictive value of ${ }^{18} \mathrm{~F}$-DCFPyL based on histopathological validation for pelvic LNs was $86.7 \%$ (95\% confidence interval [Cl] 70-95). Only one patient in cohort A underwent a biopsy of a ${ }^{18} \mathrm{~F}$-DCFPyL detected spinal $\mathrm{M} 1$ finding and was positively confirmed by histopathology.

Conclusions: A total of $22 \%$ of high-risk PCa patients planned for RP-PLND had regional or distant metastatic lesions detected on ${ }^{18} \mathrm{~F}-\mathrm{DCFP} Y \mathrm{~L}$ PET/ $\mathrm{CT}$. These results suggest the potential utility of ${ }^{18} \mathrm{~F}$-DCFPyL PET/CT in the staging of men with newly diagnosed high risk PCa to develop optimized treatment paradigms. NCT02981368.

\section{POD-2.3}

A prospective provincial prostate specific membrane antigenpositron emission tomography (PET) registry for recurrent prostate cancer (PREP): Preliminary results of lesion detection rates and PET-directed changes in clinical management

L.K. Joseph Chin ${ }^{1,8}$, Ur Metser ${ }^{2}$, Antonio Finelli ${ }^{3}$, Laurence H. Klotz ${ }^{3}$, Anil Kapoor $^{4}$, Katherine Zukotynski ${ }^{5}$, Luke T. Lavallée ${ }^{6}$, Marlon Hagerty ${ }^{7}$, Wei Liu $^{8}$, Glenn Bauman ${ }^{8}$

${ }^{1}$ Division of Urology, Department of Surgery, Western University, London, ON, Canada; ${ }^{2}$ Department of Medical Imaging, University of Toronto, Toronto, ON, Canada; ${ }^{3}$ Division of Urology, Department of Surgery, University of Toronto, Toronto, ON, Canada; ${ }^{4}$ Division of Urology, Department of Surgery, McMaster University, Hamilton, ON, Canada; ${ }^{5}$ Department of Medicine and Radiology, McMaster University, Hamilton, ON, Canada; ${ }^{6}$ Division of Urology, Department of Surgery, University of Ottawa, Ottawa, ON, Canada; ${ }^{7}$ Radiation Oncology, Thunder Bay Regional Health Sciences Centre, Thunder Bay, ON, 
Canada; ${ }^{8}$ Department of Oncology, Western University, London, ON, Canada.

Support: Cancer Care Ontario and the Ontario Ministry of Health and Long-Term Care

Introduction: The prostate specific membrane antigen-positron emission tomography (PSMA-PET) Registry for Recurrent Prostate Cancer (PREP) was launched in Ontario in 2018 and is intended to record real-world outcomes with the PSMA tracer ${ }^{18} \mathrm{~F}$-DCFPyL used for restaging men with suspected recurrent prostate cancer after primary treatment.

Methods: PREP was organized through Cancer Care Ontario as a registry study (NCT03718260), with six participating sites throughout Ontario and a target accrual of 1500 men. Eligibility included biochemical failure with no or limited ( $\leq 4$ sites) disease on conventional imaging ( $\mathrm{Cl}$ : computed tomography [CT] of the abdomen and pelvis and bone scintography) and biochemical failure after primary prostatectomy (plus or minus adjuvant or salvage radiotherapy) or primary radiotherapy. Following PSMA $\mathrm{PET} / \mathrm{CT}$, frequency of disease detection, anatomic sites of disease, and changes in management compared to $\mathrm{Cl}$ were collected using standardized questionnaires.

Results: From December 1, 2018 to October 31, 2019, a total of 410 men were enrolled on PREP and had PSMA PET/CT after CI. Overall, 261/410 (64\%) had PET-detected disease. PET detection rates among men with negative conventional imaging was 174/310 (56\%). Among men with lesions on $\mathrm{Cl}$, new lesions were seen on PET in 63/100 (63\%). Among men with either positive or negative $\mathrm{Cl}$, PSMA-PET detection rates stratified by prostate-specific antigen (PSA) on study entry were 54/139 (39\%) PSA <0.5; 49/78 (63\%) PSA 0.5-1.0; and 157/191 (82\%) PSA > 1.0. On PSMA-PET, 144/410 (35\%) had recurrent disease localized to the pelvis. Changes in management as a consequence of the PSMA$\mathrm{PET} / \mathrm{CT}$ findings were noted in $66 \%(226 / 341)$ of men with completed post-PET/CT questionnaires.

Conclusions: Access to the PSMA-PET imaging in Ontario has been facilitated by the introduction of the PREP registry study. A high detection rate and frequent impact on management suggests ${ }^{18} \mathrm{~F}$-DCFPy $\mathrm{LET}$ adds value to $\mathrm{Cl}$ for men with suspected recurrent prostate cancer.

\section{POD-2.4}

Salvage lymph node dissection for prostate-specific membrane antigen (PSMA) positron emission tomography-identified oligometastatic disease

Adam Bobrowski ${ }^{1}$, Ur Metser ${ }^{2}$, Antonio Finelli ${ }^{3}$, Neil E. Fleshner ${ }^{3}$, Alejandro Berlin ${ }^{4}$, Nathan Perlis ${ }^{3}$, Girish S. Kulkarni ${ }^{3}$, Peter Chung ${ }^{4}$, Kopika Kuhathaas ${ }^{3}$, Robert J. Hamilton ${ }^{3}$

${ }^{1}$ Faculty of Medicine, University of Toronto, Toronto, ON, Canada; ${ }^{2}$ Department of Diagnostic Radiology, Princess Margaret Hospital, University Health Network, University of Toronto, Toronto, ON, Canada; ${ }^{3}$ Departments of Surgery and Surgical Oncology (Urology), Princess Margaret Hospital, University Health Network, University of Toronto, Toronto, ON, Canada; ${ }^{4}$ Department of Radiation Oncology, Princess Margaret Hospital, University Health Network, University of Toronto, Toronto, ON, Canada

Introduction: The availability of prostate-specific membrane antigenpositron emission tomography (PSMA-PET) imaging, particularly in the setting of rising prostate-specific antigen (PSA) after definitive treatment, has led to oligometastatic disease being increasingly identified. However, despite the enthusiasm for identifying and treating oligometastatic disease, it has been relatively understudied. We sought to review our salvage lymphadenectomy (sLND) experience in the PSMA-PET era at Princess Margaret Hospital.

Methods: We performed a retrospective review of patients undergoing sLND after curative-intent primary therapy with rising PSA who had undergone a ${ }^{18} \mathrm{~F}$-DCFPyL (PSMA)-PET/computed tomography (CT) identifying oligometastatic disease (defined as $\leq 5$ PSMA avid lesions) between January 2009 and July 2019. The primary endpoint was complete response, defined as achieving a PSA $<0.2$ without the use of concomitant androgen deprivation therapy (ADT).

Results: Twenty-one patients were included. Eighteen (86\%) had prior radical prostatectomy, while three $(14 \%)$ had received primary radiation therapy (RT). At the time of salvage surgery, 14 patients $(67 \%)$ underwent pelvic sLND and seven patients (33\%) underwent retroperitoneal sLND. Median PSA at sLND was 1.85. Median node yield was 10, with a median of one positive node on pathology. Eight patients (38\%) achieved PSA $<0.2$, with six $(29 \%)$ remaining with PSA $<0.2$ at a median followup of 113 days. Six (29\%) had an initial PSA decline, but nadired above 0.2 , and in six $(29 \%)$, the PSA rose immediately after sLND. Five patients relapsed on repeat PSMA-PET. Overall, ADT was started in six patients (29\%) at a median of 257 days post-sLND. Four patients had complications (two with Clavien-Dindo $\leq 2$ and two with Clavien-Dindo 3b).

Conclusions: While there is a rationale to treat oligometastatic prostate cancer with SLND, it is important for clinicians to provide realistic expectations. It appears only approximately one-third of patients have a meaningful PSA response. Long-term benefits of treating oligometastatic disease remain unknown.

\section{POD-2.5}

Detectable prostate-specific antigen value between 0.01 and $0.1 \mathrm{ng} / \mathrm{ml}$ following robotic-assisted radical prostatectomy: Does it correlate with future biochemical recurrence?

Russell Schwartz', Ahmed S. Zakaria', Amr Hodhod', Hanna Shahine', Felix Couture ${ }^{3}$, David-Dan Nguyen ${ }^{2}$, Côme Tholomier ${ }^{2}$, Cristina Negrean ${ }^{1}$, Kyle Law², Pierre Karakiewicz', Assaad El-Hakim', Kevin C. Zorn

'Urology, Centre hospitalier de l'Université de Montréal, Montreal, QC, Canada; ${ }^{2}$ Urology, McGill University, Montreal, QC, Canada; ${ }^{3}$ Urology, Centre hospitalier de I'Université Sherbrooke, Sherbrooke, QC, Canada Introduction: Ultrasensitive prostate-specific antigen (PSA) assay is widely used in the early detection of biochemical recurrence (BCR) after roboticassisted radical prostatectomy (RARP), setting a value of $<0.01 \mathrm{ng} / \mathrm{ml}$ rather than the regular value of $<0.1 \mathrm{ng} / \mathrm{ml}$. Yet, the impact on future $B C R$ of having a nadir and persistently detectable followup PSA between 0.01 and $0.1 \mathrm{ng} / \mathrm{ml}$, is still unclear. Our aim was to characterize PSA changes over time in this setting and to assess for further progression.

Methods: We conducted a retrospective review of a prospectively maintained cohort of 1359 men who underwent RARP in three high-volume centers between 2006 and 2019. Patients were followed with PSA at one, three, six, nine, $12,18,24,30$, and 36 months, and then annually thereafter. We included patients with PSA nadir value between 0.01 and $0.1 \mathrm{ng} / \mathrm{ml}$ within six months of surgery and with at least two followup measurements within the same range. Within our cohort, we divided patients based on their BCR status and analyzed oncological outcomes and PSA evolution. Multivariable Cox (MVC) hazard models were used to analyze variables predicting BCR-free survival (BCR-FS).

Results: A total of $167(12.3 \%)$ subjects were identified for analyses, with a mean followup time of $60.2 \pm 31.4$ months (Table 1). In our cohort, fiveyear BCR-FS rate was $91.6 \%$; 32 (19.1\%) patients had BCR, with a mean time to BCR of $43.7 \pm 24.3$ months (Table 2). Subsequently, $26(15.5 \%)$ patients received salvage radiotherapy. During followup, BCR-free patients had stable mean PSA values $\leq 0.03 \mathrm{ng} / \mathrm{ml}$, while patients who developed BCR showed a slowly rising trend over time, with a significant difference between groups starting at nine months $(p<0.023)$, even when values $\geq$ $0.10 \mathrm{ng} / \mathrm{ml}$ were excluded (Fig.1). In the MVC model, rising PSA (continuous) starting at nine months was a significant predictor of BCR (hazard

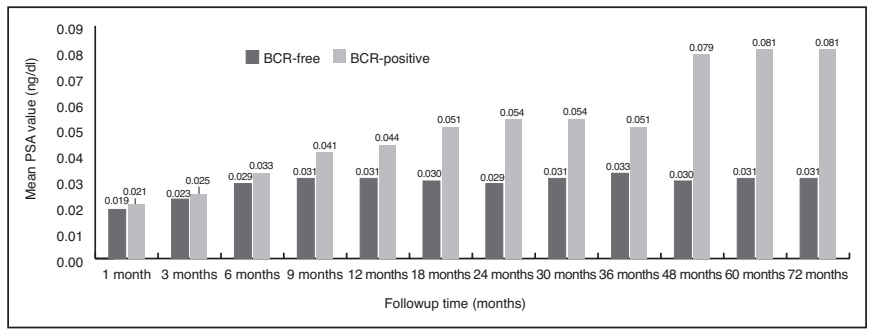

POD-2.5. Fig. 1. Average PSA value per month of followup for the two study groups (excluding values $\geq 0.10 \mathrm{ng} / \mathrm{ml})(\mathrm{p}<0.001)$. 
POD-2.5. Table 1. Whole cohort statistics and comparison of preoperative, intraoperative, and postoperative variables between BCR negative and BCR positive groups using Pearson's chi-squared test or independent-samples Mann-Whitney $\mathbf{U}$ hypothesis testing

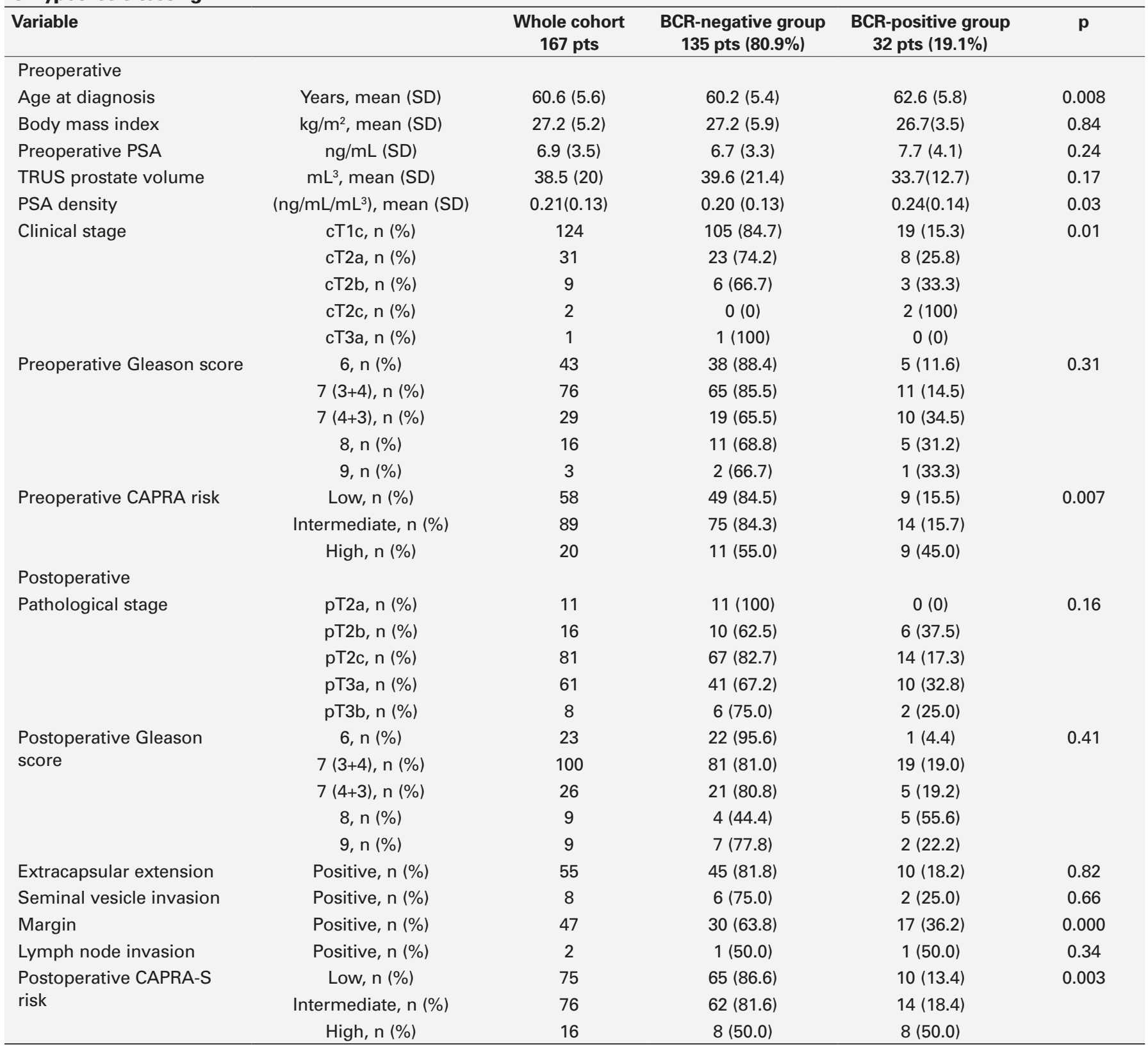

ratio [HR] 2.7; 95\% confidence interval [Cl] 1.6-3.82; $\mathrm{p}=0.013$ ] (Table 3). Conclusions: Our study demonstrated that a considerable number of men have detectable PSA values ranging between 0.01 and $0.1 \mathrm{ng} / \mathrm{ml}$ postRARP. They can still be followed regularly to avoid patients' anxiety and unnecessary radiotherapy. Close followup is still required. 
POD-2.5. Table 2. Cox proportional hazard regression models approximating univariate and multivariate hazard ratios (HR) for the ability of preoperative, perioperative, and postoperative variables to predict biochemical recurrence

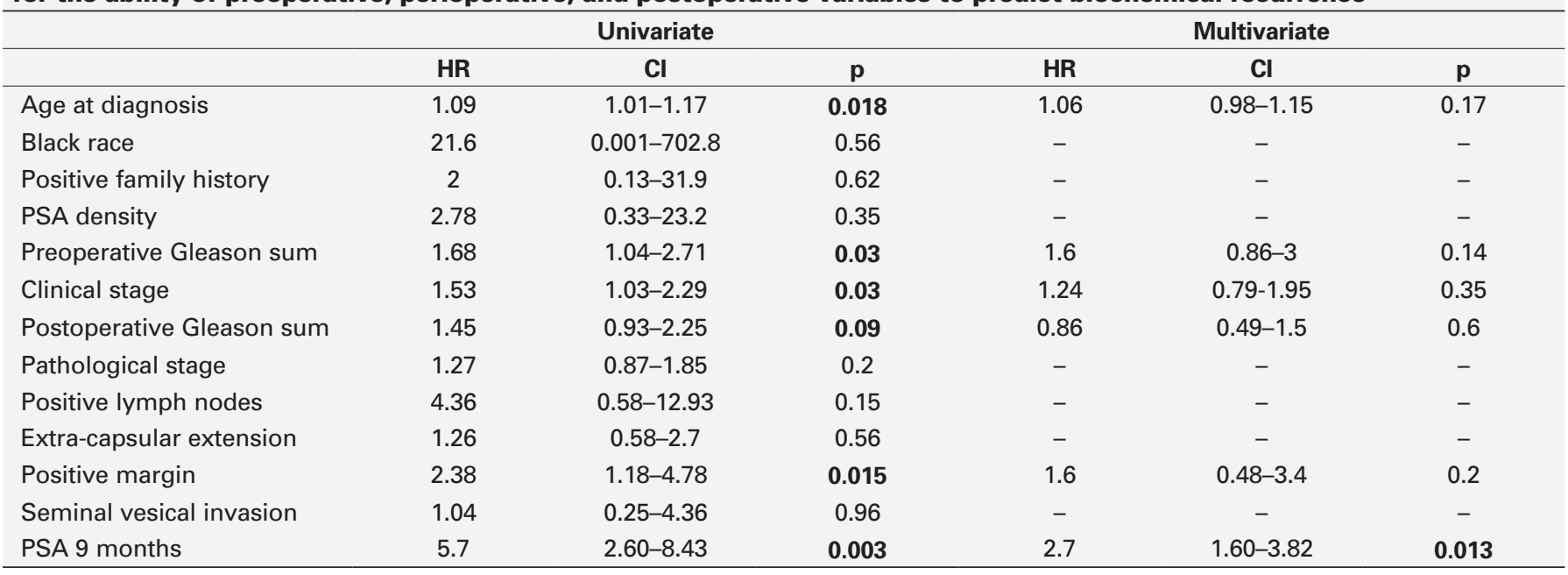

POD-2.5. Table 3. ROC curve data showing PSA cutoff points $(0.01-0.1 \mathrm{ng} / \mathrm{mL})$ at followup times of 9, 12, 18, $24 \mathrm{months}$ postoperatively

\begin{tabular}{|c|c|c|c|c|c|c|c|}
\hline \multirow[t]{2}{*}{ PSA at followup time } & \multirow{2}{*}{$\begin{array}{l}\text { PSA cutoff points } \\
\text { (ng/mL) }\end{array}$} & \multirow[t]{2}{*}{ Sensitivity (\%) } & \multirow{2}{*}{$\begin{array}{c}\text { Specificity } \\
\text { (\%) }\end{array}$} & \multirow[t]{2}{*}{ AOC } & \multirow{2}{*}{$\begin{array}{l}\text { Asymptotic } \\
\text { significance }\end{array}$} & \multicolumn{2}{|c|}{ Asymptomatic $95 \% \mathrm{Cl}$} \\
\hline & & & & & & Lower bound & Upper bound \\
\hline \multirow[t]{7}{*}{9 months } & 0.00 & 93.8 & 6.2 & 0.653 & $\mathrm{p}=0.08$ & 0.540 & 0.767 \\
\hline & 0.01 & 90.6 & 12.5 & & & & \\
\hline & 0.03 & 53.1 & 69.6 & & & & \\
\hline & 0.04 & 40.6 & 86.6 & & & & \\
\hline & 0.05 & 28.1 & 90.2 & & & & \\
\hline & 0.08 & 3.1 & 95.5 & & & & \\
\hline & 0.09 & 3.1 & 97.3 & & & & \\
\hline \multirow[t]{6}{*}{12 months } & 0.00 & 93.8 & 8.9 & 0.684 & $\mathrm{p}=0.001$ & 0.573 & 0.796 \\
\hline & 0.01 & 87.5 & 17.0 & & & & \\
\hline & 0.02 & 78.1 & 51.8 & & & & \\
\hline & 0.03 & 62.5 & 66.1 & & & & \\
\hline & 0.08 & 6.3 & 98.2 & & & & \\
\hline & 0.09 & 3.1 & 99.1 & & & & \\
\hline \multirow[t]{10}{*}{18 months } & 0.00 & 96.9 & 10.7 & 0.737 & $p<0.001$ & 0.634 & 0.839 \\
\hline & 0.01 & 93.8 & 19.6 & & & & \\
\hline & 0.02 & 84.4 & 47.3 & & & & \\
\hline & 0.03 & 65.6 & 64.3 & & & & \\
\hline & 0.04 & 56.3 & 75.0 & & & & \\
\hline & 0.05 & 50.0 & 84.8 & & & & \\
\hline & 0.06 & 46.9 & 91.1 & & & & \\
\hline & 0.07 & 37.5 & 93.7 & & & & \\
\hline & 0.08 & 31.3 & 94.6 & & & & \\
\hline & 0.09 & 25.0 & 97.3 & & & & \\
\hline
\end{tabular}




\begin{tabular}{|c|c|c|c|c|c|c|c|}
\hline & $\begin{array}{l}\text { PSA cutoff points } \\
\text { (ng/mL) }\end{array}$ & Sensitivity (\%) & $\begin{array}{c}\text { Specificity } \\
(\%)\end{array}$ & AOC & $\begin{array}{l}\text { Asymptotic } \\
\text { significance }\end{array}$ & Lower bound & Upper bound \\
\hline \multirow{7}{*}{24 months } & 0.01 & 90.6 & 21.9 & & & & \\
\hline & 0.02 & 84.4 & 52.4 & & & & \\
\hline & 0.03 & 75.0 & 65.7 & & & & \\
\hline & 0.06 & 62.5 & 83.8 & & & & \\
\hline & 0.07 & 59.4 & 90.5 & & & & \\
\hline & 0.08 & 40.6 & 93.3 & & & & \\
\hline & 0.09 & 37.5 & 93.3 & & & & \\
\hline
\end{tabular}

\section{POD-2.6}

Adjuvant vs. salvage radiotherapy: Meta-analysis of the effect of comparator salvage approach on study conclusions

Christopher I.D. Wallis ${ }^{1}$, Zachary W. Klaassen ${ }^{2}$, Amy Luckenbaugh ${ }^{1}$, Aaron Laviana', Bimal Bhindi ${ }^{3}$

${ }^{1}$ Urologic Surgery, Vanderbilt University Medical Center, Nashville, TN, UnitedStates; ${ }^{2}$ Division of Urology, Medical College of Georgia at Augusta University, Augusta, GA, United States; ${ }^{3}$ Division of Urology, University of Calgary, Calgary, $\mathrm{AB}$, Canada

Introduction: Early salvage radiotherapy following radical prostatectomy is associated with improved biochemical control when compared to late treatment. To date, guidelines advocating adjuvant radiotherapy have relied on randomized controlled trials (RCTs) using late salvage therapy, while many clinicians have advocated a salvage approach based on observational data using early salvage therapy. Recently, RCTs comparing adjuvant and early salvage radiotherapy have been presented. We quantitatively explored the effect of changing salvage strategies on the apparent efficacy of adjuvant radiotherapy.

Methods: We performed a systematic review of RCTs assessing the role of adjuvant radiotherapy in men following radical prostatectomy for prostate cancer. On an a priori basis, we classified trials as using an early or late salvage strategy as a comparator to adjuvant radiotherapy. We performed stratified meta-analysis based on early or late salvage strategy and assessed, using the chi-squared test, whether this quantitatively affected study conclusions.

Results: We identified seven eligible trials. Among four studies (published as full manuscripts) using a late salvage strategy, use of adjuvant radiotherapy conferred a significant improvement in biochemical-recurrence free survival (hazard ratio $[\mathrm{HR}] 0.47 ; 95 \%$ confidence interval $[\mathrm{Cl}]$ $0.41-0.54)$ with no significant heterogeneity between studies $\left(I^{2}=0 \%\right)$. In contrast, among three studies using an early salvage strategy (presented only in abstract form to date), biochemical-recurrence-free survival was not improved with the use of adjuvant radiotherapy ( $\mathrm{HR} 1.12 ; 95 \% \mathrm{Cl}$ $0.88-1.42)$, again with no significant heterogeneity $\left(I^{2}=0 \%\right)$. There was a quantitatively significant difference in these estimates of the benefit of adjuvant radiotherapy $\left(p<0.00001, I^{2}=97.4 \%\right)$, as well as a qualitative difference in the synthesized results of these studies.

Conclusions: The apparent efficacy of adjuvant radiotherapy in prostate cancer depends on the nature of the salvage therapy administered to patients initially undergoing observation. Consumers of the urological literature should consider the validity of study interventions, in addition to study design and methodology, when appraising a study and applying the results to patient care. 


\section{CUA ABSTRACTS}

\section{Podium Session 3: Urinary Incontinence, Voiding Dysfunction}

\section{POD-3.1}

Are urodynamic studies necessary for AdVance sling placement for post-prostatectomy stress urinary incontinence?

Lennifer A. Locke ${ }^{1}, Y u$ Zheng ${ }^{2}$, Goran Rac ${ }^{2}$, Nicholas Major ${ }^{2}$, Jennifer Rolef ${ }^{2}$ Lauren Rittenberg², Arthur Mourtzinos ${ }^{3}$, Matthew Moynihan², O. Lenaine Westney ${ }^{4}$, Mike Metro ${ }^{5}$, Sender Herschorn' ${ }^{1}$ Sarah R. Ferrara ${ }^{1}$, Caitlin Lim², Brittani Barrett-Harlow ${ }^{2}$, Ross Rames ${ }^{2}$, Lindsey Cox ${ }^{2}$, Eric Rovner ${ }^{2}$

${ }^{1}$ Urology, Sunnybrook Health Sciences Centre, University of Toronto, Toronto, ON, Canada; ${ }^{2}$ Urology, Medical University of South Carolina, Charleston, SC, United States; ${ }^{3}$ Urology, Lahey Health, Burlington, MA, United States; ${ }^{4}$ Urology, MD Anderson, Houston, TX, United States; ${ }^{5}$ Urology, Temple Health, Philadelphia, PA, United States

Introduction: Urinary retention (UR) following AdVance sling placement for post-prostatectomy stress urinary incontinence (SUI) is a known complication. Urodynamic studies (UDS) are often performed prior to evaluate bladder function, however, its ability to predict post-operative urinary UR is unknown. Therefore, our study sought to identify risk factors for UR following AdVance sling placement using preoperative UDS.

Methods: Following IRB approval, a retrospective review of medical records of patients from multiple institutions who underwent an AdVance sling for post-prostatectomy SUI from 2007-2019 was performed. Acute postoperative UR was defined as the complete inability to void or elevated post-void residual (PVR) leading to Foley catheter placement or intermittent catheterization teaching.

Results: A total of 389 patients were included in this study, $52(13.4 \%)$ had acute UR. By the second postop visit, 75\% had resolution of UR, and at the last recorded postop visit, $1.5 \%$ had persistent UR (median followup 544 days). On UDS, average Pdet $\left(27.3 \mathrm{cmH}_{2} 0\right.$ vs. $\left.27 \mathrm{cmH}_{2} 0, \mathrm{p}=0.95\right)$ maximum urinary flow rate (Qmax) $(16 \mathrm{ml} / \mathrm{s}$ vs $16.2 \mathrm{ml} / \mathrm{s}, \mathrm{p}=0.91)$ and PVR $(21.7 \mathrm{ml}$ vs. $27.5 \mathrm{ml}, \mathrm{p}=0.64)$ were similar for patients with or without UR following AdVance sling. Preoperative pad test (odds ratio [OR] 1.00; confidence interval $[\mathrm{Cl}] 0.99-1.00 ; \mathrm{p}=0.7)$, prior radiation $(\mathrm{OR} 2.22 ; \mathrm{Cl}$ $0.65-7.71 ; p=0.20)$, bladder neck contracture (OR 2.52; Cl 0.75-0.842; $\mathrm{p}=0.13$ ), or diabetes (OR $0.87 ; \mathrm{Cl} 0.37-2.05 ; \mathrm{p}=0.74)$ were not significantly associated with risk of UR. Thirty-two patients $(8.2 \%)$ had persistent stress urinary incontinence. Pdet, Qmax, and PVR from UDS were similar in patients with persistent SUI compared to those whose incontinence resolved after AdVance sling placement.

Conclusions: The rate of acute postoperative UR after AdVance sling placement for post-prostatectomy SUI was $13.4 \%$ but the long-term rate of UR was quite low at $1.5 \%$. Pdet, Qmax, and PVR obtained from UDS were not predictive of UR or persistent SUI after AdVance sling placement.

\section{POD-3.2}

Multicentered assessment of clinical outcomes and factors associated with failure of the adjustable transobturator male system (ATOMS)

Elaine Redmond ${ }^{1}$, Geneviève Nadeau ${ }^{2}$, Le Mai Tu ${ }^{3}$, R. Christopher Doiron ${ }^{4}$, Stephen S. Steele ${ }^{4}$, Dean S. Elterman ${ }^{5}$, Jennifer A. Locke ${ }^{5}$, Sender Herschorn ${ }^{5}$, Conrad Maciejewski ${ }^{6}$, Neil T. Dwyer ${ }^{7}$, Lysanne Campeau $^{8}$, Kevin V. Carlson ${ }^{9}$, Keith F. Rourke ${ }^{l}$

${ }^{1}$ University of Alberta, Edmonton, $\mathrm{AB}$, Canada; ${ }^{2}$ Université Laval, Quebec City, QC, Canada; ${ }^{3}$ University of Sherbrooke, Sherbrooke, QC, Canada; ${ }^{4}$ Queen's University, Kingston, ON, Canada; ${ }^{5}$ University of Toronto, Toronto, ON, Canada; ${ }^{6}$ University of Ottawa, Ottawa, ON,
Canada; 'Dalhousie University, Moncton, NB, Canada; ${ }^{8}$ Université de Montréal, Montreal, QC, Canada; ${ }^{9}$ University of Calgary, Calgary, AB, Canada

Introduction: The adjustable transobturator male system (ATOMS) is a transobturator device with a non-circumferential adjustable hydraulic cushion used to treat sphincteric incontinence after prostate cancer treatment. The aim of this multicenter study was to assess postoperative outcomes from the ATOMS device and to identify factors influencing failure to achieve continence.

Methods: Men who underwent insertion of ATOMS for the treatment postprostatectomy incontinence between September 2015 and August 2019 at nine Canadian centers were reviewed. The primary outcome was continence, defined as requiring $\leq 1$ pad postoperatively for patients requiring $\geq 2$ pads preoperatively and 0 pads for those requiring one pad preoperatively. Secondary outcomes included improvement $(>50 \%$ change in pad use), patient satisfaction, explantation, and 90-day complications. Other patient demographics included age, obesity (body mass index [BMI] $\geq 35$ ), comorbidities, concurrent radiotherapy, prior incontinence surgery, previous urethral stenosis, type of prostatectomy, and pads per day (ppd).

Results: A total of 295 patients with a mean age of 69.9 years were analyzed. Preoperatively, mean pad use was $4.2 \mathrm{ppd}(1-12), 32.5 \%$ of patients reported severe incontinence ( $\geq 5$ pads/day), $34.2 \%$ had concurrent radiotherapy, and $20.0 \%$ had failed previous incontinence surgery. Initial postoperative pad use was 1.4 ppd before cushion adjustment $(p<0.0001)$ and $0.9 \mathrm{ppd}$ following adjustments $(\mathrm{p}<0.0001)$. Most $(71.2 \%, \mathrm{n}=210)$ patients underwent adjustment a mean of $2.1(0-9)$ times for a mean total volume of $12.0 \mathrm{~mL}$. Overall continence rate was $72.9 \%(\mathrm{n}=215)$ at a mean followup of 19.7 months. The majority $(89.2 \%, \mathrm{n}=263)$ of patients experienced $>50 \%$ improvement; $84.4 \%(n=249)$ of patients were satisfied with the results of surgery. A total of $8.1 \%(n=24)$ required device explantation. On multivariate Cox regression analysis, concurrent radiotherapy (hazard ratio [HR] 2.5, $\mathrm{p}<0.0001$ ), diabetes (HR 2.2, $\mathrm{p}=0.006$ ) and increased pre-operative pad usage (HR 1.1, $\mathrm{p}=0.03$ ) were associated with failure to achieve continence, while patient age $(p=0.51)$, obesity $(p=0.08)$, prior urethral stenosis $(p=0.66)$, and prior incontinence surgery $(p=0.17)$ were not. Radiation therapy was also associated with device explantation (HR 3.1, $\mathrm{p}=0.007$ ). Conclusions: In the largest cohort study to date, ATOMS is a safe and efficacious device for the treatment of a broad spectrum of patients with post-prostatectomy incontinence. Our study found a durable response on continued followup, with high rates of continence, improvement, and patient satisfaction. Patients with prior radiation, increased preoperative pad use, and diabetes are less likely to achieve continence.

\section{POD-3.3}

Evaluation of benefits and harms of surgical treatments for postradical prostatectomy urinary incontinence: Systematic review and meta-analysis

Roselyne Choiniere ${ }^{1}$, Philippe D. Violette ${ }^{2,3}$, Melanie Morin ${ }^{4}$, Le Mai Tu' Christine Reed ${ }^{1}$, Philie Camille-Ariane ${ }^{7}$, Benjamin Legault ${ }^{1}$, Marie-Michele Beaudry ${ }^{1}$, Gordon H Guyatt ${ }^{2}$, Patrick Richard ${ }^{1}$

${ }^{1}$ Surgery, Division of Urology, University of Sherbrooke, Sherbrooke, QC, Canada; ${ }^{2}$ Health Research Methods Evidence and Impact, McMaster University, Hamilton, ON, Canada; 'Surgery, Division of Urology, Western, London, ON, Canada; ${ }^{4}$ Faculté de médecine et des sciences de la santé FMSS École de réadaptation, University of Sherbrooke, Sherbrooke, QC, Canada 


\begin{tabular}{|c|c|c|c|c|c|c|c|c|c|}
\hline Intervention & \multirow{2}{*}{$\begin{array}{c}\begin{array}{c}\text { No of } \\
\text { studies }\end{array} \\
\text { using the }\end{array}$} & $\begin{array}{c}\text { No of } \\
\text { participants }\end{array}$ & $\begin{array}{l}\text { Mean followup } \\
\text { (months) }\end{array}$ & \multirow[t]{2}{*}{ Proportion } & \multicolumn{2}{|c|}{$\begin{array}{c}95 \% \text { confidence } \\
\text { interval }\end{array}$} & \multirow[t]{2}{*}{$\mathbf{I}^{2}(\%)$} & \multirow[t]{2}{*}{$p$} & \multirow[t]{2}{*}{$\begin{array}{l}\text { Quality of } \\
\text { evidence }\end{array}$} \\
\hline Benefits - Cure ra & & number of pa & ds per day at $12 \mathrm{n}$ & & & & & & \\
\hline Slings & 20 & 1956 & 16.9 & 0.58 & 0.51 & 0.65 & 89.1 & $<0.001$ & Moderate \\
\hline ProACT & 6 & 406 & 17.1 & 0.63 & 0.58 & 0.68 & 22.5 & 0.264 & Low \\
\hline Bulking agents & $-^{*}$ & - & - & - & - & - & - & - & - \\
\hline Slings & 16 & 1865 & 15.4 & 0.07 & 0.04 & 0.12 & 91.5 & $<0.001$ & Low \\
\hline ProACT & 3 & 259 & 11.3 & 0.23 & 0.05 & 0.61 & 95.5 & $<0.001$ & Low \\
\hline AUS & 12 & 1596 & 19.2 & 0.22 & 0.15 & 0.31 & 92.3 & $<0.001$ & Low \\
\hline
\end{tabular}

*No data was available for bulking agents' reoperation rate.

Introduction: Surgery for post-prostatectomy urinary incontinence (PPI) can have a major impact on patients' lives. Treatment is highly sensitive to patient values and preferences. We provide estimates of effect and certainty for efficacy and harms outcomes.

Methods: We searched Medline, Embase, and Cochrane central for trials and observational studies of bulking agents, male synthetic slings, compressive balloon systems (ProACT), and artificial urinary sphincters (AUS) up to September 3, 2018. Efficacy outcome was cure (pads per day) and harms was reoperation. Secondary outcomes: Clavien 3-5, Incontinence Quality of Life (IQOL), and Patient Global Impression of Improvement (PGI-I). These were assessed at 12 months and longest followup available. Analyses were performed using random effect models. Risk of bias was assessed with Cochrane and CLARITY tools and quality of evidence using GRADE (PROSPERO 42018073923).

Results: We identified no eligible trials and 85 observational studies: three for bulking agents, 35 for slings, 10 for ProACT, and 37 for AUS, involving 13100 patients. Data quality was low to very low for most outcomes for each procedure. Data for slings and AUS were moderate quality for efficacy outcomes. Cure rates at 12 months were $26.1 \%$ for bulking agents, $58.6 \%$ for slings, $63.2 \%$ for ProACT, and $74.0 \%$ for AUS. Reoperation rates at 12 months were $5.0 \%$ for slings, $23.8 \%$ for ProACT and $22.2 \%$ for AUS (Table 1). Clavien 3-5 complications occurred in $2.8 \%$ of slings, $2.3 \%$ of ProACT, and $9.0 \%$ of AUS. IQOL score was reported for slings and ProACT only, with increases of 32 points and 35 points, respectively. Similarly, PGI-I score was only reported for slings, with an increase of 1.6 . Conclusions: Overall, the quality of evidence supporting available interventions is quite limited for both efficacy and harms. This calls into question the rigor with which devices for PPI are evaluated prior to clinical use. The systematic summary provided can facilitate shared decision-making for men with PPI.

\section{POD-3.4}

Intraperitoneal administration of mesenchymal stem cells is effective at mitigating detrusor deterioration after partial bladder outlet obstruction

Rutuja Kadam ${ }^{1}$, Bridget Wiafe', Peter D. Metcalfe

${ }^{1}$ Department of Surgery, University of Alberta, Edmonton, AB, Canada Introduction: Partial bladder outlet obstruction (pBOO) results in stress on the bladder and is associated with morbidity. The increased pressure generated by the detrusor muscle, required to empty the bladder, results in tissue hypoxia, mechanical stretch, smooth muscle cell hypertrophy, and eventually, fibrosis. ${ }^{1,2}$ Prior studies show that mesenchymal stem cells (MSCs) can mitigate this progression in vitro and in vivo in a rat model via intravenous (IV) administration. ${ }^{3-5}$ Systemic IV administration carries some concerns. ${ }^{6,7}$ Thus, the current investigation focuses on an indirect intraperitoneal (IP) administration of MSCs. We hypothesize that IP administration would be useful at mitigating deterioration after pBOO but require a higher dose.
Methods: pBOO was surgically induced in female Sprague-Dawley rats, which were simultaneously treated with a dose of one million or 5 million MSCs. Both two and four weeks after obstruction, mRNA and protein markers were visualized in pBOO treated and pBOO-non-treated bladders via PCR and immunohistochemistry. These results were correlated with a urodynamic measure of bladder function.

Results: After pBOO, HIF1 $\alpha$ and HIF3 $\alpha$ were localized in the lamina propria and detrusor layer. After obstruction, transcripts for TGFB1, SMAD2, HIF1 $\alpha$, HIF3 $\alpha$, VEGF, mTOR, P70 S6K, COL1, and COL3 increased significantly above normal levels, while IL-10 levels decreased below normal levels. MSC treatment was effective at returning the transcripts and proteins to normal levels, especially with 5 million MSCs at four weeks. Additionally, high pressure generated by $\mathrm{pBOO}$ decreased in treatment groups.

Conclusions: This data confirms that the IP administration of MSCs is effective at mitigating $\mathrm{pBOO}$ associated deterioration. Thus, we have provided further insight into the pathophysiology of $\mathrm{pBOO}$, its treatment with MSCs, and believe this will aid in the progression to clinical trials and, ultimately, improve patient care.

\section{References}

1. Fusco F, Creta M, De Nunzio C, et al. Progressive bladder remodeling due to bladder outlet obstruction: A systematic review of morphological and molecular evidences in humans. BMC Urol 2018;18:15. https://doi.org/10.1186/s12894-018-0329-4

2. Metcalfe PD, Wang J, Jiao $\mathrm{H}$, et al. Bladder outlet obstruction: progression from inflammation to fibrosis. BJU Int 2010;106:1686-94. https://doi.org/10.1111/j.1464-410X.2010.09445.x

3. Al-Saikan B, Ding J, Tredget E, et al. Benefits of mesenchymal stem cells after partial bladder outlet obstruction. Can Urol Assoc J 2016;10:1. https://doi.org/10.5489/cuaj.3257

4. Wiafe B, Adesida A, Churchill T, et al. Mesenchymal stem cells inhibit hypoxia-induced inflammatory and fibrotic pathways in bladder smooth muscle cells. World J Urol 2018;36:1157-65. https://doi.org/10.1007/s00345-018-2247-1

5. Wiafe B, Adesida A, Churchill T, et al. Mesenchymal stem cell therapy inhibited inflammatory and profibrotic pathways induced by partial bladder outlet obstruction and prevented high-pressure urine storage. J Pediatr Urol 2019;15:254.e1-10. https://doi.org/10.1016/j. jpurol.2019.03.003

6. Djouad F, Plence P, Bony $C$, et al. Immunosuppressive effect of mesenchymal stem cells favors tumor growth in allogenic animals. Blood 2003;102:3837-44. https://doi.org/10.1182/blood-2003-04-1193

7. Spees JL, Olson SD, Ylostalo J, et al. Differentiation, cell fusion, and nuclear fusion during ex vivo repair of epithelium by human adult stem cells from bone marrow stroma. Proc Natl Acad Sci USA 2003;100:2397-2402. https://doi.org/10.1073/pnas.0437997100 
Podium 3: Urinary Incontinence, Voiding Dysfunction

POD-3.5

Mirabegron vs. placebo add-on therapy in men with overactive bladder symptoms receiving tamsulosin for underlying benign prostatic hyperplasia: A safety analysis from the PLUS study Sender Herschorn ${ }^{7}$, Kevin T. McVary², Javier Cambronero Santos ${ }^{3}$, Steve Foley ${ }^{4}$, Rita M. Kristy ${ }^{5}$, Nurul Choudhury ${ }^{6}$, John Hairston ${ }^{5}$, Steven A. Kaplan ${ }^{7}$

'Department of Surgery/Urology, University of Toronto, Sunnybrook Health Sciences Centre, Toronto, ON, Canada; ${ }^{2}$ Department of Urology, Stritch School of Medicine, Loyola University Medical Center, Maywood, IL, United States; ${ }^{3}$ Department of Urology, Infanta Leonor Hospital, Madrid, Spain; ${ }^{4}$ Department of Urology, Royal Berkshire Hospital, Reading, United Kingdom; ${ }^{5}$ Astellas Pharma Global Development Inc., Northbrook, IL, United States; ${ }^{6}$ Astellas Pharma Europe Ltd., Chertsey, United Kingdom; ' Department of Urology, Icahn School of Medicine at Mount Sinai, New York City, NY, United States

Introduction: The PLUS study (NCT02757768) investigated the use of mirabegron (MIRA) vs. placebo (PL) for treating overactive bladder symptoms in men concurrently receiving tamsulosin (TAM) for lower urinary tract symptoms (LUTS) due to underlying benign prostatic hyperplasia $(\mathrm{BPH})$. The safety findings from this study are reported herein.

Methods: This 12-week, phase 4, randomized, double-blind, multicenter (North America/Europe) study enrolled men ( $\geq 40$ years old) receiving TAM for $\geq 2$ months. After a four-week TAM run-in period, patients were randomized to MIRA $25 \mathrm{mg}$ or PL. At four weeks, all patients were titrated to MIRA $50 \mathrm{mg}$ or PL equivalent for eight more weeks. Safety assessments were treatment-emergent adverse events (TEAEs), vital signs, 12-lead electrocardiograms (ECGs), and changes in post-void residual (PVR) volume and maximum urinary flow (Qmax).

Results: For the 706 men, mean age was $65.0 \pm 8.9$ years. Hypertension was the most common comorbidity (409 [57.9\%] patients). A slightly higher frequency of overall TEAEs was observed with TAM+PL, although a higher incidence of drug-related TEAEs was noted with TAM+MIRA (Table 1). Most patients reported mild (123 [60.9\%]) or moderate (66 [32.7\%]) TEAEs. Three patients experienced drug-related serious TEAEs (two $[0.6 \%]$

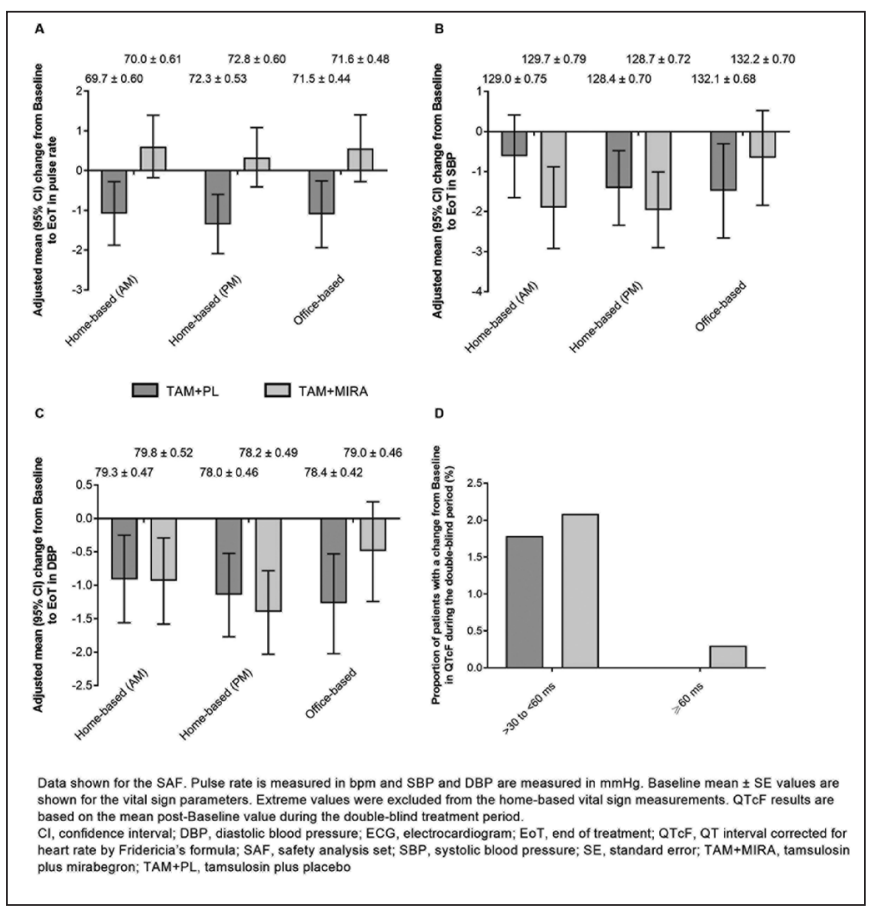

POD-3.5. Fig. 1. Vital sign overview: (A) pulse rate; (B) SBP; (C) DBP; and (D) ECG (QTCF).
TAM+MIRA patients: acute myocardial infarction with cerebral infarction and angina pectoris, one [0.3\%] TAM+PL patient: lacunar stroke). The most common TEAEs were hypertension, headache, and nasopharyngitis.

\begin{tabular}{|c|c|c|}
\hline \multicolumn{3}{|l|}{ POD-3.5. Table 1. TEAE summary } \\
\hline Parameter, no. of patients (\%) & $\begin{array}{c}\text { TAM+PL } \\
(\mathrm{n}=354)\end{array}$ & $\begin{array}{c}\text { TAM+MIRA } \\
(n=352)\end{array}$ \\
\hline Overall TEAEs & $111(31.4)$ & $91(25.9)$ \\
\hline Drug-related TEAEs & $21(5.9)$ & 42 (11.9) \\
\hline Deaths & 0 & 0 \\
\hline Serious TEAEs & $8(2.3)$ & $10(2.8)$ \\
\hline Drug-related serious TEAEs & $1(0.3)$ & $2(0.6)$ \\
\hline $\begin{array}{l}\text { TEAEs leading to study drug } \\
\text { discontinuation }\end{array}$ & $4(1.1)$ & $7(2.0)$ \\
\hline $\begin{array}{l}\text { Drug-related TEAEs leading to study } \\
\text { drug discontinuation }\end{array}$ & $2(0.6)$ & $6(1.7)$ \\
\hline $\begin{array}{l}\text { Common TEAEs ( } \geq 1 \% \text { in any treatment } \\
\text { group), no. of patients }(\%)\end{array}$ & $\begin{array}{l}\text { TAM+PL } \\
(\mathrm{n}=354)\end{array}$ & $\begin{array}{c}\text { TAM+MIRA } \\
(n=352)\end{array}$ \\
\hline Infections and infestations & $14(4.0)$ & $13(3.7)$ \\
\hline Nasopharyngitis & $6(1.7)$ & $7(2.0)$ \\
\hline Bronchitis & $5(1.4)$ & $2(0.6)$ \\
\hline Influenza & $3(0.8)$ & $4(1.1)$ \\
\hline Vascular disorders & $11(3.1)$ & $6(1.7)$ \\
\hline Hypertension & $11(3.1)$ & $6(1.7)$ \\
\hline Nervous system disorders & $8(2.3)$ & $6(1.7)$ \\
\hline Headache & $8(2.3)$ & $6(1.7)$ \\
\hline Metabolism and nutrition disorders & $4(1.1)$ & $3(0.9)$ \\
\hline Hyperglycemia & $4(1.1)$ & $3(0.9)$ \\
\hline Renal and urinary disorders & $1(0.3)$ & $6(1.7)$ \\
\hline Urinary retention & $1(0.3)$ & $6(1.7)$ \\
\hline $\begin{array}{l}\text { TEAEs of special interest, no. of patients } \\
\text { (\%) }\end{array}$ & $\begin{array}{l}\text { TAM+PL } \\
(\mathrm{n}=354)\end{array}$ & $\begin{array}{c}\text { TAM+MIRA } \\
(n=352)\end{array}$ \\
\hline Urinary retention & $1(0.3)$ & $6(1.7)$ \\
\hline Infections and infestations & $2(0.6)$ & $2(0.6)$ \\
\hline Urinary tract infection enterococcal & $1(0.3)$ & $1(0.3)$ \\
\hline Escherichia urinary tract infection & 0 & $1(0.3)$ \\
\hline Urinary tract infection & 0 & $1(0.3)$ \\
\hline Urinary tract infection bacterial & $1(0.3)$ & 0 \\
\hline Investigations & $3(0.8)$ & $1(0.3)$ \\
\hline Blood pressure increased & $2(0.6)$ & $1(0.3)$ \\
\hline Heart rate increased & $1(0.3)$ & 0 \\
\hline Cardiac disorders & $2(0.6)$ & $1(0.3)$ \\
\hline Acute myocardial infarction & 0 & $1(0.3)$ \\
\hline Arrhythmia & $1(0.3)$ & 0 \\
\hline Cerebral infarction & 0 & $1(0.3)$ \\
\hline Lacunar stroke & $1(0.3)$ & 0 \\
\hline Non-APTC/MACE & $1(0.3)$ & 0 \\
\hline Arrhythmia, no evidence of ischemia & $1(0.3)$ & 0 \\
\hline APTC/MACE & 0 & 0 \\
\hline BPO requiring surgery & 0 & 0 \\
\hline
\end{tabular}

Data shown for the SAF. APTC/MACE: Antiplatelet Trialists' Collaboration/Major Adverse Cardiovascular Events; BPO: benign prostatic obstruction; SAF: safety analysis set; TAM+MIRA: tamsulosin plus mirabegron; TAM+PL: tamsulosin plus placebo; TEAE: treatment-emergent adverse event. 
The only special interest TEAE reported by $\geq 1 \%$ of patients was urinary retention; two $(0.6 \%)$ TAM+MIRA patients required catheterization (neither required discontinuation). No major changes in mean blood pressure or pulse rate were noted and similar ECG parameters were observed for both groups (Fig. 1). Changes in mean PVR volume and $\mathrm{Q}_{\max }$ were not clinically meaningful.

Conclusions: No unexpected safety concerns were observed in men receiving treatment with TAM for LUTS due to BPH who subsequently received add-on MIRA or PL.

\section{POD-3.6}

Is the $\mathbf{5 0} \%$ improvement threshold adequate for progression to implantation in sacral neuromodulation?

David K. Charles' ${ }^{1}$ Ross G. Everett ${ }^{1}$, Zachary J. Prebay', Truman P. Landowski', R. Corey $\mathrm{O}^{\prime} \mathrm{Connnor}^{1}$, Michael L. Guralnick'

${ }^{1}$ Urologic Surgery, Medical College of Wisconsin, Milwaukee, WI, United States

Introduction: $\mathrm{A}>50 \%$ subjective improvement in urinary symptoms during sacral nerve stimulation testing (St1) is currently used as indication for progression to stage two implantation (St2). While approximately $66-90 \%$ of patients will have successful St1 and proceed to St2, some deterioration in efficacy over time has been reported. It is unclear if this is related to the initial degree of improvement. We sought to determine if $>50 \%$ improvement after St1 is sufficient to predict long-term success.

Methods: The records of 213 patients who underwent sacral neuromodulation (SNM) for overactive bladder (OAB) were reviewed. Subjects were divided into those who reported $50-75 \%$ improvement and $>75 \%$ improvement after St1 who went on to St2. Differences in clinical variables, including patient-reported outcome measures, diary and pad test information, and urodynamic characteristics, were compared between groups. Cox proportional hazard regression was performed to assess for associations between these variables, reported improvement after St1, and long-term success with SNM after St2.

Results: Of 213 OAB patients who underwent St1, 76 (35.7\%) reported $50-75 \%$ improvement and $70(92.1 \%$ ) of those progressed to St2 (group 1 ), whereas 61 patients $(28.6 \%)$ reported $>75 \%$ and $57(93.4 \%)$ of those progressed to St2 (group 2). The remaining initial 76 patients reported $<50 \%$ during St1 and were excluded. Baseline characteristics (comorbidities, symptom scores, bladder diaries, pad usage, and urodynamic parameters) were not significantly different between groups except that neuropathy was more prevalent in group 1 and SUI in group 2. After St 1 , improvements in symptom scores and diary/pad usage were not significantly different between groups apart from a greater improvement in AUASS bother score in group $2(3.43[\mathrm{n}=7]$ vs. $1.6[\mathrm{n}=5], \mathrm{p}=0.04)$. After St2, group 2 patients were more likely to report a $>75 \%$ improvement $(71.2 \%$ vs. $34.2 \%, \mathrm{p}<0.01)$. With a mean followup of 46 months, $44.3 \%$ of group 1 patients and $68.4 \%$ of group 2 patients still had a functioning device providing symptomatic benefit $(p=0.007)$. This was confirmed on logistic regression with the only variable that significantly predicted long-term functional success being the degree of improvement during St1 (50-75\% vs. $>75 \%$ improvement, odds ratio $0.367, \mathrm{p}=0.0072$ ). Furthermore, the group 2 patients had improved censored device survivability on Kaplan-Meier curve (hazard ratio 1.757, $\mathrm{p}=0.037$ ).

Conclusions: We found no significant differences in the majority of baseline characteristics and clinical data between patients with a $50-75 \%$ improvement and those with $>75 \%$ improvement after St 1 . However, with longer-term followup, patients who achieved a $>75 \%$ improvement during St 1 were more likely to maintain device efficacy. Additional study is warranted to determine if a higher threshold of improvement during St1 should be considered before proceeding to St 2 .

\begin{tabular}{|c|c|c|c|}
\hline \multicolumn{4}{|c|}{$\begin{array}{l}\text { POD-3.6. Table 1. Baseline preoperative parameters } \\
\text { between two main cohorts (St1 } 50-75 \% \text {, St1 >75\%) }\end{array}$} \\
\hline & St1 50-75\% & St1 >75\% & p \\
\hline Patients (n) & 76 & 61 & \\
\hline Age (mean, yrs) & 50.7 & 50 & 0.96 \\
\hline Sex (n=\#female, \%) & $68(89.5 \%)$ & $53(86.9 \%)$ & 0.64 \\
\hline OAB category ( $n=w e t, \%)$ & $60(78.9 \%)$ & $50(82 \%)$ & 0.95 \\
\hline Neuropathy present & $21(27.6 \%)$ & $7(11.9 \%)$ & 0.025 \\
\hline SUI (i.e., MUI; n=\#pts) & $31(40.8 \%)$ & $39(63.9 \%)$ & 0.007 \\
\hline Diabetes present & $17(22.4 \%)$ & $16(26.2 \%)$ & 0.59 \\
\hline CAD present & $4(5.3 \%)$ & $1(1.6 \%)$ & 0.38 \\
\hline HTN present & $26(34.2 \%)$ & $21(34.4 \%)$ & 0.98 \\
\hline Neurogenic bladder & $10(13.3 \%)$ & $7(11.7 \%)$ & 0.77 \\
\hline Hx back surgery & $14(18.4 \%)$ & 9 (15\%) & 0.59 \\
\hline Hx trauma & $8(10.5 \%)$ & $8(13.3 \%)$ & 0.61 \\
\hline Multiple sclerosis & $1(1.3 \%)$ & $4(6.7 \%)$ & 0.17 \\
\hline Stroke & $2(2.6 \%)$ & $3(5.0 \%)$ & 0.65 \\
\hline Parkinson's & $2(2.6 \%)$ & $1(1.7 \%)$ & 1.0 \\
\hline AUASS total $($ mean \pm SD) & $22.4 \pm 8.0$ & $20.7 \pm 8.2$ & 0.56 \\
\hline UDI6 total $($ mean \pm SD $)$ & $13.9 \pm 6.2$ & $14.3 \pm 6.0$ & 0.89 \\
\hline Average void $(o z$, mean $\pm S D)$ & $5.6 \pm 3.1$ & $6.3 \pm 3.9$ & 0.49 \\
\hline $\begin{array}{l}\text { Maximum void (oz, mean } \pm \\
\text { SD) }\end{array}$ & $11.5 \pm 5.8$ & $12.1 \pm 6.6$ & 0.97 \\
\hline $\begin{array}{l}\text { Urinary frequency }(\# / 24 \mathrm{~h} \text {, } \\
\text { mean } \pm \text { SD) }\end{array}$ & $12.7 \pm 5.5$ & $12.7 \pm 5.6$ & 0.73 \\
\hline $\begin{array}{l}\text { UUI episodes (\#/24h, mean } \\
\pm \text { SD) }\end{array}$ & $3.6 \pm 3.1$ & $4.5 \pm 3.4$ & 0.31 \\
\hline $\begin{array}{l}\text { Pad test (\#pads/24h, mean } \pm \\
\text { SD) }\end{array}$ & 4. $1 \pm 3.1$ & $3.5 \pm 1.7$ & 0.82 \\
\hline $\begin{array}{l}\text { Pad test (pad wt }(\mathrm{g} / 24 \mathrm{~h}, \text { mean } \\
\pm \mathrm{SD})\end{array}$ & $442.1 \pm 490.4$ & $432.1 \pm 469.6$ & 0.93 \\
\hline $\begin{array}{l}\text { Maximum systometric } \\
\text { capacity (oz, mean } \pm \mathrm{SD})\end{array}$ & $328.8 \pm 234.7$ & $321.4 \pm 232.1$ & 0.813 \\
\hline Detrusor overactivity present & $32(43.8 \%)$ & $30(53.6 \%)$ & 0.27 \\
\hline $\begin{array}{l}\text { Patients progressed to St2 } \\
\text { ( } \mathrm{n}=\# \text { pts) }\end{array}$ & $70(92.1 \%)$ & 57 (93.4\%) & 0.482 \\
\hline
\end{tabular}

\section{POD-3.6. Table 2. Long-term followup SNM information}

\begin{tabular}{lccc}
\hline & St1 50-75\% & St1 >75\% & p \\
\hline $\begin{array}{l}\text { Mean followup time } \\
\text { (months) }\end{array}$ & 45.8 & 46.2 & 0.96 \\
$\begin{array}{l}\text { Continued SNM-related } \\
\text { improvement (n=\#pts, \%) }\end{array}$ & $31(44.3 \%)$ & $39(68.4 \%)$ & 0.007 \\
$\begin{array}{l}\text { Initiated alternative therapy } \\
\text { (i.e., SNM failure, } \mathrm{n}=\# \text { pts, \%) }\end{array}$ & $17(24.3 \%)$ & $7(12.3 \%)$ & 0.002 \\
$\begin{array}{l}\text { Device explanted (n=\#pts, \%) } \\
\text { (n) }\end{array}$ & $22(31.4 \%)$ & $11(19.3 \%)$ & 0.34 \\
\hline
\end{tabular}




\section{CUA ABSTRACTS}

\section{Podium Session 4: Oncology - Other}

\begin{abstract}
POD-4.1
Diagnostic test accuracy for ultrasound, computed tomography urography, and cytology in the detection of bladder cancer: Results from a global, multicenter analysis

Taeweon Lee ${ }^{1,2}$, Miles Mannas ${ }^{1,2}$, Peter C. Black ${ }^{1,2}$, Mark Assmus ${ }^{3}$, Tim Wollin ${ }^{3}$, Sinan Khadhouri ${ }^{4}$, Kevin Gallagher ${ }^{5}$, Kenneth MacKenzie ${ }^{6}$, Taimur Shah ${ }^{7}$, Chuanyu Gao ${ }^{8}$, Sacha Moore ${ }^{9}$, Eleanor Zimmermann ${ }^{10}$, Eric Edison ${ }^{11}$, Matthew Jefferies ${ }^{12}$, Arjun Nambiar ${ }^{6}$, Matthew Nielsen ${ }^{13}$, John McGrath ${ }^{14}$, Veeru Kasivisvananthan ${ }^{15}$

'Department of Urologic Sciences, University of British Columbia, Vancouver, BC, Canada; ${ }^{2}$ Vancouver Prostate Centre, Vancouver General Hospital, Vancouver, BC, Canada; ${ }^{3}$ Division of Urology, University of Alberta, Edmonton, AB, Canada; ${ }^{4}$ Department of Urology, Aberdeen Royal Infirmary, Aberdeen, United Kingdom; ${ }^{5}$ Department of Urology, Western General Hospital, Edinburgh, United Kingdom; ${ }^{6}$ Department of Urology, Freeman Hospital, Newcastle, United Kingdom; ${ }^{7}$ Department of Surgery and Cancer, Charing Cross Hospital, London, United Kingdom; ${ }^{8}$ Department of Urology, Peterborough City Hospital, Peterborough, United Kingdom; ' Department of Urology, Wrexham Maelor Hospital, Wrexham, United Kingdom; ${ }^{10}$ Department of Urology, Weston General Hospital, Weston-super-Mare, United Kingdom; ${ }^{11}$ Department of Urology, North Middlesex Hospital, London, United Kingdom; ${ }^{12}$ Department of Urology, Morriston Hospital, Swansea, United Kingdom; ${ }^{13}$ University of North Carolina, Dept. of Urology, Chapel Hill, North Carolina, United States; ${ }^{14}$ Department of Urology, University of Exeter Medical School, Exeter, United Kingdom; ${ }^{15}$ Department of Urology, University College London, London, United Kingdom; The IDENTIFY Study Group

Introduction: We evaluated the diagnostic performance of tests used in bladder cancer detection in a global study of patients referred to secondary care for suspected urinary tract cancer.

Methods: The IDENTIFY group prospectively reviewed 10896 patients (27 countries). Those with previous urological malignancy were excluded.
\end{abstract} Diagnostic test (e.g., cystoscopy, imaging) performance was assessed using the predetermined reference standard (i.e., bladder cancer detection with histology or accepted clinical gold standard diagnosis) with resultant index test outcomes. Equivocal outcomes were deemed positive findings, as they prompted further workup. Test adequacy was determined by individual collaborators and only those considered adequate were included in the analysis.

Results: Bladder cancer detection rate was $17.9 \%(n=1951)$. Median age was 72 years. Prevalence rate was higher in men $(21.8 \%, n=1485)$ vs. women $(11.3 \%, \mathrm{n}=463)$. Nine patients did not receive gender allocation, three of whom were diagnosed with bladder cancer. The rate was $22.4 \%(n=1598)$ in patients with visible hematuria $(\mathrm{VH}), 5.23 \%(n=165)$ in non-visible hematuria $(\mathrm{NVH})$, and $30.6 \%(\mathrm{n}=188)$ in no hematuria $(\mathrm{NH})$. Ultrasound (USS) and computed tomography urography (CTU) showed similar performance in the detection of bladder cancer. Sensitivity of CTU was greater than USS in NVH, albeit with lower number of tests performed (Table 1). For each imaging modality, the test performance varied by whether hematuria was visible or not. The sensitivity and overall performance of cytology alone was poor but when used in combination with USS in patients with either $\mathrm{VH}$ or $\mathrm{NVH}$, had a higher overall negative predictive value than CTU alone.

Conclusions: Both USS and CTU ruled out bladder cancer with high accuracy. These results suggest bladder biopsy is warranted if USS or CTU is suspicious for the presence of a bladder tumor. Further analysis of the IDENTIFY dataset will determine which patients can be classified as lowrisk, with the possibility that these patients could avoid flexible cystoscopy if upper tract imaging is negative.

POD-4.1. Table 1. The diagnostic test performance of ultrasound (USS), computed tomography urography (CTU), and cytology in bladder cancer detection

\begin{tabular}{|c|c|c|c|c|c|c|c|}
\hline & & $\mathbf{n}$ & $\begin{array}{c}\text { Accuracy } \\
{[(T P+T N) / n]}\end{array}$ & Sensitivity & Specificity & $\begin{array}{c}\text { Positive } \\
\text { predictive value }\end{array}$ & $\begin{array}{c}\text { Negative } \\
\text { predictive value }\end{array}$ \\
\hline \multirow[t]{3}{*}{ USS } & Overall & 5993 & $89.4 \%$ & $78.3 \%$ & $92.3 \%$ & $72.1 \%$ & $94.3 \%$ \\
\hline & In VH & 3671 & $88.2 \%$ & $78.7 \%$ & $91.5 \%$ & $76.9 \%$ & $92.3 \%$ \\
\hline & In NVH & 1953 & $95.2 \%$ & $59.0 \%$ & $97.6 \%$ & $62.1 \%$ & $97.3 \%$ \\
\hline \multirow[t]{3}{*}{ CTU } & Overall & 4007 & $89.8 \%$ & $77.2 \%$ & $92.9 \%$ & $72.6 \%$ & $94.3 \%$ \\
\hline & In VH & 3177 & $89.7 \%$ & $76.8 \%$ & $93.1 \%$ & $74.8 \%$ & $93.8 \%$ \\
\hline & In $\mathrm{NVH}$ & 696 & $94.3 \%$ & $81.5 \%$ & $95.3 \%$ & $59.5 \%$ & $98.4 \%$ \\
\hline \multirow[t]{5}{*}{ Cytology } & Overall & 3742 & $83.4 \%$ & $56.4 \%$ & $88.7 \%$ & $49.4 \%$ & $91.2 \%$ \\
\hline & In VH & 2439 & $81.9 \%$ & $58.6 \%$ & $87.9 \%$ & $55.7 \%$ & $89.1 \%$ \\
\hline & In NVH & 1132 & $89.1 \%$ & $52.4 \%$ & $91.2 \%$ & $26.0 \%$ & $97.0 \%$ \\
\hline & USS + cytology (VH + NVH) & 2176 & $83.7 \%$ & $84.8 \%$ & $83.7 \%$ & $50.7 \%$ & $96.3 \%$ \\
\hline & $\mathrm{CTU}+$ cytology $(\mathrm{VH}+\mathrm{NVH})$ & 1586 & $81.1 \%$ & $84.4 \%$ & $80.4 \%$ & $47.6 \%$ & $96.1 \%$ \\
\hline
\end{tabular}

Accuracy was determined by dividing the sum of true positive (TP) and true negative (TN) by the total (n). 


\section{POD-4.2}

Diagnostic test performance of ultrasound and cytology in upper tract cancer: Results from a global, multicenter analysis

Taeweon Lee ${ }^{1,2}$, Miles Mannas ${ }^{1,2}$, Peter C. Black ${ }^{1,2}$, Mark Assmus ${ }^{3}$, Tim Wollin ${ }^{3}$, Sinan Khadhouri ${ }^{4}$, Kevin Gallagher ${ }^{5}$, Kenneth MacKenzie ${ }^{6}$, Taimur Shah $^{7}$, Chuanyu Gao ${ }^{8}$, Sacha Moore ${ }^{9}$, Eleanor Zimmermann ${ }^{10}$, Eric Edison ${ }^{11}$, Matthew Jefferies ${ }^{12}$, Arjun Nambiar ${ }^{6}$, Matthew Nielsen ${ }^{13}$, John McGrath ${ }^{14}$, Veeru Kasivisvananthan ${ }^{15}$

'Department of Urologic Sciences, University of British Columbia, Vancouver, BC, Canada; ${ }^{2}$ Vancouver Prostate Centre, Vancouver General Hospital, Vancouver, BC, Canada; ${ }^{3}$ Division of Urology, University of Alberta, Edmonton, AB, Canada; ${ }^{4}$ Department of Urology, Aberdeen Royal Infirmary, Aberdeen, United Kingdom; ${ }^{5}$ Department of Urology, Western General Hospital, Edinburgh, United Kingdom; ${ }^{6}$ Department of Urology, Freeman Hospital, Newcastle, United Kingdom; ${ }^{7}$ Department of Surgery and Cancer, Charing Cross Hospital, London, United Kingdom; ${ }^{8}$ Department of Urology, Peterborough City Hospital, Peterborough, United Kingdom; ${ }^{9}$ Department of Urology, Wrexham Maelor Hospital, Wrexham, United Kingdom; ${ }^{10}$ Department of Urology, Weston General Hospital, Weston-super-Mare, United Kingdom; ${ }^{11}$ Department of Urology, North Middlesex Hospital, London, United Kingdom; ${ }^{12}$ Department of Urology, Morriston Hospital, Swansea, United Kingdom; ${ }^{13}$ University of North Carolina, Dept. of Urology, Chapel Hill, North Carolina, United States; ${ }^{14}$ Department of Urology, University of Exeter Medical School, Exeter, United Kingdom; ${ }^{15}$ Department of Urology, University College London, London, United Kingdom; The IDENTIFY Study Group

Introduction: Upper tract urothelial carcinoma (UTUC) and renal cell carcinoma (RCC) are rare in patients with hematuria. There is limited data to guide the most appropriate upper tract evaluation in these patients. We evaluated the diagnostic test performance of investigations for UTUC and RCC in a global study of patients referred to secondary care for suspected urinary tract cancer.

Methods: The IDENTIFY group prospectively assessed 10896 patients (27 countries). Those with previous urological malignancy were excluded. Diagnostic test performance (e.g., cystoscopy, imaging) was assessed using the predetermined reference standard (i.e., detection of UTUC/ RCC with histology or accepted clinical gold standard diagnosis) with resultant index test outcomes. Computed tomography urography (CTU) was considered the reference test, thus its performance was not observed. Equivocal outcomes were deemed positive findings, as these prompted further workup. Test adequacy was determined by individual collaborators and only those deemed adequate were included in the analysis.

Results: UTUC prevalence was $1.17 \%(n=128)$. It was more prevalent in patients with visible hematuria $(\mathrm{VH})(1.60 \%, \mathrm{n}=114)$ vs. non-visible hematuria $(\mathrm{NVH})(0.29 \%, \mathrm{n}=9)$ and no hematuria $(\mathrm{NH})(0.81 \%, \mathrm{n}=5)$. Among those with NVH under the age of $60(n=1313)$, no one was found to have UTUC. RCC prevalence was $0.98 \%(n=107)$. Similarly, RCC was more prevalent in $\mathrm{VH}(1.26 \%, \mathrm{n}=90)$ vs. $\mathrm{NVH}(0.41 \%, \mathrm{n}=13)$ and $\mathrm{NH}$ $(0.65 \%, n=4)$. Ultrasound (USS) performed better in detecting RCC in the NVH group than in the $\mathrm{VH}$ group (Table 1). USS was poor at detecting UTUC alone, however, improved in sensitivity when combined with cytology. All UTUCs in the NVH group without bladder cancer were detected using a combination of USS, cytology, and flexible cystoscopy. Conclusions: UTUC and RCC are rare. USS is a good diagnostic performance test in detecting RCC, especially in patients with $\mathrm{NVH}$. Combined non-invasive tests improve diagnostic accuracy in detecting UTUC. These can be considered in place of CTU in selected patients in a risk-stratified model.

\section{POD-4.3}

The prognostic and predictive implications of the 12-Chemokine Score in muscle-invasive bladder cancer

Logan Zemp ${ }^{1 *}$, Anders Berglund ${ }^{2 *}$, Jasreman Dhillon ${ }^{3}$, RyanPutney $^{2}$, Youngchul Kim², Rohit K. Jain', G. Daniel Grass', Jingsong Zhang', Mic hael A. Poch ${ }^{1}$, Julio Pow-Sang ${ }^{1}$, Wade J. Sexton', Scott M. Gilbert ${ }^{1}$, Shari Pilon-Thomas ${ }^{5}$, José Conejo-Garcia ${ }^{5}$, Colin P.N. Dinney ${ }^{6}$, James J. Mulés, Roger $L i, M D^{1,5}$

${ }^{1}$ Department of Genitourinary Oncology, H. Lee Moffitt Cancer Center, Tampa, FL, United States; ${ }^{2}$ Department of Biostatistics and Bioinformatics, H. Lee Moffitt Cancer Center, Tampa, FL, United States; ${ }^{3}$ Department of Pathology, H. Lee Moffitt Cancer Center, Tampa, FL, United States; ${ }^{4}$ Department of Radiation Oncology, H. Lee Moffitt Cancer Center, Tampa, FL, United States; ${ }^{5}$ Department of Immunology, H. Lee Moffitt Cancer Center, Tampa, FL, United States; ${ }^{6}$ Department of Urology, MD Anderson Cancer Center, Houston, TX, United States

*Contributed equally to the study

Introduction: Adaptive anti-tumor immunity can be orchestrated by lymph node-like immune cell aggregates within the tumor microenvironment (TME) called tertiary lymphoid structures (TLSs). TLSs are postulated to be the gateway of lymphocyte infiltration into the TME. A 12-chemokine (12-CK) metagene grouping (has previously been described that correlates with the presence of TLSs in other solid tumor types and response to immune checkpoint blockade (ICB). In this study, we explored the prognostic implication of the 12-CK score in bladder cancer and its correlation with the presence of TLSs.

Methods: Cystectomy specimens from 130 patients with bladder cancer were arrayed on Affymetrix microarrays. 12-CK scores were normalized with $>1$ denoting high scores $(12-\mathrm{CKHi})$. Overall survival was estimated using the Kaplan-Meier method. Findings were validated using 12-CK scores extracted from TCGA data. Immunohistochemistry antibody staining was performed for CD4, CD8, CD20, LAMP3. A genitourinary (GU) pathologist scored TLSs into types I-III, with type III representing fully

POD-4.2. Table 1. The diagnostic test evaluation of ultrasound (USS) and cytology in renal cell carcinoma (RCC) and upper tract urothelial cancer (UTUC)

\begin{tabular}{|c|c|c|c|c|c|c|}
\hline & & $\mathbf{n}$ & Sensitivity & Specificity & Positive predictive value & Negative predictive value \\
\hline \multicolumn{7}{|l|}{ Renal cell carcinoma } \\
\hline \multirow[t]{3}{*}{ USS } & Overall & 6913 & $81.6 \%$ & $98.0 \%$ & $22.6 \%$ & $99.9 \%$ \\
\hline & In VH & 4165 & $82.1 \%$ & $97.8 \%$ & $26.0 \%$ & $99.8 \%$ \\
\hline & In NVH & 2380 & $87.5 \%$ & $98.3 \%$ & $14.6 \%$ & $100 \%$ \\
\hline \multicolumn{7}{|c|}{ Upper tract urothelial cancer } \\
\hline USS & & $* *$ & $50.0 \%$ & $97.9 \%$ & $16.9 \%$ & $99.6 \%$ \\
\hline Cytology & & ** & $58.8 \%$ & $89.4 \%$ & $8.4 \%$ & $99.2 \%$ \\
\hline USS + cytology & & 5798 & $78.7 \%$ & $92.3 \%$ & $9.8 \%$ & $99.8 \%$ \\
\hline \multirow{3}{*}{$\begin{array}{l}\text { USS + cytology + } \\
\text { cystoscopy* }\end{array}$} & Overall & ** & $80.0 \%$ & $92.1 \%$ & $10.3 \%$ & $99.8 \%$ \\
\hline & in $\mathrm{VH}$ & 3265 & $79.2 \%$ & $91.2 \%$ & $13.0 \%$ & $99.6 \%$ \\
\hline & in NVH & 2282 & $100 \%$ & $94.2 \%$ & $3.7 \%$ & $100 \%$ \\
\hline
\end{tabular}

${ }^{*}$ Cystoscopy findings suspicious for tumour (e.g., hematuric jet from ureteric orifice, bulge in the transmural ureter). ${ }^{*}$ Final data pending distribution from the IDENTIFY study group. 


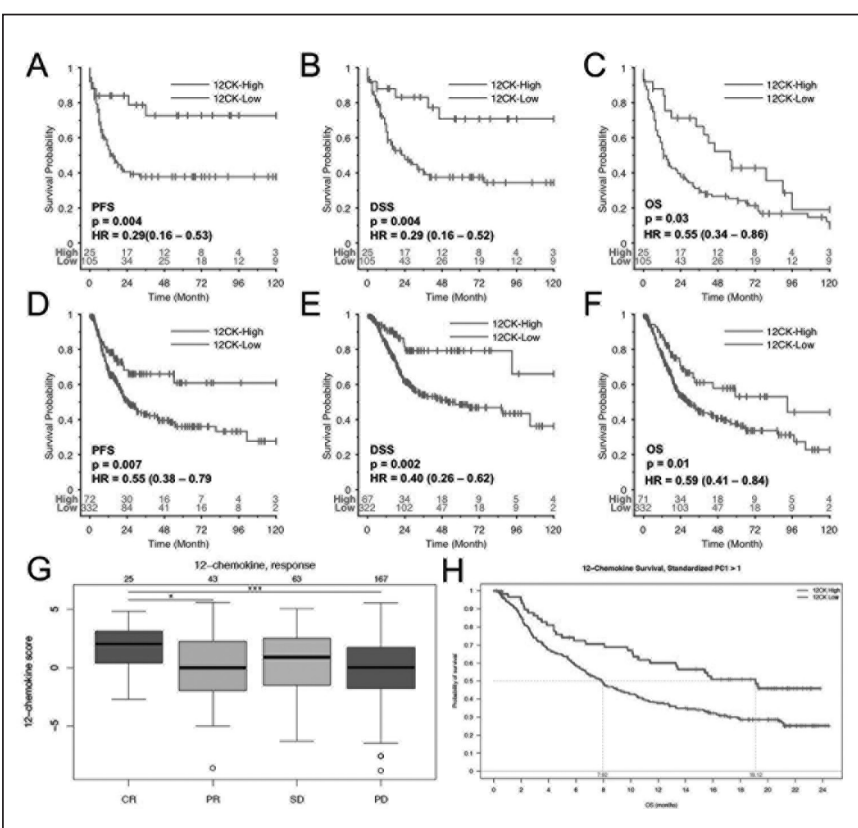

POD-4.3. Fig. 1. The prognostic and predictive implications of 12-CK score. Kaplan-Meier survival analyses revealed improved (A) PFS (HR 0.29; $p=0.004$ ); (B) DSS (HR 0.29; $p=0.004$ ); and (C) OS (HR 0.55; $p=0.03$ ) in 12-CK-high MIBC patients treated with radical cystectomy at Moffitt Cancer Center. The favorable prognosis in (D) PFS (HR 0.55; $p=0.007$ ); (E) DSS (HR 0.40; $p=0.002$ ); and (F) OS (HR 0.59; $p=0.01$ ) were confirmed in patients from TCGA. (G) From the INVIGOR-210 study testing the efficacy of atezolizumab in chemotherapyrefractory locally advanced or metastatic bladder cancer patients, complete responders were found to have significantly higher 12 -CK scores than the other cohorts. $(\boldsymbol{H})$ Stratified by the 12-CK score, 12-CK-high patients were found to have an 11.2-month OS benefit (19.1 vs. 7.9 months) after treatment using atezolizumab. ${ }^{*} \mathrm{p}<0.05 ;{ }^{* * *} \mathrm{p}<0.01 ;{ }^{* * *} \mathrm{p}<0.001$.

developed TLSs. Chi-squared and Fisher exact was used to compare immune cell infiltrates type I-III TLSs $12-\mathrm{CKHi}$ vs. 12-CKLo. Finally, we examined the association between high 12-CK scores and response to atezolizumab in the IMVIGOR 210 study

Results: Twenty-five (19.2\%) of patients were categorized as $12-\mathrm{CKHi}$. Pathological review of 43 bladder tumor specimens confirmed higher levels of type III TLSs $(p=0.02)$ and immune cell infiltrates CD4 ( $p=0.1)$, CD8 ( $p=0.02)$, CD20 (0.008), and LAMP3 (0.047) in 12-CKHi patients. $12-\mathrm{CK}$ had improved progression-free survival (PFS, $p=0.004)$, diseasespecific survival (DSS, $p=0.004)$, and overall survival (OS, $p=0.03$ ) in the Moffitt cohort (Figs. 1A-C) These findings were confirmed in the TCGA BLCA (Figs. 1D-F). With the IMvigor 210 trial, 12-CK-Hi patients had an 11.2-month OS, suggesting it as a potential marker for ICB response. Conclusions: This is the first study to link high 12-CK scores to the presence of TLSs in the TME of bladder cancer and is associated with better patient survival outcome and response to ICB. This 12-CK score may prove to be a useful prognostic marker in bladder cancer.

\section{POD-4.4}

\section{A renal tumor prediction tool using the Canadian Kidney Cancer} information system

Ameeta Nayak', Luke T. Lavallée², Ranjeeta Mallick', Simon Tanguay³, Antonio Finelli ${ }^{4}$, Anil Kapoor ${ }^{5}$, Alan I. So ${ }^{6}$, Bimal Bhindi, Ricardo A. Rendon $^{8}$, Adrian Fairey ${ }^{9}$, Frédéric Pouliot ${ }^{10}$, Lori A. Wood ${ }^{11}$, Rodney $\mathrm{H}$. Breau $^{2}$

${ }^{1}$ Faculty of Medicine, University of Ottawa, Ottawa, ON, Canada; ${ }^{2}$ Division of Urology, University of Ottawa, Ottawa, ON, Canada; ${ }^{3}$ Division of Urology, McGill University, Montreal, QC, Canada; ${ }^{4}$ Division of Urology, University of Toronto, Toronto, ON, Canada; ${ }^{5}$ Division of Urology, McMaster University, Hamilton, ON, Canada; ${ }^{6}$ Department of Urologic Sciences, University of British Columbia, Vancouver, BC, Canada; ${ }^{7}$ Department of Urology, Mayo Clinic, Rochester, MN, United States; ${ }^{8}$ Department of Urology, Dalhousie University, Halifax, NS, Canada; ${ }^{9}$ Division of Urology, University of Alberta, Edmonton, $\mathrm{AB}$, Canada; ${ }^{10}$ Division of Urology, Université Laval, Quebec City, ON, Canada; ${ }^{11}$ Division of Medical Oncology, Dalhousie University, Halifax, NS, Canada

Introduction: With the increased use of cross-sectional images, incidental kidney tumors are frequently discovered. However, many surgically removed kidney tumors are found to be benign, and some cancerous tumors are not aggressive and may not require treatment. We sought to determine the clinical and radiographical predictive factors of kidney tumor malignancy and develop a nomogram to distinguish between benign and malignant renal masses.

Methods: Patients diagnosed with solitary renal masses were identified from the Canadian Kidney Cancer information system (CKCis). Demographic, clinical, and imaging data were compared to the pathological diagnosis from surgery or biopsy. Tumors were categorized into malignant or benign, and aggressive (high-grade malignant) or indolent (low-grade malignant and benign). Logistic regression models were constructed to identify predictors of each category. Nomograms were created using statistically significant risk factors and were internally validated using bootstrap methods.

Results: Of 3991 patients diagnosed with a solitary kidney tumor between 2011 and 2017, 93\% patients had malignant tumors and 43\% had highgrade tumors. Factors associated with cancer and high-grade cancer were age (odds ratio [OR] 0.99; 95\% confidence interval [Cl] 0.98-1.00; OR $1.01,95 \% \mathrm{Cl} 1.00-1.02$, respectively) and tumor size (OR $1.25 ; 95 \%$ Cl 1.18-1.32; OR 1.28; $95 \% \mathrm{Cl} 1.25-1.31$, respectively). Male sex was also predictive of high-grade cancer (OR 1.48; 95\% Cl 1.26-1.73). The nomograms were able to discriminate between malignant/benign tumors (area under the curve $[\mathrm{AUC}] 0.71 ; 95 \% \mathrm{Cl} 0.68-0.74$ ), and aggressive/ indolent tumors (AUC $0.77 ; 95 \% \mathrm{Cl} 0.75-0.78$ ).

Conclusions: Patient and tumor characteristics are independently associated with cancer risk and high-grade cancer risk. The CKCis nomograms presented have good discriminative accuracy and this prediction tool can be used by physicians and patients with kidney tumors to help determine an optimal management plan.

\section{POD-4.5}

Trimodal therapy vs. radical cystectomy for T2 bladder cancer: Real-world evidence from Ontario

Marian S. Wettstein ${ }^{1,2,3}$, Alejandro Berlin ${ }^{4}$, Song Pham², Srikala S. Sridhar ${ }^{5}$, Peter Chung, Shabbir M.H. Alibhai ${ }^{6}$, Theodorus van der Kwast ${ }^{7}$, Syed R. Qadri', Kathy $\mathrm{Li}^{1}$, Ning $\mathrm{Li}^{2}{ }^{2}$, Thomas Hermanns ${ }^{3}$, Girish S. Kulkarni, ${ }^{1,2}$ ${ }^{1}$ Division of Urology, Department of Surgery, Princess Margaret Cancer Centre, University Health Network, University of Toronto, Toronto, ON, Canada; ${ }^{2} \mathrm{ICES}$, Toronto, ON, Canada; ${ }^{3}$ Department of Urology, University Hospital of Zurich, University of Zurich, Zurich, Switzerland; ${ }^{4}$ Radiation Medicine Program, Princess Margaret Cancer Centre, University Health Network, University of Toronto, Toronto, ON, Canada; ${ }^{5}$ Division of Medical Oncology, Department of Medicine, Princess Margaret Cancer Centre, University Health Network, University of Toronto, Toronto, ON, Canada; ${ }^{6}$ Division of Geriatric Medicine, Department of Medicine, Princess Margaret Cancer Centre, University Health Network, University of Toronto, Toronto, ON, Canada; 'Department of Pathology, Princess 
Margaret Cancer Centre, University Health Network, University of Toronto, Toronto, ON, Canada

Introduction: Comparative effectiveness research between trimodal therapy (TMT) and radical cystectomy (RC) for muscle-invasive bladder cancer is conflicting. Prior systematic reviews and meta-analyses in favor of RC were mainly driven by large U.S.-based registry studies at high risk of bias. Hence, we aimed to compare the survival among patients diagnosed with $\mathrm{T} 2$ bladder cancer who either initiated TMT or underwent $\mathrm{RC}$ in a Canadian population-based cohort adjusted for a wide range of assumed confounders.

Methods: Province-wide pathology reports (April 2004 to December 2015) were linked with health administrative data to identify patients diagnosed with T2 bladder cancer. We compared 90-day mortality and cancer-specific survival (CSS) between patients who initiated TMT and patients who underwent RC by multivariable regression analysis. Effect sizes (reference: $\mathrm{RC}$ ) were presented as adjusted odds ratios (aOR) or hazard ratios (aHR) (95\% confidence interval $[\mathrm{Cl}])$.

Results: We identified 1890 patients who were diagnosed with T2 bladder cancer, of which 188 (9.9\%) initiated TMT (median dose: 60 Gray; most common radiosensitizer: cisplatin; salvage RC rate: $9 \%$ ) and 1702 $(90.1 \%)$ underwent RC. Median followup time was 1.9 years. Ninety-day mortality was significantly lower in patients who initiated TMT compared to patients who underwent RC (crude rate: $2.7 \%$ vs. $7 \%$, $p=0.03$; adjusted aOR $0.26[0.10-0.66])$. A statistically significant difference in CSS could not be observed (aHR 0.96 [0.59-1.54]).

Conclusions: To our knowledge, this cohort study conducted in a setting with regionalized cancer care is the first population-based study that confirms, in comparison to prior U.S.-based registry cohorts, the similar survival outcomes between TMT and RC observed in single-center, comparative studies. However, we detected a 90-day mortality rate among patients who underwent RC that was more than twice as high as the one seen among patients who initiated TMT.

\section{POD-4.6}

WATChmAN: Interim results of a randomized trial of virtual surveillance vs. standard in-person care for clinical stage I testicular cancer

Robert I. Hamilton', Lauren Landoni², Kopika Kuhathaas², Peter W. M. Chung ${ }^{3}$, Philippe L. Bedard ${ }^{4}$, Padraig R. Warde ${ }^{3}$, Aaron R. Hansen ${ }^{4}$, Tran Truong $^{5}$, Ezra Hahn ${ }^{3}$, Michael A.S. Jewett ${ }^{1}$

'Division of Urology, Department of Surgical Oncology, Princess Margaret Cancer Centre, University Health Network, University of Toronto, Toronto, ON, Canada; ${ }^{2}$ Princess Margaret Cancer Centre, University Health Network, Toronto, ON, Canada; ${ }^{3}$ Department of Radiation Oncology, Princess Margaret Hospital, University Health Network, University of Toronto, Toronto, ON, Canada; ${ }^{4}$ Department of Medical Oncology, Princess Margaret Hospital, University Health Network, University of Toronto, Toronto, ON, Canada; ${ }^{5}$ Techna Institute, University Health Network, University of Toronto, Toronto, ON, Canada.

Support: MSH-UHN innovation fund. Meekison-Keystone-Posen family fund

Introduction: Most guidelines recommend active surveillance (AS) as initial management for stage I testis cancer (TC). AS entails blood work and imaging at regular intervals requiring multiple clinic visits spanning five years. This is time-consuming, costly, and requires high compliance. To address these issues, we innovated a secure online platform, WATChmAN (Web-based virtuAl Testicular CANcer clinic), to enable asynchronous communication between patients, results, and physician team. This is an interim report of results.

Methods: We conducted an RCT (NCT03360994) with patients on AS randomized to virtual (WATChmAN) vs. standard in-person care. Primary endpoint is safety: loss to followup, compliance, incidence of relapse, delay in detection, and burden of relapse. Non-compliance represents: a) delay in visit; or b) followup visit with incomplete testing. Secondary endpoints include patient/physician satisfaction and cost savings.

Results: At present, 112 of a planned 144 patients are enrolled. More patients in the virtual arm have been compliant with AS schedules $(79 \%$ vs. $66 \%$ ) with shorter median compliance delays (14 vs. 17.5 days). Fourteen patients have relapsed: eight virtual $(14.3 \%)$ and six standard $(10.7 \%)$. Median time to relapse was shorter for the virtual arm (8 vs. 9.5 months), with no difference in burden of disease at relapse. Response rates to six-month surveys were $80 \%$ and $65 \%$ for virtual and standard arms, respectively. When asked if satisfied with their care, on the virtual arm $61 \%$ reported "extremely satisfied" and 39\% "satisfied," compared to $39 \%$ and $57 \%$, respectively, for the standard arm. When WATChmAN patients were asked if the application was able to provide the same excellence of care as in-person appointments; $84 \%$ reported "strongly agree" or "agree." Conclusions: Interim results suggest virtual care in stage I TC is feasible and safe with improvements in patient satisfaction. This may serve as a potential model for virtual care for other cancers. 


\section{CUA ABSTRACTS}

\section{Podium Session 5: Training, Reconstruction}

\section{POD-5.1}

Defining which outcomes are associated with patient satisfaction after urethroplasty

Lordan Bekkema ${ }^{1}$, Keith F. Rourke

${ }^{1}$ Division of Urology, University of Alberta, Edmonton, AB, Canada Support: Dr. Rex Boake Studentship in Urology

Introduction: Outcomes after urethroplasty can be assessed by multiple measures, both surgeon-reported and patient-reported. We aimed to determine which clinical outcomes are associated with patient satisfaction after urethroplasty.

Methods: From 2012-2018, 387 patients enrolled in this prospective, single-center study. Patient-reported outcomes were assessed preoperatively and six months postoperatively. Voiding function was assessed with the International Prostate Symptom Score (IPSS), erectile function with the International Index of Erectile Function (IIEF-5), and ejaculatory function with a hybrid of the brief sexual function inventory. While patient satisfaction, penile curvature/appearance, genitourinary pain, post-void dribbling, and standing voiding function were assessed using literature-derived threeor five-point Likert scales. Urethroplasty success was defined as the easy passage of a $16 \mathrm{Fr}$ flexible cystoscope. Descriptive statistics were used to summarize findings, while multivariate binary logistic regression was used to determine the association between outcomes and patient satisfaction.

Results: At six months' followup, $96.1 \%$ of patients were stricture-free on cystoscopy while $81.7 \%$ reported being satisfied. On multivariate analysis, improvement in IPSS (odds ratio [OR] 1.1; 95\% confidence interval [CI] $1.1-1.2 ; p=0.04)$, de novo erectile dysfunction (OR $0.5 ; 95 \% \mathrm{Cl} 0.2-0.9$; $\mathrm{p}=0.04)$, de novo penile curvature (OR $0.4,95 \% \mathrm{Cl} 0.2-0.9 ; \mathrm{p}=0.03)$, and improved standing voiding function (OR $1.3 ; 95 \% \mathrm{Cl} 1.1-1.5 ; \mathrm{p}=0.004)$ were associated with patient satisfaction. Cystoscopic success $(p=0.60)$, change in pain score $(p=0.14)$, post-void dribbling $(p=0.69)$, change in penile length $(p=0.44)$, and ejaculatory dysfunction $(p=0.51)$ were not. Conclusions: Improved voiding function, de novo penile curvature, de novo erectile dysfunction, and improved standing voiding function are associated with patient satisfaction and should be included in a patient-centered approach to urethral stricture. While perhaps important to surgeons, cystoscopic success is not associated with patient satisfaction.

\section{POD-5.2}

Perioperative management of urethroplasty patients: A survey of the Society of Genitourinary Reconstructive Surgeons R. Christopher Doiron', Keith F. Rourke ${ }^{2}$

${ }^{1}$ Department of Urology, Queen's University, Kingston, ON, Canada; ${ }^{2}$ Department of Surgery, Division of Urology, University of Alberta, Edmonton, $\mathrm{AB}$, Canada

Introduction: Practice patterns in the field of reconstructive urology have been poorly described. We hypothesize significant heterogeneity exists within the field. We sought to survey fellowship-trained reconstructive urologists with respect to perioperative practice preferences for patients undergoing urethroplasty.

Methods: An online survey examining perioperative management of urethroplasty patients was administered to members of the Society of Genitourinary Reconstructive Surgeons (GURS) between August and October, 2019. The survey focused on anterior urethroplasty and inquired about preferences regarding tissue transfer, use of antibiotic and venous thromboembolism (VTE) prophylaxis, urinary catheter use, drain placement, and patient disposition.
Results: A total of 248 GURS members were invited to participate in the survey, with a response rate of $57.3 \%(n=142)$. The majority of participants perform $>20$ urethroplasties per year $(n=108,76.0 \%)$. Almost all respondents $(97.9 \%, n=139)$ reported using intraoperative intravenous (IV) antibiotic prophylaxis while a minority of surgeons use intraoperative pharmacological VTE prophylaxis $(n=57,40.1 \%)$. Most respondents routinely perform a midline incision $(n=124,87.3 \%)$ while a small minority of surgeons prefer a lambda incision $(n=17,12.0 \%)$. With respect to tissue transfer, most surgeons prefer buccal mucosa harvested from the cheek $(n=138,97.2 \%)$ in a rectangular shape $(n=79,55.6 \%)$ or, less commonly, oval shape $(n=53$, $37.3 \%$ ). Respondents were more ambivalent on graft site closure, with a majority leaving the site open $(n=76,53.5 \%)$. Perineal drains are placed routinely by $25.3 \%$ of respondents $(n=36)$ and of those left in situ, most are removed within 48 hours $(\mathrm{n}=31,86.1 \%)$. A majority of urethroplasty patients are admitted to hospital for $<24$ hours $(n=100,70.4 \%)$. Only $21.3 \%(n=30)$ of surgeons routinely prescribe bedrest for patients. In terms of postoperative antibiotic prophylaxis, a minority continue IV prophylaxis postoperatively $(n=60,42.3 \%)$, but most only do so for $<24$ hours $(n=34,56.7 \%)$. Oral antibiotic prophylaxis, however, is routinely administered by most urologists $(\mathrm{n}=98,69.0 \%)$, and most continue until the urinary catheter is removed $(n=70,72.2 \%)$. Postoperatively, most patients are left with a urethral catheter for a period of $2-3$ weeks $(n=72,58.5 \%)$ or $3-4$ weeks $(n=37,30.1 \%)$. At the time of catheter removal, most surgeons routinely perform urethral imaging with contrast $(n=96,67.6 \%)$. In terms of evaluating for stricture recurrence, a majority of urologists prefer some form of objective investigation $(n=111$, $78.2 \%)$, with uroflowmetry $(n=91,82.0 \% \%)$ and post-void residual $(n=88$, $79.3 \%$ ) being the most commonly reported methods, while cystoscopy was also commonly performed $(n=64,57.7 \%)$. Although timing of these investigations varied, most of them are routinely performed either $2-3$ months $(n=49$, $44.1 \%)$ or $4-6$ months $(n=38,34.2 \%)$ postoperatively.

Conclusions: Though there appears to be majority consensus on most urethroplasty management decisions, significant heterogeneity remain in some areas, including antibiotic use, VTE prophylaxis, donor site management, catheter management, and followup assessment. With a lack of evidence in this space, decisions will continue to be made based on clinical experience and best practice principles.

\section{POD-5.3}

Triamcinolone acetonide injections for the treatment of recalcitrant post-radical prostatectomy vesicourethral anastomotic stenosis: A large, modern-day series

Sarah R. Ferrara' ${ }^{7}$, Humberto R. Vigil', Jennifer A. Locke', Sender Herschorn ${ }^{1}$ ${ }^{1}$ Urology, Sunnybrook Health Sciences Centre, University of Toronto, Toronto, ON, Canada

Introduction: We sought to evaluate the success of bladder neck injections of triamcinolone at the time of transurethral bladder neck incision (BNI) for prevention of recurrent or recalcitrant post-radical prostatectomy (RP) vesicourethral anastomotic stenosis (VUAS)

Methods: Patients with recurrent VUAS post-RP \pm radiation were offered triamcinolone injections at the time of BNI. VUAS was diagnosed after RP by symptoms, followed by cystoscopy or urethrography. The outpatient procedures were done under general anesthesia. Cold knife incisions were made at the 3, 9, and 12 o'clock bladder neck (BN) positions, followed by triamcinolone injections $(4 \mathrm{mg} / \mathrm{mL})$ into the 3 and 9 o'clock incision sites. Postoperative catheterization was 5-7 days. Treatment outcomes were determined by clinical followup and cystoscopy. 
Results: A total of 18 men underwent 25 procedures over a four-year period. Mean age at diagnosis of VUAS was 64 , and mean time to VUAS was 13.8 months after RP. Fourteen patients $(77.8 \%)$ had undergone some form of radiation treatment. The men had undergone 128 prior unsuccessful VUAS treatments, with a mean of 7.1 failed treatments per patient. Failed treatments included dilation, $\mathrm{BNI}, \mathrm{BN}$ injection of mitomycin $\mathrm{C}$, or ALLIUM stent placement. The overall success rate after a mean of 16.2 months from the time of triamcinolone injection was $83.3 \%$. Six patients went on to have successful incontinence surgery. Five patients $(27.8 \%)$ had treatment complications (bleeding, urinary tract infection, pain, and urinary extravasation). The three patients who did not respond to treatment are stable and awaiting re-treatment with triamcinolone injection. Conclusions: Triamcinolone bladder neck injections for post-RP VUAS are a useful and safe treatment for recurrent or recalcitrant stenosis. Associated incontinence can subsequently be treated.

\section{POD-5.4}

Results of a surgical decision management protocol to treat incontinent pediatric neurogenic bladder patients: A 10-year, single-institution, retrospective series

Karan Gandhi', Jacob Davidson ${ }^{2}$, Zhan Tao (Peter) Wang ${ }^{3}$, Sumit Dave ${ }^{3}$ ${ }^{1}$ Schulich School of Medicine \& Dentistry, Western University, London, ON, Canada; ${ }^{2}$ Department of Surgery, Division of Pediatric Surgery, Western University, London, ON, Canada; ${ }^{3}$ Department of Surgery, Division of Urology and Pediatric Surgery, Western University, London, ON, Canada

Introduction: Incontinent neurogenic bladder (NB) patients are socially ostracized and are additionally often at risk of upper tract renal damage. Surgical management of NB incontinence requires assessment of the bladder and outlet as components responsible for incontinence. ${ }^{1}$

Methods: This is a retrospective chart review of a prospective protocol examining 22 incontinent NB patients who underwent one of two procedures over a 10-year period between October 2009 and June 2019. Augmentation cystoplasty (AC) with a Mitrofanoff procedure (MP) was performed for all NB patients who had a high detrusor leak point pressure (DLPP) over $40 \mathrm{~cm} \mathrm{H} \mathrm{O}$ and showed significant bladder trabeculations (AC+MP), while those with a low DLPP below $40 \mathrm{~cm} \mathrm{H} \mathrm{H}_{2} \mathrm{O}$ and a relatively smooth bladder underwent a bladder outlet procedure, including a modified Young-Dees-Leadbetter procedure with a $360^{\circ}$ rectus fascial sling and bladder neck suspension procedure with concomitant $\mathrm{AC}$ and MP $(\mathrm{BOP}+\mathrm{AC}+\mathrm{MP})$. Postoperative success was defined as being completely dry for a three-hour period without any incontinent appliance/pad or wet. Upper tract status and renal function was simultaneously assessed. Results: The mean age at the time of the procedure was 12.1 years (standard deviation $=6$ years), with 14 undergoing $A C+M P$ and eight undergoing $\mathrm{BOP}+\mathrm{AC}+\mathrm{MP}$ based on our preoperative criteria. Overall, $13(59 \%)$ patients were completely dry and nine $(41 \%)$ patients still had some ongoing leakage. Success rates for continence as defined was $71 \%$ in the $\mathrm{AC}+\mathrm{MP}$ group vs. $38 \%$ in the $\mathrm{BOP}+\mathrm{AC}+\mathrm{MP}$ group. The overall complication rate was $45 \%$, defined as those requiring surgical intervention following their index procedure.

Conclusions: Surgical treatment of NB incontinence is fraught with a high complication rate and relatively poor outcomes, despite concomitant AC. Bladder neck closure is possibly a reliable way to achieve socially acceptable continence in this population. Despite a three-procedure BOP, success rates are still not acceptable after a concomitant AC.

\section{Reference}

1. Amarenco G, Sheikh Ismael S, Chesnel C, et al. Diagnosis and clinical evaluation of neurogenic bladder. Eur / Phys Rehabil Med 2017;53:975-80.

\section{POD-5.5}

Experience and results of the first three years of implementing a competency-based didactic and simulation-focused boot camp for incoming urology residents

Yuding Wang ${ }^{l}$, Jen Hoogenes ${ }^{l}$, Hark Randhawa ${ }^{1}$, Catherine Anne Lovatt ${ }^{l}$ Kevin Kim ${ }^{1,2}$, Roderick Clark ${ }^{3}$, Courtney Moore ${ }^{1}$, Bobby Shayegan ${ }^{1}$, Edward D. Matsumoto ${ }^{1}$

'Department of Surgery, Division of Urology, McMaster University, Hamilton, ON, Canada; ${ }^{2}$ Department of Health Research Methods, Evidence and Impact, McMaster University, Hamilton, ON, Canada; ${ }^{3}$ Department of Surgery, Division of Urology, Western University, London, ON, Canada

Support: Centre for Minimal Access Surgery (CMAS), McMaster University and St. Joseph's Healthcare Hamilton

Introduction: The launch of the CanMEDS Competence by Design (CBD) initiative presents challenges for curricula design to ensure residents achieve proficiency as they progress through training. Surgical boot camps have been used to improve the learning process by orienting and preparing new residents. We developed, implemented, and evaluated an intensive didactic and simulation-focused boot camp for first-year urology residents and report our experience and findings of its first three years. Methods: Urology residents from two Canadian universities participated in the two-day boot camp within the first three weeks of residency. The boot camp includes 11 didactic lectures and six simulation sessions that allow for instruction and deliberate practice with feedback. Pre-and post-boot camp multiple choice questionnaires (MCQs) were administered. At the end of day two, a six-station objective structured clinical exam (OSCE) and feedback session were conducted. For initial validation, after year one, three PGY2 residents served as historical controls and completed the MCQ and OSCE.

Results: A total of 19 residents completed boot camp. The mean age was 26.4 years $( \pm 2.8)$ and 13 were male. Prior urology and simulation experience was minimal. Participants markedly improved on the preand post-MCQs (year 1: $62 \%$ and $91 \%$; year 2: $55 \%$ and $89 \%$; year 3: $58 \%$ and $86 \%$, respectively), while historical controls scored $66 \%$. Participants scored marginally higher than the controls on four of the six OSCE stations. Total OSCE scores remained $>88 \%$ over the three cohorts. All participants reported higher confidence levels and felt boot camp was an excellent preparation for residency.

Conclusions: During its first three years, our urology boot camp has demonstrated high feasibility and utility. Knowledge and technical skills uptake were established via MCQ and OSCE results, with participants' scores near or above those of PGY2 controls. This boot camp will remain in our CBD curriculum, and it can provide a framework for use by other urology residency programs.

\section{POD-5.6}

Organized basic laparoscopic urological skills (BLUS) course objectively improves trainee performance in a single session David M. Mikhail', Aaron Tabibzadeh ${ }^{1}$, Zachary Kozel', Arun Rai', Joseph Sarcona ${ }^{1}$, Patrick Samson', Christopher Hartman', Jessica Kreshover', Michael Schwartz' ${ }^{1}$ Louis Kavoussi', Domenico Veneziano ${ }^{2}$, Lee Richstone ${ }^{1}$ ${ }^{1}$ Urology, Northwell Health, New York, NY, United States; ${ }^{2}$ Urology, Azienda Ospedaliera Bianchi-Melacrino-Morelli di Reggio Calabria, Calabria, Italy

Introduction: Validated in 2012, the American Urological Association (AUA) Basic Laparoscopic Urological Skills (BLUS) curriculum has not been widely adopted. Simulation and objective evaluation of technical skills are essential to surgical training. We established the first standardized BLUS course for urology residents in North America. We aimed to establish whether expert-taught BLUS curriculum can lead to objective and measurable skills improvement in a single session.

Methods: Trainees self-reported laparoscopic experience and performed baseline evaluations for five BLUS skills tasks: peg transfer, circle-cutting, needle-guiding, lap-suturing, and vessel-clipping. They were evaluated by two standardized metrics validated for BLUS tasks - the Performance Improvement (Pi) score and Crowd-Sourced Assessment of Technical Skills (CSATS). $\mathrm{Pi}$ is calculated based on time and error improvements. CSATS 
uses crowd-sourcing (30-40 evaluations per video) to score technical skills. Following baseline trials, they were instructed in BLUS through live demonstration. They then practiced each task three times with feedback. A final performance of each task was once again evaluated by both metrics. Results: A total of 55 residents participated; 41 (79\%) were male and median age was 29 years. There was no correlation between self-perceived technical experience and any outcome metrics. Most residents (71\%, 39/55) showed an 'average' Pi score, while 27\% (15) had 'low' Pi scores. All residents improved in time score for all tasks with a median improvement of 25\% (interquartile range [IQR] 16-33\%). Baseline aggregate CSATS scores improved significantly across all five tasks (12.7 to 13.4; average improvement 0.7 [IQR $0.3-1.1 ; \mathrm{p}<0.03$ for all]).

Conclusions: This inaugural AUA course shows that the BLUS curriculum shows measurable and objective improvements in a single teaching session. As robotic surgery continues to overtake laparoscopic surgical volume, BLUS and similar curriculums are an important investment for training programs to teach and evaluate resident competency.

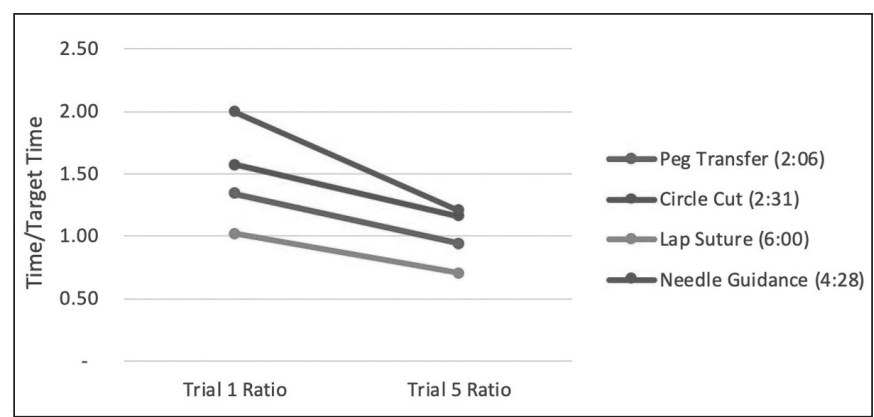

POD-5.6. Fig. 1. Target times used to evaluate procedures. All had significant decreases, with an average of $25 \%$ improvement in average times for all trainees $(p<0.001)$. This was used to calculate the "time score" aspect of the validated $\mathrm{Pi}$ (performance improvement) scoring tool. 


\section{Unmoderated Poster Session 1: Prostate Cancer, Endourology, BPH}

\begin{abstract}
UP-1.1
A pilot study to assess efficacy and safety of methoxyflurane for pain control during convective thermal therapy using Rezūm system in benign prostatic hyperplasia (BPH) - Preliminary results Dean S. Elterman ${ }^{1}$, Kevin C. Zorn², Naeem Bhojani ${ }^{2}$

${ }^{1}$ Urology, University Health Network, Toronto, ON, Canada; ${ }^{2}$ Urology, Centre hospitalier, Université de Montréal, Montreal, QC, Canada

Introduction: The optimal pain control protocol for Rezūm in benign prostatic hyperplasia (BPH) has not been determined. The use of low-dose methoxyflurane administered via a portable, disposable, single-use handheld inhaler (Penthrox ${ }^{\mathrm{TM}}$ ) was assessed in this study.

Methods: An open-labeled, single-center pilot study was conducted to demonstrate the safety and efficacy in patients undergoing Rezūm using methoxyflurane inhaler on top of standard oral analgesia, including oral lorazepam, oral oxycodone, acetaminophen and intra-urethral lidocaine gel. Intravenous propofol was used as rescue analgesia. Patients assessed current pain intensity using visual analog scale (VAS) of pain at four timepoints including: 1) before any medication; 2) initially after insertion of the rigid cystoscope and before any Rezūm treatment; 3 ) immediately after final injection of Rezūm treatment; and 4) at discharge. Patients were asked to rate on treatment satisfaction questionnaire for medication (TSQM 1.4) and one question about pain relief at discharge. Treating physician also assessed on TSQM 1.4.

Results: Ten patients were planned for this study. Up to December 2019, five patients were recruited. The average prostate volume was $49.5 \mathrm{ml}$ (range $24-73 \mathrm{ml}$ ). Patients received average 10 Rezūm injections (range 10-15), and procedure lasted average four minutes. Average VAS scores were $0,1.1,1.5$ (primary efficacy outcome) and 1.8, respectively, at the four timepoints. TSQM scores on effectiveness, side effects, convenience, and global satisfaction rated by patients were 77.8, 95.0, 81.1, and 88.6, respectively, while ratings by treating physician were $96.7,100,93.3$, and 92.9, respectively. Treatment satisfaction on pain relief was rated as 4.0 (very good). There have been no adverse events so far.

Conclusions: Methoxyflurane inhaler on top of standard oral analgesia was a feasible and easy to administer pain management in Rezūm therapy. Further data on a larger comparative study is required after completing this pilot study.
\end{abstract}

\section{UP-1.2}

Evolution of prostate-specific antigen according to prostate volume after photoselective vaporization of the prostate with GreenLight XPS 18W: A five-year experience

Rhea Coriaty ${ }^{1}$, Iman Sadri', Côme Tholomier ${ }^{3}$, Félix Couture ${ }^{4}$, David-Dan Nguyen ${ }^{2}$, Kyle Law ${ }^{2}$, Ahmed S. Zakaria ${ }^{5}$, Naeem Bhojani ${ }^{5}$, Dean S. Elterman ${ }^{6}$, Esteban Rijo ${ }^{5}$, V. Misrai ${ }^{7}$, Kevin C. Zorn ${ }^{5}$

${ }^{1}$ Faculty of Medicine, Université de Sherbrooke, Sherbrooke, QC, Canada; ${ }^{2}$ Faculty of Medicine, McGill University, Montreal, QC, Canada; ${ }^{3}$ Department of Urology, McGill University, Montreal, QC, Canada; ${ }^{4}$ Department of Urology, Université de Sherbrooke, Sherbrooke, QC, Canada; ${ }^{5}$ Department of Urology of University of Montreal Hospital Centre, Université de Montréal, Montreal, QC, Canada; ${ }^{6}$ Urology of University Health Network, University of Toronto, Toronto, ON, Canada; ${ }^{7}$ Department of Urology, Clinique Pasteur, Toulouse, France

Introduction: Prostate-specific antigen (PSA) testing remains the standard biochemical marker used both during screening and monitoring of prostate cancer. This study aims to clarify the changes in PSA kinetics in patients after photoselective vaporization of the prostate (PVP) with Greenlight XPS 180W (GL XPS).

Methods: A retrospective analysis was conducted on patients who underwent PVP GL XPS at the Centre hospitalier de I'Université de Montréal. Data regarding patient characteristics, preoperative prostate volume, medications, operative parameters, and PSA values were gathered for a period of five years after the operation. Patients with a history of prostate cancer were excluded. Data was stratified based on prostate volumes of $<80 \mathrm{cc}$ and $>80$ cC.

Results: A total of 559 patients were included. Median age was 67 years, with a median prostatic volume of $71 \mathrm{cc}$ (interquartile range [IQR] 61-74). Median preoperative PSA was $3.9 \mathrm{ng} / \mathrm{ml}$ (IQR 1.95-6.7). The PSA nadir was reached at 12 months postoperatively, with a median of $1.1 \mathrm{ng} / \mathrm{ml}(60.7 \%$ reduction). No significant differences were recorded for mean PSA and PSA change according to the use of 5-ARI or alpha-blockers preoperatively. Preoperative PSA was significantly higher in patients with a prostate volume $>80 \mathrm{cc}(\mathrm{p}<0.001)$. Compared to patients with $<80 \mathrm{cc}$ prostates, postoperative PSA in patients in the $>80$ cc group was significantly higher at six, 12 , 24,36 , and 60 months. For all prostate volumes, energy density of $>4 \mathrm{~kJ} /$ cc yielded significantly greater changes in median PSA at six, 12, 24, and 36 months, with $66.6 \%, 69.7 \% 65.6 \%$, and $65.4 \%$ PSA drop, respectively. In the $<80 \mathrm{cc}$ prostate group, greater median PSA changes were recorded with a $>4 \mathrm{~kJ} / \mathrm{cc}$ energy density, compared to $3-4 \mathrm{~kJ} / \mathrm{cc}$ and $<3 \mathrm{~kJ} / \mathrm{cc}$, with $65.1 \%, 55.4 \%$, and $52.1 \%$ median PSA changes at six months, respectively. Similar trends were recorded at 12 and 24 months. Higher energy similarly correlated with significantly higher median PSA changes at 6 and 12 months for prostates $>80 \mathrm{cC}$, with $70.0 \%$ and $68.3 \%$, respectively.

Conclusions: PSA levels reached the nadir at one year following PVP GL XPS, with slight increases during subsequent followups. Patients with $>80$ cc prostates experienced a greater drop of PSA with every energy density that the $<80$ cc group. Overall, an energy density of $>4 \mathrm{~kJ} / \mathrm{cc}$ consistently yielded greater drops in PSA changes at six, 12, 24, and 36 months.

\section{UP-1.3}

Rate of bladder stone recurrence in patients undergoing cystolitholapaxy with and without concurrent transurethral resection of the prostate

Emily Whelan' ${ }^{1}$, Amanda Hird', Diana E. Magee', Sidney B. Radomski ${ }^{1}$ Department of Urology, University of Toronto, Toronto, ON, Canada Introduction: To date, there is no long-term data on the rate of bladder stone recurrence in patients who undergo concurrent cystolitholapaxy and transurethral resection of the prostate (TURP) and those who undergo cystolitholapaxy alone. The objective of this study was to report on the natural history of patients undergoing cystolitholapaxy with and without concurrent TURP.

Methods: This prospective cohort study included males who presented with bladder stones secondary to bladder outlet obstruction between April 2001 and January 2017. All patients underwent initial cystolitholapaxy with or without concurrent TURP at a single institution. Patient outcomes reported included rate and time to stone recurrence. Risk factors for stone recurrence will also be examined.

Results: Of an initial cohort of 190 patients with bladder stones, 137 were males who developed bladder stones secondary to bladder outlet obstruction. Of the patients who previously underwent a TURP, $17 \%$ of them developed recurrent bladder stones, compared to $29 \%$ of patients who did not have a TURP. Overall, 34 patients had recurrent bladder stones with 
seven $(21 \%)$ of those patients having undergone previous concurrent TURP while $27(79 \%)$ had not. The average time to stone recurrence for patients with a previous TURP was 92.9 months and 103.1 months for the non-TURP group. In the non-TURP group, seven of the 27 patients underwent repeat cystolitholapaxy alone, with three patients ultimately developing recurrent stones.

Conclusions: Time to stone recurrence following cystolitholapaxy was similar between patients who underwent concurrent TURP and those that did not; however, the rate of recurrence was greater amount patients without a previous TURP.

\section{UP-1.5}

Prospective study evaluating ureteroscopy under conscious sedation

Amanda Eng ${ }^{1}$, M. Eric Saltel ${ }^{1}$, Premal Patel ${ }^{1}$

${ }^{1}$ Urology, University of Manitoba, Winnipeg, MB, Canada

Introduction: Flexible ureteroscopy (fURS) to treat renal or ureteric stones is typically performed in the operating room with spinal or general anesthesia. However, as endoscopic technology has improved and with limited operating room resources, the ability to perform fURS under conscious sedation may expedite patient care and reduce healthcare costs. We sought to prospectively evaluate our experience of fURS under conscious sedation at a tertiary care center as a proof of principle.

Methods: Beginning November 2019, we prospectively collected data on patients undergoing fURS with the use of intravenous sedation (fentanyl and midazolam) administered by nursing staff. Surgical variables include previous ureteric stenting or other stone procedures; whether or not an access sheath, dilator, laser, or basket was used; and if a ureteric stent was placed post-stone extraction. Stone-free rates are determined by the surgeon's ability to remove the stones and on examination of the ureter, as well as on followup imaging.

Results: From November to December 2019, a total of 11 patients underwent fURS for stone management under conscious sedation. Preliminary results demonstrate successful stone-free rate in $82 \%(9 / 11)$. Two were unsuccessful, as the ureter was too tight and a ureteric stent was inserted for definitive stone management at a later time, and in one case a stone had already passed on its own. The average analgesics used are $2.5 \mathrm{mg}$ of midazolam and $90 \mu \mathrm{g}$ of fentanyl. No procedure was discontinued due to patient intolerability.

Conclusions: Our preliminary prospective results demonstrate the feasibility of performing fURS for the treatment of renal/ureteric calculi under conscious sedation. Based on our current collected data, ureteroscopy can be performed using short-acting anesthetics in a safe and efficient way with good tolerability and stone-free rates. Over the next few months, we hope to increase our study size and analyze other variables.

\section{UP-1.7}

\section{The routine use of tranexamic acid during percutaneous} nephrolithotomy: A systematic review

Morgan MacDonald ${ }^{1}$, Stewart Whalen ${ }^{1}$, Robyn Parker ${ }^{2}$, Ross Mason', Andrea G. Lantz Powers ${ }^{7}$

${ }^{1}$ Department of Urology, Dalhousie University, Halifax, NS, Canada; ${ }^{2}$ W.K. Kellogg Health Sciences Library, Dalhousie University, Halifax, NS, Canada

Introduction: Percutaneous nephrolithotomy (PCNL) is the gold standard procedure for management of large nephrolithiasis. Bleeding and infection are the most concerning and common complications associated with PCNL. Transfusion rates post-PCNL vary by center but have been estimated to be $2-12 \% .^{1}$ Tranexamic acid (TXA) is an antifibrinolytic agent that has been used safely in many other surgical specialties. We sought to determine whether the routine administration of TXA during PCNL reduces blood loss, transfusion rates, and operative time, or increases stone-free clearance.

Methods: Systematic review of PubMed, Scopus, Embase, CENTRAL, and clinicaltrials.gov up to June 20, 2019 was performed with assistance of a professional librarian (RP). Randomized controlled trials (RCTs) compar- ing the intravenous use of TXA vs. routine care were identified. Reviewers independently screened titles (SW, MM) and extracted data (SW, MM). Primary outcomes were requirement of blood transfusion and estimated blood loss. Secondary outcomes included rates of sepsis, stone-free rate, time in hospital and procedure time. Risk of bias (RoB) was assessed with Cochrane RoB 2 tool (RCT).

Results: We identified five RCTs, with most studies demonstrating some RoB and some studies demonstrating high RoB. All studies demonstrated a decreased blood transfusion rate in the TXA group, however, only two of the five studies showed statistical significance. No statistically significant difference was found in estimated blood loss between patients receiving TXA and those who did not (mean difference -0.74 [units]; $95 \%$ confidence interval $[\mathrm{CI}]-1.56,0.08 ; \mathrm{z}=1.76 ; \mathrm{p}=0.08$ ). Patient receiving TXA had slightly lower operative times than those not receiving TXA (mean difference $-12.46 \mathrm{~min} ; 95 \% \mathrm{Cl}-14.08,-10.83 ; z=14.99 ; p<0.001)$. No statistically significant difference was found in hospital length of stay between patients receiving TXA and those not (mean difference -1.09 days; $95 \% \mathrm{Cl}-2.68,0.51 ; \mathrm{z}=0.33, \mathrm{p}=0.18$ ). There was not enough data extracted to comment on stone-free rate.

Conclusions: The studies currently available examining the routine use of TXA during PCNL are of poor quality and insufficient to comment on the utility. There may be a trend to decreased rates of blood transfusions and decreased operative time with the routine use of TXA during PCNL.

\section{Reference}

1. Fenner A. Surgery: Tranexamic acid reduces bleeding during percutaneous nephrolithotomy. Nature reviews. Urology 2013;10:2. https://doi.org/10.1038/nrurol.2012.224

\section{UP-1.8}

Urinary and sexual function outcomes at 12 months post-radical prostatectomy: Robotic-assisted vs. laparoscopic approach

Courtney Moore', Len Hoogenes ${ }^{1}$, Jaskirat Saini', Adel Moalwi', Bobby Shayegan ${ }^{7}$,Edward D. Matsumoto ${ }^{7}$

${ }^{1}$ Department of Surgery, Division of Urology, McMaster University, Hamilton, ON, Canada

Introduction: The objective of this study was to examine urinary and sexual function in prostate cancer (PC) patients at the 12-month mark following robotic-assisted (RARP) or laparoscopic (LRP) prostatectomy to identify any significant differences between groups. All cases were conducted by a single surgeon with expertise using both approaches (>1000 LRPs and >500 RARPs).

Methods: Eligible patients underwent RARP or LRP at a single center and were followed in clinic for at least 12 months. Patients completed the validated UCLA PC Index and the five-item International Index of Erectile Function (IIEF-5) at the 12-month mark. Clinical characteristics and scores were evaluated between groups, with an additional focus on three domains: sexual bother, urinary bother, and daily pad usage.

Results: A total of 90 patients were eligible (RARP $n=45$; LRP $n=45$ ) and had a mean age of $62.2( \pm 6.7) ; 55.6 \%$ of RARP and $40.0 \%$ of LRP patients underwent bilateral nerve-sparing (NS) and an equal percentage of patients in both groups $(64.4 \%)$ received postoperative erectile dysfunction (ED) treatment. RARP patients had higher (more favorable) IIEF-5 scores $(p<0.05)$ and used fewer pads $(p<0.05)$. LRP patients reported higher levels of sexual bother on the UCLA PC Index $(p<0.05)$. Patients with bilateral NS in both groups had significantly higher scores on the IIEF-5 ( $p=0.042$ ). There were no differences between groups for age, preand postoperative prostate-specific antigen, overall UCLA PC Index score, or the urinary bother domain.

Conclusions: RARP patients had significantly higher IIEF-5 scores, lower levels of sexual bother, used fewer pads, and had a higher rate of bilateral NS than LRP patients. The high rate of ED treatment and bilateral NS in all patients may relate to higher IIEF-5 scores, despite surgical approach. Continued followup past the 12-month mark, along with a larger sample size, will allow for a greater understanding of urinary and sexual function in this patient population. 
UP-1.9

Robotic-assisted laparoscopic technique for repair of iatrogenic ureteric injuries from gynecologic surgeries - the Edmonton experience

Alexandra Bain ${ }^{1}$, Blair A. St Martin', Michael G. Hobart

${ }^{1}$ Urology, University of Alberta, Edmonton, $\mathrm{AB}$, Canada

Introduction: The incidence of iatrogenic genitourinary injuries occurring at the time of gynecologic surgery for benign disease is estimated at $1 \%$, with approximately $70 \%$ of injuries involving the bladder and $30 \%$ involving the ureters. ${ }^{1}$ Recently, centers performing high volumes of robotic-assisted laparoscopic surgery have converted to using a roboticassisted approach to repair these injuries, but only a handful of series have been published on this novel approach. Edmonton has one of the highest volumes of robotic-assisted laparoscopic surgery in Canada and has been using a robotic approach for repair of these injuries. We performed a review of the indications, technique, and postoperative outcomes of these repairs at our center.

Methods: A case study of patients from January 2018 to September 2019 who underwent robotic assisted laparoscopic repair of iatrogenic ureteric injuries from gynecologic surgery for benign disease were reviewed.

Results: Six patients were identified that had iatrogenic ureteric injuries from gynecologic surgery that were repaired with a robotic-assisted laparoscopic approach. Five of six cases were a delayed repair with an average of three months (94 days) between time of injury and time of repair. All injuries were in the distal ureter requiring ureteroneocystostomy. Followup for all patients included a cystogram two weeks postoperatively, cystoscopy, stent removal 4-8 weeks postoperatively, and renal scan at 3-4 months. All patients had complete resolution of ureteric obstruction by six months, with no evidence of leak, stricture, or persistent obstruction (Table 1).

Conclusions: Robotic-assisted laparoscopic repair of iatrogenic injuries post-gynecologic surgery is an effective technique, with all patients having successful resolution of obstruction. In the era of minimally invasive surgery and patient expectations, this technique offers patients an option to resolve a surgical complication with potentially less morbidity than a traditional open repair.

\section{Reference}

1. Gellhaus PT, Bhandari A, Monn MF, et al. Robotic management of genitourinary injuries from obstetric and gynecological operations: A multi-institutional report of outcomes. BJ Int 2015;115:430-6. https://doi.org/10.1111/bju.12785

\begin{tabular}{lc}
\hline $\begin{array}{l}\text { UP-1.9. Table 1. Intraoperative and postoperative results of } \\
\text { robotic-assisted laparoscopic repair of iatrogenic ureteric } \\
\text { injuries from gynecological surgery }\end{array}$ \\
\hline \multicolumn{2}{l}{ Number of patients } \\
\hline $\begin{array}{l}\text { Location of ureteric injury } \\
\text { Proximal }\end{array}$ & $0 / 6$ \\
Mid & $0 / 6$ \\
Distal & $6 / 6$ \\
Operative technique used for repair & $6 / 6$ \\
Ureteroneocystostomy & \\
Postoperative followup & $6 / 6$ \\
Cystogram 2 weeks & $6 / 6$ \\
Cystoscopy and stent removal 4-6 weeks & $6 / 6$ \\
Renal scan 3 months & \\
Resolution of obstruction & $6 / 6$ \\
Yes & $0 / 6$ \\
No & $1 / 6$ \\
Clavien Dindo complications >grade II & 2 days \\
Average length of hospital stay post-repair &
\end{tabular}

UP-1.10

Urological use of nephrostomy tubes for ureteric obstruction Nick Dean ${ }^{1}$, Gillian Shiau', Shubhadip (Shubha) K. De ${ }^{1}$, Tim Wollin ${ }^{1}$

${ }^{1}$ Urology, University of Alberta, Edmonton, AB, Canada; ${ }^{2}$ Interventional Radiology, University of Alberta, Edmonton, $A B$, Canada

Introduction: We aimed to assess the use of nephrostomy tube drainage with and without antegrade stenting over a six-year period. The primary objective was to identify if there was a change in the volume of nephrostomy tubes placed over time and to describe the indication for placement for both benign and malignant causes of ureteric obstruction. The secondary objective of our study was to better delineate the role of the urologist in the setting of patients with both benign and malignant ureteric obstruction.

Methods: We performed a retrospective analysis of percutaneous nephrostomy requisition data provided by the Interventional Radiology department in Edmonton, Alberta from 2013-2019. Using patient identifiers, long-term outcome data was tracked from 2013 to present using the electronic medical record at the Kipnes Urology Center and compiled in our database.

Results: From 2013 to present, a total of 1247 nephrostomy tubes were inserted in Edmonton. The incidence of nephrostomy tube insertion increased linearly from 156 in 2013 to 246 in 2018. The main indication for new nephrostomy tube placement was malignancy in $45 \%$ and stone disease in $22 \%$ in $2018 ; 50 \%$ (18/36) of patients who had a nephrostomy tube placed for non-prostate cancer malignant hydronephrosis were dead within one year and 35\% (12/34) of patients receiving a nephrostomy tube for an obstructing ureteric stone had a previous failed cystoscopic placement of a retrograde stent. For other services that initiated a nephrostomy tube insertion, urology became involved in $95 \%$ of cases last year. Conclusions: Our system's use of nephrostomy tubes is increasing. Using this database, we will attempt to discover predictors of failed retrograde stent placement by cystoscopy in the setting of obstructing ureteric stones, failed indwelling stents in the setting of malignant hydronephrosis, evaluate one- vs. two-stage nephrostomy tubes and antegrade stents, and shed light on the optimal treatment strategy for treating causes of ureteric obstruction.

\section{UP-1.11}

Effect of 2012 and 2017 United States Preventive Services Task Force prostate-specific antigen screening guidelines on prostate cancer grade and age of diagnosis: A single-center, retrospective study Samantha McGirr' ', Luke Wang', Jinfeng Jiang ', Bryant Van Leeuwen' ${ }^{1}$ Chad A. LaGrange', Shawna L. Boyle

'Department of Urology, University of Nebraska Medical Center, Omaha, NE, United States

Introduction: In 2017, the United States Preventive Services Task Force (USPSTF) updated its prostate-specific antigen (PSA) testing guidelines, issuing a grade " $\mathrm{C}$ " recommendation for men 55-69 years old, making whether to undergo routine testing a personal decision. ${ }^{1}$ This changed from the USPSTF's 2012 Grade "D" recommendation, which recommended against routine testing. After the 2012 recommendation, a 3-10\% decline in PSA screening was seen in all age groups. ${ }^{2}$ The effects of the 2012 and 2017 guideline on age and cancer severity at initial diagnosis is unclear. Methods: This study analyzed all patients with biopsy-confirmed prostate cancer at the University of Nebraska Medical Center from 2005-2019 $(n=647)$. Patients were split into those diagnosed prior to the 2012 recommendation (group $1 ; n=179$ ), between the 2012 and 2017 recommendations (group 2; $\mathrm{n}=276$ ), and after the 2017 recommendation (group 3; $n=192$ ). Age of diagnosis and biopsy Gleason were obtained for each group and compared against each other. A Bonferroni correction was employed, with statistically significant p-value set at 0.017 .

Results: Mean age at diagnosis was $61.7 \pm 6.6$ years. Group 1's mean age $(60.3 \pm 6.4)$ did not significantly differ from group $2(61.6 \pm 6.6 ; p=0.046)$ but was less than group $3(63.3 \pm 6.5 ; p<0.001)$. Group 2's mean age was less than group 3 ( $p=0.007)$.

Conclusions: The USPSTF updates have been associated with significant rise in age of cancer diagnosis and a decrease in the diagnosis of GGG1 cancer, as expected. However, with each update, there is a significant increase in the rates of both GGG4 and GGG5. Furthermore, GGG3 rates 
increased significantly with the second update. The impact of USPSTF recommendations on prostate cancer severity and age at diagnosis should be examined in further studies, as well as exploring the pathologic details after treatment and longer-term outcomes.

References

1. U.S. Preventive Services Task Force. Final recommendation statement: Prostate cancer: Screening. October 2018.

2. Fleshner K, Carlsson SV, Roobol MJ. The effect of the USPSTF PSA screening recommendation on prostate cancer incidence patterns in the USA. Nat Rev Urol 2017;14:26-37. https://doi.org/10.1038/ nrurol.2016.251

\section{UP-1.12}

North American urology resident perspectives on their exposure, experience, and ability to interpret multiparametric magnetic resonance imaging of the prostate using PI-RADS V2

Kashif Visram ${ }^{1}$, D. Robert Siemens ${ }^{1}$, Craig Rodrigues

'Urology, Queen's University, Kingston, ON, Canada

Introduction: Multiparametric magnetic resonance imaging (mpMRI) of the prostate with PI-RADS V2 grading is a tool recently introduced into the armamentarium of urologists for identifying localized prostate cancer. Despite high uptake in use, there exist knowledge gaps in the urologist's ability to interpret these. Our objective was to obtain the perspective of North American residents on their comfort with mpMRI and PI-RADS V2 grading to determine whether there is a need to develop learning tools. Methods: A survey was created to obtain information about exposure, experience, and comfort with interpretation of mpMRI. This survey was completed by all chief residents that attended the QUEST urology course in Kingston and residents that attended the Upstate New York Urological Association resident research meeting. Demographic information, as well as Likert scale questions (1, strongly disagree to 5, strongly agree) were obtained to capture respondent perspectives.

Results: Fifty-three surveys were completed by PGY-1 to PGY- 5 residents, with 35 being PGY-5. Of these, only 12 had received formal training in $\mathrm{mpMRI}$ interpretation via course or lecture. Forty-eight of these residents had performed prostate biopsies and 43 had thoroughly reviewed mpMRIs. Residents disagreed with understanding the components of PI-RADS V2 scoring for T2W films (2.45 \pm 1.01$)$, DWI films (2.26 \pm 0.90$)$, and DCE films $(2.21 \pm 0.99)$ and their ability to interpret T2W films $(2.43 \pm 1.06)$ and DCE films $(2.92 \pm 1.11)$. They felt neutral towards interpreting DCE films $(3.08 \pm 1.30)$. Residents agreed that they are interested in learning opportunities to enhance their ability to interpret mpMRI films $(4.21 \pm 0.91)$. They suggested lectures and online modules as preferred modalities.

Conclusions: $\mathrm{mpMRI}$ is becoming more frequently used in urology to diagnose localized prostate cancer. However, residents feel that their ability to interpret these images is not optimal. To improve this, there is interest in tools that could help develop these skills. Online modules and lectures were suggested options for this.

\section{UP-1.13}

Results of magnetic resonance-targeted transperineal biopsy in patients with grade group 1 prostate cancer

Kevin Martell ${ }^{1,2}$, Hans Chung ${ }^{1}$, Gerard Morton ${ }^{1}$, Danny Vesprini ${ }^{1}$, Eric Tseng ${ }^{1}$, Ewa Szumacher ${ }^{1}$, Patrick Cheung ${ }^{1}$, Will Chu' ${ }^{1}$, Stanley Liu' ${ }^{7}$, Andrew Loblaw $^{1}$

${ }^{1}$ Radiation Oncology, University of Toronto, Toronto, ON, Canada; ${ }^{2}$ Oncology, University of Calgary, Calgary, AB, Canada

Introduction: With increased use of magnetic resonance (MR) imaging in patients on active surveillance for prostate cancer, targeted biopsies (TBx) of MR nodule(s) at the time of restaging biopsy is becoming commonplace. ${ }^{1,2}$ This study described a cohort of patients on active surveillance for grade group (GG) 1 disease who had MR imaging and then TBx of the nodule(s) found. It aimed to determine the minimum nodule crosssectional ellipsoid area on MR (eA) that best predicts for TBx revealing GG2 or higher disease.

Methods: Forty-six patients receiving cognitively fused, MR-guided, transperineal TBx alongside six random biopsies (RBx) between 2015 and 2019

\begin{tabular}{lcc}
\hline $\begin{array}{l}\text { UP-1.13. Table 1. Targeted biopsy results for } \\
\text { receiving targeted transperineal biopsies }\end{array}$ \\
\hline & $\begin{array}{c}\text { First target } \\
(\mathbf{n}=\mathbf{4 6})\end{array}$ & $\begin{array}{c}\text { Second } \\
\text { target (n=6) }\end{array}$ \\
\hline $\begin{array}{l}\text { Number of cores taken within target } \\
\text { Number of targeted cores positive }\end{array}$ & $1(1-2)$ & $1(1-2)$ \\
Percentage of biopsy tissue positive & $35(5-60)$ & $1(0-1)$ \\
$(\%)$ & & \\
Grade group of targeted biopsies & $11-50)$ \\
0 & $11(24 \%)$ & $2(33 \%)$ \\
1 & $17(37 \%)$ & $2(33 \%)$ \\
2 & $17(37 \%)$ & $2(33 \%)$ \\
3 & $1(2 \%)$ & $0(0 \%)$ \\
\hline *Values are the median (interquartile range) or number (\%). \\
\hline
\end{tabular}

were included in this study. A Youden cutpoint was used to determine the eA in the axial plan (perpendicular to the plane of biopsy) best predicting for $\geq$ GG 2 disease within the TBx cores. Logistic regression, including PIRADS score, nodule location, and number of TBx cores, was then used to determine predictive power of the cutpoint.

Results: Median time from MR imaging to TBx was 2.4 (1.4-5.5) months. Forty of $46(87 \%)$ patients had one nodule and six of $46(13 \%)$ had two separate nodules on MR that received TBx. Of the 52 nodules, five $(10 \%)$, $33(63 \%)$, and $14(27 \%)$ were PIRADS 3,4 , and 5, respectively. Thirteen $(25 \%)$, six $(12 \%)$, and $33(64 \%)$ were in the anterior, medial, and posterior regions of the prostate, respectively. Median eA was $0.72(0.49-1.29) \mathrm{cm}^{2}$ (average diameter $9.5 \mathrm{~mm}$ ). TBx results are presented in Table 1. Fifteen of $46(33 \%)$ patients had $\geq 1 \mathrm{RBx}$ core and 20/52 (38\%) nodules had $\geq 1$ TBx revealing $\geq$ GG 2 disease. The optimal eA cutpoint was $\geq 0.7 \mathrm{~cm}^{2}$, with an area under the curve (AUC) of $0.671(0.510-0.832)$. Sixteen of $28(57 \%)$ nodules with eA $\geq 0.7 \mathrm{~cm}^{2}$ and four of $24(17 \%)$ nodules with eA $<0.7 \mathrm{~cm}^{2}$ harbored $\geq \mathrm{GG} 2$ disease (Fisher's $\left.\mathrm{p}=0.004\right)$. On logistic regression, $\mathrm{eA} \geq 0.7 \mathrm{~cm}^{2}$ was solely predictive of TBx revealing $\geq \mathrm{GG} 2$ disease (hazard ratio 6.5 [1.3-32.4]; $p=0.022$ ).

Conclusions: Having an $\mathrm{eA} \geq 0.7 \mathrm{~cm}^{2}$ may predict for transperineal-based TBx being positive for $\geq G G 2$ disease when 1-2 cores are taken.

References

1. Stefanova V, Buckley R, Flax S, et al. Transperineal prostate biopsies under local anesthetic: Experience with 1287 patients prostate cancer detection rate, complications, and patient tolerability. J Urol 2019;201:1121-6. https://doi.org/10.1097/JU.0000000000000156

2. Klotz L, Pond G, Loblaw A, et al. Randomized study of systematic biopsy vs. magnetic resonance imaging and targeted and systematic biopsy in men on active surveillance (ASIST): 2-year postbiopsy followup. Eur Urol 2019;10:007. https://doi.org/10.1016/j. eururo.2019.10.007

\section{UP-1.14}

Utility of magnetic resonance imaging-targeted biopsy in predicting post-radical prostatectomy NCCN risk stratification Kevin Hanna ${ }^{7}$, Amber McMahon ${ }^{1}$, Richard L Sleightholm ${ }^{1}$, Bryant VanLeeuwen', Shawna L. Boyle ${ }^{1}$

${ }^{1}$ Department of Surgery, Division of Urologic Surgery, University of Nebraska Medical Center, Omaha, NE, United States

Introduction: Accurate grading and staging of prostate cancer is paramount in guiding treatment for men with prostate cancer. Historically, there has been significant heterogeneity in NCCN risk stratification from initial biopsy to post-radical prostatectomy (RP). ${ }^{1}$ In recent years, magnetic resonance imaging (MRI)-targeted biopsy has emerged as an improved means of detecting prostate cancer compared to systematic transrectal ultrasonography-guided biopsy (TRUS). ${ }^{2}$ We seek to determine if MRItargeted biopsy more accurately grades prostate cancer compared to systematic TRUS. 


\begin{tabular}{|c|c|c|}
\hline & $\begin{array}{c}\text { TRUS } \\
(n=503)\end{array}$ & $\begin{array}{c}\text { MRI-fusion } \\
\text { biopsy ( } n=74)\end{array}$ \\
\hline Median age (range) & $62(41-78)$ & $65(48-75)$ \\
\hline \multicolumn{3}{|l|}{ Race (\%) } \\
\hline White & $440(88)$ & $67(91)$ \\
\hline Black & $52(10)$ & $4(5)$ \\
\hline Other & $11(2)$ & $3(4)$ \\
\hline $\mathrm{PSA}(\mathrm{ng} / \mathrm{dl})($ mean $\pm \mathrm{SD})$ & $9.22 \pm 8.6$ & $9.98 \pm 8.87$ \\
\hline \multicolumn{3}{|l|}{ Biopsy Gleason grade group (\%) } \\
\hline 1 & $73(14.5)$ & $0(0)$ \\
\hline 2 & $237(47)$ & $32(44)$ \\
\hline 3 & 78 (15.5) & $12(16)$ \\
\hline 4 & $42(8.5)$ & $9(12)$ \\
\hline 5 & $73(14.5)$ & $21(28)$ \\
\hline \multicolumn{3}{|l|}{ RP Gleason grade group (\%) } \\
\hline 1 & $28(5.5)$ & $1(1.3)$ \\
\hline 2 & $239(47.5)$ & $31(42.7)$ \\
\hline 3 & $108(21.5)$ & $21(28)$ \\
\hline 4 & $43(8.5)$ & $3(4)$ \\
\hline 5 & $85(17)$ & $18(24)$ \\
\hline \multicolumn{3}{|c|}{ Biopsy NCCN risk stratification (\%) } \\
\hline 1-Very-low & $23(4.5)$ & $0(0)$ \\
\hline 2-Low & $34(6.75)$ & $0(0)$ \\
\hline 3-Favorable-intermediate & $186(37)$ & $25(34.7)$ \\
\hline 4-Unfavorable-intermediate & $124(24.65)$ & $19(25.3)$ \\
\hline 5-High & $88(17.5)$ & $11(14.7)$ \\
\hline 6-Very-high & $48(9.6)$ & $19(25.3)$ \\
\hline \multicolumn{3}{|l|}{ RP NCCN risk stratification (\%) } \\
\hline 1-Very-low & $1(0.2)$ & $1(0.2)$ \\
\hline 2-Low & $7(1.4)$ & $7(1.4)$ \\
\hline 3-Favorable-intermediate & $190(37.8)$ & $190(37.8)$ \\
\hline 4-Unfavorable-intermediate & $114(22.6)$ & $114(22.6)$ \\
\hline 5-High & $124(24.7)$ & $124(24.7)$ \\
\hline 6-Very-high & $67(13.3)$ & $67(13.3)$ \\
\hline
\end{tabular}

Methods: A retrospective analysis of prostate cancer patients who underwent radical prostatectomy at UNMC between December 2007 and November 2018 identified 503 and 75 patients who received either TRUS or an MRI-fusion biopsy, respectively. Comparisons were made between preoperative and postoperative NCCN risk stratification score, and the use of multivariable analysis (MVA) was used to assess the ability to preoperatively predict NCCN Risk and adjust for potential demographic influences. Results: The mean age of the patients was 62 years and the mean prostatespecific antigen (PSA) level was $9.3 \mathrm{ng} / \mathrm{ml}$ (Table 1). MRI-targeted biopsy correctly predicted post-RP NCCN risk stratification in $70.0 \%$ of patients compared to $10.6 \%$ in the TRUS cohort (Table 2). When stratifying by NCCN risk, the ability of TRUS to accurately predict NCCN risk ranged from $1.1-34.7 \%$, with nearly all incorrect assessments being underpredictions. Conversely, accurate NCCN risk for MRI-targeted biopsy ranged from $57.1-93.3 \%$, with risk scores 3 and 4 showing slight over prediction

\begin{tabular}{|c|c|c|c|}
\hline & $\begin{array}{l}\text { Odds } \\
\text { ratio }\end{array}$ & $95 \% \mathrm{Cl}$ & $\mathbf{p}$ \\
\hline TRUS vs. MRI-targeted biopsy & 20.064 & $11.301-35.621$ & $<0.0001$ \\
\hline PSA & 0.998 & $0.972-1.024$ & 0.8706 \\
\hline Age & 1.035 & $1.001-1.071$ & 0.0414 \\
\hline
\end{tabular}

and risk scores 5 and 6 showing slight underprediction. Upstaging raged from $0.0-37.5 \%$ in the MRI-targeted biopsy vs. $65.0-100.0 \%$ in the TRUS cohort. In the TRUS cohort, $28.03 \%$ of patients were upstaged by more than one stage post-RP compared to only $4.05 \%$ in the MRI-targeted biopsy cohort. On MVA, the odds ratio for predicting NCCN risk was 20.1 times greater for MRI-targeted biopsy versus TRUS $(p<0.0001)$ (Table 3$)$. Conclusions: MRI-targeted biopsy was superior to TRUS in correctly predicting post-RP NCCN risk stratification. Additionally, upstaging occurred much more often in those undergoing TRUS compared to MRI-targeted biopsy. Ultimately, these outcomes have significant implications for the diagnosis and staging of prostate cancer and emphasize the need for transition from TRUS biopsies to MRI-fusion biopsies as the standard of care. References

1. $\mathrm{Xu} \mathrm{N}, \mathrm{Wu} Y \mathrm{P}, \mathrm{Li} \mathrm{XD}$, et al. Risk of upgrading from prostate biopsy to radical prostatectomy pathology: Is magnetic resonance imagingguided biopsy more accurate. J Cancer 2018;9:3634-9. https://doi. org/10.7150/jca.26791

2. Drost FH, Osses D, Nieboer D, et al. Prostate magnetic resonance imaging, with or without magnetic resonance imaging-targeted biopsy, and systematic biopsy for detecting prostate cancer: A Cochrane systematic review and meta-analysis. Eur Urol 2020;77:7894. https://doi.org/10.1016/j.eururo.2019.06.023

\section{UP-1.15}

Is confirmatory biopsy useful prior to radical prostatectomy for patients on active surveillance who receive magnetic resonance imaging?

Paulo H Werlang', Michael Horrigan', Luke T. Lavallée', Ilias Cagiannos', Christopher G. Morash', Rodney H. Breau'

${ }^{1}$ Urology, University of Ottawa, Ottawa, ON, Canada

Introduction: Patients on active surveillance for low-grade prostate cancer may receive prostate magnetic resonance imaging (MRI) as part of routine followup or due to suspicion of an occult higher-grade lesion. In patients who underwent MRI and subsequently were treated with radical prostatectomy (RP), we aimed to determine if pre-prostatectomy prostate biopsy was useful prior to treatment.

Methods: As part of the prostate cancer Surgeon Report Card (SuRep) study, we identified active surveillance patients who had an MRI prior to RP. Based on clinical discretion, some patients received a post-MRI biopsy prior to RP, while others did not. We compared pathologic outcomes between these groups.

Results: Between 2015 and 2019, 70 patients met inclusion criteria. Twenty-three (33\%) received a prostate biopsy prior to RP. Of the biopsy cohort, the PIRADS were $\leq 3,4$, and 5 in four $(17 \%), 11(48 \%)$, and eight (35\%) patients, respectively. Corresponding PIRADS in the no-biopsy cohort was six (13\%), $11(23 \%)$, and $30(64 \%)$, respectively. In the biopsy cohort $(n=23)$, only one $(4 \%)$ had a post-MRI/pre-treatment biopsy with

\begin{tabular}{|c|c|c|c|c|c|c|c|c|c|}
\hline & & -4 & -3 & -2 & -1 & 0 & 1 & 2 & Total \\
\hline \multirow[t]{2}{*}{ TRUS } & $\#$ & 6 & 37 & 98 & 307 & 53 & 2 & 0 & 503 \\
\hline & $\%$ & 1.19 & 7.36 & 19.48 & 61.03 & 10.54 & 0.4 & 0 & 100 \\
\hline \multirow[t]{2}{*}{ MRI } & \# & 0 & 0 & 3 & 3 & 52 & 7 & 9 & 74 \\
\hline & $\%$ & 0 & 0 & 4.05 & 4.05 & 70.27 & 9.46 & 12.16 & 100 \\
\hline
\end{tabular}


grade group $\geq 2$. All of these patients had RP due to an upgraded biopsy ( 1 ; $4 \%$ ) or clinical judgment, such as rising prostate-specific antigen or concerning MRI (22; 96\%). Prostatectomy pathology revealed grade group $\geq 2$ in $15(65 \%)$ patients in the biopsy cohort. In the no-biopsy cohort $(n=47)$, $39(82 \%)$ had grade group $\geq 2$ on prostatectomy pathology. For patients with PIRADS $\leq 3$, two (50\%) had high-grade cancer in the biopsy group compared to five $(83 \%)$ in the no-biopsy group. For patients with PIRADS 4 , eight $(73 \%)$ had high-grade cancer in the biopsy group compared to seven $(64 \%)$ in the no-biopsy group. For patients with PIRADS 5, five $(63 \%)$ had high-grade cancer in the biopsy group compared to $27(90 \%)$ in the no-biopsy group.

Conclusions: In this cohort, we found that omitting post-MRI biopsy prior to RP did not result in significant over treatment regardless of MRI findings. Validation in other cohorts or in a randomized trial is encouraged.

\section{UP-1.16}

Multidisciplinary clinics in prostate cancer management: Knowledge, attitudes, and utilization among urologists

Connor Hoge ${ }^{1}$, Matt Kasson ${ }^{1}$, Tianyuan Guan', Rand Naffouje ${ }^{2}$, Timothy Struve', Sadhna Verma ${ }^{4}$, Abhinav Sidana

'Department of Urology, University of Cincinnati College of Medicine, Cincinnati, $\mathrm{OH}$, United States; ${ }^{2}$ Department of Hematology and Oncology, University of Cincinnati College of Medicine, Cincinnati, $\mathrm{OH}$, United States; ${ }^{3}$ Department of Radiation Oncology, University of Cincinnati College of Medicine, Cincinnati, OH, United States; ${ }^{4}$ Department of Radiology, University of Cincinnati College of Medicine, Cincinnati, $\mathrm{OH}$, United States

Introduction: Multidisciplinary clinics (MDCs) have a growing role in the evaluation and treatment of prostate cancer (PCa). Such a clinic allows for

\section{UP-1.16. Table 1. Characteristics and responses on use of} MDC survey

\begin{tabular}{|c|c|}
\hline \multicolumn{2}{|c|}{ Participate in a MDC? $(n=211)$} \\
\hline Yes & $107(51.0)$ \\
\hline No & $104(49.3)$ \\
\hline Age $(n=172)$, mean $( \pm S D)$ & $50.6(12.1)$ \\
\hline Gender $(n=208)$ & $41(47.1)$ \\
\hline Male & $203(97.6)$ \\
\hline Female & $5(2.4)$ \\
\hline \multicolumn{2}{|l|}{ Practice type $(n=210)$} \\
\hline Academic & $129(61.4)$ \\
\hline Non-academic & $81(38.6)$ \\
\hline \multicolumn{2}{|l|}{ Years in practice $(n=211)$} \\
\hline $0-5$ & $31(14.7)$ \\
\hline $6-10$ & $35(16.6)$ \\
\hline $11-20$ & $60(28.4)$ \\
\hline$>20$ & $85(40.3)$ \\
\hline \multicolumn{2}{|c|}{ Fellowship-trained in urologic oncology $(n=210)$} \\
\hline No & $105(50.0)$ \\
\hline Yes & $105(50.0)$ \\
\hline \multicolumn{2}{|c|}{$\begin{array}{l}\text { Number of patients seen monthly with newly } \\
\text { diagnosed PCa? }(n=211)\end{array}$} \\
\hline None & $4(1.9)$ \\
\hline $1-5$ & $68(32.2)$ \\
\hline $6-10$ & $68(32.2)$ \\
\hline $11-20$ & $46(21.8)$ \\
\hline$>20$ & 25 (11.9) \\
\hline
\end{tabular}

\begin{tabular}{lc}
\hline UP-1.16. Table 2. Responses of survey respondents \\
\hline How beneficial do you feel MDCs are in \\
management of PCa? ( $\mathrm{n}=211)$ & $12(5.7)$ \\
No benefit & $32(15.2)$ \\
Slight benefit & $51(24.2)$ \\
Moderate benefit & $74(35.1)$ \\
Very beneficial & $42(19.9)$ \\
Extremely beneficial & \\
How beneficial do you feel PCa MDCs are in & \\
promoting research ( $=210)$ & $8(4.8)$ \\
No benefit & $21(10.0)$ \\
Slight benefit & $47(22.4)$ \\
Moderate benefit & $95(45.2)$ \\
Very beneficial & $39(18.6)$ \\
Extremely beneficial & \\
MDC patient population ( $\mathrm{n}=107)$ & $73(68.2)$ \\
Gleason 6 & $86(80.4)$ \\
Gleason 7 & $89(83.2)$ \\
Gleason 8-10 & $61(57.0)$ \\
Active surveillance & $78(72.9)$ \\
Advanced/metastatic & $40(37.4)$ \\
Recurrent disease & \\
Reasons for not participating in MDC ( $\mathrm{n}=104)$ & $11(10.6)$ \\
Lack of benefit & $34(32.7)$ \\
Insufficient time & $23(22.1)$ \\
Cost & $68(65.4)$ \\
Lack of infrastructure & \\
\hline
\end{tabular}

synchronous care, where multiple specialists see the patient at the same location and day. We aim to evaluate the current knowledge, attitude, and practice patterns of urologists regarding PCa MDC, as well as analyze predictors for their utilization.

Methods: A 14-item questionnaire was designed to collect information on urologists' preferences and practice patterns regarding MDCs. The survey was sent to the members of the Society of Urologic Oncology and the Endourological Society. Data was analyzed using R (R Core team, 2017). Results: A total of 211 responses were received and summarized in Tables 1 and 2 . Overall, only $50.1 \%$ of respondents use MDCs, of which the majority $(65.4 \%)$ of participants practice in an academic institution. However, only $53.3 \%$ of oncology fellowship-trained urologists reported participating in a MDC; $94.3 \%$ of all urologists surveyed felt MDCs were useful for PCA treatment. A large majority of surgeons feel that MDCs are at least moderately beneficial for PCa treatment $(79.0 \% ; \mathrm{p}<0.001)$. Additionally, surgeons participating in MDCs were $2.58 x$ more likely to choose "extremely" beneficial than those who do not (odds ratio 2.58; $\mathrm{p}=0.008$ ). Clinically localized high-risk PCa (Gleason 8-10) constitute the most common reason for referral to MDC $(83.2 \%)$. Of those who do not use a MDC, the most common reasons cited were lack of infrastructure $(65.4 \%)$ and time $(32.7 \%)$. Age, practice type, years of practice, and number of newly diagnosed PCa patients seen per month were not significant predictors for use of MDCs.

Conclusions: Despite literature indicating the utility of MDCs, as well as widespread belief in their efficacy, many surgeons deny participating in them. Further studies are necessary to pursue potential solutions to major barriers of MDC adaptation. 


\section{UP-1.17}

Pathologic markers of prostate cancer disease severity in patients with a family history of breast cancer

Kevin Hanna ${ }^{1}$, Amber McMahon ${ }^{1}$, Bryant VanLeeuwen', Shawna L. Boyle ${ }^{1}$ Department of Surgery, Division of Urologic Surgery, University of Nebraska Medical Center, Omaha, NE, United States

Introduction: Family history is a well-described risk factor for prostate cancer, and recent studies have shown an association of familial breast cancer in first-degree relatives with increased risk of prostate cancer and lethal disease. ${ }^{1}$ This study seeks to identify markers of high-risk prostate cancer in patients with a family history of breast cancer in first-degree relatives. Methods: This was a single-institution, retrospective study of men with prostate cancer who underwent biopsy and a subsequent radical prostatectomy between December 2007 and November 2018. Men with a family history of breast cancer $(\mathrm{FHx})$ in a first-degree relative $(n=60)$ were matched based on age and race to men without family history of breast cancer $(n=60)$. Analysis for pathologic markers of high-risk disease, including prostate-specific antigen (PSA) at time of diagnosis, positive surgical margins, extra-prostatic extension, perineural invasion, Gleason grade group greater than 3 , and NCCN risk stratification greater than favorable-intermediate were made using univariate analysis.

Results: The median age of the patients in both cohorts was 60 years. The mean PSA in the FHx cohort was 9.12, while the mean PSA in the controls was $7.02(p=.026)$ (Table 1). FHx was associated with an increased risk of extra-prostatic extension (odds ratio [OR] 2.45; 95\% confidence interval $[\mathrm{CI}] 1.13-5.32 ; \mathrm{p}=0.023)$. $\mathrm{FHx}$ was not associated with positive surgical margins (OR 1.76; 95\% Cl 0.74-4.17; $\mathrm{p}=0.198$ ), perineural invasion (OR 0.54; 95\% Cl 0.26-1.126; $\mathrm{p}=0.101)$, Gleason grade group greater than 3 (OR 1.71; 95\% Cl 0.831-3.536; $p=0.145)$, or NCCN risk stratification greater than favorable-intermediate (OR 1.76 95\% Cl 0.838-3.72; $\mathrm{p}=0.135$ ) (Table 2).

Conclusions: Family history of breast cancer was associated with higher PSA at time of diagnosis and extra-prostatic extension upon final surgical

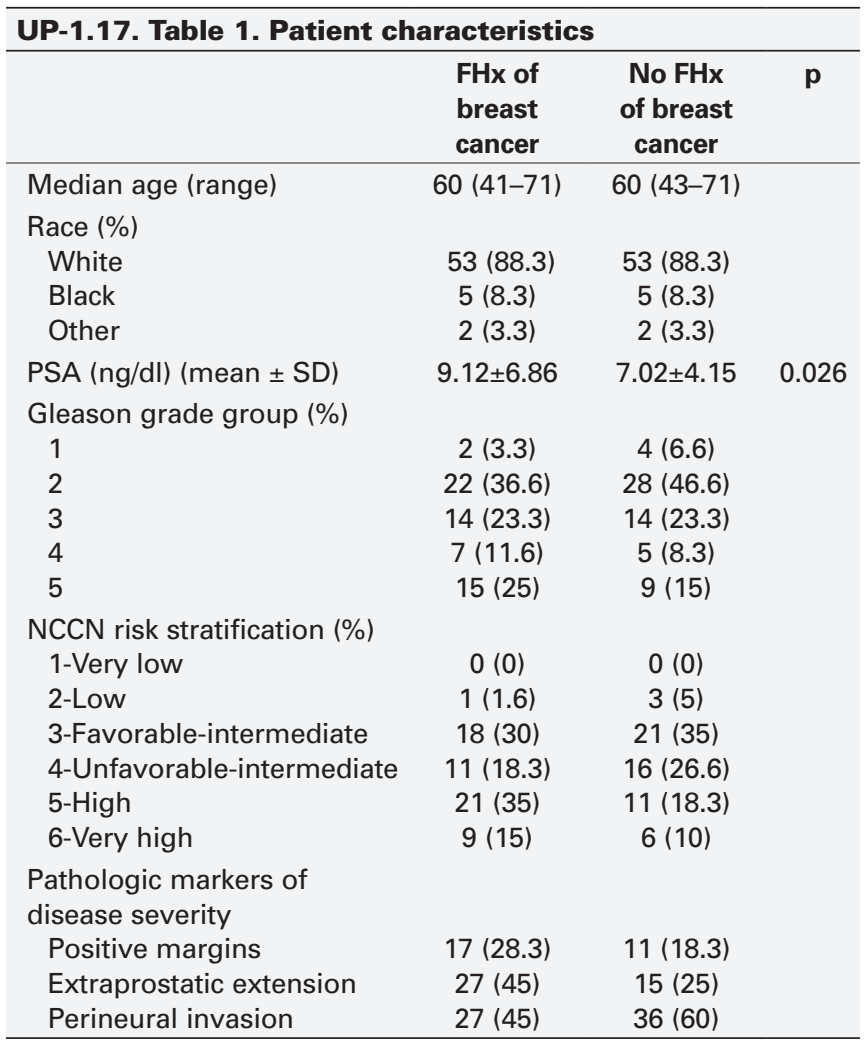

\begin{tabular}{|c|c|c|c|}
\hline Pathologic marker & Odds ratio & $95 \% \mathrm{Cl}$ & $\mathbf{p}$ \\
\hline Positive margins & 1.76 & $0.74-4.17$ & 0.198 \\
\hline Extraprostatic extension & 2.45 & $1.13-5.32$ & 0.023 \\
\hline Perineural invasion & 0.54 & $0.264-1.126$ & 0.101 \\
\hline Grade group $>3$ & 1.71 & $0.831-3.536$ & 0.145 \\
\hline NCCN risk $>3 *$ & 1.76 & $0.838-3.72$ & 0.135 \\
\hline
\end{tabular}

*for NCCN risk, 1=very low, 2=low, 3=favorable-intermediate, 4=unfavorable-intermediate, $5=$ high, $6=$ very high.

pathology. These findings further suggest the need for specified surveillance or genetic counselling for men with such familial history.

Reference

1. Barber L, GerkeT, Markt SC, et al. Family history of breast or prostate cancer and prostate cancer risk. Clin Cancer Res 2018;24:5910-7. https://doi.org/10.1158/1078-0432.CCR-18-0370

\section{UP-1.18}

Association between pre-treatment total testosterone and oncological outcomes following radical prostatectomy lin Kvu (lustin) Kim ${ }^{1}$, Antonio Finelli' ${ }^{1}$, Eshetu Atenafu ${ }^{2}$, Neil E. Fleshner ${ }^{1}$, Nathan Perlis', Greg Nason', Girish S. Kulkarni', Robert J. Hamilton' ${ }^{1}$ Division of Urology, Department of Surgery, Princess Margaret Cancer Centre, Toronto, ON, Canada; ${ }^{2}$ Biostatistics, Princess Margaret Cancer Centre, Toronto, ON, Canada

Introduction: Studies examining the association between pre-prostatectomy serum total testosterone and outcomes after radical prostatectomy (RP) have been conflicting. We aimed to add clarity by exploring this association in our large RP database.

Methods: A retrospective review of patients with available pre-treatment serum total testosterone from 2005-2015 was performed using the institutional RP database. The time period was chosen as pre-treatment serum total testosterone value was reliably collected from 2005-2015. Patients who underwent neoadjuvant hormonal therapy or undergoing salvage RP were excluded. Clinical/demographic data were retrieved from electronic patient records. Testosterone was modelled as quintiles in categorical analyses and log-transformed for continuous analyses. Association between testosterone and pathologic outcomes, time to biochemical recurrence, metastases, and initiation of androgen deprivation therapy (ADT) or radiation were explored with univariate and multivariate analyses.

Results: A total of 2654 patients were included. Baseline characteristics were balanced across quintiles of testosterone (Table 1). Lower pretreatment testosterone quintile was associated with lesser likelihood of upgrade from biopsy to prostatectomy $(20.8 \%, 20.3 \%, 22.1 \%, 27.0 \%$, $26.0 \%$, respectively; $\mathrm{p}=0.026$ ), but higher rate of positive surgical margins $(24.7 \%, 18.8 \%, 18.8 \%, 20.5 \%, 16.1 \%$, respectively; $p=0.012)$ (Table 2$)$. After multivariate adjustment, only the association with surgical margins remained significant (odds ratio [OR] $0.654 ; 95 \%$ confidence interval [Cl] 0.511-0.838; $\mathrm{p}<0.001$ ) (Table 3). No association was noted between testosterone and time to biochemical recurrence, metastasis, salvage ADT, or radiation (Table 4).

Conclusions: Among patients undergoing RP, lower pre-treatment testosterone was associated with a greater risk of positive surgical margins, but this did not translate into adverse distant outcomes. Therefore, the utility of total testosterone for pre-treatment risk calculation may be limited. 


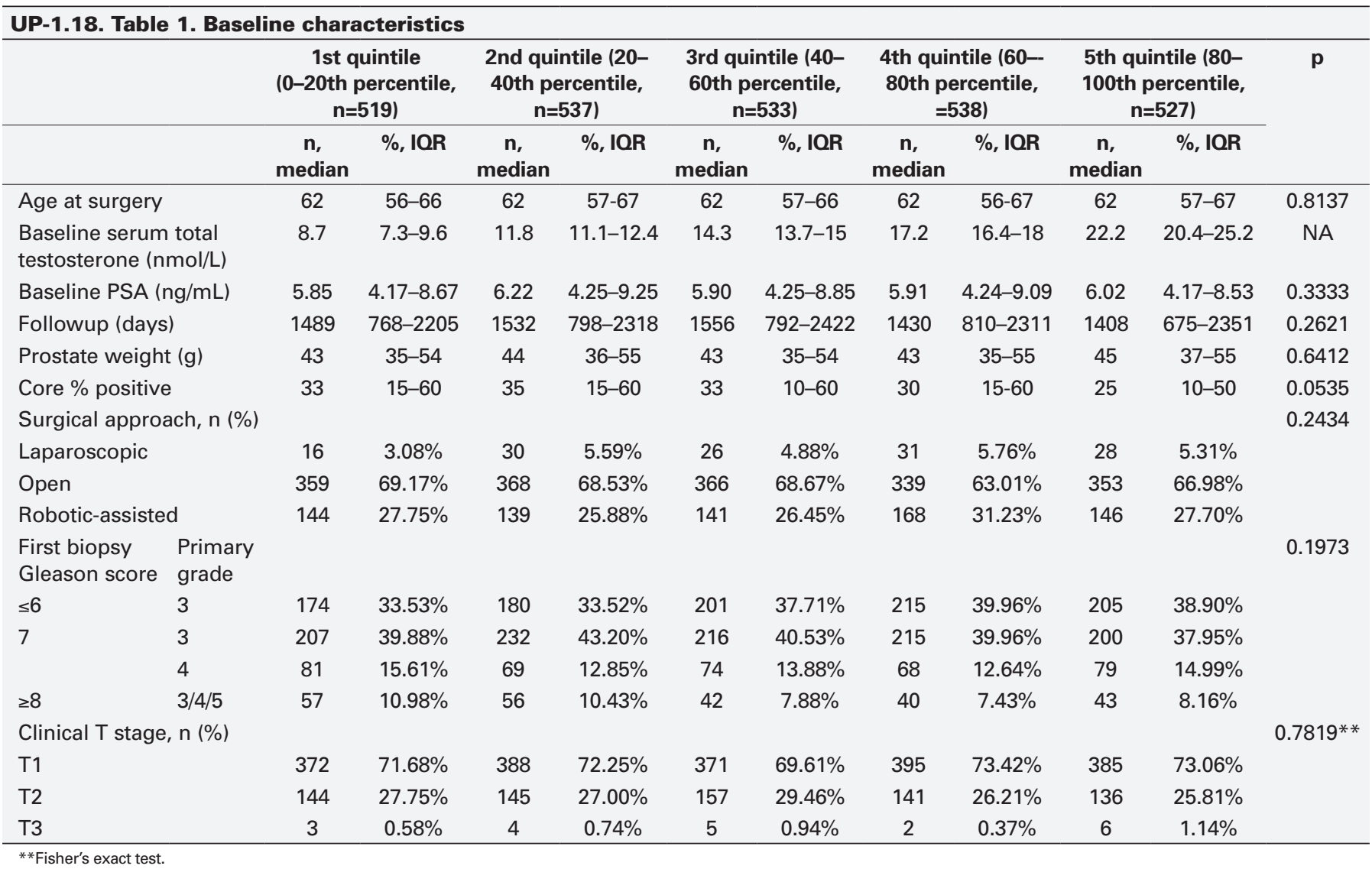

\section{UP-1.19}

A phase $2 \mathrm{~b}$, randomized, double-blind, placebo-controlled trial evaluating the effects of monoacylglyceride-conjugated eicosapentaenoic acid (MAG-EPA)-rich fish oil supplementation before radical prostatectomy on prostate cancer proliferation Marie-Hélène Guertin ${ }^{1,2}$, Karine Robitaille', Jean-François Pelletier ${ }^{1}$, Molière Nguile-Makao ${ }^{1}$, Hélène Hovington' ${ }^{1}$, Caroline Léger ${ }^{1}$, Yves Fradet ${ }^{1,3}$ Arnaud Marien ${ }^{3}$, Frédéric Pouliot $t^{1,3}$, Louis Lacombe ${ }^{1,3}$, Rabi Tiguert $^{3}$, Yves $^{2}$ Caumartin ${ }^{3}$, Thierry Dujardin ${ }^{3}$, Paul Toren ${ }^{1,3}$, Michele Lodde ${ }^{3}$,Etienne Racine ${ }^{4}$, Dominique Trudel ${ }^{5}$, Martine Périgny ${ }^{4}$, Thierry Duschesne 6 , Josée Savard ${ }^{1}$, Pierre Julien ${ }^{7}$, Isabelle Bairati ${ }^{1}$, Vincent Fradet ${ }^{1,3}$

${ }^{1}$ Oncology Unit, Centre de recherche du CHU de Québec-Université Laval, Quebec City, QC, Canada; ${ }^{2}$ Institut National de Santé Publique du Québec, Quebec City, QC, Canada; ${ }^{3}$ Centre de Recherche Clinique et Évaluative en Oncologie de L'Hôtel-Dieu de Québec, Quebec City, QC, Canada; ${ }^{4}$ Pathology, CHU de Québec-Université Laval, Quebec City, QC, Canada; ${ }^{5}$ Pathology and Cellular Biology, Université de Montréal, Montreal, QC, Canada; ${ }^{6}$ Mathematics and Statistics, Université Laval, Quebec City, QC, Canada; 'Endocrinology and Nephrology Unit, Centre de recherche du CHU de Québec-Université Laval, Quebec City, QC, Canada

Introduction: Some environmental factors, such as a diet rich in longchain omega-3 polyunsaturated fatty acids ( $\mathrm{LCn} 3)$, are thought to beneficially impact prostate cancer. Our team conducted a randomized controlled trial (RCT) to assess whether a short-term supplementation with concentrated monoacylglyceride-conjugated eicosapentaenoic acid (MAG-EPA), an LCn3 subtype, compared to placebo, affected the cancer proliferation index measured by nuclear Ki-67 expression in the prostate. Methods: A double-blind, phase 2b RCT was conducted at the CHU de Québec-Université Laval (NCT02333435). Participants were men diag- nosed with prostate cancer grade group $\geq 2$, for which radical prostatectomy (RP) was the chosen primary treatment. Participants were randomized to receive either $3 \mathrm{~g}$ of MAG-EPA daily $(n=65)$ or the equivalent in placebo $(n=65)$ for $4-10$ weeks prior to RP. The primary outcome for the study was nuclear Ki-67 expression in prostate tumor cells at RP, assessed as a percentage of total tumor cells measured by software image analysis (CaloPix, TRIBVN) on standardized tissue microarrays.

Results: Final analyses included 60 patients $(92 \%)$ in the intervention arm and 62 patients (95\%) in the placebo arm. Average Ki-67 expression in tumor sites was not different between the intervention $(4.88 \%)$ and the placebo $(4.18 \%)$ group (t-test $p=0.16)$. Average Ki-67 expression in the primary tumor site was also not different for the intervention $(4.70 \%)$ and placebo $(4.10 \%)$ group (t-test $\mathrm{p}=0.21)$. Per-protocol analyses and analyses adjusted for cancer characteristics at baseline (grade and prostate-specific antigen level) did not suggest differences between the two groups either.

Conclusions: A MAG-EPA intervention for 4-10 weeks before RP did not affect cancer proliferation in prostate tissue compared to placebo. This trial will also provide information on other important outcomes, such as quality of life and inflammatory biomarkers, as the intervention was pursued for one-year post-RP. 
UP-1.18. Table 2. Pathological outcomes

\begin{tabular}{|c|c|c|c|c|c|c|c|c|c|c|c|c|}
\hline & & \multicolumn{2}{|c|}{$\begin{array}{c}\text { 1st quintile (0- } \\
\text { 20th percentile, } \\
n=519)\end{array}$} & \multicolumn{2}{|c|}{$\begin{array}{c}\text { 2nd quintile (20- } \\
\text { 40th percentile, } \\
\quad n=537)\end{array}$} & \multicolumn{2}{|c|}{$\begin{array}{l}\text { 3rd quintile (40- } \\
60 \text { th percentile, } \\
\quad n=533)\end{array}$} & \multicolumn{2}{|c|}{$\begin{array}{c}\text { 4th quintile (60-- } \\
\text { 80th percentile, } \\
n=538)\end{array}$} & \multicolumn{2}{|c|}{$\begin{array}{c}\text { 5th quintile (80- } \\
\text { 100th percentile, } \\
\quad n=527)\end{array}$} & \multirow[t]{2}{*}{$\mathbf{p}$} \\
\hline & & $\mathbf{n}$ & $\%$ & $\mathbf{n}$ & $\%$ & $\mathbf{n}$ & $\%$ & $\mathbf{n}$ & $\%$ & $\mathbf{n}$ & $\%$ & \\
\hline$\leq 6$ & 3 & 97 & $18.69 \%$ & 114 & $21.31 \%$ & 125 & $23.50 \%$ & 123 & $22.86 \%$ & 116 & $22.10 \%$ & \\
\hline$\geq 8$ & $3 / 4 / 5$ & 40 & $7.71 \%$ & 33 & $6.17 \%$ & 25 & $4.70 \%$ & 36 & $6.69 \%$ & 31 & $5.90 \%$ & \\
\hline \multicolumn{2}{|c|}{ Upgrade in Gleason score } & & & & & & & & & & & 0.0256 \\
\hline \multicolumn{2}{|l|}{ No } & 411 & $79.19 \%$ & 428 & $79.70 \%$ & 415 & $77.86 \%$ & 393 & $73.05 \%$ & 390 & $74.0 \%$ & \\
\hline \multicolumn{2}{|l|}{ Yes } & 108 & $20.81 \%$ & 109 & $20.30 \%$ & 118 & $22.14 \%$ & 145 & $26.95 \%$ & 137 & $26.0 \%$ & \\
\hline \multicolumn{2}{|c|}{ Increase in score $\geq 2$} & 4 & $0.77 \%$ & 5 & $0.93 \%$ & 3 & $0.56 \%$ & 9 & $1.67 \%$ & 6 & $1.14 \%$ & 0.4310 \\
\hline \multirow{2}{*}{ Yes } & pT3b & 39 & $7.51 \%$ & 45 & $8.38 \%$ & 44 & $8.26 \%$ & 51 & $9.48 \%$ & 47 & $8.92 \%$ & \\
\hline & pT4 & 0 & $0.00 \%$ & 1 & $0.19 \%$ & 2 & $0.38 \%$ & 0 & $0.0 \%$ & 0 & $0.0 \%$ & \\
\hline No & $\mathrm{pT} 2$ & 312 & $60.1 \%$ & 337 & $62.76 \%$ & 350 & $65.67 \%$ & 338 & $62.83 \%$ & 340 & $64.52 \%$ & \\
\hline \multicolumn{2}{|c|}{ Pathology N stage } & & & & & & & & & & & 0.0781 \\
\hline \multicolumn{2}{|l|}{$\mathrm{pNX}$} & 281 & $54.14 \%$ & 315 & $58.66 \%$ & 295 & $55.66 \%$ & 313 & $58.18 \%$ & 327 & $62.29 \%$ & \\
\hline \multicolumn{2}{|l|}{$\mathrm{pNO}$} & 229 & $44.12 \%$ & 206 & $38.36 \%$ & 223 & $42.08 \%$ & 214 & $39.78 \%$ & 181 & $34.48 \%$ & \\
\hline \multicolumn{2}{|l|}{$\mathrm{pN} 1$} & 9 & $1.73 \%$ & 16 & $2.98 \%$ & 12 & $2.26 \%$ & 11 & $2.04 \%$ & 17 & $3.24 \%$ & \\
\hline \multicolumn{2}{|l|}{ Upstage } & & & & & & & & & & & 0.4806 \\
\hline \multicolumn{2}{|l|}{ No } & 331 & $63.78 \%$ & 357 & $66.48 \%$ & 367 & $68.86 \%$ & 359 & $66.73 \%$ & 358 & $67.93 \%$ & \\
\hline \multicolumn{2}{|l|}{ Yes } & 188 & $36.22 \%$ & 180 & $33.52 \%$ & 166 & $31.14 \%$ & 179 & $33.27 \%$ & 169 & $32.07 \%$ & \\
\hline
\end{tabular}

UP-1.18. Table 3. Multivariate analysis of positive surgical margin involvement and upgrade

\begin{tabular}{ll}
\hline $\begin{array}{l}\text { Upgrade } \\
\text { Effect }\end{array}$ & Odds ratio (95\% Cl) \\
$\begin{array}{l}\text { Pre-treatment serum total testosterone } \\
\text { (log-transformed) }\end{array}$ & $1.273(0.954-1.698)$ \\
Age & $1.029(1.013-1.046)$ \\
Baseline PSA & $1.051(1.035-1.068)$ \\
Clinical T stage & $1.212(1.001-1.468)$ \\
First positive biopsy Gleason score & $0.051(0.039-0.066)$ \\
Surgical margins & \\
Effect & Odds ratio (95\% Cl) \\
$\quad$ Pre-treatment serum total testosterone & $\mathbf{0 . 6 2 8}(0.491-0.804)$ \\
(log-transformed) & \\
Age & $1.004(0.990-1.018)$ \\
Baseline PSA & $1.057(1.042-1.072)$ \\
Clinical T stage & $1.315(1.149-1.504)$ \\
First positive biopsy Gleason score & $1.106(0.959-1.277)$ \\
\hline
\end{tabular}

UP-1.20

Predicting adverse pathological outcomes and biochemical recurrence in pre-prostatectomy patients using different quantification methods of percentage Gleason grade $4 / 5$ disease on prostate biopsy

Lethro C.C. Kwong ${ }^{7}$, Simona Minotti ${ }^{2,3}$, Adrian Cozma ${ }^{4}$, Ashkan Javidan ${ }^{1}$, Amna Ali ${ }^{2}$, Munir A. Jamal ${ }^{5}$, Thomas Short ${ }^{5}$, Frank F. Papanikolaou ${ }^{5}$, John R. Srigley ${ }^{6}$, Andrew H. Feifer ${ }^{2,5}$

${ }^{1}$ Faculty of Medicine, University of Toronto, Toronto, ON, Canada; ${ }^{2}$ Institute for Better Health, Trillium Health Partners, Mississauga, ON, Canada; ${ }^{3}$ Department of Statistics and Quantitative Methods, University of Milano-Bicocca, Milano, Italy; ${ }^{4}$ Department of Radiation Oncology, University of Toronto, Toronto, ON, Canada; ${ }^{5}$ Department of Surgery, University of Toronto, Toronto, ON, Canada; ${ }^{6}$ Department of Laboratory Medicine and Pathobiology, University of Toronto, Toronto, ON, Canada Introduction: Emerging evidence suggests that percentage Gleason grade $4 / 5$ disease $(\% 4 / 5)$ adds prognostic value to predicting adverse pathological outcomes and biochemical recurrence (BCR) in pre-prostatectomy patients. We compared three quantification methods of $\% 4 / 5$ with conventional Gleason parameters in predicting these outcomes.

Methods: Patients who underwent an open retropubic radical prostatectomy from 2002-2016 were included. Adverse pathological outcomes included extra-prostatic extension (EPE), regional lymph node involvement (RLNI), seminal vesicle invasion (SVI), lymphovascular invasion (LVI), and positive margins (PSM). BCR was defined as prostate-specific antigen (PSA) $>0.2 \mathrm{ng} / \mathrm{mL}$. We used multivariate logistic regression to 


\begin{tabular}{ll}
\hline $\begin{array}{l}\text { UP-1.18. Table 4. Cox regression analysis of clinical } \\
\text { outcomes assessed }\end{array}$ \\
\hline Time to biochemical recurrence & \\
Effect & Odds ratio (95\% Cl) \\
$\quad$ Pre-treatment serum total testosterone & $0.959(0.774-1.130)$ \\
$\quad$ (log-transformed) & \\
Age & $1.009(0.996-1.022)$ \\
Baseline PSA & $1.022(1.016-1.029)$ \\
Clinical T stage & $1.159(1.040-1.292)$ \\
First positive biopsy Gleason score & $1.523(1.362-1.705)$ \\
Time to radiation & \\
Effect & Odds ratio (95\% Cl) \\
Pre-treatment serum total testosterone & $0.925(0.683-1.252)$ \\
(log-transformed) & \\
Age & $1.005(0.987-1.023)$ \\
Baseline PSA & $0.986(0.965-1.007)$ \\
Clinical T stage & $0.747(0.600-0.931)$ \\
First positive biopsy Gleason score & $1.204(1.007-1.439)$ \\
Time to androgen deprivation therapy & \\
Effect & Odds ratio (95\% Cl) \\
Pre-treatment serum total testosterone & $0.834(0.571-1.218)$ \\
(log-transformed) & \\
Age & $1.004(0.981-1.028)$ \\
Baseline PSA & $1.019(1.012-1.026)$ \\
Clinical T stage & $1.484(1.283-1.715)$ \\
First positive biopsy Gleason score & $2.076(1.725-2.498)$ \\
Time to metastasis & \\
Effect & Odds ratio (95\% Cl) \\
Pre-treatment serum total testosterone & $0.781(0.446-1.370)$ \\
(log-transformed) & \\
Age & $1.020(0.982-1.059)$ \\
Baseline PSA & $1.011(0.998-1.024)$ \\
Clinical T stage & $(1.302-1.989)$ \\
First positive biopsy Gleason score & $(1.661-2.882)$ \\
\hline
\end{tabular}

build predictive models based on conventional parameters (i.e., PSA, Gleason score) with or without quantitative, quartile, or decile $\% 4 / 5$. The Memorial Sloan Kettering Cancer Center (MSKCC) pre-prostatectomy nomogram was used as a reference standard for our base model. We set sensitivity $\geq 0.80$ and compared performance metrics for each outcome. Results: In all, 756 patients were included. Patient disease characteristics were: $43.8 \%$ EPE, $2.3 \%$ RLNI, $11.5 \%$ SVI, $11.8 \%$ LVI, $33.6 \%$ PSM, and $14.6 \%$ BCR. Our models compared favorably to the MSKCC nomogram (Table 1). For SVI, inclusion of quartile and decile $\% 4 / 5$ achieved areas under curve (AUC) of 0.811 and 0.829 vs. 0.779 for the MSKCC nomogram ( $\mathrm{p}=0.05$ and 0.01 , respectively). For BCR, the model, which included decile $\% 4 / 5$, outperformed the MSKCC nomogram with AUC 0.744 vs. $0.711(p=0.04)$. For all other outcomes, inclusion of $\% 4 / 5$ to the base model generally resulted in improvements across all performance metrics, however, this did not reach statistical significance.

Conclusions: Our findings support the use of $\% 4 / 5$, particularly quartile and decile $\% 4 / 5$, to improve predictions of adverse pathological outcomes and BCR. These parameters may improve the performance of future iterations of widely used nomograms.

\section{UP-1.21}

Economic evaluation of robot-assisted radical prostatectomy compared to open radical prostatectomy for prostate cancer treatment in Ontario, Canada

Anna Parackal' ${ }^{1}$, Jean-Eric Tarride ${ }^{1,2,3}$, Feng Xie ${ }^{2,3}$, Gord Blackhouse ${ }^{1,2}$, Jen Hoogenes $^{2,4}$, Paul Cheon ${ }^{4}$, Daniell Hylton ${ }^{5}$, Edward D. Matsumoto ${ }^{4}$, Wael Hanna ${ }^{5}$, Anthony Adili ${ }^{6}$, Bobby Shayegan ${ }^{4}$

${ }^{1}$ Programs for Assessment of Technology in Health, McMaster University, Hamilton, ON, Canada; ${ }^{2}$ Department of Health Research Methods, Evidence and Impact, McMaster University, Hamilton, ON, Canada; ${ }^{3}$ Centre for Health Economics and Policy Analysis, McMaster University, Hamilton, ON, Canada; ${ }^{4}$ Department of Surgery, Division of Urology, McMaster University, Hamilton, ON, Canada; ${ }^{5}$ Department of Surgery, Division of Thoracic Surgery, McMaster University, Hamilton, ON, Canada; ${ }^{6}$ Department of Surgery, Division of General Surgery, McMaster University, Hamilton, ON, Canada

Introduction: Recent health technology assessments (HTAs) of robotassisted radical prostatectomy (RARP) in Ontario and Alberta, Canada, resulted in opposite recommendations, calling into question whether benefits of RARP offset the upfront investment. Therefore, the study objectives were to conduct a cost-utility analysis from a Canadian public payer perspective to determine the cost-effectiveness of RARP.

Methods: Using a 10-year time horizon, a five-state Markov model was developed to compare RARP to open radical prostatectomy (ORP). Clinical parameters were derived from Canadian observational studies and a recently published systematic review. Costs, resource utilization, and utility values from recent Canadian sources were used to populate the model. Results were presented in terms of increment costs per quality-adjusted life years (QALYs) gained. A probabilistic analysis was conducted, and uncertainty was represented using cost-effectiveness acceptability curves (CEACs). Deterministic sensitivity analyses were also conducted. Future costs and QALYs were discounted at 1.5\%

Results: Total costs of RARP and ORP were \$57 925.44 and \$55 524.18, respectively. Total estimated QALYs were 7.180 and 7.114 for RARP and ORP, respectively. The estimated incremental cost-utility ratio (ICUR) was $\$ 36564.69$ in the base-case analysis. At a willingness-to-pay threshold of $\$ 50000$ and $\$ 100000$ per QALY gained, the probability of RARP being cost-effective was 0.57 and 0.77 , respectively. The model was most sensitive to the time horizon.

Conclusions: The results of this analysis suggest that RARP is likely to be cost-effective in this Canadian patient population. The results are consistent with Alberta's HTA recommendation and other economic evaluations, but challenges Ontario's reimbursement decision.

\section{UP-1.22}

Effects of omega-3 fatty acid supplements on perioperative blood loss during radical prostatectomy

Samuel Fradet ${ }^{1}$, Karine Robitaille ${ }^{1}$, Jean-François Pelletier ${ }^{1}$, Louis Lacombe ${ }^{1,2}$, Frédéric Pouliot ${ }^{1,2}$, Paul Toren ${ }^{1,2}$, Yves Fradet ${ }^{1,2}$, Michele Lodde $^{2}$, Rabi Tiguert ${ }^{2}$, Thierry Dujardin' ${ }^{2}$, Josée Savard', Isabelle Bairati', Narcisse Singbo ${ }^{3}$, Vincent Fradet ${ }^{1,2}$

'Oncology Unit, Centre de Recherche du CHU de Québec-Université Laval, Quebec City, QC, Canada; ${ }^{2}$ Centre de Recherche Clinique et Évaluative en Oncologie de L'Hôtel-Dieu de Québec, Quebec City, QC, Canada; ${ }^{3}$ Plateforme de Recherche Clinique et Évaluative, $\mathrm{CHU}$ de Québec-Université Laval, Quebec City, QC, Canada

Introduction: Many dietary supplements, including long-chain omega-3 fatty acids (LC $\Omega 3$ ), are suspected to affect blood coagulation and platelet function. Despite no clinical evidence, discontinuation is recommended before radical prostatectomy (RP). However, LC $\Omega 3$ could be beneficial against prostate cancer progression. This study aims to determine the effects of LC $\Omega 3$ supplements on perioperative bleeding, hemoglobin, platelets, and postoperative complications at RP.

Methods: This is a planned secondary analysis of all 130 patients diagnosed with prostate cancer grade group 2 or greater enrolled in a randomized controlled trial (NCT02333435) testing the effects of LC 23 , 


\begin{tabular}{|c|c|c|c|c|c|c|c|c|}
\hline Outcome & Accuracy & $\begin{array}{c}\text { Misclassification } \\
\text { error }\end{array}$ & Sensitivity & Specificity & PPV & NPV & AUC (95\% Cl) & p-value ${ }^{2}$ \\
\hline \multicolumn{9}{|l|}{ EPE (incidence $=43.8 \%$ ) } \\
\hline MSKCC & 0.62 & 0.38 & 0.80 & 0.47 & 0.56 & 0.73 & $0.697(0.648-0.746)$ & Reference \\
\hline Base & 0.61 & 0.39 & 0.80 & 0.47 & 0.55 & 0.72 & $0.695(0.646-0.743)$ & 0.28 \\
\hline Base + decile $\% 4 / 5$ & 0.65 & 0.35 & 0.80 & 0.52 & 0.58 & 0.76 & $0.719(0.672-0.767)$ & 0.08 \\
\hline \multicolumn{9}{|l|}{ RLNI (incidence $=2.3 \%$ ) } \\
\hline MSKCC & 0.52 & 0.48 & 0.93 & 0.51 & 0.05 & 1.00 & $0.781(0.720-0.842)$ & Reference \\
\hline Base & 0.50 & 0.50 & 0.93 & 0.48 & 0.05 & 1.00 & $0.775(0.709-0.841)$ & 0.30 \\
\hline Base + quantitative $\% 4 / 5$ & 0.56 & 0.44 & 0.86 & 0.55 & 0.06 & 0.99 & $0.790(0.729-0.850)$ & 0.24 \\
\hline Base + quartile $\% 4 / 5$ & 0.64 & 0.36 & 0.93 & 0.63 & 0.08 & 1.00 & $0.793(0.732-0.855)$ & 0.46 \\
\hline Base + quantitative $\% 4 / 5$ & 0.68 & 0.32 & 0.80 & 0.66 & 0.27 & 0.96 & $0.798(0.740-0.857)$ & 0.14 \\
\hline Base + quartile $\% 4 / 5$ & 0.70 & 0.30 & 0.80 & 0.69 & 0.28 & 0.96 & $0.811(0.754-0.868)$ & 0.05 \\
\hline Base + decile $\% 4 / 5$ & 0.68 & 0.32 & 0.80 & 0.66 & 0.26 & 0.96 & $0.829(0.776-0.882)$ & 0.01 \\
\hline \multicolumn{9}{|l|}{ LVI (incidence = 11.8\%) } \\
\hline Base & 0.49 & 0.51 & 0.80 & 0.44 & 0.18 & 0.94 & $0.689(0.613-0.766)$ & Reference \\
\hline Base + quantitative $\% 4 / 5$ & 0.55 & 0.45 & 0.80 & 0.52 & 0.18 & 0.95 & $0.713(0.636-0.789)$ & 0.21 \\
\hline Base + quartile $\% 4 / 5$ & 0.55 & 0.45 & 0.80 & 0.51 & 0.18 & 0.95 & $0.710(0.636-0.784)$ & 0.27 \\
\hline Base + decile $\% 4 / 5$ & 0.56 & 0.44 & 0.80 & 0.53 & 0.18 & 0.95 & $0.728(0.653-0.802)$ & 0.10 \\
\hline \multicolumn{9}{|l|}{ PSM (incidence = 33.6\%) } \\
\hline Base & 0.48 & 0.52 & 0.80 & 0.31 & 0.39 & 0.75 & $0.642(0.587-0.696)$ & Reference \\
\hline Base + quantitative $\% 4 / 5$ & 0.51 & 0.49 & 0.80 & 0.35 & 0.40 & 0.77 & $0.644(0.589-0.698)$ & 0.54 \\
\hline
\end{tabular}

on prostate cancer biological outcomes at RP as primary outcomes. The LC $\Omega 3$ intervention (3 g/day of monoacylglyceride-conjugated eicosapentaenoic acid [MAG-EPA]) or placebo was given 4-10 weeks prior to RP. Patients taking dietary supplements were excluded. Patients' charts were reviewed for data extraction. Variables were normalized to preferentially use parametric models (linear regression); otherwise, non-parametric quantile regression models were fitted. All models were adjusted for seven confounding variables judged clinically relevant (surgical approach, body mass index [BMI], waist size, NCCN risk, and preoperative intake of aspirin, vitamin-K-agonists, or direct oral anticoagulants).

Results: We found no effect of $\mathrm{LC} \Omega 3$ vs. placebo on perioperative estimated blood loss (EBL) (adjusted EBL difference of $30.0 \mathrm{~mL} ; \mathrm{p}=0.75$ ), lab tests (adjusted hemoglobin difference of $2.6 ; \mathrm{p}=0.48$ ) or postoperative complications (adjusted difference of $0.1 ; p=0.4$ ). In contrast, as expected, we found a significant increase in perioperative EBL in open retropubic RP compared to robot-assisted RP (adjusted difference of $115.8 \mathrm{~mL} ; \mathrm{p}=0.04$ ). Conclusions: Our results suggest that $\Omega 3$ supplements can be safely taken before RP intervention without increasing the risk of surgical bleeding. These findings are particularly relevant since $\Omega 3$ are thought to decrease prostate cancer recurrence after RP.
UP-1.23

Determinants of the perceived financial burden associated with prostate cancer: $A$ remote region patient perspective

Abir El-Haouly' ${ }^{1}$, Anaïs Lacasse ${ }^{1}$, Hares El-Rami², Frédéric Liandier ${ }^{2}$, Alice Dragomir $^{3}$

${ }^{1}$ Sciences de la santé, Université du Québec en Abitibi-Témiscamingue, Rouyn-Noranda, QC, Canada; ${ }^{2}$ Chirurgie, Centre intégré de santé et de services sociaux de l'Abitibi-Témiscamingue, Rouyn-Noranda, QC, Canada; Université du Québec en Abitbi-Témiscamingue; ${ }^{3}$ Surgery, McGill University, Montreal, QC, Canada

Introduction: In publicly funded healthcare systems, patients do not pay for medical visits but can experience costs stemming from travel or overthe-counter drugs. ${ }^{1,2}$ We lack information about the extent of this burden in Canadian remote regions. This study aimed to describe perceived financial burden and identify factors associated with such a perceived burden among prostate cancer patients living in a remote region of the province of Quebec (Canada).

Methods: A cross-sectional study was conducted among 171 prostate cancer patients who consulted at the outpatient clinic of the Centre hospitalier de Rouyn-Noranda.

Results: A total of $22.3 \%$ of patients reported a moderate, considerable, or unsustainable burden. Multivariable analysis revealed that having 
incurred higher cancer-related out-of-pocket costs (odds ratio [OR] 1.001; $95 \%$ confidence interval $[\mathrm{Cl}] 1.001-1.002)$ and a private drug insurance (vs. public, OR 5.23; 95\% Cl 1.13-24.17) were associated with a greater perceived financial burden. Having better physical health-related quality of life (OR $0.95 ; 95 \% \mathrm{Cl} 0.913-0.997)$, a university education (vs. elementary/high school level, OR $0.03 ; 95 \% \mathrm{Cl} 0.00-0.79$ ), and an income between $\$ 40000$ and $\$ 79999$ (vs. $\leqslant \$ 39,999$, OR 0.15; $95 \% \mathrm{Cl}$ 0.03-0.69) were associated with a lower perceived burden.

Conclusion: Patients perceived financial prostate cancer burden as significant. Greater attention should be paid to the development of services to help patients manage this burden. Conversations between health professional and patients about the financial consequences of prostate cancer treatment are fundamental and may help address patients' perceived financial burden. Optimizing coverage by the public health system and private insurance companies is also essential.

References

1. Régie de l'assurance maladie du Québec. Services couverts et frais accessoires 2019. Available at: www.ramq.gouv.qc.ca. Accessed April 21, 2020.

2. Gouvernement du Canada. Système de soins de santé du Canada 2016. Available at: www.canada.ca. Accessed April 21, 2020.

\section{UP-1.24}

Magnetic resonance imaging-guided transurethral ultrasound ablation (TULSA) in men with localized prostate cancer: Fiveyear followup of a prospective phase 1 study

L.K. Joseph Chin ${ }^{1}$, Shiva Nair', Gencay Hatiboglu' ${ }^{2}$, James Relle ${ }^{3}$, Jason Hafron ${ }^{3}$, Matthias Roethke'2, Maya Mueller-Wolf², David Bonekamp², Zahra Kassam ${ }^{1}$, Robert Staruch ${ }^{4}$, Mathieu Burtnyk ${ }^{4}$, Heinz-Peter Schlemmer ${ }^{2}$, Sascha Pahernik ${ }^{2}$

${ }^{1}$ Division of Urology, Department of Surgery, London Health Sciences Centre, London, ON, Canada; ${ }^{2}$ German Cancer Research Center, University Hospital, Heidelberg, Germany; ${ }^{3}$ Beaumont Health System, Royal Oak, MI, United States; ${ }^{4}$ Profound Medical Inc., Toronto, ON, Canada

Introduction: Magnetic resonance imaging (MRI)-guided transurethral ultrasound ablation (TULSA) is a minimally invasive procedure for customized ablation of prostate tissue. We report five-year outcomes of a single-arm, prospective, phase 1 study in men with localized prostate cancer (PCa).

Methods: The study enrolled 30 men at three sites, with median (interquartile rage [IQR]) age $69(67-71)$ years, stage T1c-T2a, prostate-specific antigen (PSA) $5.8(3.8-8.0) \mathrm{ng} / \mathrm{ml}$, Gleason grade group 1 (GG1) disease in $80 \%$, and GG2 in $20 \%$. TULSA was delivered with $3 \mathrm{~mm}$ margins sparing $10 \%$ viable prostate at the gland periphery. Primary endpoints were safety (adverse events [AE]) and feasibility (spatial precision of ablation). Exploratory outcomes included one-year MRI, one-year and three-year 12-core biopsy, plus five-year PSA, quality of life, and survival.

Results: $\mathrm{AE}$ to one year included urinary tract infection (10 men G2), acute retention (three G1, five G2), and epididymitis (one G3), with no rectal injuries. From one year to five years, there were no new serious/ severe AE. MRI thermometry confirmed ablation precision of $\pm 1.4 \mathrm{~mm}$, with $90 \%$ coverage of the prostate. By one year, PSA decreased $90 \%$ to a nadir of $0.6(0.3-0.8) \mathrm{ng} / \mathrm{ml}$, prostate volume decreased $88 \%(83-95 \%)$. Biopsy at one year identified significant PCa in 9/29 men (31\%), and GG $\geq 1$ in 16/29 (55\%). At three years, 3/22 refused biopsy, $7 / 22$ were positive (two significant). By five years, 16 men completed protocol followup, three withdrew with PSA $<0.4 \mathrm{ng} / \mathrm{ml}, 10$ had salvage therapy without complications (six prostatectomy, three radiation, one laser), and one died of unrelated cause. Of 16 men with complete data, PSA remained at $0.55(0.4-1.2) \mathrm{ng} / \mathrm{ml}$ at five years. International Prostate Symptom Score (IPSS) of $6(5-13)$ returned to $5(4-10)$ by three months and $6.5(6-9)$ at five years. Erections sufficient for penetration were reported by $9 / 16$ at baseline, 11/16 at one year, and 7/16 at five years. Leak-free, pad-free continence was reported by $16 / 16$ at one and five years. Predictors of salvage therapy included lower ablation coverage and higher PSA nadir. Conclusions: MRI-guided TULSA in men with localized PCa demonstrates low toxicity and stable quality of life, while maintaining salvage treatment options.

\section{UP-1.25 \\ Functional and oncological outcomes after prostate cancer focal cryoablation using magnetic resonance imaging-ultrasound fusion technique \\ Jeffrey R. Talarek ${ }^{1}$, Sean Maynor ${ }^{1}$, Juliana Tobler ${ }^{2}$, Sadhna Verma², Abhinav} Sidana

'Department of Urology, University of Cincinnati, Cincinnati, $\mathrm{OH}$, United States; ${ }^{2}$ Department of Radiology, University of Cincinnati, Cincinnati, $\mathrm{OH}$, United States

Introduction: Focal cryoablation (FC) of prostate cancer (PCa) has traditionally used a predefined template or a cognitive interpretation of imaging. These imprecise methods excessively destroy benign tissue because the cancerous area is not localized intraoperatively. magnetic resonance imaging-ultrasound (MRI-US) fusion directs cryoablation, improving accuracy while preserving genitourinary structures and function. Here, we evaluate the short-term functional and oncological outcomes of prostate FC using MRI-US fusion to treat clinically significant (CS) PCa.

Methods: We conducted a retrospective review of consecutive patients undergoing FC using MRI-US fusion with the Artemis transperineal needle attachment, which enables guidance of interventional needles through the perineum. Men with localized intermediate-risk PCa (grade 2 and 3) stage $\mathrm{T} 1-\mathrm{T} 2 \mathrm{cN} 0 \mathrm{M} 0$ disease and prostate-specific (PSA) $\leq 20$ were offered FC. Patients were followed every three months to assess PSA, functional outcomes, and complications. At six months, patients received repeat prostate MRI and MRI-US fusion biopsy of the ablated area, any new lesion, and systematic biopsy.

Results: A total of 20 patients with median followup of 7.9 months (1.520.7 ) were included in the analysis. Median age and pre-procedure PSA were 62.5 (60-70) and $6.0(4.8-10.1)$, respectively. Median post-ablation PSA decline at three, six, and 12 months were $69.6 \%, 65.0 \%$, and $37.2 \%$, respectively. Median change in Sexual Health Inventory for Men (SHIM) and International Prostate Symptom Score (IPSS) at three, six, and 12 mon were $-2.0,-1.5$, and -8.0 , and $-2.0,0.0$, and -4.0 , respectively. No patient developed urinary incontinence or grade 3 or 4 complications; $61.5 \%$ of patients had absence of any PCa and $76.9 \%$ had absence of CS PCa (any pattern 4) at six-month biopsy. Thirty-three percent of the failures were 'in field,' while $67 \%$ were 'selection failures.'

Conclusions: MRI-US fusion FC therapy results in excellent preservation of genitourinary function with encouraging oncological outcomes. Additional long-term research is needed to further establish and optimize MRI-US fusion for FC of PCa.

\section{UP-1.26}

Prostatic dystrophic calcification following salvage cryotherapy: An under-reported entity

Arnon Lavi ${ }^{1}$, Shiva M Nair ${ }^{1}$, L.K. Joseph Chin ${ }^{7}$

${ }^{1}$ Urology Division, Department of Surgery, Schulich School of Medicine and Dentistry, Western University, London, ON, Canada

Introduction: Adverse effects (AE) following salvage cryoablation (SC) for radio-recurrent prostate cancer include urethral sloughing and urethrorectal fistula. ${ }^{1,2}$ Prostate dystrophic calcifications (DC) following SC have rarely been reported. ${ }^{3,4}$ Our mature SC database (median followup 12 years) revealed this phenomenon in $9.4 \%$, which is herein reported.

Methods: A database was prospectively maintained on 187 patients who underwent whole-gland SC from March 1995 to September 2004. All patients had biopsy-proven, clinically localized, radio-recurrent cancer. Urinary functional followup data were available in 85 patients.

Results: Among those with severe urinary obstructive symptoms requiring surgical intervention, heavy prostatic DC was encountered in eight patients $(9.4 \%)$ either at cystoscopy or attempted transurethral resection. The bladder outlet became impassable, necessitating urinary drainage with permanent suprapubic catheter (seven patients) and nephrostomy (one patient). Mean time from SC to emergence of severe obstructive symptoms was 8.6 years (standard deviation [SD] 6 years). With the small sample size as a limitation, all DC patients developed biochemical failure, whereas failure rate for the remaining cohort was $57.1 \%(p=0.01)$. Surprisingly, mean time from SC to death was 17.7 years for DC vs. 12.4 years for the remaining cohort $(p=0.02)$. No correlations were found with 
pre-/post-SC prostate-specific antigen time from radiation to biochemical recurrence, time to SC failure, and overall survival.

Conclusions: Prostate DC following SC appears to be under-reported Our long followup has revealed this $\mathrm{AE}$, which appears to have delayed onset (>8 years). Proposed etiology includes dystrophic calcium deposition following chronic inflammation superimposed on radiated tissues. This previously unappreciated AE can significantly impact quality of life (requiring long-term catheter drainage) and should be part of preoperative counselling.

\section{References}

1. Williams AK, Martínez CH, Lu C, et al. Disease-free survival following salvage cryotherapy for biopsy-proven radio-recurrent prostate cancer. Eur Urol 2011;60:405-10. https://doi.org/10.1016/j. eururo.2010.12.012

2. Touma NJ, Izawa JI, Chin JL. Current status of local salvage therapies following radiation failure for prostate cancer. J Urol 2005;173:3739. https://doi.org/10.1097/01.ju.0000150627.68410.4d

3. Dru C, Bender L. Dystrophic calcification of the prostate after cryotherapy. Case Rep Urol 2014;2014:1-4. https://doi. org/10.1155/2014/471385

4. Zumstein V, Betschart P, Müllhaupt G, et al. Recurrent dystrophic calcification of the prostatic resection cavity after transurethral resection of the prostate: Clinical presentation and endoscopic management. J Endouro/ Case Reports 2017;3:81-3. https://doi.org/10.1089/ cren.2017.0058

\section{UP-1.28}

Does distance to prostate cancer assessment center predict location for radiation therapy treatment center?

Mustafa Andkhoie', Michael Szafron ${ }^{1}$

'School of Public Health, University of Saskatchewan, Saskatoon, SK, Canada

Support: Telus Ride For Dad. Prostate Cancer Fight Foundation

Introduction: In Saskatchewan (SK), prostate cancer (PC) assessment centers are in the cities of Regina and Saskatoon, respectively located in the southern and central portions of SK with approximately $250 \mathrm{~km}$ between them. We hypothesize that patients undergo treatment in the city with the closest (by distance) PC assessment center.

Methods: PC patient data (2010-2014) from the SK Cancer Registry included the treatment location for patients $(n=888)$ living in south and central SK who underwent radiation therapy (RT). The study region was subdivided into 82 geographical areas (GAs) and patients were categorized per the GAs in which they lived at the time of diagnosis. For each patient, we identified the "closest" center to be Regina or Saskatoon using the Euclidean distance between the centroids of the GAs and the two cities. The proportions of patients undergoing RT in each center were calculated for each GA. The "observed" center for a GA was then the center with more than $50 \%$ of the RT treatments. Odds ratios were used to determine if any associations exist between the closest and observed centers. We conducted a sensitivity analysis on our observed center definition using $60 \%, 80 \%$, and $100 \%$.

Results: PC patients have 108 (95\% confidence interval [CI] 22.5-517) times the odds of undergoing RT in their corresponding closest center $(\mathrm{p}<0.001)$. Among the 40 and 39 GAs closest to Saskatoon and Regina, respectively, 37 (93\%) and 35 (90\%) GAs had Saskatoon and Regina as their respective observed center. The sensitivity analysis showed $36(90 \%)$, $35(88 \%)$, and $30(75 \%)$ GAs had Saskatoon and $35(90 \%), 30(77 \%)$, and $26(67 \%)$ GAs had Regina as the observed center at the $60 \%, 80 \%$, and $100 \%$ cutoffs, respectively. Three GAs with no observed values were excluded from the analysis.

Conclusions: This analysis shows that PC patients in south and central SK undergo RT at a PC assessment center closest to them in distance. Closest treatment center can be a proxy for where a patient undergoes PC treatment.

\section{UP-1.29}

Robotic-assisted radical prostatectomy as salvage procedure after abandoned open radical prostatectomy

Ellen $O^{\prime}$ Connor ${ }^{1,2,3}$, Samantha S. Kosche ${ }^{2,3}$, Niranjan N. Sathianathen ${ }^{3}$, Isaac I. Thangasamy3, Daniel D. Moon ${ }^{3}$, Declan D. Murphy,3

'Department of Surgery, University of Melbourne, Austin Hospital, Heidelberg, Australia; ${ }^{2}$ Young Urology Research Organization, (YURO), Melbourne, Australia; ${ }^{3}$ Division of Cancer Surgery, Peter MacCallum Cancer Centre, Melbourne, Australia; ${ }^{4}$ Sir Peter MacCallum Department of Oncology, University of Melbourne, Parkville, Australia

Introduction: Robotic-assisted radical prostatectomy (RARP) has largely replaced open radical prostatectomy (ORP) where robotic facilities are available, with functional and oncological outcomes reported as equivalent in studies published thus far. To the best of our knowledge, no series examining abandoned ORP where subsequent salvage RARP has then been successfully performed, has been published. We aim to evaluate the reasons for abandoning ORP and technical aspects of performing salvage RARP in our series of five patients.

Methods: A retrospective review of five patients who had ORP abandoned and were then referred for RARP was performed. Analysis of patient and operative characteristics, and oncological outcomes were performed and reasons for abandonment of ORP were described.

Results: Five patients were included for analysis. Mean age was 60 years, mean presenting prostate-specific antigen (PSA) was $6.6 \mathrm{ng} / \mathrm{ml}$, and mean body mass index (BMI) was $32.26 \mathrm{~kg} / \mathrm{m}^{2}$. All patients had intermediate-risk prostate cancer. Small prostate and deep pelvis was given as the reason for abandoning ORP in $80 \%$ of cases, with $3 / 4$ of these also citing increased $\mathrm{BMI}$ as a factor. Extensive mesh from previous bilateral inguinal hernia repair was cited as the reason in the final patient. Mean operating time was 157 minutes (range 135-190) and mean estimated blood loss was $200 \mathrm{ml}$ (range 100-300). Extensive adhesions were noted at RARP in all five patients, with small bladder lacerations encountered in the patient with mesh hernia repairs.

Conclusions: Abandoning ORP is rare, but this case series demonstrates that subsequent RARP, while more technically difficult, can be safely performed. Increased BMI with a small prostate in a deep pelvis and previous mesh hernia repair appear to be catalysts for abandonment of open surgery in this cohort. Identifying these patients early at time of prostate cancer diagnosis and potentially referring on to a center with robotic expertise may be preferential. 


\section{CUA ABSTRACTS}

\section{Unmoderated Poster Session 2: Uro-Oncology, Pediatric Urology, Sexual Dysfunction, Transplant}

\begin{abstract}
UP-2.1
Development of a 3D-printed testicular cancer model for testicular examination education

Rebecca Power ${ }^{1}$, Jason A. Hearn' ${ }^{1}$, Charles J. Gillis', David R. Harvey², Christopher G. French², Michael K. Organ ${ }^{2}$

${ }^{1}$ Faculty of Medicine, Memorial University, St John's, NL, Canada; ' 2 Department of Urology, Discipline of Surgery, Faculty of Medicine, Memorial University, St John's, NL, Canada

Introduction: Testicular cancer is the most commonly diagnosed malignancy in young males. ${ }^{1}$ Most patients present with a painless nodule on one testicle; ${ }^{2}$ thus, self-examination is a non-invasive and inexpensive method that could enable early detection of malignancy. ${ }^{3,4} 3 \mathrm{D}$-printed teaching models can improve patient education and surgical planning. ${ }^{5,6}$ The objective of this project is to develop a teaching tool for medical learners and patients to gain a better understanding of testicular malignancies and to promote testicular examination (TE).

Methods: Four scrotum models were designed, a control model with healthy testes and three models containing a healthy testicle and a testicle with an endophytic mass of varying size. The anatomy, texture, and composition of the models were refined using an iterative process between the design team and urologists. The models were designed in OpenJSCAD and printed using a Prusa MKS3S printer. The material used for the scrotum and testes was Smooth-On Ecoflex 00-30 silicone, and the malignancies were casted in a polylactic acid polymer. The models were assessed by a team of urologists, urology nurse practitioners, and medical learners. Participants were asked to visually inspect and palpate each model and to subsequently provide feedback regarding the realism and clinical utility of the developed models. Results: Participants agreed that the models would be useful teaching tools for both medical learners and patients. Respondents felt that the models enabled accurate simulation of a TE involving both healthy and pathologic testes. Qualitative feedback concluded that the majority of respondents would use these models as a teaching aid in clinic and incorporate it into their practice.
\end{abstract}

Conclusions: 3D-printed models can effectively simulate palpation of both healthy and pathologic testes. These models have the potential to be a useful adjunct in teaching TE and in demonstrating abnormal findings that require further investigation.

References

1. Trabert B, Chen J, Devesa SS, et al. International patterns and trends in testicular cancer incidence, overall and by histologic subtype, 1973-2007. Andrology 2015;3:4-12. https://doi.org/10.1111/andr.293

2. Kobayashi K, Saito T, Kitamura Y, et al. Effect of the time from the presentation of symptoms to medical consultation on primary tumor size and survival in patients with testicular cancer: Shift in the last 2 decades. Urol Oncol 2014;32:43.e17-43. https://doi.org/10.1016/j. urolonc.2013.05.007

3. Thornton C.P. Best practice in teaching male adolescents and young men to perform testicular self-examinations: A review. J Ped Health Care 2016;30:518-27. https://doi.org/10.1016/j.pedhc.2015.11.009

4. Rovito MJ, Leone JE, Cavayero CT. "Off-label" usage of testicular self-examination (TSE): Benefits beyond cancer detection. Am J Mens Health 2018;12:505-13. https://doi.org/10.1177/1557988315584942
5. Schmit C, Matsumoto J, Yost K, et al. Impact of a 3D printed model on patients' understanding of renal cryoablation: A prospective pilot study. Abdom Radiol 2019;44:304-9. https://doi.org/10.1007/s00261018-1710-1

6. Teishima J, Takayama Y, Iwaguro S, et al. Usefulness of personalized three-dimensional printed model on the satisfaction of preoperative education for patients undergoing robot-assisted partial nephrectomy and their families. Int Urol Nephrol 2018;50:1061-6. https:// doi.org/10.1007/s11255-018-1881-2

\section{UP-2.2}

Development of a 3D-printed renal malignancy model for surgical planning and patient education Lason Hearn', Rebecca J. Power', Charles Gillis', Jennifer Young², Michael K. Organ

'Faculty of Medicine, Memorial University of Newfoundland, St. John's, NL, Canada; ${ }^{2}$ Discipline of Radiology, Faculty of Medicine, Memorial University of Newfoundland, St. John's, NL, Canada; ${ }^{3}$ Department of Urology, Discipline of Surgery, Faculty of Medicine, Memorial University of Newfoundland, St. John's, NL, Canada

The authors would like to thank MUN MED 3D for their technical support on this project.

Introduction: Kidney cancer is a common diagnosis, with a five-year survival rate of $71 \%{ }^{1}$ Surgery plays a critical role in treating cancerous renal masses. ${ }^{2}$ Given the complex anatomy of the kidney, the surgical treatment of renal cancer requires careful preoperative planning and patient counselling. ${ }^{3}$ The incorporation of 3D-printed anatomical models has shown promise in facilitating the planning of surgical interventions, ${ }^{4-6}$ as well as the explanation of such procedures to patients. ${ }^{6,7}$ The objective of this project was to develop a 3D-printed renal malignancy model for use in both surgical planning and patient education.

Methods: A team of urologists, radiologists, and engineers developed a 3D-printed model of a kidney with a renal mass. A 3D-rendered image was first reconstructed using a computed tomography $(\mathrm{CT})$ scan of a patient diagnosed with a renal mass. This image was used to develop a 3D-printed model that simulated the pathologic kidney. The model was designed in AutoCAD, finalized with Meshmixer, and casted in silicone using an Ultimaker printer. A urologist verified and evaluated the anatomical accuracy of the 3D-printed model, as well as assessed the applicability of the model for surgical planning and patient education.

Results: The developed model effectively demonstrated the size, location, and overall severity of the renal mass present in the original CT scan. The model also facilitated discussions surrounding the surgical intervention that would be used to treat the lesion. The clinical experts agreed that the model would improve patient understanding and allow for better informed preoperative consent.

Conclusion: A 3D-printed renal malignancy model was developed based on a CT scan, as well as expert input from both a urologist and a radiologist. Future work will involve improving the structural accuracy of the simulated renal vasculature to enable detailed surgical planning, as well as assessing the utility of the model in clinical practice. 


\section{References}

1. Canadian Cancer Statistics Advisory Committee. Canadian Cancer Statistics 2019. Toronto; 2019.

2. Rini Bl, Campbell SC, Escudier B. Renal cell carcinoma. Lance 2009;373:1119-32. https://doi.org/10.1016/S0140-6736(09)60229-4

3. Rendon RA, Kapoor A, Breau R, et al. Surgical management of renal cell carcinoma: Canadian Kidney Cancer Forum Consensus. Can Urol Assoc J 2014;8:E398-412. https://doi.org/10.5489/cuaj.1894

4. Valverde I, Gomez G, Coserria JF, et al. 3D printed models for planning endovascular stenting in transverse aortic arch hypoplasia. Catheter Cardiovasc Interv 2015;85:1006-12. https://doi. org/10.1002/ccd.25810

5. Zheng Y, Yu D, Zhao J, et al. 3D printout models vs. 3D-rendered images: Which is better for preoperative planning? J Surg Educ 2016;73:518-23. https://doi.org/10.1016/j.jsurg.2016.01.003

6. Malik HH, Darwood ARJ, Shaunak S, et al. Three-dimensional printing in surgery: A review of current surgical applications. J Surg Res 2015;199:512-22. https://doi.org/10.1016/j.jss.2015.06.051

7. Liew Y, Beveridge E, Demetriades AK, et al. 3D printing of patientspecific anatomy: A tool to improve patient consent and enhance imaging interpretation by trainees. Br J Neurosurg 2015;29:712-4. https://doi.org/10.3109/02688697.2015.1026799

UP-2.3

One-year outcomes of dorsal buccal mucosal graft urethroplasty for vesicourethral anastomotic stenosis post-radical prostatectomy

Walid Shahrour ${ }^{1}$, Ahmed Kotb ${ }^{1}$, Owen Prowse ${ }^{1}$, Hazem Elmansy ${ }^{1}$

${ }^{1}$ Clinical Sciences, Northern Ontario School of Medicine, Thunder Bay, ON, Canada

Introduction: We aimed to evaluate the one-year outcomes of using dorsal buccal mucosal graft (BMG) for the repair of refractory vesicourethral anastomotic stenosis (VUAS) post-open radical prostatectomy.

Methods: Patients' demographics, preoperative continence status, number of dilations, and urine flow preoperatively and postoperatively were collected. Patients were discharged home on day 1 . Trial of void and retrograde urethrogram were done after three weeks. Flow and post-void residual were done at three months. Cystoscopy was done at six months to assess patency.

Results: The cohort included five patients between July 2018 and February 2019. The median age was $67(59-72)$ years. Four of five patients $(80 \%)$ had received adjuvant radiotherapy. The median number of preoperative endoscopic procedures, including dilations and incisions, was seven (4-10). The median stricture length was $2.5 \mathrm{~cm}(2-3)$. All patients were incontinent preoperatively with median preoperative flow of $5 \mathrm{~mL} / \mathrm{s}(3-7)$. Median operative time and blood loss were 150 minutes and $250 \mathrm{~mL}$, respectively. Mean postoperative urine flow at 16 months was $20 \mathrm{~mL} / \mathrm{s}$ (17-23). Median followup was 16 (9-16) months. All patients were incontinent postoperatively. Success rate was $100 \%$ at 16 months. One patient had artificial urinary sphincter postoperatively and it is successful at the 16-month time point.

Conclusions: BMG urethroplasty in vesicourethral anastomotic stricture/ stenosis is a new technique that can provide a safe perineal approach while eliminating the potential risk of rectal injury and urethral atrophy from extensive urethral mobilization. It can also decrease the need for laparotomy or the need to perform a combined abdominal-perineal approach. Long-term followup is warranted with a larger cohort of patients.

\section{UP-2.4}

\section{Outcomes of pelvic fracture urethral injury reconstruction}

Sarah R. Ferrara ${ }^{7}$, Taylor Remondini ${ }^{1}$, Amanda Hird ${ }^{1}$, Jennifer A. Locke ${ }^{1}$ Sender Herschorn ${ }^{1}$, Ronald T. Kodama

${ }^{1}$ Urology, Sunnybrook Health Sciences Centre, University of Toronto, Toronto, ON, Canada

Introduction: Urethral injury is a complication of pelvic fracture in males, with variable rates of erectile dysfunction (ED) and stricture recurrence following reconstruction. Our objective was to analyze pelvic fracture ure- thral injury (PFUI) urethroplasty outcomes and to determine risk factors for complications.

Methods: A total of 119 patients who had undergone reconstruction for PFUI at our center from 1998-2018 were identified using billing codes. Descriptive statistics were completed and used to compare the characteristics of those who experienced a complication and those who had not. Multivariable Cox proportional hazard analysis was used to assess the association between age at time of surgery, primary intervention, and pubectomy on the risk of having a complication.

Results: Median age at time of surgery was 44 years (interquartile range [IQR] 29-56), and the most common mechanism of injury was motor vehicle accident (MVA) (53.4\%). Eighty-five percent of patients had urethral injury distal to the external sphincter. Initial management was most often primary realignment with urethral catheter $(61.5 \%)$ or suprapubic tube alone $(38.5 \%)$. Ultimately, $97.5 \%$ of patients underwent primary perineal anastomosis after a median of seven months (IQR 5-14). Overall, $26 / 116$ evaluable patients $(22.4 \%$ ) had a long-term complication after a median 25 months (IQR 7-66), including stricture recurrence, de novo ED, and post-void dribbling. Only 5/116 patients (4.3\%) had stricture recurrence after a median time of eight months (IQR 5-12); all were managed with urethral dilations as needed. Nineteen patients developed some degree of de novo ED, and this was more common among patients who had a pubectomy $(79 \%$ vs. $21 \%$; $p=0.044)$. On multivariable analysis, no predetermined variables predicted for complications.

Conclusions: The rate of stricture recurrence after primary anastomosis for PFUI is low. Pubectomy was associated with de novo ED. Despite a diverse patient population, we did not identify predictors of complications following reconstruction.

\section{UP-2.5}

Predictive factors for recurrent urinary tract infections in patients with a history of spinal cord injury

Ross Everett', David Charles', Halle Foss' ${ }^{1}$, Michael Avallone', R. Corey $\mathrm{O}^{\prime} \mathrm{Connor}^{1}$, Michael Guralnick'

${ }^{1}$ Urologic Surgery, Medical College of Wisconsin, Milwaukee, WI, United States

Introduction: We sought to assess associations between demographic, clinical, and urodynamic variables and recurrent urinary tract infection (UTI) in spinal cord injury (SCl) patients performing clean intermittent catheterization $(\mathrm{CIC})$

Methods: The records of $136 \mathrm{SCl}$ patients who perform $\mathrm{CIC}$ for bladder management were retrospectively reviewed. All had a video urodynamics study (VUDS) available for analysis. Subjects were divided into recurrent (rUTI) UTI (>3/year) or non-rUTI (<3/year) groups. Differences between the cohorts were analyzed. Multivariable logistic regression was performed to assess for associations between demographic, clinical, and VUDS variables and rUTI, both self-reported and culture-proven.

Results: Self-reported rUTI were noted in 58 of 136 (42.6\%) patients. A higher body mass index (BMI) was identified in patients with self-reported rUTI (26.4 vs. 24.8; $p=0.048)$. Culture-proven rUTI were recorded in 30 of $124(24.2 \%)$ patients. Differences between groups with and without rUTI are listed in Table 1. Non-white race (African American or other) was significantly more prevalent in the culture-proven rUTI group. Additionally, those with rUTI were closer to the time of their SCI. On multivariable logistic regression, women demonstrated a stronger association with rUTI than men (odds ratio $[O R]$ 4.96; $\mathrm{p}=0.011$ ), African American was more strongly associated with rUTI compared to Caucasian race (OR 5.16; $\mathrm{p}=0.002$ ), and a longer time since injury showed a decreased association (OR 0.91; $p=0.036$ ) with rUTI. There were no significant differences in urodynamic variables between groups and none were significant on regression as predictors of rUTI.

Conclusions: Race, gender, and time since $\mathrm{SCl}$ appear to play a role in the risk of rUTI in SCI patients using CIC. Urodynamic variables, however, were not found to be predictive of rUTI in this population. 


\begin{tabular}{|c|c|c|c|c|}
\hline & $\begin{array}{c}\text { Total } \\
\mathrm{N}=124\end{array}$ & $\begin{array}{c}\text { No } \\
n=94\end{array}$ & $\begin{array}{c}\text { Yes } \\
n=30\end{array}$ & $\mathbf{p}$ \\
\hline Age, mean \pm SD & $37.3 \pm 13.9$ & $38.4 \pm 14.1$ & $34.0 \pm 13.0$ & 0.142 \\
\hline \multicolumn{5}{|l|}{$\operatorname{Sex}(\%)$} \\
\hline Female & $17(13.7 \%)$ & $10(10.6 \%)$ & $7(23.3 \%)$ & 0.123 \\
\hline Male & 107 (86.3\%) & $84(89.4 \%)$ & $23(76.7 \%)$ & \\
\hline $\mathrm{BMI}$, mean $\pm \mathrm{SD}$ & $25.7 \pm 6.3$ & $25.3 \pm 6.1$ & $26.7 \pm 6.8$ & 0.336 \\
\hline \multicolumn{5}{|l|}{ Race (\%) } \\
\hline Black & $34(27.4 \%)$ & $21(22.3 \%)$ & $13(43.3 \%)$ & 0.033 \\
\hline Other & $12(9.7 \%)$ & $8(8.5 \%)$ & $4(13.3 \%)$ & \\
\hline White & $78(62.9 \%)$ & $65(69.1 \%)$ & $13(43.3 \%)$ & \\
\hline \multicolumn{5}{|l|}{ SCI level of injury, $\mathrm{n}$} \\
\hline Cervical & $42(33.9 \%)$ & $32(34.0 \%)$ & $10(33.3 \%)$ & 1.000 \\
\hline Thoracic & $72(58.1 \%)$ & $54(57.4 \%)$ & $18(60.0 \%)$ & \\
\hline Lumbar & $10(8.1 \%)$ & $8(8.5 \%)$ & $2(6.7 \%)$ & \\
\hline Years since $\mathrm{SCl}$, mean $\pm \mathrm{SD}$ & $5.0 \pm 6.7$ & $5.6 \pm 7.0$ & $3.1 \pm 5.2$ & 0.018 \\
\hline History of diabetes mellitus (\%) & $6(4.8 \%)$ & $4(4.3 \%)$ & $2(6.7 \%)$ & 0.631 \\
\hline History of decubitus ulcers (\%) & $26(21.0 \%)$ & $20(21.3 \%)$ & $6(20.0 \%)$ & 0.881 \\
\hline Bladder stone(s) present (\%) & $8(6.5 \%)$ & $7(7.4 \%)$ & $1(3.3 \%)$ & 0.678 \\
\hline Renal stone(s) present (\%) & $15(12.1 \%)$ & $13(13.8 \%)$ & $2(6.7 \%)$ & 0.358 \\
\hline Hydronephrosis present (\%) & $4(3.2 \%)$ & $2(2.1 \%)$ & $2(6.7 \%)$ & 0.246 \\
\hline Vesicoureteral reflux present (\%) & $4(3.2 \%)$ & $4(4.3 \%)$ & $0(0.0 \%)$ & 0.571 \\
\hline Reports urinary incontinence (\%) & $80(64.5 \%)$ & $59(62.8 \%)$ & $21(70.0 \%)$ & 0.471 \\
\hline Anticholinergic medication use (\%) & $68(54.8 \%)$ & $51(54.3 \%)$ & $17(56.7 \%)$ & 0.837 \\
\hline Antibiotic prophylaxis use (\%) & $9(7.3 \%)$ & $7(7.4 \%)$ & $2(6.7 \%)$ & 1.000 \\
\hline DO present (\%) & $88(71.0 \%)$ & $68(72.3 \%)$ & $20(66.7 \%)$ & 0.551 \\
\hline Volume at first $\mathrm{DO}^{\dagger}$, median (range) & $262.0(38-850)$ & $275.0(38-850)$ & $225.0(56-550)$ & 0.287 \\
\hline Max Pdet during $\mathrm{DO}^{\ddagger}$, median (range) & $50.0(9-180)$ & $50.0(9-180)$ & $55.0(25-149)$ & 0.240 \\
\hline Cystometric capacity, median (range) & $475.0(35-1346)$ & $486.5(73-1346)$ & $395.0(35-1225)$ & 0.424 \\
\hline End fill detrusor pressure, median (range) & $7.5(1-74)$ & $7.5(1-74)$ & $7.5(1-46)$ & 0.699 \\
\hline Compliance, median (range) & $44.7(1.1-475.0)$ & $42.4(4.5-475.0)$ & $50.0(1.1-375.0)$ & 0.641 \\
\hline Number of UTI in previous year*, mean \pm SD & $1.6 \pm 1.7$ & $0.8 \pm 0.8$ & $4.1 \pm 1.7$ & 0.000 \\
\hline
\end{tabular}

\section{UP-2.6}

The Contino ${ }^{\circledR}$ urethral insert in the treatment of male stress urinary incontinence

Dean S. Elterman' ${ }^{1}$ Richard W. Casey², Thomas J. De los Reyes', Michael Holloway ${ }^{3}$

${ }^{1}$ Urology, University Health Network, Toronto, ON, Canada; ${ }^{2}$ The Fe/ Male Health Centres, Oakville, ON, Canada; ${ }^{3}$ Life360 Innovations Inc., Vancouver, BC, Canada

Support: Life360 Innovations Inc. sponsored this study
Introduction: Surgical interventions continue to be the mainstay treatment for men with stress urinary incontinence (SUI). For patients with comorbidities that preclude them from surgery or those that are unwilling to undergo invasive therapy, non-surgical options are limited to external compression or collection devices coupled with behavioral modifications. These are often not satisfactory in controlling leakage. The Contino ${ }^{\circledR}$ is a self-administered device placed into the distal urethra to inhibit urine flow, which is then removed prior to urination. This prospective, non-randomized, single-arm trial was used to assess the efficacy of the Contino ${ }^{\circledast}$ device in treating male SUI.

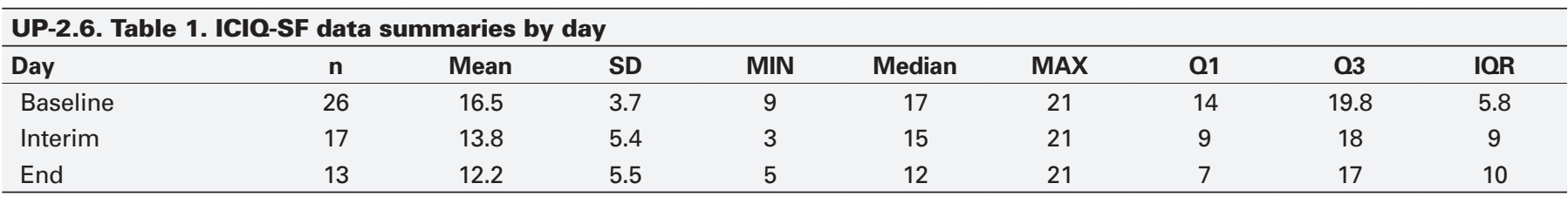




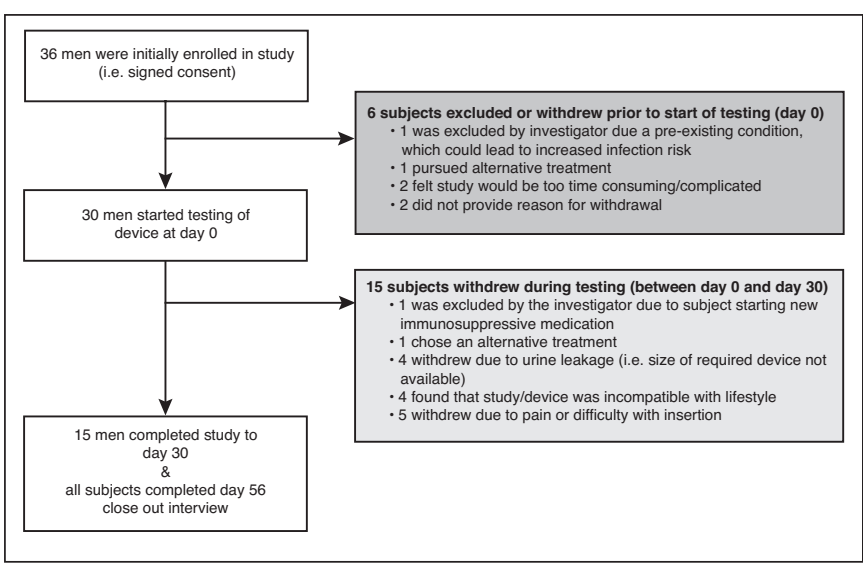

UP-2.6. Fig. 1. Study participants.

Methods: This multisite study enrolled 36 men with SUI. Each patient subsequently underwent personalized device sizing and teaching. The primary endpoints of the study were a change from baseline to interim/end of study on the following: 1) International Consultation on Incontinence Questionnaire-Short Form (ICIQ-SF); 2) pad weight to assess urine loss; and 3) adverse events. The trial was 30 days, with data collected at the start, interim, and end of study.

Results: A total of 15 participants completed the study (Fig. 1). Repeat subjects or those with missing data were removed from the statistical analysis completed post-hoc by UBC. The use of the device resulted in the reduction of mean scores of ICIQ-SF from baseline (16.5) to day 30 (12.2) (Table 1). Mean pad weight also decreased from baseline (471.4 g) to day 30 (149.1 g) (Table 2). The t-distribution is assumed for calculating confidence intervals. One-sided 95\% confidence intervals were used and gave an upper bound of the estimates (Tables 3,4). The adverse events (AE) were primarily mild and non-serious (Fig. 5).

Conclusions: The Contino ${ }^{\circledast}$ device resulted in a reduction in urine loss and improved patient perception with urinary incontinence. It is well-tolerated with minimal AE. The Contino ${ }^{\circledR}$ device may serve as an alternative, noninvasive option for men with SUI.

\section{UP-2.7}

Measuring the quality of mobile apps for lower urinary tract health

Louisa Ho ${ }^{1}$, Lynn Stothers ${ }^{1}$

${ }^{1}$ Department of Urologic Sciences, University of British Columbia, Vancouver, BC, Canada

Introduction: Overactive bladder $(\mathrm{OAB})$ is a common symptom complex that causes significant detriment to quality of life in patients. A mainstay of first-line treatment is behavioral modifications, focusing on lifestyle changes. Urological mobile health (mHealth) applications are gaining popularity among clinicians and patients as an adjunct to these interventions. ${ }^{1}$ However, few of these apps have been scientifically evaluated. The purpose of the study was review and measure the quality of mHealth apps targeted to the management of $O A B$ and lower urinary tract symptoms. Methods: A systematic search of the Apple iTunes store was performed. We included all apps related to lower urinary tract health for health professionals and patients. Expert raters trialed each of the apps and independently rated their quality using a Mobile App Rating Scale (MARS). ${ }^{2}$

\begin{tabular}{lcc}
\hline $\begin{array}{l}\text { UP-2.6. Table 3. Estimated mean difference and } \mathbf{9 5 \%} \\
\text { upper limit in ICIQ-SF from baseline by day }\end{array}$ \\
\hline Day & Estimate & Upper \\
\hline Interim & -2.471 & -0.796 \\
End & -4 & -1.827 \\
\hline
\end{tabular}

\begin{tabular}{lcc}
\hline \multicolumn{3}{l}{$\begin{array}{l}\text { UP-2.6. Table 4. Estimated mean percent change and } \mathbf{9 5 \%} \\
\text { upper limit in pad weight from baseline by day }\end{array}$} \\
\begin{tabular}{lcc}
\hline Day & Estimate & Upper \\
\hline Interim & -54.21 & -38.32 \\
End & -71.76 & -54.3 \\
\hline
\end{tabular}
\end{tabular}

Apps were rated on the the quality of information, functionality, engagement, and esthetics, as well as overall impact and impression on a Likert scale of $0-5$.

Results: There were 37 apps reviewed, listed under the categories "Medical," "Health and Fitness," and "Education." Most apps were available free $(82 \%)$. The developer credentials were unknown for $70 \%$ of apps, and $64 \%$ of apps were affiliated with a commercial organization. The average global quality star rating was 3.1. The top-rated section was functionality at 4.3 , while the worst-rated was engagement at 3.2. Only $9 \%$ of apps had been trialed and verified by evidence in scientific literature.

Conclusions: There are several publicly available apps related to lower urinary tract health marketed towards various demographics. Most apps have not been trialed and are not based on scientific evidence. Many apps are not of high quality and fail to engage the user. Future apps should improve on these aspects and exploit the capabilities of current mobile devices.

References

1. Pereira-Azevedo N, Carrasquinho E, Cardoso de Oliveira E, et al. mHealth in urology: A review of experts' involvement in app development. PLoS One 2015;10:e0125547. https://doi.org/10.1371/journal.pone.0125547

2. Stoyanov SR, Hides L, Kavanagh DJ, et al. Mobile app rating scale: A new tool for assessing the quality of health mobile apps. JMIR Mhealth Uhealth 2015;3:e27. https://doi.org/10.2196/mhealth.3422

\section{UP-2.8}

Do larger cuff sizes with artificial urinary sphincter placement increase the risk of leakage postoperatively?

Thomas I. De los Reyes ${ }^{1}$, Sidney B. Radomski

'Division of Urology, Toronto Western Hospital, University Health Network, Toronto, ON, Canada

Introduction: The artificial urinary sphincter (AUS) remains the gold standard for post-radical prostatectomy (RP) stress incontinence. Appropriate AUS cuff size is an intraoperative decision impacted by measured urethral circumference, history of radiation, and surgeon experience. Cuff sizes that are too tight are at risk of erosion, whereas persistent leakage is a concern if the cuff is too loose. The aim of this study is to determine whether AUS cuff sizes of $\geq 5.0 \mathrm{~cm}$ have an impact on urinary incontinence after AUS implantation.

Methods: A retrospective review of AUS implants at our institution from 1991 to present was performed. All patients who had an AUS cuff size

\begin{tabular}{lccccccccc}
\hline UP-2.6. Table 2. Pad weight data summaries by day & & & & & \\
\hline Day & $\mathbf{n}$ & Mean & SD & Min & Median & Max & Q1 & Q3 & IQR \\
\hline Baseline & 26 & 471.4 & 310.9 & 33 & 403.4 & 1007 & 186 & 778.5 & 592.5 \\
Interim & 11 & 291.2 & 261.5 & 2.7 & 231.7 & 721 & 76.5 & 472 & 395.5 \\
End & 11 & 149.1 & 159.7 & 1.3 & 120 & 433 & 7.8 & 261 & 253.2 \\
\hline
\end{tabular}


UP-2.6. Table 5. Adverse events (AEs), causality, severity, and type

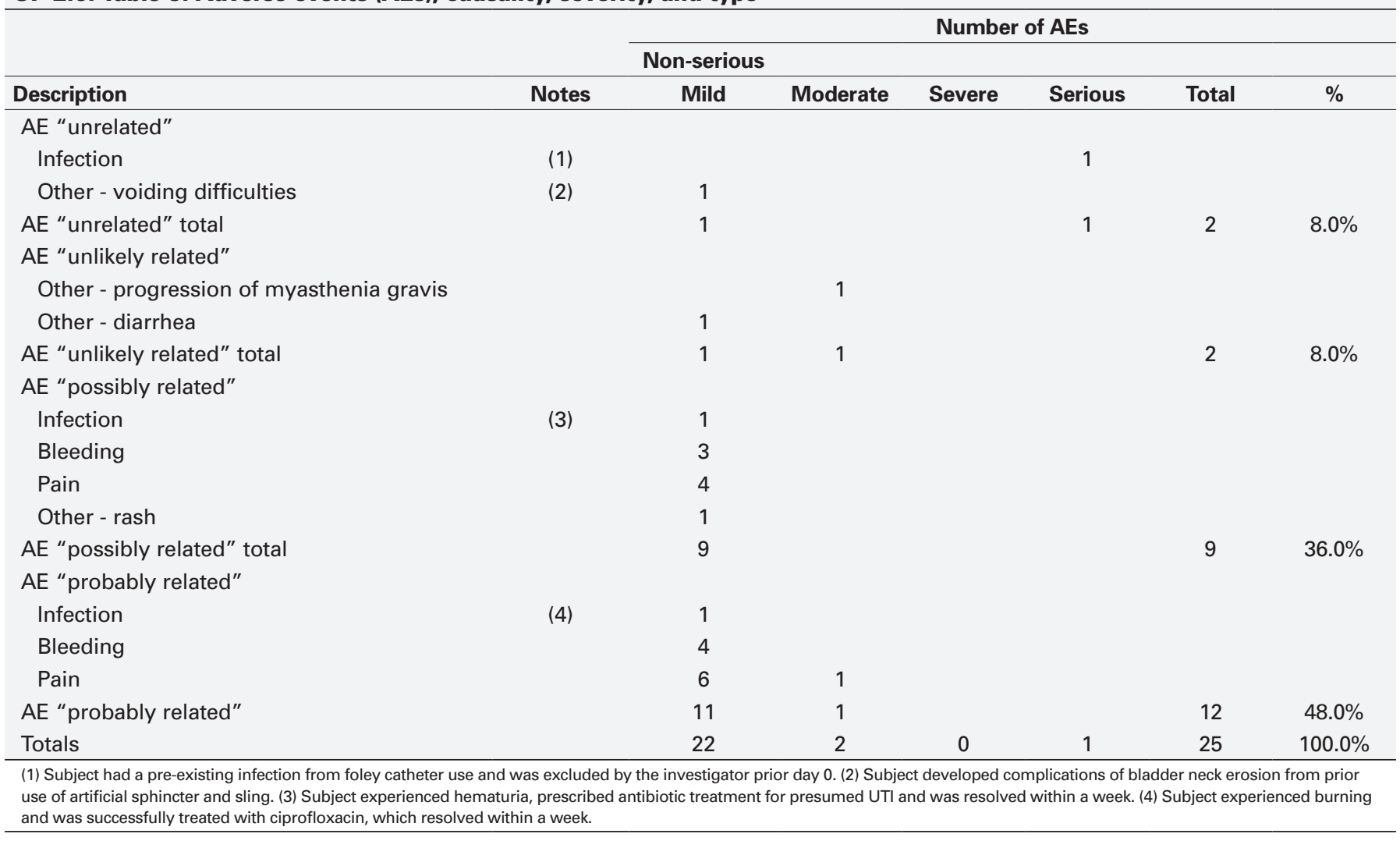

of $\geq 5.0 \mathrm{~cm}$ were included for analysis. Medical records were reviewed for demographics, including body mass index (BMI), cause of incontinence, history of radiation, Valsalva leak point pressure on urodynamics, degree of leakage preoperatively, at six months and at one year post-AUS surgery, and AUS revisions to date. Degree of leakage was quantified by patient-reported number of pads per day. The main study outcome was the degree of leakage at six months and one year post-AUS implantation. Results: A total of 35 patients had a cuff size of $\geq 5.0 \mathrm{~cm}$. The main reason for incontinence was RP for prostate cancer. Thirty patients had a cuff size of $5.0 \mathrm{~cm}$ and five patients had a cuff size of $5.5 \mathrm{~cm}$. Pre-AUS surgery, patients used an average of five pads/day. Pad use was reduced to a mean of 0.9 pads/day at six months and one year after AUS placement, with $86 \%$ reporting $0-1$ pads/day usage. There were five revisions performed to date (one downsizing of cuff, two erosions, one reservoir leak, one infection). History of radiation, BMI, number of pads preoperatively, and anticholinergic use postoperatively were not associated with degree of leakage one year post-implantation.

Conclusions: AUS cuff sizes of $\geq 5.0 \mathrm{~cm}$ does not appear to have a negative impact on the degree of leakage at six months and one year post-implantation. Radiation itself did not have an impact on leakage in these patients.

\section{UP-2.9}

Location of residence and mortality for patients diagnosed with Fournier's gangrene

Rachel Wong' ${ }^{1}$ Uday Mann ${ }^{1}$, Amanda Eng' ${ }^{1}$ Ruben Blachman-Bruan², Premal Patel ${ }^{1}$

${ }^{1}$ Section of Urology, Department of Surgery, University of Manitoba, Winnipeg, MB, Canada; ${ }^{2}$ Department of Urology, University of Miami Miller School of Medicine, Miami, FL, United States

Introduction: Fournier's gangrene (FG) is a multi-bacterial infection that leads to necrotizing fasciitis of the perineum and genitalia. Previously described prognostic factors include patient's metabolic state and time from clinical onset to surgical intervention. We sought to investigate whether patients from rural regions in Manitoba have worse clinical outcomes.

UP-2.9. Table 1. Baseline characteristics and mortality outcomes of rural and urban patients admitted to ICU with Fournier's gangrene

\begin{tabular}{lccc}
\hline Variable & $\begin{array}{c}\text { Rural } \\
\mathbf{n = 1 8}(\%)\end{array}$ & $\begin{array}{c}\text { Urban } \\
\mathbf{n = 2 2}(\%)\end{array}$ & $\mathbf{p}$ \\
\hline Age & $55.7 \pm 13.9$ & $55.9 \pm 10.8$ & 0.962 \\
Sex & $3(16.7)$ & $3(13.6)$ & \\
$\quad$ Female & $15(83.3)$ & $19(86.4)$ & 1.000 \\
$\quad$ Male & $15(83.3)$ & $20(90.9)$ & \\
Colostomy & $3(16.7)$ & $2(9.1)$ & 0.642 \\
$\quad$ No & $11(61.1)$ & $17(77.3)$ & \\
$\quad$ Yes & $7(38.9)$ & $5(22.7)$ & 0.267 \\
Scrotal debridement & $1.5(1-3.3)$ & $2(1.8-6)$ & 0.099 \\
$\quad$ No & $4.7(2.4-7.1)$ & $4(1.5-11.5)$ & 0.957 \\
$\quad$ Yes & & & \\
Charlson score & $16(88.9)$ & $17(77.3)$ & \\
LOS & $2(11.1)$ & $5(22.7)$ & 0.427 \\
Mortality & & & \\
$\quad$ Alive & &
\end{tabular}




\begin{tabular}{|c|c|c|c|}
\hline Variable & $\begin{array}{c}\text { Alive } \\
\mathrm{n}=33(\%)\end{array}$ & $\begin{array}{c}\text { Death } \\
n=7(\%)\end{array}$ & $\mathbf{p}$ \\
\hline Age & $56.2 \pm 12.2$ & $54 \pm 12.6$ & 0.667 \\
\hline \multicolumn{4}{|l|}{ Sex } \\
\hline Female & $5(15.2)$ & $1(14.3)$ & \\
\hline Male & $28(84.8)$ & $6(85.7)$ & 1.000 \\
\hline \multicolumn{4}{|l|}{ Location } \\
\hline Rural & $16(48.5)$ & $2(28.6)$ & \\
\hline Urban & 17 (51.5) & 5 (71.4) & 0.427 \\
\hline \multicolumn{4}{|l|}{ Colostomy } \\
\hline No & $28(84.8)$ & $7(100)$ & \\
\hline Yes & $5(15.2)$ & 0 & 0.565 \\
\hline \multicolumn{4}{|c|}{ Scrotal debridement } \\
\hline No & $21(63.6)$ & $7(100)$ & \\
\hline Yes & $12(36.4)$ & 0 & 0.081 \\
\hline Charlson score & $4.4(1.7-6.9)$ & $5.4(3.2-17.3)$ & 0.233 \\
\hline LOS & $58(49.5-64.5)$ & $52(49-66)$ & 0.521 \\
\hline
\end{tabular}

Methods: The Manitoba intensive care unit (ICU) prospective registry began in 1999. We identified patients admitted to the ICU with a diagnosis of FG from February 1999 to October 2019. Patient age, gender, Charlson comorbidity index $(\mathrm{CCl})$, presence of colostomy and scrotal debridement, length of stay (LOS), and mortality outcomes were obtained. Only patients with a record of their residence were analyzed and categorized as being rural or urban. Categorical variables were presented as absolute values and frequencies and analyzed with the Fisher's exact test. Continuous variables were presented as means and standard deviations $( \pm$ SD) or medians and interquartile ranges (25-75) and analyzed with the Mann-Whitney $U$ test or Student's t-test. A $p<0.05$ was considered statistically significant. Statistical analysis was performed with SPSS software (version 24).

Results: A total of 87 patients were diagnosed and admitted to the ICU with FG. Location was obtained for 40 patients, with $18(45 \%)$ and 22 $(55 \%)$ residing in rural and urban regions, respectively. There was no difference in baseline characteristics between the rural and urban groups with respect to age, gender, $\mathrm{CCl}$, need for colostomy, scrotal debridement, LOS, or mortality (Table 1). When evaluating mortality outcomes, there was no difference in baseline characteristics nor region of residency (Table 2).

Conclusions: Location of residence (urban or rural) was not predictive of death from FG in Manitoba. In addition, baseline characteristics, such as age, gender, $\mathrm{CCl}$, surgical interventions, or LOS, were also not found to be associated with mortality.

\section{UP-2.10}

Urological outcomes following diagnosis of prostate cancer in nonagenarians

Joshua White', Lenna Coles ${ }^{2}$, Ross Mason ${ }^{1}$, Karthik Tennankore ${ }^{3}$, Ashley R. $\operatorname{Cox}^{1}$

'Department of Urology, Dalhousie University, Halifax, NS, Canada; ${ }^{2}$ Postgraduate Medical Education, Dalhousie University Medical School, Halifax, NS, Canada; ${ }^{3}$ Department of Nephrology, Dalhousie University, Halifax, NS, Canada

Introduction: Nonagenarians are one of the fastest-growing population groups in Canada. ${ }^{1,2}$ They represent a challenging patient population that has previously not been evaluated in terms of prostate cancer treatment and outcomes. We sought to review the treatment selection and outcomes of our nonagenarian population diagnosed with prostate cancer.

Methods: A retrospective chart review of nonagenarians was conducted at a single academic institute. We reviewed patients who were at least 90 years of age at the time of referral to 11 urologists from 2007-2018. Men with a diagnosis of prostate cancer were included. Patient demographics, presentation of disease, treatments received, and outcomes were assessed. Results: The median patient age at time of referral was 91 (interquartile range [IQR] 3); $95.5 \%$ of patients presented with urinary symptoms $(n=42)$ and $47.7 \%$ were diagnosed on the basis of an elevated prostatespecific antigen (PSA) $(n=21)$. Skeletal-related events occurred in $29.5 \%$ of patients $(n=13)$, with spinal cord compression occurring in $4.5 \%$ of patients $(n=2)$. Surprisingly, only $20.5 \%$ of patients received a referral to palliative care $(n=9)$. A total of $79.5 \%$ of patients underwent at least one urological intervention $(n=35)$. Cystoscopy and catheter insertion were the most common interventions at $72.7 \%$ and $75.0 \%$, respectively. Most $(70.5 \%)$ patients received androgen deprivation therapy as the primary form of treatment $(n=31)$. The median age at death was 95 (IQR 3). Death from any cause was seen in $47.4 \%$ of patients $(n=19)$, and death due to prostate cancer was observed in only $18.2 \%$ of patients $(n=8)$.

Conclusions: This study highlights that nonagenarian patients with prostate cancer frequently present with urinary complaints and are diagnosed based on PSA. The majority of this cohort underwent an operative intervention. Many of these patients may live with urological complaints for many years after referral. Awareness of prostate cancer outcomes in this patient population is important due to the increasing number of nonagenarians.

\section{References}

1. Decady Y, Greenberg L; Statistics Canada, issuing body, publisher. (2014). Ninety years of change in life expectancy (Health at a glance). Ottawa, Ontario: Statistics Canada.

2. World Bank. (July 8, 2018). World development indicators: Canadian life expectancy. Available at: https://data.worldbank.org/indicator/ SP.DYN.LE00.MA.IN? locations=CA. Accessed April 21, 2020.

\section{UP-2.11}

Incisional hernia at specimen extraction site for robot-assisted laparoscopic radical prostatectomy: A single-surgeon operator series Brennan Timm ${ }^{1,2}$, Peter Liodakis ${ }^{1,2}$

${ }^{1}$ Urology, Austin Health, Heidelberg, Australia; ${ }^{2}$ North Eastern Urology, Heidelberg, Australia

Introduction: Robot-assisted laparoscopic radical prostatectomy (RALP) continues to gain traction as a patient-driven means of managing prostate cancer via a minimally invasive approach, with excellent cosmetic outcomes, shorter convalescence, and equivalent functional and oncological outcomes compared to open surgery. ${ }^{1}$ However, there is growing evidence that RALP is significantly associated with incisional hernia $(\mathrm{IH})$ at specimen extraction site compared to open surgery. ${ }^{2,3}$ This retrospective study aims to review local rates of these complications where all contemporary hernia-sparing techniques are applied in the Australian population.

Methods: We reviewed a single-surgeon series of 186 RALP patients between August 2012 and August 2018, where 1-7 years of followup was observed. The study endpoint was $\mathrm{IH}$ rates at the supraumbilical specimen extraction site used by the surgeon.

Results: $\mathrm{IH}$ rate at extraction site was $8.6 \%$, with an incidental $\mathrm{IH}$ rate of $1.1 \%$ at a lateral port site (not associated with specimen removal). Average age at operation was 60.9 years old and hernias were diagnosed at a mean of 11.8 and a median of 12 months. Common demographics in the population suffering $\mathrm{IH}$ were previous abdominal surgery, adhesiolysis, history of smoking (current or quit), and obesity.

Conclusions: Supraumbilical extraction-site hernias continue to possibly be an under-reported complication of RALP, which may impact quality of life and prompt further surgical correction. Further large-scale, prospective research is required.

\section{References}

1. Parsons JK, Bennett JL. Outcomes of retropubic, laparoscopic, and robotic-assisted prostatectomy. Urology 2008;72:412-6. https://doi. org/10.1016/j.urology.2007.11.026

2. Chennamsetty A, Hafron J, Edwards L, et al. Predictors of incisional hernia after robotic-assisted radical prostatectomy. Adv Urol 2015;2015:7. https://doi.org/10.1155/2015/457305

3. Seveso M, Melegari S, Bozzini G, et al. Does site of specimen extraction affect incisional hernia rate after robot-assisted laparoscopic radical prostatectomy? Int / Surg 2017;47:96-100. https://doi. org/10.1016/j.ijsu.2017.09.065 


\section{UP-2.12}

Patient-reported quality of life measures 15 years post-radical prostatectomy: A matched comparison of open retropubic and robot-assisted techniques

Tristan Juvet ${ }^{1}$, Malek Meskawi ${ }^{2}$, Laureano / Rangel ${ }^{1}$, Paige Nichols ${ }^{1}$, Matthew T. Gettman'

${ }^{1}$ Urology, Mayo Clinic Rochester, Rochester, MN, United States; ${ }^{2}$ Urology, Université de Montréal, Montreal, QC, Canada

Introduction: Robot-assisted radical prostatectomy (RARP) has become the most common surgical treatment option for clinically localized prostate cancer in the United States. Controversy continues to exist over the benefits of a robotic approach vs. open radical retropubic prostatectomy (RRP). Previous studies have failed to show a difference in oncological or functional outcomes. Short-term quality-of-life studies have also failed to show a significant difference between the two options. We report data on 15 years of followup using standardized quality-of-life questionnaires. Methods: Survey data was obtained from patients who underwent treatment for clinically localized prostate cancer from August 2002 to December 2005. Patients were matched 2:1 for surgical year, age, preoperative prostate-specific antigen (PSA), clinical stage, and biopsy Gleason grade. Patients were asked to fill out and return three standardized questionnaires.

Results: A total of 452 patients completed and returned the survey. This included 156 patients treated with RARP, matched to 296 patients treated by RRP. Response rate was $70 \%$. There were no significant differences in clinicopathologic characteristics between both groups. In review of all questionnaires, a significant difference was only noted in the number of pads/diapers required in the past four weeks $(p=0.04)$. The overall rate of patients reporting surgical treatments for incontinence $(2.1 \%)$ and erectile dysfunction $(0.7 \%)$ were very low, with no significant difference between RARP and RRP.

Conclusions: There continues to exist few differences in quality-of-life measures between RARP and RRP at long-term followup. Key components of the survey involving sexual function and continence showed no significant difference at 15 years' followup. This represents one of the longest followup periods to date comparing these treatment modalities. All additional variables regarding multiple facets of quality-of-life measures showed no long-term differences between both subgroups.

\section{UP-2.13}

Depression, psychological distress, self-rated mental health and prostate cancer: An examination of a population-based sample of Canadian men

Louise Moodie', Gabriela llie ${ }^{1,2,3}$, Susan Kirkland ${ }^{1}$, Rob Rutledge

${ }^{1}$ Community Health and Epidemiology, Dalhousie University, Halifax, NS, Canada; ${ }^{2}$ Urology, Dalhousie University, Halifax, NS, Canada; ${ }^{3}$ Radiation Oncology, Dalhousie University, Halifax, NS, Canada

Introduction: Having a prostate cancer diagnosis is often only the beginning of a multitude of inter-related health issues that challenge the quality of life of survivors and affect oncological outcomes. ${ }^{1-3}$ Here, we examine the association between prostate cancer and depression, self-rated mental health, and psychological distress in a national sample of Canadian men. Methods: The analytical sample was based on cross-sectional data from 25183 men (ages 47-65) who participated in either the tracking or comprehensive cohorts of the Canadian Longitudinal Study of Aging (CLSA) survey. The CLSA is a national cohort study of adults from 10 provinces across Canada, who were surveyed (baseline) between 2010 and 2015. Logistic regression analyses, controlled for the complexity of the design, demographics, and lifestyle factors (multimorbidity and substance use), evaluated the association between history of a lifetime prostate cancer diagnosis, depression (CES-D10), psychological distress (K10), and self-rated mental health.

Results: The prevalence of lifetime history of prostate cancer diagnosis in this national sample of Canadian men was $4 \%$ (95\% confidence interval [CI] 3.7,4.4). Among survivors of prostate cancer, odds of presenting with clinical psychological distress (adjusted odds ratio [aOR] 1.52; 95\% $\mathrm{Cl} 1.09,2.11)$ and/or depressive symptoms (aOR $1.24 ; 95 \% \mathrm{Cl} 1.02$, 1.51 ) was statistically significantly higher compared with men who never had a prostate cancer diagnosis, even when demographics, multimorbidity, and substance use were statistically controlled. Self-rated mental health showed no differences between the two groups.

Conclusions: While treatments make it possible for survivors of prostate cancer to live long lives, it does not preclude the associative increase of mental health issues in this population compared to men who did not have a prostate cancer diagnosis. Results here suggest that preventative efforts to reduce mental health burden among prostate cancer survivors is a health priority.

\section{References}

1. Fervaha G, Izard JP, Tripp DA, et al. Depression and prostate cancer: A focused review for the clinician. Urol Oncol 2019;37:282. https:// doi.org/10.1016/j.urolonc.2018.12.020

2. Zenger M, Lehmann-Laue A, Stolzenburg JU, et al. The relationship of quality of life and distress in prostate cancer patients compared to the general population. Psychosoc Med 2010;7:Doc02.

3. Courneya KS, Katzmarzyk PT, Bacon E. Physical activity and obesity in Canadian cancer survivors. Cancer 2008;112:2475-82. https:// doi.org/10.1002/cncr.23455

\section{UP-2.14}

\section{Anxiety and depression symptoms in prostate cancer survivors} from Atlantic Canada

\section{Gabriela Ilie $^{1,2}$, Rob Rutledge ${ }^{2}$, Ellen Sweeney ${ }^{3}$}

${ }^{1}$ Community Health and Epidemiology, Dalhousie University, Halifax, NS, Canada; ${ }^{2}$ Radiation Oncology, Dalhousie University, Halifax, NS, Canada; ${ }^{3}$ Atlantic Path, Dalhousie University, Halifax, NS, Canada

Introduction: Side effects associated with current forms of prostate cancer treatment, particularly when combined forms of treatment are administered, are extensive. ${ }^{1}$ Among them, mental health outcomes during survivorship appear to be quite prevalent., ${ }^{2,3}$ Here, we examine the association between depression and anxiety symptoms in a population-based sample of men living in Atlantic Canada and we compare current mental health outcomes among prostate cancer survivors, survivors of any other form of cancer except prostate, and men who never had a prostate cancer diagnosis. Methods: The analytical sample consisted of 6585 male participants aged 49-69 years from the 2009-2015 survey cycle of the Atlantic PATH. Mild, moderate, or severe depression or anxiety indicators were primary outcomes and were assessed using the Generalized Anxiety Disorder (GAD-7) scale and the Patient Health Questionnaire (PHQ-9). The presence of a lifetime history of prostate cancer, any other form of cancer except prostate, or absence any diagnosed form of cancer was main predictor variable. Analyses were controlled for demographic factors and survivorship time since diagnosis.

Results: An estimated 3.9\% of men reported having had a history of prostate cancer diagnosis, $11.3 \%$ of men reported having had a history of any other form of cancer except prostate, and $84.9 \%$ reported never having had a history of a diagnosed form of cancer in their lifetime. Prostate cancer survivors had 2.45 and 2.05 statistically significantly higher odds of screening positive for current anxiety or depressive symptoms, respectively, compared to controls. Cancer survivors (not including prostate), however, had similar current mental health outcomes compared to controls.

Conclusions: Results may point to a silent epidemic of mental health issues among survivors of prostate cancer compared to other cancer survivors, and highlight the importance of a multidisciplinary effort to prioritize and deliver comprehensive mental health support to prostate cancer survivors in Canada.

\section{References}

1. Canadian Cancer Society's Advisory Committee on Cancer Statistics. Canadian Cancer Statistics 2015. 2015.

2. Fervaha G, Izard JP, Tripp DA, et al. Depression and prostate cancer: A focused review for the clinician. Urol Oncol 2019;37:282. https:// doi.org/10.1016/j.urolonc.2018.12.020

3. Zenger M, Lehmann-Laue A, Stolzenburg JU, et al. The relationship of quality of life and distress in prostate cancer patients compared to the general population. Psychosoc Med 2010;7:Doc02. 


\section{UP-2.15 \\ Mental health outcomes in men with a history of radical prostatectomy}

Loshua White' ', Gabriela Ilie', David G. Bell', Gregory G. Bailly', Ricardo A. Rendon', Ross Mason', Joseph Lawen ${ }^{1}$, Rob Rutledge ${ }^{2}$

${ }^{1}$ Department of Urology, Dalhousie University, Halifax, NS, Canada; ${ }^{2}$ Department of Radiation Oncology, Dalhousie University, Halifax, NS, Canada

Support: Soillse Prostate Cancer Quality of Life Research Lab. Dalhousie Research Medical Foundation

Introduction: Survivors of radical prostatectomy (RP) report poor qualityof-life outcomes. ${ }^{1,2}$ We report the burden of mental health issues in a population-based cohort of men with a history of prostate cancer ( $\mathrm{PCa}$ ) who underwent RP to determine risk factors associated with poor mental health outcomes.

Methods: A total of 144 men (aged 48-86 years, median 68) with a history of PCa and RP completed an online survey from 2017-2018 assessing patient-reported quality-of-life outcomes. The primary outcome was a validated assessment of mental health disorder: the Kessler Psychological Distress Scale (0-50, with $>20$ being likely to have a mental health issue). Urinary problems were assessed using the International Prostate Symptom Score (0-35, with >7 indicating moderate/severe urinary symptoms). Relationship satisfaction was assessed using the validated Dyadic Adjustment Scale (0-151, with $>97$ indicating relationship satisfaction). Analyses were controlled for age, comorbidities, and survivorship time. The analytical sample was 126. A multiple logistic analysis assessed the association between the stated predictors and current mental health status. Results: A total of $16.4 \%$ men scored positive for current mental health issues; $60.3 \%$ of respondents reported mild urinary problems, while $39.7 \%$ reported moderate/severe urinary problems. The odds ratio (OR) was $4.49(95 \%$ confidence interval $[\mathrm{Cl}] 1.41,14.28)$ for presenting with mental health issues among men with moderate/severe urinary problems. Being satisfied in a relationship was a protective factor for mental health issues (OR $0.2 ; 95 \% \mathrm{Cl} 0.05,0.86$ ), as was being older (OR $0.87 ; 95 \% \mathrm{Cl}$ $0.79,0.96)$. Survivorship time did not contribute statistically significantly to the model.

Conclusions: Men with prostate cancer undergoing RP are at increased risk of mental health illness. ${ }^{2,3}$ In this study, we showed that younger men, men with moderate/severe urinary symptoms, and men reporting relationship dissatisfaction were found to be at higher risk for mental health issues. References

1. Canadian Cancer Society's Advisory Committee on Cancer Statistics. (2017). Canadian Cancer Statistics 2017. Toronto, ON: Canadian Cancer Society.

2. Fervaha G, Izard JP, Tripp DA, et al. Depression and prostate cancer: A focused review for the cliician. Urol Oncol 2019;37:282-8. https://doi.org/10.1016/j.urolonc.2018.12.020

3. Isbarn H, Wanner M, Salomon G, et al. Long-term data on the survival of patients with prostate cancer treated with radical prostatectomy in the prostate-specific antigen era. BJU Int 2010;106:37-43. https://doi.org/10.1111/j.1464-410X.2009.09134.x

\section{UP-2.16}

An examination of the relationship between oncological treatment and biopsychosocial quality-of-life outcomes in Maritime prostate cancer survivors actively treated for their malignancy

Tarek Lawen ${ }^{1}$, Gabriela Ilie $\mathrm{i}^{1,2,3}$, David Bell', Ross Mason', Gregory G. Bailly', Ricardo A. Rendon', Joseph Lawen', Rob Rutledge ${ }^{2}$

'Department of Urology, Dalhousie University, Halifax, NS, Canada; ${ }^{2}$ Department of Radiation Oncology, Dalhousie University, Halifax, NS, Canada; ${ }^{3}$ Department of Community Health and Epidemiology, Dalhousie University, Halifax, NS, Canada

Introduction: Prostate cancer is the second most common cancer in men internationally and the fifth leading cause of cancer death. ${ }^{1}$ Yet, prostate cancer carries one of the most favorable five-year survival rates of all malignancies. ${ }^{2}$ With over 1.1 million men diagnosed annually, focus on survivorship issues and patient-reported outcomes is becoming increas- ingly important. ${ }^{3}$ Prostate cancer treatment, albeit effective, is plagued by a host of life-altering side effects, such as bladder, bowel, and sexual dysfunction, as well as significant mental health issues. ${ }^{4}$ Approximately $60 \%$ of men with prostate cancer experience mental health distress, ${ }^{5}$ with $10-40 \%$ having clinically significant depression. ${ }^{6}$ The goal of our study was to evaluate the associative role of age at diagnosis, urinary function, multimorbidity, number of active forms of treatment, survivorship time, and social well-being on current mental health outcomes.

Methods: A total of 129 men (mean age 62 years) with a history of prostate cancer who received active treatment for their diagnosis completed an online survey between May 2017 and December 2018, assessing patientreported quality-of-life outcomes. The primary outcome was a validated assessment of mental health disorder: the Kessler Psychological Distress Scale (K10). Urinary problems and social well-being were assessed using the UCLA Prostate Cancer Index and the Functional Assessment of Cancer Therapy-Prostate (FACT-P), respectively. Analyses were controlled for age at diagnosis, multimorbidity, number of received treatments to date, and survivorship time.

Results: A total of $14.7 \%$ men scored positive for current mental health issues. The fitted logistic model was statistically significant X2(8)=28.67; $\mathrm{p}<0.001$. A logistic model to help predict who is more likely to screen positive for mental health issues revealed that better urinary function, increased social well-being, higher age at diagnosis, and longer survivorship time were associated with a decreased probability of having mental health issues at 0.97 (95\% confidence interval [CI] 0.95, 0.99), 0.15 (95\% Cl 0.03, 0.73), 0.91 (95\% Cl 0.83, 0.99), 0.98 (95\% Cl 0.97, 0.99), respectively. Multimorbidity, bowel, and sexual function did not contribute statistically significantly to the model.

Conclusions: Here, we show an association between a prostate cancer diagnosis and increased rates screening positive for mental distress among men in the Maritime provinces. These findings highlight the importance of a multidisciplinary approach to the prostate cancer care and survivorship, using the skillset of physicians, nurses, mental health experts, and physiotherapists.

\section{References}

1. Torre LA, Bray F, Siegel RL, et al. Global cancer statistics, 2012. CA Cancer J Clin 2015;65:87-108. https://doi.org/10.3322/caac.21262

2. Siegel RL, Miller KD, Jemal A. Cancer statistics, 2018. CA Cancer J Clin 2018;68:7-30. https://doi.org/10.3322/caac.21442

3. Donovan JL, Hamdy FC, Lane JA, et al. Patient-reported outcomes after monitoring, surgery, or radiotherapy for prostate cancer. N Engl J Med 2016;375:1425-37. https://doi.org/10.1056/NEJMoa1606221

4. Spiegel D, Giese-Davis J. Depression and cancer: Mechanisms and disease progression. Biol Psychiatry 2003;54:269-82. https://doi. org/10.1016/S0006-3223(03)00566-3

5. Steginga SK, Occhipinti S, Gardiner RA, et al. Prospective study of men's psychological and decision-related adjustment after treatment for localized prostate cancer. Urology 2004;63:751-6. https://doi. org/10.1016/j.urology.2003.11.017

6. Mohamed NE, Bovbjerg DH, Montgomery GH, et al. Pretreatment depressive symptoms and treatment modality predict post-treatment disease-specific quality of life among patients with localized prostate cancer. Urol Oncol 2012;30:804-12. https://doi.org/10.1016/j. urolonc.2011.02.002

\section{UP-2.17}

The androgen deprivation therapy educational program: A Canadian resource that supports prostate cancer patients in managing hormone therapy side effects

Lauren M. Walker 1,2, Erik Wibowo ${ }^{3}$, Carly S. Sears ${ }^{2}$, John W. Robinson 1,2, Andrew G. Matthew ${ }^{4}$, Deborah McLeod ${ }^{5}$, Richard J. Wassersug ${ }^{6}$

${ }^{1}$ Oncology, University of Calgary, Calgary, AB, Canada; ${ }^{2}$ Oncology, Division of Psychosocial Oncology, Tom Baker Cancer Centre, Calgary, AB, Canada; ${ }^{3}$ Anatomy, University of Otago, Dunedin, New Zealand; ${ }^{4}$ Department of Surgical Oncology, Princess Margaret Cancer Centre, Toronto, ON, Canada; ${ }^{5}$ Nursing, Dalhousie University, Halifax, NS, Canada; ${ }^{6}$ Department of Cellular and Physiological Sciences, University of British Columbia, Vancouver, BC, Canada 
Introduction: Androgen deprivation therapy (ADT) is a common prostate cancer $(\mathrm{PCa})$ treatment but has numerous adverse effects that reduce patients' quality of life. The ADT Educational Program helps prepare patients to manage ADT side effects. The program consists of a single, 1.5-hour, professionally facilitated class plus the CUA-endorsed book Androgen Deprivation Therapy: An Essential Guide for Prostate Cancer Patients and their Loved Ones. Here, we describe the dissemination of the program in both an in-person and online format.

Methods: Launched in person in 2014, the initial implementation study collected pre- and post-intervention data from 94 patients about side effect bother, self-efficacy in managing side effects, and patient satisfaction with the program. Feasibility and acceptability of the in-person program were assessed in a national dissemination strategy across 14 program sites. Launched in 2018, the online program has been evaluated for feasibility and acceptability, with a full-scale evaluation currently being conducted. Results: Evaluation data support the feasibility and acceptability of the ADT Education Program. Data demonstrate that the in-person program is associated with significant improvements in patients' self-efficacy to manage ADT side effects. Patient satisfaction with the in-person and online programs are similarly high. A total of 14 sites and 29 facilitators have been trained to offer the program; 13 sites in Canada and one in the United States. The program is currently offered monthly online and in-person at six sites.

Conclusions: The ADT Educational Program is feasible and acceptable to patients and cancer care institutions. The program improves patient self-efficacy and their uptake of evidence-based strategies for managing ADT side effects. Explorations have begun to bring the program to Europe and Australia.

\section{UP-2.18}

Has androgen deprivation therapy for patients receiving radical prostatectomy been adequately studied? A systematic review and meta-analysis

Ameeta Nayak ${ }^{1,2}$, Ana Flaman ${ }^{3}$, Ranjeeta Mallick ${ }^{1,2}$, Luke T. Lavallée $e^{1,2,4}$ Dean Fergusson ${ }^{1,2}$, Rodney H. Breau ${ }^{1,2,4}$

${ }^{1}$ Clinical Epidemiology Program, Ottawa Hospital Research Institute, Ottawa, ON, Canada; ${ }^{2}$ Faculty of Medicine, University of Ottawa, Ottawa, ON, Canada; ${ }^{3}$ Ottawa Urologic Oncology Research, Ottawa, ON, Canada; ${ }^{4}$ Division of Urology, Department of Surgery, The Ottawa Hospital, Ottawa, ON, Canada

Introduction: Androgen deprivation therapy (ADT) improves outcomes for patients with clinically localized disease receiving radiation. We reviewed the evidence evaluating neoadjuvant and adjuvant ADT for patients receiving radical prostatectomy.

Methods: MEDLINE, EMBASE, and the Cochrane Library were searched from inception to September 2019. Randomized trials comparing ADT with radical prostatectomy vs. prostatectomy alone in patients with prostate cancer were included. The primary outcomes were cancer recurrence and overall survival. Pathologic outcomes in patients treated with ADT prior to surgery were also evaluated.

Results: Eleven studies evaluated the effects of 3-6 months of neoadjuvant ADT ( $n=2322$ ). Neoadjuvant ADT significantly decreased the rate of positive surgical margins (relative risk [RR] $0.48 ; 95 \%$ confidence interval $[\mathrm{Cl}] 0.41-0.56)$ and T3 disease (RR $0.75 ; 95 \% \mathrm{Cl} 0.64-0.89$ ). There was no difference in pooled overall or progression free survival with neoadjuvant ADT (RR 1.3; 95\% Cl 0.8-2.1; RR 0.99; 95\% Cl 0.7-1.3, respectively). Four studies evaluated the effects of adjuvant ADT ( $n=5198)$, of which two studies evaluated anti-androgen and one studied diethylstilbestrol monotherapy. The use of adjuvant ADT demonstrated no difference in pooled overall or progression free survival (RR 1.0; 95\% Cl 0.9-1.1; RR 0.8; 95\% Cl 0.6-1.0, respectively).

Conclusions: For patients treated with radical prostatectomy, neoadjuvant trials only evaluated short-term ADT and adjuvant trials have not assessed contemporary ADT. Longer-term, contemporary ADT have not been adequately studied for patients treated with radical prostatectomy.

\section{UP-2.19}

Real-world practice patterns of androgen deprivation therapy (ADT): How do physician characteristics and socio-demographics affect intermittent ADT use?

Douglas C. Cheung ${ }^{1}$, Lisa Martin', Maria Komisarenko', Christopher Dharma ${ }^{2}$, Girish S. Kulkarni', Shabbir M. Alibhai' ${ }^{3}$, Antonio Finellit 'Department of Surgery, Division of Urology, University Health Network, Toronto, ON, Canada; ${ }^{2} \mathrm{ICES}$, Toronto, ON, Canada; ${ }^{3}$ Division of Internal Medicine and Geriatrics, University Health Network, Toronto, ON, Canada

Introduction: Level 1 evidence supports intermittent androgen deprivation therapy (IADT) as non-inferior to continuous ADT (CADT) in nonmetastatic biochemically recurrent prostate cancer (PC), while sparing adverse events. However, due to concerns about the trial definitions, clinical practice and guidelines vary. We evaluate the real-world use of IADT. Methods: We used population-level data at ICES to identify Ontario men age $\geq 65$ who were diagnosed with PC (1997-2012) and initiated ADT for $\geq 6$ months. Neoadjuvant/adjuvant therapy was excluded. After induction, lapses in ADT $\geq 6$ months (initial gap) and $\geq 3$ months (subsequent gaps) were used to identify IADT. A five-year minimum followup ensured patients had the opportunity to become intermittent. Adjustment for disease stage/likely metastatic disease was based on a de novo presentation with ADT vs. prior local therapy. Predictors of IADT were assessed using multivariable logistic regression.

Results: We identified 8544 patients with 1404 (16\%) ever on IADT. Use varied from $11.4-24.8 \%$ across health regions. IADT patients were younger, less comorbid, had higher income, and were more likely to have prior therapy. They were more often started in later calendar years, by higher-volume physicians and by those who graduated more than 10 years previously. After a mean followup of 8.3 years, $81 \%$ and $47 \%$ of IADT patients experienced second and third gaps (14 and 20 months long), respectively. In multivariable analyses, highest income quintile (vs. lowest; odds ratio [OR] 1.44, 1.19-1.74) and prior local therapy (OR $1.85 ; 1.57-2.18)$ predicted IADT. Physicians over 10 years in practice were more likely to prescribe IADT (OR 1.52; 1.18-1.97), as were radiation oncologists (vs. urologists; OR 2.13; 1.70-2.68). Case volume was associated with IADT for radiation oncologists (OR 1.65; 1.07-2.54).

Conclusions: IADT remains underuses. Radiation oncologists, higher case volume, and greater experience predicted IADT as did patient income quintile and prior local therapy.

\section{UP-2.20}

Health-related quality of life in advanced prostate cancer patients on androgen deprivation therapy

Douglas C. Cheung ${ }^{1,2}$, Ayesha Syeda ${ }^{2}$, Diana E. Magee', Amanda Hird', Lisa Martin ${ }^{1}$, Maria Komisarenko ${ }^{1}$, Karen Bremner ${ }^{2}$, Shabbir M. Alibhai ${ }^{3}$, Girish S. Kulkarni ${ }^{1}$, Antonio Finelli', Murray Krahn²

'Department of Surgery, Division of Urology, University Health Network, Toronto, ON, Canada; ${ }^{2}$ Toronto Health Economics and Technology Assessment Collaborative, University of Toronto, Toronto, ON, Canada; ${ }^{3}$ Division of Internal Medicine and Geriatrics, University Health Network, Toronto, ON, Canada

Introduction: Patient-reported outcomes (PRO; both preference- and nonpreference-based) are critical to measuring health-related quality of life (HRQoL) and providing effective and cost-effective care. However, limited longitudinal information is available to evaluate the HRQoL associated with advanced prostate cancer (PC) and androgen deprivation therapy (ADT). The Patient Oriented Prostate Utility Scale (PORPUS) is a validated disease-specific instrument to assess patient preferences across ten domains of prostate cancer care.

Methods: PORPUS-P (psychometric) and PORPUS-U (utility) scores were prospectively measured in prostate cancer patients at our tertiary care center (2003-2016). All patients that started ADT were included and stratified by those receiving primary ADT vs. after local therapy. Scores were pooled based on pre/post-ADT timing and type. Mixed models were completed to assess patient and disease characteristics predictive of PORPUS scores, after adjusting for repeated patient measurements. 
Results: A total of 7156 observations from 990 patients were identified (7.2 measurements/patient, range 1-43) over a mean followup of 7.5 years (4.3 years on ADT). Mean age at ADT initiation was 71 years. PORPUS-P and PORPUS-U scores were $67.2 \pm 13.2$ and $0.925 \pm 0.077$ for patients starting oral ADT, $67.9 \pm 11.9 \& 0.926 \pm 0.064$ on gonadotropinreleasing hormone (GNRH) agonist/antagonist agents, and $60.5 \pm 11.1$ \& $0.891 \pm 0.070$ for patients transitioning onto abiraterone/enzalutamide. After adjusting for age, income, rurality, marital status, comorbidity, local therapy, prostate-specific antigen (PSA), Gleason score and stage, ADT therapy significantly decreased psychometric and utility scores (all $p<0.001):-3.3$ and -0.015 with oral ADT, -5.0 and -0.023 on GNRH agents, and -11.0 and -0.055 after abi/enza.

Conclusions: ADT is associated with a significant decrease in HRQOL and was most pronounced following abi/enza. However, these effects are likely mediated by both the drug and disease process. These results represent the largest cohort of PRO in advanced prostate cancer, and will be critical to economic and policy model application.

\section{UP-2.21}

Outcomes of abiraterone plus prednisone vs. docetaxel in metastatic hormone-sensitive prostate cancer in a real-world setting

Paulo Werlang ${ }^{1}$, Tom Ying ${ }^{1}$, Christopher Knee ${ }^{1}$, Igal Kushnir ${ }^{1}$, Christopher G. Morash', Rodney H. Breau', Luke T. Lavallée', Ilias Cagiannos ${ }^{\dagger}$ 'Urology, University of Ottawa, Ottawa, ON, Canada

Introduction: We compared outcomes of patients treated with docetaxel $(\mathrm{DOC})$ or abiraterone + prednisone $(\mathrm{AA}+\mathrm{P})$ plus androgen deprivation therapy (ADT) in patients with metastatic hormone-sensitive prostate cancer (MHSPC) in a real-world setting.

Methods: We conducted a retrospective chart review of patients treated with either DOC or AA+P at The Ottawa Hospital from January 1, 2014 to April 1, 2017 (DOC group), and from January 1, 2017 to March 14, 2019 (AA+P group). We included patients with hormone-sensitive disease who received $\mathrm{ADT}$ for the first time. The primary outcome was overall survival (OS) rate. Secondary outcomes included radiological progression-free survival (rPFS) and prostate-specific antigen (PSA) progression-free survival (pPFS). We also identified factors associated with survival.

Results: We identified 102 eligible patients with MHSPC treated with $\mathrm{DOC}$ or $\mathrm{AA}+\mathrm{P}$. Mean age was 66 years in the DOC group and 72 years in the $A A+P$ group $(p<0.05)$. The median PSA at diagnosis in the DOC group was 175 , and 151 in $A A+P$ group $(p=0.3)$. Most patients in both groups had Gleason $\geq 8$ (DOC 95\%, AA+P 86\%). The mean followup in the DOC group was 26 months and in the $\mathrm{AA}+\mathrm{P}$ group was 15 months $(p<0.05)$. For the DOC group, nadir PSA levels six months post-initiation of ADT were $<0.2,0.2-1,1-4$, and $>4 \mathrm{ng} / \mathrm{mL}$ in $18 \%, 35 \%, 15 \%$, and $32 \%$, respectively. For the $\mathrm{AA}+\mathrm{P}$ group, nadir PSA levels six months postinitiation of ADT were $<0.2,0.2-1,1-4$, and $>4 \mathrm{ng} / \mathrm{mL}$ in $38 \%, 19 \%$, $15 \%$, and $28 \%$, respectively. Patients receiving abiraterone had superior pPFS ( $p=0.0117)$. rPFS was not statistically different $(p=0.3634)$. The only predictor of pPFS and rPFS was PSA six months post-initiation of ADT. Patients with PSA $>4$ vs. $<0.2$, and PSA $>1-4$ vs. $<0.2$ had increasingly greater results of failure.

OS did not differ significantly between groups $(\mathrm{p}=0.16)$. The only predictor of OS was the PSA at six months $>4$ vs. $<0.2$ hazard ratio $13.85(95 \%$ confidence interval [Cl] 1.06-179.73).

Conclusions: In our cohort study, DOC and AA+P groups had comparable survival endpoints. PSA nadir six months post-initiation of ADT appears to be an important predictor of OS, pPFS, and rPFS. Patients who failed to reach PSA $<1$ had worse rPFS and pPFS. Patients who did not reach PSA $<4$ had a poor OS rate.
UP-2.22

Population-based study of baseline bone density screening in prostate cancer patients treated with long-term androgen deprivation therapy

Jason Hu ${ }^{1}$, Armen-G. Aprikian ${ }^{1}$, Marie Vanhuyse ${ }^{2}$, Alice Dragomir ${ }^{1}$

${ }^{1}$ Division of Urology, McGill University, Montreal, QC, Canada; ${ }^{2}$ Division of Medical Oncology, McGill University, Montreal, QC, Canada

Introduction: Androgen deprivation therapy (ADT) is a cornerstone of advanced prostate cancer ( $\mathrm{PCa}$ ) treatment, however, it accelerates the loss of bone mineral density (BMD), which increases fracture risk. Guidelines recommend BMD testing when initiating ADT to properly assess baseline fracture risk. The objective was to examine the proportion of BMD testing in men initiating ADT in Quebec and to identify factors associated with the receipt of $\mathrm{BMD}$ testing.

Methods: The cohort consists of men extracted from Quebec public healthcare insurance administrative databases who initiated continuous ADT from 2000-2015 for over 12 months. The primary study outcome was the receipt of BMD testing in the period from six months prior to and up to 12 months after ADT initiation. Multivariable generalized linear mixed regression modeling with a logit link was performed to identify variables associated with BMD testing.

Results: We identified 22033 patients, of which 3910 (17.8\%) underwent BMD testing. Rates of BMD testing increased from $4.1 \%$ in 2000 to $23.4 \%$ in 2015. Following multivariable analyses, prior history of osteoporosis (odds ratio [OR] 1.81; 95\% confidence interval [CI] 1.29-2.52; $\mathrm{p}=0.001$ ), rheumatoid arthritis (OR 1.60; 95\% Cl 1.13-2.28; $\mathrm{p}=0.009)$, use of bisphosphonates (OR 1.46; 95\% Cl 1.25-1.72; $\mathrm{p}<0.001$ ), and chronic corticosteroids (OR $1.60 ; 95 \% \mathrm{Cl} 1.13-2.27 ; \mathrm{p}=0.008)$ were associated with higher odds of $\mathrm{BMD}$ testing. Patient age $>80$ (OR $0.67 ; 95 \% \mathrm{Cl} 0.59-0.75 ; \mathrm{p}<0.001)$, metastases (OR 0.79; $95 \% \mathrm{Cl} 0.70,0.89 ; \mathrm{p}<0.001$ ), greater Charlson comorbidity score (OR $0.71 ; 95 \% \mathrm{Cl} 0.62-0.81 ; \mathrm{p}<0.001$ ), and rural residence (OR $0.77 ; 95 \%$ Cl $0.67-0.87 ; p<0.001)$ were associated with lower odds of BMD testing. Conclusions: In our study population, BMD testing rates in men initiating ADT were low, although they increased over the years. Potential gaps identified include the older, more comorbid, and rural areas. Overall, additional efforts emphasizing the importance of BMD testing in PCa guidelines may be needed.

\section{UP-2.23}

Population-based study of abiraterone and enzalutamide in the post-chemotherapy setting in metastatic castration-resistant prostate cancer

Lason Hu' ${ }^{1}$, Armen G. Aprikian', Marie Vanhuyse², Soukaina Ouizzane', Alice Dragomir

${ }^{1}$ Division of Urology, McGill University, Montreal, QC, Canada; ${ }^{2}$ Division of Medical Oncology, McGill University, Montreal, QC, Canada

Introduction: Novel hormonal agents, such abiraterone (ABI) and enzalutamide (ENZA), have both demonstrated survival benefits in the postchemotherapy setting in metastatic castration-resistant prostate cancer (mCRPC). However, there are currently no randomized head-to-head comparisons of both agents. The objective was to compare the effectiveness of $\mathrm{ABI}$ and ENZA as second-line treatments in the post-chemotherapy setting in patients with mCRPC.

Methods: A retrospective, population-based cohort was extracted from Quebec public healthcare administrative databases. Patients were selected based on having sequentially received androgen deprivation therapy and chemotherapy prior to initiating a novel hormonal agent (ABI or ENZA) between 2012 and 2016. The index date corresponded to the date of the first prescription of $\mathrm{ABI}$ or ENZA. The primary outcome of interest was overall survival and evaluated with Kaplan-Meier analysis and multivariable Cox proportional hazards regression.

Results: The cohort is comprised of 621 patients, with 542 in the ABI group and 79 in the ENZA group. Median age at initiation was similar (ABI: 73, ENZA: 74; $p=0.449$ ). There were more patients in the $A B I$ group with a time from last chemotherapy to index date $<6$ months (ABI: $72.5 \%$, ENZA: $57.0 \%$; $p=0.005$ ). Median duration of treatment was similar in both groups at six months (interquartile range [IQR] 3-12) $(\mathrm{p}=0.317)$. Median overall survival was 15.4 months in the $\mathrm{ABI}$ group and 17.9 months in 
the ENZA group (log-rank $\mathrm{p}=0.822$ ). On multivariable analysis, the hazard ratio (HR) for $\mathrm{ABI}$ vs. ENZA was 1.08 (95\% confidence interval $[\mathrm{Cl}]$ $0.79-1.38 ; \mathrm{p}=0.721)$. Age greater than 75 (HR $1.41 ; 95 \% \mathrm{Cl} 1.18-1.70$; $\mathrm{p}<0.001)$, Charlson comorbidity scores greater than $4(\mathrm{HR} 1.33 ; 95 \% \mathrm{Cl}$ $1.08-1.63 ; p=0.008)$, presence of symptoms (HR 1.69; 95\% Cl 1.40-2.04; $\mathrm{p}<0.001)$, time from prostate cancer diagnosis $<3$ years $(\mathrm{HR} 1.65 ; 95 \% \mathrm{Cl}$ $1.31-2.09 ; p<0.001)$, and time from last chemotherapy $<6$ months (HR $1.30 ; 95 \% \mathrm{Cl} 1.40-2.04 ; \mathrm{p}<0.001)$ were associated with worse survival. Conclusions: In our study population, there was no difference in overall survival between $\mathrm{ABI}$ and ENZA as second-line treatments in the postchemotherapy setting in mCRPC. Further evaluation of both drugs using real-world data is necessary to assess differences in other health outcomes, such treatment-related complications and use of health services.

\section{UP-2.24}

\section{Comparing testosterone levels in prostate cancer patients treated} with enzalutamide or abiraterone

Zoe Glase' ${ }^{1}$, Mohamad Baker Berjaou ${ }^{2}$, Karen Hersey², Heidi Wagner ${ }^{2}$, Miran Kenk', Yazan Qaoud², Saranya Kulendran², Susan Nguyen², Alisha Bhimani ${ }^{2}$, Neil E. Fleshner ${ }^{2}$

${ }^{1}$ Carelton University, Ottawa, ON, Canada; ${ }^{2}$ Urological Oncology, University Health Network - Princess Margaret Cancer Centre, Toronto, ON, Canada

Introduction: Enzalutamide (Xtandi ${ }^{\circledR}$ ) and abiraterone acetate (Zytiga ${ }^{\circledR}$ ) are second-generation hormone therapy drugs used for the treatment of advanced prostate cancer $(\mathrm{PCa})$. Serial serum prostate specific antigen (PSA) and testosterone (T) testing is commonly used to monitor therapeutic response to most PCa therapies. There has been recent interest in interpreting and understanding how $\mathrm{T}$ levels are affected by exposure to this class of drugs. The purpose of this study was to determine if there is a significant difference between $T$ levels of patients treated with abiraterone compared to patients treated with enzalutamide.

Methods: Data from the electronic patient records (EPR) and the GU BioBank of University Health Network (UHN) (Princess Margaret Cancer Centre) was used. We examined the EPR charts of men diagnosed with advanced PCa who had been exposed to either abiraterone or enzalutamide. A total of 150 patients were identified to be on one of the two drugs: 75 patients on abiraterone and 75 patients on enzalutamide. The $\mathrm{T}$ levels of patients, measured at both UHN and external laboratories, were then analyzed and compared.

Results: The median T levels were $0.74(<0.1-11.5)$ and $0.23(<0.1-11)$ $\mathrm{nmol} / \mathrm{L}$ for enzalutamide and abiraterone, respectively. A total of $830 \mathrm{~T}$ levels were recorded for patients using enzalutamide, while 726 were recorded for patients on abiraterone. One hundred forty patients were being treated concurrently with a luteinizing hormone-releasing hormone (LHRH) agonist or antagonist; 10 patients showed no record of being on a concurrent LHRH therapy. There was a statistically significant difference in the T levels between the two drugs using the t-Test $(p=1.19 \times 10-34)$.
Conclusions: Our study suggests that there is a statistically significant difference between the T levels of patients treated with abiraterone compared to those treated with enzalutamide. Abiraterone appears to suppress $\mathrm{T}$ to lower levels than enzalutamide. There is not a clinical significance due to the drugs still having very similar effects as a treatment of $\mathrm{PCa}$, therefore, measuring $\mathrm{T}$ levels of patients on these drugs may not be required.

\section{UP-2.27}

The bacterial DNA content of normal and prostate human tumor tissues and its modulation by a single nutrient

Gabriel Lachance ${ }^{1}$, Karine Robitaille" , Hélène Hovington ${ }^{1}$, Alain Bergeron ${ }^{1}$, Yves Fradet ${ }^{\prime}$, Vincent Fradet ${ }^{\dagger}$

${ }^{1} \mathrm{CHU}$ de Québec, Unversité Laval Research Center, Quebec City, QC, Canada

Introduction: Cancer immunotherapy success was recently shown to be dependent on microbiota. Since few prostate cancer patients respond to immunotherapy, we used metagenomic sequencing to identify microbeassociated molecular patterns (MAMP) that could be linked to prostate tumor.

Methods: A double-blind, phase $2 \mathrm{~b}$, randomized controlled trial was conducted in our team to test the effects of monoacylglyceride-conjugated eicosapentaenoic acid (MAG-EPA)-rich fish oil in men with prostate cancer treated by radical prostatectomy (NCT02333435). In that study, participants were randomized to take either $3 \mathrm{~g} /$ day of MAG-EPA or placebo for 4-10 weeks before surgery. Several biological samples were collected at baseline and surgery, including blood, stool, and prostate tissue. We analyzed the bacterial composition of prostate tissue at surgery from 30 patients using 16srRNA metagenomic.

Results: Phylogenetic analysis showed that the vast majority of bacterial sequences found in all tissues and controls were associated with Proteobacteria phylum, which inhabit several ecological niches. However, sequences associated with Firmicutes phylum, which is naturally enriched in humans, were significantly higher in prostatic tissues compared to controls $(p=0.031)$. Oscillibacter genus was the only signal to be significantly different between normal and tumor samples. We also observed that sequences from Clostridia were significantly reduced in patients who received MAG-EPA compared to placebo. In contrast, sequences associated with Corynebacterium genus were enriched in MAG-EPA group with a stronger trend in tumor compared to normal samples. Interestingly, Bacteroides and Phascolarctobacterium genus showed a trend to be specifically depleted in tumor samples from the placebo group.

Conclusions: Taken together, our results support the idea that MAMPs can be modulated at the tumor site by a targeted dietary intervention. This approach could be used to provide immune cofactors and beneficially modulate the anti-tumor response in prostate cancer. 


\section{Unmoderated Poster Session 3: Prostate Cancer, Functional Urology, Other Urology Topics}

\begin{abstract}
UP-3.1
Does urethral catheter education improve confidence in community pediatric care providers? A single-institution experience

Ming-Hsien Wang ${ }^{1}$, Kathy Luong ${ }^{1}$, Alaina Dozar ${ }^{1}$

'Urology, Baylor College of Medicine/Texas Children's Hospital, Woodlands, TX, United States

Support: APPs in division of urology. Research support in urology

Introduction: Placement of urethral catheters (UC) is a common practice in the management of ill patients. Risk associated with catheters include urinary tract infection and trauma resulting in long-term morbidity. The incidence of urethral trauma remains unclear in the pediatric population. As catheter placement technique is a modifiable risk factor for urethral injury, we assessed the knowledge and comfort level of clinical staff who work with children via a pre- and post-questionnaire after a continuing medical education (CME) supported educational seminar.

Methods: This prospective pilot study was conducted at a Texas Children's community hospital. The Urology Advanced Practice Providers (APPs) created a CME-approved lecture that was reviewed by fellowship-trained urologists. The objectives of the presentation were to: 1 ) review new developments in catheter placement and removal; 2) troubleshoot; 3) recognize when urology involvement is appropriate; and 4) support a safer environment. We provided eight-question pre and post-tests, which were distributed prior to and immediately following the lecture. Each answer was given a value of 0 or 1, with 0 indicating an incorrect answer and 1 indicating a correct answer (maximum score 12).

Results: Thirteen of 23 participants completed pre- and post-test questionnaires. The mean pre-test score was 7 (range 4-10), and the mean post-test score was 10.2 (range 8-12). The mean improvement in score was 3.2 points (range 6-13). The mean improvement in overall confidence after lecture was 1.3 points (range $0-3$ ), with seven of 13 participants denoting an improvement in overall comfort.

Conclusions: This pilot study demonstrates modest improvement in provider comfort after CME-supported education on proper UC placement techniques. Further studies will be needed to optimize the educational approach to decreasing the incidence and morbidity of urethral trauma from UC placement.
\end{abstract}

\section{UP-3.2}

Home-based video tele-urology e-visit: "Doctor's house call" is back - a community urology practice experience O. Emmanuel Abara ${ }^{1}$, Valerie Lamontagne ${ }^{2}$, Ogechi Abara ${ }^{3}$, Nickolas Syvestre ${ }^{4}$

${ }^{1}$ Division of Clinical Sciences, Surgery (Urology), Northern Ontario School of Medicine/RHUPPI, Sudbury/Richmond Hill, ON, Canada; ${ }^{2}$ Nursing, North Centennial Manor, Kapuskasing, ON, Canada; ${ }^{3}$ Family Medicine/ Geriatrics, Yuma Regional Medical Center, Yuma, AZ, United States; ${ }^{4}$ Family Practice, Sensenbrenner Hospital, Kapuskasing, ON, Canada

Support: Ontario Telemedicine Network (OTN)

Introduction: The uptake of telemedicine in clinical practice is on the rise among specialties at different paces in Ontario. We studied home-based video e-visits in an office urology practice between June 6, 2018 and August 8, 2019.

Method: Following approval by the Ontario Telemedicine Network (OTN), training of the urologist and office staff was completed. Enrolment of patients was by informed consent. Participants needed to have internet access, an email account, and a computer or a tablet/smart phone. Steps to prepare for an e-visit include email invitation, testing connection/device, and installation of the app. On the day of visit, patients open the invitation email at the scheduled time and click on "join video visit" to meet the urologist. A post-visit survey was completed. Patients underage and those with limited technical skills were assisted by parents, relatives, or primary healthcare providers. Patients' demographics, diagnoses, reason for encounter, and feedback were recorded.

Results: The study included a total of 156 patients: 108 male and 48 females, aged between seven days and 96 years. Patient encounters included followup to review test results and surgical outcomes $(n=107)$, counselling $(n=43)$, and 'assessments' $(n=6)$. There were a variety of diagnoses. Participants lived near and far, in both rural and urban settings. Patients rated their experience as: time-saving, convenient, and no traffic jam. In nine patients, video visit was completed by telephone.

Conclusions: Home-based video visit is going to grow in urology practices. Collaborative enrollment of more participants in multiple sites is required to better understand the benefits and limitations of this technology-driven "doctor's house call."

\section{UP-3.3}

Association between perioperative blood transfusion and survival in patients undergoing radical cystectomy for bladder cancer Nick Dean' ${ }^{1}$, Jan K. Rudzinski ${ }^{1}$, Graham Follett ${ }^{1}$, Derrick Tilley², Niels-E. B. Jacobsen', Adrian Fairey

${ }^{1}$ Urology, University of Alberta, Edmonton, AB, Canada; ${ }^{2}$ Cancer Control Alberta, Alberta Health Services, Calgary, AB, Canada

Introduction: Perioperative blood transfusion has been associated with adverse cancer outcomes in colorectal and head and neck cancers. Radical cystectomy for bladder cancer is a high-risk surgical oncology procedure associated with high blood transfusion rates. The objective of the current study was to determine the association between receipt of perioperative blood transfusion and survival outcomes in patients undergoing radical cystectomy for primary bladder cancer at a Canadian center.

Methods: The study was a retrospective analysis of prospectively collected data. Eligible subjects were those with clinical Ta-4N0-3M0 urothelial carcinoma of the bladder undergoing curative intent radical cystectomy at the University of Alberta between 1994 and 2019. Patients were classified as having received a blood transfusion if they received any blood product within 90 days of surgery. The main outcome measures were overall survival (OS) and bladder cancer-specific survival (BCSS). The Kaplan-Meier method and Cox proportional regression models were used to analyze the association between receipt of blood transfusion and survival outcomes. Statistical tests were two-sided $(\mathrm{p} \leq 0.05)$.

Results: Between 1994 and 2019, 1094 patient underwent radical cystectomy with curative intent; 851 patients $(78.3 \%)$ were male. Median age was 67 years (range $27-89$ years). A total of 448 patients ( $44 \%$ ) had organconfined disease ( $£$ pT2N0) on final radical cystectomy pathology; 346 patients $(36 \%)$ received a perioperative blood transfusion. After adjustment for case mix, receipt of perioperative blood transfusion was independently associated with poorer OS (hazard ratio [HR] 1.26; 95\% confidence interval [CI] 1.03-1.54; $\mathrm{p}=0.024)$ and BCSS (HR 1.52; 95\% Cl 1.10-2.11; $\mathrm{p}=0.012)$. Conclusions: Perioperative blood transfusion was independently associated with poorer survival after radical cystectomy for bladder cancer. Efforts to minimize blood loss and restrictive blood product transfusion strategies seem justified. 


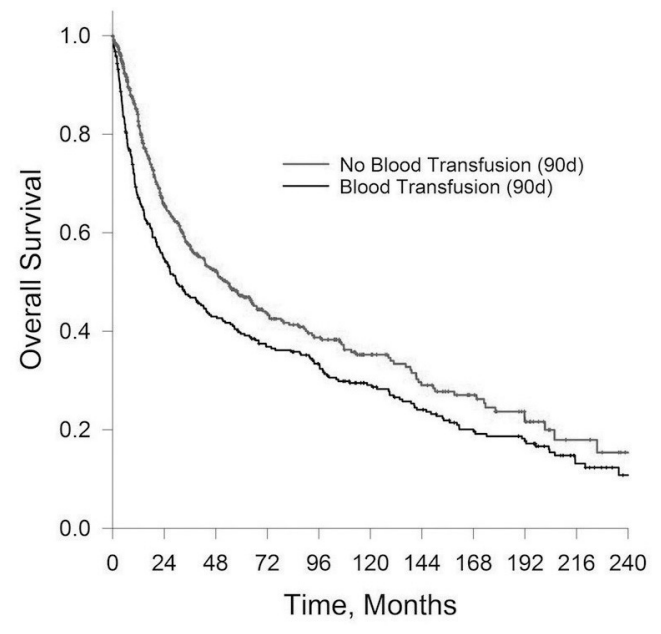

UP-3.3. Fig. 1. Kaplan-Meier curve for bladder cancer-specific survival (BCSS) stratified by perioperative transfusion.

\section{References}

1. Schuller DE, Scott C, Wilson KM, et al. The effect of perioperative blood transfusion on survival in head and neck can cer. Arch Otolaryngol Head Neck Surg 1994;120:711-6. https://doi.org/10.1001/archotol.1994.01880310017004

2. Wang YL, Jiang B, Yin FF, et al. Perioperative blood transfusion promotes worse outcomes of bladder cancer after radical cystectomy: A systematic review and meta-analysis. PLoS One 2015;10:e0130122. https://doi.org/10.1371/journal.pone.0130122

\section{UP-3.5}

Contemporary use of neoadjuvant chemotherapy for bladder cancer in a tertiary care center

Gagan Fervaha ${ }^{1,2}$, Jaime Herrera-Caceres ${ }^{2,3}$, Khaled Ajib2, ${ }^{2,3}$, Aravinth Jebanesan", Alexandre Zlotta2,3, Neil E. Fleshner, ${ }^{2,3}$, Girish S. Kulkarni 2,3

${ }^{1}$ School of Medicine, Queen's University, Kingston, ON, Canada; ${ }^{2}$ Surgical Oncology, Princess Margaret Hospital, Toronto, ON, Canada; ${ }^{3}$ Urology, University of Toronto, Toronto, ON, Canada

Introduction: Neoadjuvant chemotherapy (NAC) prior to radical cystectomy $(\mathrm{RC})$ is the standard of care for eligible patients with muscle-invasive bladder cancer (MIBC). In this study, we sought to examine the rate of NAC use in eligible patients and examine variables predictive of use. Methods: In this retrospective cohort study, we used our institutional database of consecutive patients who underwent RC for MIBC and had data of chemotherapy receipt available. Data were included from individuals undergoing their RC between 2003 and 2015. Baseline characteristics were extracted from the electronic medical charts. Chemotherapy ineligibility was defined as presence of any of the following: poor performance status (Eastern Cooperative Oncology Group score $>2$ ), chronic kidney disease (CKD stage $>3$ ), hearing loss, peripheral neuropathy, and severe heart failure (CHF; NYHA class >III). Multivariable regression was computed to examine predictors of NAC receipt.

Results: Data from 272 patients with MIBC were available; 209 patients $(76.8 \%)$ were male. Mean age at time of RC was 68.2 (standard deviation [SD] 10.4). Ten patients (3.7\%) had poor performance status, 17 $(6.3 \%)$ had CKD, $18(6.6 \%)$ had hearing loss, three $(1.1 \%)$ had peripheral neuropathy, and three (1.1\%) had severe CHF. A total of 236 patients $(86.8 \%)$ were, therefore, eligible for chemotherapy. Of those eligible, $51(21.6 \%)$ went on to receive NAC. Multivariable analysis examining predictors of NAC revealed a significant association only with year of surgery $(p<0.001)$, with those having surgery in later years more likely to receive NAC. Age, sex, kidney function, medical comorbidities, and performance status were not independently predictive.

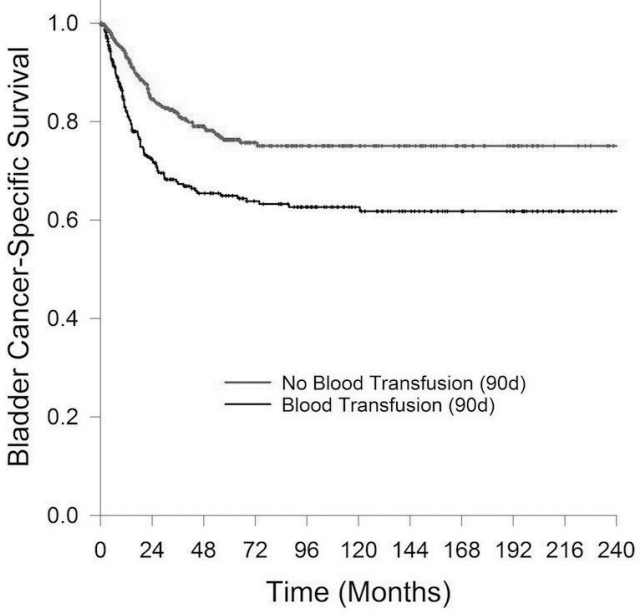

UP-3.3. Fig. 2. Kaplan-Meier curve for overall survival stratified by perioperative transfusion.

Conclusions: Despite guideline recommendations, less than one in four patients who were eligible for NAC receive this treatment modality. Increasing numbers of patients are receiving NAC in recent years, but rates continue to remain low. Future studies should examine system factors beyond clinical variables to further shed light on our understanding of practice patterns of NAC use.

\section{UP-3.7}

Assessment of health-related quality of life and supportive care needs in radical cystectomy patients through a comprehensive cancer survivorship program

Julie G Trudel', Conrad Maciejewski²

${ }^{1}$ The Ottawa Hospital Research Institute, The Ottawa Hospital, Ottawa, ON, Canada; '2Department of Surgery, Division of Urology, The Ottawa Hospital, Ottawa, ON, Canada

Introduction: Cancer survivors report unmet needs throughout their treatment journey, and these are poorly understood in the setting of bladder cancer. We aimed to understand cystectomy patients' unmet needs and health-related quality of life (HRQOL) in a comprehensive survivorship program by employing a mixed methods approach.

Methods: Cystectomy patients were comprehensively enrolled in our survivorship program starting January 2019. Patients completed standardized instruments and structured interviews prior to and 2-3 months post-RC (time 1 and 2). Questionnaires included the Cancer Survivors Unmet Needs Measure (CaSUN) and HRQOL measures (FACT-BI, COST, SF-36, and NCCN Distress Thermometer).

Results: Twenty-five patients were assessed at time 1 and 17 at time 2 . At time 1,44\% of patients reported significant stress and 38\% required emotional and financial support. At time 2, the most frequent unmet needs included financial support (44\%), addressing body image, sexual/ intimacy issues $(33 \%)$, and transitioning to a cancer survivor (33\%). At time 1, RC patients had lower HRQOL scores for emotional well-being and pain compared to US norms and exhibited clinical distress, while at time 2 they had lower HRQOL scores for physical health and energy and fatigue compared to US norms. Qualitative data at time 1 showed that some RC patients reported anxiety over the impending surgery, pain, financial, and informational needs. At time 2, some indicated low appetite, low energy, shortness of breath, psychological distress, and needed home assistance for activities of daily living.

Conclusions: RC patients report significant unmet needs and impaired HRQOL both preoperatively and postoperatively, although these differ as patients progress. Emotional and financial needs, low energy level, body image, and sexual/intimacy issues were factors that had the greatest 
impact on their HRQOL. The results highlight the importance of dedicated HRQOL survivorship assessment and tailored intervention in this population.

\section{UP-3.8}

Opioid use after nephrectomy for kidney cancer in Ontario: A population-based study

Douglas C. Cheung ${ }^{1}$, Lisa Martin ${ }^{1}$, Maria Komisarenko' ${ }^{1}$ Naheed Jivraj ${ }^{2}$ Christina Diong 3 , Olli Saarela, Madhur Nayan', Hance Clarke², Duminda Wijeysundera ${ }^{2}$,Tara Gomes ${ }^{5}$, Girish S. Kulkarni', Shabbir M. Alibhai ${ }^{6}$, Antonio Finelli

'Department of Surgery, Division of Urology, University Health Network, Toronto, ON, Canada; ${ }^{2}$ Department of Anesthesia, University of Toronto, Toronto, ON, Canada; ${ }^{3}$ ICES, Toronto, ON, Canada; ${ }^{4}$ Department of Biostatistics, University of Toronto, Toronto, ON, Canada; ${ }^{5}$ Unity Health, Toronto, ON, Canada; ${ }^{6}$ Division of Internal Medicine and Geriatrics, University Health Network, Toronto, ON, Canada

Introduction: Adequate control of postoperative pain is important to allow for optimal recovery; however, $5-10 \%$ of patients develop persistent opioid use. This effect may be more pronounced following open vs. minimally invasive (MIS) nephrectomy due to the incisional morbidity. In this population-based study, we compare the rates of early and prolonged postoperative opioid use in patients undergoing open vs. MIS nephrectomy.

Methods: Opioid-naive patients who underwent nephrectomy for kidney cancer in Ontario (1994-2018) were identified from the ICES databases. Published definitions of early (opioid prescription [Rx] 1-90 days after surgery) and prolonged (1: recurrent Rx use in both 1-90 and 91-180 days after surgery; and 2: Rx duration for $\geq 60$ days between $90-365$ days after surgery) opioid use were measured. Two cohorts were generated: using the Ontario Drug Benefits database (ODB; primarily patients over age $65 ; \mathrm{n}=5263$ ) and Narcotics Monitoring System (NMS; available after 2012 in all ages; $n=4472$ ). Predictors of opioid use were assessed by multivariable logistic regression.

Results: Early opioid Rx was higher following open vs. MIS nephrectomy in the NMS cohort only (74 vs. $69 \%$, p $<0.01$ ). Overall, prolonged opioid use was low (1-5\%). In the ODB cohort, prolonged Rx was higher for open vs. MIS in one of two definitions ( 5.3 vs. $3.9 \%$, $p=0.02 ; 1.9$ vs. $1.4 \%$, $\mathrm{p}=0.19$ ). In the NMS cohort, prolonged Rx was not different between surgeries. After adjustment for patient, surgical, physician, and hospital characteristics, the odds of early opioid Rx were significantly higher for open vs. MIS in both cohorts (odds ratio [OR] 1.19 [1.03-1.37], $\mathrm{p}=0.02$; OR 1.35 [1.15-1.56], p<0.01). Surgery type was not significantly associated with prolonged opioid Rx in either cohort or definition.

Conclusions: Early opioid Rx was higher for patients who underwent open vs. MIS nephrectomy. Prolonged opioid Rx ranged from $1-5 \%$ of patients, depending on the definition, but did not differ significantly by type of surgery.
UP-3.9

Adrenalectomy during radical nephrectomy: Oncological outcomes from the Canadian Kidney Cancer Information System (CKCis) - a modern-era, nationwide, multicenter, Canadian cohort

Arnon Lavi ${ }^{1}$, Rodney H. Breau ${ }^{2}$, Ranjeeta Mallick ${ }^{3}$, Anil Kapoor ${ }^{4}$, Antonio Finelli ${ }^{5}$, Alan I. So ${ }^{6}$, Frédéric Pouliot ${ }^{7}$, Simon Tanguay ${ }^{8}$, Luke T. Lavallée ${ }^{2}$, Ricardo A. Rendon ${ }^{9}$, Adrian Fairey ${ }^{10}$, Darrel E. Drachenberg ${ }^{11}$, Jean-Baptiste Lattouf ${ }^{12}$, Ranjena Maloni ${ }^{5}$, Nicholas E. Power ${ }^{7}$

${ }^{1}$ Urology Division, Department of Surgery, Schulich School of Medicine and Dentistry, Western University, London, ON, Canada; ${ }^{2}$ Department of Urology, The Ottawa Hospital, Ottawa, ON, Canada; ${ }^{3}$ School of Epidemiology and Public Health, University of Ottawa, Ottawa, ON, Canada; ${ }^{4}$ McMaster Institute of Urology, St. Joseph's Healthcare, Hamilton, ON, Canada; ${ }^{5}$ Division of Urologic Oncology, Princess Margaret Hospital, University of Toronto, Toronto, ON, Canada; ${ }^{6}$ Department of Urologic Sciences, University of British Columbia, Vancouver, BC, Canada; 'Department of Surgery, Division of Urology, Université Laval, Quebec City, QC, Canada; ${ }^{8}$ Department of Urology, McGill University Health Centre, Montreal, QC, Canada; ${ }^{9}$ Department of Urology, QEII Health Sciences Centre, Dalhousie University, Halifax, NS, Canada; ${ }^{10}$ Division of Urology, University of Alberta, Edmonton, $A B$, Canada; ${ }^{11}$ Division of Urology, University of Manitoba, Winnipeg, MB, Canada; ${ }^{12}$ Division of Urology, University of Montreal Hospital Centre (CHUM), Montreal, QC, Canada

Support: Kidney Cancer Research Network; The Canadian Kidney Cancer information system (CKCis)

Introduction: Ipsilateral adrenal involvement of renal cell carcinoma occurs in up to $4 \%$ of radical nephrectomies. ${ }^{1}$ However, incidental ipsilateral adrenalectomy is commonly performed during radical nephrectomy. A benefit of incidental adrenalectomy has not been demonstrated..$^{2-5}$ The objective of this study was to identify the association between adrenalectomy and oncological outcomes in a contemporary cohort.

Methods: We identified a cohort of radical nephrectomy patients in the Canadian Kidney Cancer information system (CKCis) who had surgery between 2011 and 2019. Patient, tumor, and surgical characteristics were obtained. The primary outcomes were renal cell carcinoma (RCC) recurrence and death.

Results: During the study period, 2726 patients were identified; 827 $(30.3 \%)$ had incidental adrenalectomy. Age, race, smoking status, body mass index (BMI), family history of RCC and preoperative creatinine were similar between the groups. The adrenalectomy group had more advanced disease features: lower Eastern Cooperative Oncology Group status $(p=0.004)$, longer procedure length $(p<0.0001)$, higher blood loss $(p<0.0001)$, higher $\%$ of tumor thrombus $(p<0.0001)$, pT $(p<0.0001)$, $\mathrm{pN}(\mathrm{p}=0.006), \mathrm{M}$ status $(\mathrm{p}<0.0001)$, Fuhrman grade $(\mathrm{p}<0.0001)$, and $\%$ of clear-cell histology $(p=0.027)$. The rate of adrenal involvement was $3.9 \%$. Median followup was 22 months (range 7.8-44.7). Adjusting for known confounders, adrenalectomy was associated with lower overall survival but not recurrence-free survival (Tables 1,2).

UP-3.9. Table 1. Univariable and multivariable association for overall survival

\begin{tabular}{|c|c|c|c|c|c|c|c|c|}
\hline \multirow{3}{*}{ Parameter } & \multicolumn{4}{|c|}{ Overall survival (univariate) } & \multicolumn{4}{|c|}{ Overall survival (multivariate) } \\
\hline & \multirow[t]{2}{*}{$\begin{array}{c}\text { Hazard } \\
\text { ratio }\end{array}$} & \multicolumn{2}{|c|}{$\begin{array}{c}95 \% \text { hazard ratio } \\
\text { confidence }\end{array}$} & \multirow[t]{2}{*}{$\mathbf{p}$} & \multirow[t]{2}{*}{$\begin{array}{c}\text { Hazard } \\
\text { ratio }\end{array}$} & \multicolumn{2}{|c|}{$\begin{array}{l}95 \% \text { hazard ratio } \\
\text { confidence }\end{array}$} & \multirow[t]{2}{*}{$\mathbf{p}$} \\
\hline & & \multicolumn{2}{|c|}{ Limits } & & & \multicolumn{2}{|c|}{ Limits } & \\
\hline Age & 1.04 & 1.02 & 1.05 & $<0.0001$ & & & & \\
\hline Charlson score & 1.27 & 1.18 & 1.37 & $<0.0001$ & 1.3 & 1.18 & 1.42 & $<0.0001$ \\
\hline Tumor grade & 2.78 & 2.04 & 3.79 & $<.0001$ & 2.17 & 1.63 & 2.88 & $<0.0001$ \\
\hline Adrenalectomy & 1.89 & 1.5 & 2.35 & $<0.0001$ & 1.28 & 1.03 & 1.6 & 0.028 \\
\hline
\end{tabular}




\begin{tabular}{|c|c|c|c|c|c|c|c|c|}
\hline \multirow[b]{2}{*}{ Parameter } & \multicolumn{4}{|c|}{ Recurrence-free survival (univariate) } & \multicolumn{4}{|c|}{ Recurrence-free survival (multivariate) } \\
\hline & Hazard ratio & \multicolumn{2}{|c|}{$\begin{array}{l}95 \% \text { hazard ratio } \\
\text { confidence }\end{array}$} & $\mathbf{p}$ & Hazard ratio & \multicolumn{2}{|c|}{$\begin{array}{l}95 \% \text { hazard ratio } \\
\text { confidence }\end{array}$} & $\mathbf{p}$ \\
\hline Tumor grade & 2.77 & 2.29 & 3.35 & $<0.0001$ & 2.04 & 2.04 & 2.86 & $<0.0001$ \\
\hline Tumor margin & 3.06 & 2.28 & 4.11 & $<0.0001$ & 2.15 & 1.62 & 2,86 & $<0.0001$ \\
\hline
\end{tabular}

Conclusions: In this contemporary cohort, incidental adrenalectomy was not associated with better outcomes and likely reflect the more advanced disease characteristics in this group. Adrenal sparing surgery should remain the standard of care when technically feasible and in the absence of imaging abnormalities.

\section{References}

1. Leibovitch I, Raviv G, Mor Y, et al. Reconsidering the necessity of ipsilateral adrenalectomy during radical nephrectomy for renal cell carcinoma. Urology 1995;46:316-20. https://doi.org/10.1016/ S0090-4295(99)80213-1

2. Weight CJ, Kim SP, Lohse CM, et al. Routine adrenalectomy in patients with locally advanced renal cell cancer does not offer oncologic benefit and places a significant portion of patients at risk for an asynchronous metastasis in a solitary adrenal gland. Eur Urol 2011;60:458-64. https://doi.org/10.1016/j.eururo.2011.04.022

3. Yap SA, Alibhai SMH, Abouassaly R, et al. Ipsilateral adrenalectomy at the time of radical nephrectomy impacts overall survival. BJU Int 2013;111:E54-8. https://doi.org/10.1111/j.1464-410X.2012.11435.x

4. Kutikov A, Piotrowski ZJ, Canter DJ, et al. Routine adrenalectomy is unnecessary during surgery for large and/or upper pole renal tumors when the adrenal gland is radiographically normal. / Urol 2011;185:1198-203. https://doi.org/10.1016/j.juro.2010.11.090

5. Weight CJ, Mulders PF, Pantuck AJ, et al. The role of adrenalectomy in renal cancer. Eur Urol Focus 2016;1:251-7. https://doi. org/10.1016/j.euf.2015.09.005

\section{UP-3.10}

Dual-energy computerized tomography for enhanced evaluation of renal cell carcinoma with inferior vena cava thrombus Ellen O'Connor ${ }^{1,2,3}$, Brennan Timm 1,2, Joseph Ischia' ${ }^{1}$, Amy Baker ${ }^{4}$, Damien Bolton ${ }^{1}$, Nathan L. Lawrentschuk',

${ }^{1}$ Department of Surgery, University of Melbourne, Austin Health, Heidelberg, Australia; ${ }^{2}$ Young Urology Research Organisation, (YURO), Melbourne, Australia; ${ }^{3}$ Division of Cancer Surgery, Peter MacCallum Cancer Centre, Melbourne, Australia; ${ }^{4}$ Department of Radiology, Austin Health, Heidelberg, Australia

Introduction: Direct endovascular extension of tumor thrombus into the inferior vena cava (IVC) occurs in $4-10 \%$ of renal cell carcinomas. Traditionally,

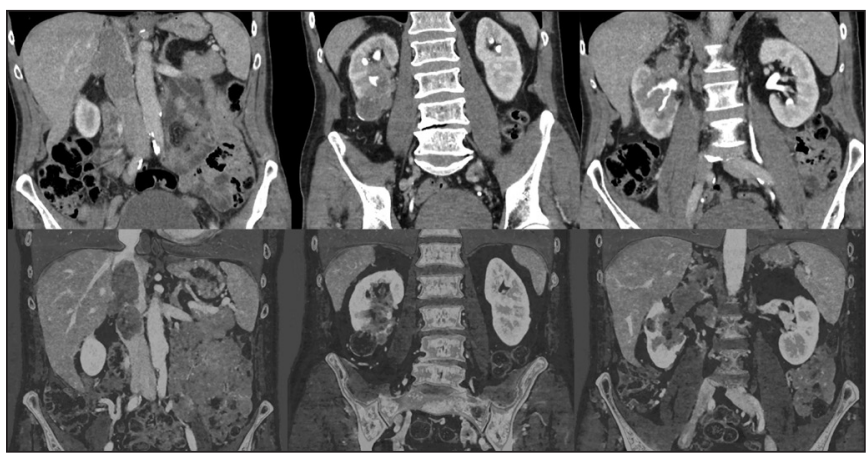

UP-3.10. Fig. 1. Comparison of standard CT (above) to dual-energy CT (below). standard computerized tomography (CT) or magnetic resonance imaging (MRI) have adequately identified IVC extension, however, proximal extent can be underestimated. Accurate and timely assessment of tumor thrombus staging is imperative prior to undertaking radical surgical resection. We aimed to assess the value of dual energy CT in the evaluation of IVC tumor thrombus in patients undergoing nephrectomy and IVC thrombectomy.

Methods: Four patients identified to have resectable IVC thrombus were included in the pilot study. All patients had undergone standard CT at external imaging services and were reviewed independently. Contrastenhanced dual-energy CT was subsequently performed within seven days prior to planned resection using an adapted CT protocol. Virtual unenhancement, blended-weighted average, and color-coded iodine overlay reconstruction was performed. All images were then re-analyzed by a radiologist, urologist, and trainee who were unaware of the original reports. Final determination of tumor thrombus level was made by consensus following intraoperative findings.

Results: Fig. 1 demonstrates comparison between standard and dual-energy $\mathrm{CT}$. The dual-energy CT results were accurate when compared to intraoperative findings. Improved delineation of proximal extent of thrombus and involvement of IVC wall was seen compared to the initial standard CT. Conclusions: Dual-energy $\mathrm{CT}$ is a reliable imaging technique for evaluation of IVC tumor thrombus. Although there are few patients in the present pilot, all cases showed accurate imaging findings when compared to final surgical pathological confirmation. Ongoing evaluation of imaging standard of care in IVC thrombus is essential to maintain an easily accessible, cost-effective, and accurate staging technique.

\section{UP-3.11}

The increasing use of renal tumor biopsy among Canadian urologists: When physician perceptions matter most

Felix Couture ${ }^{1}$, Rodney H. Breau ${ }^{2,3}$, Adrian Fairey ${ }^{4}$, Antonio Finelli ${ }^{5}$, Daniel YC Heng ${ }^{6}$, Luke T. Lavallée ${ }^{2,3}$, Ricardo A. Rendon', Simon Tanguay ${ }^{8}$, Patrick Richard'

'Department of Urology, Centre Hospitalier Universitaire de Sherbrooke, Sherbrooke, QC, Canada; ${ }^{2}$ Department of Urology, University of Ottawa, Ottawa, ON, Canada; ${ }^{3}$ Ottawa Hospital Research Institute, University of Ottawa, Ottawa, ON, Canada; ${ }^{4}$ Department of Urology, University of Alberta, Edmonton, AB, Canada; ${ }^{5}$ Department of Urology, Princess Margaret Hospital, Toronto, ON, Canada; ${ }^{6}$ Department of Oncology, Tom Baker Cancer Centre, Calgary, AB, Canada; ${ }^{7}$ Department of Urology, Dalhousie University, Halifax, NS, Canada; ${ }^{8}$ Department of Urology, McGill University Health Centre, Montreal, QC, Canada

Introduction: The role renal tumor biopsy (RTB) in the management of small renal masses (SRMs) is progressively being recognized. While an increasing number of studies assessing its role as a diagnostic tool are becoming available, the use of RTB remains variable among patients diagnosed with a SRM. Many factors may influence urologists on whether to perform a RTB to help guide management. We aimed at identifying factors associated with the use of RTB in the Canadian population diagnosed with a SRM.

Methods: Data from the Canadian Kidney Cancer information system of 3371 patients diagnosed with a SRM $(\leq 4 \mathrm{~cm})$ between January 2011 and September 2019 was retrospectively reviewed. Patients were stratified based on whether a RTB was performed prior to the primary 
therapeutic intervention. Variables such as age, American Society of Anesthesiologists (ASA) class, Eastern Cooperative Oncology Group (ECOG) score, lesion size, year of diagnosis, and patient-volume at the referring center were analyzed as possible factors associated with RTB use. Univariable and multivariable logistic regression models were used. Results: A total of 807 patients $(23.9 \%)$ underwent RTB. There was an overall increase in RTB use with time $(p=0.002)$, with patients diagnosed in later years (2015-2019) undergoing RTB more often than in earlier years (2011-2014) $(27.7 \%$ vs. $20.7 \%$, respectively; $p<0.001)$. On multivariable analysis, increasing year of diagnosis was significantly associated with more RTB use (odds ratio $1.12 ; 95 \%$ confidence interval $1.02-1.22 ; p=0.017)$. Patients managed in centers from the highest patient-volume quartile had RTB more frequently than patients from low-volume centers $(p<0.001)$.

Conclusions: Our results reveal an increasing popularity and overall use of RTB among Canadian urologists. Our findings also suggest a greater use of RTB in larger-volume centers. This study highlights the large influence of physician perceptions in the decision to use RTB and stresses the importance of providing urologists with evidence-based information on the role of RTB in the management of SRMs.

\section{UP-3.12}

A 21-year, population-based comprehensive review of surgically treated kidney cancer in Manitoba

Ryan Sun', Benjamin Shiff', Oksana Harasemiw', Navdeep Tangri', Lourens Lacobs ${ }^{1}$, Jeffery W. Saranchuk', Rahul K. Bansal', Darrel E. Drachenberg', Jasmir (Jay) G. Nayak'

${ }^{1}$ Urology, University of Manitoba, Winnipeg, MB, Canada

Introduction: Partial nephrectomy (PN) has become an increasingly used option for localized kidney cancer compared to radical nephrectomy (RN), due to its advantage of renal preservation and seemingly equivalent oncological efficacy in several studies. However, there are conflicting reports of survival benefits, and the technical challenges of PN also lead to potential for increased perioperative complications. In this study, we aim to compare the survival and perioperative outcomes between patients who underwent PN and RN for renal cell carcinoma (RCC) using a large, population-based cohort database.

Methods: We performed a retrospective cohort study by analyzing patientlevel data of all patients within the province of Manitoba who underwent either RN or PN for RCC from 1995-2015, by linking multiple provincial administrative health registries.

Results: Of 2604 patients identified who underwent surgery for RCC, 565 (21.7\%) underwent PN and 2039 (78.3\%) underwent RN. PN patients were significantly younger (mean 59.6 vs. 63.0 years, $p<0.001$ ), had a lower mean Charlson comorbidity index (CCl) (6.2 vs. 7.8, p<0.001), and a smaller mean tumor size $(3.3$ vs. $6.5 \mathrm{~cm}, \mathrm{p}<0.01)$. Most $(86.6 \%)$ of PN tumors were pT1, compared to $47.5 \%$ of RN tumors. PN patients were less likely to require initiation of dialysis postoperatively compared to RN patients $(4.07 \%$ vs. $8.63 \%, \mathrm{p}=0.0003)$. After a median followup of $5.0(2.1-9.7)$ years, the overall survival rate was significantly higher in PN $(85.54 \%)$ than RN (54.10\%). The five-year estimated survival rates were $69.46 \%$ for RN compared with $87.60 \%$ for PN $(p<0.0001)$. There were no significant differences in 30-day and 90-day readmission rates between the two groups.

Conclusions: Within the provincial population cohort, PN was associated with improved survival and yielded similar 30-day or 90-day readmission rates compared to $\mathrm{RN}$. These results will require further evaluation with comparativeness effectiveness methodology.
UP-3.13

Anatomical characterization of the inguinal lymph nodes using microcomputed tomography to inform radical inguinal lymph node dissections for metastatic penile cancer

Kaitlin Marshall', Nicholas E. Power ${ }^{2}$, Shiva Nair ${ }^{2}$, Katherine E Willmore', Tyler S Beveridge

'Department of Anatomy \& Cell Biology, Schulich School of Medicine \& Dentistry, Western University, London, ON, Canada; ${ }^{2}$ Department of Surgery, Divisions of Urology and Surgical Oncology, Schulich School of Medicine \& Dentistry, Western University, London, ON, Canada

Introduction: In men with penile cancer, a radical inguinal lymph node dissection (rILND) is integral to improve disease-specific survival. Unfortunately, lymphocele and lymphedema are severe postoperative complications that can follow this procedure. A modified inguinal lymph node dissection was developed to reduce complications; however, it is not suited for patients with advanced-stage disease and further modification is limited by our understanding of the lymphatic anatomy in this region. Therefore, this study aims to elucidate the lymphatic anatomy within the current surgical borders of a rlLND using human cadavers.

Methods: To visualize the position of the lymph nodes, tissue packets excised from the inguinal region were imaged using microcomputed tomography $(154 \mu \mathrm{m}$ Locus Ultra CT scanner; $80 \mathrm{kVp}, 55 \mathrm{~mA}, 0.36$ degrees per view). To characterize the distribution of lymph nodes within each tissue packet, lymph nodes were segmented by grayscale values in 3D using a modified seed-growing algorithm (Region Growing v1.5; Kellner, 2011) in MATLAB. To compare anatomy between specimens, each lymph node packet was registered in a common coordinate system by aligning four landmarks (anterior superior iliac spine, pubic tubercle, saphenofemoral junction and the apex of the femoral triangle) using a generalized Procrustes analysis.

Results: Preliminary findings from five samples ( $\mathrm{n}=3$ cadavers; two male, one female) show a median of six lymph nodes (range 3-7) and their distribution is illustrated in Fig. 1.

Conclusions: This study provides the first standardized comparison of lymph node anatomy in the inguinal region, and uses a novel imaging methodology validated by our lab to study the anatomy of lymphatic tissue in 3D and in situ. In doing so, the anatomy elucidated in this study will help inform refinement to the borders of the radical surgical template, to limit unnecessary resection in an attempt to reduce the incidence of post-surgical lymphedema and/or lymphocele.

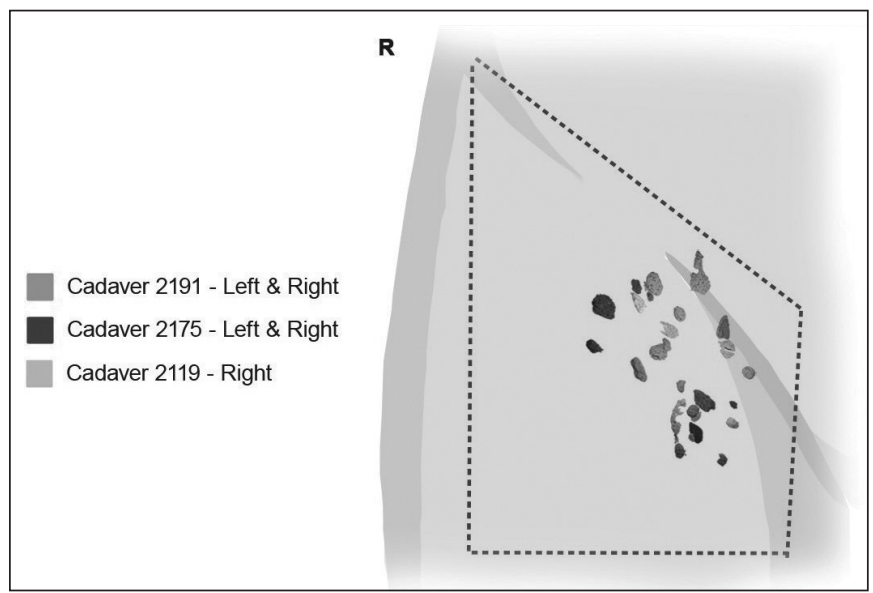

UP-3.13. Fig. 1. The standardized lymph node data after having generalized Procrustes analysis applied. The dotted lines represent the rILND surgical borders. 


\section{UP-3.14}

Patient perspectives on the value of WATChmAN (Web virtuAL Testicular CANcer clinic) for clinical stage I patients on active surveillance

Adam Bobrowski', Robert J. Hamilton ${ }^{2}$, Lauren Landoni², Kopika Kuhathaas ${ }^{2}$, Peter Chung, Philippe L. Bedard ${ }^{4}$, Padraig Warde ${ }^{3}$, Aaron Hansen $^{4}$, Tran Truong 5 , Ezra Hahn ${ }^{3}$, Michael A. Jewett ${ }^{2}$, Jackie Bender ${ }^{6}$ ${ }^{1}$ Faculty of Medicine, University of Toronto, Toronto, ON, Canada; ${ }^{2}$ Departments of Surgery Departments of Surgery and Surgical Oncology and Surgical Oncology (Urology), Princess Margaret Hospital, University Health Network, University of Toronto, Toronto, ON, Canada; ${ }^{3}$ Department of Radiation Oncology, Princess Margaret Hospital, University Health Network, University of Toronto, Toronto, ON, Canada; ${ }^{4}$ Department of Medical Oncology, Princess Margaret Hospital, University Health Network, University of Toronto , Toronto, ON, Canada; ${ }^{5}$ Techna Institute, University Health Network, University of Toronto, Toronto, ON, Canada; ${ }^{6}$ Cancer Rehabilitation and Survivorship Program, Princess Margaret Hospital, University Health Network, University of Toronto, Toronto, ON, Canada

Introduction: Adherence to stage I active surveillance (AS) schedules can present compliance issues, as well as financial and logistical challenges for testicular cancer patients. We innovated a secure online platform, WATChmAN (Web-based virtuAI Testicular CANcer clinic) allowing asynchronous communication between patients, results, and physician team. In this study, we explore patient perspectives regarding the value of virtual clinics.

Methods: Patients enrolled in the WATChmAN trial were invited to participate in semi-structured telephone interviews to discuss their experience. Eligible patients had at least two interactions with virtual care. All interviews were audio recorded, transcribed verbatim, and analyzed using thematic analysis.

Results: All 13 eligible approached patients agreed to participate in an interview. This cohort was comprised of $46 \%$ seminoma and $54 \%$ nonseminoma patients. Subjects valued virtual care because it addressed the logistical challenges they experienced with traditional care, specifically attending frequent appointments, long distance travel, parking, and wait times. While less than half $(46 \%)$ believed they felt the same level of connection to their provider as they would with in-person visits, all patients perceived the quality of care on the virtual care platform to be at least equivalent. No patients had privacy concerns, nor did any cite any challenges communicating with their healthcare team via virtual care. Challenges with understanding medical jargon and laboratory values in AS updates was common and often required explanation from the medical team. Nearly all patients preferred to learn about disease relapse virtually, but all wanted prompt face-to-face clinical followup with their provider to address questions. None of the patients believed virtual care could entirely replace face-to-face medical care.

Conclusions: Testicular cancer patients valued the convenience of virtual care AS and perceived their care to be of equivalent quality to in-person visits.

\section{UP-3.15}

Management of localized penile cancer with an organ-sparing approach using split thickness skin grafting results in excellent oncological and functional outcomes

\section{Benjamin Beech' ${ }^{1}$ David W. Chapman' ${ }^{1}$, Keith F. Rourke'}

${ }^{1}$ Division of Urology, University of Alberta, Edmonton, $A B$, Canada

Introduction: Penectomy as the traditional surgical treatment of penile cancer, has substantial adverse functional and psychological impact. Glansectomy with split thickness skin graft (STSG) reconstruction, aims to provide curative resection while maximizing functional outcomes, and minimizing psychological harm. We describe our outcomes of glansectomy with STSG reconstruction for penile cancer in a Canadian setting. Methods: We identified patients undergoing glansectomy with STSG genital reconstruction for squamous cell carcinoma of the penis from July 2006 to July 2019 at a single center. Patients undergoing glansectomy for reasons other than penile cancer were excluded. We collected clinical and pathological data including patient demographics, 90-day complica- tions, positive margin rate, local recurrence rate, disease-specific survival, and functional outcomes. Descriptive statistics were used to characterize our cohort, and to examine outcomes.

Results: Twelve men met study criteria with a median age of 62 years. Seven patients had failed prior treatment. The 90-day complication rate (Clavien-Dindo $>2$ ) was $0 \%$ and graft take was excellent in all cases. The positive margins rate was $16.7 \%(n=2)$. Local recurrence occurred in two patients $(16.7 \%)$, one of whom underwent a repeat organ-sparing surgery for salvage, while the other underwent radical penectomy for high-risk pathological features. Disease-free survival at a median followup of 14 months was $91.7 \%$ (11/12). Standing voiding and erectile function, as well as satisfactory cosmesis were preserved in all patients.

Conclusions: Glansectomy with STSG reconstruction is a safe and effective treatment for men with localized penile cancer with simultaneous preservation of cosmesis, urinary, and sexual function.

\section{UP-3.16}

Long-term outcomes of cecostomy tube insertion for patients with bowel dysfunction: A retrospective review

David W. Chapman ${ }^{1}$, Steven Tong ${ }^{1}$, Peter D. Metcalfe ${ }^{1}$, Philippe Sarlieve ${ }^{2}$, Nathan Hoy', Darcie A. Kiddoo

${ }^{1}$ Urology, University of Alberta, Edmonton, $\mathrm{AB}$, Canada; ${ }^{2}$ Radiology and Diagnostic Imaging, University of Alberta, Edmonton, $A B$, Canada

Introduction: Antegrade continence enemas are the standard treatment for refractory fecal incontinence and constipation. Cecostomy tubes (CT) are considered less invasive than Malone Antegrade Continence Enemas (MACE), but are associated with mechanical breakage, dislodgement, and require annual changes. The aim was to describe the continence and complication rates of $\mathrm{CT}$ insertion.

Methods: A retrospective review was conducted on patients that underwent insertion of CT for fecal incontinence. Demographics included age, gender, presence of developmental delay, and etiology of bowel dysfunction. Primary outcomes included continence rates and unplanned exchanges. Secondary outcomes include number of anesthetics required and length of hospital (LOS) stay. Descriptive statistics and Student t-test was performed when appropriate.

Results: Forty-one patients were reviewed with a mean age of 9.9 years and LOS of 3.47 days. Bowel dysfunction was a result of spina bifida (20/41), imperforate anus (13/41), functional (4/41), hypotonia/motor dysfunction (3/41), and a spinal cord tumor (1/41). Continence was achieved in $90 \%(37 / 41)$ of patients and failures were managed by conversion to MACE (1/4) and distal insertion of a second cecostomy tube $(2 / 4)$. Unplanned CT exchanges occurred at least once in $73.2 \%$ (30/41), however, the average rate of exchange was $1.3 /$ patient/year. Developmental delay, gender, and age at the time of insertion were not associated with an increase in complication rate. Patients required an average of 3.6 general anesthetics (GAs) (range 0-15), and GAs were no longer required at the age of 14.9 years old (average). A laparotomy was required in two patients. Conclusions: CT insertion is a safe and effective method for the treatment of fecal incontinence, however, unplanned exchange of tube due to malfunction, mechanical breakage, or dislodgment occurs frequently. This data will ensure that families are counselled appropriately about the long-term commitment that may be required to achieve a high rate of continence.

\section{UP-3.17}

How should we measure the anteroposterior diameter (APD) of the renal pelvis in congenital hydronephrosis?

Amr Hodhod', Francis Petrella', Mohamed El-Sherbiny', Iman Sadri', Roman Jednak' J. J.-P. Capolicchio

${ }^{1}$ Urology, McGill University Health Centre, Montreal, QC, Canada Introduction: APD is commonly used in hydronephrosis management. However, there is no consensus on the optimal APD measurement technique, including whether it should be measured supine or prone. Some suggest the APD should be measured at the intrarenal part of the renal pelvis, while others suggest that it should be done at the renal contour. This study evaluates six different techniques of APD measurements, in 
supine and prone positions, in an attempt to identify the optimal method for the prediction of pyeloplasty.

Methods: Patients with initial high-grade hydronephrosis (Society of Fetal Urology [SFU] grade 3 and 4) from 2008-2014 were retrospectively reviewed. All ultrasounds were reviewed by one blinded investigator. In the mid-renal transverse plane, the APD was measured at the innermost, renal contour and maximum extrarenal regions of the renal pelvis both supine and prone. The six APD measurements were compared based on the outcome (pyeloplasty vs. conservative management). A ROC curve evaluated the ability of the various APD measurements to predict surgery, the cutoff value being the lowest diameter with $100 \%$ specificity.

Results: We included 124 patients (150 units) (Table 1): 50 units (47 patients) with pyeloplasty and 100 renal units (77 patients) managed conservatively. The comparison based on management choice showed a significant difference in every APD measurement $(p<0.001$ for all). Using the ROC curve, all APD measurements were effective in predicting pyeloplasty (Fig.1). Among all measurements, the supine extrarenal APD was the most reliable predictor (AUC 0.90, $p<0.001$ ) with a cutoff value $25 \mathrm{~mm}$ (100\% specificity and $35.4 \%$ sensitivity). The AUCs of other measurements ranged from $0.86-0.89$ and their cutoff values ranged from $18-27 \mathrm{~mm}$

Conclusions: APD measurements differ based on the technique, but they can all predict pyeloplasty well. This implies that any APD technique can be used, but the technique should be consistent when comparing different ultrasounds. The statistical superiority of the supine extrarenal measurement is likely not clinically significant.

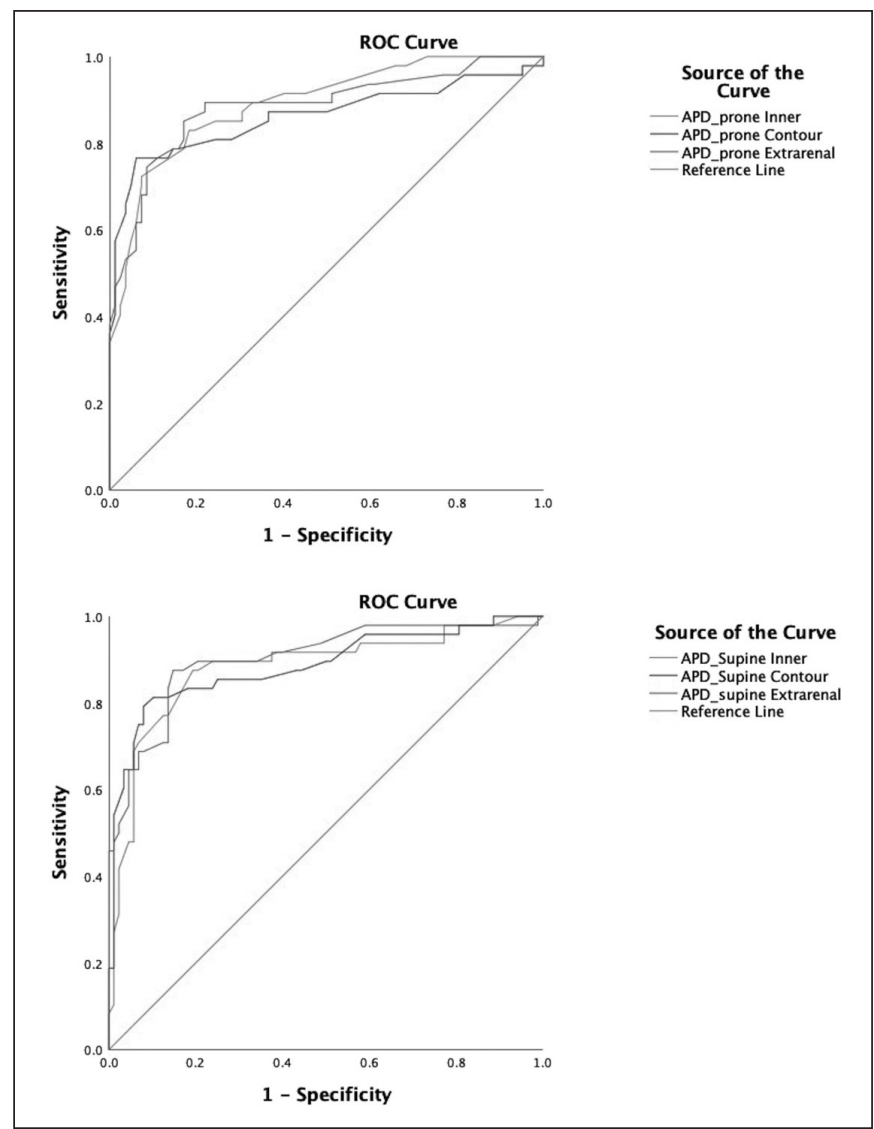

UP-3.17. Fig. 1. The ability of different supine and prone APD measurements in predicting pyeloplasty using ROC curve.

UP-3.17. Table 1. Patients' demographics and APD measurements in both supine and prone positions regarding the need for surgery

\begin{tabular}{|c|c|c|c|c|}
\hline \multicolumn{2}{|l|}{ Parameter } & \multirow{2}{*}{$\begin{array}{c}\text { Pyeloplasty group } \\
34(91.9)\end{array}$} & \multirow{2}{*}{$\begin{array}{c}\text { Conservative group } \\
63(81.8)\end{array}$} & \multirow{2}{*}{\begin{tabular}{c|}
$\mathbf{p}$ \\
0.22
\end{tabular}} \\
\hline Gender & Male, n (\%) & & & \\
\hline & Female, n (\%) & $13(8.1)$ & $14(18.2)$ & \\
\hline \multirow[t]{2}{*}{ Laterality } & Unilateral, n (\%) & $30(63.8)$ & $46(59.7)$ & 0.65 \\
\hline & Bilateral, n (\%) & $17(36.2)$ & $31(40.3)$ & \\
\hline \multirow[t]{2}{*}{ Side } & Right, n (\%) & $17(34)$ & $20(20)$ & 0.06 \\
\hline & Left, n (\%) & $33(66)$ & $80(80)$ & \\
\hline \multicolumn{2}{|c|}{ Age at presentation median (range) } & 0.3 mon $(0.1-11.7)$ & 0.8 mon $(0.03-6.3)$ & $<0.001$ \\
\hline \multirow{2}{*}{\multicolumn{2}{|c|}{ Followup median (range) }} & 43.2 mon (10.9-95.2) & 37.7 mon (8.9-107.8) & 0.79 \\
\hline & & APD measurements & & \\
\hline \multirow[t]{2}{*}{ Supine } & Inner median (range) & $16.3 \mathrm{~mm}(5-47)$ & $8 \mathrm{~mm}(3-24)$ & $<0.001$ \\
\hline & Contour median (range) & $22 \mathrm{~mm}(6.5-49)$ & $10.6 \mathrm{~mm}(3-27)$ & $<0.001$ \\
\hline Prone & Extrarenal median (range) & $23 \mathrm{~mm}(8-58)$ & $11 \mathrm{~mm}(4.5-27)$ & $<0.001$ \\
\hline
\end{tabular}




\section{UP-3.18}

The association of neurofibromatosis type 1 and lower urinary tract dysfunction in the pediatric population

Bruce GaO ${ }^{1}$, Keara Decotiis' ${ }^{2}$, Adam Bobrowski ${ }^{3}$, Martin A. Koyle ${ }^{2}$, Fardod $\mathrm{O}^{\prime} \mathrm{Kell}^{2}$

${ }^{1}$ Urology, University of Toronto, Toronto, ON, Canada; ${ }^{2}$ Urology, The Hospital for Sick Children, Toronto, ON, Canada; ${ }^{3}$ Undergraduate Medical Education, University of Toronto, Toronto, ON, Canada

Introduction: Neurofibromatosis type 1 (NF1) is an autosomal-dominant, multisystem, neurocutaneous disorder. ${ }^{1}$ This condition has been associated with lower urinary tract dysfunction due to either direct genitourinary organ involvement or spinal cord compression. ${ }^{2,3}$ Based on current literature, there are no reviews examining the relationship between NF1 and lower urinary tract dysfunction (LUTD) in the pediatric population. Methods: A critical review of the literature was conducted using a systematic search of MEDLINE, PubMed, and Embase yielding a total of 1285 manuscripts published up to 2019. Two independent reviewers selected studies for screening, eligibility, and inclusion into the review (Fig. 1). Following title, abstract, and full-text review, 45 articles were analyzed. Results: Within these 45 articles, 79 cases were presented (Fig. 2). The mean patient age at the time of presentation was $6.97 \pm 9.19$ years. The most common urological presentations were irritative lower urinary tract symptoms (30\%) and a newly discovered abdominopelvic mass $(21 \%)$. Diagnostic investigations commonly demonstrated a retrovesical mass with direct invasion of the bladder in 58 cases $(73 \%)$ and other genitourinary organ involvement in 39 cases (39\%). Throughout the total case volume, LUTD was present in $49 \%$. Pathology of malignancy was most commonly malignant peripheral nerve sheath tumor and rhabdomyosarcoma ( $20 \%$ and $14 \%$, respectively). When indicated, surgical management of the lower urinary tract included both radical and partial cystectomy. Conservative management of urinary retention included clean intermittent catheterization (56\%), suprapubic catheterization $(22 \%)$, vesicostomy creation $(11 \%)$, and Mitrofanoff creation $(11 \%)$.

Conclusions: A complete urological evaluation, including clinical, radiological, and possibly pathological investigation, is warranted and should

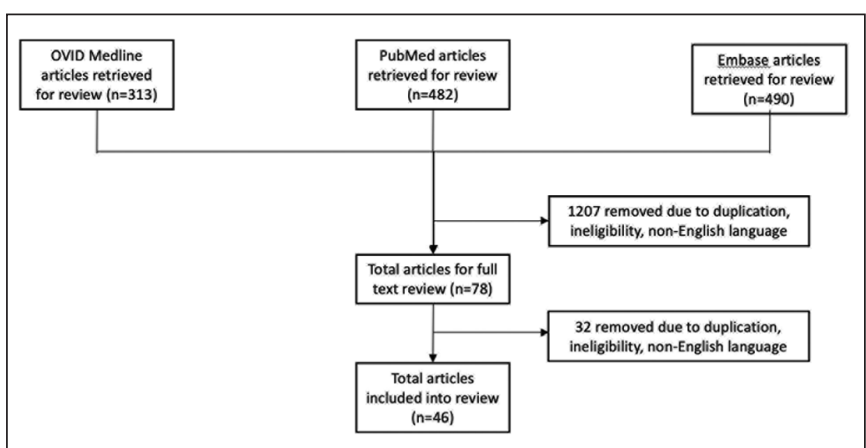

UP-3.18. Fig. 1. Study flowchart.

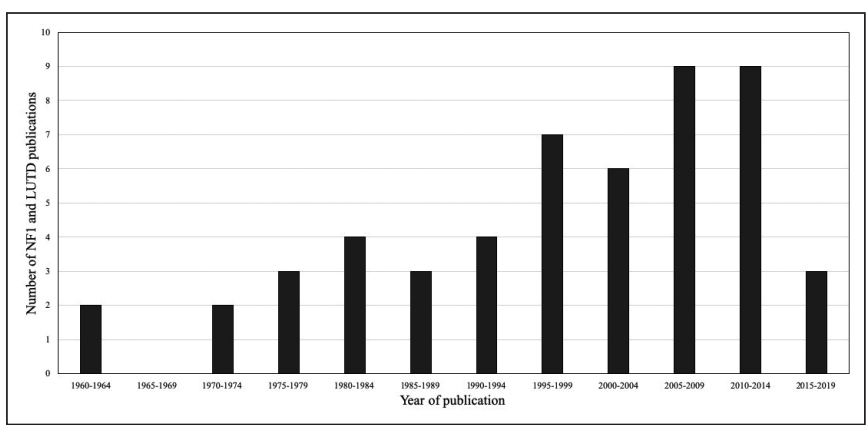

UP-3.18. Fig. 2. Publication trend. be performed for both diagnosis and appropriate management of LUTD in patients with NF1.

\section{References}

1. Tonsgard JH. Clinical manifestations and management of neurofibromatosis type 1. Semin Pediatr Neurol 2006;13:2-7. https://doi. org/10.1016/j.spen.2006.01.005

2. Kaefer M, Adams MC, Rink RC, et al. Principles in management of complex pediatric genitourinary plexiform neurofibroma. Urology 1997;49:936-40. https://doi.org/10.1016/S0090-4295(97)00098-8

3. Bouty A, Dobremez E, Harper L, et al, Bladder dysfunction in children with neurofibromatosis type I: Report of four cases and review of the literature. Urol Int 2018;100:339-45. https://doi. org/10.1159/000487193

\section{UP-3.19}

Use of smartphone camera and telemedicine in the management of neonatal priapism

O. Emmanuel Abara ${ }^{1}$, Natasha Samarasinghe ${ }^{2}$, Maurice Levy ${ }^{3}$

${ }^{1}$ Division of Clinical Sciences, Surgery (Urology), Northern Ontario School of Medicine/RHUPPI, Sudbury/Richmond Hill, ON, Canada; ${ }^{2}$ Community Pediatric Practice, Richmond Hill Pediatrics, Richmond Hill, ON, Canada; ${ }^{3}$ Pediatrics, North York General Hospital/Bathurst Medical Centre, Toronto/Thornhill, ON, Canada

Introduction: Priapism in the neonate is rare, poorly understood, and not commonly reported in the literature. Parents and primary healthcare providers are concerned and seek expert opinion. The use of smartphone camera and telemedicine on two cases seen between February 14, 2018 and June 15, 2019 is presented.

Methods: Case 1: A five-day old baby was referred on February 14, 2018 with a history of persistent intermittent penile erections since birth. A term baby, the child had no pain and urinated normally. The parents were concerned whether this will affect their decision for the child to be circumcised on the eight day of life, as done in their culture. The mother documented episodes of prolonged erections with her smart phone camera. Occurrence of priapism during the office visit matched the digital photo appearance. Watchful waiting was adopted. He had a successful circumcision on the eight day of life. Followup has included telemedicine e-visits with no reported recurrence in 18 months. Case 2: A 19-day-old baby was referred on October 30, 2018 with suspected penile chordee. Parents, however, expressed concerns of baby having prolonged intermittent penile erections since birth. The mother's smart phone images of prolonged penile erections were similar in appearance as during the office visit. Watchful waiting with periodic assessment through telemedicine was done. Priapism resolved before age 28 days, with no recurrence in 12 months.

Results: Parents were concerned about the prolonged penile erections and documented these episodes with their smart phone cameras. The digital photos were helpful in assessment and treatment. The outcome of watchful waiting management was satisfactory. This is in keeping with the reported cases in the literature. ${ }^{1}$ Telemedicine as a platform for followup was attractive to the mothers.

Conclusions: Neonatal priapism causes anxiety for the parents. The use of smart phone cameras by mothers to document neonatal priapism can be useful in the watchful waiting management strategy. Telemedicine was attractive, time saving and convenient.

Reference

1. Dust N, Dahoval T, Guerra L. Evaluation and management of priapism in a newborn: A case report and review of the literature. Paediatr Child Health 2011:16:e6-e8. https://doi.org/10.1093/ pch/16.1.e6 
UP-3.20

Baseline measurements of physical function and frailty in pretransplantation end-stage renal disease patients

Colin Cavey ${ }^{1}$, Katie Lyman ${ }^{1}$, Rohit Singla ${ }^{7}$, David I. Harriman', Christopher Y. Nguan'

${ }^{1}$ University of British Columbia, Vancouver, BC, Canada

Introduction: End-stage renal disease (ESRD) is a debilitating chronic condition that affects roughly $1-2 \%$ of the Canadian population. ${ }^{1}$ Renal transplantation (RT) remains the gold standard therapy, while waitlists exceed five years in some regions. Individuals with ESRD are prone to functional decline and muscle-wasting. ${ }^{2,3}$ As a result, poor physical function before RT is common and is associated with higher mortality and morbidity. ${ }^{4,5}$ This work proposes a set of baseline measures as a tool to assess physical functional status of preoperative RT recipients.

Methods: Renal transplant preassessments $(n=36)$ at Vancouver General Hospital were recruited and all patients completed a supervised baseline physical assessment to assess cardiovascular health and frailty, including a six-minute walk test, 30-second sit to stand, and the Fried frailty score evaluation.

Results: Thirty-six recipients were identified with a mean age of 57 years (range 27-80, M:F 42\%:58\%) Mean distance covered during the sixminute walk test was 360.0 meters for elderly males $(68 \%$ of the expected distance of a healthy individual) and 281.0 meters for elderly females (64\% of normal). Mean distance for non-elderly males was 367.7 meters ( $60 \%$ of normal) and 381.4 meters for non-elderly females (69\% of normal). ${ }^{6,7}$ Mean number of repetitions for the 30-second sit to stand was 9.4 for elderly and 13.2 for non-elderly. There was no significant difference in Fried frailty score between age groups. The mean Frailty score for both groups was 2.0, which is considered intermediately frail (2/5).

Conclusions: Preliminary results indicate potential utility for quantifying baseline cardiovascular health, physical functioning, and frailty status in this comorbid population. Ongoing data collection and assessment will enable fine-grained baselines for different demographics, including gender, ethnicity, age, and body mass index. This work is the first step towards initiating a prehabilitation exercise intervention.

\section{References}

1. Arora P, Vasa P, Brenner D, et al. Prevalence estimates of chronic kidney disease in Canada: Results of a nationally representative survey. CMA/ 2013;185:E417-23. https://doi.org/10.1503/cmaj.120833

2. Takahashi A, Hu SL, Bostom A. Physical activity in kidney transplant recipients: A review. Am / Kidney Dis 2018;72:433-43. https://doi.org/10.1053/j.ajkd.2017.12.005

3. Hill NR, Fatoba ST, Oke JL, et al. Global prevalence of chronic kidney disease - a systematic review and meta-analysis. PLOS ONE 2016;11:e0158765. https://doi.org/10.1371/journal.pone.0158765

4. McAdams-DeMarco MA, Ying H, Olorundare I, et al. Individual frailty components and mortality in kidney transplant recipients. Transplantation 2017;101:2126-32. https://doi.org/10.1097/ TP.0000000000001546

5. Nastasi AJ, McAdams-DeMarco MA, Schrack J, et al. Pre-kidney transplant lower extremity impairment and post-kidney transplant mortality. Am J Transplant 2018;8:189-96. https://doi.org/10.1111/ajt.14430

6. Enright PL, McBurnie MA, Bittner V, et al. The 6-min walk test: A quick measure of functional status in elderly adults. Chest 2003;123:387-98. https://doi.org/10.1378/chest.123.2.387

7. Enright PL, Sherrill DL. Reference equations for the six-minute walk in healthy adults. Am / Resp Crit Care Med 1998;158:1384-7. https://doi.org/10.1164/ajrccm.158.5.9710086

\section{UP-3.22}

Effect of transendocardial stem cell injection on erectile function in men with cardiomyopathy: Results from the TRIDENT, POSEIDON, and TAC-HFT trials

Lesse Ory 1,3, Russell G Saltzman², Ruben Blachman-Braun², Simon Dadoun ${ }^{2}$, Darcy L DiFede², Courtney Premer ${ }^{2}$, Barry Hurwitz ${ }^{4}$, Gavin Langille', Joshua M Hare², Ranjith Ramasamy ${ }^{3}$

${ }^{1}$ Urology, Dalhousie University, Halifax, NS, Canada; ${ }^{2}$ Interdisciplinary Stem Cell Institute, Miller School of Medicine, University of Miami,
Miami, FL, United States; ${ }^{3}$ Urology, Miller School of Medicine, University of Miami, Miami, FL, United States; ${ }^{4}$ Behavioral Medicine Research Center, University of Miami, Miami, FL, United States

Introduction: There is a growing interest in the use of stem cell therapy (SCT) for erectile dysfunction (ED). Human studies thus far have been limited to phase $1 / 2$ trials, with few men with ED receiving SCT. The effect of systemically delivered SCT and its effects on ED have not been studied. Our aim is to determine the effect of transendocardial injection (TESI) of SCT on erectile function (EF) using randomized, placebo-controlled data. Methods: We used International Index of Erectile Function (IIEF) scores collected from men enrolled in three separate randomized controlled trials: POSEIDON, TAC-HFT, and TRIDENT. ${ }^{1-3}$ All three trials included men with ischemic cardiomyopathy undergoing TESI of SCT or placebo via cardiac catheterization. Followup was one year. IIEF data was collected at baseline and at multiple time points in each trial. We investigated EF over time based on cell dose, cell source (autologous versus allogenic), cell type (mesenchymal stem cells vs. bone marrow mononuclear cells), as well as comparing men who received SCT to placebo.

Results: A total of 36 men were identified from the three trials with complete IIEF data. Eight men received placebo injection and 28 received SCT. Median age was 66.5 years. Comorbidities were similar among all men. Analysis was performed on men with ED, defined by an IIEF-EF score of 24 or less. In the placebo and SCT group, median IIEF-EF score was 5 (1-8) and $5(1-15)$ at baseline, and was $3.5(3-5.8)$ and $7(1-18)$ at 12 months, respectively $(p>0.05)$. None of our subgroup analyses revealed a change in

\begin{tabular}{|c|c|c|}
\hline & $\begin{array}{l}\text { Placebo } \\
n=8(\%)\end{array}$ & $\begin{array}{c}\text { Stem cell } \\
\text { therapy } \\
\mathbf{n}=28(\%)\end{array}$ \\
\hline Age \pm SD & $65 \pm 11.75$ & $65 \pm 10.94$ \\
\hline $\mathrm{BMI} \pm \mathrm{SD}$ & $28.67 \pm 4.94$ & $29.6 \pm 3.96$ \\
\hline History of coronary interventions & 7 (87.5) & $27(96.4)$ \\
\hline History of hypertension & $6(75)$ & $18(64.3)$ \\
\hline $\begin{array}{l}\text { History of congestive heart } \\
\text { failure }\end{array}$ & $6(75)$ & $21(75)$ \\
\hline History of valvular heart disease & $2(25)$ & $4(14.3)$ \\
\hline \multicolumn{3}{|l|}{ Smoking history } \\
\hline Never smoked & $2(25)$ & $7(25)$ \\
\hline Former smoker & $4(50)$ & $20(71.4)$ \\
\hline Current Smoker & $2(25)$ & $1(3.6)$ \\
\hline History of hyperlipidemia & $6(75)$ & $21(75)$ \\
\hline History of diabetes mellitus & $1(12.5)$ & $5(17.9)$ \\
\hline History of renal insufficiency & $2(25)$ & $1(3.6)$ \\
\hline History of cancer & $1(12.5)$ & $3(10.7)$ \\
\hline History of TIA or CVA & 0 & $3(10.7)$ \\
\hline \multicolumn{3}{|l|}{$\begin{array}{l}\text { Specify genitourinary } \\
\text { reproductive condition }\end{array}$} \\
\hline $\mathrm{BPH}$ & 0 & $3(10.7)$ \\
\hline Nocturia & 0 & $1(3.6)$ \\
\hline Urolithiasis & 0 & $1(3.6)$ \\
\hline Baseline IIEF-EF (IOR) & $5.5(1.5-8.5)$ & $5(1.3-14)$ \\
\hline 3-month IIEF-EF (IQR) & $6.5(2.5-20)$ & $6(1-20.8)$ \\
\hline 6-month IIEF-EF (IQR) & $6(1.5-16)$ & $4(1-21)$ \\
\hline 12-month IIEF-EF (IQR) & $3.5(3-5.8)$ & $7(1.520)$ \\
\hline
\end{tabular}

Data pooled from POSEIDON, TRIDENT, and TAC-HFT. 


\begin{tabular}{lcccc}
\hline UP-3.22. Table 2. IIEF data in men with ED stratified by number of stem cells received & \\
\hline & Placebo, $\mathbf{n = 8}$ & $\mathbf{2 0}$ million, $\mathbf{n = 9}$ & $\mathbf{1 0 0}$ million, $\mathbf{n = 8}$ & $\mathbf{2 0 0} \mathbf{~ m i l l i o n , ~} \mathbf{n = 1 1}$ \\
\hline Age \pm SD & $65 \pm 11.7$ & $70.3 \pm 9.9$ & $71 \pm 3.8$ & $56.3 \pm 9.8$ \\
Baseline IIEF-EF (IQR) & $5.5(1.5-8.5)$ & $4(1-12)$ & $2(1-5.8)$ & $14(4-23)$ \\
3-month IIEF-EF (IQR) & $6.5(2.5-20)$ & $2(1-13)$ & $2.5(1-8.5)$ & $21(7-23)$ \\
6-month IIEF-EF (IQR) & $6(1.5-16)$ & $1(1-18.5)$ & $1(1-4.5)$ & 0.005 \\
12-month IIEF-EF (IQR) & $3.5(3-5.8)$ & $1(1-15)$ & $3(2-9.3)$ & 0.079 \\
\hline
\end{tabular}

Data pooled from POSEIDON, TRIDENT, and TAC-HFT trials, restricted to men with IIEF $<25$.

\begin{tabular}{lccc}
\hline UP-3.22. Table 3. IIEF data in men with ED stratified by the source of stem cells received \\
\hline & Placebo, $\mathbf{n = 8}$ & Autologous, $\mathbf{n = 1 0}$ & Allogenic, $\mathbf{n = 1 8}$ \\
\hline Age \pm SD & $55.6 \pm 10.1$ & $70.2 \pm 7.5$ & $65 \pm 11.7$ \\
Baseline IIEF-EF (IQR) & $5.5(1.5-8.5)$ & $14(3.8-23.3)$ & $3.5(1-11.5)$ \\
3-month IIEF-EF (IQR) & $6.5(2.5-20)$ & $20(5.5-22.3)$ & $2.5(1-12)$ \\
6-month IIEF-EF (IQR) & $6(1.5-16)$ & $16.5(2.8-24.8)$ & 0.001 \\
12-month IIEF-EF (IOR) & $3.5(3-5.8)$ & $20(12-22)$ & 0.138 \\
\hline
\end{tabular}

Data pooled from POSEIDON, TRIDENT, and TAC-HFT trials, restricted to men with IIEF <25. Autologous: stem cells derived from each individual patient. Allogenic: stem cells derived from healthy stem cell donors.

erectile function in any groups (Figs. 1, 2, 3). This trial is the first to investigate the use of SCT on ED using randomized, placebo-controlled data Conclusions: Erectile function does not appear to improve following TESI of SCs in men with ischemic cardiomyopathy and at least mild ED. The optimal route and source of SCT to enact a change in erectile function has yet to be determined and deserves further research.

\section{References}

1. Hare JM, Fishman JE, Gerstenblith G, et al. Comparison of allogeneic vs autologous bone marrow-derived mesenchymal stem cells delivered by transendocardial injection in patients with ischemic cardiomyopathy: The POSEIDON randomized trial. JAMA 2012;308:236979. https://doi.org/10.1001/jama.2012.25321

2. Heldman AW, DiFede DL, Fishman JE, et al. Transendocardial mesenchymal stem cells and mononuclear bone marrow cells for ischemic cardiomyopathy: The TAC-HFT randomized trial. JAMA 2014;311:62-73. https://doi.org/10.1001/jama.2013.282909

3. Florea V, Rieger AC, DiFede DL, et al. Dose comparison study of allogeneic mesenchymal stem cells in patients with ischemic cardiomyopathy (The TRIDENT Study). Circ Res 2017;121:1279-90. https://doi.org/10.1161/CIRCRESAHA.117.311827

\section{UP-3.23}

Insurance approval rates and patterns for collagenase clostridium histolyticum for the treatment of Peyronie's disease: A Canadian single-center experience

\section{Benjamin Shiff', Premal Patel ${ }^{1}$}

${ }^{1}$ Section of Urology, Department of Surgery, University of Manitoba, Winnipeg, MB, Canada

Introduction: Peyronie's disease (PD) is a common urological condition that can have significant impact on male quality of life. Until recently, no Health Canada-approved pharmacological therapy for PD existed, and surgical intervention has been the mainstay of treatment. Collagenase clostridium histolyticum $(\mathrm{CCH})$ received Health Canada approval for the treatment of PD in 2018. CCH treatment is expensive, with most patients requiring insurance approval. However, to date the subject of insurance approval rates for $\mathrm{CCH}$ in Canada has not been examined.

Methods: A prospective dataset of all patients with PD was established in July 2019. Patients eligible for $\mathrm{CCH}$ included adult men with chronic phase PD with a palpable plaque with a penile curvature of $\geq 30$ and $<90$ degrees. Patients with complex PD or ventral curvatures were not offered $\mathrm{CCH}$ therapy. Chart review was performed to identify patients for which insurance coverage submissions had been made for $\mathrm{CCH}$ and which patients had obtained approval.
Results: A total of 40 patients had been evaluated with PD since July 2019. Mean age was 52.5 years (standard deviation [SD] 13.1 years). Insurance coverage applications for $\mathrm{CCH}$ were submitted for nine of these patients, of which two were approved $(22.2 \%)$ and seven were denied $(77.8 \%)$. Applications were submitted to four insurance companies, of which six submissions (66.7\%) were made to Manitoba Blue Cross, all of which were denied.

Conclusions: Despite Health Canada approval for $\mathrm{CCH}$ use for the treatment of PD, rates of insurance coverage for this treatment are low. The rationale for coverage denial must be examined and addressed to ensure that men have fair access to this proven treatment.

\section{UP-3.25}

Anatomical 3D printed silicone prostate models and digital rectal examination task trainer for the training of medical residents and undergraduate medical students

Lasmine DeZeeuw ${ }^{1}$, Michael K. Organ ${ }^{1}$, Christine Goudie ${ }^{1}$, Adam Dubrowski ${ }^{1}$

'Faculty of Medicine, Memorial University of Newfoundland, St. John's, NL, Canada

Support: MUN Med 3D Network

Introduction: There is a scarcity of affordable, validated, and anatomically correct silicone prostate models for the rehearsal of the digital rectal examination (DRE). The purpose of this technical report is to describe and validate evidence for silicone prostate models and a DRE task trainer created from 3D printed molds for medical student and resident training and clinical skills maintenance.

Methods: A pre-existing 3D human model and five different prostate models from open-source, royalty-free websites were converted using Fusion360 ${ }^{\mathrm{TM}}$ (Autodesk Inc., San Rafael, Ca, U.S.) into stereolithography files and altered to produce negative molds. Fig. 1A illustrates the designed prostate models. The prostate molds were filled with silicone and polylactic acid (PLA) filament "nodules." The buttocks were isolated from the human model and an anal canal was designed with a larger cavity on the interior to hold the silicone prostate models to simulate a real DRE (Figs. 1B, 1C). Five practicing urologists were recruited to evaluate the 3D printed silicone prostate models and the DRE task trainer. The participants were provided with a qualitative survey and asked to rate the perceived realism and educational effectiveness.

Results: The silicone models and task trainer were considered useful in simulation training when attempting DRE techniques. The feedback from the participants was positive overall and included thoughts about stabilizing the prostate models in the task trainer, smoothing the transition 


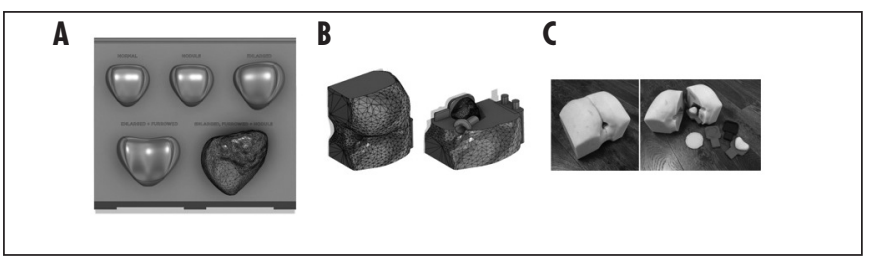

UP-3.25. Fig. 1. (A) Designed prostate models; $(\boldsymbol{B}, \boldsymbol{C})$ The buttocks were isolated from the human model and an anal canal was designed with a larger cavity on the interior to hold the silicone prostate models to simulate a real DRE. between the rectum and the prostate, and adding an additional "normal" prostate model.

Conclusions: Silicone prostate models and DRE task trainers created from 3D molds are an economical anatomically and tactically accurate training tool to teach and maintain DRE skills as compared to commercially available, cost-prohibitive models. With suggested modifications made, the prostate models and DRE task trainer could potentially be used as a future tool for clinical skills training and maintenance and for patient education. 


\section{CUA ABSTRACTS}

\section{Moderated Poster Session 1: Endourology I}

\begin{abstract}
MP-1.2
Safety and efficacy of synchronous bilateral percutaneous nephrolithotomy: A matched retrospective cohort study Colin Lundeen', Victor KF Wong ${ }^{1}$, Kymora Scotland ${ }^{1,2}$, Ben H. Chew ${ }^{\prime}$

${ }^{1}$ Department of Urologic Sciences, University of British Columbia, Vancouver, BC, Canada; ${ }^{2}$ Department of Urology, University of California Los Angeles, Los Angeles, CA, United States

Introduction: Percutaneous nephrolithotomy $(\mathrm{PCNL})$ is the gold standard treatment for renal stones greater than $2 \mathrm{~cm}$. For patients with substantial bilateral nephrolithiasis requiring stone treatment, there is debate regarding the safety and efficacy of synchronous vs. staged bilateral procedures. At our tertiary hospital, nearly all bilateral PCNL procedures are performed in a synchronous fashion. In this study, we investigated synchronous PCNL for the treatment of bilateral renal stones.

Methods: Using the institutional review board-approved PCNL database from our hospital, patients who underwent bilateral synchronous PCNL were retrospectively matched by age, gender, and American Society of Anesthesiologists physical status classification to patients with unilateral stones undergoing PCNL. Procedures were performed in the prone position using balloon dilation up to $30 \mathrm{~F}$, ultrasonic lithotripsy followed by flexible nephroscopy \pm laser lithotripsy. Non-contrast computed tomography $(\mathrm{CT}$ was performed on postoperative day one to assess for residual fragments. Results: A total of 90 subjects were included with 45 in each group. Subjects undergoing bilateral synchronous PCNL had longer operating room (OR) times (249.5 vs. 179.2 minuyes; $\mathrm{p}<0.001)$ and hospital stay (3.60 vs. 2.56 days; $p=0.020)$, as well as more residual fragments (1.63 vs. $1.28 ; p=0.001$ ). No differences were seen in complications, narcotic use, readmission rates, or postoperative hemoglobin change ( $p>0.05)$.

Conclusions: Bilateral synchronous PCNL is safe in stone patients but may lead to longer OR times and hospital stay, in addition to more residual fragments compared to unilateral PCNL. Further prospective data is required to determine the optimal treatment timing for patients with substantial bilateral stone burden.
\end{abstract}

\section{MP-1.3}

Early assessment and outcomes of tubeless outpatient percutaneous nephrolithotomy: A retrospective, contemporaneous analysis to standard percutaneous nephrolithotomy at a single center

Zachary Valley', John W. Dushinski', Richard E. Barr', Patricia A. Dere ${ }^{1}$, Derek Bley', Charles B. Metcalfe', Kamaljot S. Kaler ${ }^{1}$

'Department of Surgery, Section of Urology, University of Calgary, Calgary, AB, Canada

Introduction: Standard percutaneous nephrolithotomy (sPCNL) is the gold standard surgical treatment for large renal calculi. Traditionally, the patient is admitted postoperatively with an indwelling nephrostomy tube, which is typically removed within 1-2 days. However, use of nephrostomy tubes has its downsides, including discomfort, urine leakage, and prolonged discharge. Recently, outpatient tubeless PCNL (oPCNL) has been described where patients are discharged on the same day as surgery without the use of a nephrostomy tube but instead with a stent. We hypothesize that oPCNL will result in lower healthcare costs when compared to SPCNL with similar outcomes.

Methods: A retrospective chart review was conducted of patients who underwent PCNL at the Rockyview General Hospital from June to September 2019 by three fellowship-trained endourologists. Patients who underwent

\begin{tabular}{|c|c|c|c|}
\hline Variable & $\begin{array}{l}\text { Outpatient } \\
\text { PCNL }\end{array}$ & $\begin{array}{l}\text { Standard } \\
\text { PCNL }\end{array}$ & $\mathbf{p}$ \\
\hline Number of patients & 20 & 20 & - \\
\hline Number of males & 15 & 10 & 0.11 \\
\hline Mean age & 52.0 & 56.0 & 0.29 \\
\hline Mean BMI & 32.6 & 31.0 & 0.61 \\
\hline Total bilateral PCNL & 2 & 5 & 0.22 \\
\hline $\begin{array}{l}\text { Total unilateral PCNL and } \\
\text { Contralateral Ureteroscopy }\end{array}$ & 4 & 0 & $0.01 *$ \\
\hline $\begin{array}{l}\text { Mean sum diameter of all } \\
\text { stones }(\mathrm{mm})\end{array}$ & 40.8 & 27.0 & $0.002^{*}$ \\
\hline Mean Guy's score & 2.4 & 2.0 & 0.10 \\
\hline
\end{tabular}

SPCNL (fluoroscopic with nephrostomy tube with postoperative nephrostogram) were retrospectively compared with the oPCNL (endoscopic-guided with stent and tripe hemostasis with same-day computed tomography [CT] scan) cohort based on age, body mass index (BMI), and stone burden (Table 1). Student's t-tests were used to determine statistical significance.

Results: Twenty patients underwent oPCNL and 20 underwent SPCNL. The oPCNL technique at a single center demonstrates a reduction in patient cost. Postoperative complications were not different (Table 2). There was no significant difference in postoperative emergency department visits or hospital readmission. Median time to discharge was 12.4 hours for oPCNL and 55.7 hours for SPCNL. For oPCNL, $64 \%$ of accesses were via the upper pole, while $84 \%$ of sPCNL was accessed via the lower pole.

Conclusions: The oPCNL group in this early cohort shows a total cost savings of \$53 420 despite higher operative case cost with majority of patients discharged same day. This represents a large percentage of upper pole oPCNL patients with no difference in complications. Long-term data is required to see if costs change with a larger patient population.

\section{MP-1.4}

Outcome of miniaturized percutaneous nephrolithotomy using hemostatic plug vs. standard percutaneous nephrolithotomy Fabiola Oquendo ${ }^{1}$, Amr Hodhod ${ }^{1}$, Loay Abbas ${ }^{1}$, Brianna Beaudry ${ }^{1}$, Amer Alaref ${ }^{2}$, Yasser Labib ${ }^{3}$, Scott Bonneville", Ahmed Kotb ${ }^{1}$, Owen Prowse', Walid Shahrour', Hazem Elmansy?

${ }^{1}$ Urology, Northern Ontario School of Medicine, Thunder Bay, ON, Canada; ${ }^{2}$ Radiology, Northern Ontario School of Medicine, Thunder Bay, ON, Canada; ${ }^{3}$ Anesthesia, Northern Ontario School of Medicine, Thunder Bay, ON, Canada

Introduction: We assessed perioperative morbidity and stone-free rate (SFR) after mini percutaneous nephrolithotomy (mPCNL) using hemostatic plug vs. standard percutaneous nephrolithotomy (sPCNL) for managing single medium-sized renal stones $(1-3 \mathrm{~cm})$

Methods: We conducted a retrospective study of prospectively collected data from 2018 to 2019. All patients underwent a preoperative non-contrast 


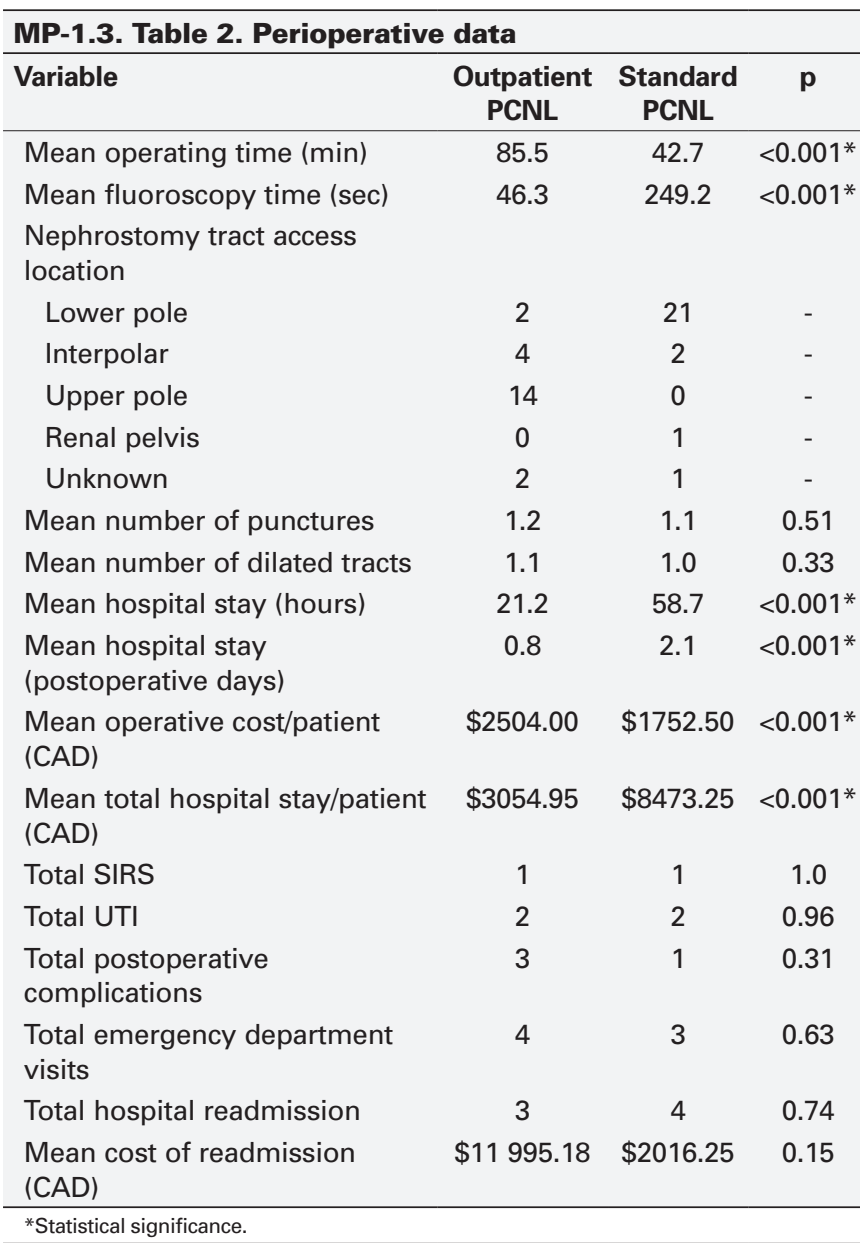

computed tomography $(\mathrm{CT})$ to determine hydronephrosis grade, stone burden, and location. A 16.5/17.5 F system (Karl Storz, Germany) was used for mPCNL and a $30 \mathrm{~F}$ sheath with a $26 \mathrm{~F}$ nephroscope (Karl Storz, Germany) was used for sPCNL. A nephrostomy tube remained after sPCNL, while a Floseal hemostatic plug was used after mPCNL. Operative data were collected. Patients undergoing $\mathrm{MPCNL}$ were discharged the same day and SPCNL patients were discharged two days after surgery. A non-contrast CT was done on postoperative day 1 to evaluate the presence of hematoma. Postoperative complications were assessed using the Clavien-Dindo system. Hematomas were graded based on size: $<10 \mathrm{~mm}$ (trace): small; 10-30 mm: moderate; and $>30 \mathrm{~mm}$ : large. At one month followup, a non-contrast CT was done to detect residual stones. Stone-free was defined as residual stone fragments $\leq 3 \mathrm{~mm}$.

Results: Twenty-five patients underwent mPCNL with hemostatic plug and 39 patients had sPCNL. Patients' characteristics are presented in Table 1. None of the patients had intraoperative complications. The mPCNL arm had less operative time (70 vs. 93 minutes) and fluoroscopic time (102 vs. 143 seconds) ( $p=0.013$ and $p<0.001$, respectively) (Table 2). Perioperative morbidity and hemoglobin loss percentages were similar in both groups $(p=0.4$ and $p=0.29$, respectively). Sixteen percent of $\mathrm{mPCNL}$ were complicated with hematomas compared to $7.7 \%$ after $\mathrm{sPCNL}(\mathrm{p}=0.3$ ) (Table 2). SFR and residual stone size were comparable among the groups. Conclusions: We found $\mathrm{mPCNL}$ with hemostatic plug to be a safe and effective procedure that reduced hospital stays.

\begin{tabular}{|c|c|c|c|c|}
\hline \multicolumn{5}{|c|}{$\begin{array}{l}\text { MP-1.4. Table 1. Patient characteristics and preoperative } \\
\text { data }\end{array}$} \\
\hline Parameter & $\begin{array}{c}\text { mPCNL } \\
25 \text { patients }\end{array}$ & & $\begin{array}{c}\text { sPCNL } \\
39 \text { patients }\end{array}$ & $\mathbf{p}$ \\
\hline \multicolumn{2}{|c|}{ Age median (range), years } & $58.5(34-72)$ & $61(23-85)$ & 0.42 \\
\hline \multirow{2}{*}{ Side } & Right, n (\%) & $11(44)$ & $15(38.5)$ & \multirow{2}{*}{0.66} \\
\hline & Left, n (\%) & $14(56)$ & $24(61.5)$ & \\
\hline \multirow[b]{2}{*}{ Gender } & Male, n (\%) & $15(60)$ & $23(59)$ & \multirow[b]{2}{*}{0.9} \\
\hline & $\begin{array}{c}\text { Female, } \mathrm{n} \\
(\%)\end{array}$ & $10(40)$ & $16(41)$ & \\
\hline \multirow[b]{2}{*}{ Hydronephrosis } & $\begin{array}{l}\text { Nil or mild, } \\
\quad \mathrm{n}(\%)\end{array}$ & $16(64)$ & $31(79.5)$ & \multirow[b]{2}{*}{0.17} \\
\hline & $\begin{array}{c}\text { Moderate } \\
\text { or severe, } \\
\mathrm{n}(\%)\end{array}$ & $9(36)$ & $8(20.5)$ & \\
\hline \multicolumn{2}{|c|}{ Stone burden median (range) } & $\begin{array}{l}19 \mathrm{~mm} \\
(10-24)\end{array}$ & $\begin{array}{l}21 \mathrm{~mm} \\
(11-29)\end{array}$ & 0.08 \\
\hline \multirow{4}{*}{ Stone location } & Pelvic, n (\%) & $5(20)$ & $11(28.2)$ & \\
\hline & $\begin{array}{l}\text { Lower } \\
\text { calyx, n (\%) }\end{array}$ & $16(64)$ & 19 (48.8) & \\
\hline & $\begin{array}{c}\text { Middle } \\
\text { calyx, } \mathrm{n}(\%)\end{array}$ & $3(12)$ & 7 (17.9) & \\
\hline & $\begin{array}{l}\text { Upper } \\
\text { calyx, } \mathrm{n}(\%)\end{array}$ & $1(4)$ & $2(5.1)$ & \\
\hline
\end{tabular}

\section{MP-1.5}

Single-center experience: Miniaturized percutaneous nephrolithotomy vs. flexible ureteroscopy in management of lower calyceal renal stones $\leq \mathbf{2} \mathbf{~ c m}$

Fabiola Oquendo' ${ }^{7}$, Amr Hodhod', Loay Abbas', Brianna Beaudry', Yasser Labib $^{2}$, Scott Bonneville ${ }^{2}$, Ahmed Kotb ${ }^{1}$, Owen Prowse ${ }^{1}$, Walid Shahrour ${ }^{1}$, Hazem Elmansy?

${ }^{1}$ Urology, Northern Ontario School of Medicine, Thunder Bay, ON, Canada; ${ }^{2}$ Anesthesia, Northern Ontario School of Medicine, Thunder Bay, ON, Canada

Introduction: The aim of this study is to compare outcomes of miniaturized percutaneous nephrolithotomy ( $\mathrm{mPCNL}$ ) and flexible ureteroscopy (fURS) at our tertiary institution. We assessed the safety of discharging patients undergoing $\mathrm{mPCNL}$ for the management of lower calyceal renal stones $\leq 2 \mathrm{~cm}$ on the same operative day.

Methods: We conducted a retrospective study of prospectively collected data from September 2018 to September 2019. Demographic data were collected. All patients underwent a preoperative evaluation using noncontrast helical computed tomography $(\mathrm{CT})$ to determine hydronephrosis grade, stone burden, and location. mPCNL was performed using either a Storz (Germany) 16.5/17.5 Fr nephroscope or fURS. Operative data included operative time, fluoroscopy time, intraoperative complications, and the need for JJ stenting. Patients were postoperatively discharged on the same day. Postoperative complications were collected and assessed using the Clavien-Dindo system. A non-contrast helical CT was ordered at one month to detect residual stones. A patient was defined as stone-free if residual stone fragments were $\leq 3 \mathrm{~mm}$.

Results: Thirty-seven patients underwent MPCNL and 54 patients had fURS. Patient demographics are presented in Table 1. There was no significant difference between the treatment modalities in terms of gender, affected kidney side, and stone burden. mPCNL was associated with shorter operating room time but longer fluoroscopy time than fURS $(p=0.03$ and $p<0.001$, respectively) (Table 2$)$. No intraoperative complications were reported for either group. fURS was associated with a higher 


\begin{tabular}{|c|c|c|c|}
\hline Parameter & MPCNL & sPCNL & $\mathbf{p}$ \\
\hline $\begin{array}{l}\text { Operation time median } \\
\text { (range) }\end{array}$ & $\begin{array}{l}70 \min \\
(19-121)\end{array}$ & $\begin{array}{l}93 \min \\
(32-194)\end{array}$ & 0.013 \\
\hline $\begin{array}{l}\text { Fluoroscopic time median } \\
\text { (range) }\end{array}$ & $\begin{array}{c}102 \mathrm{sec} \\
(49-424)\end{array}$ & $\begin{array}{c}143 \mathrm{sec} \\
(61-521)\end{array}$ & $<0.001$ \\
\hline $\begin{array}{l}\text { Intraoperative } \\
\text { complications, n (\%) }\end{array}$ & 0 & 0 & - \\
\hline JJ stent, n (\%) & $10(40)$ & $19(48.7)$ & 0.49 \\
\hline $\begin{array}{l}\% \text { of hemoglobin loss } \\
\text { median (range) }\end{array}$ & $6.5 \%(0-13.2)$ & $8.3 \%(0-16)$ & 0.29 \\
\hline \multicolumn{4}{|l|}{ Perioperative Clavien } \\
\hline I & 0 & $2(5.1)$ & \multirow[t]{2}{*}{0.4} \\
\hline $\begin{array}{l}\text { III A } \\
\text { III B }\end{array}$ & $1(4)$ & $3(7.7)$ & \\
\hline $\begin{array}{l}\text { Hospital stay median } \\
\text { (range) }\end{array}$ & 0 days $(0-3)$ & $2(2-6)$ & $<0.001$ \\
\hline $\begin{array}{l}\text { Hospital readmission, } \mathrm{n} \\
(\%)\end{array}$ & $3(12)$ & $6(15.4)$ & 0.7 \\
\hline $\begin{array}{l}\text { Causes of readmission } \\
\text { Intolerable pain, n (\%) } \\
\text { Postoperative fever, n } \\
(\%) \\
\text { Leak, n (\%) }\end{array}$ & $\begin{array}{c}3(12) \\
0 \\
0\end{array}$ & $\begin{array}{c}4(10.3) \\
1(2.6) \\
1(2.6)\end{array}$ & 0.53 \\
\hline \multicolumn{4}{|l|}{ Hematoma } \\
\hline Total, n (\%) & 4 (16) & $3(7.7)$ & \\
\hline Small, n (\%) & $3(12)$ & $3(7.7)$ & 0.3 \\
\hline Moderate, n (\%) & 0 & 0 & \\
\hline Large, n (\%) & $1(4)$ & 0 & \\
\hline SFR ( $\leq 3 \mathrm{~mm}), \mathrm{n}(\%)$ & $21(84)$ & $35(89.7)$ & 0.5 \\
\hline $\begin{array}{l}\text { Residual stone (>3 mm), } \\
\mathrm{n}(\%)\end{array}$ & $4(16)$ & $4(10.3)$ & 0.5 \\
\hline
\end{tabular}

MP-1.5. Table 1. Patients' characteristics and preoperative data

\begin{tabular}{|c|c|c|c|c|}
\hline \multicolumn{2}{|l|}{ Parameter } & $\begin{array}{l}\text { Mini-PCNL } \\
37 \text { patients }\end{array}$ & $\begin{array}{c}\text { fURS } \\
54 \text { Patients }\end{array}$ & $\mathbf{p}$ \\
\hline \multicolumn{2}{|c|}{ Age median (range), years } & $58(32-70)$ & $58 y(30-83)$ & 0.86 \\
\hline \multirow[t]{3}{*}{ Side } & $\begin{array}{l}\text { Right, } \mathrm{n} \\
(\%)\end{array}$ & $16(43.2)$ & $17(31.5)$ & \multirow[t]{3}{*}{0.2} \\
\hline & Left, n (\%) & $21(56.8)$ & $37(68.5)$ & \\
\hline & Male, n (\%) & $19(51.4)$ & $25(46.3)$ & \\
\hline Gender & $\begin{array}{l}\text { Female, } \mathrm{n} \\
(\%)\end{array}$ & $18(48.6)$ & $29(53.7)$ & 0.63 \\
\hline \multicolumn{2}{|c|}{ Previous PCNL n (\%) } & $6(16.2)$ & $6(11.1)$ & \multirow[t]{2}{*}{0.48} \\
\hline \multirow[b]{2}{*}{ Hydronephrosis } & $\begin{array}{l}\text { No or mild } \\
\mathrm{n}(\%)\end{array}$ & $19(51.4)$ & $47(87)$ & \\
\hline & $\begin{array}{c}\text { Moderate } \\
\text { or severe } \\
\mathrm{n}(\%)\end{array}$ & $18(48.6)$ & $7(13)$ & $<0.001$ \\
\hline \multicolumn{2}{|c|}{$\begin{array}{l}\text { Stone burden median } \\
\text { (range) }\end{array}$} & $\begin{array}{l}17 \mathrm{~mm} \\
(10-20)\end{array}$ & $\begin{array}{l}15 \mathrm{~mm} \\
(9-20)\end{array}$ & 0.12 \\
\hline
\end{tabular}

MP-1.5. Table 2. Intraoperative and postoperative data

\begin{tabular}{|c|c|c|c|}
\hline Parameter & Mini-PCNL & fURS & p \\
\hline $\begin{array}{l}\text { Operation time median } \\
\text { (range) }\end{array}$ & $\begin{array}{l}69 \min \\
(19-121)\end{array}$ & $\begin{array}{l}88 \min \\
(29-157)\end{array}$ & 0.03 \\
\hline $\begin{array}{l}\text { Fluoroscopic time median } \\
\text { (range) }\end{array}$ & $\begin{array}{c}102 \mathrm{sec} \\
(49-424)\end{array}$ & $\begin{array}{c}62 \mathrm{sec} \\
(15-247)\end{array}$ & $<0.001$ \\
\hline Ureteral sheath, n (\%) & - & $51(94.4)$ & - \\
\hline $\begin{array}{l}\text { Intraoperative } \\
\text { complications, n (\%) }\end{array}$ & 0 & 0 & - \\
\hline JJ stent, n (\%) & $12(32.4)$ & $50(92.6)$ & $<0.001$ \\
\hline \multicolumn{4}{|l|}{$\begin{array}{l}\text { Perioperative Clavien- } \\
\text { Dindo classification }\end{array}$} \\
\hline I & $2(5.4)$ & 0 & - \\
\hline II & 0 & 0 & \\
\hline III & $1(2.7)$ & 0 & \\
\hline Hospital readmission, n (\%) & $3(8.1)$ & $1(1.9)$ & 0.15 \\
\hline SFR ( $\leq 3 \mathrm{~mm}), \mathrm{n}(\%)$ & $28(78.4)$ & 41 (75.9) & 0.98 \\
\hline $\begin{array}{l}\text { Residual stone (>3 mm), } \\
\mathrm{n}(\%)\end{array}$ & 9 (21.6) & $13(24.1)$ & 0.12 \\
\hline $\begin{array}{l}\text { Size of residual stone } \\
\text { median (range) }\end{array}$ & $3 \mathrm{~mm}(4-8)$ & $3 \mathrm{~mm}(4-8)$ & 0.29 \\
\hline \multicolumn{4}{|l|}{ Stone composition } \\
\hline monohydrate, $\mathrm{n}(\%)$ & $10(27)$ & $22(40.7)$ & \\
\hline Calcium oxalate & $9(24.3)$ & $8(14.8)$ & \\
\hline dihydrate, n (\%) & $1(2.7)$ & $2(3.7)$ & 0.66 \\
\hline Calcium phosphate, n (\%) & $6(16.3)$ & $8(14.8)$ & \\
\hline Urate, n (\%) & 0 & $1(1.9)$ & \\
\hline Struvite, n (\%) & $11(29.7)$ & $13(24.1)$ & \\
\hline
\end{tabular}

stenting rate $(p<0.001)$. Three patients in the $\mathrm{mPCNL}$ group were readmitted for intolerable pain. Two patients were managed medically and one required stenting. Stone-free rate (SFR) and the presence of residual stones were comparable among both groups ( $\mathrm{p}=0.98$ and $\mathrm{p}=0.12$, respectively). Conclusions: Ambulatory mPCNL is feasible, safe, and has similar SFR to fURS. Further prospective, randomized trials are needed to confirm these results.

\section{MP-1.6}

Evaluation of the 3 French Trilogy pneumatic/ultrasonic probe: Comparison to holmium laser lithotripsy during mini-PCNL with a $10 \mathrm{~F}$ nephroscope

Brennan Timm ${ }^{1}$, Greg Jack', Damien Bolton

${ }^{1}$ Urology, Austin Health, Heidelberg, Australia

Introduction: Mini percutaneous nephrolithotomy ( $\mathrm{mPCNL}$ ) continues to gain popularity due to its low morbidity, high efficacy, and low costs. A limitation of $\mathrm{mPCNL}$ is the small working lumens size of mini-nephroscopes allowing only for limited lithotripter instrumentation with under $5 \mathrm{~F}$ instruments. We evaluated the efficacy of the ultra-small Trilogy dual energy $3 \mathrm{~F}$ lithotripter probe for $\mathrm{mPCNL}$ and compared it to a matched cohort using holmium laser for clearance.

Methods: We performed a prospective analysis of mPCNL cases trialing the Trilogy lithotripter fragmentation efficacy with holmium laser. A Stortz $10 \mathrm{~F}$ MIPS nephroscope was used for all cases, with a $16.5 \mathrm{~F}$ sheath and a $5 \mathrm{~F}$ single-flow working channel. The Swiss Lithoclast ${ }^{\circledR}$ Trilogy (EMS) was used with the $3 \mathrm{~F}$ mini-PCN $550 \mathrm{~mm}$ probe and dual ultrasonic and electromagnetic ballistic settings at maximal energy. The holmium laser (Convergent, U.S.), was used at a max of 20 W, with a 270-550 um fiber. 
Results: Twenty-seven consecutive mPCNL cases performed by five surgeons were analyzed, 12 using the trilogy, 15 using the holmium laser. Trilogy and laser settings varied intraoperatively a to a maximal power or frequency of $2.0 \mathrm{~J}$ or $18 \mathrm{Hertz}$ with the holmium laser and a $100 \%$ electromagnetic ballistic and $100 \%$ ultrasonic energy for the Trilogy. The median surgical time for Trilogy vs. holmium was 131 (interquartile range [IQR] 102-159) minutes vs. 125 minutes (IQR 97-153), respectively. The $3 \mathrm{~F}$ Trilogy probe performed well on soft stones, with a clearance rate of $177.0 \mathrm{~mm}^{3} / \mathrm{min}$ for stones $<1000 \mathrm{HU}$, but $24.3 \mathrm{~mm}^{3} / \mathrm{min}$ for stones $>1000$ $\mathrm{HU}$. There was one infective complication in the holmium cohort and none in the Trilogy cohort. There were no device failures in either modality. Conclusions: The combined ultrasonic, electromagnetic ballistic, and suction features of the $3 \mathrm{~F}$ probe made it effective for $\mathrm{mPCNL}$ on soft and infection stones. Holmium laser was superior to $3 \mathrm{~F}$ Trilogy for large, dense stones in our limited trial.

\section{MP-1.9}

\section{Ultra-smooth ureteral stent material decreases encrustation} Khatereh Aminoltejari ${ }^{1}$, Ben H. Chew ${ }^{1}$, Dirk Lange

'Department of Urologic Sciences, University of British Columbia, Vancouver, BC, Canada

Introduction: Ureteral stents are one of the most used devices in urology and are associated with complications that limit their long-term use, including infection and encrustation. Previous studies have shown uneven surfaces to promote bacterial and crystal deposition, resulting in increased risk for complications. Recently, a new ureteral stent with an ultra-smooth surface was developed via a modified extrusion process to address the accumulation of urine salts and decrease encrustation. This study assessed the ability for the Tria Soft and Firm materials to decrease struvite and calcium oxalate encrustation compared to other commercially available stents.

Methods: Overall, the encrustation of Tria Soft and Firm was compared to that of the InLay Optima and Contour stent materials. Stent pieces of $2 \mathrm{~cm}$ were exposed to artificial urine containing Proteus mirabilis to induce struvite crystal deposition and encrustation. Incubation in artificial urine in the absence of bacteria served as a non-struvite encrustation control. Encrustation was assessed after two weeks of incubation via the quantification of total calcium and magnesium from the stent surface using Atomic Absorption Spectroscopy analysis. For samples incubated with P. mirabilis, the accumulation of bacteria on the device surface was also assessed via colony forming unit counts.

Results: Overall, accumulation of struvite/CaPO on the surface of Tria Soft and Firm was significantly decreased compared to the InLay Optima and Contour materials. Similarly, non-struvite crystal deposition was significantly decreased on the Tria material compared to InLay Optima and Contour materials.

Conclusions: The novel ultra-smooth Tria Soft and Firm material developed using an altered extrusion process was shown to be more effective at resisting the accumulation of struvite and non-struvite encrustation compared to other commercially available stent materials. Further studies are required to validate this beneficial effect in patients.

\section{MP-1.10}

Scatter radiation exposure to the surgical team during supine and prone percutaneous nephrolithotomy

David W. Chapman', Devin Demitor', Shubhadip (Shubha) K. De

${ }^{1}$ Urology, University of Alberta, Edmonton, $A B$, Canada; ${ }^{2}$ Diagnostic Imaging, University of Alberta, Edmonton, $\mathrm{AB}$, Canada

Introduction: Radiation is central to diagnostic and therapeutic procedures in urology. Many strategies help reduce radiation exposure during percutaneous nephrolithotomy (PCNL), however, little has been studied in the contribution of patient positioning and $\mathrm{C}$-arm orientation. The aim of this study was to compare the radiation exposure to the surgical team during supine and prone positions with common $\mathrm{C}$-arm configurations. Methods: A benchtop experiment was conducted using a Siemens C-arm, anthropomorphic model ( $80 \mathrm{~kg}$ human torso) and a $180 \mathrm{cc}$ ionization chamber to directly measure radiation scatter $(\mathrm{mR} / \mathrm{min})$ to the team dur- ing different configurations of the C-arm (AP, 150 oblique, lateral) for prone and supine (Bart's free flank 150 decubitus) PCNL. Exposure was measured using regular and high dose fluoroscopy settings, for all personnel for six C-arm orientations at eye and gonad level.

Results: Standard radiation dose was defined as detected scatter with the patient in traditional prone position, and C-arm in AP (eyes: URO 0.59 , ANS 0.00, SN 0.00 , XRT $0.00 \mathrm{mR} / \mathrm{min}$; gonads URO 1.3, ANS 0.00 , SN 0.22 , XRT $0.00 \mathrm{mR} / \mathrm{min}$ ). With the C-arm in lateral orientation and patient in supine, the average dose increased by $6.49-21.13$ times compared to the oblique and AP configuration, respectively. When dose reduction methods were employed: positioning the surgical team opposite to the image intensifier, exposure was reduced by 3.14-3.58 times, whereas rotating the $\mathrm{C}$-arm over the table (into lateral position) reduced exposure by 1.49-1.46 times (as compared to standard 'under-the-table' lateral positioning).

Conclusions: Radiation is of increasing concern and current guidelines do not recommend physical strategies of dose reduction. This study highlights the ability for a surgical team to drastically alter radiation exposure by considering positioning as part of the contributing factors. Knowing relative exposure increases may empower surgeons to choose alternative orientations to accomplish tasks while minimizing scatter.

\section{MP-1.11}

The risk of postoperative sepsis in patients undergoing ureteroscopy or percutaneous nephrolithotomy for urinary tract stones

Jennifer Bjazevic ${ }^{1}$, Noah Stern ${ }^{1}$, Hassan Razvi ${ }^{1}$

${ }^{1}$ Surgery, Western University, London, ON, Canada

Introduction: The incidence of urinary stone disease continues to rise. Co-existent with this, there is also evidence to suggest patient comorbidities and frailty are becoming more common, potentially placing patients at higher risk for postoperative complications. Postoperative sepsis remains a leading cause of morbidity and mortality following surgical intervention for stone disease. We aimed to quantify the postoperative complication rate of urinary sepsis following ureteroscopy (URS) and percutaneous nephrolithotomy (PCNL), and to identify predictive risk factors.

Methods: The American College of Surgeons National Surgical Quality Improvement Database (NSQIP) database was used to identify URS and PCNL procedures performed from 2005-2017. Postoperative sepsis was defined as the presence of two or more systemic inflammatory response (SIRS) criteria. Multivariate logistic regression analysis was performed to identify potential patient and operative risk factors predictive of sepsis development.

Results: A total of 1517 procedures were identified, including 1148 URS and 369 PCNLs. The overall sepsis rate in patients undergoing URS was $1.0 \%$ compared to a rate of $2.7 \%$ in those undergoing PCNL. A trend towards an increasing rate of sepsis among the URS cohort was observed $(\mathrm{p}=0.05)$. Factors associated with the occurrence of postoperative sepsis for the entire cohort included a higher American Society of Anesthesiologists (ASA) score (odds ratio [OR] 1.12; $\mathrm{p}=0.027$ ), active smoking status (OR 1.35; $\mathrm{p}=0.008$ ), and dialysis (OR 3.66; $\mathrm{p}<0.001$ ). Conclusions: The overall incidence of postoperative sepsis following URS and PCNL remains low, however, a trend towards increasing rates of sepsis among patients undergoing URS was noted. Several preoperative patient characteristics were also identified as potential risk factors. Further investigation should be conducted to determine the reason(s) for this increasing trend, and to aid in the development of reliable predictive models to help mitigate the risk of postoperative sepsis. 


\section{MP-1.12}

Predictors of postoperative urosepsis in struvite stone patients Lustin Y.H. Chan ${ }^{1}$, Victor K.F. Wong ${ }^{7}$, Julie Wong ${ }^{7}$, Kymora Scotland ${ }^{1}$, Ben H. Chew

'Department of Urologic Sciences, University of British Columbia, Vancouver, BC, Canada

Introduction: Struvite stones account for $7-15 \%$ of all urinary calculi and are associated with urease-splitting bacteria. ${ }^{1}$ Due to their association with bacteria, struvite patients are at risk of developing urosepsis. ${ }^{2}$ Our study aims to identify predictor variables for urosepsis in patients that have undergone percutaneous nephrolithotomy (PCNL) for struvite stones. Methods: A retrospective review was performed on struvite stone patients. The Systemic Inflammatory Response Syndrome (SIRS) criteria and quick Sepsis-related Organ Failure Assessment (qSOFA) were used to identify patients with suspected infection and increased risk for urosepsis. Univariable and multivariable analysis was performed to identify predictor variables for urosepsis.

Results: Chart review identified 96 patients with struvite stones who were treated with PCNL. Postoperatively, 38 patients were SIRS-positive $(\geq 2$ criteria) and 11 were considered high-risk patients for in-hospital mortality (qSOFA score $\geq 2$ ). Univariable analysis determined that patients with prior stone procedures $(p=0.04)$, stenting $(p=0.01)$, elevated white blood cell count $(p=0.01)$, and longer operative times $(p<0.0001)$ were more likely to be SIRS-positive. With qSOFA, patients at higher risk for urosepsis had other medical comorbidities ( $p=0.02)$, a blood transfusion $(p=0.0002)$, a longer operation $(p<0.0001)$, lengthier hospitalization $(p=0.007)$, and received antibiotics within three months prior to stone surgery $(p=0.04)$. Multivariable analysis did not reveal any factors that may predispose patients to being SIRS-positive. Patients with bladder outlet obstruction (odds ratio $[O R] 2.86 ; p \leq 0.0001)$ or stenting (OR 1.28; $p=0.004)$ significantly increased the odds of having a qSOFA score $\geq 2$.

Conclusions: Struvite stone patients with bladder outlet obstruction and stenting postoperatively may be at an increased risk for progressing to urosepsis. Further research using the Sepsis-3 SOFA criteria as a measure of urosepsis is needed to confirm the findings of this study.

\section{References}

1. Flannigan $\mathrm{R}$, Choy $\mathrm{WH}$, Chew $\mathrm{B}$, et al. Renal struvite stones pathogenesis, microbiology, and management strategies. Nat Rev Urol 2014;11:333. https://doi.org/10.1038/nrurol.2014.99

2. Rivera M, Viers B, Cockerill P, et al. Pre- and postoperative predictors of infection-related complications in patients undergoing percutaneous nephrolithotomy. J Endourol 2016;30:982-6. https:// doi.org/10.1089/end.2016.0191

\section{MP-1.13}

Predictors of mortality for patients admitted to the intensive care unit with obstructing septic stones

Uday Mann', Zack Li ${ }^{1}$, Amanda Eng ${ }^{1}$, Rachel Wong ${ }^{1}$, Ruben BlachmanBraun $^{2}$, Premal Patel

${ }^{1}$ Section of Urology, Department of Surgery, University of Manitoba, Winnipeg, MB, Canada; ${ }^{2}$ Department of Urology, University of Miami, Miami, FL, United States

Introduction: Urosepsis with obstructing calculi or septic stone is a urological emergency requiring urgent intervention. These patients are at significant risk of morbidity and mortality should source control through drainage be delayed and are often admitted to intensive care units (ICU) for hemodynamic support. ${ }^{1-4}$ The purpose of this study was to determine patient factors that may predict mortality in patients admitted to ICU with septic stone, particularly whether rural patients at a greater distance from a tertiary care center were at greater risk of mortality given the inherent delay in intervention.

Methods: The Manitoba Intensive Care Unit prospective registry began in 1999 and includes all patients admitted to ICU across Manitoba. Baseline characteristics, such as age, gender, vital signs, creatinine, Charlson comorbidity index $(\mathrm{CCl})$, mortality outcomes, and location of residency were obtained for those admitted to ICU for septic stone. Comparison

\begin{tabular}{|c|c|c|c|}
\hline \multicolumn{4}{|c|}{ MP-1.13. Table 1. Patient demographics by location } \\
\hline Variable & Urban $n=68(\%)$ & Rural $n=138(\%)$ & $\mathbf{p}$ \\
\hline Age & $61.0 \pm 15.6$ & $62.1 \pm 16.1$ & 0.661 \\
\hline \multicolumn{4}{|l|}{ Sex } \\
\hline Male & $29(42.6)$ & $59(42.8)$ & \\
\hline Female & $39(57.4)$ & $79(57.2)$ & 0.988 \\
\hline Temperature & $37.3(36.5-38.4)$ & $37.2(36.4-38.1)$ & 0.355 \\
\hline \multicolumn{4}{|l|}{$\begin{array}{l}\text { Temperature } \\
\text { codified }\end{array}$} \\
\hline$<36^{\circ} \mathrm{C}$ & $3(4.4)$ & $16(11.6)$ & \\
\hline $36-38^{\circ} \mathrm{C}$ & $45(66.2)$ & $87(63)$ & \\
\hline$>38^{\circ} \mathrm{C}$ & $20(29.4)$ & $35(25.4)$ & 0.235 \\
\hline SBP & $80(70-93)$ & $79(69.8-89.3)$ & 0.394 \\
\hline DBP & $46(40.3-50)$ & $47(40-52)$ & 0.655 \\
\hline $\mathrm{HR}$ & $119(104.5-140)$ & $116(100.8-132)$ & 0.292 \\
\hline Total GCS & $14(10-15)$ & $14(9-15)$ & 0.385 \\
\hline \multicolumn{4}{|l|}{ Intubation status } \\
\hline Not intubated & $45(66.2)$ & $92(66.7)$ & \\
\hline Intubated & $23(33.8)$ & 46 (33.3) & 0.944 \\
\hline $\mathrm{pH}^{*}$ & $7.28 \pm 0.11$ & $7.29 \pm 0.12$ & 0.770 \\
\hline WBC & $19.4(14.3-28.1)$ & $18.9(11.8-26)$ & 0.425 \\
\hline Creatinine & $197(120.3-262.8)$ & 163 (104.3-284) & 0.338 \\
\hline \multicolumn{4}{|l|}{ AKI } \\
\hline No AKI & $50(73.5)$ & $98(71)$ & \\
\hline AKI & $18(26.5)$ & $40(29)$ & 0.706 \\
\hline Charlson score & $2(0-3.8)$ & $2(0.8-3)$ & 0.791 \\
\hline LOS & $3.3(1.2-6.1)$ & $3.8(1.7-7.2)$ & 0.141 \\
\hline \multicolumn{4}{|l|}{ Mortality } \\
\hline Alive & $56(82.4)$ & $122(88.4)$ & \\
\hline Death & $12(17.6)$ & $16(11.6)$ & 0.233 \\
\hline
\end{tabular}

of variables between groups was performed using the Mann-Whitney $U$, Student t-test, chi-squared, and Fisher's exact test. Association between death and clinical/demographic variable was performed with adjusted multivariable logistical regression analysis. Analysis was performed with SPSS version 24.0 (Chicago, U.S.).

Results: A total of 206 patients with septic stone with location data available were admitted to the ICU (68 urban and 138 rural). Baseline characteristics were similar between groups ( $p>0.05)$. There was a difference in HR, GCS, AKI between groups (Table 1). On multivariable adjusted logistical regression, the presence of AKI $(p<0.001)$ and intubation $(p$ $<0.001$ ) was associated with mortality (Table 2 ). There was no difference in mortality attributable to location ( $>>0.05)$, vital signs, or $\mathrm{CCl}$.

Conclusions: Among patients admitted to the ICU for septic stones in Manitoba, we demonstrate an association between AKI and intubation with mortality. Other factors, such as distance from a tertiary care center and baseline patient characteristics, were not predictive of mortality.

\section{References}

1. Yoshimura K, Utsunomiya N, Ichioka K, et al. Emergency drainage for urosepsis associated with upper urinary tract calculi. J Urol 2005;173:458-62. https://doi.org/10.1097/01. ju.0000150512.40102.bb

2. Hamasuna R, Takahashi S, Nagae H, et al. Obstructive pyelonephritis as a result of urolithiasis in Japan: Diagnosis, treatment and prognosis. Int J Urol 2015;22:294-300. https://doi.org/10.1111/iju.12666 
MP-1.13. Table 2. Univariable and multivariable adjusted logistical regression considering the outcome of mortality

\begin{tabular}{|c|c|c|c|c|c|c|}
\hline \multirow[b]{2}{*}{ Variable } & \multicolumn{3}{|c|}{ Univariable } & \multicolumn{3}{|c|}{ Multvariable } \\
\hline & OR & $95 \% \mathrm{Cl}$ & $\mathbf{p}$ & OR & $95 \% \mathrm{Cl}$ & $\mathbf{p}$ \\
\hline Age & 1.021 & 0.994-1.049 & 0.133 & 1.023 & $0.987-1.061$ & 0.211 \\
\hline \multicolumn{7}{|l|}{ Sex } \\
\hline Male & 1 & & & 1 & & \\
\hline Female & 0.603 & $0.271-1.342$ & 0.215 & 0.508 & $0.174-1.483$ & 0.216 \\
\hline \multicolumn{7}{|c|}{ Temperature codified } \\
\hline SBP & 0.989 & $0.967-1.011$ & 0.316 & 0.988 & $0.964-1.012$ & 0.314 \\
\hline $\mathrm{HR}$ & 1.018 & $1.003-1.033$ & 0.015 & 1.012 & $0.995-1.029$ & 0.163 \\
\hline \multicolumn{7}{|l|}{ Intubation status } \\
\hline Not intubated & 1 & & & 1 & & \\
\hline Intubated & 5.404 & $2.292-12.744$ & $<0.001$ & 4.131 & $1.386-12.311$ & 0.011 \\
\hline$<7.1$ & 9.500 & $1.895-47.614$ & 0.006 & 2.755 & $0.340-22.315$ & 0.342 \\
\hline WBC & 0.978 & $0.942-1.016$ & 0.256 & 0.964 & $0.921-1.010$ & 0.120 \\
\hline \multicolumn{7}{|l|}{ AKI } \\
\hline No AKI & 1 & & & 1 & & \\
\hline AKI & 6.210 & $2.656-14.520$ & $<0.001$ & 4.794 & $1.544-14.890$ & 0.007 \\
\hline Charlson score & 1.132 & $0.954-1.343$ & 0.155 & 1.090 & $0.858-1.385$ & 0.480 \\
\hline LOS & 1.008 & 0.994-1.021 & 0.269 & 1.018 & 0.999-1.038 & 0.059 \\
\hline \multicolumn{7}{|l|}{ Location } \\
\hline Urban & 1 & & & 1 & & \\
\hline Rural & 0.612 & $0.272-1.379$ & 0.236 & 0.506 & $0.172-1.490$ & 0.217 \\
\hline
\end{tabular}

3. Sammon JD, Ghani KR, Karakiewicz PI, et al. Temporal trends, practice patterns, and treatment outcomes for infected upper urinary tract stones in the United States. Eur Urol 2013;64:85-92. https://doi.org/10.1016/j.eururo.2012.09.035

4. Liang $X$, Huang J, Xing $M$, et al. Risk factors and outcomes of urosepsis in patients with calculous pyonephrosis receiving surgical intervention: A single-center retrospective study. BMC Anesthesiol 2019;19:61. https://doi.org/10.1186/s12871-019-0729-3

\section{MP-1.14}

Correlating crowd-sourced assessment of technical skills with postoperative complication rates in urological robotic surgery David M. Mikhail', Joseph Sarcona ${ }^{1}$, Aaron Tabibzadeh², Daniel Nassau ${ }^{1}$ Zachary Kozel', Michael Feuerstein ${ }^{1}$, Louis Kavoussi ${ }^{1}$, Lee Richstone ${ }^{1}$ ${ }^{1}$ Urology, Northwell Health, New York, NY, United States; ${ }^{2}$ Zucker School of Medicine, Hofstra University, Hempstead, NY, United States Introduction: Crowd-sourced assessment of technical skills (CSATS) is a voluntary, validated crowd-sourced system for surgeon feedback and improvement based on the five-domain global evaluative assessment of robotic skills (GEARS) ${ }^{1}$ score out of 25 . While CSATS has correlated with technical outcomes (i.e., anastomotic leaks after robotic-assisted laparoscopic prostatectomy), there is a paucity of data comparing it to surgical complications. We aimed to compare CSATS score with complications after robotic urological surgery.

Methods: We retropsectively reviewed our prospectively maintained robotic surgery complication database and compared with our CSATS data. Fellowship-trained surgeons with a minimum of 10 cases submitted to CSATS with complete complication data were included for analysis.
CSATS score and surgeon quartiles were compared to total and $\geq$ Clavien grade 3 complications.

Results: In 2018-19, a total 196 urology cases were submitted to CSATS by our group. Of these, 125 cases by four fellowship-trained urologists had complete complication data from a historical robotic complications database. These CSATS cases were $75 \%$ prostate, $13 \%$ upper tract, and $10 \%$ bladder. These four surgeons completed 315 robotic cases from 2015-18 with mean complications of $20.3 \%(13-32 \%)$ and $8.9 \%(6-14 \%)$ Clavien $\geq 3$ complications. Mean CSATS score was 20.26 (20.18-20.43). Of the four surgeons, two were in the third and two in the top quartile. CSATS score

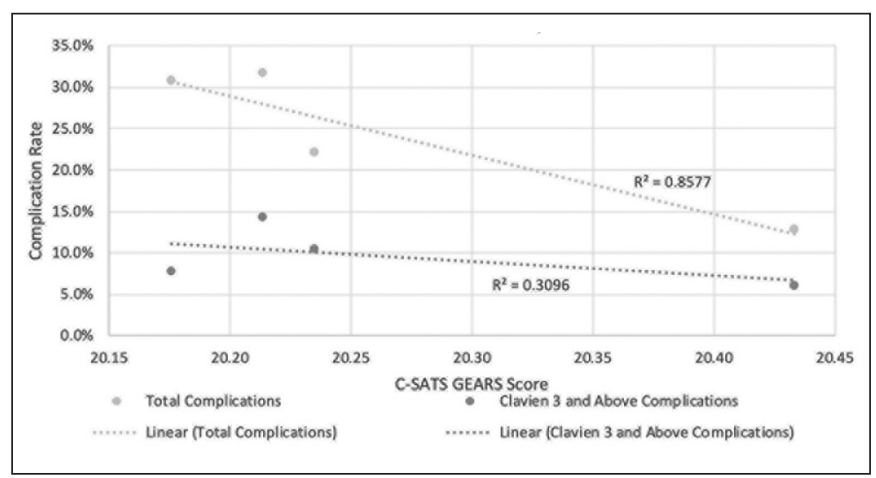

MP-1.14. Fig. 1. C-SATS GEARS score vs. complications. 
correlated with total and Clavien $\geq 3$ complications with Pearson coefficients of $-0.926\left(R^{2} 0.86\right)$ and $-0.556\left(R^{2} 0.31\right)$ (Fig. 1). Being in the top quartile did not significantly reduce Clavien $\geq 3$ complications ( $6.3 \%$ vs. $12.1 \%$; $p=0.07)$. Conclusions: This is the first assessment comparing CSATS scores to complication rates in urological surgery. ${ }^{2}$ Overall complication rate was strongly correlated to CSATS score. Significant complications weakly correlated to CSATS score but approached significance with surgeon quartiles. We are currently evaluating the correlation of technical skills and complication rates across all robotics in our health system.

\section{References}

1. Kowalewski T, Comstock B, Sweet R, et al. Crowd-sourced assessment of technical skills for validation of basic laparoscopic urologic skills tasks. J Urol 2016;195:1859-65. https://doi.org/10.1016/j. juro.2016.01.005

2. Birkmeyer JD, Finks JF, O'Reilly A, et al. Surgical skill and complication rates after bariatric surgery. N Engl J Med 2013;369:1434-42. https://doi.org/10.1056/NEJMsa1300625 


\section{Moderated Poster Session 2: Trauma, Reconstruction, Pelvic Pain}

\begin{abstract}
MP-2.1
Assessing the management of collecting system injuries in renal trauma grade $\mathbf{4}$ and 5 in a large, retrospective cohort Lennifer A. Locke', Roshan Navaratnam', Sarah R. Ferrara' ', Andrea Phillips ${ }^{1}$ Avery Nathens', Sender Herschorn ${ }^{1}$, Ronald T. Kodama

'Urology, Sunnybrook Health Sciences Centre, Toronto, ON, Canada Support: Tory Trauma Program

Introduction: Collecting system injuries coincide with $50 \%$ of AAST grade $4 / 5$ renal injuries and $29 \%$ of these collecting system injuries are stented. ${ }^{1}$ AUA guidelines suggest that, "surgeons perform urinary drainage (via stent or percutaneous nephrostomy tube) in the presence of complications, such as enlarging urinoma, fever, increasing pain, ileus, fistula, or infection." ${ }^{2}$ In this retrospective study, we question if ureteral stenting is necessary in the management of collecting system injuries after grade 4/5 renal trauma. Methods: After REB approval, we conducted a chart review of grade $4 / 5$ renal traumas with collecting system injuries at our institution from 1998-2019. Results: We identified 149 patients with grade $4 / 5$ renal traumas; 98 had immediate trauma imaging leading to the diagnosis of urinary extravasation in $46(47 \%)$. Three patients underwent eventual stent insertion and two had a late drain placed for a urinoma. Five patients underwent immediate trauma laparotomy (two nephrectomy, three operative repair of the kidney). The remaining $36(78 \%)$ did not undergo any interventions. Of those who did not undergo an intervention for a collecting system injury, 31 (86\%) had repeat imaging (ultrasound [US]: five; computed tomography [CT], non-contrast: three; CT with IV contrast: 13; and CT urogram: 10; median time four days; range 1-112). Of those reimaged, 20 (65\%) demonstrated no extravasation and $10(32 \%)$ demonstrated continued urinary extravasation. Resolution of extravasation on subsequent imaging was observed in nine of those, while one was lost to followup.

Conclusions: Our results suggest that collecting system injuries do not require stent intervention and will resolve with time. This supports the role for conservative management of high-grade renal traumas with collecting system injuries and questions the necessity for routine early CT with IV contrast reimaging in the stable and asymptomatic patient.
\end{abstract}

References

1. Keihani $\mathrm{S}, \mathrm{Xu} \mathrm{Y}$, Presson AP, et al. Contemporary management of highgrade renal trauma: Results from the American Association for the Surgery of Trauma Genitourinary Trauma study. I Trauma Acute Care Surg 2018;4:418-25. https://doi.org/10.1097/TA.0000000000001796

2. Morey AF, Brandes S, Dugi DD 3rd, et al. Urotrauma: AUA guideline. J Urol 2014;192:327-35. https://doi.org/10.1016/j.juro.2014.05.004

\footnotetext{
MP-2.2

Single-stage reconstruction of fossa navicularis strictures using a "sliding-T" dorsal inlay urethroplasty with buccal mucosal graft Lordana Fersovich ${ }^{1}$, Alvaro Saavedra ${ }^{7}$, Keith F. Rourke

${ }^{1}$ Department of Urology, University of Alberta, Edmonton, AB, Canada Introduction: Fossa navicularis strictures are challenging to treat. Endoscopic treatments typically offer temporary relief and require lifelong instrumentation. Reconstruction requires establishing an unobstructed urethra while preserving cosmesis and sexual function. Various urethroplasty techniques exist, including single- and multi-staged approaches. We describe a singlestage urethroplasty for fossa navicularis strictures using a "sliding-T" dorsal inlay technique with buccal mucosa.

Methods: A retrospective review of a prospectively maintained reconstruction database identified patients undergoing urethroplasty of fossa navicu-
}

laris strictures using our technique. The primary outcome was urethroplasty success defined as easy passage of a $16 \mathrm{Fr}$ flexible cystoscope with a minimum of 12 months' followup. Secondary outcomes included 90-day complications, de novo erectile dysfunction, and chordee at six months postoperatively. Comparisons were made using Cox regression, t-test, or chi-squared as appropriate.

Results: From January 2013 to May 2018, 27 patients underwent fossa navicularis reconstruction with the "sliding- $\mathrm{T}$ " technique. Etiologies were lichen sclerosus $(70.4 \%)$, iatrogenic $(18.5 \%)$, or idiopathic $(11.1 \%)$. Mean stricture length was $3.2 \pm 1.2 \mathrm{~cm}$ and mean patient age was 47.3 years (19-71); $92.6 \%$ of patients failed prior endoscopic treatment, $7.4 \%$ failed prior urethroplasty. At a mean followup of 29.6 (12-60) months, the success rate was $92.6 \%$. Both recurrences were treated with meatotomy. Ninety-day complications (Clavien $\geq 2$ ) occurred in two patients $(7.4 \%)$ (treated surgical site infections). Functionally, one patient experienced de novo erectile dysfunction, one reported mild chordee, and $96.3 \%$ of patients reported being satisfied with surgical outcome.

Conclusions: Reconstruction of fossa navicularis strictures using buccal mucosa with a single-stage "sliding-T" dorsal inlay technique provides satisfying anatomic and functional outcomes with a low rate of complications.

\section{MP-2.3}

Augmented anastomotic urethroplasty in the treatment of long bulbar urethral strictures is independently associated with stricture recurrence

Elaine Redmond ${ }^{1}$, Dylan Hoare ${ }^{1}$, Nathan Hoy ${ }^{1}$, Keith F. Rourke ${ }^{1}$ Department of Urology, University of Alberta, Edmonton, AB, Canada Introduction: Controversy exists regarding the optimal urethroplasty technique for bulbar urethral strictures requiring buccal mucosal graft (BMG). Augmented anastomotic urethroplasty (AAU) involves partial stricture excision to remove the most fibrotic portion, with subsequent onlay of the remaining stricture. However, this technique may risk ischemic recurrence through transection of the urethra. "Pure" dorsal onlay (DO) grafting avoids transection of the urethra but may suboptimally treat obliterative segments at risk of recurrence. The aim of our study was to assess the relative outcomes of AAU vs. DO in the setting of bulbar urethroplasty requiring buccal mucosal graft.

Methods: A retrospective review was performed on all patients who underwent urethroplasty with BMG for long $(2-10 \mathrm{~cm})$ bulbar strictures between October 2003 and March 2019. In approximately 2011, institutional technique shifted from routinely performing a transecting augmented anastomotic urethroplasty with dorsal BMG to a non-transecting DO. Exclusion criteria included anastomotic urethroplasty without BMG, ventral onlay, staged, flap, or circumferential reconstructions. Patients were assessed with routine cystoscopy at six months postoperatively and symptomatically thereafter. Recurrence was defined as stricture $<16 \mathrm{Fr}$ on cystoscopy. Those who failed to attend for cystoscopy were excluded from analysis. Secondary outcomes included 90-day complications. Multivariate Cox regression analysis was performed to assess the impact of technique and other relevant clinical factors on stricture recurrence.

Results: Of the 836 patients who underwent bulbar urethroplasty during the study period, 507 met inclusion criteria. Of these, 221 patients received an AAU with dorsal BMG, while 286 underwent DO with BMG. Mean patient age and stricture length was $45.4 \pm 14.8$ years and $4.4 \pm 1.5 \mathrm{~cm}$, respectively. Overall success rate was $93.9 \%$, with a mean followup of 78.9 (5-189) months. On multivariate Cox regression analysis AAU (hazard ratio [HR] 
$4.8 ; 95 \%$ confidence interval $[\mathrm{CI}] 1.8-13.3 ; \mathrm{p}=0.002)$, increasing stricture length ( $\mathrm{HR} 1.2 ; 95 \% \mathrm{Cl} 1.1-1.4 ; \mathrm{p}=0.002)$, and iatrogenic strictures (HR $3.2 ; 95 \% \mathrm{Cl} 1.1-9.1 ; p=0.03$ ) were independently associated with stricture recurrence, while comorbidity $(p=0.06)$, prior endoscopic treatment $(p=0.41)$, prior urethroplasty $(p=0.89)$, and other etiologies were not. There was no significant difference between the cohorts with respect to 90-day complications (Clavien $\geq 2)(3.6 \%$ vs. $4.2 \%$; $p=0.74)$.

Conclusions: AAU is independently associated with stricture recurrence when compared to a pure DO technique. This may be related to urethral ischemia. We recommend that AAU should be reserved for longer strictures where DO is precluded due to areas of complete luminal obliteration.

\section{MP-2.4}

Improvement of storage-associated lower urinary tract symptoms following urethroplasty: A prospective, single-cohort study Dylan Hoare ${ }^{7}$, Jordan Bekkema', Keith F. Rourke

'Division of Urology, University of Alberta, Edmonton, AB, Canada Introduction: Storage symptoms tend to induce the greatest degree of bother in patients with lower urinary tract symptoms (LUTS). When associated with lower urinary tract obstruction, such as urethral stricture, it is unclear how these specific symptoms respond to surgery. This study aims to evaluate the prevalence and effect of urethroplasty on patientreported storage LUTS.

Methods: This was a prospective, single-cohort, descriptive study evaluating patients undergoing urethroplasty for urethral stricture from 2012 2019. Patients were administered the International Prostate Symptom Score (IPSS) preoperatively and six months post-urethroplasty. Outcome measures included the frequency, urgency, and nocturia domains of the IPSS and a combined score of the aforementioned symptoms. Symptoms were considered clinically significant if they were graded $\geq 3$ for the individual domains and $>6$ for the combined score. Significant change was defined as $\mathrm{a} \geq 2$ points on the combined scale. Survey results were compared using the Wilcoxon-signed rank test. Binary logistic regression was used to assess the association between clinical factors and non-responders.

Results: A total of 387 patients completed IPSS surveys pre- and postoperatively. Mean age was 50 years, with an average stricture length of $4.5 \mathrm{~cm}$. Cystoscopic success at six months was $96 \%$. Preoperatively, urinary frequency $(53 \%)$, urgency $(49 \%)$, and nocturia $(42 \%)$ were common symptoms. Postoperatively, these rates decreased to $12 \%, 11 \%$, and $11 \%$, respectively $(p<0.0001)$. Likewise, overall storage symptom scores improved (median 8 vs. 2 ; $\mathrm{p}<0.0001$ ) with $75.2 \%$ of patients reporting significant improvement in storage LUTS. On binary logistic regression, no factor was associated with a lack of response (stricture recurrence, age, stricture length, etc.).

Conclusions: Urethroplasty yields clinically and statistically significant improvements in storage LUTS in men with urethral strictures.

\footnotetext{
MP-2.5

Urinary symptom non-response ("LUTS failure") after urethroplasty: Incidence and associations

David W. Chapman' , Jordan Bekkema', Keith F. Rourke

${ }^{1}$ Urology, University of Alberta, Edmonton, AB, Canada

Introduction: Urethroplasty is a highly effective treatment for recurrent urethral stricture. However, some patients fail to experience significant improvement in lower urinary tract symptoms (LUTS) despite being stricture-free. We aim to identify the incidence of "LUTS failure" after urethroplasty and examine associated factors.

Methods: Patients undergoing urethroplasty over a six-year period (January 2012 to December 2018) were offered enrollment in a study examining urinary function after urethroplasty. Urinary function and urinary quality of life (UQOL) were assessed preoperatively and at six months postoperatively using the international prostate symptoms score (IPSS). "LUTS failure" was defined as less than three-point improvement in IPSS. Stricture recurrence was defined as the inability to pass a $16 \mathrm{Fr}$ flexible cystoscope. Patients with incomplete data were excluded. Descriptive statistics were used to summarize findings while multivariate binary logistic regression was used to determine the association between clinical factors and LUTS failure.
}

Results: Of the 828 patients undergoing urethroplasty over this period, 387 patients completed IPSS scores pre- and postoperatively and of these $372(96.1 \%)$ were stricture free on cystoscopy at six months. Mean patient age was 49.5 years, mean stricture length was $4.3 \mathrm{~cm}$, and $84.4 \%$ of patients failed prior endoscopic treatment. Stricture location was most commonly bulbar $(60.2 \%)$, penile $(19.6 \%)$, posterior $(13.7 \%)$, and pan-urethral (6.5\%). Techniques included buccal mucosa graft onlay $(50.5 \%)$, anastomotic $(31.7 \%)$ or staged $(12.4 \%)$ reconstruction. Mean IPSS (19.0 vs. $5.4 ; p<0.0001)$ and median UQOL (5 vs. $1 ; p<0.0001)$ were improved postoperatively. Despite being stricture-free on cystoscopy, $13.7 \%$ of patients did not report improvement in IPSS (LUTS failure) and $10.2 \%$ did not report improvement in UQOL. On multivariate binary logistic regression, increasing patient age (odds ratio [OR] 1.04; 95\% confidence interval $[\mathrm{Cl}] 1.01-1.06$; $\mathrm{p}=0.006)$, lichen sclerosus (OR 7.6; $95 \% \mathrm{Cl} 1.1-51.0 ; \mathrm{p}=0.04$ ), and hypospadias strictures (OR $8.3 ; 95 \%$ Cl 1.3-54.0; $p=0.03$ ) were associated with LUTS failure, while stricture location $(p=0.46)$, length $(p=0.07)$, previous urethroplasty $(p=0.86)$, prior endoscopic treatment $(p=0.50)$, type of urethroplasty $(p=0.17)$, and other urethroplasties did not. Increasing patient age was also the only factor associated with a lack of improvement in UQOL (OR 1.03; $95 \% \mathrm{CI}$ $1.01-1.06 ; p=0.02$ ).

Conclusions: While the majority of patients experience improvement in lower urinary tract function after urethroplasty, 13.7\% experience "LUTS failure" despite being stricture-free on cystoscopy, and 10.2\% report no improvement in UQOL. Both occurrences are independently associated with increasing patient age and may be related to concurrent benign prostatic hyperplasia or alternately detrusor dysfunction related to longstanding bladder outlet obstruction.

\section{MP-2.6}

Two-year outcomes of a paclitaxel-coated balloon for treatment of male bulbar urethral stricture

Sean Elliott ${ }^{1}$, Ramón Virasoro ${ }^{2}$, Jessica DeLong ${ }^{2}$, Rafael Estrella ${ }^{3}$, Merycarla Pichardo ${ }^{4}$, Ramón Rodriguez Lay ${ }^{5}$, Gustavo Espino ${ }^{6}$, George Suarez $^{3}$, George Webster ${ }^{7}$, Gerald Jordan², Mélanie Aube-Peterkin ${ }^{8}$

1 Urology, University of Minnesota, Minneapolis, MN, United States; ${ }^{2}$ Urology, Eastern Virginia Medical School, Norfolk, VA, United States; ${ }^{3}$ Urology, Baptist Hospital, Miami, FL, United States; ${ }^{4}$ Urology, Hospital General de la Plaza de la Salud, Santo Domingo, Dominican Republic; ${ }^{5}$ Urology, Royal Center, Panama City, Panama; ${ }^{6}$ Urology, Centro Especializado San Fernando, Panama City, Panama; ${ }^{7}$ Urology, Duke University Medical Center, Durham, NC, United States; ${ }^{8}$ Urology, McGill University Health Centre, Montreal, QC, Canada

Introduction: We report two-year outcomes of ROBUST I, a multicenter, single-arm, open-label study on the safety and efficacy of a paclitaxel drug-coated balloon (DCB) for treatment of urethral stricture.

Methods: Men with bulbar urethral strictures of $2 \mathrm{~cm}$ with 1-3 prior endoscopic treatments were enrolled at four study sites in the Dominican Republic and Panama following ethics committee approvals. The DCB was inflated under cystoscopic visualization and placement confirmed via fluoroscopy. International Prostate Symptom Score (IPSS) was documented at three, six, 12, and 24 months. Cystoscopy was documented at six and 12 months. Primary efficacy endpoint was improvement in IPSS and primary safety endpoint was serious complications through three months. We also report updated results of our composite definition of success: 1) no retreatment; 2) no recurrence on cystoscopy; and 3) IPSS 11 at last followup. For the two-year outcomes data, men were considered successful if they had a normal cystoscopy at 12 months and IPSS remained 11 at two years. If IPSS was $>11$ at two years, men were considered a failure and cystoscopy was not repeated inside the study protocol.

Results: A total of 53 subjects were enrolled and all were successfully treated. Average subject age was 51 years (range 22-81). Average number of prior treatments was 1.8 per subject (range $1-4$ ). There were no serious or unexpected device related adverse events. Mean IPSS decreased from $25.2 \pm 4.5$ (baseline) to $7.3 \pm 8.10(\mathrm{p}<0.001)$ in 41 men with 24 -month data. Two-year success based on the composite definition was 30/45 $(67 \%)$ compared to $32 / 46(70 \%)$ at one year. Failures in year 2 included 
two men with a new increase in IPSS $>11$; censors in year 2 included one man lost to followup. No man was retreated in year 2 .

Conclusions: Two-year data indicate that in men with short bulbar urethral stricture refractory to standard endoscopic management, the paclitaxel DCB is safe and produces a durable improvement in IPSS.

\section{MP-2.7 Lower urinary tract reconstruction after radiation therapy for pelvic cancer \\ Sender Herschorn ${ }^{1}$, Geneviève Nadeau ${ }^{2}$}

${ }^{1}$ Urology, University of Toronto, Toronto, ON, Canada; ${ }^{2}$ Urology, $\mathrm{CHU}$ de Québec - Université Laval, Quebec City, QC, Canada Support: University of Toronto Functional Urology Research

Introduction: Pelvic radiation therapy (RT) for malignancies may compromise the lower urinary tract (LUT). Reconstruction can be quite challenging, especially when combined with surgical extirpation. As an alternative to cystectomy, enterocystoplasty with or without ureteral re-implantation or continent stoma creation brings the potential benefit of preserving the LUT. Methods: The records of patients treated between 1994 and 2019 who underwent LUT reconstruction after pelvic RT were reviewed. Complications and functional results were recorded.

Results: Thirty-one patients (15 women, 16 men) who had received RT for advanced or recurrent pelvic cancer were identified. Ten patients underwent reconstruction as part of primary tumor excision after chemo-rads (group 1) and 21 had surgery due to radiation complications (group 2). Mean age at surgery was 58.4 years (range 33-76). All patients underwent enterocystoplasty and $19(61 \%)$ of these had simultaneous ureteral re-implants either into an intestinal limb (12) or into the bladder (7). Re-implants were done for obstruction due to cancer or post-RT. Eight patients had continent abdominal stomas performed with the augmentation due to devastated urethras. After a mean of 49 months (range 2-170), the re-operation rate was $30 \%$ and was similar in both groups. Nine patients had re-operations; stoma problems (4), bladder stones (3), and vesicovaginal fistula (1). One anastomotic leak was treated with diversion. Two developed ureteral anastomotic strictures that responded to temporary stents. No renal deterioration was seen. Seventeen of 24 non-stoma patients void spontaneously, two do intermittent catheterization (IC), and two have Foley catheters. All eight stoma patients do IC. Urinary incontinence was noted in five patients. Twenty-eight of 31 patients considered their reconstruction successful.

Conclusions: Prior pelvic RT is not a contraindication to enterocystoplasty with or without ureteral reimplantation or continent abdominal stoma. Satisfactory functional outcomes and acceptable morbidity can be achieved.

\section{MP-2.8}

Urethrovaginal fistula repair with or without concurrent fascial sling placement

\section{Sarah R. Ferrara' Jennifer A. Locke' Sender Herschorn}

${ }^{1}$ Urology, Sunnybrook Health Sciences Centre, University of Toronto, Toronto, ON, Canada

Introduction: Urethrovaginal fistula (UVF), a rare diagnosis in the developed world, is most often due to iatrogenic causes. We reviewed our 29-year experience on the management and outcomes of UVF repair, with or without concomitant fascial sling placement.

Methods: All patients diagnosed with UVF at our center from 1988-2017 were included in this study through a prospectively kept database. Chisquare or Fisher's exact test were used to compare postoperative stress urinary incontinence (SUI) rates and complication rates between patients with or without fascial sling placement at the time of UVF repair.

Results: We identified 41 cases of UVF, 40 of whom underwent surgical repair. Mean age at diagnosis was $47.8(21-81)$. All patients had undergone pelvic surgery; 20 patients $(48.8 \%)$ having had SUI surgery. Etiology of UVF was secondary to SUI surgery in 16 patients $(39 \%)$ and urethral diverticulum repair in eight patients (19.5\%). The most com- mon presenting symptom was continuous incontinence in 19 patients $(46.3 \%)$. Nineteen patients had a fascial sling placed at the time of surgery $(47.5 \%)$. Concomitant sling placement was associated with a statistically significant decrease in postoperative SUI $(10.5 \%$ vs. $38.1 \%$; $p=0.0481)$ and no significant difference in postoperative complication rates $(26.3 \%$ vs. $23.8 \%$; $\mathrm{p}=0.855$ ). Two patients had Clavien-Dindo grade I complications (5\%) and two patients had grade III complications (5\%). Four patients had long-term complications $(10 \%)$, including urinary retention, chronic pain, and urethral stricture. Two patients had UVF recurrence (5\%). Mean followup after surgery was 3.44 years $(0.11-17.04)$.

Conclusions: Although UVF is rare, it should be suspected in patients with continuous incontinence following a surgical procedure, and patients should be counselled on the risk of UVF associated with SUI procedures. Most UVF surgical repairs are successful, with or without simultaneous fascial sling placement.

\section{MP-2.9}

Management of female vesicovaginal fistula Canadian tertiary center experience

Samer Shamout ${ }^{1}$, Richard J. Baverstock', Kevin V. Carlson ${ }^{1}$

'Department of Surgery, Division of Urology, University of Calgary, Calgary, AB, Canada

Introduction: Surgical repairs of vesicovaginal fistula (VVF) are most commonly performed vaginally, abdominally, or laparoscopically. Treatment approach from either abdominal or vaginal is often dictated by complexity of the fistula and surgeons' preference. The study aims to present the outcomes of all patients referred with vesico-vaginal fistulae to a tertiary center, and to investigate the patient, fistula, and surgical factors relevant to success.

Methods: This is a retrospective study analyzing the database registry of 63 consecutive patients who underwent vesicovaginal fistula repair between 2005 and 2019. Participants underwent clinical evaluation, including demographic, fistula profile, and surgical approach (vaginal or abdominal). Operative data, postoperative outcome, and followup were recorded. Two surgical groups were compared, including abdominal and vaginal repair.

Results: A total of 63 women with a mean age of $47.7( \pm 10.7)$ years and mean body mass index of $28.8 \mathrm{~kg} / \mathrm{m}^{2}( \pm 7.27)$ were included in the study. The etiology of fistula was secondary to malignancies in six $(9.5 \%)$ patients and gynecological/obstetric procedure related in $57(90.4 \%)$ patients. Thirteen $(20.6 \%)$ patients had prior repair and $50(79.3 \%)$ were naive. The mean period from onset of leakage to time of repair was 44.47 $( \pm 85.1)$ days. Forty-two patients had transvaginal repair, whereas $21 \mathrm{VVF}$ had abdominal repair. The two groups did not show a significant difference in demographics (Table 1). Transvaginal approach had a significant shorter operative time, less intraoperative blood loss, and reduced postoperative hospital stay $(p<0.005)$. Transabdominal repair was associated with increased complications after surgery $(p<0.05)$. Length of operative time and estimated blood loss were positively and significantly correlated with age $(r=0.392 ; p=0.005$ and $r=0.394, p=0.002$, respectively) and time to surgery $(r=0.0386 ; p=0.01$ and $r=0.416 ; p=0.002$, respectively). The success rates of transvaginal and abdominal techniques were $97.6 \%$ and $85.7 \%$ respectively.

Conclusions: Transvaginal repair of VVF is a technically feasible approach with a high success rate and low morbidity. Despite varied etiology and different surgical approach, age, and time of VVF repair are significant determinants of operative complexity. 


\begin{tabular}{|c|c|c|c|}
\hline \multicolumn{4}{|c|}{$\begin{array}{l}\text { MP-2.9. Table 1. Demographic and surgery characteristics } \\
\text { for the total study population and the transabdominal and } \\
\text { transvaginal approaches }\end{array}$} \\
\hline & $\begin{array}{l}\text { VVF } \\
(n=63)\end{array}$ & $\begin{array}{l}\text { TA } \\
\text { approach } \\
(\mathbf{n}=21)\end{array}$ & $\begin{array}{l}\text { TV } \\
\text { approach } \\
(\mathrm{n}=42)\end{array}$ \\
\hline \multicolumn{4}{|l|}{$\begin{array}{l}\text { Demographic } \\
\text { characteristics }^{\text {a }}\end{array}$} \\
\hline Age, year $($ mean $\pm S D)$ & $47.7(10.7)$ & $49.1(13.3)$ & $47.1(9.4)$ \\
\hline BMI (Mean \pm SD) & $28.8(7.27)$ & $25.5(10.2)$ & $29.4(7.1)$ \\
\hline Cigarette smoking status & $9(14.3 \%)$ & $1(4.8 \%)$ & $8(19 \%)$ \\
\hline \multicolumn{4}{|l|}{ VVF etiology } \\
\hline Malignancies & & & $4(9.5 \%)$ \\
\hline Gynecological procedures & $56(88.9 \%)$ & $15(71.4 \%)$ & $41(97.6 \%)$ \\
\hline Obstetric & $1(1.6 \%)$ & $1(4.7 \%)$ & 0 \\
\hline Previous fistula repair & $13(20.6)$ & $3(14.3 \%)$ & $10(23.8 \%)$ \\
\hline Timing of repair (days) & $\begin{array}{l}44.47 \\
(85.1)\end{array}$ & $\begin{array}{c}56.9 \\
(115.2)\end{array}$ & $38.9(68.7)$ \\
\hline $\begin{array}{l}\text { Comorbidities (frequency, } \\
\% \text { ) }\end{array}$ & $24(38.1 \%)$ & $10(47.6 \%)$ & $14(33.3 \%)$ \\
\hline \multicolumn{4}{|l|}{ Surgery characteristics } \\
\hline Operative time $(\min )^{*}$ & $\begin{array}{l}134.94 \\
(87.7)\end{array}$ & $\begin{array}{c}185.2 \\
(122.7)\end{array}$ & $\begin{array}{c}108.2 \\
(44.95)\end{array}$ \\
\hline $\begin{array}{l}\text { Estimated blood loss } \\
(\mathrm{ml})^{* *}\end{array}$ & $\begin{array}{c}160.1 \\
(200.7)\end{array}$ & $\begin{array}{l}343.75 \\
(261.3)\end{array}$ & $\begin{array}{c}88.4 \\
(109.56)\end{array}$ \\
\hline Length of stay (days) ${ }^{* *}$ & $4.2(5.6)$ & $8.7(7.7)$ & $2(2.18)$ \\
\hline Duration of leakage (days) & $\begin{array}{c}300.9 \\
(686.8)\end{array}$ & $391.8(962)$ & $\begin{array}{c}257.9 \\
(520.89)\end{array}$ \\
\hline \multicolumn{4}{|c|}{$\begin{array}{l}\text { "No significant differences were found between transabdominal and transvaginal } \\
\text { approaches patients' demographics using independent } t \text {-test or chi square test. * } p<0.05 \text {; } \\
*^{* * p}<0.005 \text { indicating a significant difference between TA and TV approaches (Mann- } \\
\text { Whitney test); }{ }^{p} p<0.05 \text { indicating a significant difference between the two approaches } \\
\text { (chi-squared test). }\end{array}$} \\
\hline
\end{tabular}

\section{MP-2.10}

Comparing outcomes of uretero-neocystotomy and ureteroureterostomy for iatrogenic ureteric injuries in an institutional dataset

Lennifer A. Locke ${ }^{1}$, Roshan Navaratnam ${ }^{1}$, Sarah R. Ferrara ${ }^{1}$, Sender Herschorn

${ }^{1}$ Urology, Sunnybrook Health Sciences Centre, Toronto, ON, Canada Introduction: latrogenic ureteric injury (IUI) is a rare but significant complication of surgery. Ureteral re-implant (UR) has been the treatment of choice for IUI near the pelvic brim because it is thought that the distal ureter has a precarious vascular supply. ${ }^{1-3}$ We hypothesize that ureteroureterostomy $(U U)$ is an equivalent method for distal IUIs. Our objective was to retrospectively compare the outcomes of UR and UU for IUI in an institutional dataset.

Methods: After REB approval, a chart review of surgical outcomes was conducted on patients with a diagnosis of IUI as identified through an institutional medical record system from 2002-2019. Chi-squared statistical analysis was performed.

Results: There were 114 patients with a total of 120 IUIs identified at our institution. The majority were repaired by UR $(74,62 \%)$, followed by UU $(31,26 \%)$, primary repair (six, 5\%), no intervention (three, $3 \%$ ), and ureteric stent (two, $2 \%$ ). As anticipated, the majority of distal and midureteric injuries were treated with UR $(61 / 76 ; 80 \%)$ and UU $(22 / 37 ; 59 \%)$, respectively. For all IUIs the incidence of subsequent ureteric stricture and hydronephrosis were $8 \%$ and $24 \%$, respectively. There were no statistically significant differences between UR and UU for ureteric stricture

\begin{tabular}{|c|c|c|c|}
\hline & $\begin{array}{l}\text { VVF } \\
(n=63)\end{array}$ & $\begin{array}{c}\text { TA } \\
\text { approach } \\
(\mathbf{n}=21)\end{array}$ & $\begin{array}{c}\text { TV } \\
\text { approach } \\
(n=42)\end{array}$ \\
\hline \multicolumn{4}{|l|}{ Fistula site } \\
\hline Base & $9(14.3 \%)$ & $6(28.6 \%)$ & $3(7 \%)$ \\
\hline Posterior wall & $7(11.1 \%)$ & $4(19.1 \%)$ & $3(7 \%)$ \\
\hline Around trigone & $34(54 \%)$ & $4(19.1 \%)$ & $30(71.4 \%)$ \\
\hline Dome & $1(1.6 \%)$ & $1(4.8 \%)$ & 0 \\
\hline Midline & $7(11.1 \%)$ & $1(4.85)$ & $6(14.3 \%)$ \\
\hline \multicolumn{4}{|l|}{ Fistula size $(\mathrm{cm})$} \\
\hline$<1.5$ (small) & $22(34.9 \%)$ & $5(23.8 \%)$ & $17(40.5 \%)$ \\
\hline 1.5-3 (medium) & $27(42.9 \%)$ & $5(23.8 \%)$ & $22(52.4 \%)$ \\
\hline$>3$ (large) & $11(17.5 \%)$ & $8(38.1 \%)$ & $3(7 \%)$ \\
\hline \multicolumn{4}{|l|}{ Tissue interposition } \\
\hline Martius flap & $7(11.1 \%)$ & 0 & $7(16.7 \%)$ \\
\hline Peritoneal & $10(15.9 \%)$ & $1(4.8 \%)$ & $9(21.4 \%)$ \\
\hline Peritoneal fat & 27 (42.9\%) & $2(9.5 \%)$ & $25(59.5 \%)$ \\
\hline Omentum & $10(15.9 \%)$ & $10(47.6 \%)$ & 0 \\
\hline None & $5(7.9 \%)$ & $4(19.1 \%)$ & $1(2.4 \%)$ \\
\hline Fistula tract excision & 15 (23.8\%) & $15(71.4 \%)$ & 0 \\
\hline Concomitant surgery & $24(38.1 \%)$ & $8(38.1 \%)$ & $16(38.1 \%)$ \\
\hline Suprapubic tube ${ }^{\#}$ & $16(25.4 \%)$ & $12(57.1)$ & $4(9.5 \%)$ \\
\hline $\begin{array}{l}\text { Intraoperative } \\
\text { complication" }\end{array}$ & $6(9.5 \%)$ & $5(23.8 \%)$ & $1(2.4 \%)$ \\
\hline $\begin{array}{l}\text { Early postoperative } \\
\text { complications }^{\#}\end{array}$ & $5(7.9 \%)$ & $5(23.8 \%)$ & 0 \\
\hline $\begin{array}{l}\text { Late postoperative } \\
\text { complications }{ }^{\#}\end{array}$ & $7(11.1 \%)$ & $5(23.8 \%)$ & $2(4.8 \%)$ \\
\hline Success rate $(\%)$ & $59(93.6 \%)$ & $18(85.7 \%)$ & $41(97.6 \%)$ \\
\hline
\end{tabular}

${ }^{a}$ No significant differences were found between transabdominal and transvaginal approaches patients' demographics using independent t-test or chi square test. ${ }^{*} \mathrm{p}<0.05$;

${ }^{* *} \mathrm{p}<0.005$ indicating a significant difference between TA and TV approaches (MannWhitney test); $\# p<0.05$ indicating a significant difference between the two approaches (chi-squared test).

and hydronephrosis, regardless of location of injury. For all IUIs, injury location (mid) and previous radiation were predictors for ureteric stricture $(p=0.002)$ and hydronephrosis $(p=0.029)$, respectively. Sex, timing of diagnosis, original surgery type, and previous surgery did not predict for these outcomes.

Conclusions: There was no difference in outcomes between UR and UU, regardless of location of injury. Therefore, $U U$ is a reasonable alternative to UR for IUI near the pelvic brim.

References

1. Watterson JD. CIS 1998.

2. Assimos D. J Urol 1994.

3. Gill H. AUA Update 1994. 


\section{MP-2.11}

Patient perspectives on cannabinoids for interstitial cystitis/ bladder pain syndrome

Kate Anderson ${ }^{1}$, Danielle Jenkins ${ }^{2}$, Mary E. Lynch ${ }^{3}$, Ashley R. Cox ${ }^{1}$ ${ }^{1}$ Urology, Dalhousie University, Halifax, NS, Canada; ${ }^{2}$ Urology, Queen's University, Kingston, ON, Canada; ${ }^{3}$ Anesthesia, Pain Management \& Perioperative Medicine, Dalhousie University, Halifax, NS, Canada Introduction: Interstitial cystitis/bladder pain syndrome (IC/BPS) is a chronic condition often causing a significant impact on quality of life. We sought to: determine if patients with IC/BPS use cannabis products for symptoms of IC/BPS, elicit perceptions of patients with IC/BPS regarding use of cannabis products, and determine if higher Pain and Urgency/Frequency Symptom Scale (PUF) scores correlate to use of cannabinoid products.

Methods: We conducted a cross-sectional survey study of IC/BPS patients at our center. Patients were asked to complete two anonymous surveys: 1) a demographics and perceptions of cannabis survey; and 2) PUF symptom scale. Descriptive statistics were used to characterize the survey responses. Results: Of the 97 patients who participated, $80 \%$ completed both surveys. Average age was $49 \pm 16.5$ years; $95 \%$ of respondents were female. Median duration of IC/BPS symptoms was nine years. Reported cannabis use was $58 \%(56 / 97)$ and $82 \%(37 / 45)$ found it to be at least slightly effective for symptom control. Cannabis use resulted in decrease or discontinuation of their other IC/BPS medications for $17 \%$ of respondents. Most patients reported no negative side effects from cannabis use $(60 \%)$. Moderate to severe side effects were reported by $12.5 \%$ of patients. The three most common modalities used were: smoked, oils, edibles. The mean total PUF score was 20.4 \pm 7.4 . There was no correlation found between cannabis use and total PUF score $(r=0.041, n=78, p=0.724)$ We found no statistically significant difference in severity of PUF total score for patients who had used cannabis within the last six months and those who had not.

Conclusions: Use of cannabis products by patients with IC/BPS is common. Respondents who used cannabis products largely perceived them to be beneficial for their symptoms with minimal side effects. Further research should be directed towards determining if there is clinical utility for cannabis as a therapy for patients with IC/BPS.

\section{MP-2.12}

Triamcinolone acetate injections as treatment for Hunner's lesions in bladder pain syndrome: Reducing pain treatment regimens Sarah R. Ferrara ${ }^{1}$, Jennifer A. Locke', Lesley Carr', Sender Herschorn ${ }^{1}$ 'Urology, Sunnybrook Health Sciences Centre, University of Toronto, Toronto, ON, Canada

Introduction: Bladder pain syndrome (BPS) is a difficult-to-manage, chronic pain syndrome often requiring multiple treatments. A subgroup of patients with BPS have Hunner's lesions - inflammatory lesions of the bladder wall. We present our experience with triamcinolone injections into Hunner's lesions for BPS management.

Methods: This is a retrospective analysis of 25 patients receiving triamcinolone injections into Hunner's lesions identified from 2010-2018. The lesions were injected endoscopically with $0.5 \mathrm{cc}$ injections of triamcinolone acetate $(40 \mathrm{mg} / \mathrm{ml}$, max $10 \mathrm{~mL})$. Pain resolution and change in pain regimens were recorded at followup. Wilcoxon signed-rank test was used to compare pain regimens before and after injections.

Results: Patients used a median of two pain treatments for BPS prior to triamcinolone injection (interquartile range [IQR] 1-4). The most common treatment was pentosan polysulfate $(54.2 \%)$, and $28 \%$ of patients were using opioids for BPS. The median age at first triamcinolone injection was 69 (IQR 56-74), and the median number of injections/patient was three (IQR 2-6). Median time between injections was 6.5 months (IQR 5-10). With a mean followup time of 3.3 years (4.5-99.6 months), all patients had improvement in pain symptoms with one or more injections. A durable effect of at least two months was seen after $85.2 \%$ of injections. Two patients had complete resolution of lesions. There was a significant decrease in the number of pain treatments required following triamcinolone injections ( 2.68 vs. $1.04 ; \mathrm{p}=0.0015)$. Fifty-two percent managed with triamcinolone injections alone, with no other pain treatments. Of the seven patients using opioids, four discontinued opioids, while one decreased to as-needed use. One patient on warfarin had gross hematuria following the procedure and required overnight admission; no other complications were found.

Conclusions: Injection of triamcinolone into Hunner's lesions as a treatment for BPS is safe and effective and decreases the need for other pain treatments.

\section{MP-2.13}

Treating the lesion in Hunner's lesion interstitial cystitis/bladder pain syndrome (HL-IC/BPS): A call to arms

R. Christopher Doiron ${ }^{1}$, Kerri-Lynn Kelly', Brad Dean ${ }^{1}$, J. Curtis Nickel

${ }^{1}$ Urology, Queen's University, Kingston, ON, Canada

Introduction: Hunner's lesion interstitial cystitis/bladder pain syndrome (HL-IC/BPS), a distinct subtype of IC/BPS only identified by cystoscopy, can be treated with transurethral resection, fulguration, and/or injection. We queried our large database to examine patterns and clinical outcomes in directed lesion treatment in HL-IC/BPS.

Methods: HL-IC/BPS subjects were retrospectively identified from a prospectively maintained IC/BPS database from 2006-2019, which included patient demographics, treatments, and ICSI/PI symptom score. Baseline visit was defined as their first visit in our tertiary care clinic while change in objective data was measured at first post-procedure clinic visit.

Results: Of a total of 469 patients, 44 were identified as HL-IC/BPS. Median age of HL-IC/BPS cohort was 70 years old, all were Caucasian, and $25 \%$ were male. Of the 44 patients with HLs, 22 received direct $\mathrm{HL}$ treatment: 15 fulguration only $(\mathrm{F})$, two triamcinolone injection only (T), and five both fulguration and triamcinolone injection (FT) over the course of the study period. Median age of F cohort was 70 years, while T and FT cohorts had median age of 54 years. F cohort was $70 \%$ female, T cohort $100 \%$ female, and the FT cohort $60 \%$ female. Baseline ICSI for $\mathrm{F}, \mathrm{T}$, and FT cohorts were 16,15 , and 17 , respectively, while their respective ICPI scores were 13,12 , and 14 . There was a $73 \%$ clinically significant subjective improvement in the F cohort patients' symptoms, with a median change in ICSI/PI scores of 4 and 3 respectively. The small T cohort subjectively improved but had missing data regarding objective evaluation. The FT cohort experienced a $100 \%$ subjective clinical improvement following both fulguration and triamcinolone treatments. The median improvement in ICSI/PI scores were 7 and 8, respectively.

Conclusions: Our clinical experience indicates that directly treating the lesion in HL-IC/BPS with fulguration and/or triamcinolone injection consistently results in high levels of objective and subjective symptom improvement. 


\section{CUA ABSTRACTS}

\section{Moderated Poster Session 3: Uro-Oncology}

\section{MP-3.1}

PDL1 expression by tumor proportion score in immunohistochemistry on the tumor tissue of urothelial carcinoma locally advanced or metastatic treated by immune checkpoint inhibitors

Ouang Loc Bui ', Ahmed Khalil', Hong Son Trinh'², Carolina Saldana 3 , Aurelien Gobert ${ }^{4}$, Maya Nourieh ${ }^{5}$, Eva Comperat ${ }^{6}$, Olivier Cussenot ${ }^{7}$ ${ }^{1}$ Oncology Medical, Tenon Hospital, Paris, France; ${ }^{2}$ Oncology Service, Viet Duc Hospital, Hanoi, Viet Nam; ${ }^{3}$ Oncology Medical, Henri-Mondor Hospital, Paris, France; ${ }^{4}$ Oncology Medical, Pitié Salpêtrière Hospital, Paris, France; ${ }^{5}$ Pathology, Henri-Mondor Hospital, Paris, France; ${ }^{6}$ Pathology, Tenon Hospital, Paris, France; ${ }^{7}$ Urology, Tenon Hospital, Paris, France

Introduction: The benefit of immune checkpoint inhibitors (ICls) is modest, with a tumor response rate of $23-29 \%$ in locally advance or metastatic urothelial carcinoma. ${ }^{1,2}$ The expression of PD-L1 is perhaps a predictive factor of tumor response of ICls.

Methods: We conducted a non-interventional, retrospective, multicenter study in three university hospitals in Paris. All patients were diagnosed with locally advanced or metastatic urothelial carcinoma and treated by antiPD1 or anti-PDL1 for at least one cycle. We classified all patients into two groups according to RECIST 1.1 criteria: the clinical benefit group, which included stable disease, partial response or complete response; and the progression group. We collected paraffin tissue blocks of these patients. Then we determined the expression of PDL-1 by immunohistochemistry using the tumor proportion score (TPS).

Results: From January 1, 2015 to June 30, 2019, 23 patients were enrolled in our study. For the primary endpoint, the mean PDL1 expression did not differ between the clinical benefit group and the progression group (TPS 32.83\%; 95\% confidence interval [CI] 3.5-67.3 vs. 26.35; 95\% Cl 7.8-48.0, respectively); the difference of PDL1 expression was $6.48 \%(95 \%$ $\mathrm{Cl}-46.2-28.3)$. Concerning the secondary endpoint, overall survival from diagnosis did not differ significantly either in the TPS $\geq 50 \%$ group or in the TPS $<50 \%$ group (hazard ratio [HR] $0.75 ; 95 \% \mathrm{Cl} 0.19-2.99 ; \mathrm{p}=0.69$ ). Patients in the clinical benefit group received ICls earlier compared to the progression disease group, with a significant difference of 1.5 lines $(95 \%$ $\mathrm{Cl} 0.8-2.3$ ). Half (50\%) of the patients received the ICls first-line and $50 \%$ second-line, benefiting the clinical group. On average, the progression disease group received ICls as third-line treatment.

Conclusions: Our results suggest that PDL1 expression is not a predictive factor of tumor response in the locally advanced or metastatic urothelial carcinoma patients treated by ICls.

References

1. Balar AV, Galsky MD, Rosenberg JE, et al. Atezolizumab as first-line treatment in cisplatin-ineligible patients with locally advanced and metastatic urothelial carcinoma: A single-arm, multicenter, phase 2 trial. Lancet 2017 07;389:67-76. https://doi.org/10.1016/S01406736(16)32455-2

2. Powles T, Durán I, van der Heijden MS, et al. Atezolizumab vs. chemotherapy in patients with platinum-treated locally advanced or metastatic urothelial carcinoma (IMvigor211): A multicenter, open-label, phase 3 randomized controlled trial. Lancet 2018 24;391:748-57. https://doi.org/10.1016/S0140-6736(17)33297-X

\section{MP-3.3}

Ureteric stents for malignant ureteric obstruction - assessing the factors associated with encrustation and inadequate drainage: $\mathrm{A}$ cohort study

Shreyas Gandhi' ${ }^{1}$ Alex Koziarz' ${ }^{2}$, Jason Y. Lee ${ }^{7}$

${ }^{1}$ Department of Urology, University of Toronto, Toronto, ON, Canada; ${ }^{2}$ Faculty of Medicine, University of Toronto, Toronto, ON, Canada

Introduction: Malignant ureteric obstruction (MUO) is a challenging clinical entity for urologists. ${ }^{1}$ Currently, no definitive guidelines exist regarding optimal management of $\mathrm{MUO}$, including the required frequency of stent changes..$^{2,3}$ Therefore, we evaluated the association of clinical factors at initial stent implantation with prognosis and stent management in patients with MUO.

Methods: A single-center, retrospective cohort study was conducted among all patients with MUO managed with a ureteral stent from August 2015 to December 2019. A multivariable regression model was used to evaluate patient factors associated with the following primary outcomes: stent encrustation requiring need for stent change, need for antegrade decompression due to inadequate drainage, and time to first stent change.

Results: Fifty patients ( $72 \%$ female) were included with mean age of 63.9 years. Mean followup was one year. The most common indication for stenting was acute kidney injury (46\%), followed by hydronephrosis without creatinine elevation $(42 \%)$, and pain $(6 \%)$. Ten patients $(20 \%)$ required antegrade decompression following first stent check. Median (interquartile range $[I Q R])$ time to first stent change was 91 (84-104) days. History of bowel resection $(p=0.018)$ and diabetes mellitus $(p<0.00001)$ was associated with increased frequency of stent change when controlling for stent location, creatinine at stent insertion, and indication. Initial ureteric obstruction at the mid-ureter was associated with fewer number of stent changes $(p=0.035)$. There was no association of stent location, creatinine at stent insertion, indication, history bowel resection, or diabetes mellitus with risk for future antegrade decompression, risk for encrustation at first check, or time to first change.

Conclusions: History of bowel resection and diabetes mellitus is associated with greater number of stent changes due to encrustation. Patients with this history may require more frequent stent surveillance, as encrustation rates may be higher.

\section{References}

1. O'Connor E, Nason GJ, Kiely E. Urological management of extramural malignant ureteric obstruction: A survey of Irish urologists. Curr Urol 2017;11:21-5. https://doi.org/10.1159/000447190

2. Sountoulides P, Pardalidis N, Sofikitis N. Endourologic management of malignant ureteral obstruction: Indications, results, and quality of life issues. J Endourol 2010;24:129-42. https://doi.org/10.1089/ end.2009.0157

3. Lang EK, Winer AG, Abbey-Mensah G, et al. Long-term results of metallic stents for malignant ureteral obstruction in advanced cervical malignancy. J Endouro/ 2013;27:646-51. https://doi.org/10.1089/ end.2012.0552 


\section{MP-3.4}

Occupational exposure and risk of genitourinary malignancies: A Canadian population study

Shiva M. Nair ${ }^{1}$, Tina Luu Ly'2, Arnon Lavi' ${ }^{1}$, Michael Haan², Yujiro Sano², Nicholas E. Power ${ }^{1}$

${ }^{1}$ Urology Division, Department of Surgery, Schulich School of Medicine and Dentistry, University of Western Ontario, London, ON, Canada; ${ }^{2}$ Department of Sociology, University of Western Ontario, London, ON, Canada

Support: Canadian Census Health and Environment Cohort

Introduction: Occupational exposure to aromatic hydrocarbons has been linked to urothelial malignancy. Examining occupational exposure in malignancy lays an important foundation for a physician to stratify the risk to individual patients. To this end, our analysis focuses on explaining the industrial effects on three forms of urological cancer: prostate, bladder, and kidney.

Methods: Data were obtained from a population-based, linked dataset, Canadian Census Health and Environment Cohort. Approximately 2.7 million people aged 25 or older who responded to the 1991 long-form Census questionnaire were linked with the Canadian Cancer Registry. Inclusion criteria included diagnosis of a urological cancer between 1991 and 2010. Individuals' industry of work was identified on the 1991 Census. Cox proportional hazards models were used to predict incidences of cancer with the agricultural industry as a reference point.

Results: Prostate cancer incidence was 36350 men. Univariate analysis found an increased incidence of prostate cancer in men with greater income (hazard ratio $[H R] 1.05 ; 1.04-1.06 ; p<0.0001$ ) and level of education (university vs. no high school, HR $1.14 ; 1.10-1.19 ; \mathrm{p}<0.0001$ ). Compared to agriculture, a number of the industries had lower risk of prostate cancer diagnosis, including accommodation and food (HR 0.77 ; $0.70-0.84 ; \mathrm{p}<0.0001)$. Bladder cancer was diagnosed in 7125 men and 1595 women. When compared to agriculture, real estate industry had higher risk for both sexes (men: HR 1.34; 95\% Cl 1.16-1.62; $\mathrm{p}<0.01$; women: HR $1.63 ; 1.12-2.37 ; \mathrm{p}<0.05)$. Renal cancer incidence was 4480 for men and 1860 for women. Health and social service had increased risk for both men (HR 1.30; $1.04-1.62 ; \mathrm{p}<0.05)$ and women (HR 1.36; $1.01-1.83 ; \mathrm{p}<0.05)$.

Conclusions: Prostate cancer diagnosis was greatest in agriculture industry, likely due to better perception of health by the primary care physician. Effects of real estate industry and health and social service were seen consistently for both sexes in bladder and renal cancer, respectively.

\section{MP-3.6}

99mTc-sestamibi SPECT/CT imaging for the risk stratification of renal masses: A preliminary study

Golmehr Sistani ${ }^{1}$, Jennifer Bjazevic ${ }^{2}, Z^{2}$ ahra Kassam ${ }^{1}$, Jonathan Romsa ${ }^{1}$ Stephen E. Pautler ${ }^{2}$

${ }^{1}$ Medical Imaging, Western University, London, ON, Canada; ${ }^{2}$ Surgery, Western University, London, ON, Canada

Introduction: Imaging characteristics of renal cell carcinoma (RCC) and oncocytoma overlap significantly, resulting in diagnostic uncertainty.

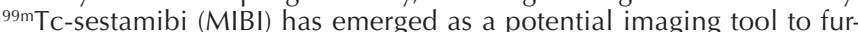
ther characterize renal masses, as it localizes within the mitochondria. This study evaluated the utility of MIBI single photon emission computed tomography/computed tomography (SPECT/CT) imaging in the assessment and risk stratification of indeterminate renal masses.

Methods: Twenty-three patients with indeterminate renal masses on crosssectional imaging underwent MIBI SPECT/CT imaging. MIBI SPECT/CT imaging characteristics, including lesion density and MIBI uptake, were correlated with histopathology from either percutaneous biopsy or surgical resection. Lesions with any degree of MIBI uptake were defined as positive and lesions with no uptake as negative (Fig. 1). Twenty-three lesions with a median size of $3.2 \mathrm{~cm}(1.8-6)$ and density ranging from $22-56$ Hounsfield units were analyzed.

Results: Twenty of the lesions were solid enhancing masses and three were Bosniak 4 cysts with measurable solid components. Histopathology demonstrated five oncocytomas, one hybrid oncocytic/chromophobe

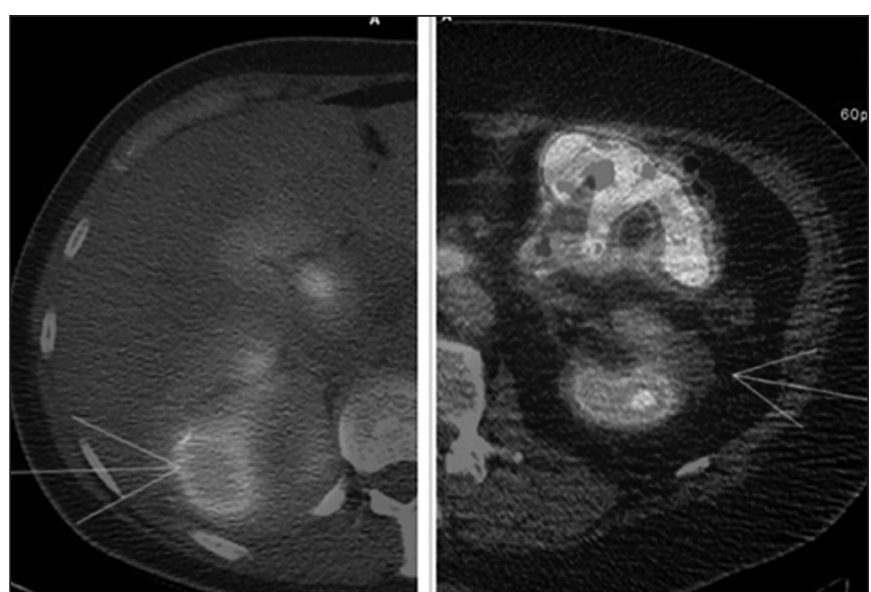

MP-3.6. Fig. 1. Appearance of oncocytoma (right) and RCC (left) on ${ }^{99 \mathrm{mTC}-}$ sestamibi SPECT/CT.

tumor (HOCT), and 17 RCCs, including 13 clear-cell, two papillary, and one chromophobe subtype. The median of mean and maximum relative tumor uptake (relative to ipsilateral renal parenchyma) in MIBI-positive tumors (oncocytomas and HOCT) was 0.80 and 0.85 , compared to 0.30 and 0.30 with RCC, respectively. Using a mean relative tumor uptake of 0.5 , all patients with oncocytoma and HOCT $(100 \%)$ had positive MIBI uptake, and all RCC patients $(0 \%)$ were negative.

Conclusions: This study demonstrates that the combination of presence or absence of MIBI uptake and lesions' density on SPECT/CT represents a novel imaging approach to risk-stratifying incompletely characterized renal masses. Further validation of this technique may reduce the need for further imaging and unnecessary biopsy or surgical resection of indeterminate renal masses.

\section{MP-3.8}

Natural history of large renal masses on active surveillance: Systematic review of available case series

Kashif Visram ${ }^{1}$, Adam Gabara ${ }^{1}$, Francisco Vera Badillo', Naji J. Touma

'Urology, Queen's University, Kingston, ON, Canada

Introduction: Active surveillance (AS) for renal masses less than $4 \mathrm{~cm}$ in size is a well-accepted management strategy in the comorbid patient. In contrast, little is known about the safety of AS in renal masses larger than $4 \mathrm{~cm}$. The objective was to evaluate existing literature on AS of large renal masses $(>4 \mathrm{~cm})$, to determine the rate of intervention, metastatic rate, cancer-specific mortality, and overall mortality. Additionally, tumor biology was evaluated using growth rate as a surrogate.

Methods: A comprehensive search of electronic databases (PUBMED, OVID, Cochane, and Web of Science) using terms: "renal mass," "kidney mass," "nephrotic mass," "renal tumor," "kidney tumor," "renal neoplasia," "kidney neoplasia," "renal neoplasm," and "kidney neoplasm" generated 19475 studies. From this database, the terms "renal mass," "surveillance," "observation," and "growth rate" provided 656 studies which were reviewed by two independent reviewers and 628 studies were excluded. The remaining 28 papers underwent full-text review and of these, six studies met inclusion criteria. This provided data on 295 large renal masses from 285 patients. The data from these papers underwent pooled analysis using weighted means to assess the outcomes of interest. A risk of bias assessment was also conducted.

Results: The mean age of these patients was 72.9 years. At presentation, the mean size of the renal masses was $5.41 \mathrm{~cm}$ and the mean surveillance time was 40.25 months. The mean linear growth rate of these masses was $0.568 \mathrm{~cm} /$ year. A total of $10.8 \%$ of large renal masses underwent biopsy and $7.28 \%$ underwent surgical intervention; $3.1 \%$ of masses progressed to metastatic disease. Cancer-specific mortality was $1.7 \%$. Overall mortality was $7.46 \%$ 
Conclusions: Large renal masses $(>4 \mathrm{~cm})$ appear to have more aggressive cancer biology than their smaller counterparts $(<4 \mathrm{~cm})$, as reflected by a higher growth rate. However, metastatic rates and cancer-specific mortality are low in carefully selected patients on AS.

\section{MP-3.9}

A novel nuclear protein as a therapeutic target to block metastasis of clear-cell renal cell carcinoma

Lan K. Rudzinski ${ }^{1,2}$, Natasha Govindasamy ${ }^{2}$, Konstantin Stoletov ${ }^{2}$, Arun Raturi' ${ }^{2}$, John D Lewis ${ }^{2}$

'Division of Urology, Department of Surgery, University of Alberta, Edmonton, $A B$, Canada; ${ }^{2}$ Department of Oncology, University of Alberta, Edmonton, $\mathrm{AB}$, Canada

Support: Kidney Cancer Research Network and Kidney Cancer Canada Research Grant

Introduction: Whole human genome screen on human squamous cell carcinoma cells identified a panel of novel functional genes required for metastatic dissemination in vivo. One such novel protein target encodes for C14orf142, which is upregulated in metastatic clear-cell RCC (CRCC). The objective was to characterize its impact on cRCC vascular extravasation and distant metastasis in vivo.

Methods: cRCC cell lines, 786-0, were obtained from ATCC. Targeted genomic editing to knockout (KO) C14orf142 was achieved with CRISPRCas 9 system. To study cancer cell vascular extravasation in vivo, 786-0 cells were injected intravenously into fertilized avian embryos. To determine the impact of C14orf142 $\mathrm{KO}$ on in vivo spontaneous metastasis, a xenograft NSG nude mouse model was used. Six million 786-0 CRISPR $\mathrm{KO}$ or scramble clones were suspended in Matrigel and injected subcu- taneously into the right flank. To quantify the metastatic burden of 786-0 C14orf142 KO or scramble cells in distal organs, qPCR was performed with primers specific for human Alu repeats. For statistical analysis, t-test was used to evaluate differences between groups with $p \leq 0.05$ accepted as statistically significant.

Results: The CRISPR-Cas 9 targeted genomic editing resulted in generation of 786-0 clones with complete KO of C14orf142. Compared to 786-0 scramble clones, C14orf142 KO clones demonstrated significant reduction in extravasation on the avian embryo model $(100 \%$ vs $46.33 \pm 8.37 \%$, respectively, $\mathrm{n}=6, \mathrm{p} \leq 0.05$ ). Compared to NSG mice injected with 786-0 scramble clones, NSG mice implanted with 786-0 C14orf142 KO clones demonstrated significant reduction in distant metastatic burden to lungs $(2 \Delta \Delta$ CT $1.00+0.34$ vs $0.08+0.01$, respectively, $n=10, p \leq 0.05)$, liver $(2 \Delta \Delta$ CT $1.00+0.04$ vs $0.52+0.14$, respectively, $n=10, p \leq 0.05)$, and brain $(2 \Delta \Delta$ CT $1.00+0.17$ vs $0.27+0.06$, respectively, $n=10, p \leq 0.05)$.

Conclusions: A novel nuclear protein, C14orf142, may play an important role in cRCC vascular extravasation and distant metastasis in vivo.

\section{MP-3.10}

DDX41 expression induces worse prognosis and sensitivity to nivolumab via sting signaling activation in clear-cell renal cell carcinoma

Kenichiro Ikeda ${ }^{1}$, Kohei Kobatake ${ }^{1}$, Tetsutaro Hayashi', Jun Teishima ${ }^{1}$, Akio Matsubara ${ }^{1}$

${ }^{1}$ Urology, Hiroshima University Hospital, Hiroshima, Japan

Introduction: Mutations of chromosome $3 p$, such as PBRM1, SETD2, and BAP1, have emerged as prognostic biomarker in clear-cell renal cell carcinoma (ccRCC). ${ }^{1,2}$ However, the response immune checkpoint

MP-3.10. Table 1. Patient demographics

\begin{tabular}{|c|c|c|c|c|c|c|c|c|c|}
\hline \multicolumn{10}{|l|}{ Gene alterations } \\
\hline mRNA expression high & 48 (10.8) & - & & $26(5.8)$ & - & & $23(5.2)$ & - & \\
\hline mRNA expression low & $1(0.2)$ & - & & $0(0)$ & - & & $0(0)$ & - & \\
\hline Female & 19 (38.8) & $137(34.5)$ & & $7(26.9)$ & $149(35.5)$ & & $8(34.8)$ & $148(35.0)$ & \\
\hline \multicolumn{10}{|l|}{ Diagnosis age } \\
\hline$\leq 50$ & $8(16.3)$ & $85(21.4)$ & 0.064 & $3(11.5)$ & $90(21.4)$ & 0.380 & $5(21.7)$ & $88(20.8)$ & 0.876 \\
\hline $51-60$ & 12 (24.5) & $118(29.7)$ & & 10 (38.5) & $120(28.6)$ & & $7(30.4)$ & $123(29.1)$ & \\
\hline $61-70$ & $9(18.4)$ & $107(27.0)$ & & $9(34.6)$ & $107(25.5)$ & & $4(17.4)$ & $112(26.5)$ & \\
\hline $\mathrm{G} 1$ & $0(0)$ & $9(2.3)$ & $<0.0001$ & $0(0)$ & $9(2.1)$ & 0.056 & $0(0)$ & $9(2.1)$ & 0.052 \\
\hline G2 & $11(22.4)$ & $178(44.8)$ & & $7(26.9)$ & $182(43.3)$ & & $5(21.7)$ & $184(43.5)$ & \\
\hline G3 & 19 (38.8) & $156(39.3)$ & & 10 (38.5) & 165 (39.3) & & $10(43.5)$ & $165(39.0)$ & \\
\hline G4 & 19 (38.8) & 49 (12.3) & & $9(34.6)$ & $59(14.1)$ & & $8(34.8)$ & $60(14.2)$ & \\
\hline NA & $0(0)$ & $5(1.3)$ & & $0(0)$ & $5(1.2)$ & & $0(0)$ & $5(1.2)$ & \\
\hline \multicolumn{10}{|l|}{ Stage } \\
\hline I & $10(20.4)$ & 206 (51.9) & 0.001 & $10(38.4)$ & $206(49.0)$ & 0.006 & $7(30.4)$ & $209(49.4)$ & 0.246 \\
\hline II & 7 (14.3) & $39(9.8)$ & & $0(0)$ & $46(11.0)$ & & $2(8.7)$ & $44(10.4)$ & \\
\hline III & $20(40.8)$ & 91 (22.9) & & 7 (26.9) & $104(24.8)$ & & $9(39.1)$ & $102(24.1)$ & \\
\hline IV & 12 (24.5) & 59 (14.9) & & 8 (30.8) & $63(15.0)$ & & $5(21.7)$ & 66 (15.6) & \\
\hline
\end{tabular}


inhibitors (ICls) are not so high in metastatic RCC. Here, we focused on DEAD/H-box helicase gene DDX41 as potential therapeutic biomarker of ICls in RCC. DDX41 functions as DNA sensor that depends on the stimulator of interferon genes (STING) to sense pathogenic DNA and directly binds DNA and STING. It functions through the STING and the downstream effectors, such as interferon regulatory factor 3 (IRF3), leading to the expression of type I IFNs.

Methods: We aimed to investigate the effect of DDX41 in ccRCC using public database and in vitro experiment. We investigated the genetic alterations in DDX41, TMEM173 (encoding STING), and IRF3 in patients with ccRCC using the data sets from The Cancer Genome Atlas (TCGA) including 446 complete samples. To assess the DDX41 function, we established DDX41 overexpressing 786-O and Caki-1 cells and performed RNA sequence.

Results: The genetic alterations in DDX41, TMEM173, and IRF3 were found respectively in $49(11 \%), 28(5.8 \%)$, and $23(5.2 \%)$ samples. Enhanced expression of DDX41 mRNA is associated with worse prognosis, as well as TMEM173 and IRF3. Each enhanced expression of DDX41, TMEM173, and IRF3 mRNA significantly correlates with increased PD-1cording gene. In vivo experiments shows that enhanced DDX41 expression cause enrichment of E2F target gene sets in both 786-O and Caki-1 cell lines.

Conclusions: Expression of DDX41 might be a potential biomarker for predicting the response of anti PD-1 therapy in cCRCC.

References

1. Liu X, Wang C. The emerging roles of the STING adaptor protein in immunity and diseases. Immunology 2016;147:285-91. https://doi.org/10.1111/imm.12561

2. Cancer Genome Atlas Research Network. Comprehensive molecular characterization of clear-cell renal cell carcinoma. Nature 2013;499:43-9. https://doi.org/10.1038/nature12222

\section{MP-3.12}

Clinical severity of resected pheochromocytoma and its relation to Pheochromocytoma of the Adrenal Gland Scaled Score (PASS) liefei Yao ${ }^{7}$, Luke Witherspoon ${ }^{1}$,Eric Belanger ${ }^{2}$, Neal E. Rowe ${ }^{1}$

${ }^{1}$ Surgery-Urology, University of Ottawa, Ottawa, ON, Canada; ${ }^{2}$ Pathology, The Ottawa Hospital, Ottawa, ON, Canada

Introduction: Pheochromocytoma (PCC) is a rare catecholamine secreting tumor that often arises from the adrenal medulla. While only a small portion of tumors are malignant, many are associated with classic signs and symptoms, including, hypertension, tachycardia, headache, and palpitations. The Pheochromocytoma of the Adrenal Gland Scale Score (PASS) was developed by Thompson in 2002. ${ }^{1}$ This scoring system is based on the presence of 12 different histological parameters to aid the identification of malignant tumors. PASS $\geq 4$ is thought to have an increased potential for malignancy, while $<4$ is favored to be benign. The relationship between PASS and other features of PCC has not been described. Our study aimed to assess the relationship between PASS and presentation severity.

Methods: We performed a retrospective chart review on all resected PCCs in a tertiary center from 2008-2018. Demographics, operative details, pre/postoperative courses, and histopathology information were collected. Any histological specimens were assigned a PASS.

Results: Sixty-four resected tumors were identified and only two cases (PASS 4 and 12) were malignant. Mean PASS was 5.4 (0-15); 61\% had PASS $\geq 4$. ICU admission occurred in seven patients, with average PASS of 8.6 vs. 5.1 in non-ICU ( $p=0.0264)$. Average max intraoperative systolic blood pressure for PASS $<4$ and $\geq 4$ was $178 \mathrm{mmHg}$ and $183 \mathrm{mmHg}$ respectively $(p=0.67)$. Average PASS for incidentally detected PCC vs. symptomatic was 5.45 and 5.44 , respectively $(p=0.99)$.

Conclusions: Patients with an asymptomatic presentation of PCC did not have different PASS than patients with symptoms. However, PASS was higher in patients identified to have the most extreme clinical presentation (ICU admission). While our data suggests a role for PASS beyond prediction of metastatic potential, further studies involving patients from multiple centers is needed.

\section{Reference}

1. Thompson L. Pheochromocytoma of the Adrenal gland Scaled Score (PASS) to separate benign from malignant neoplasms: A clinicopathologic and immunophenotypic study of 100 cases. Am J Surg Pathol 2002;26:551-66. https://doi.org/10.1097/00000478200205000-00002

\section{MP-3.13}

Role of lactate dehydrogenase (LDH) in identifying relapse for patients with stage I testis cancer on active surveillance

Adam Bobrowski', Lauren Landoni ${ }^{2}$, Lynn Anson-Cartwight ${ }^{2}$, Aaron Hansen $^{3}$, Peter Chung 4 , Phillippe Bedard 3 , Padraig Warde ${ }^{4}$, Michael A. Jewett ${ }^{2}$, Martin $\mathrm{O}^{\prime}$ Malley $^{5}$, Joan Sweet ${ }^{6}$, Robert J. Hamilton ${ }^{2}$

${ }^{1}$ Faculty of Medicine, University of Toronto, Toronto, ON, Canada; ${ }^{2}$ Departments of Surgery and Surgical Oncology (Urology), Princess Margaret Hospital, University Health Network, University of Toronto, Toronto, ON, Canada; ${ }^{3}$ Department of Medical Oncology, Princess Margaret Hospital, University Health Network, University of Toronto, Toronto, ON, Canada; ${ }^{4}$ Department of Radiation Oncology, Princess Margaret Hospital, University Health Network, University of Toronto, Toronto, ON, Canada; ${ }^{5}$ Department of Diagnostic Radiology, Princess Margaret Hospital, University Health Network, University of Toronto, Toronto, ON, Canada; ${ }^{6}$ Department of Pathology, Princess Margaret Hospital, University Health Network, University of Toronto, Toronto, ON, Canada

Introduction: Tumor markers alpha-fetoprotein (AFP), human chorionic gonadotropin (hCG), and lactate dehydrogenase (LDH) have played a key role in diagnosis, staging, prognosis, and therapeutic response in testis cancer. Nonetheless, LDH has weak sensitivity and very weak specificity, which can lead to falsely elevated results that cause anxiety for patients on surveillance. We explored the utility of LDH in identifying relapse among stage I seminomatous and non-seminomatous germ cell tumors (NSGCT) on surveillance.

Methods: Subjects were selected from a prospectively maintained database from December 1980 to August 2019 and followed according to published surveillance algorithms. The link between LDH elevation and relapse was examined. We noted if $\mathrm{LDH}$ elevation was the sole indicator of relapse. The rate of false positive LDH elevation was assessed in the non-relapsed cohort.

Results: A total of 1753 patients (1077 seminoma and 676 NSGCT) were studied. Among seminomas, 183 patients (17\%) relapsed at a median of 15 months. The most common mode of relapse detection was imaging alone (83\%). Tumor marker elevation alone was noted in $1.6 \%$. LDH was elevated in $27(15 \%)$ cases at relapse but never in isolation as the sole indicator of relapse. Of the 676 NSGCT patients, 175 (26\%) experienced disease relapse at a median of 7.9 months. The most common mode of relapse detection was imaging alone (42\%). LDH was elevated in 26 $(15 \%)$ cases at relapse and, like in seminoma, never in isolation as the sole indicator of relapse. Among non-relapsing NSGCT patients diagnosed from January $2000(n=312), 234(75 \%)$ registered at least one false positive $\mathrm{LDH}$, and $160(52 \%)$ had multiple false positive $\mathrm{LDH}$ values. This translates into an average of 2.9 false positives per patient.

Conclusions: LDH did not independently contribute to early relapse detection in stage I seminoma or NSGCT. False positive values were commonly documented in non-relapsing NSGCT. Based on these results, we advocate no longer measuring LDH in stage I surveillance. 
MP-3.14

Long-term testis cancer survivors in Canada: Mortality risks in a large, population-based cohort

Roderick Clark', Arnon Lavi', Tina Luu Ly' ${ }^{2}$, Shiva M. Nair ${ }^{1}$, Khalil Hetou', Michael Haan ${ }^{2}$, Nicholas E. Power ${ }^{1}$

${ }^{1}$ Division of Urology, Department of Surgery, Schulich School of Medicine and Dentistry, Western University, London, ON, Canada; ${ }^{2}$ Department of Sociology, Western University, London, ON, Canada

Introduction: Introduction of cisplatin chemotherapy revolutionized treatment of testis cancer (TC) ${ }^{1,2}$ Cohorts received these revolutionary treatments are now reaching middle and later stages of their lives. Early and long-term sequelae of these treatments have been defined, ${ }^{3-5}$ but very longterm (>10-year) mortality risks are still a topic of debate. Our objective was to compare cause of death for TC patients to those without TC in Canada. Methods: We conducted a population-level, retrospective cohort study using the Canadian Census Health and Environment Cohort (CanCHEC). Exposed individuals were those diagnosed with TC from 1969-2010. Main outcome was cause of death between 1991 and 2010. Exposed individuals were matched with unexposed individuals (not diagnosed with TC by 1991). Demographic characteristics were compared using $\chi^{2} / t$-tests. Cox proportion hazards models were generated. Forward selection hazard ratios (HR) explored interaction between covariates and main effect.

Results: We identified 2678410 individuals (1950 exposed and 2676 460 unexposed) meeting our inclusion criteria (median age: 44 years, interquartile range [IQR] 25). Mean followup was 19.6 years. There were numerous demographic differences between exposed and unexposed individuals (Table 1). Majority of individuals with TC had seminoma histology $(n=1170,74.1 \%)$. Individuals with non-seminoma TC were more likely to die of cardiovascular disease (Table 2) during the study period (HR 4.46; 95\% confidence interval [Cl] 2.10-9.43; $\mathrm{p}<0.01$ ), while individuals with seminoma were not (HR 2.34; 95\% Cl 0.98-5.49; $p=0.054)$. Risk of death from non-TC cancer was increased for both seminoma (HR 7.38; 95\% Cl 4.53-12.06; $\mathrm{p}<0.01)$ and non-seminoma ( $\mathrm{HR} 7.03 ; 95 \% \mathrm{Cl}$ 4.58-10.78; $\mathrm{p}<0.01)$. Multivariate analysis controlling for demographic parameters confirmed these findings (Table 3 ).

Conclusions: TC survivorship is associated with increased risk of death from other malignancies. Non-seminoma survivorship is associated with an increased risk of death from cardiovascular disease.

\section{References}

1. Hanna N, Einhorn LH. Testicular cancer: A reflection on 50 years of discovery. J Clin Oncol 2014;32:3085-92. https://doi.org/10.1200/ JCO.2014.56.0896

2. Verdecchia A, Francisci S, Brenner H, et al. Recent cancer survival in Europe: A 2000-02 period analysis of EUROCARE-4 data. Lancet Oncol 2007;8:784-96. https://doi.org/10.1016/S14702045(07)70246-2

MP-3.14. Table 1. Demographic baseline characteristics

\begin{tabular}{|c|c|c|c|c|}
\hline & $\begin{array}{c}\text { Cohort } \\
(n=2678410)\end{array}$ & $\begin{array}{c}\text { Testis cancer } \\
(\mathrm{n}=1950)\end{array}$ & $\begin{array}{c}\text { Non-testis cancer } \\
(n=2676460)\end{array}$ & $\mathbf{p}$ \\
\hline Age (median years, IQR) & $44(25)$ & $36(15)$ & $44(25)$ & $<0.0001$ \\
\hline Male sex & 1302245 (48.6\%) & $1950(100 \%)$ & $1300295(48.6 \%)$ & $<0.0001$ \\
\hline Single & $459845(17.2 \%)$ & $535(27.4 \%)$ & $459310(17.2 \%)$ & \\
\hline Married & $1734475(64.7 \%)$ & $1220(62.3 \%)$ & $1733250(64.7 \%)$ & \\
\hline Education & & & & $<0.0001$ \\
\hline No high school & $996010(37.2 \%)$ & $525(26.7 \%)$ & $995485(37.2 \%)$ & \\
\hline High school & $949325(35.4 \%)$ & 790 (40.4\%) & $948540(35.4 \%)$ & \\
\hline Post-secondary, non-university & 390775 (14.6\%) & $280(14.4 \%)$ & 390495 (14.6\%) & \\
\hline University & 342295 (12.8\%) & $360(18.5 \%)$ & $341940(12.8 \%)$ & \\
\hline West Coast (BC) & $331350(12.4 \%)$ & $265(13.6 \%)$ & 331085 (12.4\%) & \\
\hline Prairies (Man, Sas, Alb) & 435475 (16.3\%) & $410(21 \%)$ & $435065(16.3 \%)$ & \\
\hline Atlantic Canada (NB, NFLD, PEI, NS) & 221005 (8.3\%) & $170(8.7 \%)$ & 220835 (8.3\%) & \\
\hline Territories (NWT, Yuk) & $6905(0.2 \%)$ & $10(0.5 \%)$ & $6895(0.2 \%)$ & \\
\hline Urbanicity & & & & 0.1939 \\
\hline Rural & $604805(22.6 \%)$ & $445(22.8 \%)$ & $604360(22.6 \%)$ & \\
\hline Small urban $(<30000)$ & $359660(13.4 \%)$ & $255(13.1 \%)$ & 359405 (13.4\%) & \\
\hline Urban (30 000-99 999) & $244220(9.1 \%)$ & $190(9.7 \%)$ & 244025 (9.1\%) & \\
\hline Urban (100 000-499 999) & $284025(10.6 \%)$ & $235(12.1 \%)$ & $283795(10.6 \%)$ & \\
\hline Urban $(500000+)$ & $1185700(44.3 \%)$ & $825(42.3 \%)$ & $1184875(44.3 \%)$ & \\
\hline
\end{tabular}


MP-3.14. Table 2. Cause of death stratified by testicular histology

\begin{tabular}{lcccccc}
\hline & \multicolumn{3}{c}{ Non-seminoma } & \multicolumn{2}{c}{ Seminoma } \\
\hline Cause of death & HR & $\mathbf{9 5 \%}$ Cl & $\mathbf{p}$ & HR & $\mathbf{9 5 \%}$ Cl & $0.98-5.49$ \\
\hline Cardiovascular & 4.46 & $2.10-9.43$ & $<0.01$ & 2.34 & 0.054 & $4.53-12.06$ \\
Other neoplasm & 7.03 & $4.58-10.78$ & $<0.01$ & 7.38 & 0.01 & $0.46-40.09$ \\
Infectious and parasitic & 6.88 & $0.892-53.07$ & 0.06 & 1.64 & 0.19 & 0.006 \\
Respiratory & 0.009 & $0-\infty$ & 0.78 & 0.006 & $1.87-14.43$ \\
Genitourinary neoplasm & 10.29 & $4.24-24.94$ & $<0.01$ & 5.2 & $<0.01$ \\
\hline
\end{tabular}

\begin{tabular}{|c|c|c|c|}
\hline Cause of death & $\begin{array}{l}\text { Testis } \\
\text { cancer }\end{array}$ & $\begin{array}{c}\text { Demographic } \\
\text { variable }\end{array}$ & Histology \\
\hline Cardiovascular & $\begin{array}{c}0.517 \\
(p<0.01)\end{array}$ & 1.047 & $\begin{array}{c}0.469 \\
\text { Seminoma: } 0.998 \\
\text { Non-seminoma: } 2.11 \\
(p<0.01)\end{array}$ \\
\hline Any neoplasm & 0.96 & $1.51(p<0.01)$ & $\begin{array}{c}0.178(p<0.01) \\
\text { Non-seminoma: } 7.03 \\
(p<0.05) \\
\text { Seminoma: } 7.39 \\
(p<0.01)\end{array}$ \\
\hline $\begin{array}{l}\text { Infectious and } \\
\text { parasitic }\end{array}$ & 1.27 & $1.92(p<0.05)$ & $\begin{array}{c}0.325 \\
\text { Non-seminoma: } 6.88 \\
\text { Seminoma: } 4.31\end{array}$ \\
\hline Respiratory & $\begin{array}{c}0.040 \\
(p<0.05)\end{array}$ & 0.843 & $\begin{array}{c}107.1 \\
\text { Non-seminoma: } 0.009 \\
\text { Seminoma: } 0.006\end{array}$ \\
\hline $\begin{array}{l}\text { Genitourinary } \\
\text { neoplasm }\end{array}$ & $\begin{array}{c}1.816 \\
(p<0.01)\end{array}$ & $\begin{array}{c}3.308 \\
(p<0.01)\end{array}$ & $\begin{array}{c}0.474 \\
\text { Non-seminoma: } 10.29 \\
(p<0.01) \\
\text { Seminoma: } 5.20 \\
(p<0.01)\end{array}$ \\
\hline
\end{tabular}

3. Haugnes HS, Wethal T, Aass $\mathrm{N}$, et al. Cardiovascular risk factors and morbidity in long-term survivors of testicular cancer: A 20-year followup study. J Clin Oncol 2010;28:4649-57. https://doi.org/10.1200/ JCO.2010.29.9362

4. Travis LB, Fosså SD, Schonfeld SJ, et al. Second cancers among 40576 testicular cancer patients: Focus on long-term survivors. $/ \mathrm{NCl}$ J Natl Cancer Inst 2005;97:1354-65. https://doi.org/10.1093/jnci/ dji278

5. van Den Belt-Dusebout AW, Nuver J, de Wit R, et al. Long-term risk of cardiovascular disease in 5-year survivors of testicular cancer. / Clin Oncol 2006;24:467-75. https://doi.org/10.1200/JCO.2005.02.7193

\section{MP-3.15}

Robotic retroperitoneal lymph node dissection for primary and post-chemotherapy testis cancer

Gregory Nason ${ }^{1}$, Kopika Kuhathaas' ${ }^{1}$ Lynn Anson-Cartwright ${ }^{1}$, Michael A. Jewett ${ }^{1}$, Martin O'Malley ${ }^{2}$, Joan Sweet ${ }^{3}$, Aaron Hansen ${ }^{4}$, Philippe Bedard ${ }^{4}$, Peter Chung 5 , Ezra Hahn ${ }^{5}$, Padraig Warde ${ }^{5}$, Robert J. Hamilton ${ }^{1}$

${ }^{1}$ Urology, Princess Margaret Cancer Centre, Toronto, ON, Canada; ${ }^{2}$ Joint Department of Medical Imaging, University Health Network, Toronto, ON, Canada; ${ }^{3}$ Laboratory Medicine and Pathobiology, University Health Network, Toronto, ON, Canada; ${ }^{4}$ Medical Oncology, Princess Margaret Cancer Centre, Toronto, ON, Canada; ${ }^{5}$ Radiation Oncology, Princess Margaret Cancer Centre, Toronto, ON, Canada

Introduction: The role of retroperitoneal lymph node dissection (RPLND) in testicular cancer is well-established. Minimally invasive approaches have had limited adoption. Robotic RPLND has evolved, but to date, only small series have reported outcomes, with analyses hampered by disproportionate inclusion of early-stage disease and the impact of adjuvant chemotherapy. Herein, we report the first 25 robotic RPLND cases performed in Canada.

Methods: A retrospective review was performed of all patients undergoing robotic RPLND, which were performed by a single surgeon at Princess Margaret Cancer Centre. Demographic, perioperative, and oncological data were analyzed using descriptive statistics.

Results: Between September 2014 and October 2019, 25 patients underwent a robotic RPLND, 17 (68\%) were primary and eight (32\%) postchemotherapy. Stage at RPLND was 2A (56\%), 2B (32\%), 2C (4\%), and 3A $(8 \%)$. Median operating room time (incision to closure) was 510 minutes (range 180-600) and median blood loss was $200 \mathrm{ml}$ (range 50-6000). Nerve-sparing was performed in all but one case (96\%). Six (24\%) adjuvant procedures were performed, including two $(8 \%)$ vascular repairs and two cases were converted to open (one for bleeding and other for rapid progression from preoperative computed tomography CT). Median length of stay was two days (range 1-8). Viable tumour was detected in $16(64 \%)$, teratoma in eight $(32 \%)$, and fibrosis/necrosis in only one $(4 \%)$. Median followup was 20 months (range 1-52). Three patients (12\%) relapsed: one brain, one retrocrural, and one combined in- and out-of-field retroperitoneal relapse 21 months post-RPLND for a seminoma. Two of three received chemo, while the brain relapse was treated with radiation alone. Conclusions: Robotic RPLND can be performed safely. A long-term followup of series such as ours, enriched with patients with viable disease and/or teratoma, and not treated with adjuvant chemotherapy, is required to ensure oncological outcomes are comparable to the open approach. 


\section{CUA ABSTRACTS}

\section{Moderated Poster Session 4: Prostate Cancer I}

\section{MP-4.1}

Relationship between omega-3 fatty acid intake and prostate cancer risk: Preliminary result of the BIOCAPPE_GRÉPEC study Hanane Moussa ${ }^{1}$, Lamoussa Diabaté ${ }^{1}$, Karine Robitaille ${ }^{1}$, Laurence Bettan ${ }^{7}$ Molière Nguile-Makao ${ }^{1}$, Hélène Hovington ${ }^{1}$, Fred Saad ${ }^{2}$, Michel Carmel ${ }^{3}$, Armen-G. Aprikian ${ }^{4}$, BIOCaPPE Network ${ }^{1,2,3,4}$, Benoit Lamarche ${ }^{5}$, Pierre Julien ${ }^{1}$, Yves Fradet ${ }^{1}$, Vincent Fradet ${ }^{1,5}$

${ }^{1}$ Centre de recherche du CHU de Québec, Université Laval, Quebec City, QC, Canada; ${ }^{2}$ Centre de Recherche du CHUM, Montreal, QC, Canada; ${ }^{3}$ Centre de Recherche du CHUS, Sherbrooke, QC, Canada; ${ }^{4}$ Institut de Recherche du CUSM, Montreal, QC, Canada; ${ }^{5}$ Institut sur la nutrition et les aliments fonctionnels (INAF), Quebec City, QC, Canada

Introduction: Prostate cancer is a major health problem worldwide. Omega-3 fatty acids $(\Omega 3)$ are among the dietary factors thought to impact prostate cancer risk. Preclinical and clinical studies suggest that a high consumption of $\Omega 3$ have protective effects on prostate cancer development and progression. The objective of this work was to explore the associations between prostate cancer incidence and consumption of $\Omega 3$ in men at risk of prostate cancer.

Methods: We conducted a multicenter, prospective cohort study with 2500 men at high risk of prostate cancer. This observational study aimed at evaluating the role of lifestyle, including diet, on five candidate biomarkers and prostate cancer incidence. At study baseline, $\Omega 3$ intake was measured using a validated food frequency questionnaire and their levels in red blood cell membranes were measured by gas chromatography. Cancer incidence was evaluated at two years' followup by biopsy. Preliminary associations between $\Omega 3$ intake and prostate cancer incidence in the first 256 participants was explored using multivariate logistic regression.

Result: Among the first 256 participants, 33\% were diagnosed with prostate cancer at two years. We observed an inverse association between prostate cancer incidence and long-chain $\Omega 3$ (LC $\Omega 3$ ) intake (odds ratio [OR] 0.33; 95\% confidence interval [Cl] 0.11-0.95; $\mathrm{p}=0.04)$, DHA intake (OR 0.33; $95 \% \mathrm{Cl} 0.11-0.93 ; \mathrm{p}=0.03)$, and $\mathrm{LC} \Omega 3: \Omega 6$ ratio intake (OR 0.35; $95 \% \mathrm{Cl}$ $0.12-0.98 ; p=0.04)$, measured in the diet. This inverse association between $\mathrm{LC} \Omega 3$ and prostate cancer incidence was accompanied by a dose-response relationship ( $\mathrm{p}$ trend $=0.02$ ).

Conclusions: These preliminary results suggest a potential link between prostate cancer incidence and LC 23 consumption in men at risk of prostate cancer. However, these relationships were not translated in the circulating measurement of $\Omega 3$. Analyses of the associations between $\Omega 3$ intake and prostate cancer incidence in the entire cohort are warranted.

\section{MP-4.2}

The times they are a-changin': The evolution of prostate cancer screening practices and beliefs among primary care physicians in Victoria, British Columbia Lesse Spooner ${ }^{1}$, Manjot Birk', Victor Espinosa², Nathan Hoag ${ }^{3}$

'Island Medical Program, University of British Columbia, Victoria, BC, Canada; ${ }^{2}$ Research and Capacity, Island Heath, Victoria, BC, Canada; ${ }^{3}$ Urological Sciences, University of British Columbia, Victoria, BC, Canada

Introduction: Prostate cancer screening practices remain controversial among primary care practitioners (PCPs). Inconsistent guidelines and publication of large prostate cancer screening trials have failed to provide definitive guidance. This study investigates the evolution of prostate cancer screening practices and beliefs over 12 years in Victoria, BC.
Methods: Questionnaires were delivered to 119 randomly selected PCPs in 2019. Descriptive analysis, together with exploratory graphs and Pearson Cchi-squared test for independence was calculated. 2008 data from the study conducted by Hoag et $\mathrm{al}^{1}$ in Victoria, BC, on a presumed similar demographic was compared by determining if their value fell within the 2019 data's 95\% confidence intervals.

Results: Response rate was $69.8 \%$ (83/119); 30.1\% reported regularly screening asymptomatic men with prostate-specific antigen (PSA) testing, and $37.3 \%$ reported regularly performing digital rectal exam (DRE). The combination of PSA and DRE was the most commonly used (48.2\%) screening modality. A total of $73.5 \%$ of PCPs reported that guidelines influence their screening practices, with the most popular choice being those published by The Canadian Task Force on Preventative Health Care (CTF) (32.5\%). Conclusions: The results demonstrate a movement away from prostate cancer screening among PCPs when compared to 2008. ${ }^{1}$ PCPs believe that DRE and PSA are less valuable as screening tools and that there is insufficient evidence to support their use. The most used initial screening modality was the combination of PSA/DRE, however, we found a decrease in their use between the two study periods. ${ }^{1}$ Clinical guidelines continue to influence PCPs screening practices, but the shift of more PCPs following the CTF guidelines since 2008 has likely led to the reciprocal decrease in prostate cancer screening. ${ }^{2}$ Reference

1. Hoag N, Davidson R, Pommerville P. Prostate cancer screening practices and attitudes among primary care physicians in Victoria, British Columbia. BCMJ 2008;50:456-60.

\section{MP-4.3}

\section{Transperineal prostate biopsy minimizes the risk of infectious} complications requiring hospital management

Veselina Stefanova ${ }^{1}$, Roger J. Buckley ${ }^{1}$, Rupayan Mukherjee ${ }^{1,2}$, Abirami Kirubarajan ${ }^{1,3}$, Nicole Golda ${ }^{1}$, Andrew Loblaw ${ }^{1,4}$, Stanley Flax ${ }^{1}$, Les Spevack ${ }^{1}$ ${ }^{1}$ Urology, North York General Hospital, Toronto, ON, Canada; ${ }^{2}$ Management and Innovation, The University of Toronto, Mississauga, ON, Canada; ${ }^{3}$ Medicine, The University of Toronto, Toronto, ON, Canada; ${ }^{4}$ Radiation Oncology, Sunnybrook Health Sciences Centre, Toronto, ON, Canada

Acknowledgement: Beverly Ann Persaud, Colleen Andersen, Tim Dong Introduction: Prostate cancer ( $\mathrm{PCa}$ ) has typically been diagnosed using the transrectal biopsy (TRBx). However, infectious complications (ICs) following TRBX are a common morbidity occurring at rising rates. ${ }^{1}$ The risk of urosepsis post-TRBx has been estimated to be from $5-7 \%$, with hospital admission rates ranging from $2-4 \% .{ }^{2}$ Growing evidence suggests that transperineal prostate biopsy (TPBx) is a feasible diagnostic alternative with a minimized risk of ICs. ${ }^{3}$ Beginning on October 12, 2016, our urology practice switched from performing TRBx to exclusively TPBx. We compared the frequency and management of ICs in patients who underwent TRBx or TPBx.

Methods: A retrospective chart review was conducted on patients who underwent TRBx and TPBx during a two-year period pre- and post-October 12 , respectively. We determined all cases of ICs managed at our hospital as outpatients (emergency room visit only) or inpatient (hospitalization and/or ICU stay) within 31 days post-biopsy. The total hospital cost for the management of post-biopsy ICs was calculated for each biopsy strategy. Results: During the study period, 1031 and 1325 patients underwent TRBx and TPBx, respectively. The overall risk of ICs post-TPBx was significantly lower than post-TRBx $(p<0.001)$. A summary of all ICs is listed in Table 1. The most common IC following TRBx and TPBx was a urinary tract infection 


\begin{tabular}{|c|c|c|c|c|}
\hline \multicolumn{5}{|c|}{$\begin{array}{l}\text { MP-4.3. Table 1. Infectious complications following TRBx } \\
\text { and TPBx requiring outpatient or inpatient management } \\
\text { at North York General Hospital }\end{array}$} \\
\hline & \multicolumn{2}{|c|}{$\begin{array}{c}\text { TRBx } \\
(\mathrm{n}=1031)\end{array}$} & \multicolumn{2}{|c|}{$\begin{array}{c}\text { TPBx } \\
(n=1325)\end{array}$} \\
\hline Fever & 1 & $(0.1)$ & 0 & (0) \\
\hline Epididymitis & 1 & $(0.1)$ & 1 & $(0.1)$ \\
\hline UTI & 20 & (1.9) & 3 & $(0.2)$ \\
\hline Bacteremia & 7 & (0.7) & 0 & (0) \\
\hline Urosepsis & 12 & (1.1) & 1 & $(0.1)$ \\
\hline Septic Shock & 2 & (1.9) & 0 & (0) \\
\hline Death & 0 & (0) & 0 & (0) \\
\hline All ICs & 43 & (4.1) & 5 & $(0.4)$ \\
\hline ER visit & 19 & (1.8) & 4 & $(0.3)$ \\
\hline Hospitalization & 26 & (2.5) & 1 & $(0.1)$ \\
\hline ICU stay & 2 & (1.9) & 0 & (0) \\
\hline
\end{tabular}

(UTI), which occurred in $1.9 \%$ and $0.2 \%$ of patients, respectively. Two patients developed septic shock post-TRBx, requiring hospitalization in both and an ICU stay in one. There was only one $(0.07 \%)$ case of urosepsis in the TP group compared to $12(1.2 \%)$ cases in the TR group. The total cost for managing ICs at our hospital post-TRBx was $\$ 76700$ compared to $\$ 4300$ in the TP group.

Conclusions: The TPBx minimizes the risk of ICs, which commonly occur post-TRBx. There is near-zero risk of urosepsis post-TPBx. The TPBX can reduce the financial burden on hospitals managing post-biopsy ICs. The TPBx improves patient safety and should be considered as a standard diagnostic modality for PCa diagnosis.

References

1. Nam RK, Saskin R, LeeY, et al. Increasing hospital admission rates for urological complications after transrectal ultrasound guided prostate biopsy. J Urol 2010;18:963-9. https://doi.org/10.1016/j.juro.2009.11.043

2. Liss MA, Ehdaie B, Loeb S, et al. An update of the American Urological Association white paper on the prevention and treatment of the more common complications related to prostate biopsy. J Urol 2017;198:329-34. https://doi.org/10.1016/j.juro.2017.01.103

3. Huang GL, Kang CH, Lee WC, et al. Comparisons of cancer detection rate and complications between transrectal and transperineal prostate biopsy approaches - a single-center preliminary study. BMC Urol 2019;19:1-8. https://doi.org/10.1186/s12894-019-0539-4

\section{MP-4.4}

Experience with 1939 transperineal prostate biopsies under local anesthesia: Prostate cancer detection rate, complications, and tolerability

Veselina Stefanova', Roger I. Buckley', Rupayan Mukherjee ${ }^{1,3}$, Abiram Kirubarajan ${ }^{1,2}$, Stanley Flax' ${ }^{1}$ Nicole Golda', Les Spevack ${ }^{1}$, David Hajek', Andrew Loblaw', ${ }^{1,4}$, Enoch Lai ${ }^{1}$, Adam Tunis ${ }^{1,5,6}$, Joseph Peltz ${ }^{1,5,6}$

${ }^{1}$ Urology, North York General Hospital, Toronto, ON, Canada; ${ }^{2}$ Medicine, University of Toronto, Toronto, ON, Canada; ${ }^{3}$ Management and Innovation, University of Toronto, Mississauga, ON, Canada; ${ }^{4}$ Radiation Oncology, Sunnybrook Health Sciences Centre, Toronto, ON, Canada; ${ }^{5}$ Radiology, North York General Hospital, Toronto, ON, Canada; ${ }^{6}$ Diagnostic Imaging, North York General Hospital, Toronto, ON, Canada

Acknowledgement: Beverly Ann Persaud, Colleen Andersen, and Tim Dong Introduction: The transrectal prostate biopsy (TRUSBx) has been the standard modality for prostate cancer (PCa) diagnosis. However, the risk of urosepsis following TRUSBx has been estimated to be from $5-7 \%$, with hospital admission rates ranging from 2-4\%. ${ }^{1}$ Recently, the transperineal prostate biopsy (TPBx) has been growing in favor due to its low risk of post-biopsy infection. ${ }^{2,3}$ We report on our experience and outcomes in patients undergoing TPBx under local anesthesia.
Methods: A retrospective chart review was conducted on consecutive patients who underwent TPBx from October 2016 to September 2019. Patient tolerability was assessed using the Visual Analog Scale (VAS). Patients reported their level of discomfort from 0 (no pain) to 10 (worst pain imaginable) for various parts of the procedure. Results: A total of 1939 patients underwent a TPBx under local anesthetic. PCa was diagnosed in 1027 (53.0\%) patients who had positive pathology. Clinically significant PCa (Gleason grade group $\geq 2$ ) was detected in 633 $(61.6 \%)$ patients and $113(11.0 \%)$ had exclusively anterior zone pathology findings. Acute urinary retention developed in $40(2.1 \%)$ patients following TPBx. Nine patients $(0.5 \%)$ presented with symptoms of a urinary tract infection (UTI) post-biopsy but only five had positive urine cultures. Only one $(0.05 \%)$ patient was admitted to the hospital post-TPBx due to presumed urosepsis; however, cultures were negative. Patient tolerability was assessed in 584 patients with VAS scores of 2.1 for ultrasound probe insertion, 2.5 for the biopsy itself, 2.9 for periprostatic local infiltration, and 3.1 for local skin infiltration.

Conclusions: The TPBx under local anesthetic is a safer alternative to the TRUSBx biopsy in the detection of PCa. The TPBx has an acceptable cancer detection rate with an added ability to detect anterior zone cancers. TPBx has a low risk of infectious complications and is well-tolerated. The TPBx should be considered in the initial diagnosis of PCa.

References

1. Liss MA, Ehdaie B, Loeb $S$, et al. An update of the American Urological Association white paper on the prevention and treatment of the more common complications related to prostate biopsy. J Urol 2017; 198:329-34. https://doi.org/10.1016/j.juro.2017.01.103

2. Pepdjonovic L, Tan GH, Huang S, et al. Zero hospital admissions for infection after 577 transperineal prostate biopsies using single-dose cephazolin prophylaxis. World J Urol 2017;35:1199-1203. https:// doi.org/10.1007/s00345-016-1985-1

3. Ristau BT, Allaway M, Cendo D, et al. Free-hand transperineal prostate biopsy provides acceptable cancer detection and minimizes risk of infection: Evolving experience with a 10-sector template. Semin Urol Oncol 2018;36:528-e15. https://doi.org/10.1016/j. urolonc.2018.09.013

\section{MP-4.5}

Assessing the clinical impact of magnetic resonance imagingultrasound fusion targeted prostate biopsies among patients with previously negative transrectal ultrasound biopsy: A community urology experience

Thomas Southall', Charlie Gillis', Michelle Anderson², Jennifer Young², Richard Hewitt ${ }^{3}$, I. Matthew Andrews ${ }^{3}$

${ }^{1}$ Faculty of Medicine, Memorial University of Newfoundland, St. John's, NL, Canada; 'Department of Radiology, Health Sciences Centre, St. John's, NL, Canada; ${ }^{3}$ Department of Surgery, Division of Urology, Health Sciences Centre, St. John's, NL, Canada

Introduction: Prostate cancer (PCa) is commonly diagnosed among Canadian men. ${ }^{1,2}$ Occasionally, men will experience a clinical suspicion of PCa and persistent prostate-specific antigen (PSA) elevation despite a negative transrectal ultrasound (TRUS) biopsy. ${ }^{3}$ This so-called elusive PSA creates a clinical dilemma for urologists. New technologies, such as magnetic resonance imaging (MRI)-ultrasound (US) fusion targeted biopsies, are designed to increase detection of clinically significant prostate cancer (csPCa)..$^{3-5}$ We report our experience with MRI-US fusion biopsies in patients with an elusive PSA.

Methods: We prospectively included 97 patients undergoing MRI-US fusion biopsies at our institution from September 2018 to December 2019. All men had clinical suspicion of PCa, prior negative TRUS biopsies, and persistent PSA elevation. Index lesions on multiparametric MRI were reviewed using PIRADS v2 scoring system. Lesions classified as PIRADS $\geq 3$ received targeted biopsy. Biopsy-naive patients and those on active surveillance were excluded. The primary outcome was detection rate of csPCa, defined as ISUP grade group $\geq 2$. Descriptive statistics were used to calculate differences in proportions.

Results: Prior to fusion biopsy, patients had a mean of $18.5 \pm 8.8$ negative core biopsies per patient, mean PSA $11.81 \pm 6.33 \mathrm{ng} / \mathrm{ml}$. MRI-US fusion 


\begin{tabular}{lc}
\hline $\begin{array}{l}\text { MP-4.5. Table 1. Demographics in patients with } \\
\text { previously negative TRUS prostate biopsy results, and } \\
\text { raised PSA undergoing MRI-US fusion prostate biopsy for } \\
\text { clinical suspicion of prostate cancer }\end{array}$ \\
\hline $\mathrm{n}$ & 97 \\
Median age (years) (range) & $65(44-80)$ \\
Mean PSA (ng/ml) & $11.81 \pm 6.33$ \\
Mean interval between MRI and & $134.01 \pm 136.71$ \\
fusion Bx (days) & \\
$\begin{array}{l}\text { Mean interval between last Bx and } \\
\text { fusion Bx (days) } \\
\text { Mean number of cores per patient prior } \\
\text { to fusion Bx }\end{array}$ & $1084.63 \pm 811.44$ \\
\hline
\end{tabular}

\begin{tabular}{|c|c|}
\hline \multicolumn{2}{|c|}{$\begin{array}{l}\text { MP-4.5. Table 2. Cancer detection by PIRADS lesion score } \\
\text { and lesion location in patients undergoing MRI-US fusion } \\
\text { prostate biopsy }\end{array}$} \\
\hline \% positive MRI-US fusion bx & $42.3 \%$ \\
\hline$\%$ negative MRI-US fusion bx & $57.7 \%$ \\
\hline Overall PCa detection ( $G G \geq 1$ ) & $42(42.9 \%)$ \\
\hline csPCa detection ( $G G \geq 2)$ & $32(32.7 \%)$ \\
\hline \multicolumn{2}{|l|}{ Lesions detected by PIRADS type } \\
\hline PIRADS 3 & 49 \\
\hline PIRADS 4 & 55 \\
\hline PIRADS 5 & 36 \\
\hline \multicolumn{2}{|l|}{ csPCa by lesion type } \\
\hline PIRADS 3 & $3(6.1 \%)$ \\
\hline PIRADS 4 & $13(23.6 \%)$ \\
\hline PIRADS 5 & $15(41.7 \%)$ \\
\hline \multicolumn{2}{|l|}{$\%$ csPCa by lesion zone } \\
\hline Peripheral & $19(59.4 \%)$ \\
\hline Transition & $12(37.5 \%)$ \\
\hline Central & $3(9.4 \%)$ \\
\hline \multicolumn{2}{|l|}{$\%$ csPCa by lesion location } \\
\hline Anterior & $17(53.1 \%)$ \\
\hline Midgland & $10(31.3 \%)$ \\
\hline Posterior & $5(15.6 \%)$ \\
\hline
\end{tabular}

MP-4.5. Table 3. Clinical outcomes of patients found to have csPCa on MRI-US fusion biopsy

\begin{tabular}{lc}
\hline csPCa Detection $(\mathrm{GG} \geq 2)$ & $32(32.7 \%)$ \\
Continue PSA surveillance & $1(3.1 \%)$ \\
Radiation treatment & $18(56.3 \%)$ \\
Radical prostatectomy & $12(37.5 \%)$ \\
Androgen-deprivation therapy alone & $0(0 \%)$ \\
Undecided & $1(3.1 \%)$ \\
\hline
\end{tabular}

biopsy resulted in diagnosis of PCa in 41/97 (42.3\%) patients. csPCa was found in three $(6.7 \%), 13(23.6 \%)$, and $15(41.7 \%)$ of PIRADS 3,4 , and 5 lesions, respectively. The location of csPCa was within the peripheral zone $(59.4 \%)$, transitional zone $(37.5 \%)$, and central zone $(9.4 \%)$. Clinical outcomes of patients with newly diagnosed csPCa show $3.1 \%, 56.3 \%$,
$37.5 \%$, receiving ongoing PSA surveillance, radiation treatment, radical prostatectomy, respectively.

Conclusions: MRI-US fusion targeted biopsy yields high detection rates for csPCa in men with elusive PSA elevation and frequently guides a change in clinical management.

\section{References}

1. Smith-Palmer J, Takizawa C, Valentine W. Literature review of the burden of prostate cancer in Germany, France, the United Kingdom, and Canada. BMC Urol 2019;19:19. https://doi.org/10.1186/s12894019-0448-6

2. LeBlanc AG, Demers A, Shaw A. Recent trends in prostate cancer in Canada. Health Rep 2019;30(4):8.

3. Drost F-JH, Osses DF, Nieboer D, et al. Prostate MRI, with or without MRI-targeted biopsy, and systematic biopsy for detecting prostate cancer. Cochrane Database Syst Rev 2019;4:CD012663. https://doi.org/10.1002/14651858.CD012663.pub2

4. Rosenkrantz Andrew B, Verma S, Choyke P, et al. Prostate magnetic resonance imaging and Magnetic Resonance Imaging Targeted Biopsy in Patients with a Prior Negative Biopsy: A consensus statement by AUA and SAR. J Urol 2016;196:1613-8. https://doi.org/10.1016/j.juro.2016.06.079

5. Schoots IG, Roobol MJ, Nieboer D, et al. Magnetic resonance imaging-targeted biopsy may enhance the diagnostic accuracy of significant prostate cancer detection compared to standard transrectal ultrasound-guided biopsy: A systematic review and metaanalysis. Eur Urol 2015;68:438-50. https://doi.org/10.1016/j. eururo.2014.11.037

\section{MP-4.6}

Is index lesion multiplicity and lesion location associated with detection of clinically significant prostate cancer during magnetic resonance imaging-ultrasound targeted biopsy?

Charlie Gillis ${ }^{1}$, Thomas M. Southall' ${ }^{1}$ Michelle Anderson'², Jennifer Young ${ }^{2}$, Richard J. Hewitt ${ }^{3}$, J. Matthew Andrews ${ }^{3}$

${ }^{1}$ School of Medicine, Memorial University of Newfoundland, St. John's, NL, Canada; ${ }^{2}$ Department of Radiology, Memorial University of Newfoundland, St John's, NL, Canada; ${ }^{3}$ Department of Surgery, Division of Urology, Memorial University of Newfoundland, St John's, NL, Canada Introduction: There is growing recommendation for use of magnetic resonance imaging (MRI) and targeted biopsies for detection of prostate cancer (PCa). ${ }^{1}$ MRI-ultrasound fusion targeted biopsy (MRI-US Bx) of index lesions has shown increased detection of clinically significant prostate cancer (csPCa) over transrectal ultrasound-guided (TRUS) biopsy alone, and detection of fewer insignificant cancers. ${ }^{2-5}$ Commonly, prostatic MRI identifies multiple suspicious PIRADS lesions within a single prostate. We investigate whether index lesion multiplicity and lesion location are associated with detection of csPCa using MRI-US Bx.

Methods: A total of 142 men were identified retrospectively, from March 2017 to December 2019, after completion of MRI-US Bx. We included biopsy-naive patients, those with previous negative TRUS biopsies, and those on active surveillance for low-risk PCa. All patients received multiparametric MRI with index lesions graded using PIRADS classification system. PIRADS $\geq 3$ lesions received MRI-US Bx using bk3000 and MIM software. Detection rate of csPCa (defined as ISUP grade group $\geq 2$ ) was analyzed. Multivariate regression was performed for lesion-specific and patient-specific factors.

Results: We identified 207 lesions with mean 2.6 cores per index lesion. Multiple lesions were found in 59 (41.5\%) of patients. CsPCa was detected in $33.8 \%$ of patients, with per-lesion detection rate of $27.6 \%$. csPCa was identified in eight $(11.3 \%), 19(23.5 \%)$, and $23(41.8 \%)$ of PIRADS 3,4 , and 5 lesions, respectively. Detection of csPCa was associated with anterior lesions $(p=0.017)$, peripheral lesions $(p=0.004)$, and higher number of cores per lesion $(p=0.015)$ but inversely associated with prostate volume $(p=0.002)$. No significant associations were found between csPCa and tumor multiplicity or maximum lesion diameter.

Conclusions: Detection of csPCa within index lesions is associated with number of core biopsies per lesion, anterior location, and smaller prostate volume, but not lesion multiplicity. 


\begin{tabular}{lc}
\hline $\begin{array}{l}\text { MP-4.6. Table 1. Baseline characteristics of } \\
\text { clinical suspicion of prostate cancer who underwent }\end{array}$ \\
MRI-US fusion biopsies \\
\begin{tabular}{lc}
\hline Patient characteristic ( $\mathbf{n}=142)$ & Value \\
\hline Age, years, median (IQR) & $66(61-70)$ \\
PSA, ng/ml, median (IQR) & $9.5(7.6-14.5)$ \\
Prostate volume, ml, median (IQR) & $54.3(37.8-94.5)$ \\
Previous negative Bx & $103(72.5 \%)$ \\
Active surveillance & $26(18.3 \%)$ \\
Bx-naive & $13(9.2 \%)$ \\
\hline
\end{tabular}
\end{tabular}

MP-4.6. Table 2. Clinically significant prostate cancer (ISUP grade group $\geq 2$ ) stratified by patient type, PSA, and age

\begin{tabular}{lcc}
\hline Patient characteristic & Value \\
\hline Overall PCa (ISUP GG $\geq 1$ ) & $68(47.9 \%)$ \\
Overall csPCa (ISUP GG $\geq 2)$ & $48(33.8 \%)$ \\
Active surveillance - change in & $8(30.8 \%)$ \\
management & & $32(31.1 \%)$ \\
Previous negative bx & $<10$ & $21(43.8 \%)$ \\
PSA & $>10$ & $27(56.3 \%)$ \\
& $<50$ & $0(0 \%)$ \\
Age & $50-54$ & $2(4.2 \%)$ \\
& $55-59$ & $6(12.5 \%)$ \\
& $60-64$ & $8(16.7 \%)$ \\
& $65-70$ & $11(22.9 \%)$ \\
& $>70$ & $21(43.8 \%)$ \\
\hline
\end{tabular}

References

1. Mottet N, van der Bergh EB, Cornford P, et al. EAU guidelines: Prostate cancer. Available at: https://uroweb.org/guideline/prostatecancer/\#note_169. Published 2019. Accessed Aug.12, 2019.

2. Drost F-JH, Osses DF, Nieboer D, et al. Prostate MRI, with or without MRI-targeted biopsy, and systematic biopsy for detecting prostate cancer. Cochrane Database Syst Rev 2019;4:CD012663. https://doi. org/10.1002/14651858.CD012663.pub2

3. van Hove A, Savoie P-H, Maurin C, et al. Comparison of imageguided targeted biopsies vs. systematic randomized biopsies in the detection of prostate cancer: a systematic literature review of well-designed studies. World J Urol 2014;32:847-58. https://doi.org/10.1007/s00345-014-1332-3

4. Schoots IG, Roobol MJ, Nieboer D, et al. Magnetic resonance imaging-targeted biopsy may enhance the diagnostic accuracy of significant prostate cancer detection compared to standard transrectal ultrasound-guided biopsy: A systematic review and metaanalysis. Eur Urol 2015;68:438-50. https://doi.org/10.1016/j. eururo.2014.11.037

\section{MP-4.7}

PI-RADS v2 aids in prediction of post-radical prostatectomy NCCN risk-stratification

Amber McMahon', Kevin Hanna', Richard Sleightholm', Bryant VanLeeuwen', Shawna L. Boyle

'Department of Surgery, Division of Urologic Surgery, University of Nebraska Medical Center, Omaha, NE, United States

Introduction: Correct staging of prostate cancer is imperative to guiding treatment and magnetic resonance imaging (MRI) has emerged as a

\begin{tabular}{|c|c|c|c|c|}
\hline Characteristic & Overall & PIRADS 3 & PIRADS 4 & PIRADS 5 \\
\hline $\mathrm{n}$ & 207 & 71 & 81 & 55 \\
\hline $\begin{array}{l}\text { Cores per } \\
\text { lesion, mean }\end{array}$ & $2.6( \pm 1.1)$ & $2.2( \pm 1.1)$ & $2.7( \pm 1.1)$ & $3.2( \pm 1.1)$ \\
\hline $\begin{array}{l}\text { Overall PCa } \\
(G G \geq 1)\end{array}$ & $86(41.5 \%)$ & $15(21.1 \%)$ & $34(42.0 \%)$ & 30 (54.5\%) \\
\hline $\begin{array}{l}\text { CsPCa (GG } \\
\geq 2 \text { ) }\end{array}$ & $55(26.6 \%)$ & $8(11.3 \%)$ & 19 (23.5\%) & 23 (41.8\%) \\
\hline $\begin{array}{l}\text { CsPCa lesion } \\
\text { max diameter, } \\
\mathrm{cm} \text {, mean }\end{array}$ & $1.5( \pm 0.7)$ & $0.9( \pm 0.2)$ & $1.1( \pm 0.3)$ & $2.1( \pm 0.6)$ \\
\hline $\begin{array}{l}\text { CsPCa by } \\
\text { lesion zone }\end{array}$ & $\mathrm{n}=55$ & & & \\
\hline Peripheral & $30(54.5 \%)$ & $4(50.0 \%)$ & $11(57.9 \%)$ & 15 (65.2\%) \\
\hline Transition & $15(27.3 \%)$ & $4(50.0 \%)$ & 7 (36.8\%) & $4(17.4 \%)$ \\
\hline Central & $2(3.6 \%)$ & $0(0 \%)$ & $0(0 \%)$ & $2(8.7 \%)$ \\
\hline $\begin{array}{l}\text { CsPCa by } \\
\text { lesion location }\end{array}$ & $n=55$ & & & \\
\hline Anterior & $18(32.7 \%)$ & 1 (12.5\%) & $6(31.6 \%)$ & 11 (47.8\%) \\
\hline Mid-gland & $12(21.8 \%)$ & $5(62.5 \%)$ & $3(15.8 \%)$ & $4(17.4 \%)$ \\
\hline Posterior & $8(14.5 \%)$ & $0(0 \%)$ & $5(26.3 \%)$ & $3(13.0 \%)$ \\
\hline
\end{tabular}

potential means of preoperatively predicting outcomes. ${ }^{1}$ In 2015, PI-RADS version 2 was created to help standardize the interpretation and reporting of MRI characteristics of prostate cancer. ${ }^{2}$ The goal of this study was to compare the preoperative (preop) NCCN risk group determined via MRI-fusion biopsy ( $\mathrm{FBx}$ ) with NCCN risk group after radical prostatectomy (RP), and to determine what correlation exists between the PI-RADS score reported during preop MRI.

Methods: This was a single-institution, retrospective study of men with suspected prostate cancer who underwent an $\mathrm{MRI}$, an $\mathrm{FBx}$, and a subsequent RP between August 2017 and November 2018 ( $n=56)$. Comparisons were made between the preop NCCN risk group determined via FBx and PI-RADS with the postoperative (postop) NCCN risk group determined via postop pathological reporting radical. Logistic regression modeling was used to identify factors associated with final NCCN risk group and multivariable analysis (MVA) was used to adjust for potential confounding variables.

Results: Both preop NCCN risk and PI-RADS were associated with final NCCN risk $(p<0.0001$ and $p=0.0015$, respectively). When combined, preop NCCN risk and PI-RADS displayed an improved model of postop NCCN risk with a c-statistic of 0.821 . On MVA, preop NCCN risk and PI-RADS remained significant after adjusting for various demographics. Furthermore, the combination of preop NCCN risk and PI-RADS was better capable of distinguishing postop high-risk (NCCN 5-6) vs. intermediate-risk (NCCN 3-4) patients (c-statistic $=0.873$ ).

Conclusions: PI-RADS score combined with NCCN risk group determined via FBx correlated with postop NCCN risk group better than either biopsy or $\mathrm{PI}$-RADS alone. Additionally, the combination resulted in better prediction of high- vs. intermediate-risk patients after surgical resection. In summary, implementation of the preop PI-RADS score may be beneficial for preoperative risk-stratification and treatment options for prostate cancer patients. References

1. Chung DY, Kim MS, Lee JS, et al. Clinical significance of multiparametric magnetic resonance imaging as a preoperative predictor of oncologic outcome in very low-risk prostate cancer. J Clin Med 2019;8:542. https://doi.org/10.3390/jcm8040542

2. Weinreb JC, Barentsz JO, Choyke PL, et al. PI-RADS Prostate Imaging - Reporting and Data System: 2015, Version 2. Eur Urol 2016;69:1640. https://doi.org/10.1016/j.eururo.2015.08.052 
MP-4.8

Correlation of prostate-specific membrane antigen (PSMA) expression with intraprostatic PSMA tracer uptake detected by positron emission tomography (18F-DCFPyL PET/CT) in high-risk prostate cancer

Frédéric Pouliot ${ }^{1,2}$, Cassandra Ringuette Goulet ${ }^{1,2}$, Hélène Hovington ${ }^{1,2}$, Hervé Brisson ${ }^{1,2}$, Bertrand Neveu ${ }^{1,2}$, François-Alexandre Buteau ${ }^{3}$

'Surgery, CHU de Québec-Université Laval, Quebec City, QC, Canada; ${ }^{2}$ Oncology, Cancer Research Center, CHU de Québec-Université Laval, Quebec City, QC, Canada; ${ }^{3}$ Medical Imaging, CHU de QuébecUniversité Laval, Quebec City, QC, Canada

Introduction: Prostate-specific membrane antigen (PSMA)-targeted positron emission tomography/computed tomography (PET/CT) imaging agents have shown great promise for localizing and staging prostate cancer (PCa), and as a predictive biomarker for PSMA-targeted radioligand therapy. ${ }^{18} \mathrm{~F}-\mathrm{DCFPyL}$, a second-generation, small molecule, PSMAtargeted imaging agent, has been extensively evaluated prospectively in high-risk, recurrent, and metastatic PCa. In this study, we aimed to correlate ${ }^{18} \mathrm{~F}$-DCFPyL-intraprostatic PET uptake with PSMA protein and mRNA expression, which has not been studied previously.

Methods: Retrospective analysis was conducted for 13 patients diagnosed with a Gleason sum $\geq 8$ prostate adenocarcinoma who underwent ${ }^{18} \mathrm{~F}$-DCFPyLPET/CT imaging before radical prostatectomy (RP) and pelvic lymphadenectomy during the OSPREY study (NCT02981368). Messenger RNA from ${ }^{18} \mathrm{~F}$-DCFPyL-uptake regions at RP was extracted and corresponding slides were obtained for immunohistochemistry (IHC) analysis. Normalized PSMA, androgen receptor (AR), and PSA mRNA expression quantification was performed by GPCR. PSMA, AR, and PSA protein expression was blindly scored after IHC using specific antibodies. Correlation between ${ }^{18} \mathrm{~F}$-DCFPyL-intraprostatic uptake regions and protein or mRNA were analyzed using the Spearman's correlation coefficient. Results: Intraprostatic ${ }^{18} \mathrm{~F}$-DCFPyL-uptake was observed in 13/13 of patients (mean \pm standard deviation SUVmax 4.7 \pm 3.4 ). After prostate histopathological analysis, PCa was found at corresponding location of ${ }^{18} \mathrm{~F}$-DCFPyL uptake for all patients. Correlation was found between ${ }^{18} \mathrm{~F}$-DCFPyL uptake and PSMA expression levels from both IHC $(r=0.608 ; \mathrm{p}<0.031)$ and $\mathrm{qPCR}(\mathrm{r}=0.589 ; \mathrm{p}<0.037)$ analyses. No correlation was found between ${ }^{18} \mathrm{~F}$-DCFPyL uptake and PSA or AR expression levels. Conclusions: We show that intraprostatic ${ }^{18} \mathrm{~F}$-DCFPyL uptake on PET/ CT correlates with PCa PSMA expression. Therefore, ${ }^{18} \mathrm{~F}$-DCFPyL PET/CT imaging can be used as an alternative marker for tissue PSMA expression levels, a property that is of great value for PSMA-targeted radioligand therapy studies.

\section{MP-4.9}

The association between income status and treatment selection for prostate cancer in a universal healthcare system: A population-based analysis

Lustin Oake', Oksana Harasemiw2,3, Navdeep Tangri' ${ }^{2,3,4}$, Thomas W. Ferguson 2,3, Jeffery W. Saranchuk', Rahul K. Bansal', Darrel E. Drachenberg', Jasmir G. Nayak

'Section of Urology, University of Manitoba, Winnipeg, MB Canada; ${ }^{2}$ Chronic Disease Innovation Centre, Seven Oaks General Hospital, Winnipeg, MB, Canada; ${ }^{3}$ Department of Internal Medicine, University of Manitoba, Winnipeg, MB, Canada; ${ }^{4}$ Department of Community Health Sciences, University of Manitoba, Winnipeg, MB, Canada

Introduction: Socioeconomic disparities are known to affect cancer treatment options, particularly in a predominantly privatized healthcare system. Whether these disparities exist in the management of localized prostate cancer in a universal healthcare system is unknown. We sought to evaluate whether income status, as a surrogate for socioeconomic status (SES), was associated with the disparate management (radical prostatectomy $[R P]$ vs. radiation therapy $[R T]$ ) for men diagnosed with nonmetastatic prostate cancer.

Methods: Men from Manitoba, Canada who were diagnosed with nonmetastatic prostate cancer between 2004 and 2016 and subsequently treated with RP or RT were identified using a provincial cancer database and linked with hospital discharge datasets and vital statistics. SES was defined as neighborhood income by postal code and divided into income quintiles (Q1-Q5, with Q1 the lowest quintile and Q5 the highest). Income quintiles were derived for entire provincial population. Multivariable logistic regression nested models were used to compare whether SES was associated with treatment type received.

Results: We identified 4560 individuals from 2004-2016 who were diagnosed with non-metastatic prostate cancer; 2554 men were treated with RP and 2006 with RT. Adjusting for age, comorbid status, rurality, and stage of disease, as income quintile increased, men were incrementally more likely to undergo RP than RT (Q3 vs. Q1: adjusted odds ratio [aOR] 1.45, 95\% cmfidence interval [CI] 1.09-1.92; Q5 vs. Q1: aOR 2.17; 95\% Cl 1.52-2.86).

Conclusions: Higher income levels were associated with an increased odds of receiving RP rather than RT for localized prostate cancer. These findings may inform initiatives to better understand the values, priorities, and barriers that patients experience when making treatment decisions in a universal healthcare system that aims to provide care equally to all residents.

\section{MP-4.10}

A comparison of cancer detection rates between template systematic biopsies obtained using magnetic resonance imagingultrasound (MRI-US) fusion machines and free-hand transrectal ultrasound-guided systematic biopsies

Connor Hoge ${ }^{1}$, Sean Maynor ${ }^{1}$, Tianyuan Guan ${ }^{1}$, Rand Naffouje ${ }^{2}$, Monzer Haj-Hamed ${ }^{1}$, Nilesh Patil', James Donovan' ${ }^{1}$, Krishnanath Gaitonde', Sadhna Verma ${ }^{3}$, Abhinav Sidana ${ }^{1}$

'Department of Urology, University of Cincinnati College of Medicine, Cincinnati, $\mathrm{OH}$, United States; ${ }^{3}$ Department of Hematology and Oncology, University of Cincinnati College of Medicine, Cincinnati, $\mathrm{OH}$, United States; ${ }^{2}$ Department of Radiology, University of Cincinnati College of Medicine, Cincinnati, $\mathrm{OH}$, United States

Introduction: There are some reports that the 12-core template systematic biopsies ( $\mathrm{SBx}$ ) obtained using software registration machines (e.g., Artemis) have higher cancer detection rates (CDRs) of prostate cancer (PCa) than that of the standard 12-core transrectal ultrasound (TRUS)guided biopsies. The goal of our study is to compare the clinically significant (CS) CDRs of SBx in two independent cohorts who underwent TRUS systematic biopsy alone (cohort A) or underwent SBx as a part of a combined magnetic resonance imaging-ultrasound (MRI-US) fusion biopsy ( $\mathrm{FBx}$ ) using a software registration machine (cohort $\mathrm{B}$ ).

Methods: A retrospective review of all patients undergoing prostate biopsies over a two-year period at the University of Cincinnati Medical Center was performed. CS cancer was defined as having a Gleason score $\geq 7$. MRI-US FBx were obtained using an Artemis software registration device (ARTEMIS ${ }^{\text {TM }}$, Eigen Inc., Grass Valley, CA). Statistical significance was considered at $\mathrm{p}<0.05$.

Results: Nine hundred and thirty men underwent SBx (474 in cohort $A$ and 456 in cohort B). The CS CDR of SBx in cohorts A and B were $39.3 \%$ and $33.8 \%$, respectively $(\mathrm{p}=0.093)$. In patients with no prior history of prostate biopsy, SBx CDR for CS disease was $40.4 \%$ and $38.0 \%$ in cohorts A and B, respectively ( $p=0.951)$. Patients in cohort A and B with a prior negative biopsy had a CS CDR of $22.7 \%$ and $25.0 \%$, respectively $(p=0.910)$. Multivariate logistic regression controlling for age, prostatespecific antigen level, abnormal digital rectal exam, and family history of PCa demonstrated comparable CS CDR by SBx in the two cohorts even when stratified by prior biopsy history (all patients $p=0.958$, biopsy-naive $p=0.291$, prior negative prostate biopsy $p=0.403$ ) (Table 1 ).

Conclusions: Our study did not find a significant difference in the CS CDRs of SBx using registration software as compared to SBX using freehand TRUS. Unless the SBx is done at the time of MRI-US fusion biopsy, use of these machines for obtaining SBx only is unlikely to result in any increase of CS CDR. 


\begin{tabular}{|c|c|c|c|}
\hline \multicolumn{4}{|c|}{$\begin{array}{l}\text { MP-4.10. Table 1. Odds ratio of free hand TRUS biopsy } \\
\text { detecting CS cancer over template guided systematic } \\
\text { biopsy after controlling for age, race, PSA, DRE, and } \\
\text { family history of PCa }\end{array}$} \\
\hline & Odds ratio & $95 \% \mathrm{Cl}$ & $\mathbf{p}$ \\
\hline All patients & 0.99 & $0.71-1.38$ & 0.958 \\
\hline Biopsy-naive & 0.79 & $0.51-1.22$ & 0.291 \\
\hline Prior negative & 0.64 & $0.21-1.75$ & 0.403 \\
\hline
\end{tabular}

MP-4.11

Explainable AI: Using machine learning to identify risk factors and explain improved predictions of extra-prostatic extension in pre-prostatectomy patients

Lethro C.C. Kwong', Simona Minotti ${ }^{2,3}$, Adrian Cozma ${ }^{4}$, Ashkan Javidan', Amna Ali ${ }^{2}$, Munir A. Jamal ${ }^{5}$, Thomas Short ${ }^{5}$, Frank F. Papanikolaou ${ }^{5}$, John R. Srigley ${ }^{6}$, Andrew H. Feifer 2,5

${ }^{1}$ Faculty of Medicine, University of Toronto, Toronto, ON, Canada; ${ }^{2}$ Institute for Better Health, Trillium Health Partners, Mississauga, ON, Canada; ${ }^{3}$ Department of Statistics and Quantitative Methods, University of Milano-Bicocca, Milano, Italy; ${ }^{4}$ Department of Radiation Oncology, University of Toronto, Toronto, ON, Canada; ${ }^{5}$ Department of Surgery, University of Toronto, Toronto, ON, Canada; ${ }^{6}$ Department of Laboratory Medicine and Pathobiology, University of Toronto, Toronto, ON, Canada Introduction: Current nomogram predictions of extra-prostatic extension (EPE) in pre-prostatectomy patients use logistic regression on a limited set of covariates. While incorporating machine learning $(\mathrm{ML})$ methodology may improve predictive accuracy, their "black-box models" limit interpretability in the clinical setting. We used explainable ML to identify additional pre-prostatectomy risk factors of EPE and explain how each feature may enhance predictive capacity compared to current tools.

Methods: Our prostatectomy database includes 351 patients with 21 covariates (known as features in ML terminology). We used random forest classification, which is an ensemble ML method composed of a collection of decision trees. Feature selection was determined by Gini impurity and permutation importance. Hyperparameter tuning included number and depth of each tree. Ten-fold cross-validation was used for model development, tuning, and validation. The reference standard used for comparison was the Memorial Sloan Kettering Cancer Center (MSKCC) pre-prostatectomy nomogram.

Results: A total of 167/351 (47.6\%) patients had EPE. The top six features were: \% tissue involvement in overall biopsy, \% involvement of most involved core, \% involvement of highest Gleason core, pre-prostatectomy prostate-specific antigen (PSA), quantitative $\%$ Gleason 4/5 on biopsy, and perineural invasion. The ML model achieved an area-under-curve (AUC) of 0.771 vs. 0.674 for the MSKCC nomogram ( $p=0.006)$. Setting sensitivity at 0.80 , the ML model achieved higher accuracy $(0.67$ vs. 0.60$)$, specificity $(0.55$ vs. 0.43$)$, positive predictive value $(0.62 \mathrm{vs} .0 .56)$, and negative predictive value ( 0.75 vs. 0.70 ) compared to the MSKCC nomogram. The ML model generates feature weights on a case-by-case basis to explain how each feature contributes to the final prediction (Table 1).

Conclusions: Our preliminary ML model performed favorably compared to our reference standard. Further studies with larger datasets are needed to validate this methodology.

\section{MP-4.12}

Preoperative neutrophil to lymphocyte ratio predicts adverse pathology at radical prostatectomy

Kush M. Joshi ${ }^{1}$, Arnon Lavi ${ }^{2}$, Ray S. Jia ${ }^{1}$, David Z. Guy ${ }^{1}$, Danielle A. Starcevic ${ }^{1}$, Sophia Moralis Frost ${ }^{1}$, Natan Veinberg ${ }^{1}$, Shiva M. Nair ${ }^{2}$, L.K. Joseph Chin ${ }^{2}$

${ }^{1}$ Schulich School of Medicine \& Dentistry, Western University, London, ON, Canada; ${ }^{2}$ Urology Division, Department of Surgery, Schulich School of Medicine and Dentistry, Western University, London, ON, Canada Introduction: Neutrophil to lymphocyte ratio (NLR) has been reported to have prognostic significance for a variety of malignancies, including urological cancers. We set to define the prognostic value of NLR before radical retropubic prostatectomy (RRP).

Methods: We analyzed our retrospective RRP database to assess the predictive value of NLR. A cutoff of 3.5 was used based on previous reports. A logistic regression was preformed to evaluate the correlation between NLR and adverse pathological characteristics at RRP.

Results: Complete data was available in 698 patients. Correlation between NLR and common adverse RRP pathology parameters were assessed (Table 1). Higher NLR was found to predict upgrading at RRP, extraprostatic extension (EPE), and seminal vesical involvement (SVI) both on univariable analysis (odds ratios [OR] 1.94, $\mathrm{p}=0.002$; OR 1.9, $\mathrm{p}<0.001$; OR $2.76, p<0.0001$, respectively) and multivariable analysis adjusted for established preoperative prognostic parameters (OR 1.74, $p=0.027$; OR 2.41, $\mathrm{p}<0.0001$; OR 3.95, $\mathrm{p}<0.0001$, respectively). Presence of positive lymph nodes and positive surgical margins were not correlated with high NLR.

Conclusions: In this current cohort, higher NLR predicts upgrading at RRP, $\mathrm{EPE}$, and SVI and may be a commonly available prognostic indicator for poorer outcome in patients undergoing radical prostatectomy.

\begin{tabular}{|c|c|c|c|c|}
\hline Patient \#1 & Patient \#2 & ML-model features & $\begin{array}{l}\text { ML-model weights } \\
\text { for Patient \#1 }\end{array}$ & $\begin{array}{l}\text { ML-model weights } \\
\text { for Patient \#2 }\end{array}$ \\
\hline & & Constant & 0.472 & 0.472 \\
\hline 20 & 5 & $\begin{array}{c}\text { Quantitative } \% \text { Gleason } \\
4 / 5 \text { on biopsy }\end{array}$ & 0.002 & -0.014 \\
\hline 20 & 5 & $\begin{array}{l}\% \text { tissue involvement in } \\
\text { overall biopsy }\end{array}$ & 0.092 & -0.065 \\
\hline 35 & 22.5 & $\begin{array}{c}\% \text { involvement of highest } \\
\text { Gleason core }\end{array}$ & -0.016 & -0.069 \\
\hline 80 & 22.5 & $\begin{array}{l}\% \text { involvement of most } \\
\text { involved core }\end{array}$ & 0.076 & -0.078 \\
\hline Yes & No & Perineural Invasion & 0.038 & -0.015 \\
\hline Yes & No & $\begin{array}{l}\text { Probability of EPE } \\
\text { (predicted) }\end{array}$ & 0.686 & 0.227 \\
\hline
\end{tabular}




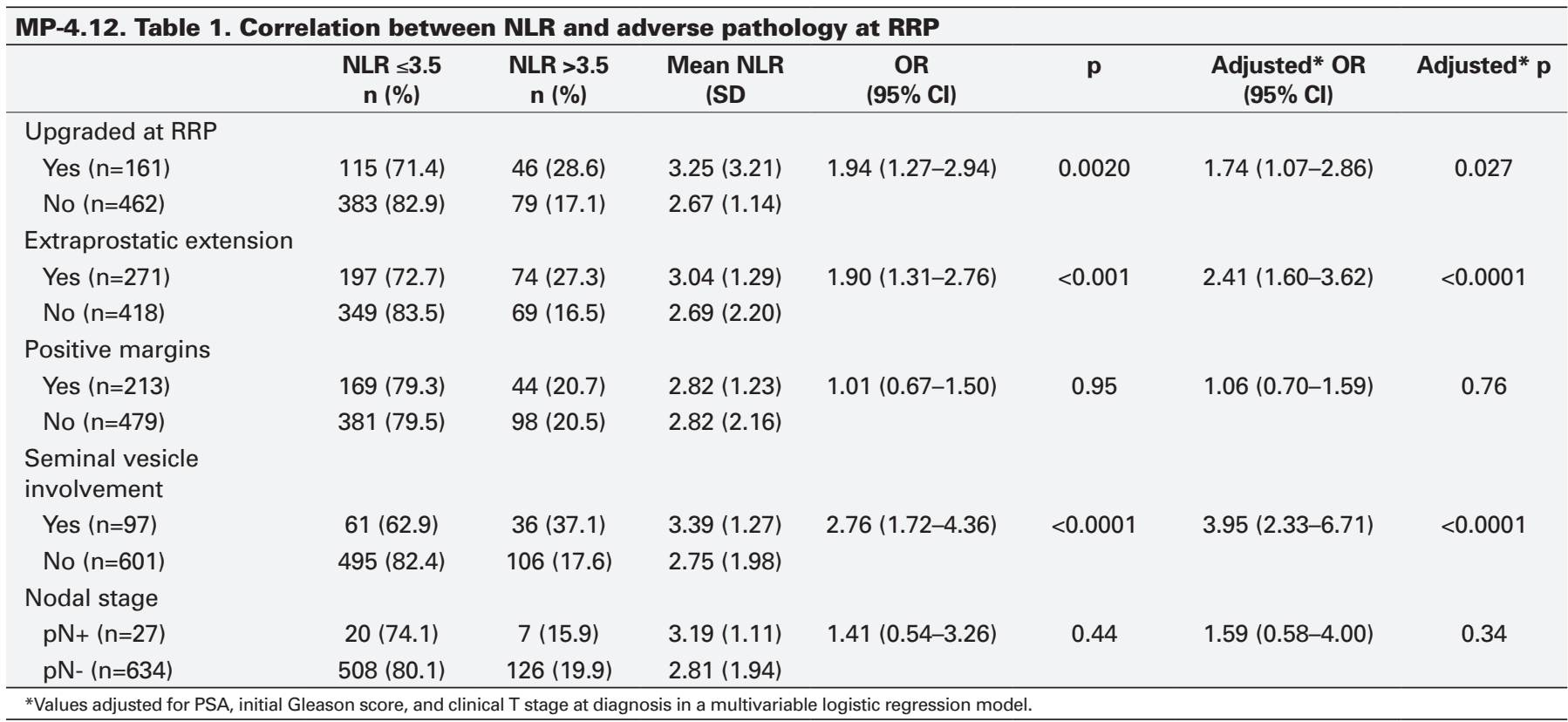

\section{MP-4.13}

Impact of surgical wait times during summer months on the oncological outcomes following robotic-assisted radical prostatectomy: 10 years' experience from a large Canadian academic center

Ahmed S. Zakaria Ahmed', Félix Couture'2, David-Dan Nguyen ${ }^{3}$, Hanna Shahine ${ }^{1}$, Côme Tholomier ${ }^{3}$, Cristina Negrean' ${ }^{1}$ Kyle Law³ ${ }^{3}$ Pierre Karakiewicz', Assaad El-Hakim', Kevin C. Zorn ${ }^{1}$

${ }^{1}$ Urology, University of Montreal Hospital Center, Montreal, QC, Canada; ${ }^{2}$ Urology, University of Sherbrooke Hospital Center, Montreal, QC, Canada; ${ }^{3}$ Urology, McGill University Health Center, Montreal, QC, Canada

Introduction: Compared to the other seasons of the year, most Canadian hospitals face significant reductions $(20-50 \%)$ in operative room access during summer months due to nursing shortages, leading to increased surgical delays. Hence, we sought to assess the impact of this extra wait time to undergo robotic-assisted radical prostatectomy (RARP) on the oncological outcomes of localized prostate cancer.

Methods: We conducted a retrospective review of a prospectively maintained RARP database in two high-volume academic centers between 2010 and 2019. Wait time was defined as the interval between surgical booking and RARP. Assessed outcomes included impact on the difference between post-biopsy USCF-CAPRA and post-surgical CAPRA-S scores, biochemical recurrence (BCR) rates, and Gleason score upgrade on surgical specimen. Multivariable analysis (MVA) with regression models was used to evaluate the effect of wait times.
Results: A total of 1057 men were included for analysis. Consistent over a 10-year period, analysis of mean surgical/operative booking volumes revealed that summer months had the lowest surgical volumes output despite above-average booking volumes. The lowest surgical volume occurred during July (7.1 case/month), which was 35\% less than the cohort average. Moreover, summer months had the longest average time between surgical booking date and RARP, with the longest wait occurring for patients booked in June (average $93 \pm 69$ days, $p<0.001$ ). On MVA, patients booked in June had significantly more chance of having an increase in CAPRA score [(hazard ratio [HR] 1.64; 95\% confidence interval $[\mathrm{Cl}] 1.02-2.63 ; \mathrm{p}=0.04)$ and in CAPRA risk group (HR 1.82; $95 \%$ $\mathrm{Cl}$ 1.04-3.19) after surgery compared to patients booked in other months. Moreover, Cohort analysis showed fair correlation between CAPRA-score difference and time between booking and RARP $(r=-0.062 ; p=0.044)$.

Conclusions: Our cohort results demonstrate that conventional RARP wait times are significantly and consistently prolonged during summer months, with worse post-RARP oncological outcomes in terms of CAPRA score, which is associated with a higher risk of BCR. Further multispecialty and large-scale national studies are required to address these delays in other oncological populations. Furthermore, other compensatory mechanisms to sustain consistent yearly operative output should be considered. 


\section{Moderated Poster Session 5: Technical Advances in Urology, Improving Patient Relations}

\begin{abstract}
MP-5.1
A randomized controlled trial of "bag squeeze" to minimize discomfort in male outpatient flexible cystoscopy

Mohamad Baker Berjaoui', Ishan Aditya ${ }^{2}$, Jaime Herrera Caceres ${ }^{1}$, Khaled Ajib', Yazan Qaoud', Jason Y. Lee ', Alexandre Zlotta' ${ }^{1}$, Antonio Finelli', Robert J. Hamilton ${ }^{1}$, Nathan Perlis ${ }^{1}$, Girish S. Kulkarni', Neil E. Fleshner ${ }^{1}$

${ }^{1}$ Urological Oncology, University Health Network, Princess Margaret Cancer Centre, Toronto, ON, Canada; ${ }^{2}$ School of Medicine, University of Toronto, Toronto, ON, Canada

Introduction: Among the most common symptoms of patients undergoing flexible cystoscopy are pain and discomfort. Hydrodistension of prostate is a commonly used, yet unproven technique used by physicians to minimize outpatient flexible cystoscopy-induced discomfort. This maneuver involves the health professional performing a "bag squeeze" of the irrigation fluid as one traverses the distal urinary sphincter until passing through the bladder neck. Herein, we report results from a single-center, single-blinded, randomized trial to determine whether use of the "bag squeeze" technique during flexible cystoscopy significantly alters pain scores.

Methods: Consenting participants were all male, outpatients, ambulatory who had undergone a prior cystoscopy and not expected to require any secondary procedures (e.g., stent, tumor fulguration, etc.). Men with prior urethral stricture or bladder neck contracture were excluded. They were randomized to group A (bag squeeze) or group B (no bag squeeze). Following the procedure, all men responded to a Likert scale pain grid. Mean pain score differences between the groups were tested using a Students t-test with two-sided alpha of 0.05 .
\end{abstract}

Results: Two hundred patients were recruited and underwent a flexible cystoscopy. Ten were deemed ineligible as they required secondary procedures. Among the 190 eligible patients, 97 were randomized to squeeze arm and 93 to no squeeze arm. Mean pain scores were 3.39 in the no bag squeeze arm and 1.91 in the bag squeeze arm $(p<0.005)$.

Conclusions: This study showed a statistically and clinically significant decrease in pain score with pericystoscopic bag squeeze and accordingly should be considered a suitable, simple, and affordable method to improve patient comfort during flexible cystoscopy.

\section{MP-5.2}

Evaluation of a cystoscopy smartphone light adaptor on a urological endoscopy simulator

\section{Yanbo Guo ${ }^{1}$, Anil Kapoor ${ }^{1}$, Edward D. Matsumoto ${ }^{7}$}

${ }^{1}$ Urology, McMaster University, Hamilton, ON, Canada

Introduction: Cystoscopy is a vital tool that is often needed at the bedside. Current practice requires a significant amount of equipment to be brought to the patient, including heavy light sources and dedicated cystoscopy towers. We developed an affordable universal adapter that uses the light from a smartphone rather than a conventional light source, minimizing the inconvenience and cost associated with bedside cystoscopy. Here, we evaluated the degradation of the video quality using this light adapter compared to a standard light source on a high-fidelity cystoscopy simulator.

Methods: A video of the urethra and bladder were produced. They were recorded using a HD video endoscopy tower (Stryker, U.S.), a flexible cystoscope (Olympus, Japan), and a urological endoscopy trainer (Limbs \& Things, U.K.). The videos were then evaluated using a double stimulus impairment scale method. ${ }^{1}$ Viewers were shown a reference and test samples, and then asked to evaluate the level of impairment using a standardized Likert scale. The reference video used a conventional light source
(Stryker) while the test video used the adaptor and an iPhone X (Apple, U.S.). They were also asked how comfortable they would be to use the light adapter to perform common cystoscopy procedures.

Results: Thirty urology trainees and staff participated in this trial. For the urethra, all respondents rated a 4 (perceptible impairment but not annoying) or 5 (no impairment) for overall video quality. For the bladder, $80 \%$ of respondents rated a 2 (impairment was very annoying) or 3 (impairment was annoying) for overall video quality. Most (96\%) of evaluators rated a 4 (often) or 5 (always) on their comfort of using this adapter for the placement of a difficult catheter and removal of a ureteric stent.

Conclusions: The novel light adaptor caused minimal impairment when visualizing the urethra but significant impairment in the bladder. Respondents were also comfortable using the adapter for common bedside cystoscopic procedures.

Reference

1. International Telecommunication Union, Methodologies for the subjective assessment of the quality of television images BT.500-14. 2019

\section{MP-5.3}

Evaluation of a novel, single-use flexible cystoscope: A multiinstitutional international study

Victor Wong ${ }^{1}$, Kymora Scotland ${ }^{2}$, Justin Y.H. Chan' ${ }^{1}$ Ehab Tawfiek ${ }^{3}$, Allen Chiura $^{4}$, Demetrius Bagley ${ }^{5}$, Ben H. Chew

${ }^{1}$ Department of Urologic Sciences, University of British Columbia, Vancouver, BC, Canada; ${ }^{2}$ Department of Urology, University of California Los Angeles, Los Angeles, CA, United States; ${ }^{3}$ Department of Urology, El-Minia University Hospital, El-Minia, Egypt; ${ }^{4}$ Parirenyatwa Hospital, Harare, Zimbabwe; ${ }^{5}$ Department of Urology, Thomas Jefferson University, Philadelphia, PA, United States

Introduction: The flexible cystoscope is an indispensable tool in urology but also requires high acquisition (including a tower), maintenance, and reprocessing costs..$^{1-3} \mathrm{~A}$ potential solution to such problems is single-use cystoscopes. The aim of this study is to evaluate the NeoFlex Single-Use Flexible Cystoscope ${ }^{\mathrm{TM}}$ (Neoscope Inc.) to standard-of-care reusable flexible cystoscopes (Olympus CYF-VH) in a bench-top and clinical setting. The single-use cystoscope is powered and visualized by direct USB connection to a laptop, therefore, obviating the need for other expensive equipment, such as controllers, towers, and monitors. This provides true portability and may provide more accessibility in international settings.

Methods: The single-use digital flexible cystoscopes (Neoscope) were prospectively evaluated against reusable digital flexible cystoscopes in 25 clinical procedures in multiple international sites. During clinical assessment, performance in maneuverability, visualization, scope deflection, image colour, and illumination were investigated. Bench-top assessments were also conducted.

Results: Bench testing revealed smaller tip diameter $(4.06 \mathrm{vs.} 6.09 \mathrm{~mm})$ in the single-use cystoscopes. Deflection of the single-use scope was superior with an empty working channel (230/220) vs. the reusable (195/95) but was more substantially decreased when an instrument was placed in the channel. Clinical use revealed satisfactory maneuverability, ease of use of instruments, deflection, and visualization between the two scopes. Most $(96 \% ; 24 / 25)$ clinical procedures conducted with the Neoflex single-use cystoscope were successful.

Conclusions: The Neoflex Single-use Flexible Cystoscope offers comparable maneuverability, imaging, and illumination to standard-of-care flexible reusable cystoscopes without the need for maintenance or repurposing. This 
would improve accessibility to flexible cystoscopy in international settings by obviating the need for expensive tower equipment.

\section{References}

2. Shah J. Endoscopy through the ages. BJU Int 2002;89:645-52. https://doi.org/10.1046/j.1464-410X.2002.02726.x

3. Phan YC, Cobley J, Mahmalji W. Cost analysis and service delivery on using Isiris $\alpha^{\mathrm{TM}}$ to remove ureteric stents. I Endolum Endourol 2018;1:e3-16. https://doi.org/10.22374/jeleu.v1i1.5

4. Ibbotson S, Dettmer T, Kara S, et al. Eco-efficiency of disposable and reusable surgical instruments-a scissors case. Int / Life Cycle Assess 2013;18:1137-48. https://doi.org/10.1007/s11367-013-0547-7

\section{MP-5.4}

\section{Clinical validation of an audio-based uroflowmetry app in adult males}

Mark Dawidek ${ }^{1}$, Rohit Singla², Louisa Ho Ho , Lucie Spooner ${ }^{1}$, Christopher Y. Nguan 1,2

'Department of Urologic Sciences, University of British Columbia, Vancouver, BC, Canada; ${ }^{2} \mathrm{MD} / \mathrm{PhD}$ Program, Faculty of Medicine, University of British Columbia, Vancouver, BC, Canada

Introduction: Uroflowmetry is a common test for evaluating voiding by measuring urine flow directly. "Sonouroflowmetry" is a novel approach that determines urine flow from acoustic measurement alone. Available as a software app on mobile devices, it is low-cost and portable. It has potential for screening and monitoring common urological pathologies, particularly in out-of-office environments. This study is the first to robustly evaluate sonouroflowmetry in a clinical setting against the gold standard.

Methods: Adult male patients $(n=69)$ attending a general urology clinic were recruited. Sonouroflowmetry was performed using the publicly available Uroflow Tracings (Traders Micro, Montreal, QC) app. Conventional uroflowmetry was performed using the Urocap IV Uroflowmeter (Laborie, Brossard, QC). MATLAB (MathWorks, Natick, MA) was used to extract flow curve data. Bland-Altman analysis was used to compare performance with respect to max flow, time to max flow, and total voiding time. Symmetric mean absolute percentage error (SMAPE) was used to quantitively compare curve shapes.

Results: The correlation coefficient for max flow was $0.25(p=0.04)$. This increased to $0.41(p<0.001)$ when normalized by the voided volume, with limits of agreement (LoA) $-123 \%$ and $-17 \%$. Correlation for time to max flow was 0.61 ( $p=0.002)$ with LoA $-120 \%$ and $165 \%$. Correlation for total voiding time was $0.92(\mathrm{p}<0.001)$ with LoA $-41 \%$ and $38 \%$. The SMAPE for curve shape was $32.7 \%$ (standard deviation 14\%), corresponding to an accuracy of $67.3 \%$.

Conclusions: Sonouroflowmetry was inconsistent in evaluating flow magnitude. This is attributable to high variability and difficult standardization for acoustic signals. Performance improved with respect to temporal variables. The curve shape was surprisingly concurrent, both subjectively and objectively. Further work evaluating intra-patient reliability and pathologyspecific performance is required to fully evaluate sonouroflowmetry as a screening or monitoring tool.

\section{MP-5.5}

\section{Causes of anxiety surveyed in patient supporters during the perioperative process}

Keesha Khehra ${ }^{1}$, Liz Burden ${ }^{1}$, Rohit Singla ${ }^{2,3}$, Angela Cho ${ }^{4}$, Christopher Y. Nguan ${ }^{4}$

${ }^{1} \mathrm{MD}$ Undergraduate Program, Faculty of Medicine, University of British Columbia, Vancouver, BC, Canada; ${ }^{2} \mathrm{MD} / \mathrm{PhD}$ Program, Faculty of Medicine, University of British Columbia, Vancouver, BC, Canada; ${ }^{3}$ School of Biomedical Engineering, University of British Columbia, Vancouver, BC, Canada; ${ }^{4}$ Department of Urologic Sciences, University of British Columbia, Vancouver, BC, Canada

Introduction: The perioperative process proves to be an anxiety- and stress-inducing ordeal for not only the patients, but their family and friends as well. While their loved one is undergoing a surgical procedure, patient supporters are left in uncertainty while having to navigate an unfamiliar clinical environment. In order to improve this process and reduce undue stress, this work investigates the causes of worry in the perioperative process, as well as supporter-staff dynamics in a breadth of surgeries. Methods: A survey was administered to family and friends in the Surgical Family Waiting Room at Vancouver General Hospital $(n=80)$. The survey consisted of questions regarding the respondents' relation to the patient, the surgical procedure taking place, and anxiety levels. Causes of such anxiety were explored, as was communication with the healthcare team. Results: Most participants were waiting during what they perceived to be a major surgery $(84 \%, n=67)$. Most frequently reported causes of worry included morbidity and mortality $(71 \%, \mathrm{n}=57)$ and logistics regarding the patient's location, status, and surgical timeline $(21 \%, n=17)$. The majority reported less than three visits to the nursing station $(82 \%, n=59)$, with $30 \%(n=24)$ reporting hesitancy in approaching staff for updates. The types of questions participants had were centered around logistics, procedural status, and timeline. When prompted on how communication could be improved, more frequent updates during surgery were desired $(44 \%, n=35)$.

Conclusions: There is a clear need for improved communication between the healthcare team and patient supporters in order to properly address the anxiety they experience. Providing more frequent updates, having an improved understanding of the perioperative period, and gaining better access to the surgical team are all avenues to ease their stress. Future work will involve determining the feasibility of a communications platform to address these identified gaps.

\section{MP-5.6}

Use of multimedia in postoperative patient education: A quality improvement initiative

Ailsa Gan' , Luke Witherspoon', Rodney H. Breau', Ranjeeta Mallick', Ilias Cagiannos ${ }^{1}$, Christopher G. Morash', Luke T. Lavallée

'Department of Surgery, Division of Urology, University of Ottawa, Ottawa, ON, Canada

Introduction: Variation in the discharge process has been found to increase discharge times and decrease quality of care. ${ }^{1}$ This quality improvement study examined if a multimedia-based resource could decrease late discharges after robotic prostatectomy (after 2 pm on postoperative day 1 ) to $<25 \%$.

Methods: From April 2018 to October 2019, all patients undergoing robotic-assisted radical prostatectomy (RARP) were asked to complete an anonymous survey using Likert scales evaluating the perioperative experience. The quality improvement (QI) intervention started in March 2019. A series of six educational videos were shown via an iPad to all patients undergoing RARP. The videos were used to supplement postoperative instruction prior to discharge. The discharge times of all patients were obtained from The Ottawa Hospital Data Repositories derived directly from the electronic medical record. A Student's t-test was used to compare mean discharge time (primary outcome). A run chart analysis was used to detect change in discharge time (QI outcome measure). Patient satisfaction and experience (QI balancing measure) was analyzed using chi-squared analysis.

Results: A total of 347 robotic prostatectomies (199 pre-intervention, 148 post-intervention) were available. Mean discharge time was not significantly reduced in the intervention group compared to non-intervention group (2:14 pm vs. $2: 37 \mathrm{pm} ; \mathrm{p}=0.12)$. Analysis of the run chart revealed that no rules were met to provide evidence for non-random change (Fig. 1). A total of 140 surveys (59 pre-intervention, 81 post-intervention) were completed, corresponding with a response rate of $29.6 \%$ and $54.7 \%$, respectively. Median score on a 10-point scale for overall satisfaction was equal between the intervention and non-intervention groups ( 9 [interquartile range (IQR) 8-10] vs. 10 [IQR 8-10]; $p=0.92$ ). There were no differences in scores for anxiety at discharge, postoperative care, and completeness of postoperative instruction between groups.

Conclusions: Use of multimedia resources did not reduce discharge times after robotic prostatectomy in this study. Patient perception of care and education is high and was not negatively impacted when postoperative instruction was delivered using multimedia resources. 


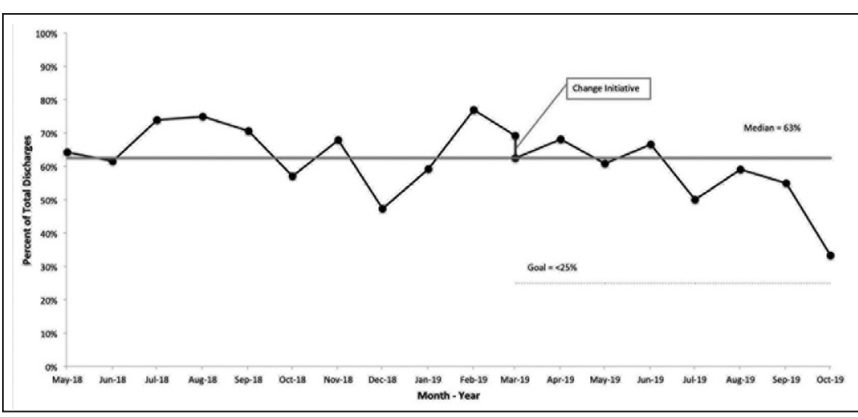

MP-5.6. Fig. 1. Run chart displaying percent of total discharges classified as "late" (after $2 \mathrm{pm}$ ) before and after implementation of video-based educational tool (change initiative) for patients undergoing robotic-assisted radical prostatectomy.

\section{Reference}

1. Arafeh M, Barghash MA, Haddad N, et al. Using six sigma DMAIC methodology and discrete event simulation to reduce patient discharge time in King Hussein Cancer Center. J Healthc Eng 2018;2018:3832151. https://doi.org/10.1155/2018/3832151

\section{MP-5.7}

PatientLink: A novel application to address stress and anxiety in patient supporters during surgical operations

Liz Burden ${ }^{1}$, Lucie Spooner ${ }^{2}$, Rohit Singla ${ }^{3,4}$, Keesha Khehra' ${ }^{1}$ Angela Cho ${ }^{2}$, Christopher Y. Nguan²

${ }^{1} \mathrm{MD}$ Undergraduate Program, University of British Columbia Faculty of Medicine, Vancouver, BC, Canada; ${ }^{2}$ Urologic Sciences, University of British Columbia, Vancouver, BC, Canada; ${ }^{3}$ School of Biomedical Engineering, University of British Columbia, Vancouver, BC, Canada; ${ }^{4} \mathrm{MD} /$ PhD Program, University of British Columbia Faculty of Medicine, Vancouver, BC, Canada

Introduction: The perioperative process is a stressful ordeal for not only patients but their supporters. Prior work revealed a need for improved communication between the healthcare team and a patient's loved ones. This pilot study proposes a means to alleviate worry in the form of a patient-centered, online communications platform. PatientLink is a webbased tool developed in-house that enables secure, one-way communication of patient status to their loved ones. An individualized operation tracker is sent to patient supporters reflecting six main stages of the perioperative process, with a statement describing each. The patient can be followed by their supporters, in hospital and remotely, as the surgical team logs in and timestamps events as real-time status updates. This pilot evaluates the viability of PatientLink.

Methods: After ethics approval and informed consent, patients participated in the PatientLink pilot. Prior to the operation date, an individualized link to a unique webpage was created and distributed via email to

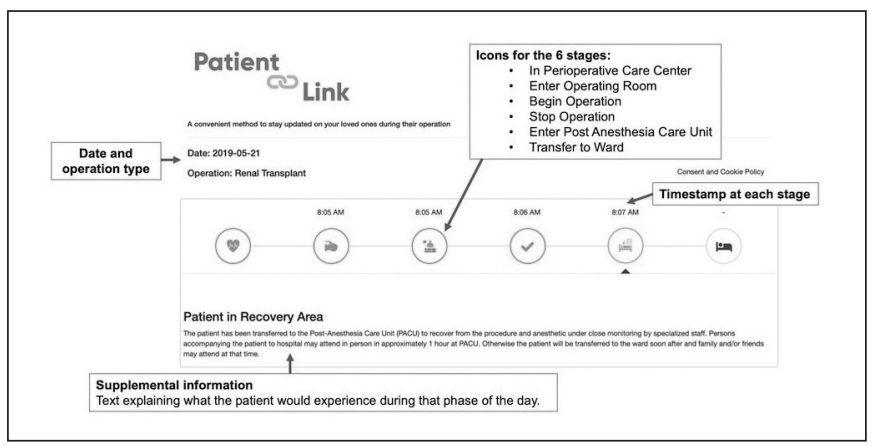

MP-5.7. Fig. 1. The annotated participant's view of Patient Link the patient and/or their loved ones. On the operation date, the surgical team provided real-time status updates to this webpage. An online survey was administered at the conclusion of the perioperative process.

Results: All patients were undergoing major surgery $(n=11)$ : five kidney recipients, three oncological nephrectomies, and three donor nephrectomies. PatientLink platform satisfaction was reported as neutral for $9 \%$ of respondents, satisfied for $28 \%$, very satisfied for $63 \%$. PatientLink was reported as reducing anxiety and stress levels in $73 \%$. In a trans-Canada case, a user stated, "I was able to access [PatientLink] comfortably and see the patient move through the process... Felt like I had a connection to her even though I was in a different province."

Conclusions: Early reports show PatientLink as a novel online platform that improves quality of care for patient supporters. Further data collection and validation of preliminary results is warranted.

\section{MP-5.8}

Outcomes of a clinical trial exploring the value of adding patient-centered prostate magnetic resonance imaging reports to traditional ones in clinical practice

Nathan Perlis ${ }^{1}$, Antonio Finelli', Mike Lovas ${ }^{2}$, Amelia Di Meo ${ }^{1}$, Alejandro Berlin', Janet Papadakos', Sangeet Ghai ${ }^{5}$, David Wiljer ${ }^{6}$, Vasiliki Bakas, Joseph Cafazzo ${ }^{8}$, Masoom Haider ${ }^{9}$

${ }^{1}$ Surgical Oncology, Division of Urology, University Health Network, Toronto, ON, Canada; ${ }^{2}$ Healthcare Human Factors, University Health Network, Toronto, ON, Canada; ${ }^{3}$ Radiation Oncology, University Health Network, Toronto, ON, Canada; ${ }^{4}$ ELLICSR: Health, Wellness \& Cancer Survivorship Centre, University Health Network, Toronto, ON, Canada; ${ }^{5}$ Joint Department of Medical Imaging (JDMI), University Health Network, Toronto, ON, Canada; ${ }^{6}$ Education Technology Innovation at UHN Digital, University Health Network, Toronto, ON, Canada; ${ }^{7}$ myUHN Patient Portal, University Health Network, Toronto, ON, Canada; ${ }^{8}$ Centre for Global eHealth Innovation, University Health Network, Toronto, ON, Canada; ${ }^{9}$ Medical Imaging, Sinai Health System , Toronto, ON, Canada. Support: Canadian Urological Association Scholarship Foundation (CUASF) Introduction: As patient autonomy is increasingly valued, having effective patient-centered tools is paramount. ${ }^{1}$ We have reported on the creation of a prototype patient-centered radiology report (PACERR) for prostate magnetic resonance imaging (MRI) (Fig. 1). In the current study, we explored its usefulness by assessing whether adding prostate PACERRs to traditional radiology reports (TRR) increases patient knowledge, facilitates better communication, and improves the patient experience.

Methods: Patients undergoing prostate MRI at Princess Margaret Cancer Centre between July and October 2019 were included. In an alternating fashion, patients were assigned to TRR only or TRR+PACERR groups,

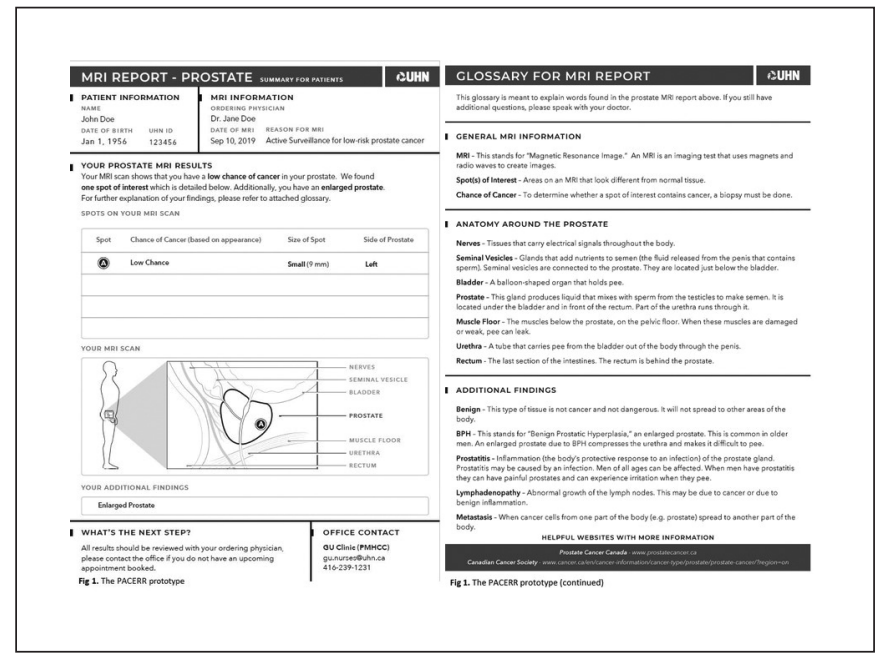

MP-5.8. Fig. 1. The PACERR prototype. 
stratified by normal or abnormal MRI result. Questionnaires exploring patient experience and knowledge acquisition were administered before and after their clinical encounter. In addition, there was a study coordinator observing each clinical encounter to see how physicians and patients interacted with the TRRs and PACERRs.

Results: Twenty-seven patients with a median age of 64.5 years were included. Men were either on active surveillance $(n=14)$, had prior negative biopsy $(n=9)$, or were biopsy-naive $(n=4)$. MRI was abnormal in 15 men (55\%). Following the clinical encounter, more patients in the PACERR arm reported improved ability to express themselves $(93 \%$ vs. $57 \%, p=0.03)$ and understand the doctor's explanation of the report $(86 \%$ vs. $50 \%, p=0.04)$. Adding the PACERR to the clinical encounter did not increase the median visit length ( 5 vs. 6 minutes). More abnormal items were correctly identified by men in the PACERR arm compared to men in the TRR arm ( $100 \%$ vs. $62 \%, p=0.001)$.

Conclusions: Using the PACERR in clinical practice empowers patients to express themselves and comprehend their results. Patients report better interaction with their medical professionals. Further work should be directed toward improving the generalizability of PACERRs to other disease sites and automation from traditional reports.

Reference

1. Barry MJ, Edgman-Levitan S. Shared decision-making - the pinnacle of patient-centered care. New Engl J Med 2012;366:780-1. https://doi.org/10.1056/NEJMp1109283

\section{MP-5.9}

Systematic review on timing of perioperative pharmacological thromboprophylaxis initiation and its effect on venous thromboembolism and bleeding outcomes

Kristen McAlpine ${ }^{1}$, Rodney H. Breau ${ }^{1,2}$, Marc Carrier ${ }^{2,3}$, Dean Fergusson ${ }^{2}$, Ilias Cagiannos ${ }^{1,2}$, Christopher G. Morash ${ }^{1,2}$, Luke T. Lavallée ${ }^{1,2}$

${ }^{1}$ Division of Urology, The Ottawa Hospital, Ottawa, ON, Canada; ${ }^{2}$ Ottawa Hospital Research Institute, Ottawa, ON, Canada; ${ }^{3}$ Department of Medicine, The Ottawa Hospital, Ottawa, ON, Canada

Introduction: Venous thromboembolism (VTE) and bleeding are important complications of urological surgery. Thromboprophylaxis guidelines for urologists are conflicting on timing of the initial dose of perioperative thromboprophylaxis and are largely based on non-urological literature. We sought to systematically assess the literature on the effect of the timing of the initial dose of perioperative thromboprophylaxis on VTE and bleeding outcomes.

Methods: EMBASE, MEDLINE, and CENTRAL databases were search on July 10, 2019 for randomized controlled trials (RCT) that evaluated VTE and/or bleeding outcomes between groups receiving the initial dose of thromboprophylaxis at different times perioperatively. Trials were limited to those that used the same medication between groups to ensure comparability. Prespecified subgroup analyses of abdominopelvic surgery (e.g., urological) and orthopedic surgery RCTs were planned.

Results: The initial search resulted in 3067 entries. After manual review, 21 RCTs including 16962 randomized patients met full inclusion criteria. Nine RCTs assessed abdominopelvic surgery, nine assessed orthopedic surgery, and three assessed other surgeries (e.g., thoracic). Only three RCTs showed a statistically significant difference between study groups in VTE (one RCT) or bleeding outcomes (two RCTs). These RCTs showed increased bleeding and decreased VTE rates with earlier initiation of thromboprophylaxis. Bleeding outcomes were reported with high variability with only three RCTs using established definitions for major and minor bleeding. Most studies were underpowered to detect a difference between study groups. Variability in the timing and medications between studies did not allow for a meta-analysis of outcomes. Conclusions: This systematic review confirms limited high-quality evidence is available on the optimal timing of the initial dose of perioperative thromboprophylaxis to prevent VTEs and bleeding. RCTs in urological patients are necessary to guide best practice.

\section{MP-5.10 \\ Early therapeutic intervention of hyperbaric oxygen improves radiation-induced hemorrhagic cystitis and proctitis}

Hayato Takeda ${ }^{1}$, Go Kimura', Shuhei Nozaki ${ }^{1}$, Mikio Shibasaki ${ }^{1}$, Yuya Hasegawa', Naoto Hodotsuka', Ryo Amakawa', Shigehito Minaguchi', Hikaru Mikami', Masato Yanagi', Jun Akatsuka', Yuki Endo', Tatsuro Hayashi', Yukihiro Kondo ${ }^{7}$

'Urology, Nippon Medical School, Tokyo, Japan

Introduction: Severe radiation-induced side effects can diminish quality of life (QOL) and cause life-threatening conditions. Hyperbaric oxygen therapy (HBOT) is an effective treatment not responding to conventional management. This study analyzed the clinical factors that affect the efficacy of HBOT.

Material: Prostate cancer patients who were treated with external beam radiation therapy (EBRT), produced Radiation Therapy Oncology Group and the European Organization for Research and Treatment of Cancer (RTOG/EORTC) grade 3 hemorrhagic cystitis and proctitis, and received HBOT were identified. Therapeutic effects were evaluated after at least six months of followup using RTOG/EORTC late radiation morbidity scoring schema. Time from radiation to onset of side effect and HBOT, as well as number of HBOT sessions were statistically analyzed by the chi-squared test. Complete resolution was defined as disappearance of macroscopic hematuria and hemorrhagic stool, and partial resolution as lower RTOG/ EORTC score.

Results: Between 2005 and 2016, out of 389 patients with sufficient data, 57 patients complained of hemorrhagic stool or hematuria. Twenty patients (12 proctitis and eight cystitis) with grade 3 side effects, mean age 78 years, received an average of 36 (range 1-224) HBOT. Complete and partial resolution rates were $50.0 \%$ and $25.0 \%$, respectively. Four patients $(20.0 \%)$ had no improvement and further surgical treatment was carried out in three patients $(15.0 \%)$. One patient $(5.0 \%)$ had a mild earache but continued therapy. When treated within one month from onset of side effect, $54.5 \%$ completely cured and $18.2 \%$ showed partial resolution ( $p=0.092)$, whereas $33.3 \%$ were partially cured with treatment delivery after a month. Side effects that occurred within 24 months after radiation showed $50 \%$ resolution $(\mathrm{p}=0.208)$, and $22.2 \%$ resolved after 20 HBO sessions $(p=0.547)$ did not affect clinical outcomes.

Conclusions: Treatment effectiveness was correlated with the time from onset of side effect to start of HBOT and associated with efficient clinical improvement. Early primary use of HBOT can successfully and safely treat severe radiation-induced cystitis and proctitis.

\section{MP-5.11}

\section{Establishing a care pathway for hydronephrosis}

Betty Wang ${ }^{1}$, Nathan Hoy', Shubhadip (Shubha) K. De', Trevor D. Schuler ${ }^{1}$, Timothy A. Wollin

'Department of Urology, University of Alberta, Edmonton, AB, Canada Introduction: The diagnostic evaluation for hydronephrosis is not uniform for every patient. Depending on the severity, acuity, and renal function, the urgency of the referral and evaluation will vary. This becomes challenging for triage staff, as there is no established care pathway. We aimed to establish a pathway for the triage of hydronephrosis patients.

Methods: For this quality improvement project, we conducted a retrospective chart review of all new adult hydronephrosis referrals to the Kipnes Urology Centre from July 2018 to September 2019. Data was abstracted from our electronic medical record and entered into a RedCap database. The following key parameters were recorded: patient demographics, type of referral, location and severity of hydronephrosis, investigations prior to referral, and additional investigations requested by urology. Final diagnosis and disposition were also included.

Results: A total of 111 charts were reviewed. Mean age at referral was 55 years. Mild, moderate, and severe hydronephrosis was reported in $38 \%, 32 \%$, and $30 \%$, respectively. Investigations prior to referral consisted of renal ultrasound ( $85 \%)$, contrast computed tomography $(37 \%)$, NCCT $(12 \%)$, and diuretic renal scan (13\%). After chart review and/or urology consultation, additional investigations included diuretic renal in $60 \%$, retrograde pyelography in $10 \%$, and no additional imaging in $23 \%$. Twenty-six patients $(23 \%)$ were diagnosed with ureteropelvic junction 
obstruction, of which six had laparoscopic pyeloplasty and 16 are being followed. Twenty-four patients $(22 \%)$ were diagnosed with non-obstructive hydronephrosis, with $88 \%(21 / 24)$ discharged to primary care. Of the remaining 61 patients, $15 \%(9 / 61)$ required intervention, 51\% (31/61) are being followed, and 34\% (21/61) were discharged from urology care. Conclusions: Based on these findings, we believe that a care pathway for hydronephrosis patients can be developed for our triage team to help expedite the referral process and prioritize patients based on clinical need.

\section{References}

1. Greco Baldea, Kristin A. Renal, Upper Tract Obstruction. American Urological Association. Oct. 2, 2018. Available at: https://university.auanet.org/modules/webapps/core/index.cfm\#/corecontent/62. Accessed April 23, 2020

2. Singh, IM, Strandhoy JW, Assimos DG. Pathophysiology of Urinary Tract Obstruction. In: Kavoussi L, Novick A, Partin A, Peters C, eds. Campbell-Walsh Urology. 10th ed. Philadelphia, PA: ElsevierSaunders; 2012:1087-1108. https://doi.org/10.1016/B978-1-41606911-9.00040-2

\section{MP-5.12}

Evaluation of the new holmium laser $\mathrm{Xpeeda}^{\mathrm{TM}}$ side-firing fiber vs. Greenlight XPS $180 \mathrm{~W}$ vapo-enucleation of the prostate for benign prostatic obstruction: An early clinical experience

Ahmed Ibrahim ${ }^{1}$, Mélanie Aube-Peterkin ${ }^{1}$, Hazem Elmansy ${ }^{2}$, Serge Carrier ${ }^{\dagger}$ ${ }^{1}$ Urology, McGill University, Montreal, QC, Canada; ${ }^{2}$ Urology, Thunder Bay Regional Health Sciences Centre, Thunder Bay, ON, Canada Introduction: Recently, holmium Lumenis Pulse ${ }^{\mathrm{TM}} 120 \mathrm{~W}$ was introduced and it stands apart from other available technologies as a combination of power and efficiency, which minimizes vaporization time. It delivers $120 \mathrm{~W}$, which represent the highest holmium power on the market, with better energy use through the new Xpeeda ${ }^{\top M}$ side-firing fiber. This technology seems to revolutionize use of the holmium power and delivering more energy directly to the tissue, due to its ability to be in contact with the tissue. Herein, we report our early clinical experience comparing both procedures in improving bothersome lower urinary tract symptoms (LUTS) secondary to benign prostatic hyperplasia $(\mathrm{BPH})$

Methods: A prospective study comparing holmium laser $\mathrm{Xpeeda}^{\mathrm{TM}}$ sidefiring fiber of the Lumenis Pulse $120 \mathrm{~W}$ to photoselective vapo-resection of the prostate-XPS $180 \mathrm{~W}$ was conducted. International Prostate Symptom Score (IPSS), flow rate, residual urine, prostate-specific antigen, and prostate volume changes, as well as perioperative complications were collected and compared.

Results: Overall, 26 and 30 patients were included in the holmium laser Expeeda $^{\mathrm{TM}}$ side-firing (group A) and photoselective vapo-resection (group B), respectively. There were no significant differences in terms of operative time, hospital stay, and time to catheter removal between both groups ( $>0.05)$. There was a significant comparable improvement in IPSS and post-void residual urine volume at one and four months. Re-intervention was needed in one and two cases in groups $A$ and $B$, respectively $(p=0.1)$. There were no significant difference between both groups in terms of intraoperative complications ( 1 vs. $2, \mathrm{p}>0.05$ )

Conclusions: Our early clinical experience suggests that the new holmium laser Xpeeda ${ }^{\mathrm{TM}}$ side-firing fiber is safe, non-inferior, and effective in treatment of BPH when compared with Greenlight XPS. Long-term followup and randomized clinical trials are definitely warranted to assess whether this new technology is more effective and more cost-effective.

\section{MP-5.13}

Hemopatch parenchymal closure technique and initial outcomes during robot-assisted partial nephrectomy

Benjamin Beech ${ }^{1}$, Ryan McLarty', Jan K. Rudzinski', Howard J. Evans ${ }^{1}$ Division of Urology, University of Alberta, Edmonton, AB, Canada Introduction: The Hemopatch is a novel polyethylene glycol-coated (PEGcoated) collagen patch that acts as a topical hemostatic agent. It has been applied to a variety of surgical techniques. Here, we present our initial series using it for robot assisted laparoscopic partial nephrectomy (RAPN). Methods: We conducted a retrospective review of all patients undergoing RAPN by a single surgeon at a tertiary Canadian medical center between July and December 2018. We excluded patients if they did not have 90 days followup or a Hemopatch was not used on the renal parenchymal defect. We collected data pertaining to demographics, renal tumor complexity (RENAL nephrometry score), and postoperative outcomes. This included 90-day complications (Clavien-Dindo classification), 90-day arterial embolization, estimated blood loss (EBL), change in postoperative hemoglobin, clamp method, and length of stay (LOS). Descriptive statistics were used to examine our outcomes. We also describe in detail our "gloved sleeping bag" technique for Hemopatch deployment.

Results: Seventeen patients met inclusion criteria, of whom 12 were male. Mean age was 63 years. Median size of renal mass was $2.85 \mathrm{~cm}$ in largest dimension, and median RENAL score was 6 . Hilar clamping was carried out in $12(70.9 \%)$ cases, with an average warm ischemic time of 16.1 minutes. The remainder of cases were performed off-clamp. Median EBL was $238 \mathrm{~mL}$ and the mean change in hemoglobin on postoperative day 1 compared to preoperatively was $21.2 \mathrm{~g} / \mathrm{L}$. Average LOS was 1.76 days. No patient underwent angioembolization for bleeding within 90 days and there were no complications greater than Clavien-Dindo grade 2 . One patient developed a urinary tract infection and a second developed postoperative urinary retention.

Conclusions: Hemopatch can be used safely and effectively in lieu of traditional two-layer renorraphy with acceptable outcomes. Larger prospective series are required to ascertain its true value and cost-effectiveness.

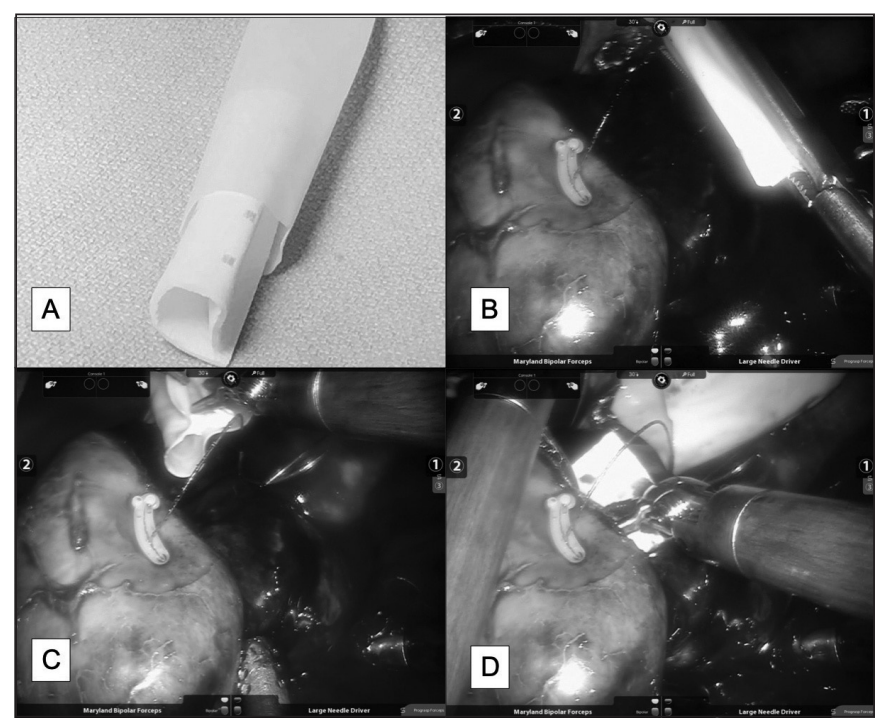

MP-5.13. Fig. 1. Preparation and deployment of Hemopatch into renal mass defect. (A)The Hemopatch is rolled with the adhesive side facing inward and placed into the cut fifth finger of a sterile glove. (B) The assistant deploys the glove finger into the field; $(\boldsymbol{C}, \boldsymbol{D})$ and the surgeon can manipulate the Hemopatch onto the defect without contact with surrounding tissues. 


\section{CUA ABSTRACTS}

\section{Moderated Poster Session 6: Training, Evaluation}

\section{MP-6.1}

A new ureteroscopy training platform that uses computed tomography urograms to replicate complex patient renal collecting system anatomies

Nuley Seo ${ }^{1}$, Bader Alsaikhan 1,2, Sylvia Koo 3 , Brian Carillo, Monica Farcas² ${ }^{1}$ Faculty of Medicine, University of Toronto, Toronto, ON, Canada; ${ }^{2}$ Department of Urology, St. Michael's Hospital, Toronto, ON, Canada; ${ }^{3}$ John A. Burns School of Medicine, University of Hawaii, Honolulu, HI, United States

Introduction: Flexible ureteroscopy (fURS) is a one-person surgical technique, limiting trainees' ability to practice intraoperatively. ${ }^{1}$ Currently, there are several simulator models, but few are able to reproduce accurate, complex collecting systems. ${ }^{2}$ We developed an anatomically accurate benchtop ureteroscopy simulator using 3D reconstructions of patient-specific computed tomography (CT) urograms and elastomer 3D printing technology. This simulator aims to reproduce realistic challenging anatomies (such as sharp infindibulo-pelvic angles and spidery complex collecting systems). Methods: Patient-specific CT urograms were used to create 3D reconstructions of the renal collecting system using Slicer ${ }^{\mathrm{TM}}$. 3D models were then modified using Blender ${ }^{\mathrm{TM}}$. Hollow elastomer kidney models for fURS were created using an Objet $3 \mathrm{D}^{\mathrm{TM}}$ printer. To test and evaluate the new fURS simulator, 25 volunteers were recruited (five novices, 13 residents, seven urologists). The model was compared to an existing fURS model (Cook Medical $^{\mathrm{TM}}$ ) on various criteria using the student paired t-test. Furthermore, volunteers were asked to explore the model with fURS and draw out the collecting system from memory.

Results: We were able to use CT urogram and 3D printing technology to create a fURS simulator that accurately replicates anatomically complex collecting systems. Using the new model and simulator, we noted that, unlike staff urologists, most novices and residents completely missed visualizing the lower pole calyces. A survey comparison between our simulator and a comparable benchtop simulator (Cook Medical ${ }^{\mathrm{TM}}$ ) revealed consistently better ratings for our simulator on all criteria $(p<0.05)$.

Conclusions: We were able to successfully create an anatomically accurate fURS simulator that can provide a more realistic scoping experience. Preliminary testing revealed that trainees will benefit from this simulator particularly with respect to learning how to navigate challenging collecting systems.

\section{References}

1. Wignall GR, Denstedt JD, Preminger GM, et al. Surgical simulation: A urological perspective. J Urol 2008;179:1690-9. https://doi.org/10.1016/j.juro.2008.01.014

2. Brunckhorst O, Aydin A, Abboudi H, et al. Simulation-based ureteroscopy training: A systematic review. J Surg Educ 2014;72:135-43. https://doi.org/10.1016/j.jsurg.2014.07.003

\section{MP-6.2}

Analysis of hand/instrument motion during ureteroscopy Sylvia Koo ${ }^{1,2}$, Bader Alsaikhann ${ }^{1,3}$, Nuley Seo ${ }^{1}$, Kai Fok', Brian Carrillo ${ }^{1}$, Monica Farcas 1,3

${ }^{1}$ University of Toronto, Toronto, ON, Canada; ${ }^{2}$ John A. Burns School of Medicine, University of Hawaii, Honolulu, HI, United States; ${ }^{3}$ Department of Urology, St. Michael's Hospital, Toronto, ON, Canada

Introduction: Hand/instrument motion-tracking in surgical simulation can provide valuable data to improve psychomotor skills, and can serve as a formative evaluative tool. ${ }^{1,2}$ Although motion analysis has been well-studied within laparoscopic surgery, it has been poorly studied in endoscopic surgery. ${ }^{3}$ There are essentially no studies looking at surgeon hand/instrument motion tracking for flexible ureteroscopy (fURS), a surgical procedure that requires a significant amount of hand dexterity. ${ }^{4,5}$ We aimed to develop a ureteroscopic surgery simulation platform that incorporates motion tracking capabilities.

Methods: Using the Polhemus ${ }^{\mathrm{TM}}$ system, we designed a motion tracking platform for a benchtop ureteroscopy simulator. This system was designed to capture specific instrument/surgeon hand motions determined to be important during fURS. Motion data was captured for a specific defined task performed on the simulator. Using this data, motion analysis metrics for fURS were established. Twenty-five volunteers were tested on this new platform and motion analysis parameters were recorded and analyzed. Results: Using motion tracking software, we analyzed three key motions during fURS: scope in-out motion, scope rotation, and scope tip flexion. The target task was to visualize an upper, inter, and lower pole calyx on a benchtop surgical simulator of the left kidney. Participants paused for 10 seconds when visualizing each papilla to help discriminate the data. Twenty-five participants were tested (five novices, 13 residents, seven urologists). While scope in-out motion and scope tip flexion were significantly different between participants of different expertise, the most discriminatory metric that differentiated experts from novices was scope rotation (Fig. 1). Conclusions: We successfully created a fURS simulation platform that captures instrument motion. Preliminary data suggests scope rotation is the most discriminatory motion parameter in fURS that differentiates between novice and expert surgeons.

\section{References}

1. Obstein KL, Patil, VD, Jayender J, et al. Evaluation of colonoscopy technical skill levels by use of an objective kinematic-based system. Gastrointest Endosc 2011;73:315-21. https://doi.org/10.1016/j. gie.2010.09.005

2. Colella S, Sondergaard Svendsen MB, Konge L, et al. Assessment of competence in simulated flexible bronchoscopy using motion analysis. Respiration 2015;89:155-61. https://doi.org/10.1159/000369471

3. Nasr A, Carrillo B, Gerstle JT, et al. Motion analysis in the pediatric laparoscopic surgery (PLS) simulator: Validation and potential use in teaching and assessing surgical skills. J Pediatr Surg 2014;49:791-4. https://doi.org/10.1016/j.jpedsurg.2014.02.063

4. Wignall GR, Denstedt JD, Preminger GM, et al. Surgical simulation: A urological perspective. J Urol 2008;179:1690-9. https://doi. org/10.1016/j.juro.2008.01.014

5. Hu D, Liu T, Wang X. Flexible ureteroscopy training for surgeons using isolated porcine kidneys in vitro. BMC Urol 2015;15:71. https://doi.org/10.1186/s12894-015-0067-9 

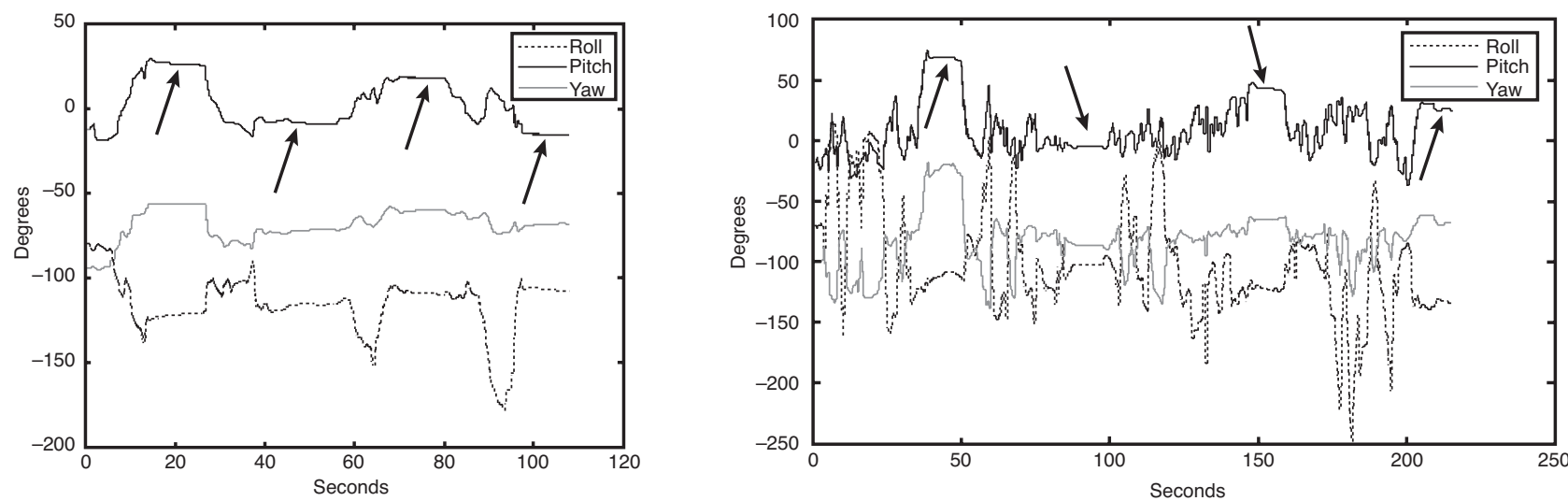

MP-6.2. Fig. 1. Scope rotation for (A) expert run; and (B) novice run.

MP-6.3

Evaluating the utility of a novel 3D immersive virtual-reality percutaneous nephrolithotomy simulator

Jason Y. Lee ${ }^{1}$, Luke Reynolds ${ }^{1}$, Monica Farcas ${ }^{1}$

${ }^{1}$ Urology, University of Toronto, Toronto, ON, Canada

Introduction: Percutaneous nephrolithotomy (PCNL) is the gold standard treatment for patients with a large stone burden. Gaining safe access into the collecting system is the most critical step of PCNL, but also the most technically challenging aspect. There are a variety of methods to teach this important endourological skill, including the use of simulation-based methods. In this study, we evaluate three PCNL simulation platforms, including a novel, 3D-immersive VR simulator, for potential use in teaching and assessing PCNL access skills.

Methods: Urology residents, fellows, and faculty from our institution were recruited to participate in this study, which included completing standardized tasks on three PCNL simulation platforms: the Marion K181 simulator (Marion Surgical), the PercMentor VR simulator (Symbionix), and a porcine inanimate PCNL model (Cook Medical). All participants were given standardized introductions to each of the three simulated platforms before being asked to complete the standardized tasks — gaining PCNL access using a fluoroscopic-guided technique. Participant demographic and performance data were recorded for analysis. Participants also evaluated the three PCNL simulation platforms.

Results: A total of 18 participants (two faculty endourologists, four endourology fellows, 12 urology residents) with varying levels of PCNL experience ( $78 \%$ had performed $<10$ cases, $17 \%$ over 50 cases) completed the study. The Marion K181 had higher ratings in all domains compared to the Perc Mentor $(p<0.05)$ but did not differ in any domain when compared to the Cook porcine PCNL model. Fluoroscopy time was the variable that most consistently correlated with participant PCNL experience and level of training across all three PCNL simulation platforms, but other performance metrics did not correlate well.

Conclusions: There are a variety of PCNL simulation platforms available for teaching PCNL access skills. Based on our initial study, there is validity evidence to support the use of the novel Marion K181 PCNL simulator as a teaching tool, with high user ratings across domains, but evidence is yet lacking for use as an assessment tool.

\section{MP-6.4}

Development, implementation, and evaluation of a point of care ultrasound course for training urology residents

Catherine Anne Lovatt ${ }^{1}$, Michael John Uy ${ }^{1}$, Jennifer Hoogenes ${ }^{1}$, Carol Bernacci ${ }^{2}$, Edward D. Matsumoto ${ }^{\prime}$

'Department of Surgery, Division of Urology, McMaster University, Hamilton, ON, Canada; ${ }^{2}$ Department of Medical Radiation Sciences, Mohawk College, Hamilton, ON, Canada

Support: Mohawk College Department of Radiation Sciences

Introduction: Point-of-care ultrasound (PoCUS) is a widely used bedside tool in urology. Applications include the assessment of an undifferentiated acute scrotum, renal colic, and the guidance of suprapubic catheter placement. However, the user-dependent nature of this modality necessitates appropriate use and competence. The objective of this study was to develop and evaluate an introductory PoCUS course for urology residents. Methods: All urology residents at our institution completed a three-hour, online course followed by a three-hour, hands-on seminar involving kidney, bladder, and testicular sonographic scanning. Simulated testicular phantoms (Fig. 1) and suprapubic catheter models (Fig. 2) were constructed and used in an ultrasound lab. Course material was developed by licensed ultrasound technologists, based on the Sonography Canada National Competency Profiles. ${ }^{1}$ Pre- and post-course surveys focused on residents' user confidence, while pre- and post-course multiple-choice questionnaires (MCQ) assessed theoretical knowledge.

Results: Fourteen urology residents (PGY 1-5) participated in the course. Theoretical knowledge in PoCUS improved significantly $(p<0.001)$, as

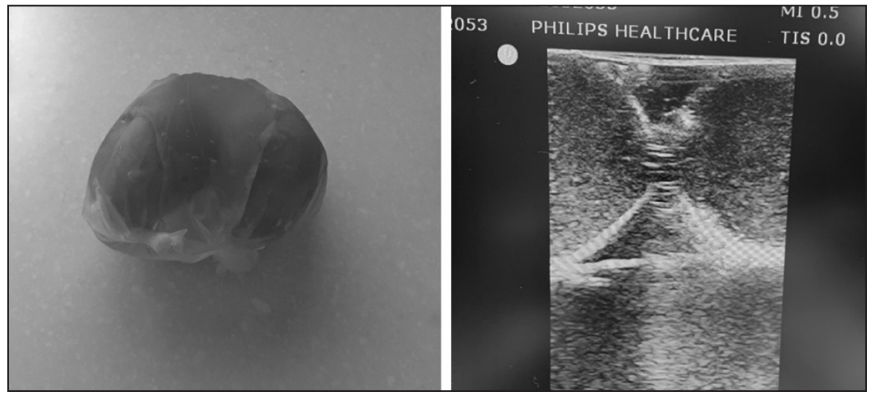

MP-6.4. Fig. 1. Simulated testicular phantoms within the scrotal case (left) and corresponding sonographic appearance (right). 


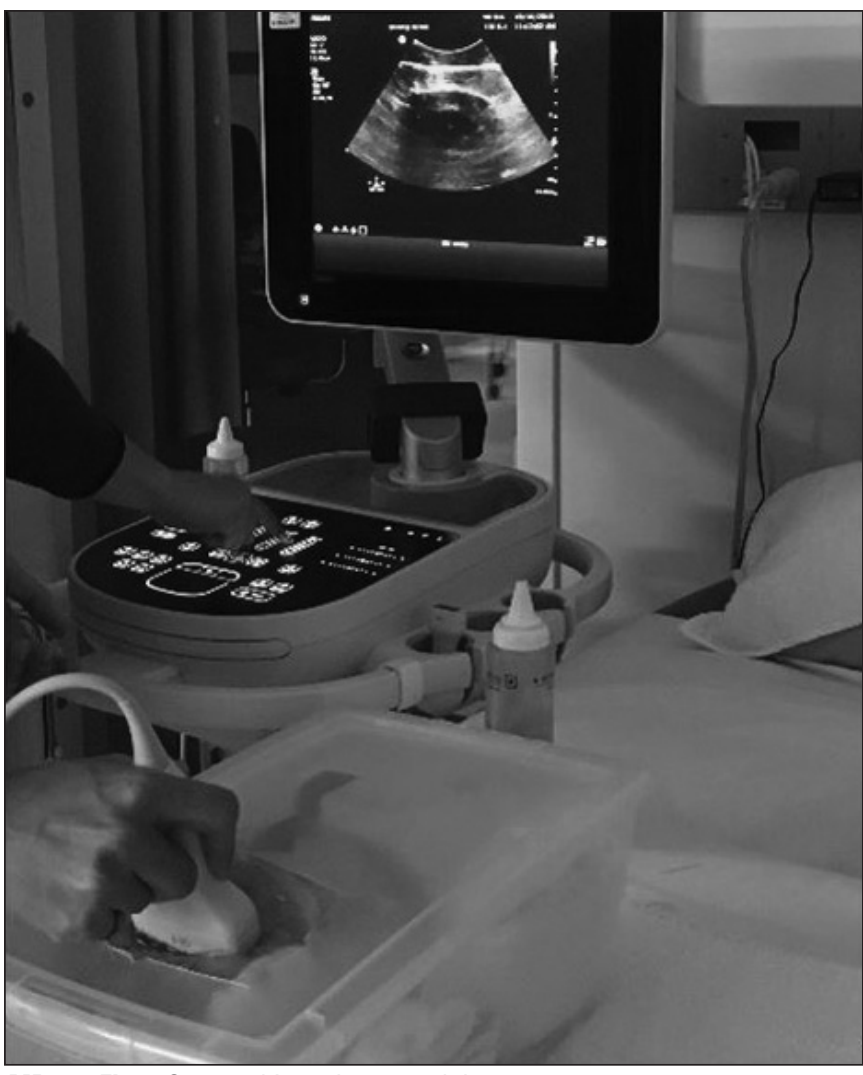

MP-6.4. Fig. 2. Suprapubic catheter model.

well as self-rated confidence in manipulation of ultrasound controls, doppler imaging, and PoCUS of the kidney, bladder, and testicles (all $\mathrm{p}<0.001$ ) (Table 1). All participants indicated that the course increased their likelihood of PoCUS use in clinical practice, and that PoCUS training should be integrated into a urology training curriculum.

Conclusions: We successfully developed and implemented a feasible foundational PoCUS course for urology residents based on national ultrasound training guidelines. Residents significantly improved in user

\section{MP-6.4. Table 1. Pre- and post-course changes in}

theoretical knowledge and user confidence

\begin{tabular}{|c|c|c|c|}
\hline & Pre-course & Post-course & $\mathbf{p}$ \\
\hline \multicolumn{4}{|l|}{ Theoretical knowledge } \\
\hline $\begin{array}{l}\text { Multiple choice assessment } \\
(\%)\end{array}$ & $52.8(11.2)$ & $72.3(6.7)$ & $<0.001$ \\
\hline Extra questions & - & $76.4(8.4)$ & - \\
\hline \multicolumn{4}{|l|}{ User confidence $^{\dagger}$} \\
\hline Ultrasound interpretation & $2.2(1.1)$ & $4.1(0.7)$ & $<0.001$ \\
\hline PoCUS controls & $2.2(0.9)$ & $4.2(0.6)$ & $<0.001$ \\
\hline Doppler imaging & $1.6(0.8)$ & $3.8(0.7)$ & $<0.001$ \\
\hline Kidney PoCUS & $1.8(0.7)$ & $4.2(0.7)$ & $<0.001$ \\
\hline Bladder PoCUS & $2.7(0.9)$ & $4.3(0.5)$ & $<0.001$ \\
\hline Testicular PoCUS & $1.8(0.9)$ & $4.1(0.6)$ & $<0.001$ \\
\hline
\end{tabular}

Standard deviation in brackets; ${ }^{\dagger}$ Confidence assessed via 5-point Likert Data: 1 = very unskilled (little to no experience), 2 = unskilled (beginner proficiency), $3=$ intermediate performer (proficient), 4 = skilled user (comfortable with use), $5=$ very skilled (expert). confidence and theoretical knowledge. This easily modifiable and reproducible course can be used as an introductory tool for urology residents to begin developing competency in ultrasound, and may act as a stepping stone for further competency-based training programs.

\section{Reference}

1. Sonography Canada. National competency profiles version 5.1. 2018. Available at: https://sonographycanada.ca/app/uploads/2019/03/ NCP-5.1-Final.pdf. Accessed Dec. 30, 2019.

\section{MP-6.5}

National discrepancies in residency training of open simple prostatectomy for benign prostatic enlargement: Redefining our gold standard

Patrice Levasseur-Fortin', Kyle W. Law², David-Dan Nguyen², Ahmed Zakaria ${ }^{3}$,Vincent Misrai ${ }^{4}$, Dean S. Elterman ${ }^{5}$, Naeem Bhojani ${ }^{3}$, Enrique Rijo ${ }^{6}$, Kevin C. Zorn ${ }^{3}$

${ }^{1}$ Faculty of Medicine, Université de Montréal, Montreal, QC, Canada; ${ }^{2}$ Faculty of Medicine, McGill University, Montreal, QC, Canada; ${ }^{3}$ Division of Urology, Centre hospitalier de I'Université de Montréal, Montreal, QC, Canada; ${ }^{4}$ Department of Urology, Clinique Pasteur, Toulouse, France; ${ }^{5}$ Division of Urology, University of Toronto, Toronto, ON, Canada; ${ }^{6}$ Department of Urology, Hospital Quiron Barcelona, Barcelona, Spain

Introduction: In light of recent urological association (CUA, AUA, EAU) recommendations for the treatment of benign prostate hyperplasia (BPH), open simple prostatectomy (OSP) remains the recommended approach for prostates with volumes over $80 \mathrm{cc} .{ }^{1}$ We sought to assess the current state of OSP and other BPH surgical training across Canadian urology residency programs and the use of guideline-recommended imagery prior to BPH surgery.

Methods: A survey was distributed among Canadian urology program directors in June 2019. We identified the various surgical modalities available for the treatment of BPH offered by each program and obtained the annual number of OSP performed at each academic residency program. Additionally, we evaluated for the routine performance of preoperative transrectal ultrasound (TRUS) of the prostate during patient counselling as recommended by 2018 CUA guidelines.

Results: All 13 program directors from the Canadian urology programs responded to our survey. OSP and monopolar transurethral resection of the prostate (TURP) are practiced across all 13 centers. Greenlight photovaporization, bipolar TURP, holmium laser enucleation of the prostate, and robot-assisted simple prostatectomy were practiced in $76.8 \%, 69.2 \%$, $23.1 \%$, and $23.1 \%$ of centers, respectively (Fig. 1). The mean number of OSP per academic training program was 4.7 cases annually (Fig. 2). Moreover, only five $(38 \%)$ academic centers routinely performed a preoperative TRUS to evaluate prostate volume for BPH counselling (Fig. 3). Conclusions: Although recognized and referenced as the $\mathrm{BPH}$ gold standard for the treatment of prostates over $80 \mathrm{cc}$, Canadian urology trainees'

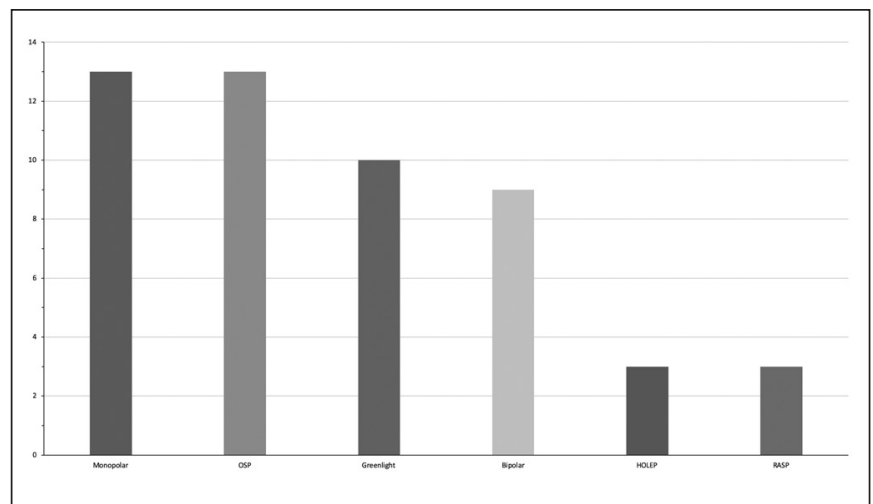

MP-6.5. Fig. 1. Variety of resection techniques of the prostate practiced in the 13 Canadian residency programs. 


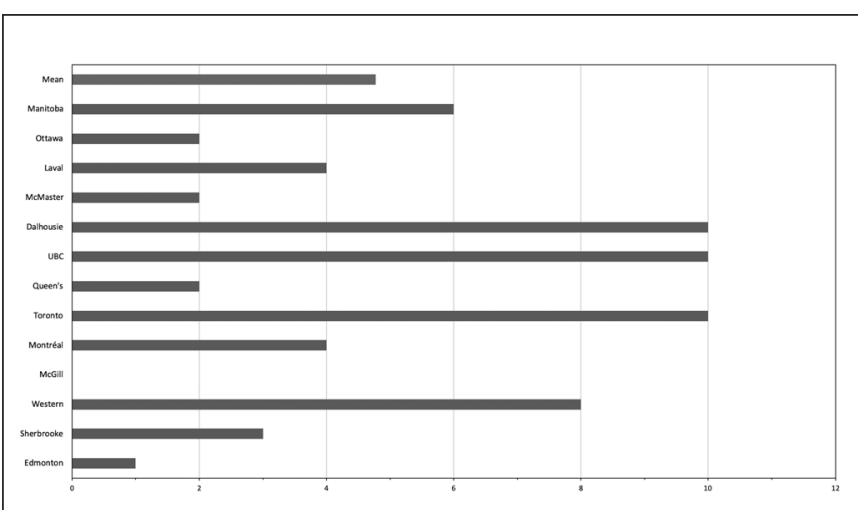

MP-6.5. Fig. 2. Number of open simple prostatectomies performed per year (based on 2018 case volume).

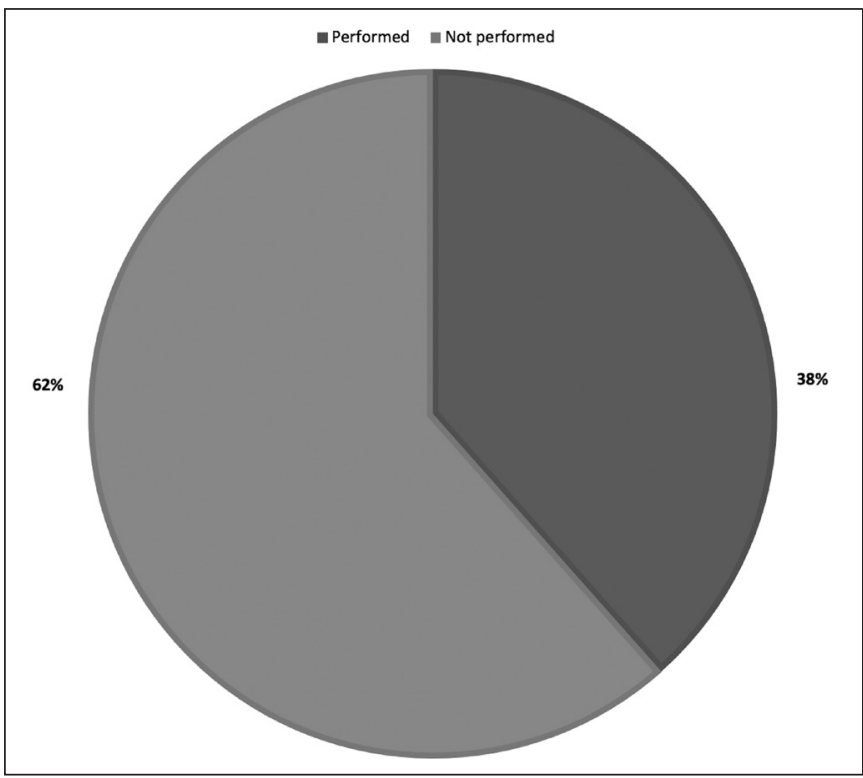

MP-6.5. Fig. 3. Use of preoperative transrectal ultrasound for prostate volume evaluation in 13 Canadian academic centers.

annual OSP exposure remains limited. While this intervention is given a high degree of importance (category A) during residency training as per the Royal College's practice guidelines, it may be unrealistic to reach these national standards considering the annual case OSP volumes in academic Canadian urology faculties.

\section{Reference}

1. Nickel JC, Aaron L, Barkin J, et al. Canadian Urological Association guideline on male lower urinary tract symptoms/benign prostatic hyperplasia (MLUTS/BPH): 2018 update. Can Urol Assoc J 2018;12:303-12. https://doi.org/10.5489/cuaj.5616

\section{MP-6.6}

Influence of musical background on surgical skills acquisition Ryan Sun ${ }^{1}$, Wendy Wang ${ }^{2}$, Cole Kubay², Sean Fernando², Andrew Fast ${ }^{2}$, Christopher Dutka², Kianna Cadogan'², Darrel E. Drachenberg ${ }^{1}$ ${ }^{1}$ Section of Urology, University of Manitoba, Winnipeg, MB, Canada; ${ }^{2}$ College of Medicine, University of Manitoba, Winnipeg, MB, Canada Introduction: Surgical skills require high levels of psychomotor, visualspatial, and depth perception functions, which are similarly applied in musical tasks. It has, therefore, been speculated that musicians and surgeons may have common cerebral specialization, and that musical background may have a positive impact on surgical training. In limited studies, correlation has been found between musical education and microsurgi$\mathrm{cal}^{1}$ and laparoscopic ${ }^{2}$ performance. In this study, we aim to investigate the influence of musical background on the acquisition of surgical skills. Methods: We conducted a prospective, cross-sectional study involving novice medical trainees with no prior surgical exposure. Each subject was asked to complete detailed questionnaire on their level of musical experience, a validated dexterity test using the Purdue Pegboard, and surgical tasks, including laparoscopic peg transfer and basic suturing. Statistical analysis was performed to compare between those with and without musical backgrounds.

Results: A total of 27 first-year medical students without surgical experience were included; 17 (63\%) had background of musical instrument play. The musical group had a faster performance on the dexterity test $(p=0.042)$, laparoscopic peg transfer $(p=0.006)$, and a faster but nonsignificant baseline suture speed compared to the non-musical group $(p=0.08)$. These differences were more pronounced in musicians with increasing years of musical experience. A second suture attempt after standardized five-minute practice showed significant improvement for both groups, with a narrowing difference between the two groups.

Conclusions: Based on this study involving trainees with no prior surgical experience, musical experience correlated with increased dexterity and faster performance of basic surgical skills at baseline. There is no observed difference in rate of improvement, and more work is underway to elucidate differences in learning curve and optimal mode of training. References

1. Moustaki M, Masud D, Hachach-Haram N, et al. Effect of computer games and musical instruments on microsurgery. J Plast Reconstr Aesthet Surg 2017;70:982-4. https://doi.org/10.1016/j. bjps.2017.02.014

2. Boyd T, Jung I, Van Sickle K, et al. Music experience influences laparoscopic skills performance. JSLS 2008;12:292-4.

\section{MP-6.7}

Is there a correlation between cognitive and technical skills during urology objective structured clinical examinations?

Ahmed Ibrahim ${ }^{1}$, Anne Yin ${ }^{1}$, Yasser Noureldin ${ }^{1}$, Serge Carrier ${ }^{1}$, Mélanie Aube-Peterkin' Sero Andonian'

${ }^{1}$ Urology, McGill University, Montreal, QC, Canada

Introduction: The worldwide paradigm shift toward competency-based medical education necessitated incorporation of simulators for objective assessment of technical skills. The aim of the present study was to assess the relationship between the technical and cognitive skills during urology objective structured clinical examinations (OSCEs)

Methods: This study was conducted after obtaining the local institutional ethics approval and informed consents. Postgraduate trainees (PGTs) from the four urology training programs in the province of Quebec were recruited for this study during a semi-annual urology OSCE. Participants were from post-graduate years (PGY) 3, 4, and 5. Technical skills were assessed by two stations (photo-selective vaporization of the prostate [PVP] simulator and laparoscopic intracorporeal knot [ICK] stations). Competency scores were noted for both tasks. PGTs who obtained competency in both tasks were considered competent in technical skills. Furthermore, cognitive skills were assessed during 13 oral OSCE stations. Correlation between technical and cognitive skills was calculated.

Results: Twenty-nine PGTs who attended the OSCE voluntarily participated in this study. The mean age of participants was $29.5 \pm 0.7$ years and five $(17.2 \%)$ were female. When compared with non-competent PGTs, 
competent PGTs in technical skills obtained significantly higher scores in the cognitive tasks. However, there was no significant correlation when comparing cognitive tasks scores with PVP task scores, nor with ICK task scores. Similarly, there was no significant correlation between both technical skills scores $(r=0.29 ; p=0.11)$.

Conclusions: Competency in technical skills is associated with higher cognitive tasks scores, although a positive correlation has not been demonstrated in this small study. Formal evaluation of urology residents should, therefore, include both cognitive and technical skills evaluations to provide a well-rounded assessment of the trainee.

\section{MP-6.8 \\ Heterogeneity in urology teaching curricula among Canadian urology residency programs \\ Uday Mann ${ }^{1}$, Jasmir G. Nayak', Premal Patel ${ }^{1}$}

${ }^{1}$ Section of Urology, University of Manitoba, Winnipeg, MB, Canada Introduction: Postgraduate education is moving to a competency-based curriculum in an effort to standardize the quality of graduating trainees. The learning experiences in each institution are likely variable, as no standard exists regarding the teaching curriculum offered in residency. The objective of this study is to examine the various curricula among Canadian urology residency programs and to identify which teaching modalities are prioritized by program directors (PDs).

Methods: A 10-question survey was sent electronically to PDs at all 12 urology residency programs across Canada. Questions were designed to quantify the time allotted for teaching and to assess the various teaching modalities prioritized by programs to ensure the successful training of their graduates. We assessed each program's perceived value of written/ oral exams, didactic sessions, and simulation sessions. Responses were assessed using a Likert scale and a ranking format. Descriptive statistics were performed.

Results: Overall survey response rate from residency PDs was 75\% (9/12); $67 \%$ of programs designated one day of teaching per week, whereas $33 \%$ split this over two days. Review of chapters directly from Campbell'sWalsh Urology textbook were deemed the most valuable teaching session. Oral exams were also prioritized, whereas most programs felt that simulation labs contributed the least to residency education. All programs included review of the core urology textbook in their weekly teaching, while $67 \%$ of programs included faculty-led didactic sessions and case presentations; $44 \%$ of programs included resident-led sessions. Oral exams and simulation labs were the least commonly included teaching sessions.

Conclusions: We found significant heterogeneity in the teaching sessions prioritized and offered in current urology residency curricula. As we move to standardize the quality of graduating trainees, understanding the impact of variable educational opportunities on residency education may become increasingly important.

\section{MP-6.9 \\ Burnout in chief residents among Canadian urology residency programs \\ leannette Johnstone' ${ }^{1}$, Adam Gabara², Naji J. Touma'}

'Faculty of Health Science/Urology, Queen's University, Kingston, ON, Canada; ${ }^{2}$ Faculty of Health Science/School of Medicine, Queen's University, Kingston, ON, Canada

Introduction: Burnout is a hot topic of discussion in medicine, including urology, which has reported higher rates than other fields. ${ }^{1,2}$ Burnout among urologists has increased from $41 \%$ to $63.6 \%$ during the $2011-2014$ interval in the AUA census. ${ }^{2} \mathrm{~A}$ recent study assessing burnout in urology trainees in the U.S. and Europe revealed that burnt out trainees were less likely to pursue urology again as compared to their non-burnt out colleagues $(46 \%$ vs. $81 \%$ and $57 \%$ vs. $85 \%$, respectively). ${ }^{3}$ Dissatisfaction with work/life balance, and lack of access to mental health services were associated with increased burnout. ${ }^{3}$ In our study, we wanted to assess the state of burnout and services available in Canadian urology residency programs.

Methods: Thirty-seven chief residents among the Canadian urology residency programs attended the Queen's Urology Exam Skills Test (QUEST) on December 13 and 14, 2019. The Maslach Burnout Inventory (MBI) questionnaire was administered to these residents, and answers were collected anonymously. Descriptive statistics were used to analyze the data. Results: Pertinent findings from the survey were that $94.6 \%$ of respondents reported fatigue, $27 \%$ are dissatisfied with the balance between their personal and professional lives, $84.8 \%$ find it difficult to engage in self-care activities, $54.1 \%$ feel emotionally drained a few times a week or more, and, $29.7 \%$ feel burnt out from their work a few times a week or more. Conclusions: This study enabled us to confirm that urology chief residents are affected by burnout. Fatigue was universal among all trainees and more than a quarter reported being dissatisfied with their current balance between professional and personal lives. Strategies proven beneficial in reducing burnout include increasing self-wellness, ${ }^{4}$ seeking professional assistance to reflect on stressors and life/career priorities, ${ }^{5}$ as well as structured mentorship programs. ${ }^{3}$ These are examples of how we can use this data to tailor interventions accordingly.

References

1. Shanafelt TD, Hasan O, Dyrbye LN, et al. Changes in burnout and satisfaction with work-life balance in physicians and the general US working population between 2011 and 2014. Mayo Clin Proc 2015;90:1600-13. https://doi.org/10.1016/j.mayocp.2015.08.023

2. North AC, McKenna PH, Sener A, et al. Burnout in urology-findings from the 2016 AUA annual census. Urol Pract 2018;5:489-94. https://doi.org/10.1016/j.urpr.2017.11.004

3. Marchalik D, Goldman CC, Carvalho FFL, et al. Resident burnout in USA and European urology residents: An international concern. BJU Int 2019;124:349-56. https://doi.org/10.1111/bju.14774

4. Waxman BP. Caring and sharing: Strategies for recognizing and surviving burnout in surgeons. ANZ / Surg 2011;81:493-4. https://doi.org/10.1111/j.1445-2197.2011.05803.x

5. Maslach C, Jackson S, Leiter M. Maslach Burnout Inventory Manual. Menlo Park, CA: Mind Garden, 2010.

\section{MP-6.10}

Pursuit of post-residency training and perception of job availability among graduating Canadian urologists

Thomas Canil', Monica Farcas

'Urology, St. Michael's Hospital, Toronto, ON, Canada

Introduction: Job availability and eventual employment has been a point of anxiety for Canadian urology graduates. Surgical graduates across Canadian residency programs have expressed increasing dissatisfaction surrounding job availability, ${ }^{1}$ with corresponding increased pursuit of fellowship-level training. ${ }^{2}$ In this study, we hope to characterize fellowship training among urology trainees and how this influenced their employment.

Methods: A survey study was circulated to Canadian urology graduates between 2010 and 2015. The names of participants were obtained for the individual universities and were cross-referenced with the CUA. A separate survey was submitted to program directors in order to obtain their perspective of graduates training.

Results: We obtained contact information for 123 eligible participants with 64 responses. Approximately $70 \%$ of respondents completed a fellowship, with the majority being one year in duration. In evaluating motivation for pursuing additional training, $11 \%$ cited lack of comfort for independent practice, $36 \%$ a lack of job availability, and $84 \%$ to obtain subspecialized training in an area of interest. As it pertains to employment, $95 \%$ of overall respondents are now employed full-time, with $80 \%$ being employed within six months of completing their training. Subspecialty training was cited by $38 \%$ as the most helpful factor in obtaining employment, however, personality was considered the most influential factor employers considered. The majority of program directors encouraged fellowship-level training for residents, with an equivalent proportion citing the opportunity for subspecialization.

Conclusions: Most trainees graduating from Canadian urology programs are seeking out fellowship training. This is largely motivated by job availability, along with the pursuit of subspecialty training - a practice that is encouraged by most program directors. Job availability overall appears to be adequate, with high overall employment rates and minimal time for job acquisition. 


\section{References}

1. Hosier GW, Touma NJ. Attitudes of graduating Canadian urology residents on the job market: Is it getting better or are we just spinning our wheels? Can Urol Assoc J 2018;12:104-9. https://doi. org/10.5489/cuaj.4765

2. Welk B, Kodama R, MacNeily A. The newly graduated Canadian urologist: Over-trained and underemployed? Can Urol Assoc J 2013;7:E10-5. https://doi.org/10.5489/cuaj.188

\section{MP-6.12}

Assessing the quality of systematic reviews in the urological literature (2016-18) using AMSTAR-2

Maylynn Ding', Leah Soderberg², Jae H Jung3, Philipp Dahm²

${ }^{1}$ Urology, McMaster University, Hamilton, ON, Canada; ${ }^{2}$ Urology, University of Minnesota, Minneapolis, MN, United States; ${ }^{3}$ Urology, Yonsei University, Wonju, Korea

Introduction: This project aimed to investigate the methodological quality of systematic reviews published in the urological literature.

Methods: PubMed ${ }^{\circledR}$ was systematically searched for systematic reviews related to questions of prevention and therapy published in five major urology journals from January 2016 to December 2018. Two reviewers followed a written a priori protocol to independently screen references in Rayyan ${ }^{\circledR}$ and extracted data using a piloted form based on the sixteen domains of AMSTAR-2. We performed pre-planned statistical hypothesis testing by journal of publication in SPSS Version 24.0.

Results: Our search identified 260 relevant references, 144 of which ultimately met inclusion criteria. The largest contributors by journal of publication were European Urology $(53 ; 36.8 \%)$ followed by Urology (36; $25.0 \%$ ), and BJU International $(24 ; 16.6 \%)$. The most common clinical topics were oncology $(64 ; 44.4 \%)$ and voiding dysfunction (32; $22.2 \%)$ followed by stones/endourology $(14 ; 9.7 \%)$. Just over one-third $(52 ; 36.2 \%)$ of reviews had a registered protocol. Nearly all studies (139; $96.5 \%)$ searched at least two databases. Less than one-third (46; 31.9\%) also searched trial registries and one-fifth (30; 20.8\%) consulted experts for additional trials. Few systematic reviews $(14 ; 10.4 \%)$ provided a list of potentially relevant but excluded studies. Only six (4.2\%) systematic reviews met all AMSTAR-2 critical domains as a prerequisite for highquality reviews.

Conclusions: Many systematic reviews are published in the urological literature each year, yet their quality is suboptimal. There is a need for educating authors, peer reviewers, and editors alike on established standards for high-quality systematic reviews to ensure improvement in the future. 


\section{CUA ABSTRACTS}

\section{Moderated Poster Session 7: Benign Prostatic Hyperplasia}

\section{MP-7.1}

Cardiac failure may be associated with long-term medical therapy of benign prostatic hyperplasia

Avril J. Lusty', D. Robert Siemens ${ }^{2}$, Mina Tohidi ${ }^{2}$, J. Curtis Nickel ${ }^{2}$

${ }^{1}$ Urology, University of Ottawa, Ottawa, ON, Canada; ${ }^{2}$ Urology, Queen's University, Kingston, ON, Canada

Acknowledgement: Marlo Whitehead

Introduction: Worrisome reports of increased risk of cardiac failure with alpha blockers $(\mathrm{ABs})$ in hypertension studies and 5-alpha reductase inhibitors (5ARIs) in prostate studies have raised safety concerns with these medications for management of benign prostatic hyperplasia (BPH). The objective of this study was to determine if $5 \mathrm{ARIs}$ and/or ABs were associated with an increased risk of cardiac failure in males with $\mathrm{BPH}$.

Methods: A retrospective cohort was created using the province of Ontario's administrative database at the Institute for Clinical Evaluative Sciences. Males aged 66 and over with a diagnosis of BPH were included. Men were categorized based on exposure to $5 \mathrm{ARI}$ and/or $A B$ exposure. Further information collected included: diagnosis of "new" cardiac failure, exposure time to 5ARIs and/or ABs, dosage, age, and comorbidities associated with cardiac disease. Subjects with a history of cardiac failure or prostate cancer were excluded. A competing risk model was used to determine if $5 \mathrm{ARI}$ and/or $\mathrm{AB}$ use was associated with an increased risk of cardiac failure.

Results: From January 1, 2005 to December 31, 2015, 175201 BPH subjects were included in the analysis: 69988 with no treatment, and 8339, 55 383, and 41491 exposed to $5 \mathrm{ARI}, \mathrm{AB}$, and combination therapy, respectively. The competing risk model comparing exposed subjects to those with no treatment, showed that subjects with $\mathrm{BPH}$ treated with $5 \mathrm{ARI}$ and $A B$, alone or in combination, had a statistically increased risk of being diagnosed with cardiac failure. Cardiac failure risk was highest for $\mathrm{ABs}$ alone (hazard ratio [HR] 1.260; 95\% confidence interval [Cl] 1.218-1.304; $\mathrm{p}<0.001$ ), intermediate for combination ABs/5ARIs (HR $1.151 ; 95 \% \mathrm{Cl}$ $1.110-1.194 ; \mathrm{p}<0.001)$, and lowest for 5ARIs alone (HR $1.074 ; 95 \% \mathrm{Cl}$ $1.003-1.151 ; \mathrm{p}=0.0412$ ).

Conclusions: This long-term, retrospective analysis of men with BPH found a statistically significant increase in "new" cardiac failure in men exposed to both $5 \mathrm{ARI}$ and $\mathrm{AB}$ therapy, with the highest risk for men exposed to $\mathrm{AB}$ (alone or in combination with 5ARI).

\section{MP-7.2}

Preoperative accuracy of transabdominal ultrasonography compared to radical prostatectomy specimen for weight and median lobe assessment: Novel use of handheld wireless pointof-care ultrasound (POCUS)

Ghizlane Moussaoui' ', Cristina Negrean'², Felix Couture ${ }^{3}$, Côme Tholomier ${ }^{4}$, David-Dan Nguyen ${ }^{1}$, Ahmed S. Zakaria'2, Kevin C. Zorn'

${ }^{1}$ Faculty of Medicine, McGill University, Montreal, QC, Canada; ${ }^{2}$ Urology, Université de Montréal, Montreal, QC, Canada; ${ }^{3}$ Urology, Université Sherbrooke, Sherbrooke, QC, Canada; ${ }^{4}$ rology, McGill University, Montreal, QC, Canada

Introduction: Prostate size estimation is a valuable clinical measure widely used in urology. Although step-section planimetry remains the gold standard, it is time-consuming and equipment-intensive, discouraging its use in the clinical setting. ${ }^{1,2}$ This study evaluated the accuracy of preoperative transabdominal ultrasound (TAUS) using the prolate ellipsoid formula in estimating prostate volume and median lobe presence compared to radical prostatectomy specimens in different size groups. The effect of the presence of a median lobe on accuracy was assessed.

Methods: A total of 79 men undergoing robot-assisted radical prostatectomy were enrolled in this study. Preoperative evaluation of prostate size was done using measurements obtained from TAUS using the Clarius $\mathrm{C} 3$ handheld, wireless POCUS device. Participants were grouped based on prostate size ( $<30 \mathrm{~g}, 30-60 \mathrm{~g}$, and $>60 \mathrm{~g}$ ). Mean absolute percentage of error (MAPE) was used to evaluate accuracy. Mean percentage error determined if there was an overestimation or underestimation. Correlation between each TAUS size group and true prostate weight was performed.

Results: TAUS estimation was found to be more accurate for larger prostates (Fig. 1). The MAPE for each group was: $49.2 \%$ (<30 g), 28.3\% (30-60 g), and $17.2 \%$ (>60 g) (Table 1). A strong correlation was found between radical prostatectomy specimen weight and large TAUS prostate size $(r=0.938 ; p<0.001)$. TAUS tended to underestimate smaller prostates $(<60 \mathrm{~g})$. Irrespective of body mass index, median lobe was identified accurately in all men with TAUS. The presence of median lobe was associated with greater accuracy (MAPE $16.6 \pm 7.4$ vs. $35.0 \pm 19.2 ; p=0.02$ ) (Table 2, Fig. 2).

Conclusions: Bedside, handheld, wireless POCUS provides rapid, inexpensive, non-invasive, and clinically accurate TAUS prostate assessments for larger prostates. Such features of identifying median lobes and prostate volumes are valuable during office patient counselling of lower urinary

MP-7.2. Table 1. Sample characteristics based on prostate size on ultrasound

\begin{tabular}{|c|c|c|c|c|c|}
\hline & $\begin{array}{c}\text { All patients } \\
(\mathrm{N}=79)\end{array}$ & $\begin{array}{c}<30 \mathrm{~g} \\
(\mathrm{n}=27 ; 34.2 \%)\end{array}$ & $\begin{array}{c}30-60 \mathrm{~g} \\
(\mathrm{n}=39 ; 49.4 \%)\end{array}$ & $\begin{array}{c}>60 \mathrm{~g} \\
(\mathrm{n}=13 ; 16.4 \%)\end{array}$ & $\begin{array}{c}p \\
\text { ANOVA }\end{array}$ \\
\hline Mean age (years) & $61.4 \pm 6.2$ & $59.4 \pm 6.8$ & $62.0 \pm 5.7$ & $64.0 \pm 5.7$ & 0.06 \\
\hline Mean BMI $\left(\mathrm{kg} / \mathrm{m}^{2}\right)$ & $27.6 \pm 4.4$ & $28.1 \pm 3.8$ & $27.1 \pm 3.9$ & $28.1 \pm 6.5$ & 0.63 \\
\hline PSA $(\mu \mathrm{g} / \mathrm{L})$ & $7.2 \pm 3.7$ & $7.0 \pm 3.2$ & $7.1 \pm 3.7$ & $8.3 \pm 4.6$ & 0.54 \\
\hline Initially on active surveillance, $\mathrm{n}$ (\%) & $25(31.6)$ & $6(22.2)$ & $16(41.0)$ & $3(23.1)$ & 0.21 \\
\hline More than 1 lifetime biopsy, n (\%) & $27(34.2)$ & $6(22.2)$ & $18(46.2)$ & $3(23.1)$ & 0.09 \\
\hline Median lobe, $\mathrm{n}(\%)$ & $6(7.6)$ & $0(0)$ & $3(7.7)$ & $3(23.1)$ & 0.04 \\
\hline Mean Gleason score on biopsy & $6.8 \pm 0.4$ & $6.8 \pm 0.4$ & $6.7 \pm 0.4$ & $6.9 \pm 0.5$ & 0.45 \\
\hline Mean Gleason score on final pathology & $6.8 \pm 0.5$ & $6.8 \pm 0.8$ & $6.8 \pm 0.4$ & $6.9 \pm 0.3$ & 0.72 \\
\hline
\end{tabular}


MP-7.2. Table 2. Analysis of accuracy and correlation between size on ultrasound and true prostate weight

\begin{tabular}{|c|c|c|c|c|c|}
\hline & All patients & $<30 \mathrm{~g}$ & $30-60 \mathrm{~g}$ & $>60 \mathrm{~g}$ & p \\
\hline Mean absolute percentage of error (\%) & $33.6 \pm 19.2$ & $49.2 \pm 17.8$ & $28.3 \pm 4.9$ & $17.2 \pm 9.2$ & $<0.001$ \\
\hline Mean percentage of error (\%) & $27.9 \pm 26.9$ & $49.2 \pm 17.8$ & $22.5 \pm 22.9$ & $-0.24 \pm 20.1$ & $<0.001$ \\
\hline Average size by US $\left(\mathrm{cm}^{3}\right)$ & $42.4 \pm 27.2$ & $21 \pm 6.3$ & $41.7 \pm 8.1$ & $87 \pm 37.5$ & $<0.001$ \\
\hline Average true prostate weight (g) & $58.6 \pm 27.3$ & $45 \pm 11.2$ & $56.7 \pm 14.2$ & $92.0 \pm 48.0$ & $<0.001$ \\
\hline Paired-sample t-test $(p)$ & $<0.001$ & $<0.001$ & $<0.001$ & 0.777 & \\
\hline Pearson bivariate correlation analysis & 0.825 & -0.043 & 0.187 & 0.938 & \\
\hline
\end{tabular}
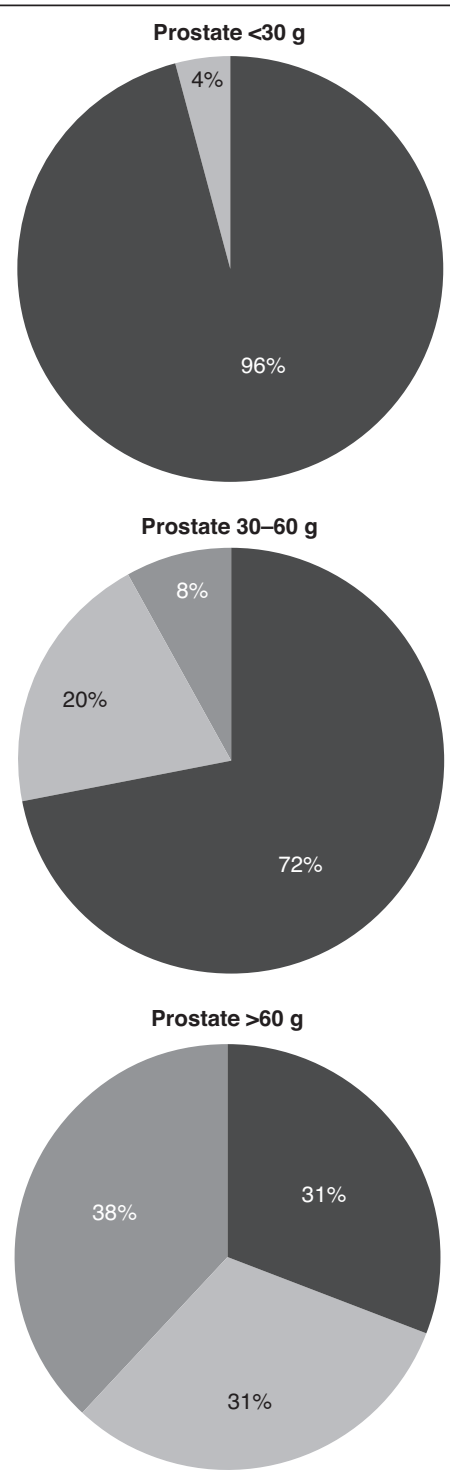

Underestimation (PE >15\%)

Accurate (MAPE $<15 \%)$

Overestimation (PE <-15\%)

MP-7.2. Fig. 1. Proportion of underestimated, accurate, and overestimated measurements stratified by prostate gland size.

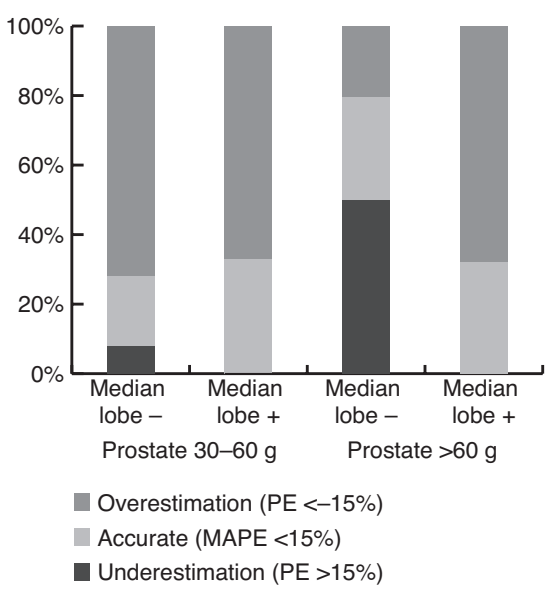

MP-7.2. Fig. 2. Proportion of underestimated, accurate, and overestimated measurements stratified by prostate gland size and presence of median lobe.

tracts symptoms, elevated prostate-specific antigen, and benign prostatic hyperplasia surgical options.

References

1. Eri LM, Thomassen H, Brennhovd B, et al. Accuracy and repeatability of prostate volume measurements by transrectal ultrasound. Prostate Cancer Prostatic Dis 2002;5:273-8. https://doi.org/10.1038/ sj.pcan. 4500568

2. Wolff JM, Boeckmann W, Mattelaer P, et al. Determination of prostate gland volume by transrectal ultrasound: Correlation with radical prostatectomy specimens. Eur Urol 1995;28:10-2. https://doi.org/10.1159/000475012

\section{MP-7.3}

Largest Canadian cohort of Rezūm therapy for large-volume benign prostatic hyperplasia

Dean S. Elterman ${ }^{1}$, Kevin C. Zorn², Naeem Bhojani ${ }^{2}$

${ }^{1}$ Urology, University Health Network, Toronto, ON, Canada; ${ }^{2}$ Urology, Centre hospitalier de I'Université de Montréal, Montreal, QC, Canada Introduction: Open prostatectomy and laser are the standard treatment for benign prostatic hyperplasia (BPH) of large-volume prostates $>80 \mathrm{ml}$. Rezūm is a minimally invasive procedure to ablate benign prostatic tissue. Methods: Rezūm was introduced to Canada in 2019, and these two hospitals were the pioneers. For all patients undergoing Rezūm, baseline medi$\mathrm{cal}$ and $\mathrm{BPH}$ history were documented. Clinical outcomes on prostate symptoms were assessed using uroflowmetry (maximum flow rate [Qmax] and post-void residual [PVR]), International Prostate Symptom Score (IPSS) and Quality of Life (IPSS QoL) and Benign Prostate Hyperplasia Impact Index (BPHII). Impact on sexuality was tracked using International Index of Erectile Function (IIEF-15) and Male Sexual Health Questionnaire ejaculatory function and bother (MSHQ-EjD function and bother). This was an analysis of prostate volumes of $\geq 80 \mathrm{ml}$.

Results: Thirty-four patients of prostate size $\geq 80 \mathrm{ml}$ were treated with Rezūm from April to mid-December 2019. The average prostate volume 


\begin{tabular}{|c|c|c|}
\hline Demographic & Mean & Count \\
\hline Age & 68.4 & 34 \\
\hline \multicolumn{3}{|l|}{ Duration of BPH, years } \\
\hline$<5$ & & 10 \\
\hline $5-7$ & & 9 \\
\hline $8-10$ & & 8 \\
\hline$>10$ & & 7 \\
\hline \multicolumn{3}{|l|}{ Median lobe } \\
\hline Yes & & 26 \\
\hline No & & 8 \\
\hline \multicolumn{3}{|l|}{ Previous BPH surgery } \\
\hline TURP & & 2 \\
\hline PAE & & 1 \\
\hline \multicolumn{3}{|l|}{ Current BPH medication } \\
\hline Alpha-blocker & & 26 \\
\hline $5 A R I$ & & 7 \\
\hline Cialis/Viagra & & 4 \\
\hline None & & 7 \\
\hline \multicolumn{3}{|l|}{ Medical history } \\
\hline Kidney/bladder stone & & 3 \\
\hline Hypertension & & 9 \\
\hline Diabetes & & 4 \\
\hline Dyslipidemia & & 7 \\
\hline History of urinary retention & & 6 \\
\hline
\end{tabular}

was $98.9 \mathrm{ml}$ (range $80-160 \mathrm{ml}$ ) and $76 \%$ had a median lobe. Six of them had a history of urinary retention. On average, patients received 13 Rezūm injections. Visibility and bleeding during the procedure were assessed using a five-point scale, and were rated as 1.7 and 1.8, respectively. Catheter was placed for 5-10 days and was extended to a month in patients with pre-existing retention. Two patients needed catheter replacement due to postoperative retention. The preliminary one-month, three-month, and six-month followup results were analyzed (Table 1). At one month and three months, Qmax had improved by $24.9 \%$ and $53.4 \%$, while PVR by $56.6 \%$ and $68.7 \%$, respectively. Improvement on prostate symptoms was also reflected by the IPSS, IPSS QoL, and BPHII scores by $32.4 \%, 38.2 \%$, and $14.4 \%$, respectively at one month, and more prominently at three months $(55.2 \%, 60.7 \%$, and $61.8 \%$, respectively). Minimal impact on sexuality was observed.

Conclusions: Herein we demonstrate that Rezūm therapy is a viable treatment modality in prostate glands $\geq 80 \mathrm{ml}$.

\section{MP-7.4 \\ Aquablation for benign prostatic hyperplasia in large prostates (80-150cc): Two-year results}

Kevin C. Zorn', Naeem Bhojani', Dean S. Elterman², S. Larry Goldenberg', Alan I. So ${ }^{3}$, Ryan F. Paterson ${ }^{3}$, Mihir Desai ${ }^{4}$, Steven Kaplan', Roehrborn Roehrborn

${ }^{1}$ Urology, Centre hospitalier de I'Université de Montréal, Montreal, QC, Canada; ${ }^{2}$ Urology, University of Toronto, Toronto, ON, Canada; ${ }^{3}$ Urology, University of British Columbia, Vancouver, BC, Canada; ${ }^{4}$ Urology, UTSW, Dallas, TX, United States; ${ }^{5}$ Urology, USC, Los Angeles, CA, United States Support: WATER2 Study Group (ClinicalTrials.gov number, NCT03123250) Introduction: We aimed to report two-year safety and effectiveness outcomes of the Aquablation procedure for the treatment of men with symptomatic benign prostatic hyperplasia $(\mathrm{BPH})$ and large-volume prostates. Methods: A total of 101 men with moderate-to-severe BPH symptoms and prostate volumes of $80-150 \mathrm{cc}$ underwent a robotic-assisted Aquablation procedure in a prospective, multicenter, international clinical trial. Functional and safety outcomes were assessed at two years postoperatively.

Results: Mean prostate volume was 107 cc (range 80-150). Mean operative time was 37 minutes and mean Aquablation resection time was eight minutes. The average length of hospital stay following the procedure was 1.6 days. International Prostate Symptom Score (IPSS) scores improved from 23.2 at baseline to 5.1 at two years (change score of 18.1 points). At baseline, maximum flow rate (Qmax) was $8.7 \mathrm{cc} / \mathrm{sec}$ and improving to $16.6 \mathrm{cc} / \mathrm{sec}$ at 24 months. Improvements in both IPSS and Qmax were immediate and sustained throughout followup. The average annual retreatment occurrence was $0 \%$.

Conclusions: The Aquablation procedure is demonstrated to be safe and effective in treating men with large prostates $(80-150 \mathrm{cc})$ after two year of followup, with an acceptable complication rate and without a significant increase in procedure or resection time compared to smaller sized glands. Two-year results held consistent with one-year outcomes.

\section{MP-7.5}

Transfusion rates after $\mathbf{8 0 0}$ Aquablation procedures using various hemostasis methods

Dean S. Elterman ${ }^{1}$, Thorsten Bach ${ }^{2}$, Enrique Rijo ${ }^{3}$, Vincent Misrai ${ }^{4}$, Paul Anderson ${ }^{5}$, Kevin C. Zorn ${ }^{6}$, Naeem Bhojani ${ }^{6}$, Albert El Hajj ${ }^{7}$, Bilal Chughtai, Mihir Desai

${ }^{1}$ Urology, University Health Network, Toronto, ON, Canada; ${ }^{2}$ Urology, Asklepios Hospital Harburg, Hamburg, Germany; ${ }^{3}$ Urology, Hospital Quirón Salud, Barcelona, Spain; ${ }^{4}$ Urology, Clinique Pasteur, Toulouse, France; ${ }^{5}$ Urology, Royal Melbourne Hospital, Melbourne, Australia; ${ }^{6}$ Urology, Centre hospitalier de l'Université de Montréal, Montreal, QC, Canada; 'Urology, American University of Beirut Medical Center, Beirut, Lebanon; ${ }^{8}$ Urology, Weill Cornell Medical College, New York, NY, United States; ${ }^{9}$ Urology, University of Southern California, Los Angeles, CA, United States

Introduction: Many studies have evaluated the bleeding complication profile postoperatively for transurethral resection of the prostate (TURP)

\begin{tabular}{|c|c|c|c|c|c|c|}
\hline & Mean & SD & Median & Count & Min & Max \\
\hline Omax $(\mathrm{ml} / \mathrm{sec})$ & 8.2 & 4.4 & 8.0 & 30 & 2.0 & 19.0 \\
\hline PVR (ml) & 194.0 & 181.5 & 146.0 & 27 & 2.0 & 820.0 \\
\hline Prostate volume (ml) & 98.9 & 18.7 & 95.0 & 34 & 80.0 & 160.0 \\
\hline PSA (ug/L) & 8.0 & 16.2 & 4.5 & 34 & 0.8 & 98.0 \\
\hline IPSS & 20.9 & 7.2 & 22.0 & 33 & 6 & 35 \\
\hline IIEF & 48.8 & 19.7 & 57 & 27 & 12 & 71 \\
\hline MSHO function & 9.4 & 3.5 & 9 & 24 & 4 & 15 \\
\hline MSHO bother & 1.7 & 1.8 & 1.5 & 24 & 0 & 5 \\
\hline
\end{tabular}




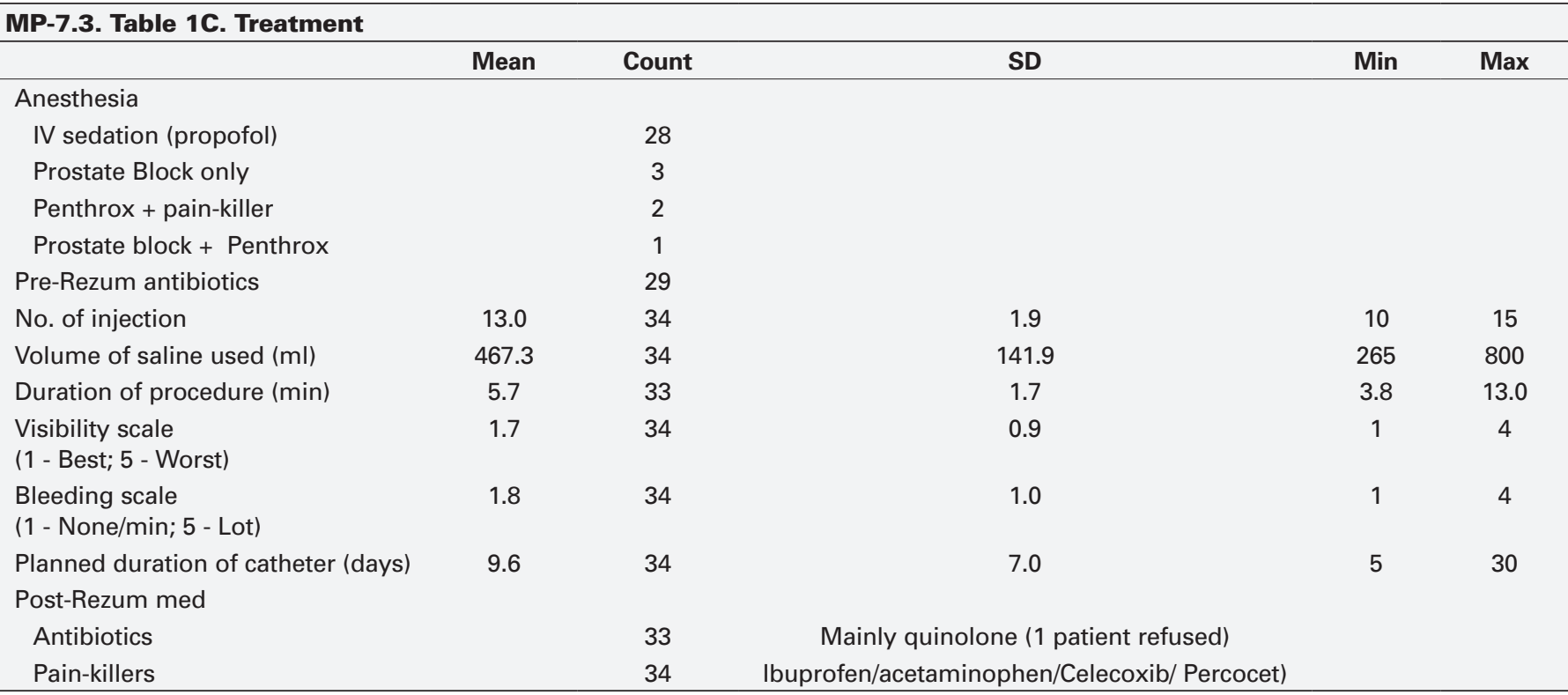

and report a range up to $7 \%$ of patients requiring a blood transfusion, but with typical limitations in treating prostates up to $80 \mathrm{~mL}$ in size. For larger prostates $(>80 \mathrm{~mL})$, open prostatectomy and holmium laser enucleation of the prostate (HoLEP) are the global reference standard surgical options with reported transfusion rates up to $24 \%$ and $4 \%$, respectively.

Methods: The current commercial AQUABEAM robot that performs Aquablation Therapy was first used in 2014. Clinical trial data from 2014 have been pooled with the early commercial procedures from France, Germany, and Spain. The objective is to determine if athermal methods are as effective in preventing blood transfusions as the use of cautery across various prostate volume sizes.

Results: A total of 801 patients were treated with Aquablation therapy from 2014 to early 2019 . The average prostate volume was $67 \pm 33 \mathrm{~mL}$ (range 20-280 mL) where $31(3.9 \%)$ transfusions were reported. The largest contributing factor to transfusion risk was prostate size and method of traction. There was an increasing risk of transfusions in larger prostates when robust traction using a catheter tensioning device without cautery ranging from $0.8-7.8 \%$ in prostates ranging from $20-280 \mathrm{~mL}$. However when standard traction (taping the catheter to the leg, gauze knot synched up to the meatus, or no traction at all) was used and where the surgeon performed bladder neck cautery only when necessary, the risk of transfusion was $1.4-2.5 \%$ in prostates ranging from $20-280 \mathrm{~mL}$.

Conclusions: While the athermal subgroup with robust traction with a catheter tension device had comparable transfusion rates for smaller prostates, the risk increased significantly as prostate volume increased. With standard traction methods and selective bladder neck cautery, the risk of transfusion is reduced to a $1.9 \%$ across all prostate sizes.

\section{MP-7.6}

Predictors of anejaculation after the Aquablation procedure for benign prostatic hyperplasia

David-Dan Nguyen ${ }^{1,2}$, Dean S. Elterman ${ }^{3}$, Kevin C. Zorn ${ }^{4}$, Naeem Bhojani ${ }^{1}$ Faculty of Medicine, McGill University, Montreal, QC, Canada; ${ }^{2}$ Health Policy and Management, Harvard T.H. Chan School of Public Health, Boston, MA, United States; ${ }^{3}$ Division of Urology, University of Toronto, Toronto, ON Canada; ${ }^{4}$ Division of Urology, Université de Montréal, Montreal, QC, Canada Introduction: The Aquablation procedure is a new, robotically executed, resective surgical treatment for lower urinary tract symptoms related to benign prostatic hyperplasia (BPH). Anejaculation following Aquablation is less frequent, presumably due to precise resection targeting through use of transrectal imaging and robotic resection. We sought to determine to what extent avoidance of key anatomic structures prevent postoperative anejaculation after Aquablation?
Methods: Sexually active participants in WATER, WATER II, and WATER FRANCAIS who had normal preoperative Male Sexual Health Questionnaire (MSHQ-EjD) scores and marked postoperative decreases in scores were each matched with 1-2 sexually active men from the same trial with similar prostate sizes whose postoperative scores were not decreased. Video logs from the procedure were scored by an expert blinded to case/control status for the following: veru cut coverage as a percent in the sagittal plane, penetration of ejaculatory ducts, approximate depth of cut below the veru on sagittal images, approximate angle offset of veru to centerline of protection zone, number of passes, and intraprostatic calcifications. Conditional logistic regression was used to calculate univariate and multivariate odds ratios relating anatomic findings to case/control status.

Results: The 24 cases and 27 controls had preoperative mean prostate volumes of 82 and $81 \mathrm{cc}$, respectively. In univariate analysis, statistically significant predictors of postoperative anejaculation were: penetration of the ejaculatory duct (odds ratio [OR] 8.6; 95\% confidence interval [CI] $1.09-67.5 ; p=0.041)$ and depth below the veru (OR 1.92; 95\% Cl 1.1-3.3; $\mathrm{p}=0.015)$. Multivariate regression showed no independent predictors but veru depth showed an elevated OR $(2.92 ; 95 \% \mathrm{Cl} 0.90-9.45)$.

Conclusions: Violation of anatomic structures involved in ejaculation during the Aquablation procedure increases the risk of postoperative anejaculation. More careful attention to anatomic structures during contour planning may help to reduce the rate of postoperative anejaculation after Aquablation.

\section{MP-7.7}

Global Greenlight group: Largest international Greenlight experience for benign prostatic hyperplasia

Kyle W. Law' ${ }^{1}$, Côme Tholomier ${ }^{2}$, Félix Couture ${ }^{3}$, Ahmed S. Zakaria', DavidDan Nguyen ${ }^{1}$, Luca Cindolo ${ }^{5}$, Giovanni Ferrari ${ }^{5}$, Carlos Vasquez-Lastra ${ }^{6}$, Tiago J. Borelli-Bovo , Edgardo F. Becher ${ }^{8}$, Hannes Cash', Maximilian Reimann ${ }^{9}$, Vincent Misrail ${ }^{10}$, Kevin C. Zorn ${ }^{4}$

${ }^{1}$ Faculty of Medicine, McGill University, Montreal, QC, Canada; ${ }^{2}$ Division of Urology, McGill University, Montreal, QC, Canada; ${ }^{3}$ Division of Urology, Université de Sherbrooke, Sherbrooke, QC, Canada; ${ }^{4}$ Department of Urology, Centre hospitalier de I'Université de Montréal, Montreal, QC, Canada; ${ }^{5}$ Department of Urology, Hesperia Hospital, Modena, Italy; ${ }^{6}$ Department of Urology, ABC Medical Center, Mexico City, Mexico; 'Department of Urology, Borelli Urologia, Ribeirão Presto, Brazil; ${ }^{8}$ Centro de Urologia, CDU, Buenos Aires, Argentina; ${ }^{9}$ Department of Urology, Universitaetsmedizin Berlin, Berlin, Germany; ${ }^{10}$ Department of Urology, Clinique Pasteur, Toulouse, France 


\begin{tabular}{|c|c|c|c|c|c|c|c|c|}
\hline & & & & & & & & \\
\hline & Mean & SD & Min & Max & Mean & SD & Min & Max \\
\hline Omax $(\mathrm{ml} / \mathrm{sec})$ & & & & & & & & \\
\hline $\mathrm{n}$ (paired value) & $n=21$ & & & & $\mathrm{n}=14$ & & & \\
\hline Baseline (mean \pm SD) & 8.4 & 4.4 & & & 9.9 & 4.9 & & \\
\hline Followup (mean $\pm \mathrm{SD}$ ) & 10.5 & 4.5 & & & 15.1 & 5.7 & & \\
\hline Change (mean \pm SD) & 2.1 & 6.1 & -7.0 & 18.0 & 5.3 & 6.3 & -3 & 17 \\
\hline$\%$ of change & $24.9 \%$ & & & & $53.4 \%$ & & & \\
\hline PVR (ml) & & & & & & & & \\
\hline $\mathrm{n}$ (paired value) & $\mathrm{n}=18$ & & & & $n=13$ & & & \\
\hline Baseline (mean \pm SD) & 176.8 & 134.4 & & & 186.5 & 155.8 & & \\
\hline Followup (mean $\pm \mathrm{SD}$ ) & 76.8 & 77.4 & & & 58.4 & 42.2 & & \\
\hline Change (mean $\pm \mathrm{SD}$ ) & -100.0 & 137.5 & -487 & 121 & -128.2 & 149.0 & -482 & 35 \\
\hline$\%$ of change & $-56.6 \%$ & & & & $-68.7 \%$ & & & \\
\hline IPSS & & & & & & & & \\
\hline $\mathrm{n}$ (paired value) & $n=29$ & & & & $\mathrm{n}=21$ & & & \\
\hline Baseline (mean \pm SD) & 19.9 & 6.8 & & & 20.7 & 7.0 & & \\
\hline Followup $($ mean $\pm S D)$ & 13.4 & 6.1 & & & 8.6 & 4.9 & & \\
\hline Change (mean $\pm \mathrm{SD}$ ) & -6.4 & 7.6 & -21 & 10 & -11.4 & 7.9 & -25 & -1 \\
\hline$\%$ of change & $-32.4 \%$ & & & & $-55.2 \%$ & & & \\
\hline IPSS QoL & & & & & & & & \\
\hline n (paired value) & $n=29$ & & & & $\mathrm{n}=21$ & & & \\
\hline Baseline (mean $\pm \mathrm{SD})$ & 4.2 & 1.2 & & & 4.2 & 1.2 & & \\
\hline Followup $($ mean $\pm S D)$ & 2.6 & 1.4 & & & 1.7 & 1.2 & & \\
\hline Change (mean $\pm \mathrm{SD})$ & -1.6 & 1.8 & -5 & 1 & -2.6 & 1.5 & -5 & 0 \\
\hline$\%$ of change & $-38.2 \%$ & & & & $-60.7 \%$ & & & \\
\hline BPHII & & & & & & & & \\
\hline $\mathrm{n}$ (paired value) & $n=27$ & & & & $\mathrm{n}=15$ & & & \\
\hline Baseline (mean $\pm \mathrm{SD}$ ) & 6.7 & 2.9 & & & 7.1 & 3.2 & & \\
\hline Followup (mean $\pm \mathrm{SD}$ ) & 5.7 & 3.3 & & & 2.7 & 2.4 & & \\
\hline Change (mean $\pm \mathrm{SD}$ ) & -1.0 & 3.5 & -7 & 7 & -4.4 & 2.8 & -9 & 0 \\
\hline$\%$ of change & $-14.4 \%$ & & & & $-61.8 \%$ & & & \\
\hline IIEF & & & & & & & & \\
\hline $\mathrm{n}$ (paired value) & $\mathrm{n}=17$ & & & & $\mathrm{n}=15$ & & & \\
\hline Baseline (mean \pm SD) & 52.0 & 17.7 & & & 49.8 & 20.0 & & \\
\hline Followup $($ mean $\pm S D$ ) & 53.1 & 17.9 & & & 55.8 & 16.4 & & \\
\hline Change (mean $\pm \mathrm{SD}$ ) & 1.1 & 12.4 & -37 & 17 & 6.0 & 11.4 & -10 & 28 \\
\hline$\%$ of change & $2.1 \%$ & & & & $12.0 \%$ & & & \\
\hline MSHO function & & & & & & & & \\
\hline n (paired value) & $n=18$ & & & & $\mathrm{n}=15$ & & & \\
\hline Baseline $($ mean $\pm \mathrm{SD})$ & 9.1 & 3.1 & & & 8.9 & 3.7 & & \\
\hline Followup $($ mean $\pm S D)$ & 10.5 & 4.2 & & & 9.4 & 4.7 & & \\
\hline Change (mean $\pm \mathrm{SD})$ & 1.4 & 3.7 & -5 & 8 & -0.2 & 4.6 & -8 & 11 \\
\hline$\%$ of change & $15.2 \%$ & & & & $-2.3 \%$ & & & \\
\hline MSHO bother & & & & & & & & \\
\hline $\mathrm{n}$ (paired value) & $\mathrm{n}=18$ & & & & $\mathrm{n}=15$ & & & \\
\hline Baseline (mean $\pm \mathrm{SD}$ ) & 1.8 & 1.7 & & & 1.7 & 1.9 & & \\
\hline Followup $($ mean $\pm S D)$ & 1.8 & 1.2 & & & 1.5 & 1.4 & & \\
\hline Change (mean \pm SD) & 0.2 & 1.8 & -3 & 3 & -0.2 & 2.6 & -5 & 4 \\
\hline$\%$ of change & $12.1 \%$ & & & & $-11.5 \%$ & & & \\
\hline
\end{tabular}




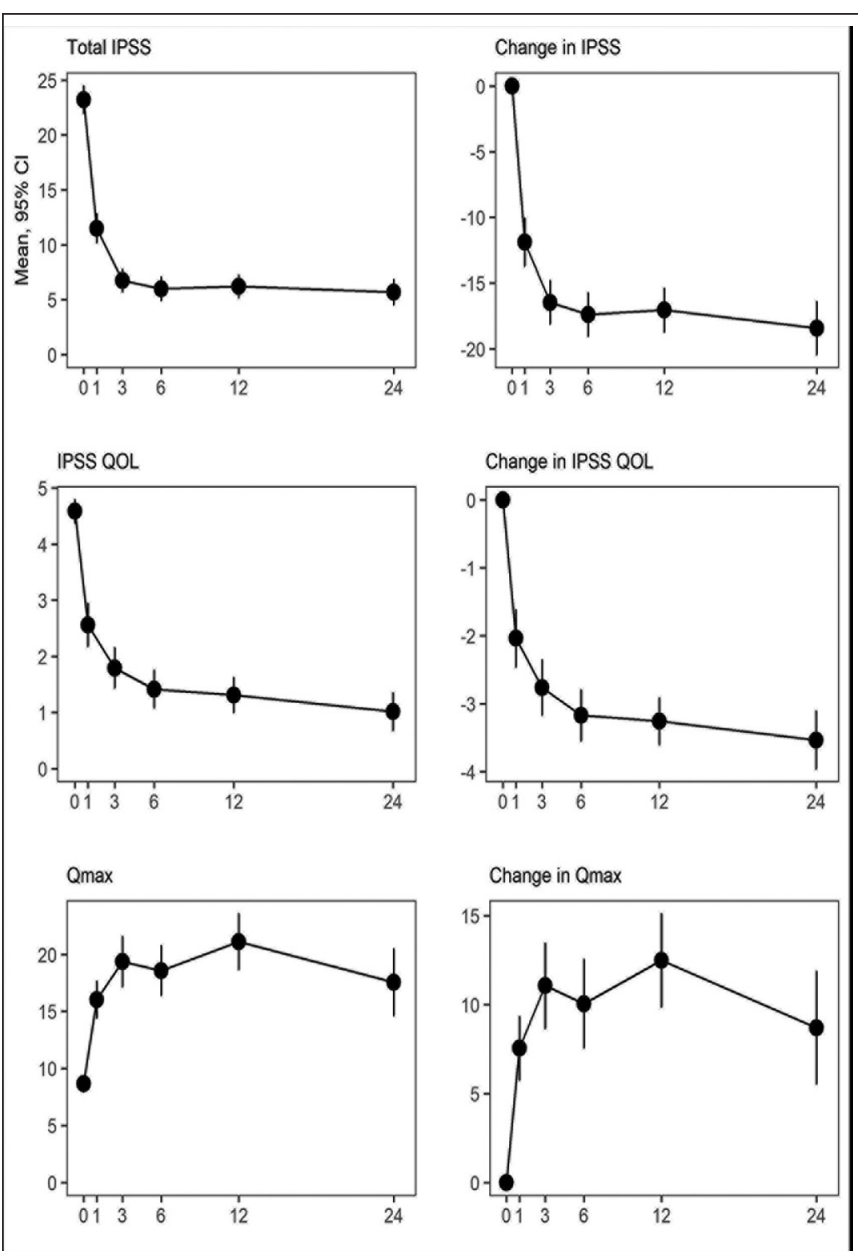

MP-7.4. Fig. 1.

Introduction Among the surgical treatment modalities available, Greenlight photo-selective vaporization of the prostate (GL-PVP) has gained international acceptance as a safe and effective alternative procedure for the treatment of benign prosatatic hyperplasia (BPH), with hemostatic benefits particularly in anticoagulated men. This descriptive analysis aims to characterize the current state of GL-PVP, pooling intraand postoperative outcomes from multiple academic centres globally.

Methods: Data from 3809 patients who underwent Greenlight 180W-XPS PVP in seven international centers by eight expert surgeons between 2011 and 2019 was retrospectively analyzed. Demographic, peri-, and postoperative data was collected, including International Prostate Symptom Score (IPSS), quality of life (QOL), maximum urinary flow rate (Qmax), post-void residual (PVR), and prostate-specific antigen (PSA). ClavienDindo postoperative adverse events were also assessed.

Results: Median age, prostate volume, PSA, and IPSS was 70 years (interquartile range [IQR] 64-77), $65 \mathrm{cc}(47-90), 3.1 \mathrm{ng} / \mathrm{mL}$ (1.8-6.0), and 22 (19-27), respectively. Median lasing time was 34 minutes (23-48) during a median 62 minutes (45-85) procedure. Median energy use was $250 \mathrm{~kJ}$ (167-367), with $92.5 \%$ of procedures being completed with one laser fiber. In $60.6 \%$ of cases, Foley catheter was removed on postoperative day 1 , with $86.2 \%$ of men being discharged within three days of admission. The 30-day postoperative readmission rate was $13.2 \%$, while major cardiac events and death occurred in $0.8 \%$ and $0.3 \%$ of cases, respectively. Prostatic adenocarcinoma was found on tissue pathology in $1.9 \%$ of cases. Median PSA reduction at 12 months, 24 months, and 60 months was $46.3 \%, 53.5 \%$, and $46.6 \%$, respectively. $\mathrm{BPH}$ recurrence requiring repeat surgical intervention was observed at $1.5 \%$ at five years of followup.

\begin{tabular}{|c|c|}
\hline Parameters & $\begin{array}{l}\text { Median (interquartile } \\
\text { range) or mean } \pm \text { SD or } \\
\text { number (percentage) }\end{array}$ \\
\hline Age & $70(64-77)$ \\
\hline BMI & $26.1(24.0-29.0)$ \\
\hline TRUS prostate volume (mL) & $65(47-90)$ \\
\hline PSA (ng/mL) & $3.11(1.8-6.0)$ \\
\hline PSA density (ng/mL/cc) & $0.048(0.028-0.082)$ \\
\hline Median lobe presence & $628(36.5 \%)$ \\
\hline SHIM & $17(12-21)$ \\
\hline IPSS & $22(19-27)$ \\
\hline QoL & $4(3-5)$ \\
\hline Omax (mL/s) & $6.3(4.0-9.0)$ \\
\hline PVR (mL) & $122(32-291)$ \\
\hline Previous TURP & $157(6.4 \%)$ \\
\hline Use of 5-ARI & $1296(35.4 \%)$ \\
\hline Use of alpha-blockers & $2826(77.4 \%)$ \\
\hline Use of anticoagulants & $1261(34.2 \%)$ \\
\hline Pre-operative Foley & $1183(35.3 \%)$ \\
\hline \multicolumn{2}{|l|}{ ASA } \\
\hline 1 & $462(21.0 \%)$ \\
\hline 2 & $1106(50.2 \%)$ \\
\hline 3 & $617(28.0 \%)$ \\
\hline 4 & $20(0.9 \%)$ \\
\hline \multicolumn{2}{|l|}{ Operative characteristics } \\
\hline Parameters & $\begin{array}{l}\text { Median (interquartile } \\
\text { range) or mean } \pm \text { SD or } \\
\text { number (percentage) }\end{array}$ \\
\hline TRUS prostate volume $(\mathrm{mL})$ & $65(47-90)$ \\
\hline OR time (min) & $62(45-85)$ \\
\hline Laser/OR time ratio & $0.44(0.31-0.55)$ \\
\hline Energy used (kJ) & $250(167-367)$ \\
\hline $\begin{array}{l}\text { Energy used/preoperative prostate } \\
\text { volume }(\mathrm{kJ} / \mathrm{mL})\end{array}$ & $3.90(2.87-5.00)$ \\
\hline$<3 \mathrm{~kJ} / \mathrm{cc}$ & $1158(31.7 \%)$ \\
\hline $3-4 \mathrm{~kJ} / \mathrm{cc}$ & $883(24.2 \%)$ \\
\hline$>4 \mathrm{~kJ} / \mathrm{cc}$ & $1610(44.1 \%)$ \\
\hline \multicolumn{2}{|l|}{ Fibers used } \\
\hline 1 & 2906 (92.5\%) \\
\hline 2 & $219(7.0 \%)$ \\
\hline $3+$ & $17(0.5 \%)$ \\
\hline Intraoperative transfusion & $23(0.8 \%)$ \\
\hline Intraoperative complication & $138(12.2 \%)$ \\
\hline Hospital stay (days) & $2(1-3)$ \\
\hline 0 & $596(16.1 \%)$ \\
\hline 1 & 1114 (30.1\%) \\
\hline 2 & $953(25.8 \%)$ \\
\hline 3 & $524(14.2 \%)$ \\
\hline 4 & $198(5.4 \%)$ \\
\hline 5 & $115(3.1 \%)$ \\
\hline $6-119$ & $195(5.3 \%)$ \\
\hline
\end{tabular}

Conclusions: To the best of our knowledge, from the largest multi-user, international experience, Greenlight PVP of the prostate is a safe, effective, and durable $\mathrm{BPH}$ procedure. 


\begin{tabular}{|c|c|}
\hline \multicolumn{2}{|c|}{ MP-7.7. Table 1 (cont'd). Patient demographics } \\
\hline \multicolumn{2}{|l|}{ Operative characteristics (cont'd) } \\
\hline Parameters & $\begin{array}{l}\text { Median (interquartile } \\
\text { range) or mean } \pm \text { SD or } \\
\text { number (percentage) }\end{array}$ \\
\hline Foley catheterization duration (days) & $1(1-2)$ \\
\hline 0 & $99(3.4 \%)$ \\
\hline 1 & $1749(60.6 \%)$ \\
\hline 2 & $602(20.9 \%)$ \\
\hline 3 & $250(8.7 \%)$ \\
\hline $4-44$ & $187(6,5 \%)$ \\
\hline Required re-catheterization & $170(5.9 \%)$ \\
\hline Adenocarcinoma on pathology & $17(1.9 \%)$ \\
\hline 30-day readmission & $209(13.2 \%)$ \\
\hline \multicolumn{2}{|l|}{ Postoperative complications ( $\leq 30$ days) } \\
\hline Parameters & Number of patients (\%) \\
\hline \multicolumn{2}{|l|}{ Minor } \\
\hline Fever & $63(4.0 \%)$ \\
\hline UTI & $126(5.4 \%)$ \\
\hline LUTS & $522(22.3 \%)$ \\
\hline $\mathrm{OAB}$ & $7(1.1 \%)$ \\
\hline Incontinence & $244(10.4 \%)$ \\
\hline Retention & $173(7.4 \%)$ \\
\hline Hematuria & $232(9.9 \%)$ \\
\hline \multicolumn{2}{|l|}{ Major } \\
\hline Urosepsis & $5(0.8 \%)$ \\
\hline Osteitis Pubis & $1(0.2 \%)$ \\
\hline Retrograde ejaculation & $35(4.4 \%)$ \\
\hline Paraphymosis & $1(0.2 \%)$ \\
\hline Prostatic capsule perforation & $8(0.5 \%)$ \\
\hline Postoperative transfusion & $21(1.3 \%)$ \\
\hline $\begin{array}{l}\text { Stenosis (urethra, meatus, bladder } \\
\text { neck) }\end{array}$ & $2(0.1 \%)$ \\
\hline False passage & $1(0,2 \%)$ \\
\hline Arrhythmia & $6(0.4 \%)$ \\
\hline Major cardiac event & $12(0.8 \%)$ \\
\hline Respiratory distress (desaturation) & $3(0.2 \%)$ \\
\hline Death & $4(0.3 \%)$ \\
\hline
\end{tabular}

\section{MP-7.8}

Top-down Greenlight laser enucleation of the prostate (GreenLEP): Early results

Fabiola Oquendo' ${ }^{1}$, Amr Hodhod ${ }^{1}$, Loay Abbas ${ }^{1}$, Brianna Beaudry' ${ }^{1}$, Ahmed Kotb', Owen Prowse', Walid Shahrour ${ }^{1}$, Hazem Elmansy'

${ }^{1}$ Urology, Northern Ontario School of Medicine, Thunder Bay, ON, Canada

Introduction: Laser prostatectomy is considered one of the best modalities for the surgical management of benign prostatic hyperplasia (BPH). Recently, the top-down technique was introduced as a modification to traditional holmium laser enucleation (HoLEP). Herein, we describe the top-down technique using Greenlight laser technology and its feasibility. Methods: Between 2018 and 2019, we prospectively collected the data of 20 patients who underwent Greenlight enucleation of the prostate using the top-down technique (top-down GreenLEP). A Greenlight XPS machine and a 2090 laser fibre were used for these procedures. One posterior groove was created at either 5 or 7 o'clock up to the verumontanum allowing enucleation of the median lobe with the attached lateral lobe. After the anterior commissure mucosa was incised at the 12 o'clock position, a top-down lateral lobe dissection was performed and extended anteroposteriorly, towards the apical adenoma at the 6 o'clock position. As the apical dissection was performed from top-down, the mucosal strip was easily visualized on the medial side of the scope. By incising the band-shaped mucosa close to the adenoma, the apex of the adenoma was completely released without damaging the ring formed by the sphincter muscle. We recorded patients' demographics, intraoperative, and postoperative data. The preoperative and postoperative International Prostate Symptom Score (IPSS), quality of life (QOL), maximum urinary flow rate (Qmax), and post-void residual (PVR) were evaluated.

Results: The median prostatic volume was 92 cc (80-150) and the median age at surgery was 63.4 years. There were no intraoperative complications or blood transfusions. All patients had their catheters removed the next day postoperatively. Only two patients $(10 \%)$ had failed a trial of voiding (TOV) initially but succeeded after three days. The one-month postoperative data are presented in Table 1.

Conclusions: Top-down GreenLEP is a feasible procedure. Early outcomes are satisfactory and promising. To better evaluate this technique, further studies with larger populations and longer followup are required.

\section{MP-7.9}

Does the complexity affect the outcome of top-down holmium laser enucleation of prostate (top-down HoLEP)?

Amr Hodhod', Fabiola Oquendo', Loay Abbas' ${ }^{1}$, Brianna Beaudry', Ahmed Kotb ${ }^{1}$, Owen Prowse', Walid Shahrour ${ }^{1}$, Hazem Elmansy

'Urology, Northern Ontario School of Medicine, Thunder Bay, ON, Canada

Introduction: In this study, we evaluated the outcome of top-down holmium laser enucleation of prostate (HoLEP) for complex cases due to repeated transurethral resection of the prostate (TURP) and prostate cancer, in comparison to non-complex prostates.

Methods: We retrospectively reviewed the charts of prospectively collected patients who underwent top-down HoLEP, by a single urologist (HE), between 2017 and 2018. We used a 100-W holmium:YAG laser (VersaPulse ${ }^{\circledast}$ PowerSuite, Lumenis) with a $550 \mu \mathrm{m}$ laser fiber and a $28 \mathrm{Fr}$ continuous flow resectoscope. Enucleated tissue was morcellated using a Karl Storz DrillCut ${ }^{\mathrm{TM}}$ Morcellator. We recorded the enucleation time, the morcellation time, and intraoperative and postoperative complications. All patients were followed up postoperatively at three, six, and 12 months. The evaluation included the International Prostate Symptom Score (IPSS), quality of life (QOL), maximum urinary flow rate (Qmax), and post-void residual (PVR).

Results: Sixty patients were enrolled in this study. The complex group consisted of 27 patients, while the control group included 33 patients. Patients' characteristics are shown in Table 1. The complexity of HoLEP was due to recurrent TURP (24 patients) and prostate cancer (three patients). The mean enucleation time for the complex group was 102.1 minutes (42-131), and 80.8 minutes (45-128) for the control group $(p=0.04)$. There was no significant difference between both groups in terms of morcellation time, resected weight, catheter duration, and hospital stay. One patient in the control group had a slight bladder mucosal injury. The postoperative outcomes regarding IPSS, QoL, Qmax, and PVR were comparable between both groups (Table 2). At one-year followup, one patient in the complex group had stress incontinence $(p=0.26)$ and one patient in each group experienced urge incontinence $(p=0.89)$.

Conclusions: Apart from a significantly longer enucleation time in the complex group, the safety and postoperative outcomes of top-down HoLEP were similar for both groups.

\section{MP-7.10}

Effect of holmium laser enucleation of the prostate on prostatespecific antigen kinetics in patients with prostate cancer on active surveillance

Ahmed Ibrahim', Ahmed Zakaria', Mélanie Aube-Peterkin', Simon Tanguay', Serge Carrier', Armen-G. Aprikian'

${ }^{1}$ Urology, McGill University, Montreal, QC, Canada

Introduction: Our aim was to evaluate the effect of holmium laser enucleation of the prostate (HoLEP) on prostate-specific antigen (PSA) kinetics for prostate cancer (PCa) patients actively managed by observation. 
MP-7.7. Table 1. (cont'd). Patient demographics

\begin{tabular}{|c|c|c|c|c|c|c|c|c|}
\hline \multicolumn{9}{|c|}{ Functional outcomes } \\
\hline \multirow[t]{2}{*}{ Outcome } & \multirow[t]{2}{*}{ Preoperative } & \multicolumn{7}{|c|}{ Months } \\
\hline & & 3 & 6 & 12 & 24 & 36 & 48 & 60 \\
\hline PSA (ng/mL) & $\begin{array}{c}3.11 \\
(1.8-6)\end{array}$ & - & $\begin{array}{c}1.6 \\
(0.8-3.14)\end{array}$ & $\begin{array}{c}1.5 \\
(0.7-2.8)\end{array}$ & $\begin{array}{c}1.7 \\
(0.7-3.7)\end{array}$ & $\begin{array}{c}1.7 \\
(0.7-3.7)\end{array}$ & $\begin{array}{c}1.7 \\
(0.7-3.7)\end{array}$ & $\begin{array}{c}1.4 \\
(0.6-3.6)\end{array}$ \\
\hline IPSS & $\begin{array}{c}22 \\
(19-27)\end{array}$ & $\begin{array}{c}6 \\
(4-9)\end{array}$ & $\begin{array}{c}5 \\
(4-9)\end{array}$ & $\begin{array}{c}4 \\
(2-7)\end{array}$ & $\begin{array}{c}4 \\
(3-7)\end{array}$ & $\begin{array}{c}4 \\
(3-7)\end{array}$ & $\begin{array}{c}4 \\
(3-7)\end{array}$ & $\begin{array}{c}5 \\
(3-8)\end{array}$ \\
\hline $\operatorname{Omax}(\mathrm{mL} / \mathrm{s})$ & $\begin{array}{c}6.3 \\
(4-9)\end{array}$ & $\begin{array}{c}19 \\
(16-22)\end{array}$ & $\begin{array}{c}18 \\
(15-22)\end{array}$ & $\begin{array}{c}18 \\
(15-22)\end{array}$ & $\begin{array}{c}19 \\
(15-23)\end{array}$ & $\begin{array}{c}18 \\
(15-22)\end{array}$ & $\begin{array}{c}17 \\
(14-22)\end{array}$ & $\begin{array}{c}17 \\
(14-21)\end{array}$ \\
\hline PVR (mL) & $\begin{array}{c}122 \\
(32-291)\end{array}$ & $\begin{array}{c}17 \\
(0-50.8)\end{array}$ & $\begin{array}{c}15 \\
(0-38)\end{array}$ & $\begin{array}{c}15 \\
(0-40)\end{array}$ & $\begin{array}{c}10 \\
(0-40)\end{array}$ & $\begin{array}{c}10 \\
(0-41)\end{array}$ & $\begin{array}{c}15 \\
(0-50.3)\end{array}$ & $\begin{array}{c}23.5 \\
(3.5-63.1)\end{array}$ \\
\hline \multicolumn{2}{|c|}{$\begin{array}{l}\text { BPH recurrence requiring } \\
\text { repeat surgery }\end{array}$} & \multicolumn{7}{|c|}{$1.5 \%$} \\
\hline
\end{tabular}

\begin{tabular}{|c|c|c|}
\hline \multicolumn{2}{|l|}{ Data } & Finding \\
\hline \multicolumn{2}{|l|}{ Age median (range) } & $\begin{array}{l}63.4 \text { years } \\
(55.9-88.3)\end{array}$ \\
\hline \multicolumn{2}{|c|}{ Size of prostatic adenoma median (range) } & 92 cc $(80-150)$ \\
\hline \multicolumn{2}{|c|}{ Preoperative PSA median (range) } & $4.7 \mathrm{ng} / \mathrm{dl}(2.1-12.6)$ \\
\hline \multicolumn{2}{|c|}{ Enucleation time median (range) } & $79(35-170)$ \\
\hline \multicolumn{2}{|c|}{ Morcellation time median (range) } & $13.1(5-26)$ \\
\hline \multicolumn{2}{|c|}{$\begin{array}{l}\text { Median resected weight }(\mathrm{g}) \text { median } \\
\text { (range) }\end{array}$} & $86(48-130)$ \\
\hline \multicolumn{2}{|l|}{ Laser energy median } & $156 \mathrm{KJ}(141-169)$ \\
\hline \multicolumn{2}{|c|}{ Stress urinary incontinence $\mathrm{n}(\%)$} & $2(10 \%)$ \\
\hline \multicolumn{2}{|c|}{ Urge urinary incontinence $\mathrm{n}(\%)$} & $2(10 \%)$ \\
\hline \multirow{2}{*}{ IPSS median (range) } & Preoperative & $22(7-30)$ \\
\hline & Postoperative & $7(0-10)$ \\
\hline \multirow{2}{*}{ QoL median (range) } & Preoperative & $5(2-6)$ \\
\hline & Postoperative & $1(0-5)$ \\
\hline \multirow{2}{*}{ Omax median (range) } & Preoperative & $7.3(5.2-14.5)$ \\
\hline & Postoperative & $22.9 \mathrm{ml} / \mathrm{s}(17.5-36.1)$ \\
\hline \multirow{2}{*}{ PVR median (range) } & Preoperative & $204 \mathrm{ml}(87-380)$ \\
\hline & Postoperative & $48 \mathrm{ml}(0-78)$ \\
\hline
\end{tabular}

Methods: A prospectively maintained database for patients undergoing HoLEP was reviewed. Patients with PCa diagnosis between 1998 and 2016 managed by active surveillance (AS) were included for analysis. Cohort demographics and perioperative data were collected and compared to a regular benign prostatic hyperplasia $(\mathrm{BPH})$ group undergoing HoLEP at baseline. Univarite and multivariate analyses were performed using linear regression models.

Results: Of the 1445 HoLEP cases, 124 (8.6\%) patients were identified to have PCa. Only $25(2 \%)$ patients met the study inclusion criteria of being on AS prior to the HoLEP; the median age at the time of surgery was $72.5(69-79)$ years and the median prostate volume was $64.5 \mathrm{~mL}$. After a median followup of 7.3 years, the absolute median decline in the first PSA measurement post-HoLEP was $4.7 \mathrm{ng} / \mathrm{ml}$ with a relative decrease of $47.4 \%$. The median lowest PSA reached post-HoLEP was $1.1 \mathrm{ng} / \mathrm{ml}$ within the first year after HoLEP. In different followup visits, there were no significant differences in terms of PSA values between the study cohort and the BPH group undergoing HoLEP (all $p>0.05$ ). On a univariate analysis, higher baseline primary Gleason score and a lower percentage of cancer in post-HoLEP specimen were found to significantly increase relative PSA changes post-HoLEP ( $p=0.004$ and $p=0.022$, respectively). Additionally, the majority of AS patients undergoing HoLEP were found to have lower postoperative PSA values when compared to the TURP group (1.1 vs.3.2 ng/ml; $\mathrm{p}=0.019$ ).

Conclusions: PCa patients on AS undergoing HoLEP had a very slow PSA velocity after HoLEP. This could be attributed to higher resection velocity (efficiency) of the HoLEP procedure. Further prospective studies are warranted to compare HoLEP with other minimally invasive procedures to determine the effect of efficient resection on postoperative PSA nadirs.

\section{MP-7.11}

Postoperative storage symptoms of holmium laser enucleation vs. Greenlight vaporization of the prostate for symptomatic benign prostatic hyperplasia

Ahmed Ibrahim ${ }^{1}$, Mélanie Aube-Peterkin' ${ }^{1}$, Serge Carrier ${ }^{1}$

${ }^{1}$ Urology, McGill University, Montreal, QC, Canada

Introduction: We aimed to compare Greenlight laser plasma-vaporization of the prostate (PVP) vs. holmium laser enucleation of the prostate (HoLEP) in improving postoperative storage lower urinary tract symptoms (LUTS) in a large series of patients.

Methods: A retrospective review for patients undergoing HoLEP or PVP for symptomatic benign prostatic hyperplasia (BPH) between March 2002 and July 2016 was performed. Patients with urethral stricture, previous prostate surgery, prostate cancer, or known history of neurogenic voiding dysfunction were excluded from the study. Patients were followed up at one, three, six, and 12 month postoperatively, including prostate volume, International Prostate Symptoms Score (IPSS), quality of life (QOL), peak flow rate (Qmax), post-void residual (PVR), and prostate- specific antigen (PSA). The subtotal storage symptom score of IPSS was used to compare within and between groups, including the frequency, urgency, and nocturia scores. Moderate or severe storage symptoms were defined as IPSS storage score $\geq 9$.

Results: Out of 1755 HoLEP and PVP procedures, 1100 were included in the final analysis: 809 HoLEP vs. 291 PVP procedures. The HoLEP group had significantly larger prostates, higher total IPSS, and longer operative times (Table 1). Postoperatively, HoLEP patients had significantly lower IPSS than PVP group up to 12 months. Storage score was significantly higher after PVP and did not improve until three months postoperatively. After then, the storage symptoms improved and became comparable with that of the HoLEP group. In contrast, the subtotal storage symptom score was significantly decreased after HoLEP compared to the baseline values. Number of patients with IPSS-storage score $\geq 9$ were significantly higher in PVP group at one and three months' followup (37.3\% vs. $15.1 \%$; $\mathrm{p}<0.001$ and $26.6 \%$ vs. $17.5 \%$; $p=0.004$, respectively).

Conclusions: Storage urinary symptoms significantly improved after HoLEP compared to PVP. Patients undergoing PVP may have to wait at least three months postoperatively to have their storage symptoms relived. 


\begin{tabular}{|c|c|c|c|c|}
\hline \multicolumn{5}{|c|}{$\begin{array}{l}\text { MP-7.9. Table 1. Patient's characteristics and } \\
\text { perioperative data }\end{array}$} \\
\hline \multicolumn{2}{|l|}{ Parameter } & Complex group & Control group & $\mathbf{p}$ \\
\hline \multicolumn{2}{|c|}{$\begin{array}{l}\text { Number of patients } \\
(\%)\end{array}$} & $27(45)$ & $33(55)$ & - \\
\hline \multicolumn{2}{|c|}{$\begin{array}{l}\text { Age at surgery, mean } \\
\text { (range) }\end{array}$} & $\begin{array}{l}74.5 \text { years } \\
(57-87.4)\end{array}$ & $\begin{array}{l}71.5 \text { years } \\
(54-88)\end{array}$ & 0.051 \\
\hline \multicolumn{2}{|c|}{$\begin{array}{l}\text { Prostate size, mean } \\
\text { (range) }\end{array}$} & $\begin{array}{l}144.7 \mathrm{~g} \\
(70-237)\end{array}$ & $\begin{array}{c}127.6 \mathrm{~g} \\
(74-266)\end{array}$ & 0.31 \\
\hline \multicolumn{2}{|c|}{$\begin{array}{l}\text { Pre-IPSS, mean } \\
\text { (range) }\end{array}$} & $24.3(17-31)$ & $19.6(10-34)$ & 0.15 \\
\hline \multicolumn{2}{|c|}{ Pre-PVR, mean (range) } & $\begin{array}{l}190.5 \mathrm{ml} \\
(11-508)\end{array}$ & $\begin{array}{l}267.9 \mathrm{ml} \\
(21-780)\end{array}$ & 0.18 \\
\hline \multicolumn{2}{|c|}{$\begin{array}{l}\text { Pre-Qmax, mean } \\
\text { (range) }\end{array}$} & $\begin{array}{l}11.3 \mathrm{ml} / \mathrm{s} \\
(8.3-14.2)\end{array}$ & $\begin{array}{l}8.1 \mathrm{ml} / \mathrm{s} \\
(5.5-15)\end{array}$ & 0.53 \\
\hline \multicolumn{2}{|c|}{ Pre-QoL, mean (range) } & $5.1(3-6)$ & $4.5(2-6)$ & 0.96 \\
\hline \multicolumn{2}{|c|}{$\begin{array}{l}\text { Preoperative PSA, } \\
\text { mean (range) }\end{array}$} & $\begin{array}{l}8.1 \mathrm{ng} / \mathrm{ml} \\
(2-17)\end{array}$ & $\begin{array}{l}9.2 \mathrm{ng} / \mathrm{ml} \\
(1.2-27.1)\end{array}$ & 0.55 \\
\hline \multicolumn{5}{|c|}{ Cause of complexity } \\
\hline \multicolumn{2}{|c|}{$\begin{array}{l}\text { Recurrent after BPH } \\
\mathrm{n}(\%)\end{array}$} & $24(88.9)$ & - & - \\
\hline \multicolumn{2}{|c|}{$\begin{array}{l}\text { Prostate cancer } n \\
(\%)\end{array}$} & $3(11.1)$ & - & - \\
\hline \multicolumn{2}{|c|}{$\begin{array}{l}\text { Enucleation time, } \\
\text { mean (range) }\end{array}$} & $\begin{array}{l}102.1 \mathrm{~min} \\
(42-131)\end{array}$ & $\begin{array}{l}80.8 \min \\
(45-128)\end{array}$ & 0.04 \\
\hline \multicolumn{2}{|c|}{$\begin{array}{l}\text { Morcellation time, } \\
\text { mean (range) }\end{array}$} & $18.1 \min (6-58)$ & $\begin{array}{l}19 \text { minutes } \\
(4-45)\end{array}$ & 0.96 \\
\hline \multicolumn{2}{|c|}{$\begin{array}{l}\text { Resected weight, } \\
\text { mean (range) }\end{array}$} & $\begin{array}{c}115.5 \mathrm{~g} \\
(46-202)\end{array}$ & $\begin{array}{c}103.5 \mathrm{~g} \\
(44-242)\end{array}$ & 0.41 \\
\hline \multicolumn{2}{|c|}{ Energy, mean (range) } & $\begin{array}{c}187.7 \mathrm{~kJ} \\
(93.6-319)\end{array}$ & $\begin{array}{c}172.7 \mathrm{~kJ} \\
(92.3-257)\end{array}$ & 0.23 \\
\hline \multirow{2}{*}{$\begin{array}{l}\text { Catheter } \\
\text { stay, } \\
\text { n (\%) }\end{array}$} & $\begin{array}{l}<24 \\
\text { hours }\end{array}$ & $26(96.3)$ & 31 (93.9) & \multirow{2}{*}{0.68} \\
\hline & $\begin{array}{l}>24 \\
\text { hours }\end{array}$ & $1(3.7)$ & $2(6.1)$ & \\
\hline \multirow{2}{*}{$\begin{array}{l}\text { Hospital } \\
\text { stay, } \\
\text { n (\%) }\end{array}$} & $\begin{array}{l}<24 \\
\text { hours }\end{array}$ & $25(92.6)$ & $32(97)$ & \multirow{2}{*}{0.44} \\
\hline & $\begin{array}{l}>24 \\
\text { hours }\end{array}$ & $2(7.4)$ & $1(3)$ & \\
\hline
\end{tabular}

This should be considered in choosing the appropriate option for management of patients presenting with severe irritative voiding symptoms and should be discussed with patients before surgery.

\section{MP-7.12}

The one-year outcomes of holmium laser vapo-resection of the prostate

Amr Hodhod ${ }^{1}$, Fabiola Oquendo ${ }^{1}$, Loay Abbas ${ }^{1}$, Brianna Beaudry ${ }^{1}$, Jill Skogberg ${ }^{1}$, Ahmed Kotb ${ }^{1}$, Owen Prowse ${ }^{1}$, Walid Shahrour ${ }^{1}$, Hazem Elmansy ${ }^{1}$

${ }^{1}$ Urology, Northern Ontario School of Medicine, Thunder Bay, ON, Canada

Introduction: We introduce the one-year outcomes of vapo-resection of the prostate using the newly introduced Xpeeda ${ }^{\mathrm{TM}}$ side-firing holmium laser fiber.

Methods: Thirty-six patients underwent holmium laser vapo-resection of the prostate (HoLVRP) from July 2018 to June 2019. We used a 100W holmium:YAG laser (VersaPulse ${ }^{\circledR}$ PowerSuite ${ }^{\mathrm{TM}}$; Lumenis) with a side-firing
MP-7.9. Table 2. Top-down postoperative outcome measures at 3, 6, 12 months

\begin{tabular}{|c|c|c|c|}
\hline Parameter & $\begin{array}{l}\text { Complex } \\
\text { group }\end{array}$ & $\begin{array}{l}\text { Control } \\
\text { group }\end{array}$ & $\mathbf{p}$ \\
\hline \multicolumn{4}{|l|}{ IPSS } \\
\hline Mean (range) 3 months & $5.9(0-21)$ & $3.1(0-11)$ & 0.11 \\
\hline Mean (range) 6 months & $3.9(0-14)$ & $3(0-12)$ & 0.55 \\
\hline Mean (range) 12 months & $3.1(0-7)$ & $2.1(0-9)$ & 0.87 \\
\hline \multicolumn{4}{|l|}{ QoL } \\
\hline Mean (range) 3 months & $0.63(0-5)$ & $0.7(0-4)$ & 0.77 \\
\hline Mean (range) 6 months & $0.88(0-4)$ & $0.4(0-4)$ & 0.44 \\
\hline Mean (range) 12 months & $0.67(0-3)$ & $0.7(0-4)$ & 0.68 \\
\hline \multicolumn{4}{|l|}{ PVR } \\
\hline $\begin{array}{l}\text { Mean (range) } \\
3 \text { months }\end{array}$ & $\begin{array}{l}56.2 \mathrm{ml} \\
(0-200)\end{array}$ & $\begin{array}{l}48.7 \mathrm{ml} \\
(0-109)\end{array}$ & 0.94 \\
\hline $\begin{array}{l}\text { Mean (range) } \\
12 \text { months }\end{array}$ & $\begin{array}{l}18.4 \mathrm{ml} \\
(0-48)\end{array}$ & $\begin{array}{l}10.8 \mathrm{ml} \\
(0-43)\end{array}$ & 0.79 \\
\hline $\begin{array}{l}\text { Qmax } \\
\text { Mean (range) } 3 \text { months }\end{array}$ & $\begin{array}{c}30.7 \mathrm{ml} / \mathrm{s} \\
(12.3-48.7)\end{array}$ & $\begin{array}{c}24.3 \mathrm{ml} / \mathrm{s} \\
(12.5-48.1)\end{array}$ & 0.13 \\
\hline Mean (range) 6 months & $\begin{array}{c}27 \mathrm{ml} / \mathrm{s} \\
(14.1-48)\end{array}$ & $\begin{array}{l}25.5 \mathrm{ml} / \mathrm{s} \\
(14.8-42)\end{array}$ & 0.28 \\
\hline Mean (range) 12 months & $\begin{array}{c}23.6 \mathrm{ml} / \mathrm{s} \\
(14.8-32.1)\end{array}$ & $\begin{array}{l}25.8 \mathrm{ml} / \mathrm{s} \\
(14-44.8)\end{array}$ & 0.86 \\
\hline $\begin{array}{l}\text { PSA } \\
\text { Mean (range) } 3 \text { months }\end{array}$ & $\begin{array}{l}0.99 \mathrm{ng} / \mathrm{ml} \\
(0.29-5.1)\end{array}$ & $\begin{array}{l}1.1 \mathrm{ng} / \mathrm{ml} \\
(0.16-9)\end{array}$ & 0.56 \\
\hline $\begin{array}{l}\text { Stress incontinence (1y) } \\
\mathrm{n}(\%)\end{array}$ & $1(3.7)$ & 0 & 0.26 \\
\hline $\begin{array}{l}\text { Urge incontinence (1y) } \\
\mathrm{n}(\%)\end{array}$ & $1(3.7)$ & $1(3)$ & 0.89 \\
\hline
\end{tabular}

Xpeeda $^{T M}$ laser fiber. The patients were managed by a single surgeon (HE). Patient demographics and preoperative data were recorded, including transrectal ultrasonography prostate sizing and preoperative prostate-specific antigen (PSA), International Prostate Symptom Score (IPSS), quality of life (QOL), maximum flow rate (Qmax), and post-void residual (PVR). The laser energy and operative times were recorded. Intra- and postoperative complications were collected in addition to catheter time and the duration of hospital stay. Trial of void (TOV) was conducted two hours after the procedure. The short-term followup data included IPSS, QoL, Qmax, and PVR at one, three, six, and 12 months postoperatively.

Results: Patient characteristics are presented in Table 1. All patients had

MP-7.12. Table 1. Patient characteristics, preoperative, and operative data

\begin{tabular}{lc} 
Parameter & Findings \\
Age, mean (range) & 72.7 years (57-91) \\
Prostate size, mean (range) & $49.3 \mathrm{ml}(30-70)$ \\
Preoperative PSA, mean (range) & $3.2 \mathrm{ng} / \mathrm{ml}(0.55-10.4)$ \\
Preoperative IPSS, median (range) & $22.3(4-34)$ \\
Preoperative QoL, median (range) & $5(3-6)$ \\
Preoperative PVR, mean (range) & $295 \mathrm{ml}(23-487)$ \\
Preoperative Omax, mean (range) & $7.8(3.8-11.7)$ \\
Recurrent after TURP, n (\%) & $4(11.1)$ \\
Vaporesection time, mean (range) & 49.4 minutes (23-80) \\
Laser energy, mean (range) & $245 \mathrm{~kJ}(70-414)$ \\
\hline
\end{tabular}




\begin{tabular}{|c|c|c|}
\hline Parameter & Findings & $\mathbf{p}$ \\
\hline \multicolumn{3}{|l|}{ IPSS } \\
\hline Median (range) 1 month & $7(1-28)$ & \\
\hline Median (range) 3 months & $5(1-23)$ & 0.018 \\
\hline Median (range) 6 months & $4(2-15)$ & \\
\hline Median (range) 12 months & $3(0-12)$ & \\
\hline \multicolumn{3}{|l|}{ QoL } \\
\hline Median (range) 1 month & $2(0-5)$ & \\
\hline Median (range) 3 months & $2(0-6)$ & 0.015 \\
\hline Median (range) 6 months & $1(0-3)$ & \\
\hline Median (range) 12 months & $0(0-3)$ & \\
\hline \multicolumn{3}{|l|}{ PVR } \\
\hline Mean (range) 1 month & $70 \mathrm{ml}(8-270)$ & \\
\hline Mean (range) 3 months & $71 \mathrm{ml}(10-287)$ & 0.13 \\
\hline Mean (range) 6 months & 75 ml (8-328) & \\
\hline Mean (range) 12 months & $44 \mathrm{ml}(5-154)$ & \\
\hline \multicolumn{3}{|l|}{ Qmax } \\
\hline Mean (range) 1 month & $19 \mathrm{ml} / \mathrm{s}(11-41)$ & \\
\hline Mean (range) 3 months & $23 \mathrm{ml} / \mathrm{s}(6.8-50)$ & \\
\hline Mean (range) 6 months & $23.6 \mathrm{ml} / \mathrm{s}(8.3-44)$ & \\
\hline Mean (range) 12 months & $24 \mathrm{ml} / \mathrm{s}(17.4-40.4)$ & \\
\hline \multicolumn{3}{|l|}{ PSA } \\
\hline Mean (range) preoperative & $3.2 \mathrm{ng} / \mathrm{ml}(0.55-10.4)$ & \\
\hline Mean (range) 3 months & 0.59 ng/ml (0.3-3.9) & \\
\hline
\end{tabular}

their catheters removed two hours postoperatively. Initially, four patients $(11.1 \%)$ failed their TOV (requiring catheter reinsertion) but succeeded within 1-3 days. Postoperatively, one patient experienced stress urinary incontinence and another patient developed urge incontinence; both symptoms disappeared at three months' followup. All preoperative parameters significantly improved postoperatively ( $p<0.001$ for all parameters) and continued to show significant improvement throughout the followup in terms of IPSS, QoL, and Qmax $(p=0.018, p=0.015$, and $p=0.003$, respectively), whereas there was slight improvement of PVR when comparing one, three, six, and 12 months' followup ( $p=0.13$ ) (Table 2). A single patient experienced intraoperative bladder perforation and was managed conservatively.

Conclusions: The one-year outcomes indicate that HoLVRP is a promising and safe technique for the management of moderate-sized prostates.

\footnotetext{
MP-7.13

Thulium fiber laser enucleation of the prostate: Initial description of new laser platform

Jonathan Moore', Raphael V Carrera², Haidar Abdul-Muhsin', Amihay Nevo Pima', Scott Cheney ${ }^{1}$, Donald Neff', Mitchell Humphreys ${ }^{\prime}$

'Department of Urology, Mayo Clinic Arizona, Phoenix, AZ, United States; ${ }^{2}$ Department of Urology, University of Kansas Medical Center, Kansas City, KS, United States

Introduction: Thulium fiber laser enucleation of the prostate (ThuFLEP) is a new laser technology with a smaller, quieter, more energy efficient generator. ${ }^{1}$ The thulium laser fiber has a wider range of frequency and energy settings than traditional holmium:YAG or thulium:YAG lasers. Thulium fiber lasers have been used for treatment of nephrolithiasis. ${ }^{1}$ Early descriptions exist of ThuFLEP from Europe, ${ }^{2,3}$ however, this represents the first series from North America.

Methods: We prospectively reviewed patients who underwent a ThuFLEP using the Olympus SOLVITE Premium Super Pulsed Laser for benign prostatic
}

\begin{tabular}{|c|c|}
\hline Parameter & $\mathrm{n}=9$ \\
\hline \multicolumn{2}{|l|}{ Age at time of surgery } \\
\hline Mean (SD) & $66.0(8.95)$ \\
\hline Median & 67.68 \\
\hline Range & $(50.1-76.69)$ \\
\hline \multicolumn{2}{|l|}{$\mathrm{BMI}\left(\mathrm{kg} / \mathrm{m}^{2}\right)$} \\
\hline Mean (SD) & $26.7(4.91)$ \\
\hline Median & 25.70 \\
\hline Range & $(20.98-34.57)$ \\
\hline \multicolumn{2}{|l|}{ Charlson index points } \\
\hline Mean (SD) & $2.33(0.87)$ \\
\hline Median & 3 \\
\hline Range & $(1-3)$ \\
\hline Preoperative indwelling catheterization & $2(22 \%)$ \\
\hline \multicolumn{2}{|l|}{ Duration of catheterization (days) } \\
\hline Mean (SD) & $128.5(19.1)$ \\
\hline Median & 128.5 \\
\hline Range & $(115-142)$ \\
\hline \multicolumn{2}{|l|}{ BPH medications } \\
\hline Alpha-blockers & $6(67 \%)$ \\
\hline 5AR inhibitors & 0 \\
\hline PDE5 inhibitors & $1(11 \%)$ \\
\hline \multicolumn{2}{|l|}{ Previous BPH surgery } \\
\hline TURP & $2(22 \%)$ \\
\hline Urolift & $2(22 \%)$ \\
\hline TUNA & $1(11 \%)$ \\
\hline HoLEP & 0 \\
\hline \multicolumn{2}{|l|}{ Prostate size $\left(\mathrm{cm}^{3}\right)$} \\
\hline Mean (SD) & $106.7(53.6)$ \\
\hline Median & 89.6 \\
\hline Range & (38-196) \\
\hline Any anticoagulation & $0(0 \%)$ \\
\hline \multicolumn{2}{|l|}{ Preoperative $\mathrm{Hgb}$} \\
\hline Mean (SD) & $14.4(1.7)$ \\
\hline Median & 14.8 \\
\hline Range & $(11.6-16.5)$ \\
\hline \multicolumn{2}{|l|}{ Preoperative PSA } \\
\hline Mean (SD) & $5.61(3.44)$ \\
\hline Median & 5.25 \\
\hline Range & $(1.4-13.2)$ \\
\hline \multicolumn{2}{|l|}{ ASA score } \\
\hline 1 & $1(11 \%)$ \\
\hline 2 & 7 (77\%) \\
\hline 3 & $1(11 \%)$ \\
\hline
\end{tabular}

hyperplasia from October to December 2019. Demographic and baseline variables were collected, including age, body mass index (BMI), comorbidities, prostate size, and preoperative bloodwork. In addition, we described perioperative outcomes, complications, and early functional outcomes. Results: A total of nine patients underwent ThuFLEP. Baseline characteristics (Table 1) included mean age 66.32 (standard deviation [SD] 9.43), BMI $23.7 \mathrm{~kg} / \mathrm{m}^{2}$ (SD 4.19), Charlson comorbidity index 2.33 (SD 0.87), 


\begin{tabular}{|c|c|c|c|}
\hline Parameter & $n=9$ & Parameter & $\mathrm{n}=9$ \\
\hline $\begin{array}{l}\text { Enucleation } \\
\text { technique }\end{array}$ & & $\begin{array}{l}\text { Catheterization } \\
\text { time (hours) }\end{array}$ & \\
\hline Bilobar & $5(55.6 \%)$ & Mean (SD) & $27(20.48)$ \\
\hline Trilobar & $4(44.4 \%)$ & Median & 22 \\
\hline $\begin{array}{l}\text { Total operative } \\
\text { time (min) }\end{array}$ & & Range & $(5-77)$ \\
\hline Mean (SD) & $123.1(72.4)$ & $\mathrm{EBL}$ & \\
\hline Median & 126.0 & Mean (SD) & $25(21.55)$ \\
\hline Range & $(30-249)$ & Median & 17.5 \\
\hline \multicolumn{2}{|c|}{ Enucleation time ( $\mathrm{min})$} & Range & $(0-50)$ \\
\hline Mean (SD) & $71.8(34.0)$ & \multicolumn{2}{|l|}{ Postoperative Hgb } \\
\hline Median & 71.0 & Mean (SD) & $12.7(1.69)$ \\
\hline Range & $(25-112)$ & Median & 12.7 \\
\hline \multicolumn{2}{|c|}{ Total laser time (min) } & Range & (10.8-15.4) \\
\hline Mean (SD) & $43.1(15.3)$ & \multicolumn{2}{|l|}{ Change in $\mathrm{Hgb}(\mathrm{g} / \mathrm{dL})$} \\
\hline Median & 39 & Mean (SD) & $-1.3(1.3)$ \\
\hline Range & $(20-62)$ & Median & -1.1 \\
\hline \multicolumn{2}{|c|}{ Total laser energy $(\mathrm{kJ})$} & Range & $(-3.8-0.2)$ \\
\hline Mean (SD) & $85.3(25.5)$ & \multirow{2}{*}{\multicolumn{2}{|c|}{$\begin{array}{l}\text { Resected prostate } \\
\text { weight }(\mathrm{g})\end{array}$}} \\
\hline Median & 76.1 & & \\
\hline Range & $(51.8-125.3)$ & Mean (SD) & $64.25(36.14)$ \\
\hline \multicolumn{2}{|c|}{ Morcellation time (min) } & Median & 56.0 \\
\hline Mean (SD) & $19.3(19.8)$ & Range & $(27-129)$ \\
\hline Median & 13.0 & \multirow{2}{*}{\multicolumn{2}{|c|}{$\begin{array}{l}\text { Resection } \\
\text { efficiency (g/min) }\end{array}$}} \\
\hline Range & $(5-69)$ & & \\
\hline \multirow{2}{*}{$\begin{array}{l}\text { Hematuria scale } \\
\text { value at } 4 \text { hours }\end{array}$} & \multirow[t]{2}{*}{$\mathrm{n}=8$} & Mean (SD) & $1.08(0.23)$ \\
\hline & & Median & 0.99 \\
\hline $0-2$ & 3 & Range & $(0.55-1.23)$ \\
\hline $3-4$ & 1 & Complications & \\
\hline $5-6$ & 3 & Clavien I or II & $1(11.1 \%)$ \\
\hline $7-8$ & 1 & Clavien III-V & $0(0 \%)$ \\
\hline \multirow{2}{*}{$\begin{array}{l}\text { Hospital stay } \\
\text { (hours) }\end{array}$} & & Blood transfusion & $0(0 \%)$ \\
\hline & & Re-catheterization & $0(0 \%)$ \\
\hline Mean (SD) & $21.4(11.8)$ & \multirow{6}{*}{$\begin{array}{l}\text { 30-day } \\
\text { re-admission }\end{array}$} & $0(0 \%)$ \\
\hline Median & 25.0 & & \\
\hline Range & $(6-40)$ & & \\
\hline \multicolumn{2}{|c|}{ Outpatient protocol } & & \\
\hline Yes & $4(44.4 \%)$ & & \\
\hline No & $5(55.6 \%)$ & & \\
\hline
\end{tabular}

and prostate size $109.1 \mathrm{~cm}^{3}$ (SD 56.8, range 38-196). Mean total operative time was 123.1 minutes (SD 72.4), with a laser time of 43.1 minutes (SD 15.3) and laser energy of 85.3 kJ (SD 23.5) (Table 2). Nearly half (44\%) of patients were discharged the same day, with a mean hospital stay of 21.4 hours (SD 11.8, range 6-40) and catheterization time of 27 hours (range 5-77). Resection efficiency was excellent, with a mean of $1.08 \mathrm{~g} /$ min (SD 0.99). There was minimal blood loss, with a mean postoperative hemoglobin change of $13 \mathrm{~g} / \mathrm{L}$, as well as no intraoperative complications, readmission, or re-catheterization.

Conclusions: ThuFLEP is a safe and efficient treatment method for BPH over a wide range of prostate sizes. Practical advantages of the device, including reduced noise, size, and power requirements and direct transfer- ability of HoLEP skills, make the thulium laser fiber an attractive option for future laser platforms.

\section{References}

1. Kronenberg P, Traxer O. The laser of the future: Reality and expectations about the new thulium fiber laser - a systematic review. Transl Androl Urol 2019;8:S398-417. https://doi.org/10.21037/ tau.2019.08.01

2. Enikeev D, Netsch C, Rapoport L, et al. Novel thulium fiber laser for endoscopic enucleation of the prostate: A prospective comparison with conventional transurethral resection of the prostate. Int I Urol 2019;26:1138-43. https://doi.org/10.1111/iju.14115

3. Enikeev D, Okhunov Z, Rapoport L, et al. Novel thulium fiber laser for enucleation of prostate: A retrospective comparison with open simple prostatectomy. J Endourol 2019;33:16-21. https://doi. org/10.1089/end.2018.0791

\section{MP-7.14}

Variations in operative time in benign prostatic hyperplasia: Comparison of Aquablation, Greenlight PVP, GreenLEP, HoLEP, and ThuLEP

Iman Sadri', David-Dan Nguyen ${ }^{1}$, Vincent Misraï ${ }^{2}$, Thorsten Bach ${ }^{3}$, Naeem Bhojani $^{4}$, James E. Lingeman ${ }^{5}$, Dean S. Elterman ${ }^{6}$, Kevin C. Zorn ${ }^{4}$

${ }^{1}$ Faculty of Medicine, McGill University, Montreal, QC, Canada; ${ }^{2}$ Department of Urology, Clinique Pasteur, Toulouse, France; ${ }^{3}$ Department of Urology, Asklepios Hospital Harburg, Hamburg, Germany; ${ }^{4}$ Division of Urology, Université de Montréal, Montreal, QC, Canada; ${ }^{5}$ Department of Urology, Indiana University School of Medicine, Indianapolis, IN, United States; ${ }^{6}$ Division of Urology, University of Toronto, Toronto, ON, Canada

Introduction: The surgical armamentarium for benign prostatic hyperplasia is composed of a rapidly expanding number of technologies and techniques. These include, but are not limited to Greenlight photovaporization of the prostate (PVP), Greenlight enucleation of the prostate (GreenLEP), holmium laser enucleation of the prostate (HoLEP), thulium laser enucleation of the prostate (ThuLEP) and, more recently, the Aquablation procedure. To our knowledge, no direct comparison in their operative time has been performed.

Methods: Data for this study were pooled from five sources. For Aquablation, patient-level data from four studies of the procedure were provided by the device manufacturer, as well as data from a high-volume commercial user. PVP, GreenLEP, HoLEP, and ThuLEP were performed by high-volume, skilled experts. Procedure characteristics included total operative time, resection time, and the fraction of total operative time spent on resection. Linear models were used to evaluate the relationship

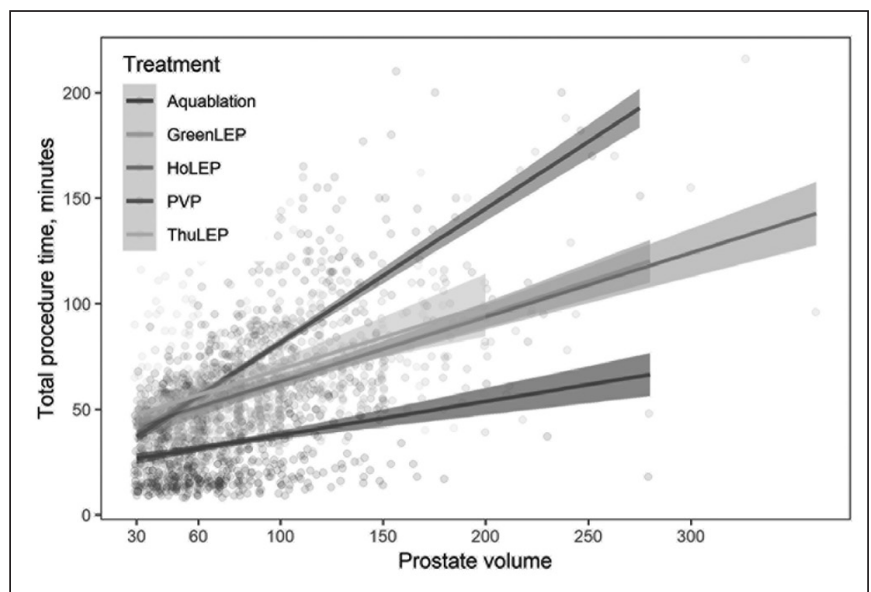

MP-7.14. Fig. 1. Total procedure time by prostate volume and treatment. Each dot represents an individual patient. Lines/shaded regions represent linear fit with $95 \%$ confidence interval. 


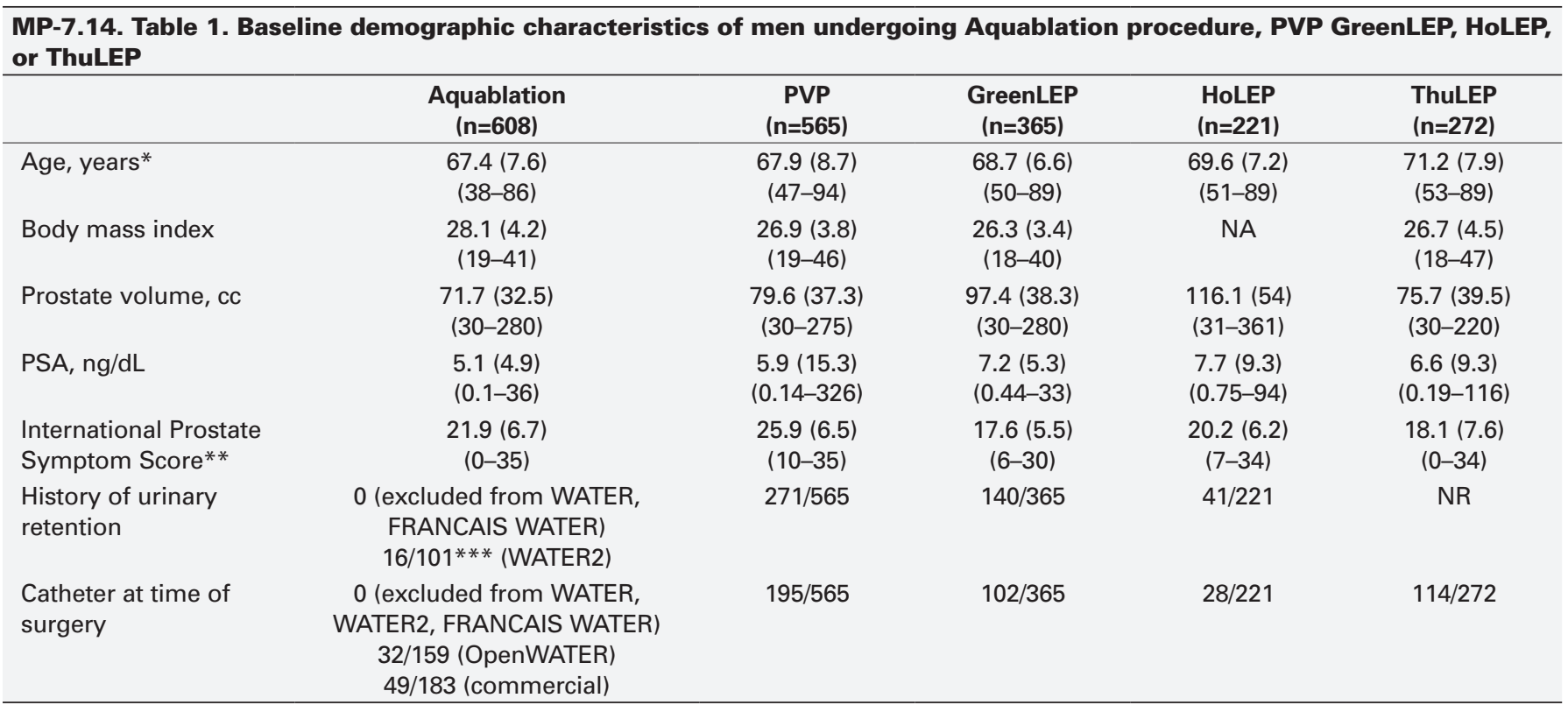

between prostate volume and procedure time. Postoperative followup consisted of International Prostate Symptom Score (IPSS) comparisons.

Results: Baseline demographics are presented in Table 1. Total procedure time was strongly related to prostate size. Except for prostates smaller than $40 \mathrm{cc}$, at any given prostate volume, procedure time was longest for PVP, intermediate for LEPs, and lowest for Aquablation (Fig. 1). The relationship between procedure time and prostate size (i.e., slope of the fitted lines) was $0.16 \mathrm{~min} / \mathrm{g}$ for Aquablation, $0.32 \mathrm{~min} / \mathrm{g}, 0.28 \mathrm{~min} / \mathrm{g}$, and $0.32 \mathrm{~min} / \mathrm{g}$ for GreenLEP, HoLEP, and ThuLEP, respectively, and $0.63 \mathrm{~min} / \mathrm{g}$ for PVP. Conclusions: Our analysis of the pooled data of multisurgical techniques and technologies by high-volume experts demonstrated that Aquablation provided the lowest operative time across all prostate volumes. PVP had the longest procedure time in prostates $>40 \mathrm{cc}$.

\section{MP-7.15}

A simple opioid education session for urologists decreases opioid prescribing following transurethral resection of the prostate Sohrab Naushad Ali', Nicholas Paterson', Kristen McAlpine', Tinghua Zhang ${ }^{2,3}$, Brian D. Blew ${ }^{1}$, Neal E. Rowe ${ }^{1,3}$

${ }^{1}$ Division of Urology, University of Ottawa, Ottawa, ON, Canada; ${ }^{2} \mathrm{Ottawa}$ Methods Centre, The Ottawa Hospital, Ottawa, ON, Canada; ${ }^{3}$ Ottawa Health Research Institute, The Ottawa Hospital, Ottawa, ON, Canada Introduction: Opioid over-prescription continues to be a driving force in the opioid crisis worldwide. ${ }^{1,2}$ According to Health Canada, there were 2861 opioid-related deaths in 2016 in Canada alone. ${ }^{3}$ In patients who undergo urological surgery, one in 1000 develop opioid dependence or have an opioid-related adverse event, including death. ${ }^{4}$ Factors that contribute to the overall problem include lack of physician training in prescribing opioids and concerns over patient perceived pain. Our study aims to identify opioid prescription trends for a common endourological procedure and assess the efficacy of an educational intervention in reducing opioid prescribing.

Methods: In March 2019, an educational rounds session was held for all staff and resident physicians in the division of urology at our institution. The dangers of opioid over-prescription and opioid reduction strategies were discussed. We reviewed all cases of transurethral resection of prostate (TURP) for one year prior and seven months following the educational intervention. Patient age, primary vs. secondary procedure, resection time, resection weight, days in hospital, opioid prescription status, morphine milligram equivalents (MME), and resident vs. staff prescriptions were evaluated in both groups. Multivariate regression analyses were performed to evaluate risk factors for opioid prescription and identify changes in opioid prescription post-educational intervention.

Results: A total of 314 TURP procedures were performed during the study period. Of these, 184 were pre-educational intervention and 130 were post-intervention. Demographics were similar between both groups. An average of 28.1 MMEs per patient were prescribed in the preintervention group compared to 11.4 MME's post-intervention. The most prescribed opioid was tramadol, followed by hydromorphone. Patients in the post-intervention group were four times less likely to receive an opioid prescription (odds ratio [OR] 0.23; 95\% confidence interval [Cl] $0.138-0.383 ; \mathrm{p}<0.0001)$. Overall, there was $60 \%$ reduction in opioid prescription post-intervention. Of all variables tested, none of the factors reached statistical significance as independent risk factors for opioid prescription.

Conclusions: Opioid over-prescription continues to be a major factor in opioid dependency, abuse, and adverse events. Our study demonstrates that a simple educational intervention can significantly reduce opioid prescription rates post-TURP. This may have a profound impact on the number of patients unnecessarily exposed to opioids while decreasing opioids in the community.

\section{References}

1. Kiechle JE, Gonzalez CM. The opioid crisis and urology. Urology 2018;112:27-8. https://doi.org/10.1016/j.urology.2017.10.009

2. Theisen K, Davies BJ. The American opioid crisis: The inexorable march to death and addiction. Eur Urol 2019;75:219-20. https:// doi.org/10.1016/j.eururo.2018.10.051

3. Belzak L, Halverson J. Evidence synthesis - the opioid crisis in Canada: A national perspective. Heal Promot Chronic Dis Prev Canada 2018;38. https://doi.org/10.24095/hpcdp.38.6.02

4. Shah AS, Blackwell RH, Kuo PC, et al. Rates and risk factors for opioid dependence and overdose after urological surgery. I Urol 2017;198:1130-6. https://doi.org/10.1016/j.juro.2017.05.037 


\section{CUA ABSTRACTS}

\section{Moderated Poster Session 8: Bladder Cancer}

\section{MP-8.1}

Risk of bladder cancer and upper tract urothelial carcinoma in Lynch syndrome patients with DNA mismatch repair gene mutations: An update

Karla Rebullar ${ }^{1,2}$, Otto Hemminki², Sean Skeldon², Kara Semotiuk ${ }^{3}$, Melyssa Aronson $^{3}$, Cynthia Kuk ${ }^{2,4}$, Steven Gallinger ${ }^{2}$, Zane Cohen ${ }^{3}$, Alexandre Zlotta ${ }^{2,4}$ ${ }^{1}$ Division of Urology, Department of Surgery, University of Toronto, Toronto, ON, Canada; ${ }^{2}$ Surgical Oncology, Urology, Princess Margaret Hospital, University Health Network, Toronto, ON, Canada; ${ }^{3}$ Familial Gastrointestinal Cancer Registry, Zane Cohen Center for Digestive Diseases, Mount Sinai Hospital, Toronto, ON, Canada; ${ }^{4}$ Urology, Mount Sinai Hospital, Toronto, ON, Canada

Introduction: Lynch syndrome (LS) is an autosomal dominant syndrome caused by mutations in DNA mismatch repair (MMR) genes, which increases risk for developing several types of cancer. ${ }^{1}$ Prior studies have shown an increased risk for upper tract urothelial carcinoma (UTUC) in this population. ${ }^{2,3} \mathrm{We}$ have previously described an increased risk for bladder urothelial carcinoma (UC) in LS patients with MSH2 mutations. ${ }^{4}$ Herein, we update our previous findings and assess the risk of bladder UC and UTUC in patients with MMR mutations by analyzing a larger cohort of LS patients within the Familial Gastrointestinal Cancer Registry (FGICR) in Toronto.

Methods: Cancer data from 1980 to present were obtained from the FGICR for 1349 LS patients with confirmed MMR mutations (MSH2, MLH1, MSH6, PMS2). Among mutation carries, the majority had MSH2 (559) and MLH1 (417) mutations. Standardized incidence ratio (SIR) was used to compare cancer risk in MSH2 patients with the general population. Results: Among the 559 patients in the registry with a confirmed MSH2 mutation, bladder UC was found in 28 patients (5.0\%), significantly above the expected rate in the general population. Seven of $417(1.8 \%)$ patients with MLH1 mutation, one patient with PMS2 and 0 for MSH6 were found to have bladder UC. UTUC was found in 26 patients with a confirmed $\mathrm{MSH} 2$ mutation $(4.7 \%)$, in five patients with MLH1 mutation $(1.2 \%)$, and in five with MSH6 (2.0\%).

Mean age at diagnosis was 58.9 and 63.9 for bladder UC and UTUC, respectively, with a male:female (M:F) ratio of 0.64 for both cancers, compared to a mean diagnosis age of $>70$ and M:F ratio of 3 for both cancers in the general population. ${ }^{5}$

Conclusions: A similar high incidence (5\%) of bladder UC and UTUC was found in patients with MSH2 mutation. Tumors were detected at an earlier age and, in contrast with sporadic cases, were more common in females. Urologists should be encouraged to recognize LS, as genetic testing can benefit patients and family members, leading to tailored surveillance and risk reduction measures.

\section{References}

1. Vasen HF, Moslein G, Alonso A, et al. Guidelines for the clinical management of Lynch syndrome (hereditary non-polyposis can- cer). J Med Genet 2007;44:353-62. https://doi.org/10.1136/jmg.2007.048991

2. Goecke T, Schulmann K, Engel C, et al. Genotype-phenotype comparison of German MLH1 and MSH2 mutation carriers clinically affected with Lynch syndrome: A report by the German HNPCC Consortium. J Clin Oncol 2006;24:4285-92. https://doi.org/10.1200/ JCO.2005.03.7333

3. Geary J, Sasieni P, Houlston R, et al. Gene-related cancer spectrum in families with hereditary non-polyposis colorectal cancer (HNPCC). Fam Cancer 2008;7:163-72. https://doi.org/10.1007/s10689-0079164-6
4. Skeldon S, Semotiuk K, Aronson M, et al. Patients with Lynch syndrome mismatch repair gene mutations are at higher risk for not only upper tract urothelial cancer but also bladder cancer. Eur Urol 2013;63:37985. https://doi.org/10.1016/j.eururo.2012.07.047

5. Jemal A, Siegel R, Ward E, et al. Cancer statistics 2009. CA Cancer J Clin 2009;59:225-49. https://doi.org/10.3322/caac.20006

\section{MP-8.2}

Oncological long-term benefit of re-transurethral resection in primary T1 bladder cancer: A population-based cohort study from Ontario

Marian S. Wettstein ${ }^{1,2,3,4}$, Nancy N. Baxter ${ }^{2,3}$, Rinku Sutradhar ${ }^{2,3}$, Muhammad Mamdani ${ }^{2,3}$, Song Pham ${ }^{3}$, Syed R. Qadri ${ }^{1}$, Kathy Li ${ }^{1}$, Ning Liu' ${ }^{3}$, Theodorus van der Kwast ${ }^{5}$, Thomas Hermanns ${ }^{4}$, Girish S. Kulkarni 1,2,3

${ }^{1}$ Division of Urology, Department of Surgery, Princess Margaret Cancer Centre, University Health Network, University of Toronto, Toronto, ON, Canada; ${ }^{2}$ Institute of Health Policy, Management and Evaluation, University of Toronto, Toronto, ON, Canada; ${ }^{3} \mathrm{ICES}$, Toronto, ON, Canada; ${ }^{4}$ Department of Urology, University Hospital of Zurich, University of Zurich, Zurich, Switzerland; ${ }^{5}$ Department of Pathology, Princess Margaret Cancer Centre, University Health Network, University of Toronto, Toronto, ON, Canada Introduction: A second transurethral resection (TUR) within 2-6 weeks after the initial resection, a so-called re-TUR, is recommended for patients diagnosed with primary T1 bladder cancer, as prior studies suggest a therapeutic, diagnostic, and prognostic benefit. Results on mortality endpoints, however, are sparse and conflicting. Hence, we aimed to provide real-world evidence by investigating the oncological long-term benefit of re-TUR at the population level.

Methods: Retrospective population-wide observational cohort study based on pathology reports linked to health administrative data. We identified patients who were diagnosed with primary T1 bladder cancer in the province of Ontario (January 2001 to December 2015) and used billing claims to ascertain whether they received re-TUR within 2-10 weeks. The time-dependent effect of re-TUR on survival outcomes was modeled by Cox proportional hazards regression (HR) (unadjusted and adjusted numerous assumed patient- and surgeon-level confounders). Potential residual confounding was verified by a trace outcome (time to cataract surgery). Effect measures were presented as hazard ratios and $95 \%$ confidence intervals $(\mathrm{Cl})$.

Results: We identified 7666 patients with non-missing covariates, of which $2162(28.7 \%)$ underwent re-TUR after a median time of 45 days (interquartile range 35-56 days). During any time of followup, patients who received re-TUR were less likely to die from any causes $(\mathrm{HR} 0.68 ; 95 \% \mathrm{CI}$ $0.63-0.74 ; \mathrm{p}<0.001$ ) or from bladder cancer (HR $0.65 ; 95 \% \mathrm{Cl} 0.57-0.76$; $\mathrm{p}<0.001$ ). After adjusting for all measured confounders, re-TUR was still associated with a lower rate of death (overall survival HR $0.88 ; 95 \% \mathrm{Cl}$ $0.82-0.95 ; p=0.002$; cancer-specific survival HR 0.87; 95\% Cl 0.75-1.02; $\mathrm{p}=0.08$ ). Based on the trace outcome, the suspicion for substantial residual confounding is low.

Conclusions: This population-wide study represents one of the largest datasets of T1 tumors assembled and provides real-world evidence supporting the use of re-TUR in primary T1 bladder cancer. 
MP-8.3

Predictors of re-resection in primary $\mathrm{T} 1$ bladder cancer: Identifying patients who do not receive guideline-concordant care at the population level

Marian S. Wettstein ${ }^{1,2,3,4}$, Nancy N. Baxter ${ }^{2,3}$, Rinku Sutradhar ${ }^{2,3}$, Muhammad Mamdani ${ }^{2,3}$, Song Pham ${ }^{3}$, Syed R. Qadri', Girima Bhalla', Kathy Li ${ }^{1}$, Ning Liu $^{3}$, Theodorus van der Kwast ${ }^{5}$, Thomas Hermanns ${ }^{4}$, Girish S. Kulkarni, 1,2,3 'Division of Urology, Department of Surgery, Princess Margaret Cancer Centre, University Health Network, University of Toronto, Toronto, ON, Canada; ${ }^{2}$ Institute of Health Policy, Management and Evaluation, University of Toronto, Toronto, ON, Canada; ${ }^{3} \mathrm{ICES}$, Toronto, ON, Canada; ${ }^{4}$ Department of Urology, University Hospital of Zurich, University of Zurich, Zurich, Switzerland; ${ }^{5}$ Department of Pathology, Princess Margaret Cancer Centre, University Health Network, University of Toronto, Toronto, ON, Canada

Introduction: Most guidelines recommend a second transurethral resection (re-TUR) within 2-6 weeks after the initial resection in T1 bladder cancer. Prior research, however, has shown that concordance with this recommendation is suboptimal. Therefore, the aim of this populationbased study was to identify predictors of re-TUR.

Methods: We linked province-wide pathology reports (January 2001 to December 2015) with health administrative data to derive a cohort of patients diagnosed with $\mathrm{T} 1$ bladder cancer in the province of Ontario and ascertained re-TUR by a billing claim for transurethral resection within 2-8 weeks after the initial resection. Logistic regression analysis was used to identify predictors of re-TUR. Effect sizes were presented as odds ratios (OR) with 95\% confidence intervals $(\mathrm{Cl})$.

Results: Overall, 1561/6681 patients (23.4\%) underwent re-TUR after a median time of 42 days. High tumor grade (OR 3.09; 95\% Cl 2.33-4.16), lack of muscularis propria (OR 1.28; $95 \% \mathrm{Cl} 1.10-1.50)$, higher age (OR $0.76 ; 95 \% \mathrm{Cl} 0.71-0.82$ per decade), higher Charlson comorbidity index (OR $0.71 ; 95 \% \mathrm{Cl} 0.56-0.90$ per 10 points), and higher health care utilization (OR 0.90; 95\% Cl0.81-0.99), as well as the prevalence of congestive heart failure (OR $0.70 ; 95 \% \mathrm{Cl} 0.53-0.92$ ), dementia (OR $0.52 ; 95 \%$ $\mathrm{Cl} 0.31-0.84]$ ), and rheumatoid arthritis (OR $0.49 ; 95 \% \mathrm{Cl} 0.26-0.88)$ were independent predictors of not receiving re-TUR within 2-8 weeks. Independent surgeon-level predictors of performing a re-TUR were female gender (OR 1.69; 95\% Cl 1.10-2.55), Canadian medical graduate (OR 2.09: $95 \% \mathrm{Cl} 1.57-2.83$ ), and less years since board certification (OR $1.60 ; 95 \% \mathrm{Cl} 1.46-1.76$ per decade).

Conclusions: Most T1 bladder cancer patients do not receive re-TUR within 2-6 weeks. Higher tumor grade and lack of muscularis propria were strong predictors of timely re-TUR, while older and sicker patients were less likely to receive guideline-concordant cancer care. Female, younger, and/or surgeons who graduated from Canadian medical schools were more likely to perform re-TUR, suggesting an influence of eminence over evidence-based medicine.

\section{MP-8.4}

Association between surgical case volume and survival in T1 bladder cancer: A plea for regionalization of care?

Marian S. Wettstein ${ }^{1,2,3}$, Song Pham², Syed R. Qadri', Kathy Li ${ }^{1}$, Ning Liu ${ }^{2}$, Theodorus van der Kwast ${ }^{4}$, Thomas Hermanns ${ }^{3}$, Girish S. Kulkarni ${ }^{1,2}$

'Division of Urology, Department of Surgery, Princess Margaret Cancer Centre, University Health Network, University of Toronto, Toronto, ON, Canada; ${ }^{2} \mathrm{ICES}$, Toronto, ON, Canada; ${ }^{3}$ Department of Urology, University Hospital of Zurich, University of Zurich, Zurich, Switzerland; ${ }^{4}$ Department of Pathology, Princess Margaret Cancer Centre, University Health Network, University of Toronto, Toronto, ON, Canada

Introduction: Prior research demonstrated an association between surgical case volume and survival in muscle-invasive bladder cancer (BC). This relationship, however, has not been investigated in the setting of T1 BC so far. Therefore, we investigated whether a higher surgical case volume of T1 BC translates into improved survival outcomes.

Methods: Province-wide pathology reports (January 2002 to December 2015) were linked with health administrative data to identify patients diagnosed with T1 BC. For each patient, we determined the T1 case volume of the involved surgeon by benchmarking (quintiles) her/him against his/her colleagues during a lookback period of one year. The volume-outcome (overall survival) relationship was then investigated by Cox proportional hazards regression (unadjusted and adjusted for a wide range of assumed confounders) that incorporated volume in three different ways (continuous [quintiles], median and higher vs. below, 80th percentile and higher vs. below). Effect sizes were presented as hazard ratios (HR) with $95 \%$ confidence interval $(\mathrm{Cl})$.

Results: We identified 7426 patients who were diagnosed with $\mathrm{T} 1 \mathrm{BC}$ and followed for 4.8 years. A third of all patients $(n=1895,34.3 \%)$ received surgery by a high-volume surgeon (80th percentile and higher). Higher T1 case volume was associated with improved survival both in unadjusted (continuous HR 0.97; 95\% Cl 0.94-0.99; median HR 0.93; 95\% Cl 0.87-0.99; 80th percentile HR 0.93; 95\% Cl 0.86-0.99]) and adjusted analysis (continuous HR; $95 \% \mathrm{Cl} 0.97$ 0.95-0.99]; median HR 0.93; 95\% Cl 0.87-0.99; 80th percentile HR 0.94; 95\% Cl 0.88-1.01).

Conclusions: This province-wide cohort study could demonstrate a volume-outcome relationship in T1 BC and raises questions regarding the regionalization of care in non-muscle-invasive $\mathrm{BC}$. The generalizability of our findings, however, is limited by the fact that the performance of the initial resection by a high-volume surgeon does not necessarily translate into downstream care by the same surgeon.

\section{MP-8.5}

No survival difference between patients with de novo muscleinvasive bladder cancer compared to those that progress in routine clinical practice

Avril I. Lusty ${ }^{1}$, D. Robert Siemens'2, Christopher Booth ${ }^{2}$

${ }^{1}$ Urology, University of Ottawa, Ottawa, ON, Canada; ${ }^{2}$ Urology, Queen's University, Kingston, ON, Canada

Introduction: Although consensus suggests that patients who progress to muscle-invasive bladder cancer (MIBC) have worse outcomes than those that present de novo, contemporary studies have been equivocal..$^{1,2}$ The concept is important for both understanding the biology of the disease and clinical decision-making. The primary objective of this study was to determine if there is a difference in cancer-specific (CSS) and overall survival (OS) between radical cystectomy (RC) patients who presented with de novo MIBC or those patients who progressed to MIBC.

Methods: This retrospective, population-based study reports early and late outcomes of all patients treated within Ontario from 2009-2013 based on their presentation of bladder cancer. All incident cases that underwent RC were identified using the Ontario Cancer Registry and linked to treatment records. Records from the Canadian Institute for Health Information were used to identify those patients treated with RC. Statistical analysis of the survival data was performed using the Kaplan-Meier method and Cox proportional hazards.

Results: A total of 1573 patients underwent RC from 2009-2013 in the province of Ontario; 893 were diagnosed with de novo MIBC and $79 \%$ had clinical T2 or greater disease. Six hundred eighty patients were identified as progressors, and $49 \%$ had clinical T2 or greater disease at the time of transurethral resection of bladder tumor. Several process indicators and early outcomes spread to be equivalent between the cohorts, including 30-day mortality with a hazard ratio of 1.69 (95\% confidence interval [CI] 0.90-3.19). There was improved CSS and OS for progressors compared to de novo MIBC patients with hazard ratios of 0.85 (95\% CI $0.74-0.98)$ and 0.90 (95\% Cl $0.79-1.03)$, respectively, in unadjusted analysis for the whole cohort. When controlled for patients with only clinical T2 or greater disease, there was no difference in either CSS or OS between groups in adjusted analysis.

Conclusions: This study shows no significant difference in CSS or OS between patients with de novo MIBC and progressors of MIBC in routine clinical practice.

\section{References}

1. Van Den Bosch S, Witjes J. Long-term cancer-specific survival in patients with high-risk, non-muscle-invasive bladder cancer and tumor progression: A systematic review. Eur Urol 2011;60:493-500. https://doi.org/10.1016/j.eururo.2011.05.045 
2. Chen J, Zhang H, Sun G, et al. Comparison of the prognosis of primary and progressive muscle-invasive bladder cancer after radical cystectomy: A systematic review and meta-analysis. IJS 2018;52:21420. https://doi.org/10.1016/j.ijsu.2018.02.049

\section{MP-8.6}

Non-muscle-invasive bladder cancer recurrences in patients managed with trimodal therapy: Conservative or radical treatment?

Khaled Ajib ${ }^{1}$, Mohammad Baker Berjaoui ${ }^{1}$, Jaime O. Herrera-Caceres ${ }^{1}$ Michael Tjong', Gregory Nason', Guan Hee Tan', Annette Erlich', Srikala Sridhar ${ }^{3}$, Neil E. Fleshner ${ }^{1}$, Alexandre Zlotta ${ }^{4}$, Charles Catton ${ }^{2}$, Alejandro Berlin², Peter Chung ${ }^{2}$, Girish S. Kulkarni

${ }^{1}$ Urology, Princess Margaret Hospital, Toronto, ON, Canada; ${ }^{2}$ Radiation Oncology, Princess Margaret Hospital, Toronto, ON, Canada; ${ }^{3}$ Medical Oncology, Princess Margaret Hospital, Toronto, ON, Canada; ${ }^{4}$ Urology, Mount Sinai Hospital, Toronto, ON, Canada

Introduction: Bladder preservation with trimodal therapy (TMT) has emerged as a feasible alternative to radical cystectomy in patients with localized, muscle-invasive bladder cancer (MIBC). We evaluated whether recurrences of non-muscle-invasive bladder cancer (NMIBC) after receiving TMT are best managed with radical or conservative therapies.

Methods: We identified all patients with recurrent non-muscle-invasive bladder cancer from 2003-2018 from the TMT database. Patients were treated with maximal transurethral resection of bladder tumor (TURBT) followed by combination chemotherapy/radiotherapy. We compared those patients to a cohort of matched controls with primary de novo NMIBC. Those patients were matched based on clinical stage and grade in a 6:1 manner (controls:cases). Recurrences in the TMT group were managed according to the standard therapy for NMIBC. Overall survival, recurrence-free, and cystectomy-free survival were calculated for each group and analyzed using the Kaplan-Meier method.

Results: From the TMT cohort, only nine patients out of 124 had NMIBC recurrence and were matched to 54 de novo NMIBC patients as a control group. Median age of the TMT group was 72.4 years vs. 66 years for the non-TMT group. Median followup for both groups was 3.8 years. The nine recurrences within the TMT group had the following clinical staging: cTa (three patients), cT1 (three patient), and carcinoma in situ (three patients). The total number of recurrences was $24 \%(13 / 54)$ in the non-TMT group compared to $11.1 \%(1 / 9)$ in the TMT group $(p=0.32)$. No patient from the TMT group required a cystectomy as compared to $16.7 \%(9 / 54)$ in the non-TMT group $(p=0.36)$. Overall survival was $77.8 \%(7 / 9)$ in TMT group compared to $100 \%$ in non-TMT group $(p<0.001)$, knowing that the disease-specific survival in the TMT group is $100 \%$.

Conclusions: We demonstrated that NMIBC recurrences post-TMT can be successfully managed with endoscopic and adjuvant intravesical therapies, with outcomes similar to de novo NMIBC patients of the same stage and grade.

\footnotetext{
MP-8.7

Trimodal therapy vs. radical cystectomy for muscle-invasive bladder cancer: A Markov microsimulation model

Diana E. Magee ${ }^{1}$, Douglas C. Cheung', Amanda Hird', Srikala S. Sridhar ${ }^{2}$, Charles Catton ${ }^{3}$, Peter Chung 3 , Padraig Warde ${ }^{3}$, Alejandro Berlin $^{3}$, Alexandre Zlotta ${ }^{4}$, Neil E. Fleshner ${ }^{4}$, Girish S. Kulkarnit

'Division of Urology, Department of Surgery, University of Toronto, Toronto, ON, Canada; ${ }^{2}$ Division of Medical Oncology, Department of Internal Medicine, University Health Network, University of Toronto, Toronto, ON, Canada; ${ }^{3}$ Department of Radiation Oncology, University Health Network, University of Toronto, Toronto, ON, Canada; ${ }^{4}$ Division of Urology, Department of Surgery, University Health Network, University of Toronto, Toronto, ON, Canada

Introduction: Radical cystectomy (RC) is the historically accepted gold standard treatment for muscle-invasive bladder cancer (MIBC), but trimodal therapy (TMT) has emerged as a valid therapeutic option. These two modalities, however, have not been directly compared head-to-head in prospective clinical trials. Therefore, we elected to assess the effectiveness of TMT and RC using decision analytic methods.
}

\begin{tabular}{|c|c|c|}
\hline Starting age of patients & TMT (QALE/LY) & RC (QALE/LY) \\
\hline 45 & $8.26 / 11.56$ & $8.45 / 12.87$ \\
\hline 55 & $8.10 / 11.20$ & $8.13 / 12.17$ \\
\hline 65 & $7.68 / 10.45$ & $7.57 / 11.08$ \\
\hline 75 & $6.67 / 8.97$ & $6.41 / 9.13$ \\
\hline 85 & $5.58 / 7.43$ & $5.19 / 7.26$ \\
\hline
\end{tabular}

Methods: A two-dimensional Markov microsimulation model was constructed using TreeAge Pro to compare RC and TMT for patients with newly diagnosed MIBC. Model probabilities and utilities were derived from published literature. Our primary outcome was quality-adjusted life expectancy (QALE). Secondary outcomes included crude life expectancy (LE) and bladder cancer recurrences. Markov cycle length was dynamic to mimic clinical practice. The simulated patient for our model was an adult with MIBC (pT2-4 NO MO) who was a candidate for either RC or TMT. Individual level sampling was completed for age, gender, and reconstruction type.

Results: TMT was the preferred modality, with an estimated mean QALE of 7.48 (95\% confidence interval [CI] 7.43-7.54) vs. 7.41 (95\% Cl 7.347.48) for RC. However, the average LE for patients treated with TMT was lower (10.20 years, $95 \% \mathrm{Cl} 10.13-10.28)$ compared with RC $(10.74$ years, $95 \% \mathrm{Cl} 10.63-10.83)$. A sensitivity analysis evaluating the impact of age showed that younger patients treated with RC had greater QALE and longer LE than those treated with TMT. However, inverse findings were observed for elderly patients (Table 1 ). Overall, $39.4 \%$ of patients in the TMT arm experienced a bladder recurrence with $27 \%$ of patients undergoing a salvage cystectomy.

Conclusions: Our study suggests that RC provides a larger gain in unadjusted life years compared to TMT (0.54 years), but with a lower quality adjusted life expectancy (-0.07 years). Differences in treatment preference were dependent on age, with a larger survival benefit with RC seen in younger patients secondary to improved oncological control.

\section{MP-8.8}

Partial cystectomy for muscle-invasive bladder cancer (MIBC): An option abandoned too soon?

\section{R. Bruce Bracken}

${ }^{1}$ Surgery/Urology, University of Cincinnati, Cincinnati, OH, United States Introduction: The 2017 guidelines for non-metastatic muscle-invasive bladder cancer (NMIBC) recommend against partial cystectomy. Our experience suggests that a urologist experienced in bladder cancer can safely select patients for partial cystectomy.

Methods: For 15 years, we performed open and robotic partial cystectomy and extended pelvic lymphadenectomy in 27 men and 16 women aged $47-83$ years. All had muscle invasion and clinical stage T2-T4. Extensive transurethral resection of bladder tumour was not employed in any patient. Thirty-eight patients had neoadjuvant chemotherapy and two patients had postoperative chemotherapy for N1 and N2 disease. The choice of partial cystectomy is biased to patients with small primary tumors and those with significant downstaging from neoadjuvant chemotherapy. Robotic partial cystectomy can be accomplished with tumors arising from all portions of the bladder. We excluded patients whose tumors were too large or when downstaging was minimal. Results: There were no deaths the first two years after surgery. Voiding and sexual function were unaffected. Postoperative hospitalization ranged from 1.5-15 days (median 3). Eleven patients have died, nine from bladder cancer 3-7 years following surgery. One patient died from a myocardial infarction seven years after surgery and one patient died suddenly at home 10 days after an uneventful salvage cystectomy for muscle-invasive bladder cancer that occurred 5.5 years after partial cystectomy. The other patient with recurrent MIBC responded to combination chemo/radiation therapy and has no evidence of disease 30 months after treatment. Ten patients had recurrent low-stage bladder cancer, which was managed 
in standard fashion. The 32 surviving patients are well, with an intact bladder six months to 15 years after surgery (median 66 months) and no patient is being treated for bladder cancer.

Conclusions: In patients properly selected by a urologist experienced in bladder cancer, partial cystectomy has comparable urological outcome to radical cystectomy, with much less morbidity and no operative mortality.

\section{MP-8.9} Characteristics and resistance patterns of infectious complications
after radical cystectomy

lames Ross ${ }^{1}$, Rodney H. Breau ${ }^{1,2}$, Ilias Cagiannos ${ }^{1}$, Christopher G. Morash ${ }^{1}$ Ranjeeta Mallick2, Duane R. Hickling ${ }^{1,2}$, Luke T. Lavallée $e^{1,2}$

${ }^{1}$ Department of Surgery, Division of Urology, University of Ottawa, Ottawa, ON, Canada; ${ }^{2}$ The Ottawa Hospital Research Institute, The Ottawa Hospital, Ottawa, ON, Canada

Introduction: Infection is common after radical cystectomy. We hypothesize that detailed characterization of infections can improve prophylaxis strategies. The objective of this study is to characterize infections postradical cystectomy, including incidence, risk factors, causative organisms, and antibiotic resistance.

Methods: A retrospective review was performed on all patients who underwent radical cystectomy at a tertiary Canadian center from January 2016 to May 2019. Thirty-day postoperative urinary tract infection (UTI), surgical site infection (SSI), and C. difficile infection were identified. Incidence, causative organism, and antibiotic resistance/sensitivities were collected. Adjusted associations between patient and perioperative factors with risk of infection were determined.

Results: In total, 135 patients underwent radical cystectomy during the study period. Mean age was 69 years, 101 were male $(75 \%)$, and 60 received neobladders (44\%). Postoperative UTI was seen in 29 (21\%), SSI in $37(29 \%)$, and C. difficile in $7(5 \%)$. The most common cause of UTI was fungal infection (31\%), Escherichia coli $(24 \%)$, Klebsiella sp $(17 \%)$, and Enterococcus sp (14\%). The most common cause of SSI was Klebsiella sp $(25 \%)$, Enterococcus sp $(17 \%)$, Enterobacter sp $(12 \%)$, and fungal infection ( $8 \%$ ). Twenty-six percent of infections were polymicrobial. There was a high antibiotic resistance rate, with $44 \%$ resistant to ampicillin, $21 \%$ to cefazolin, $18 \%$ to amoxicillin/clavulin, and $14 \%$ to ceftriaxone; $22 \%$ of infections were multidrug-resistant. Intraoperative transfusion and diabetes were independently associated with risk of infection $(p<0.05)$. Conclusions: Infectious complications are common post-radical cystectomy. There is a high rate of polymicrobial infection, fungal infection, and multidrug resistance, which may not be covered by most standard prophylactic antibiotic regimens. This should be considered when choosing perioperative prophylaxis and for early treatment of suspected infection.

\section{MP-8.11}

The utility of postoperative serial hemoglobin measurements in patients undergoing radical cystectomy for urothelial carcinoma Stewart Whalen ${ }^{1}$, Ricardo A. Rendon', David G. Bell', Morgan MacDonald ${ }^{\prime}$, Jon Duplisea ${ }^{1}$, Ashley R. Cox ${ }^{1}$, Gregory G. Bailly', Ross Mason ${ }^{1}$ 'Department of Urology, Dalhousie University, Halifax, NS, Canada Introduction: Routine serial measurements of serum hemoglobin ( $\mathrm{sHgb}$ ) are common after major abdominal surgery. However, prolonged serial measurements may be associated with patient anxiety and discomfort, increased costs, and prolonged hospitalization without significant clinical benefit. We examined packed red blood cell (pRBC) transfusion rates and timing of transfusion after radical cystectomy (RC) for urothelial carcinoma (UC) in order to determine the utility of serial sHgb measurements.

Methods: We retrospectively reviewed our institutional records to identify all patients who underwent RC for UC between 2009 and 2019. The number of sHgb measurements for each patient was examined and pRBC transfusion rates were calculated intraoperatively and on postoperative days (POD) $0,1,2$, and beyond POD 2. Multivariable logistic regression was used to determine factors associated with transfusion beyond POD 2 . Results: The median number of sHgb measurements throughout hospital admission was 9 (interquartile range [IQR] 7,25). Overall, $69(28.7 \%)$ patients received a postoperative transfusion at some point during their hospital stay, with $14(5.8 \%), 18(7.5 \%), 15(6.2 \%)$, and $46(19.2 \%)$ patients receiving a pRBC transfusion on POD $0,1,2$, and beyond POD 2, respectively. Among patients with a sHgb level $>100 \mathrm{~g} / \mathrm{L}$ on POD 2, only $7 / 85(8.2 \%)$ went on to receive a transfusion beyond POD 2 compared with 39/155 (25.2\%) patients with $\mathrm{sHgb}<100 \mathrm{~g} / \mathrm{L}$. In multivariable analysis, risk factors associated with transfusion beyond POD 2 included older age, lower sHgb on POD 2, and longer duration of hospitalization. Conclusions: pRBC transfusion rates after radical cystectomy remain high, with many patients requiring transfusions several days after surgery. However, patients with $\mathrm{sHgb}>100 \mathrm{~g} / \mathrm{L}$ on POD 2 are at a low risk of requiring transfusion. Discontinuing further sHgb checks in these patients may serve to decrease patient anxiety, unnecessary investigations, and healthcare costs.

\section{MP-8.12}

Cost-effectiveness analysis of the IMvigor 130 study evaluating atezolizumab as monotherapy or combined with platinum-based chemotherapy vs. chemotherapy alone in advanced urothelial cancer

Diana E. Magee ${ }^{1}$, Douglas C. Cheung ${ }^{1}$, Amanda Hird', Srikala S. Sridhar ${ }^{2}$, Nazanin Fallah-Rad ${ }^{2}$, Neil E. Fleshner ${ }^{3}$, Girish S. Kulkarni ${ }^{3}$

${ }^{1}$ Division of Urology, Department of Surgery, University of Toronto, Toronto, ON, Canada; ${ }^{2}$ Division of Medical Oncology, Department of Internal Medicine, University Health Network, University of Toronto, Toronto, ON, Canada; ${ }^{3}$ Division of Urology, Department of Surgery, University Health Network, University of Toronto, Toronto, ON, Canada Introduction: Platinum-based chemotherapy is the standard first-line therapy for patients with metastatic urothelial cancer, but immunotherapies are emerging as a treatment option. The implications on cost have not been previously explored, therefore, we developed a cost-effectiveness model using the interim results from IMvigor130 trial.

Methods: A Markov microsimulation model using TreeAgePro was developed to compare the cost and effectiveness of atezolizumab-gemcitabine/platinum (AGP) combination, gemcitabine/platinum (GP) alone, and atezolizumab alone. Model probabilities and utilities were derived from published literature. Cost of patient care and pharmaceuticals were obtained from Medicare and Medicaid Services, and the Medicare Physician Fee Schedule. Simulated patients included those with metastatic or locally advanced urothelial cancer and incremental cost-effectiveness ratios were compared with a willingness-to-pay threshold of \$100 000 per quality-adjusted life year (QALY).

Results: AGP combination was the most effective modality, resulting in 1.19 QALYs compared to 1.16 for atezolizumab alone and 0.85 for GP alone at a mean treatment cost of $\$ 116492, \$ 93662$, and $\$ 60328$, respectively. Factored together, however, AGP combination was not costeffective; the modest increase in QALY was offset by substantial cost $(\$ 761$ 000/QALY vs. atezolizumab and \$165 188/QALY vs. GP). Atezolizumab alone represented a moderate option at \$107 530/QALY vs. GP.

Conclusions: Our cost-effectiveness model based on the IMvigor130 trial illustrates that, while the AGP combination results in the greatest net QALY benefit, it comes at a significant cost. Atezolizumab may represent a more cost-effective option for appropriately selected patients (namely high PDL1 expressors) since it leaves well-established therapeutic options to be used in the second-line space. Further maturation of the subgroup data (cisplatin use and PDL1 rates) may allow for further refinement of these results. 


\section{MP-8.14}

FOXF1 is a potentially novel regulator of metastases in bladder cancer through regulation of cell proliferation and migration

Andrea Kokorovic ${ }^{1}$, Sharada Mokkapati ${ }^{1}$, Debasish Sundi', Amy Lim ${ }^{1}$, Vikram M. Narayan', Sima Porten ${ }^{3}$, Beat Roth ${ }^{4}$, Matthew Wszolek ${ }^{5}$, Neema Navai ${ }^{1}$, Colin P Dinney ${ }^{7}$

${ }^{1}$ Urology, The University of Texas MD Anderson Cancer Center, Houston, TX, United States; ${ }^{2}$ Urology, The Ohio State University, Colombus, $\mathrm{OH}$, United States; ${ }^{3}$ Urology, The University of California San Francisco, San Francisco, CA, United States; ${ }^{4}$ Urology, Centre Hospitalier Universitaire Vaudois, Bern, Switzerland; ${ }^{5}$ Urology, Massachusetts General Hospital, Boston, MA, United States

Introduction: FOXF1 is a forkhead transcription factor whose role in tumorigenesis is controversial. We investigated for the first time the functional role of FOXF1 in bladder cancer (BC).

Methods: Whole genome mRNA gene expression profiling was performed on paired primary tumors and nodal metastases from a cystectomy cohort and FOXF1 was found to be a top differentially expressed gene. Effect of FOXF1 expression on survival was determined using independent clinical cohorts. mRNA sequencing and qPCR were used to determine FOXF1 expression in parental BC cell lines. Knockdown (KD) of FOXF1 in RT112 cell lines was performed using FOXF1 small interfering RNA (siRNA) or siRNA control (ctrl). Tryptan blue dye exclusion method and annexin $\mathrm{V}$ staining were used to determine cell viability and apoptosis at 72 hours post-transfection, respectively. Migration potential was tested using the scratch migration assay, with wound closure measured at 24, 48, and 72 hours post-transfection.

Results: FOXF1 expression was 3.6-fold lower in metastatic lesions compared to primary $(p<0.001)$. Low FOXF1 was associated with worse survival in two independent cohorts $(p=0.046$ and $p=0.006)$. FOXF1 expression was generally higher in luminal $\mathrm{BC}$ cells compared to mesenchymal. Using the high FOXF1 expressing luminal RT112 cell line, we found that FOXF1 KD decreased number of viable cells $(p=0.023)$ without a difference in overall \% cell viability. There was no difference in apoptosis between the FOXF1 ctrl and KD group, suggesting the difference in cell viability is due to decreased cell proliferation. Scratch migration assay demonstrated decreased propensity for wound closure in the FOXF1 KD cells. KD cells demonstrated increased gene expression of e-cadherin and TGFB1, while VEGFA expression was decreased.

Conclusions: FOXF1 may be a novel driver of BC metastases and appears to regulate cellular proliferation and migration. In vivo animal studies are required to elucidate potential mechanisms.

\section{MP-8.15}

\section{Patient-derived organoids as a tool for use of precision medicine} in bladder cancer

Paria Saadat ${ }^{1,2,3}$, Patrick B Thomas ${ }^{1,2,3}$, Kate Zimmerman ${ }^{4}$, Saied Alinezhad ${ }^{1,3}$, Tony J. Kenna ${ }^{4}$, Elizabeth D. Williams ${ }^{2,3}$, Ian Vela ${ }^{1,2,3}$

${ }^{1}$ Urology, Princess Alexandra Hospital, Brisbane, Australia; ${ }^{2}$ Queensland Bladder Cancer Initiative ( $\mathrm{QBCl})$, Institute of Health and Biomedical Innovation, Brisbane, Australia; ${ }^{3}$ Australian Prostate Cancer Research Centre-Queensland, Institute of Health and Biomedical Innovation, Brisbane, Australia; ${ }^{4}$ Translational Genomics Group, Queensland University of Technology (QUT), Brisbane, Australia

Introduction: Bladder cancer (BC) is the 10th most common malignancy worldwide. ${ }^{1}$ There is a need to develop in vitro models of $\mathrm{BC}$ to provide an avenue for predictive drug response testing. ${ }^{2}$ Here, we aim to develop a comprehensive $\mathrm{BC}$ biobank, using patient-derived organoids (PDOs) as a tool for rapidly predicting therapeutic responses in individuals with BC. Methods: Following ethics approval, patient specimens - including bladder tumor tissue, serum, urine, and peripheral blood mononuclear cells (PBMCs) - and demographic data were collected. Following transurethral resection of bladder tumor or cystectomy, 3D ex vivo cultures of PDOs were developed by enzymatic digestion of the $\mathrm{BC}$ tissue and suspended in a fully defined organoid medium-Matrige ${ }^{\mathrm{TM}}$ (Fig.1). PDO growth and cell viability were inspected using time-lapse and dual-parameter fluorescence microscopy. Following suspension in a serum-free, modified natural polymer suspension (Happy Cell ${ }^{\circledR}$ Advanced Suspension Medium; Vale Life Sciences), BC PDOs were treated with combinations of docetaxel,

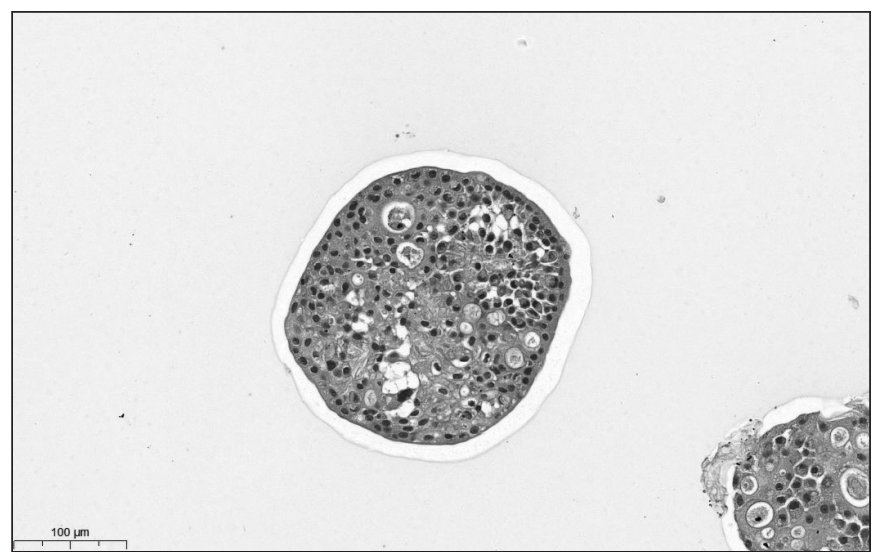

MP-8.15. Fig. 1.

cisplatin, and gemcitabine over a six-day period, with response monitored longitudinally and endpoint survival measured.

Results: Fifteen viable PDO cultures were established (65\% success rate) from low- and high-grade urothelial cancers comprising multiple variant histologies. Here, we highlight results from four clinical cases. Preliminary studies reveal complex remodeling of $\mathrm{BC}$ organoids within days of formation. We show, for the first time, Happy Cell ASM as a useful medium to maintain organoid viability and monitor response to chemotherapies. Finally, this response is heterogeneous in in vitro cultures and may reflect the complex clinical therapeutic responses seen in patients.

Conclusions: Our pilot study demonstrates that BC PDOs can be feasibly generated as part of a Biobank storage pipeline and may provide an ability to predict an individual's therapeutic response.

References

1. Bray F, Ferlay J, Soerjomataram I, et al. Global cancer statistics 2018: GLOBOCAN estimates of incidence and mortality worldwide for 36 cancers in 185 countries. CA Cancer J Clin 2018;68:394-424. https:// doi.org/10.3322/caac.21492

2. Lee SH, Hu W, Matulay JT, et al. Tumor evolution and drug response in patient-derived organoid models of bladder cancer. Cell 2018;173:515-28.e17. https://doi.org/10.1016/j.cell.2018.03.017 


\section{Moderated Poster Session 9: Endourology II}

\begin{abstract}
MP-9.1
Clinical outcomes of the small acute ureteral stone protocol at an ambulatory urology clinic

Conrad Bayley', Tim Wollin'2, Shubhadip (Shubha) K. De ${ }^{2}$, Trevor D. Schuler ${ }^{1}$ University of Alberta Faculty of Medicine and Dentistry, Edmonton, AB, Canada; ${ }^{2}$ Department of Surgery, University of Alberta Faculty of Medicine and Dentistry, Edmonton, $\mathrm{AB}$, Canada

Introduction: It was noted that many patients with small ( $\leq 5 \mathrm{~mm}$ stones) often missed their acute stone clinic (ASC) appointments due to symptom resolution, which led to underuse of appointments and decreased access for other patients. To improve access to the ASC, the small acute ureteral stone (SAUS) protocol was developed. A nurse case manager contacts patients to review their symptoms and the results of a followup ultrasound. Disposition to an ASC, elective stone clinic, or followup with a family physician is determined based on symptoms, imaging findings, and patient preferences. The objective of this study was to determine the disposition of patients enrolled in the SAUS protocol. In addition, this study provides further data on the natural history of small ureteral stones and the impact of the SAUS protocol on ASC resources.

Methods: We completed a retrospective electronic medical record review of all 216 patients enrolled in the SAUS protocol over the 12 months since its inception. Data was stored in a REDCap database.

Results: The 216 patients had a mean age of 49 , with a male to female ratio of $2: 1$ and a mean stone size of $3.89 \mathrm{~mm} ; 17 \%$ (37) were recurrent stone-formers. At initial phone contact by the RN, $66.2 \%$ (143) reported symptom resolution and $78.7 \%$ (170) had no evidence of hydronephrosis or ureteral stone(s) on followup imaging. The disposition distribution was 46.8\% (101) ASC, 34.3\% (74) general practitioner (GP), 13.0\% (28) ESC, and $5.9 \%$ (13) other. At last contact with urology, 73.5\% (159) had passed their stone spontaneously and, thus, didn't require intervention. Stone passage rate was found to be independent of position along the ureter.

Conclusions: Introduction of the SAUS protocol to acute ureteral stone management led to a reduction in the need for ASC appointments by $52.8 \%$ for patients with ureteral stones $\leq 5 \mathrm{~mm}$. Moreover, this confirms that few stones $\leq 5 \mathrm{~mm}$ require intervention. Our results support the utility of the SAUS protocol as a tool to determine patient disposition in this population.
\end{abstract}

\section{MP-9.5}

Extending the reach of a stone clinic: Can nurse extenders run a stone clinic?

Alexander Hengel', Stephanie Fodness ${ }^{1}$, Trevor D. Schuler ${ }^{1}$, Tim Wollin ${ }^{1}$ Shubhadip (Shubha) K. De ${ }^{1}$

${ }^{1}$ Division of Urology, University of Alberta, Edmonton, AB, Canada

Introduction: Limited literature surrounds medical extenders' roles in the management of specific patient populations. Our stone clinic uses a registered nurse extender (NE) to review and communicate details of new patients to the treating urologist. The objective of this study was to characterize the accuracy of NE-generated management plans, when compared to those of the treating urologists.

Methods: A retrospective review of acute stone clinic (ASC) patients between June and September 2019 was performed. A standardized "clinical summary and disposition planning" document was used by the NE for all new ASC patients. Management plans were then compared between $\mathrm{NE}$ and urologist, and categorical data was compared using chi-squared, Fisher's exact, and Kappa tests, when appropriate.
Results: Of the 226 patients reviewed $(67.7 \%$ male, mean age 54 years) disposition plans included: followup imaging (34.5\%), ureteroscopy (URS, $28.3 \%)$, shockwave lithotripsy (SWL, 20.4\%), observation $(10.2 \%)$, and other $(6.6 \%)$. Comparing NE to urologist plans, overall agreement was $55.3 \%$ with moderate agreement $(\mathrm{Kappa}=0.506)$. When patients were to be followed with imaging, the NE was correct in $85.4 \%$. When choosing intervention vs. observation, the NE was correct in $75.0 \%$, although the correct intervention was only selected in $55.1 \%$. When NE incorrectly chose an intervention $(n=61), 55.7 \%$ of cases were actually managed with observation and $44.3 \%$ with an alternate intervention modality. When observation was incorrectly chosen $(n=14), 21.4 \%$ of patients were selected to undergo URS and $21.4 \%$ SWL. The NE selected multiple possible management options in $18.6 \%$ and did not select any treatment options in $11.5 \%$ of cases. NE was more likely to select intervention than surgeon when patients were symptomatic $(84.0 \%$ vs. $64.5 \% ; \chi 2=12.54 ; p<0.001)$. There was no difference in intervention rates when the patients were asymptomatic $(49 . \%$ vs. $40.9 \% ; \chi^{2}=1.22 ; p=0.269$ ).

Conclusions: This study demonstrates that within our clinic, extenders are able to predict the management plan in $55.3 \%$ of cases. Further work is required in understanding which factors may contribute to errors in disposition planning and where opportunities for education may exist.

\section{MP-9.6}

\section{Do patients follow the guidelines? Understanding stone clinic} attrition rates and patterns

Runhan Ren ${ }^{1}$, Tim Wollin ${ }^{1}$, Trevor D. Schuler ${ }^{1}$, Dariusz (Derek) Bochinski ${ }^{1}$, Shubhadip (Shubha) K. De

${ }^{1}$ Urology, University of Alberta, Edmonton, AB, Canada

Intrduction: Best practice guidelines suggest that by providing an opportunity to serially investigate and re-engage stone patients, long-term followup can be crucial to reducing the risk of future complications. Missing appointments not only represent wasted resources but may also be detrimental to patients. Little is known about what kinds of patients are eventually lost to followup (LTF). Our our objective was to evaluate rates and patterns of LTF in our stone clinics.

Methods: We retrospectively reviewed two years (2017-2019) of stone clinic patients who missed/cancelled their last appointment without further followup. Patients were identified using administrative and billing codes and reviewed for demographic and clinical information.

Results: A total of 4548 acute and 1419 elective stone clinic visits were completed at our center from 2017-2019. The overall rate of cancelled/ missed visits was $31.4 \%$, with true LTF being confirmed in 525 patients $(8.8 \%)$. LTF mean age was 50 years (19-97), and 63\% were male. LTF was not affected by season and inversely related to distance $(52 \%$ local, $32 \%$ $<2$ hours, $12 \%<5$ hours travel time). Visits were missed $47 \%$ and cancelled $53 \%$ of the time. Patients attended an average of four visits over $9 \mathrm{~m}$, and 1.15 procedures prior to LTF. Thirty-six percent of LTF ( $n=205)$ was postoperative re-checks with the majority being after ureteroscopy ( $72 \%$ vs. $18 \%$ shockwave lithotripsy [SWL], 10\% percutaneous nephrolithotomy [PCNL]). Reassessment of stone passage accounted for $9 \%(n=49)$ of LTF and of $45 \%$ of these patients completed their investigations. Nineteen percent LTF were high-risk patients (mean 1.3 procedures over $19 \mathrm{~m}, 95 \% \geq 1$ abnormality on 24 -hour urine collection), $23 \%$ of whom completed their investigations. If LTF was for a visit where 24 -hour urines were involved $(14 \%, n=110)$; $97 \%$ did not complete their urine collection. 
Conclusions: These results identify where challenges may exist in providing stone patients with recommended long-term care. Further evaluation will provide insight into system-based approaches to help tailor followup and resource allocation.

\section{MP-9.7}

Assessing novel preoperative indicators of failed retrograde ureteric stent insertion in patients requiring urgent decompression Patrick Albers ${ }^{3}$, Nick Dean ${ }^{1}$, Ryan McLarty ${ }^{1}$, Tim Wollin' ${ }^{1}$, Gillian Shiau ${ }^{2}$, Shubhadip (Shubha) K. De ${ }^{1}$

${ }^{1}$ Department of Surgery, University of Alberta, Edmonton, $A B$, Canada; ${ }^{2}$ Department of Radiology, University of Alberta, Edmonton, $\mathrm{AB}$, Canada; ${ }^{3}$ University of Alberta, Edmonton, $\mathrm{AB}$, Canada

Introduction: The primary objective of our study is to identify patient and stone characteristics that may help predict failed retrograde ureteric stent placement in patients that require urgent upper urinary tract drainage. Methods: A retrospective analysis of patients undergoing urgent retrograde ureteric stent insertions for obstructing ureteral calculi was completed (2013-19). Eligible patients were identified from our nephrostomy tube (NT) database and were matched to controls (who underwent successful stent insertions) from our electronic medical records. All patients required preoperative computed tomography (CT) scans, and variables collected included patient demographics, stone and anatomic characteristics (i.e., stone size/location, ureteral wall thickness, ureteric density, degree of hydronephrosis, renal asymmetry/atrophy), time since stone diagnosis, and indication for stenting. Univariate and binary variate logistic regression were performed using SPSS to identify novel preoperative predictors of failed ureteric stent placement.

Results: We identified 34 patients with failed stent placement (FSP) who required NT insertions. Compared to 72 successfully stented controls, no significant differences in mean age: FSP 59.3 years vs. control 60.7, or gender (percent female FSP $41 \%$ vs. control 53\%) were identified. Of the 106 patients, $81 \%$ were stented for septic stones, $14 \%$ acute kidney injury, and $5 \%$ for preoperative. Our controls had a higher rate of stenting due to sepsis ( $93 \%$ vs. $55 \%)$. Univariate analyses showed time since diagnosis of stone $(p=0.002)$, ureteral wall thickness (UWT) $(p<0.001)$, size of stone $(p=0.001)$, renal atrophy $(p=0.012)$, and indication for stenting $(p=0.001)$ were found to be significant. Our multivariate analysis however, only identified UWT to differ significantly between groups (odds ratio 0.32; 95\% Cl 0.17-0.61; $\mathrm{p}=0.001$ ).

Conclusions: This analysis suggests UWT may be a useful predictor to identify patients that are more likely to fail retrograde ureteric stenting and may benefit from upfront percutaneous drainage in patients with ureteric stones causing obstructive renal failure or sepsis.

\section{References}

1. Yamashita S, Kohimoto $\mathrm{Y}$, Iguchi $\mathrm{T}$, et al. Ureteral wall volume at ureteral stone site is a critical predictor for shockwave lithotripsy outcomes: comparison with ureteral wall thickness and area. Urolithias 2019 August 23. [Epub ahead of print]. https://doi.org/10.1007/ s00240-019-01154-w

2. Sarica K, Eryildirim B, Akdere H, et al. Could ureteral wall thickness have an impact on the operative and postoperative parameters in ureteroscopic management of proximal ureteral stones? Actas Urol Esp 2019;43:474-9. https://doi.org/10.1016/j.acuroe.2018.10.007

\section{MP-9.8}

\section{Determination of optimal stent length: A survey of urologic} surgeons

Lustin S. Kwong', R.J. D'A Honey', Jason Y Lee', Michael Ordon'

'Division of Urology, Department of Surgery, St. Michael's Hospital, University of Toronto, Toronto, ON, Canada

Introduction: Ureteral double-J stent length is an important factor affecting stent-related symptoms. Stents that are too long are associated with more severe symptoms, while stents that are too short have a higher rate of migration. Several techniques exist to determine the ideal stent length for a given patient with some techniques being more accurate than others, however, little is known about what techniques urologists rely on. The objective of this study was to identify how urologists determine optimal stent length.

Methods: An online survey was emailed to all members of the Endourology Society. The survey sought to assess what methods are commonly used to determine choice of stent length, along with frequency of stent placement post-ureteroscopy (URS), duration of stenting, availability of different stent lengths, and the use of stent tether.

Results: A total of 301 urologists (15.1\%) responded to our survey. Most respondents practiced in North America $(40.9 \%)$ followed by Europe $(26.2 \%)$. Following ureteroscopy (URS), $84.5 \%$ of respondents would stent at least $50 \%$ of the time and $34.8 \%$ would stent $>90 \%$ of the time. Following uncomplicated URS, most respondents $(52.0 \%)$ would leave a stent for 2-7 days. Patient height was most commonly ranked first as the method of choice in determining stent length $(47.0 \%)$, followed by estimation based on experience only $(20.6 \%)$ and intraoperative direct measurement of ureteric length $(19.1 \%)$. Most respondents used multiple methods in determination of optimal stent length. Most respondents (66.5\%) were interested in a simple intraoperative technique using a special ureteral catheter that would help choose the most appropriate stent length.

Conclusions: Post-URS stent insertion is common and patient height is the most common method of choice used in determining optimal stent length. Most respondents were interested in using a simple, novel ureteral catheter device that would allow them to more accurately select optimal stent length.

\section{MP-9.9}

Computed tomography measurements of stone impaction can successfully predict extracorporeal shockwave lithotripsy outcomes for proximal ureteral stones

Ryan McLarty', Callum Lavoie', Trevor D. Schuler', Tim Wollin', Shubhadip (Shubha) K. De

${ }^{1}$ Urology, University of Alberta, Edmonton, AB, Canada

Introduction: Extracorportal shockwave lithotripsy (ESWL) is a first-line treatment option for proximal ureteric calculi but patient access is geographically limited in Canada. There continues to be a need for novel preoperative predictors of treatment success to optimize ESWL use. We sought to evaluate whether preoperative computed tomography (CT) measurements of stone impaction - ureteric wall thickness and ureteric wall density - can predict the clinical outcomes of ESWL.

Methods: We identified all patients from January 2017 to December 2018 who underwent ESWL for a single proximal ureteric stone. Patients were excluded if they had prior treatment of their stone or no preoperative CT evaluation. CT images were reviewed to measure stone size, stone volume, stone density, skin to stone distance (SSD), hydronephrosis (0-3), ureteric wall thickness (UWT), and the Hounsfield units (HU) of the ureter distal and proximal to the stone. Successful ESWL was defined as normal radiographic investigations (KUB X-ray and ultrasound) at the time of routine two-week scheduled followup. Multivariable logistic regression was performed to determine independent predictions of successful ESWL. Results: A total of 224 patients with proximal ureteric stones were identified, of which 141 met inclusion criteria. Seventy-six patients had successful ESWL at two weeks' followup. On univariate analysis, patients with successful ESWL were younger and had smaller measurements for stone size, UWT, ureter density distal to the stone, and ureter HU distal/ proximal ratio. Multivariate analysis demonstrated that UWT (odds ratio [OR] $0.64 ; 95 \%$ confidence interval $[\mathrm{Cl}] 0.47-0.89 ; \mathrm{p}<0.01)$ and the distal 
ureteric density (OR 0.86; 95\% Cl 0.81-0.94; $\mathrm{p}<0.01)$ were independent predictors of successful ESWL. UWT size less than $3.4 \mathrm{~mm}$ had a sensitivity of $68.4 \%$ and specificity of $80.1 \%$ for and distal ureteric density values less than $19.7 \mathrm{HU}$ had a sensitivity of $70.1 \%$ and specificity of $84.0 \%$ for successful ESWL.

Conclusions: This study suggests preoperative CT measurements of stone impaction can predict ESWL outcomes for proximal ureteric stones. UWT and distal ureteric density values of less than $3.4 \mathrm{~mm}$ and $19.7 \mathrm{HU}$, respectively, are associated with successful ESWL at two weeks. These measurements may help predict patients more likely to benefit from ESWL.

\section{MP-9.10}

Dissolution of uric acid stones quantification by automated computed tomography stone volume measurement

Lonathan Moore ${ }^{1}$, Amihay Nevo Pima', Haidar Abdul-Muhsin', Karen Stern ${ }^{1}$, Mitchell Humphreys ${ }^{1}$

'Department of Urology, Mayo Clinic Arizona, Phoenix, AZ, United States Introduction: An effective management option for uric acid nephrolithiasis includes oral dissolution therapy (ODT) with urinary alkalization. ${ }^{1}$ In vivo rate of uric acid dissolution is not well-described. We present the quantification of uric acid dissolution using software-generated 3D images and stone volume measurements. ${ }^{2,3}$

Methods: We retrospectively reviewed a sample of patients treated with ODT at a single institution. Baseline patient demographics, metabolic urine testing, and stone characteristics were collected. Computed tomography $(\mathrm{CT})$ scan images were evaluated using the quantitative Stone Analysis Software (qSAS) 3 software to obtain total stone volume (TSV), maximum diameter (MD), and stone number.

Results: Thirteen stones in nine patients were analyzed (Table 1). Baseline patient characteristics included mean age 65.7 years (standard dveitauion [SD] 14.3), body mass index (BMI) 30.4 (SD 3.76), and daily urine volume $1.9 \mathrm{~L}$ (SD 0.36). All patients had $100 \%$ uric acid stones except one with $80 \%$ uric acid and $20 \%$ calcium oxalate. Patients were treated with potassium citrate $(n=4)$, bicarbonate $(n=1)$, or combination $(n=4)$. Mean urine $\mathrm{pH}$ on treatment was 6.9 (SD 0.55, range 6.4-8.0) and treatment duration was 180 days (SD 131; range 41-531). Pre-treatment stone burden had a MD of $16.6 \mathrm{~mm}$ (SD 8.7, range 4.5-40.4), attenuation 354.8 HU (SD 72, range 22-455), and TSV $1014 \mathrm{~mm}^{3}$ (SD 1333, range 27-4939) (Table 2). Absolute change in TSV on treatment was $441 \mathrm{~mm}^{3}$ (range $19-4939$ ) and relative change was $84.75 \%$ (range $41-100 \%$ ). Six $(46 \%)$ of the stones completely resolved on followup imaging. Rate of change was $3.72 \mathrm{~mm}^{3}$ or $0.56 \%$ of TSV per day. Calculated T for dissolution based on TSV was 12.85 weeks.

Conclusions: Use of software-calculated TSV is an effective method of measuring uric acid stone response to alkalization. Stone volume decreased by $50 \%$ after 12 weeks of treatment. Further studies with a larger sample size are needed to confirm if this can be used to guide surveillance schedules for dissolution therapy.

References

1. Gridley CM, Sourial MW, Lehman A, et al. Medical dissolution therapy for the treatment of uric acid nephrolithiasis. World / Urol 2019;37:2509-15. https://doi.org/10.1007/s00345-019-02688-9

2. Selby MG, Vrtiska TJ, Krambeck AE, et al. Quantification of asymptomatic kidney stone burden by computed tomography for predicting future symptomatic stone events. Urology 2015;85:45-50. https:// doi.org/10.1016/j.urology.2014.08.031

3. O'Brien Urology Research Center, funded by the National Institutes of Health (DK100277), and the CT Clinical Innovation Center at the Mayo Clinic
MP-9.10. Table 1. Baseline demographics for nine patients treated with urine alkalization for uric acid stones

\begin{tabular}{|c|c|}
\hline Parameter & \\
\hline Male & $8(89 \%)$ \\
\hline \multicolumn{2}{|l|}{ Age (years) } \\
\hline Mean & $65.7(14.3)$ \\
\hline Median & 68.0 \\
\hline Range & $(43.0-84.0)$ \\
\hline \multicolumn{2}{|l|}{$\mathrm{BMI}\left(\mathrm{kg} / \mathrm{m}^{2}\right)$} \\
\hline Mean & $30.4(3.8)$ \\
\hline Median & 29.6 \\
\hline Range & $(28.0-40.0)$ \\
\hline $\mathrm{Hx}$ DM & $3(33 \%)$ \\
\hline Hx HTN & $7(78 \%)$ \\
\hline Stone burden & $\mathrm{n}=10$ stone episodes \\
\hline Solitary & $5(50 \%)$ \\
\hline Multiple unilateral & $3(33 \%)$ \\
\hline Bilateral & $1(11 \%)$ \\
\hline Staghorn & $1(11 \%)$ \\
\hline \multicolumn{2}{|l|}{ \# stones per episode } \\
\hline 1 & $5(50 \%)$ \\
\hline 2 & $5(50 \%)$ \\
\hline$>2$ & 0 \\
\hline \multicolumn{2}{|l|}{ Urine volume (L) } \\
\hline Mean & $1.9(0.4)$ \\
\hline Median & 1.8 \\
\hline Range & $(1.4-2.4)$ \\
\hline \multicolumn{2}{|l|}{ Treatment $\mathrm{pH}$} \\
\hline Mean & $6.9(0.5)$ \\
\hline Median & 6.6 \\
\hline Range & $(6.4-8.0)$ \\
\hline Supersaturation uric acid & $\mathrm{n}=6$ \\
\hline Mean & $1.1(1.1)$ \\
\hline Median & 0.8 \\
\hline Range & $(0.2-2.8)$ \\
\hline $24 \mathrm{~h}$ urinary uric acid (g/day) & $\mathrm{n}=6$ \\
\hline Mean & $0.7(0.19)$ \\
\hline Median & 0.7 \\
\hline Range & $(0.3-0.8)$ \\
\hline
\end{tabular}

\section{MP-9.11}

Intermittent dosing of rapamycin decreases calcium oxalate stone burden in a dietary Drosophila melanogaster model of urolithiasis

Mike Pignanelli ${ }^{1,2}$, Anna-Lena Spierling ${ }^{2,3}$, Kait Al2 , Jeremy Burton², Jennifer Bjazevic ${ }^{1}$, Hassan Razvi'

${ }^{1}$ Urology, Western University, London, ON, Canada; ${ }^{2}$ Microbiology, Lawson Health Research Institute, London, ON, Canada; ${ }^{3}$ Molecular Biology, Bielefeld University, Bielefeld, Germany

Support: Lawson Health Research Institute. Western Masters of Surgery Program

Introduction: Metabolic syndrome is correlated with an increased risk of urinary stone disease. ${ }^{1}$ At the individual level, patients with a higher body mass index (BMI) have increased prevalence of recurrent symptom- 


\begin{tabular}{|c|c|}
\hline \multicolumn{2}{|l|}{ Parameter } \\
\hline \multicolumn{2}{|c|}{ Treatment duration (days) } \\
\hline Mean (SD) & $180.2(131.3)$ \\
\hline Median & 135 \\
\hline Range & $(41-532)$ \\
\hline \multicolumn{2}{|c|}{ \# of CTs per patient } \\
\hline 2 & 4 \\
\hline 3 & 4 \\
\hline 4 to 5 & 2 \\
\hline \multicolumn{2}{|c|}{ Pre-treatment: } \\
\hline \multicolumn{2}{|c|}{ Max diameter (mm) } \\
\hline Mean (SD) & $16.6(8.7)$ \\
\hline Median & 14.9 \\
\hline Range & $(4.5-40.5)$ \\
\hline \multicolumn{2}{|l|}{ Mean HU } \\
\hline Mean (SD) & $354.8(73.0)$ \\
\hline Median & 365 \\
\hline Range & (224-455) \\
\hline \multicolumn{2}{|c|}{ Volume $\left(\mathrm{mm}^{3}\right)$} \\
\hline Mean (SD) & $1014.3(1333.7)$ \\
\hline Median & 502 \\
\hline Range & (27-4939) \\
\hline \multicolumn{2}{|c|}{ Post treatment: } \\
\hline \multicolumn{2}{|c|}{ Max diameter (mm) } \\
\hline Mean (SD) & $5.5(6.7)$ \\
\hline Median & 2.6 \\
\hline Range & $(0-22.2)$ \\
\hline \multicolumn{2}{|c|}{ Volume $\left(\mathrm{mm}^{3}\right)$} \\
\hline Mean (SD) & $169.5(384.8)$ \\
\hline Median & 8 \\
\hline Range & $(0-1400)$ \\
\hline \multicolumn{2}{|c|}{ Change volume $\left(\mathrm{mm}^{3}\right)$} \\
\hline Mean (SD) & $-844.8(1283.3)$ \\
\hline Median & -441 \\
\hline Range & (-19 to -4939$)$ \\
\hline \multicolumn{2}{|c|}{ Change $\%$ volume } \\
\hline Mean (SD) & $-83.6 \%(20.2)$ \\
\hline Median & $-84.8 \%$ \\
\hline Range & $(-41.4 \%$ to $-100 \%)$ \\
\hline \multicolumn{2}{|c|}{ Change max diameter (mm) } \\
\hline Mean (SD) & $-11.1(10.5)$ \\
\hline Median & -8.8 \\
\hline Range & (1.1 to -40.4$)$ \\
\hline
\end{tabular}

atic urolithiasis, 24-hour urinary excretion of oxalate, sodium, uric acid,

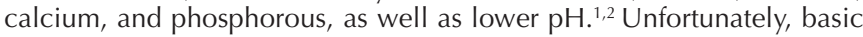
science mechanisms to explain this relationship have been scarce. ${ }^{3}$ There have been suggestions that the mammalian target of rapamycin (mTOR) pathway has been implicated in the pathogenesis of obesity-associated renal tubulointerstitial inflammation, autophagy defects, and urolithiasis. ${ }^{4}$

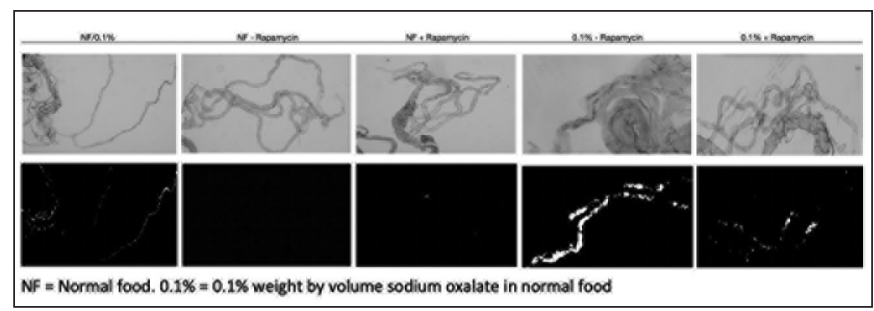

MP-9.11. Fig. 1. Malpighian tubule dissections under bright field (white background) and birefringent (black background) light microscopy for Drosophilafed control diets and experimental diets.

In this study, we are the first to examine the effect of short-term intermittent rapamycin dosing on calcium oxalate concretion in a dietary model of urolithiasis in Drosophila melanogaster. ${ }^{5}$

Methods: Canton $\mathrm{S}$ wild strain D. melanogaster were reared under standard conditions. They were fed either a lithogenic diet containing $0.1 \%$ sodium oxalate or normal food supplemented with either control ethanol or $200 \mathrm{uM}$ rapamycin on $1.5 \%$ agar. All diets were alternated with normal control food every 24 hours. Treatment effect was assessed with life expectancy and stone burden was measured with pixel intensity of calcium oxalate crystals within dissected Malpighian tubules under birefringent microscopy on day 10.

Results: Life expectancy was not significantly different between the different treatment groups. Preliminary results show a trend towards lower calcium oxalate Malpighian tubule concretions in both male and female D. melanogaster with rapamycin treatment (Fig. 1).

Conclusions: Rapamycin may reduce calcium oxalate stone burden in a dietary model of $D$. melanogaster of urolithiasis. Further investigation is required to confirm these results. Furthermore, mutant $D$. melanogaster strains are required to further delineate the downstream effectors by which the mTOR pathway may influence calcium-based stone formation.

References

1. Taylor EN, Curhan GC. Body size and 24-hour urine composition. Am J Kidney Dis 2006;48:905-15. https://doi.org/10.1053/j. ajkd.2006.09.004

2. Taylor EN, Stampfer MJ, Curhan GC. Diabetes mellitus and the risk of nephrolithiasis. Kidney Int 2005;68:1230-5. https://doi.org/10.1111/ j.1523-1755.2005.00516.x

3. Chi T, Taylor E, Stoller ML. The link between metabolic syndrome and nephrolithiasis: A white whale for understanding urinary stone disease. Transl Androl Urol 2014;3:296.

4. Unno R, Kawabata T, Taguchi K, et al. Deregulated MTOR (mechanistic target of rapamycin kinase) is responsible for autophagy defects exacerbating kidney stone development. Autophagy 2020;16:70923. https://doi.org/10.1080/15548627.2019.1635382

5. Ali SN, Dayarathna TK, Ali AN, et al. Drosophila melanogaster as a function-based high-throughput screening model for anti-nephrolithiasis agents in kidney stone patients. Dis Model Mech 2018;16:11. https://doi.org/10.1242/dmm.035873

\section{MP-9.12}

Association between visceral adipose tissue loss post-bariatric surgery and 24-hour urinary profile changes

Michael John Uy' ${ }^{1}$ Richard D. Di Lena', Jen Hoogenes ${ }^{1}$, Badr Al-Harbi ${ }^{2}$, Aiden Woodward ${ }^{3}$, Edward D. Matsumoto

${ }^{1}$ Department of Surgery, Division of Urology, McMaster University, Hamilton, ON, Canada; ' 2 Department of Surgery, College of Medicine, Qassim University, Qassim, Saudi Arabia; ${ }^{3}$ Department of Health Sciences, Western University, London, ON, Canada

Introduction: Obesity is a known risk factor for abnormal urinary profiles and nephrolithiasis. Body mass index $\left(\mathrm{BMI}, \mathrm{kg} / \mathrm{m}^{2}\right)$ has been the traditional objective measure of obesity; however, visceral adipose tissue $\left(\right.$ VAT, $\left.\mathrm{cm}^{2}\right)$ has been shown to be more accurate. Postoperatively, bariatric patients often have a pronounced decrease in VAT, yet the association of VAT loss 


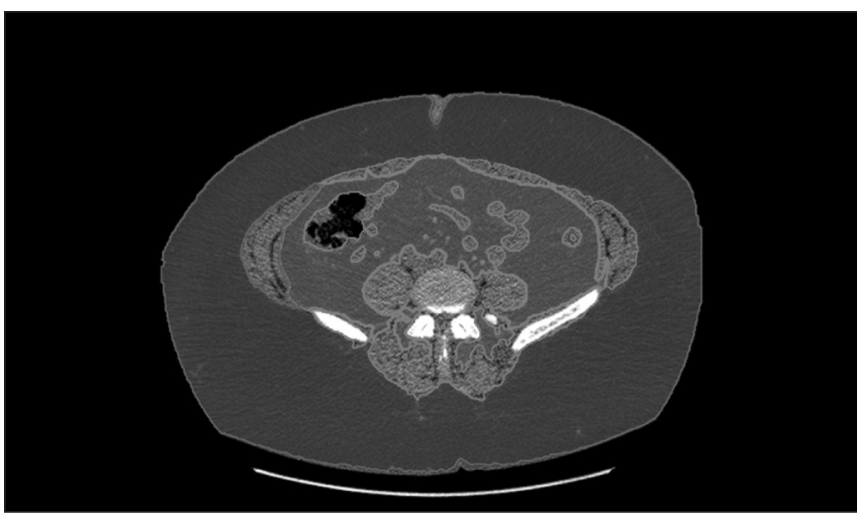

MP-9.12. Fig. 1. Axial computed tomography slice at the level of VAT 1 (L4 and L5).

and changes to urinary profiles has not been well-studied. We explored the relationship between the percentage of VAT loss post-bariatric surgery and metabolic urinary changes.

Methods: Records of patients with a history of nephrolithiasis who underwent bariatric surgery at our center were reviewed. Eligible patients had pre- and postoperative 24 -hour urine $(24 \mathrm{HU})$ analysis and a computed tomography of the kidneya-uretera-bladder (CT KUB). A CT-based fat delineation program, AnalyzePro ${ }^{\circledR}$, was used to measure pre- and postoperative VAT at levels L4 and L5 (VAT1) (Fig. 1), and L1-L2 (VAT2). Univariate and multivariate analyses examined associations between VAT and $24 \mathrm{HU}$ values, comorbidities, and urinary profiles.

Results: A total of 88 patients met the inclusion criteria. The mean age was $51.9( \pm 9.2)$ and $68.2 \%$ were female. Preoperatively, VAT1 was significantly correlated with hypertension, dyslipidemia, and metabolic syndrome ( $p=0.006,0.015$, and 0.004 , respectively), and VAT2 was correlated with hypertension and metabolic syndrome $(p=0.012$ and 0.014 , respectively). VAT2 was significantly associated with all $24 \mathrm{HU}$ values $(p<0.001-0.030)$, while VAT1 did not correlate with phosphate or calcium. The $\%$ of both VAT1 and 2 loss was a significant predictor for decrease in $24 \mathrm{HU}$ urate ( $p=0.002$ and 0.003 , respectively) (Fig. 2). Conclusions: Within this patient cohort, VAT measurements were significantly correlated with preoperative $24 \mathrm{HU}$ values and multiple comorbidities. The $\%$ of VAT loss was able to predict postoperative $24 \mathrm{HU}$ urate changes. These findings indicate that VAT measurements can be used to inform the clinical management of bariatric patients with a history of nephrolithiasis.

\section{MP-9.13}

\section{Optimal sedation dosing for shockwave lithotripsy: Assessing} dose reduction strategies

Callum Lavoie ${ }^{\prime}$, Tim Wollin', Trevor D. Schuler ${ }^{1}$, Shubhadip (Shubha) K. De

${ }^{1}$ Division of Urology, University of Alberta, Edmonton, $A B$, Canada Introduction: Sedation with narcotics and anxiolytics is a common analgesic strategy for shockwave lithotripsy (SWL). With no consensus on optimal sedation doses, our center recently revised its protocol, reducing the initial and prn doses of benzodiazepines and narcotics by $50 \%$. Therefore, our objective was to quantify the outcomes of anesthetic dose reduction on SWL patients at our center.

Methods: A retrospective review of patients undergoing renal/ureteral SWL was performed. Data from two distinct patient cohorts from June 2019 and January 2017 were compared. Cohort 1 (2019) represents a dose-reduced protocol, whereas cohort 2 (2017) represents a standard dosing protocol. Patient demographics, stone characteristics, and perioperative fentanyl, midazolam, and ketorolac doses were recorded. The primary outcome was stone-free rates at followup; secondary outcomes were time in the recovery room, self-reported pain at discharge, nor-

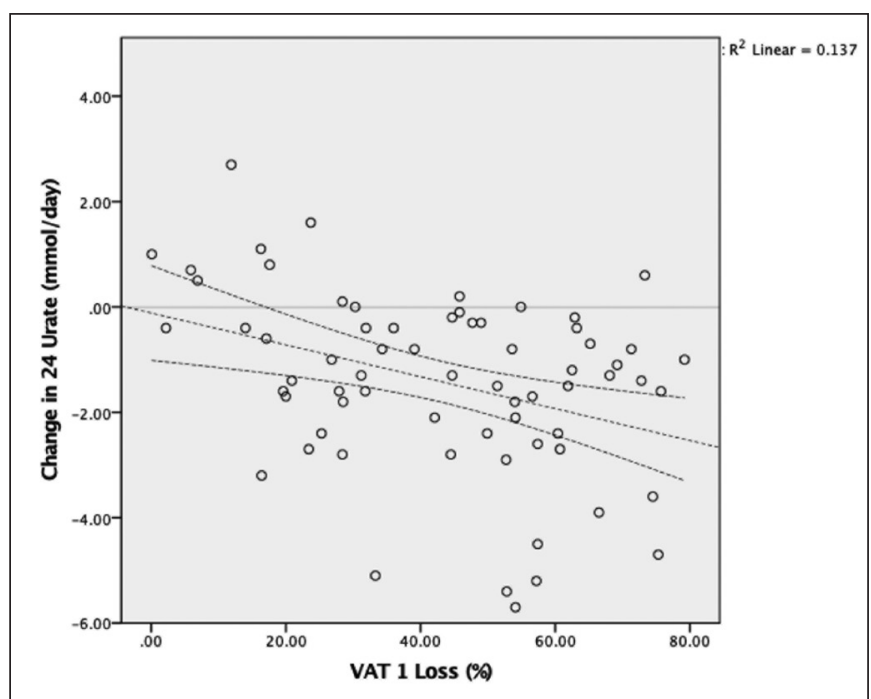

MP-9.12. Fig. 2. 24-hour urinary urate change by \%VAT 1 loss.

malization of sedation score, and requirement of any airway support or maintenance. Statistical analysis was performed using SPSS Software v26. Results: A total of 100 patients were analyzed, with 50 from each cohort. There was a statistically significant decrease in midazolam dosages ( 5.5 vs. $2.7 \mathrm{mg} ; \mathrm{p}<0.001)$ in cohort 1 , with an increased use of ketorolac ( $18 \%$ vs. $46 \%$; $p=0.003)$. No difference in stone-free rates, or treatment success was identified at two-week followup ( $64 \%$ vs. $62 \%$; $p=0.836)$. There was no difference in need for airway support intra or postoperatively $(16 \%$ vs. $10 \% ; p=0.372)$ or in time to discharge (61.6 vs. 58.2 minutes; $\mathrm{p}=0.505)$. Pain scores in both groups were minimal at discharge $(0.84$ vs. $1.62 ; p=0.031$ ) with no increased complications associated with the use of ketorolac.

Conclusions: In reducing the doses of sedative agents during SWL, we were able to maintain treatment efficacy and comfort, without increasing side effects. Though post-procedure non-steroidal anti-inflammatory drugs were used more frequently with decreased sedation dosing, there were no increases in associated morbidity.

\section{MP-9.14}

\section{Immediate vs. delayed shockwave lithotripsy at 90 shocks per} minute

Alex Koziarz ${ }^{1}$, Bader Alsaikhan ${ }^{1,2,3}$, Rheanne Sbrocca ${ }^{1}$, Daniela Ghiculete2 Michael Ordon ${ }^{1,2,3}$, Kenneth T. Pace ${ }^{1,2,3}$

${ }^{1}$ Faculty of Medicine, University of Toronto, Toronto, ON, Canada; ${ }^{2}$ Division of Urology, St. Michael's Hospital, Toronto, ON, Canada; ${ }^{3}$ Department of Surgery, University of Toronto, Toronto, ON, Canada

Introduction: Shockwave lithotripsy (SWL) rate plays an important role in treatment success and stone fragmentation. Multiple studies demonstrated rates of 60 shocks per minute (SPM) and 90 SPM to be superior to 120 SPM. Rate reduction after the first 1000 shocks is a common practice at our institution if initial fragmentation at a faster rate is poor. In this study, we compare outcomes of SWL rate reduction from 120 SPM to 90 after an initial 1000 shocks vs. a complete treatment at a reduced rate of 90 SPM. Methods: A retrospective review of patients treated at our center for renal and ureteric stones using Storz Modulith SLX-F2 was performed. Group 1 was treated with 3000 shocks at 90 SPM $(n=88)$. Group 2 was treated with 1000 shocks at 120 SPM, followed by rate reduction to 90 SPM for the remaining 2000 shocks $(n=87)$. The primary outcome was fragmentation success rates after first SWL treatment. Demographic, stone, treatment, and followup parameters were collected. A multivariate logistic regression was performed adjusting for patient body mass index (BMI), stone 
area, and location. Other variables were compared with ANOVA and chi-squared analysis where appropriate.

Results: There was no difference in stone location, age, BMI, or gender between the two groups. Stones were significantly larger in group 1 (93.4 vs. $59.4 \mathrm{~mm}^{2} ; \mathrm{p}<0.001$ ). Overall single SWL treatment success rate was $58.9 \%$. Logistic regression demonstrated no difference in SWL success rates between the two groups $(p=0.221)$ after adjusting for stone area $(p<0.001)$ and stone location $(p=0.076)$. There were no differences in complications, re-treatment rates, or rates of auxiliary procedures.
Conclusions: In our small cohort, immediate SWL rate reduction to 90 SPM did not show a significant difference in terms of treatment success rate compared to delayed reduction of SWL rate after an initial 1000 shocks at 120 SPM. Stone area was strongly predictive of outcome, with a trend towards a difference based on stone location. 


\section{Moderated Poster Session 10: Prostate Cancer II}

\begin{abstract}
MP-10.1
A pilot window-of-opportunity study of preoperative fluvastatin in localized prostate cancer

Robert I. Hamilton 1,3, Joseph Longo ${ }^{1,2}$, Mehdi Masoomian $^{4}$, Najia Khurram ${ }^{1,3}$, Peter Mullen ${ }^{1}$, Karen Hersey 1,3, Dianne Chadwick ${ }^{4}$, Sangeet Ghai ${ }^{1}$, Eric Chen ${ }^{1}$, Theodorus van der Kwast ${ }^{4}$, Neil E. Fleshner ${ }^{1,3}$, Linda Penn $n^{1,2}$

${ }^{1}$ Princess Margaret Cancer Centre, University Health Network, Toronto, ON, Canada; ${ }^{2}$ Department of Medical Biophysics, University of Toronto, Toronto, ON, Canada; ${ }^{3}$ Division of Urology, Department of Surgical Oncology, University Health Network \& University of Toronto, Toronto, ON, Canada; ${ }^{4}$ Department of Pathology, Laboratory Medicine Program, University Health Network, Toronto, ON, Canada

Introduction: Epidemiological and pre-clinical evidence support an association between statins and improved prostate cancer (PCa) outcomes. We evaluated the effects of neoadjuvant fluvastatin on markers of cell proliferation and apoptosis in men with localized PCa.

Methods: Thirty-three men were treated daily with $80 \mathrm{mg}$ fluvastatin for 4-12 weeks in a single-arm, window-of-opportunity study between diagnosis of intermediate or high-risk PCa and radical prostatectomy (RP) (NCT01992042). Percent Ki67 and cleaved Caspase-3 (CC3)-positive cells in tumor tissues were evaluated in 23 patients by immunohistochemistry before and after fluvastatin treatment. Serum and intraprostatic fluvastatin concentrations were quantified by liquid chromatography-mass spectrometry.

Results: Median prostate-specific antigen (PSA) was $6.48 \mathrm{ng} / \mathrm{mL}$ (range 2.17-28.30) and median duration of fluvastatin treatment was 49 days (range 27-102). A median 35.4\% decrease in low-density lipoprotein (LDL) cholesterol after treatment indicated compliance. Fluvastatin treatment did not significantly alter serum PSA or intratumoral Ki67 positivity. Secondary endpoint analyses revealed a statistically significant, median 2.7-fold increase in intratumoral CC3 positivity (95\% confidence interval [Cl] 1.9-5.0; $p=0.007$ ) in post-statin RP tissues compared to matched pretreatment biopsy specimens. In a subset analysis, this response was more pronounced in men on fluvastatin for at least 50 days (median 3.3-fold increase, $95 \% \mathrm{Cl} 0.8-8.0 ; \mathrm{p}=0.025$ ). Mean fluvastatin concentration in the serum and prostate was $63.4 \mathrm{ng} / \mathrm{mL}$ (range 0.0-437.0) and $3.5 \mathrm{ng} / \mathrm{g}$ (range $0.0-31.7)$, respectively.

Conclusions: Fluvastatin prior to RP achieves measurable drug concentrations in prostatic tissue. Despite the lack of significant changes in proliferation indices, immunohistochemical data indicate promising effects on tumor cell apoptosis. Combined, these data warrant further investigation into the anti-neoplastic effects of statins in prostate tissue.
\end{abstract}

\footnotetext{
MP-10.2

The perioperative outcomes in prostatectomy patients on neoadjuvant apalutamide

Raj Tiwari ${ }^{1}$, Lui Shiong Lee ${ }^{1}$

${ }^{1}$ Urology, Sengkang General Hospital, Singapore, Singapore

Introduction: We demonstrated the surgical and functional outcomes in a series of patients who received neoadjuvant apalutamide prior to radical prostatectomy. Our aim was to show equivalence in outcomes between our series and other large conventional prostatectomy series.

Methods: After IRB approval, 17 patients were prospectively recruited and received neoadjuvant apalutamide (ARN 509) for 12 weeks, subsequently undergoing robotic radical prostatectomy. While on apalutamide, their prostate-specific antigen (PSA) results, quality of life, and erectile function was studied using the European Organization for the Research and
}

Treatment of Cancer Quality of Life Questionnaire (EORTC QLQ-C30) and Sexual Health Inventory for Men (SHIM) scores. PSA values were measured pre and postoperatively. Intraoperative dissection videos were collated and postoperative pathological and surgical outcomes were studied.

Results: While on apalutamide, there was a durable and significant PSA decrease in patients both prior to prostatectomy $(p=0.0005)$ and six months postoperatively $(p=0.037)$. SHIM scores showed a significant drop ( $p=0.002)$, while QLQ-C30 scores were maintained while on apalutamide. Our pathological outcomes were comparable to preceding large robotic series, with most patients $(80 \%)$ being intermediate-risk and an $18 \%$ rate of upgrade on whole mount. Other operative outcomes, such as blood loss, catheter time, length of stay, and Clavian-Dindo complications, were comparable. Positive surgical margin rate was significantly less $(11.8 \%$ vs. $42.9 \%)$ with a higher lymph node dissection count and node positivity rate when compared to our preceding prostatectomy series.

Conclusions: Neoadjuvant apalutamide followed by prostatectomy has shown at least non-inferiority in most surgical and functional outcomes when compared to conventional prostatectomy series. A durable PSA response up to six months postoperatively combined with improved positive surgical margin rates are key findings. Disadvantages would include worsened erectile function while on therapy.

\section{MP-10.3}

Delivery of surgeon report cards does not improve individual surgeon outcomes for radical prostatectomy

Avril J. Lusty', Rodney H. Breau', Luke T. Lavallée', Ravi Kumar ${ }^{1}$

'Urology, University of Ottawa, Ottawa, ON, Canada

Introduction: Radical prostatectomy aims for complete oncological resection while optimizing patient continence and potency. Previous reports are mixed on whether presenting surgeons their patient outcome data modifies their operative performance or improves patient outcomes. The primary objective of this study was to determine if delivery of surgeon report cards improves individual surgeon outcomes for men treated with radical prostatectomy. The four co-primary outcomes included: pT2 and pT3 margin status, continence, and potency.

Methods: This prospective, cohort study collected data from July 2015 to December 2018, of eight urologists from The Ottawa Hospital. The prefeedback cohort included 12 months of patient data, including baseline, six-month and 12-month data. Report cards were delivered to surgeons in August 2016 and every three months thereafter, with patient data collected after this comprising the post-feedback cohort. Report cards presented to surgeons included: pT2 and pT3 margin status, continence, and potency data, while other surgeons' data were de-identified.

Results: A total of 754 men aged 59-69 underwent radical prostatectomy, with a mean prostate-specific antigen (PSA) of $8.7 \mathrm{ng} / \mathrm{mL}$. Patients' pathological T stage was: $48.3 \%$ and $51.7 \%$ for pT2 and pT3, respectively. Most $(52.7 \%)$ had bilateral nerve-spare, $24.1 \%$ had unilateral nerve-spare, and $23.2 \%$ had bilateral nerve resection. Of the four co-primary outcomes, only continence changed after report card feedback, with 12 -month continence decreasing from $94.2 \%$ to $83.0 \%$ ( $p<0.0001$ ). Pre- and post-report card data of pT2 margin status, pT3 margin status, and potency were not significantly different, with pT2 positive margins of $23.3 \%$ vs. $24.9 \%$, pT3 positive margins of $42.5 \%$ vs. $47.8 \%$, and potency at 12 months of $38.7 \%$ vs. $32.1 \%$. Radar plots of individual surgeon outcomes showed both minor improvement and worsening of patient outcome data. 
Conclusions: Distribution of surgeon report cards did not improve individual surgeon patient outcomes of men treated with radical prostatectomy at 12 months' followup.

\section{MP-10.5}

Functional and perioperative outcomes in elderly men after robotic-assisted radical prostatectomy for localized prostate cancer

Ahmed S. Zakaria Ahmed', Samer Traboulsi', David-Dan Nguyen², Kyle Law ${ }^{2}$, Hanna Shahine ${ }^{1}$, Malek Meskawi ${ }^{1}$, Cristina Negrean ${ }^{7}$, Pierre I. Karakiewicz ${ }^{1}$, Assaad El-Hakim ${ }^{1}$, Kevin C. Zorn ${ }^{1}$

'Urology, Centre hospitalier de l'Université de Montréal, Montreal, QC, Canada; ${ }^{2}$ Urology, McGill University Health Centre, Montreal, QC, Canada

Introduction: Robotic-assisted radical prostatectomy (RARP) is a feasible and safe procedure in elderly men. However, urinary incontinence and erectile dysfunction are of concerns due to considerable impact on healthrelated quality of life. We aimed to compare preoperative and postoperative functional outcomes following RARP in elderly men with localized prostate cancer $(\mathrm{PCa})$

Methods: A retrospective review of a prospectively maintained database of men who underwent RARP between January 2007 and November 2018 was performed. Patients over 65 years of age were selected $(n=302)$ and then stratified by age group: $66-69$ years old $(n=214)$ and $\geq 70$ years old $(n=88)$. Full continence was defined as strict 0 -pad per day usage. Preoperative potency included those with a Sexual Health Inventory for Men (SHIM) score $\geq 17$. Preoperative and postoperative functional outcomes were assessed. Kaplan-Meier analysis was used to estimate time to recovery of continence in both groups.

Results: Both groups had comparable preoperative parameters. Continence rates at one, three, six, nine, 12, 18, and 24 months in the 66-69-yearold group were $6 \%, 34 \%, 61 \%, 70 \%, 74 \%, 80 \%$, and $87 \%$, respectively. Comparatively, in the $\geq 70$-year-old group, continence rates were significantly lower at all time points $(3 \%, 22 \%, 50 \%, 56 \%, 66 \%, 69 \%$, and $75 \%$, respectively). Men in the 66-69-year-old group (Fig. 1) were significantly more likely to be continent after RARP when compared to patients 70 years of age and above (hazard ratio [HR] 0.73; 95\% confidence interval $[\mathrm{Cl}]$ 0.54-0.97; $\mathrm{p}=0.035$ ).

Conclusions: Our results suggest that RARP is feasible in all groups of elderly patients. Nevertheless, elderly patients in the $>70$ years age group had significantly inferior continence rates postoperatively compared to patients aged $66-69$ years. Such information is valuable when counselling men during preoperative RARP planning for realistic postoperative expectations.

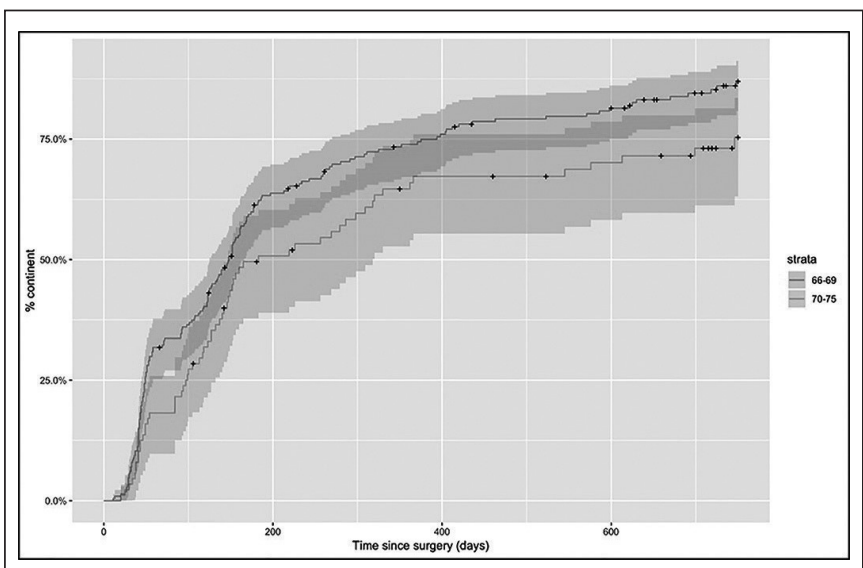

MP-10.5. Fig. 1. Incidence plot of time to recovery of continence, comparing ages $66-69$ to $\geq 70$ years old.
MP-10.6

Baseline patient-reported outcomes data shows high prevalence of overactive bladder, sexual dysfunction, depression, and anxiety in men with newly diagnosed localized prostate cancer Henry Han-I Yao ${ }^{1,2,3}$, Trafford Crump ${ }^{1}$, Camille Charbonneau', Asher Khan ${ }^{\prime}$, Carly Barton ${ }^{1}$, Hilary L. Brotherhood ${ }^{1,2}$, Jing Jiang ${ }^{1}$, Kevin V. Carlson 1,2, Richard I. Baverstock ${ }^{1,2}$

${ }^{1}$ vesia (Alberta Bladder Centre), Calgary, AB, Canada; ${ }^{2}$ Department of Surgery, University of Calgary, Calgary, AB, Canada; ${ }^{3}$ Eastern Health Clinical School, Monash University, Melbourne, Australia

Support: This work was awarded by Prostate Cancer Canada, and is proudly funded by the Movember Foundation

Introduction: We aimed to elucidate the prevalence of lower urinary tract symptoms (LUTS), overactive bladder (OAB), sexual dysfunction, depression, and anxiety in Canadian men with newly diagnosed localized prostate cancer (PCa).

Methods: This was a single-centre, prospective study of men with newly diagnosed localized PCa. Patients were recruited from June 2017 to July 2018. The patient-reported outcomes (PRO) instruments used included the International Prostate Symptoms Score (IPSS), ${ }^{1}$ OAB-V8, ${ }^{2}$, EQ-5DTM, ${ }^{3}$ and the Expanded Prostate Cancer Index Composite short form (EPIC26) ${ }^{4}$ Clinico-pathological data were extracted from medical records. The prevalence of LUTS, OAB, sexual dysfunction, depression, and anxiety were determined from the PROs.

Results: Eighty-three patients were included in this study. The median age was 63 years. The median prostate-specific antigen (PSA) was 6.7 $\mathrm{ng} / \mathrm{ml}$. The median prostate volume was $35 \mathrm{ml}$. The proportion of men with International Society of Urological Pathology grade group 1, 2, 3, 4, and 5 was $36.1 \%, 37.3 \%, 12.0 \%, 10.8 \%$, and $3.6 \%$, respectively. Based on IPSS, $55.3 \%$ of men had mild LUTS, $36.8 \%$ had moderate LUTS, and $7.9 \%$ had severe LUTS. Based on OAB-V8, $55.8 \%$ of men had a score of 8 or higher, suggestive of OAB. Only $55.8 \%$ of men reported erections adequate for intercourse; $23.1 \%$ of men reported to have a moderate to big problem with depression; and $28.8 \%$ of men reported to have a degree of anxiety or depression.

Conclusions: $O A B$ is a significant problem affecting half of Canadian men with newly diagnosed localized PCa. Baseline sexual dysfunction in this population of men is also significant, with only half of the men able to achieve an erection adequate for intercourse. Anxiety and depression also affect a quarter of these men. It is important to identify these pretreatment, as they can impact management decisions and counselling of patients on functional outcome. Management of these issues starting pre-treatment can potentially improve the quality of life for men following PCa treatment.

References

1. Barry MJ, Fowler Jr. FJ, O'Leary MP, et al. The American Urological Association symptom index for benign prostatic hyperplasia. The Measurement Committee of the American Urological Association. J Urol 1992;148:1549-57; discussion 1564. https://doi.org/10.1016/ S0022-5347(17)36966-5

2. Coyne KS, Zyczynski T, Margolis MK, et al. Validation of an overactive bladder awareness tool for use in primary care settings. Adv Ther 2005;22:381-94. https://doi.org/10.1007/BF02850085

3. Desroziers K, Aballea S, Maman K, et al. Estimating EQ-5D and OAB-5D health state utilities for patients with overactive bladder. Health Qual Life Outcomes 2013;11:200. https://doi. org/10.1186/1477-7525-11-200

4. Wei JT, Dunn RL, Litwin MS, et al. Development and validation of the expanded prostate cancer index composite (EPIC) for comprehensive assessment of health-related quality of life in men with prostate cancer. Urology 2000;56:899-905. https://doi.org/10.1016/ S0090-4295(00)00858-X 


\section{MP-10.7}

The comparative outcomes of radical prostatectomy vs. radiotherapy for non-metastatic prostate cancer: A longitudinal, population-based analysis

Justin Oake ${ }^{1}$, Benjamin Shiff', Oksana Harasemiw'w', Navdeep Tangri'2,3,4, Thomas W. Ferguson ${ }^{2,3}$, Bimal Bhindi ${ }^{5}$, Jeffery W. Saranchuk', Rahul K. Bansal', Darrel E. Drachenberg', Jasmir (Jay) G. Nayak'

${ }^{1}$ Section of Urology, University of Manitoba, Winnipeg, MB, Canada; ${ }^{2}$ Chronic Disease Innovation Centre, Seven Oaks General Hospital, Winnipeg, MB, Canada; ${ }^{3}$ Department of Internal Medicine, University of Manitoba, Winnipeg, MB, Canada; ${ }^{4}$ Department of Community Health Sciences, University of Manitoba, Winnipeg, MB, Canada; ${ }^{5}$ Section of Urology, University of Calgary, Calgary, AB, Canada Introduction: The comparative effectiveness of radical prostatectomy (RP) vs. radiation therapy (RT) for prostate cancer remains a largely debated topic. Using a provincial, population-based, linked dataset from an equalaccess, universal healthcare system, we sought to compare outcomes among patients treated with either RT or RP for non-metastatic prostate cancer.

Methods: We performed a retrospective cohort study by linking several administrative datasets to identify patients who were diagnosed with prostate cancer from 2004-2016 in Manitoba, Canada, and who were subsequently treated with either RP or RT. Cox proportional hazard models with inverse probability of treatment weighting (IPTW) were used to compare rates of all-cause mortality, as well as prostate cancer-specific mortality (PCSM) between patients who underwent RP vs. RT.

Results: During the study period, 2540 patients underwent RP and 1895 underwent RT for prostate cancer. Unadjusted overall survival (OS) was higher for RP vs. RT (five-year OS $95.52 \%$ for RP compared with $84.55 \%$ for RT; $\mathrm{p}<0.0001)$. In IPTW-adjusted Cox regression analysis, compared to patients in the RP groups, patients in the RT group had an increased rate of all-cause mortality (hazard ratio [HR] 1.93; 95\% confidence interval [Cl] 1.65-2.26; $\mathrm{p}<0.0001)$ and PCSM (HR 3.98; 95\% Cl 2.89-5.49; $\mathrm{p}<0.0001)$.

Conclusions: RT was associated with lower OS and higher PCSM rates compared with RP. These findings highlight the importance of comparative effectiveness research to identify treatment disparities and warrant further investigation.

\section{MP-10.8}

Biochemical definition of cure after low dose rate prostate brachytherapy

Juanita Crook ${ }^{1}$, Chad Tang ${ }^{2}$, Howard Thames ${ }^{2}$, Pierre Blanchard ${ }^{2}$, Jeremiah Sanders' ${ }^{2}$, Jay Ciezki ${ }^{3}$, Mira Keyes ${ }^{4}$, Gregory Merrick ${ }^{5}$, Charles Catton ${ }^{6}$, Hamid Raziee ${ }^{6}$, Richard Stock 7 , Frank J Sullivan ${ }^{8}$, Jeremy Millar ${ }^{9}$, Mitch Anscher ${ }^{2}$, Steven Frank

${ }^{1}$ Radiation Oncology, BC Cancer, Kelowna, BC, Canada; ${ }^{2}$ Radiation Oncology, MD Anderson, Houston, TX, United States; ${ }^{3}$ Radiation Oncology, Case Western Reserve University, Cleveland, $\mathrm{OH}$, United States; ${ }^{4}$ Radiation Oncology, BC Cancer, Vancouver, BC, Canada; ${ }^{5}$ Radiation Oncology, Schiffler Cancer Center, Wheeling, WV, United States; ${ }^{6}$ Radiation Oncology, University Health Network, Toronto, ON, Canada; 'Radiation Oncology, Mt Sinai School of Medicine, New York, NY, United States; ${ }^{8}$ Galway Clinic, National University of Ireland, Galway, Ireland; ${ }^{9}$ Alfred Health, Melbourne, Australia

Introduction: The incorporation of brachytherapy into the radiation management of prostate cancer is associated with extremely low and stable prostate-specific antigen (PSA) values. Prior consensus has defined failure after radiation with high specificity, but not cure. We sought to identify a PSA threshold value, at an intermediate followup time, associated with cure as defined by long term (10-15-year) freedom from prostate cancer. Methods: We analyzed prospectively collected outcome data from seven institutions for 14220 patients with localized prostate cancer treated with low dose rate (LDR) brachytherapy (BT) either alone $(n=8552$ : $61 \%)$ or in combination with external beam radiotherapy $(n=1175: 8 \%)$, androgen deprivation $(n=3165: 22 \%)$ or both $(n=1,328: 9 \%) ; 42 \%$ were low-risk, $50 \%$ intermediate-, and $8 \%$ high-risk. PSA measured at four years (range 3.5-4.5) in 8746 patients with minimum 3.5-year PSA followup, without

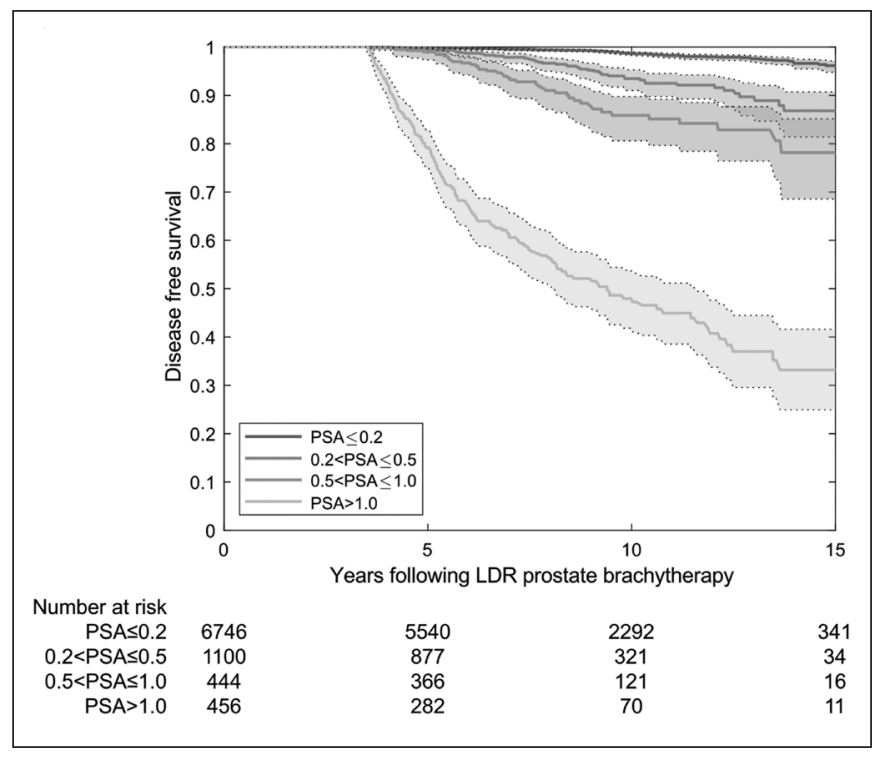

MP-10.8. Fig. 1. Disease-free survival.

prior clinical failure, was correlated with disease status at 10-15 years. Kaplan-Meier analysis was carried out using clinical failure (local, distant, regional, or biochemical triggering salvage) as endpoints for each of four PSA categories: PSA $\leq 0.2 \mathrm{ng} / \mathrm{ml}, \mathrm{PSA}>0.2$ to $\leq 0.5$, PSA $>0.5$ to $\leq 1.0$, and PSA $>1.0 \mathrm{ng} / \mathrm{ml}$. Results were compared to three independent validation cohorts: low-risk ( $n=366$ : Australia), intermediate-risk $(n=221$ : MD Anderson Phase 2 trial), and high-risk ( $n=160$ : AscendeRT Phase 3 trial BT arm).

Results: Most (77.1\%) patients had a PSA $\leq 0.2 \mathrm{ng} / \mathrm{ml}$ at four years. KaplanMeier analysis showed $98.7 \%$ free of recurrence at 10 years (95\% confidence interval [CI] 98.3-99.0) and 96.1\% at 15 years (95\% CI 94.8-97.2). Successive PSA categories were associated with diminished disease-free rates at 10 and 15 years (Fig. 1). PSA range was strongly associated with treatment success $(\mathrm{p}<0.0005)$. Median PSA at $10(\mathrm{n}=4864$, readings $)$ and 15 years $(\mathrm{n}=1137)$ was $0.01 \mathrm{ng} / \mathrm{ml}$. For the three validation cohorts, $\mathrm{PSA} \leq 0.2 \mathrm{ng} / \mathrm{ml}$ at four years was associated with $99.0 \%, 99.4 \%$, and $96.7 \%$ freedom from failure at 10 years, respectively.

Conclusions: As over $80 \%$ of patients achieve a PSA $\leq 0.2 \mathrm{ng} / \mathrm{ml}$ at four years post-LDR BT, and this is associated with $97-99 \%$ being disease-free beyond 10 years, we suggest that this biochemical definition of cure be adopted for LDR brachytherapy patients with $\geq 4$ years' followup.

\section{MP-10.10}

Does salvage whole gland ablation therapy confer survival advantage to patients who failed primary radiotherapy for prostate cancer?

Shiva M. Nair', Andrew Warner ${ }^{2}$, Arnon Lavi' ${ }^{1}$, George B. Rodrigues², L.K. Joseph Chin

'Departments of Urology and Oncology, Schulich School of Medicine and Dentistry, Western University, London, ON, Canada; ${ }^{2}$ Department of Radiation Oncology, Schulich School of Medicine and Dentistry, Western University, London, ON, Canada

Support: Pan-Canadian Prostate Cancer Risk Stratification (ProCaRS)

Introduction: Men experiencing prostate cancer recurrence after radiotherapy usually progress to systemic therapies, most often with androgen deprivation therapy (ADT). Selected men may be candidates for local salvage therapy, avoiding or at least delaying ADT. We compared long-term outcomes of post-radiation-salvage cryoablation $(\mathrm{sCT})$ and high-intensity focused ultrasound (sHIFU) vs. standard of care (SOC) without local salvage therapy in a large radiation therapy database.

Methods: Men undergoing ablation for localized radio-recurrent prostate cancer at Western University between 1995 and 2018 were identified. The 
SOC group was identified from the pan-Canadian Prostate Cancer Risk Stratification (ProCaRS) database treated between 1994 and 2008. Men were matched using propensity score-based pre-radiation characteristics: age, prostate-specific antigen (PSA), Gleason score, T-stage, and ADT use. Primary endpoints were overall survival (OS) and cancer-specific survival (CSS).

Results: A total of 982 men developed biochemical failure in the SOC group; 186 men were treated with sCT and 113 men with sHIFU. Median followup from radiation treatment was 11.6, 25.1, and 14.3 years following external beam radiation therapy (EBRT), sCT, and sHIFU, respectively. In the first comparison, the propensity-matched cohorts consisted of 196 EBRT vs. 98 sCT. Prostate cancer-specific deaths occurred in 24 of 80 deaths in the SCT group and 49 of 78 deaths in the SOC group. Propensity-matched analysis showed significant benefit in CSS $(p<0.001)$ and OS ( $\mathrm{p}<0.001$ ) favoring $\mathrm{sCT}$. In the second comparison, the propensitymatched cohort consisted of 177 SOC vs. 59 sHIFU. There were 52 deaths (31 prostate cancer) for SOC vs. 18 deaths (nine prostate cancer) for sHIFU. There were no significant differences in CSS or OS, likely attributed to shorter followup of sHIFU cohort.

Conclusions: In select men with radio-recurrent prostate cancer, further local treatment may lead to benefits in CSS and OS. Careful patient selection remains critical in the decision-making process of salvage therapy.

\section{MP-10.11}

Real-world testosterone suppression outcomes in a Canadian population of prostate cancer patients treated with degarelix Miran Kenk ${ }^{1}$, Virna Cepero², Lorne E. Aaron ${ }^{3}$, Bobby Shayegan ${ }^{4}$, Richard Sioufi ${ }^{5}$, Anil Kapoor ${ }^{4}$, Victor Mak', Zeid Mohamedali ${ }^{7}$, Jeffrey M. Spodek ${ }^{8}$, Paul Jr. Ouellette ${ }^{9}$, Neil T. Dwyer ${ }^{10}$, George Vrabec ${ }^{11}$, Lee M. Jonat ${ }^{12}$, Sergio Dalla Nora², Neil E. Fleshner

${ }^{1}$ Surgical Oncology, Princess Margaret Cancer Centre, University Health Network, Toronto, ON, Canada; ${ }^{2}$ Ferring Pharmaceuticals, North York, ON, Canada; ${ }^{3}$ Service d' Urologie, Hôpital Charles LeMoyne, Longueuil, QC, Canada; ${ }^{4}$ Institute of Urology, Juravinski Cancer Centre, Hamilton, ON, Canada; ${ }^{5}$ Hôpital Anna Laberge, Châteauguay, QC Canada; ${ }^{6}$ Department of Surgery, Mackenzie Health, Richmond Hill, ON, Canada; ${ }^{7}$ Department of Urological Sciences, University of British Columbia, Port Alberni, BC, Canada; ${ }^{8}$ Scarborough Health Network, Scarborough, ON, Canada; ${ }^{9}$ Centre de recherche TheraDev, Granby, QC, Canada; ${ }^{10}$ The Moncton Hospital, Moncton, NB, Canada; ${ }^{11}$ Abbotsford Regional Hospital and Cancer Centre, Abbotsford, ON, Canada; ${ }^{12}$ Royal Inland Hospital, Kamloops, BC, Canada

Acknowledgements: We gratefully acknowledge doctors, nurses and clinical personnel from all centers participating in this program; also Dan Toma (Pharmec) for data collection and processing

Introduction: Testosterone ( $\mathrm{T}$ ) control is key for advanced prostate cancer (PCa) patients. Data suggest that lower T levels correlate with better outcomes. While the traditional target is $T \leq 1.7 \mathrm{nmol} / \mathrm{L}$, data support a cutoff of $\mathrm{T} \leq 0.7 \mathrm{nmol} / \mathrm{L}$. Degarelix showed fast and sustained $\mathrm{T}$ suppression in clinical studies. However, little data are available from its real-world use. Here, we report T suppression outcomes in a prospective cohort of Canadian patients.

Methods: A prospective survey-based program assessing real-world use of degarelix was conducted in 88 centers across Canada from April 2016 to April 2019. Aggregate data were collected monthly, at treatment initiation and followup visits. T values were collected as ranges: $T \leq 0.7 \mathrm{nmol} / \mathrm{L}, \mathrm{T}$ $>0.7$ to $\leq 1.7 \mathrm{nmol} / \mathrm{L}, \mathrm{T}>1.7 \mathrm{nmol} / \mathrm{L}$.

Results: Data from 1340 initial and 17321 followup visits were collected. At degarelix initiation, $72 \%$ of patients were $\geq 70$ years; $58 \%$ were newly diagnosed, $20 \%$ had prostate-specific antigen (PSA) rise after failure of local therapy, $13 \%$ had PSA rise on luteinizing hormone releasing hormone agonist, and 9\% were reported as other/unknown; $46 \%$ were metastatic. During followup, $10656 \mathrm{~T}$ measurements were obtained on degarelix. Of these, $99 \%$ had $\mathrm{T} \leq 1.7 \mathrm{nmol} / \mathrm{L}$, including $81 \%$ with $\mathrm{T} \leq 0.7$ $\mathrm{nmol} / \mathrm{L} ; 0.9 \% \mathrm{had} \mathrm{T}>1.7 \mathrm{nmol} / \mathrm{L}$. Of $3095 \mathrm{~T}$ measurements on degarelix monotherapy, only one measurement of $\mathrm{T}>1.7 \mathrm{nmol} / \mathrm{L}$ was reported.
Conclusions: This is the largest report on real-world use of degarelix in PCa patients. Current use of degarelix in clinical practice provides optimal $\mathrm{T}$ control in PCa patients.

\section{MP-10.12 \\ Development of a panel of circulating DNA methylation biomarkers predictive of treatment response in castration- resistant prostate cancer}

Madonna Peter ${ }^{1,2}$, Misha Bilenky ${ }^{3}$, Ruth Isserlin ${ }^{4}$, Aaron Hansen ${ }^{5}$, Gary Bader $^{4}$, Martin Hirst ${ }^{3}$, Anthony Joshua $a^{5,6}$, Neil E. Fleshner ${ }^{7}$, Bharati Bapat ${ }^{1,2,8}$ ${ }^{1}$ Lunenfeld-Tanenbaum Research Institute, Sinai Health System, Toronto, ON, Canada; ${ }^{2}$ Department of Laboratory Medicine and Pathobiology, University of Toronto, Toronto, ON, Canada; ${ }^{3}$ Canada's Michael Smith Genome Sciences Centre, BC Cancer Agency, Vancouver, BC, Canada; ${ }^{4}$ Terrence Donnelly Centre for Cellular and Biomolecular Research, University of Toronto, Toronto, ON, Canada; ${ }^{5}$ Division of Medical Oncology and Hematology, Princess Margaret Cancer Centre, Toronto, ON, Canada; ${ }^{6}$ Department of Medical Oncology, Kinghorn Cancer Centre, Darlinghurst, Australia; ${ }^{7}$ Departments of Surgery and Surgical Oncology, Division of Urology, University Health Network, Toronto, ON, Canada; ${ }^{8}$ Department of Pathology, University Health Network, Toronto, ON, Canada

Introduction: Androgen-targeting agents continue to be a major therapeutic avenue for castration-resistant prostate cancer (CRPC). ${ }^{1}$ However, de novo or therapy-driven resistance is inevitable, especially among those patients that develop neuroendocrine-CRPC (NE-CRPC). Biomarkers that identify this subgroup upfront are needed to guide optimal therapy sequences. Circulating cell-free DNA (cfDNA) can harbor tumor-derived mutations (i.e., androgen receptor) associated with treatment resistance. ${ }^{2}$ Beyond genomic aberrations, changes in DNA methylation are also a major hallmark of prostate cancer. ${ }^{3}$ In this study, we assessed genomewide changes in the cfDNA methylome to identify markers of treatment response.

Methods: We collected 45 blood samples from 16 metastatic (m) CRPC patients receiving either enzalutamide or abiraterone acetate. We analyzed sequentially collected plasma cfDNA samples, from prior to treatment initiation (baseline), 12 weeks during treatment, and upon clinical progression. We used methylated cfDNA immunoprecipitation sequencing (cfMeDIP-seq) and developed a novel analysis pipeline that identifies changes in methylation between treatment visits.

Results: Overall, cfDNA harbors changes in well-established prostate cancer methylation markers and alterations in key pathways, such as Wnt and neuronal development. Patients that maintained methylation changes from baseline to week 12 and until progression tended to have a longer time to clinical progression (TTP). Importantly, we observed that markers associated with NE-CRPC could be detected prior to initiating treatment and were correlated with a faster TTP. Currently, we are developing a targeted panel to further validate these candidate markers.

Conclusions: This study highlights the potential of monitoring the cfDNA methylome during therapy in mCRPC. Our findings also suggest that detection of NE-CRPC-associated methylation signatures in earlier stages of treatment may serve as predictive markers.

1. Saad F, Aprikian A, Finelli A, et al. 2019 Canadian Urological Association (CUA)-Canadian Uro Oncology Group (CUOG) guideline: Management of castration-resistant prostate cancer (CRPC). Can Urol Assoc / 2019;13:307-14. https://doi.org/10.5489/cuaj.6136

2. Vandekerkhove G, Chi KN, Wyatt AW. Clinical utility of emerging liquid biomarkers in advanced prostate cancer. Cancer Genet 2018;228-229:151-8. https://doi.org/10.1016/j.cancergen.2017.08.003

3. Massie CE, Mills IG, Lynch AG. The importance of DNA methylation in prostate cancer development. / Steroid Biochem Mol Biol 2017;166:1-15. https://doi.org/10.1016/j.jsbmb.2016.04.009 
MP-10.14

Edmonton Prostate Interdisciplinary Cancer Clinic (EPICC): Real-world efficacy outcomes of a multidisciplinary clinic for metastatic castration-resistant prostate cancer

Graeme Follett ${ }^{3}$, Derek Tilley², Naveen Basappa ${ }^{3}$, Brita Danielson ${ }^{4}$, Michael P. Chetner ${ }^{1}$, Michael Kolinsky ${ }^{3}$, Scott North ${ }^{3}$, Sarah Rayner-Myers', Gerry Todd ${ }^{1}$, Adrian Fairey ${ }^{1}$

'Division of Urology, Department of Surgery, University of Alberta, Edmonton, $A B$, Canada; ${ }^{2}$ Holy Cross Hospital, Calgary, AB Canada; ${ }^{3}$ Department of Medical Oncology, University of Alberta, Edmonton, $\mathrm{AB}$, Canada; ${ }^{4}$ Department of Radiation Oncology, University of Alberta, Edmonton, $\mathrm{AB}$, Canada

Support: Graeme Follett is supported by a research fellowship from Janssen Inc

Introduction: Multidisciplinary management improves complex treatment decision-making in cancer care but its impact for metastatic castrationresistant prostate cancer (M1 CRPC) has not been documented. The Edmonton Prostate Interdisciplinary Cancer Clinic (EPICC) is a multidisciplinary, specialized clinic focused on the delivery of novel therapeutics (androgen receptor axis therapy [ARAT]) to men with chemotherapy-naive M1 CRPC. The objective of the current study was to assess the efficacy of ARAT in the EPICC.

Methods: The study was a retrospective, quality-assurance analysis. Eligible patients had a new diagnosis of chemotherapy-naive M1 CRPC with minimal symptoms. EPICC patients were assessed and treated by a multidisciplinary cancer control team that included nursing oncology, pharmacy oncology, and physician oncology (urological, medical, and radiation). Patients were treated first-line with an ARAT (abiraterone [AA] or enzalutamide [EZ]) from October 2017 to March 2018. The main efficacy outcome was overall survival (OS). The Kaplan-Meier method and Cox regression model were used to analyze survival data. Statistical tests were two-sided $(\mathrm{p} \leq 0.05)$.

Results: From October 2017 to March 2018, 160 chemotherapy-naive M1 CRPC patients were assessed in the EPICC. Median age at EPICC admission was 77 years (range 54-92). Median prostate-specific antigen (PSA) level at EPICC admission was $26.6 \mathrm{ng} / \mathrm{mL}$ (range 0.1-5000). 84 Eight-four of 160 (53\%) patients had received prior radical local therapy (RLT) with curative intent; $83(57 \%)$ patients were treated with EZ and $64(43 \%)$ patients were treated AA. Median OS for the entire cohort was 23 months. In multivariable analysis, absence of prior RLT (hazard ratio [HR] 3.6; 95\% confidence interval [CI] 1.9-6.6; $\mathrm{p}<0.001), \mathrm{PSA}>20 \mathrm{ng} /$ $\mathrm{mL}$ (HR 3.2; 95\% Cl 1.4-7.2; $\mathrm{p}=0.004)$, and higher ECOG performance status ( 1 vs. 0 : HR $2.4 ; 95 \% \mathrm{Cl} 1.3-4.4 ; \mathrm{p}=0.005 ; 2$ vs. 0 : HR $3.5 ; 95 \%$ $\mathrm{Cl} 1.5-8.0 ; p=0.003$; and 3 vs. 0 : HR $12.7 ; 95 \% \mathrm{Cl} 2.5-63.8 ; p=0.002$ ) were independently associated with poorer OS.

Conclusions: Multidisciplinary management of chemotherapy-naive M1 CRPC with ARAT is feasible. Real-world efficacy of ARAT in EPICC are similar to data reported in phase 3 trials. 


\section{CUA ABSTRACTS}

\section{Moderated Poster Session 11: Urinary Incontinence, Voiding Dysfunction, Sexual Dysfunction, Transplant}

\begin{abstract}
MP-11.2
LIBERATE International: Evaluation of the safety and efficacy of the Viveve treatment for stress urinary incontinence

Blayne K. Welk', Sean Peterson ${ }^{2}$, Sender Herschorn ${ }^{3}$

${ }^{1}$ Western University, London, ON, Canada; ${ }^{2}$ Bluewater Clinical Research Group, Sarnia, ON, Canada; ${ }^{3}$ University of Toronto, Toronto, ON, Canada Introduction: Energy-based vaginal treatments have been proposed as a non-invasive option to treat stress urinary incontinence (SUI), however, there is no high-quality evidence to support their efficacy. The primary objective of this study was to evaluate the efficacy of the Viveve treatment, SUI protocol (non-ablative, cryogen-cooled monopolar radiofrequency), in improving mild-moderate SUI in premenopausal women as measured with the one-hour pad weight test (PWT).

Methods: This was a randomized, double blind, sham-controlled trial (RCT) of six months' duration. Ninety-nine subjects at nine sites with mild-moderate SUI meeting the inclusion/exclusion criteria were randomized 2:1 to either the active $\left(90 \mathrm{~J} / \mathrm{cm}^{2} R F\right.$ and cryogen cooling) or sham $(\leq 1 \mathrm{~J} / \mathrm{cm} 2$ RF and cryogen cooling) group. Treatment involved delivering 220 intravaginal pulses in two circumferential rings. At baseline and followup visits, subjects completed the one-hour PWT, additional objective endpoints, and patient-reported outcomes (PROs). Safety assessments were completed throughout the study.

Results: Patient baseline demographics were similar in both groups. At six months post-treatment, the active and sham groups both had clinically relevant, but not statistically different decreases in the one-hour PWT of $77 \%$ and $81 \%$, respectively ( $\mathrm{p}=0.403$ ). The active group reported greater improvements in all PROs over sham at six months, however, this did not reach statistical significance (I-QOL, $p=0.456$; UDI-6, $p=0.887$; ICIQ-UI-SF $\mathrm{p}=0.817)$. No device-related safety issues were reported.

Conclusions: Blinded RCTs are essential to obtain high-quality data to evaluate energy-based treatments for SUI. This trial was one of the first to report objective and subjective data in support of these treatments. The active group reported clinically relevant changes in leakage volume and SUI symptoms at six months post-treatment. Due to the magnitude of the sham response, further studies are needed to determine the effect of cryogen cooling alone on vaginal tissue.
\end{abstract}

\section{MP-11.3}

Efficacy and safety of polyacrylamide hydrogel (Bulkamid) injections for the treatment of leakage after continent urinary diversion

Geneviève Nadeau' ${ }^{1}$, Michelle Bergeron', Stéphane J. Bolduc

${ }^{1}$ Urology, Laval University, Quebec, QC, Canada

Introduction: Patients with a continent cutaneous urinary diversion (CCUD can develop leakage as a consequence of valve incompetence. Injection of bulking agents has been used as an alternative to open surgical revision. Case series on the use of various bulking agents (collagen, hyaluronic acid, polydimethylsiloxane) after CCUD have been published, but none has described the use of polyacrylamide hydrogel (Bulkamid) injections. Since PDMS is no longer available in Canada, we have been using Bulkamid as our bulking agent of choice for the last decade. Our objective was to assess the efficacy and safety of Bulkamid injections for the treatment of incontinence in patients with CCUD.

Methods: We retrospectively analyzed the medical records of patients who underwent polyacrylamide hydrogel injections for urinary incontinence after CCUD between 2010 and 2019 at our institution.
Results: Eight patients with a neurogenic bladder underwent polyacrylamide hydrogel injections of their CCUD. Five patients had a concomitant bladder augment while three had their native bladder. Five patients had a Monti ileal tube and three patients had an appendix Mitrofanoff. The mean volume injected was $2 \mathrm{ml}$ per treatment. At a median (range) followup of 30 (3-48) months, one patient with a Mitrofanoff was completely dry after two sets of injections, three patients achieved partial success ( $>50 \%$ reduction in leakage; three Monti, one Mitrofanoff), and four patients failed the treatment (two Monti, one Mitrofanoff). Of the four patients who did not respond to the injections, three underwent an open surgical revision of the channel, while the fourth patient is currently awaiting a second set of injections. The only complication was a urinary tract infection for one patient.

Conclusions: The use of Bulkamid injections for the treatment of urinary incontinence after CCUD surgery cured or improved only half of our cohort, but with a low complication rate. It should be considered a less invasive alternative to avoid or delay major reconstructive surgery.

\section{MP-11.4}

Obesity, weight gain, and new comorbidities in patients with urinary incontinence following prostate cancer surgery

Elaine Redmond ${ }^{7}$, R. Christopher Doiron ${ }^{7}$, Keith F. Rourke

${ }^{1}$ Department of Urology, University of Alberta, Edmonton, AB, Canada Introduction: Approximately $3-5 \%$ of patients develop urinary incontinence following prostate cancer surgery. Post-prostatectomy incontinence (PPI) can have debilitating social consequences that may deter the patient from engaging in physical activity and increase the risk of developing comorbidities. The aim of this study was to examine the effect of PPI on the development of weight gain and new comorbidities in patients with PPI undergoing anti-incontinence surgery.

Methods: Patients who underwent surgery for PPI at a single center from 2004-2018 were identified. A retrospective review was performed to document patient characteristics as noted at the time of prostate cancer treatment and compare these with parameters recorded at the time of anti-incontinence surgery. Demographics included weight, body mass index (BMI), individual medical comorbidities, and Charlson comorbidity index (CCI). Multivariate regression analysis was performed to identify factors which might influence differences in health outcomes following prostatectomy. Results: A total of 229 patients were included in the study with a mean age of 68.8 years at the time of incontinence surgery. Median duration of incontinence was $3.5 \pm 4.6$ years. There was no change in weight $(91.1 \mathrm{vs} .91 .8 \mathrm{~kg}$; $p=0.34)$, obesity ( $43.6 \%$ vs. $41.8 \% ; p=1.0)$, or BMI ( 29.6 vs. $30.0 ; p=0.18)$ between prostate cancer surgery and PPI surgery. There was a significant increase in $\mathrm{CCl}$ between prostate cancer surgery and PPI surgery $(2.7 \pm 1.5$ vs. $4.1 \pm 1.9 ; p<0.0001)$. Almost half of patients $(45.2 \%)$ developed a new comorbidity while awaiting incontinence surgery including an increase in the incidence of diabetes ( $21.9 \%$ vs. $12.7 \%$; $\mathrm{p}<0.0001)$, hypertension $(56.2 \%$ vs. $36.7 \% ; \mathrm{p}<0.0001)$, coronary artery disease $(14.6 \%$ vs. $8.9 \% ; \mathrm{p}=0.008)$, and arrhythmia ( $11.0 \%$ vs. $3.8 \%$; $=0.008)$. On multivariate analysis, the duration of incontinence (years) significantly predicted the likelihood of developing a new comorbidity (odds ratio [OR] 1.2; 95\% confidence interval [Cl] 1.1-1.4; $p<0.01)$ while age $(p=0.20)$ and severity of incontinence $(p=1.0)$ did not. Conclusions: Patients with PPI may be at higher risk of developing new comorbidities while awaiting anti-incontinence surgery, which may be related to the duration of incontinence. Strategies which expedite return of continence, such as early surgical intervention, may facilitate the resumption of physical activity and minimize the risk of future comorbidity. 


\section{MP-11.5 \\ Increased risk of dementia among overactive bladder patients treated with an anticholinergic medication compared to a beta-3 agonist \\ Blayne K. Welk ${ }^{1,2}$, Eric McArthur ${ }^{2}$}

${ }^{1}$ Surgery, Western University, London, ON, Canada; ${ }^{2} \mathrm{ICES}$, London, ON, Canada

Introduction: Research has suggested that anticholinergic medications are associated with an increased risk of dementia, however, this has not been well-studied in the overactive bladder population. Our objective was to determine if there is an increased risk of dementia among overactive bladder patients starting anticholinergic medication compared to those starting a beta-3 agonist.

Methods: This was a population-based, retrospective, matched cohort study using linked administrative data from Ontario, Canada. We matched 47324 new users of anticholinergics to 23662 new users of a beta-3 agonists; all the included medications are only indicated for the treatment of overactive bladder. We measured 75 baseline variables (including comorbid conditions, recent medications, and prior healthcare use) and used these to create a propensity score, which was used in the matching. The primary exposure was the class of overactive bladder medication (anticholinergic or beta-3 agonist). The primary outcome was the new diagnosis of dementia.

Results: The most common anticholinergics used were tolterodine $(40 \%)$, oxybutynin $(29 \%)$, and solifenancin $(26 \%)$. The median duration of use among anticholinergics was 30 (interquartile range [IQR] 30-170) days. The median duration of use of a beta-3 agonist (mirabegron) was 64 (IQR 30-317) days. There was an increased risk of dementia among anticholinergic users compared to beta-3 agonist users (hazard raio [HR] 1.23; 95\% confidence interval [Cl] 1.12-1.35). There was significant effect modification with gender and age; men and those $\leq 75$ years of age on anticholinergics had the highest risk of dementia relative to similar beta-3 agonist users. An exploratory analysis did not show significant differences in dementia risk between specific anticholinergics.

Conclusions: The use of anticholinergic medications among overactive bladder patients was associated with an increased risk of new onset dementia compared to beta-3 agonist users. This may have implications during the selection of initial medical therapy for patients with overactive bladder.

\section{MP-11.6}

Multimodal magnetic resonance imaging assessment of brain networks associated with visually evoked urgency urinary incontinence (VE-UUI)

Lennifer A. Locke' ${ }^{1}$, Andrew Macnab ${ }^{1}$, Mark K. Nigro', Saurabh Garg ${ }^{2}$ Sepideh Allahdadian², Martin McKeown'2, Lynn Stothers

${ }^{1}$ Urologic Sciences, University of British Columbia, Vancouver, BC, Canada; ${ }^{2}$ Neurology, University of British Columbia, Vancouver, BC, Canada

Support: Canadian Urological Association - Pfizer Resident Grant Program has graciously funded this project

Introduction: Patients with urgency urinary incontinence (UUI) often describe visual, somatic, and tactile triggers that initiate symptoms. The periaqueductal gray (PAG) region provides innervation to the bladder and urethra but the structure and dynamics of the upstream brain networks modulating the PAG, especially in people with visually evoked (VE)-UUI, are unknown.

Methods: VE-UUI subjects were identified within a subspecialty urology practice. Controls were community dwelling men and women without a history of lower urinary tract symptoms (LUTS). We developed a novel functional magnetic resonance imaging (fMRI) protocol to evaluate cortical pathways associated with VE-UUI. We first asked subjects to provide a picture of their "trigger" stimulus (typically a part of their bathroom). We then randomly sampled smaller regions of the trigger picture to provide unique "trigger stimuli." To create "neutral" stimuli, we also created images of different parts of their house. We then incorporated an individualized visual display stream consisting of alternate 30 -second blocks of five trigger stimuli and five neutral stimuli into a block-design fMRI experiment. All MRI scans were performed on a 3T Philips Elition scanner. Diffusion tensor imaging and myelin water fraction (MWF) were also acquired for tractography purposes. Connectivity between cortical and PAG were computed with a novel partial least squares (PLS) approach. ${ }^{1}$ Results: Ten subject were consented; cases-UUI and key in the door or visual trigger history; controls-IPSS=0 or genitourinary distress inventory $=0$. Debriefing of participants suggested that this fMRI paradigm effectively elicited the desired response during the "trigger" blocks. Tractography demonstrated robust structural connectivity between the anterior cingulate cortex and the PAG.

Conclusions: While preliminary, the aforementioned paradigm may provide a powerful method to evaluate cortical-PAG connectivity and dynamics in subjects with VE-UUI.

Reference

1. Cai J, Lee S, Ba Fet al. Galvanic vestibular stimulation (GVS) augments deficient pedunculopontine nucleus (PPN) connectivity in mild Parkinson's disease: fMRI effects of different stimuli. Front Neurosci 2018;12:101. https://doi.org/10.3389/fnins.2018.00101

\section{MP-11.7}

Monitoring cortical activity in outpatients to evaluate brain mediated voiding mechanisms

Lynn Stothers ${ }^{1}$, Andrew Macnab ${ }^{1}$, Jennifer A. Locke ${ }^{1}$, A Klausner $^{2}$, I Speich ${ }^{2}$ 1 Urologic Sciences, University of British Columbia, Vancouver, BC, Canada; ${ }^{2}$ Urology, Virginia Commonwealth University, Richmond, VA, United States.

Introduction: Functional magnetic resonance imaging (fMRI) studies have identified a network of brain regions integral to bladder sensation and voiding control, where dysfunction may contribute to phenotypes of urge incontinence (UI). The term "brain overactive bladder $(\mathrm{OAB})$ " has been coined for a subgroup of patients identified to require non-conventional treatment. Problematically, urologists currently lack a practical means to evaluate brain-mediated voiding mechanisms in an everyday clinical context. However, since the optical technique functional near-infrared spectroscopy (fNIRS) was shown to identify brain activity in the frontal micturition area originally identified using fMRI during natural voiding, we hypothesized that a newly available wireless multichannel brain fNIRS system could provide urologists with simultaneous anterior cortical data during voiding studies.

Methods: Subjects (controls IPSS or UGDI $=0$ and cases with $\mathrm{OAB}$ ) were monitored using a commercially available, 23-channel, portable fNIRS system with dual wavelength (750 and $860 \mathrm{~nm}$ ) emitters and photodiode detectors worn over the anterior cortex in a neoprene headcap. Changes in oxy, deoxy, and total hemoglobin concentration were measured at 50 $\mathrm{Hz}$ during natural bladder-filling and spontaneous emptying.

Results: Six subjects (ages 24-76, two female, four male) were studied. Increases in oxyhemoglobin concentration occurred in localized geographic areas of the cortex associated with specific phases of the filling voiding cycle. The prefrontal cortex showed strong localized oxyhemoglobin concentration signals when bladder-filling reached a sensation of urgency. These increases occurred in locations previously linked to voiding through fMRI by use of the Polhemus system, which linked NIRS

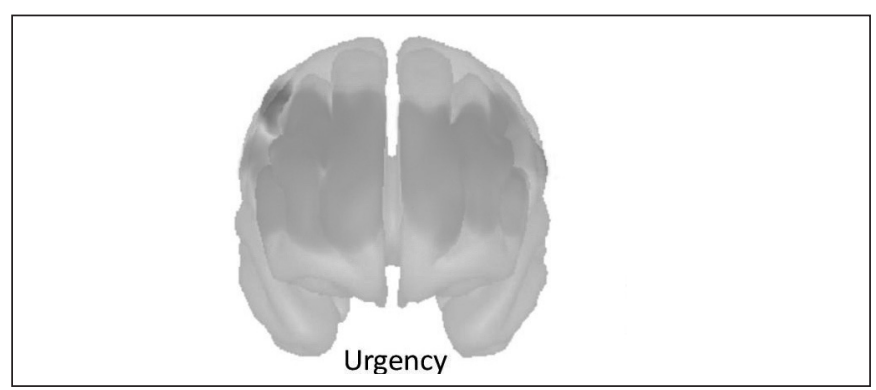

MP-11.7. Fig. 1. fNIRS activity in the brain during urgency. 
to cerebral anatomy using a magnetic pencil to bony skull landmarks (Fig. 1). Voiding reduced the micromolar colorographic regional change. Conclusions: fNIRS of the brain can identify the location, timing, and magnitude of cortical activity linked to voiding; the importance of brainmediated signaling is now recognized. The technique described offers those interested in investigating the central pathophysiological mechanisms of OAB and UI a way to study a broader population than if they had to rely $\mathrm{fMRI}$ alone. $\mathrm{fNIRS}$ is now a feasible addition to studies investigating voiding dysfunction.

\section{MP-11.8}

High risk of Clostridium difficile infection from the use of antibiotics commonly used to treat urinary tract infections in spinal cord injury patients

Bonnie Liu ${ }^{1}$, Jennifer Reid ${ }^{2}$, Michael Silverman ${ }^{3,4}$, Blayne K. Welk ${ }^{1,2,4}$

'Department of Surgery, Western University, London, ON, Canada; ${ }^{2} \mathrm{ICES}$, London, ON, Canada; ${ }^{3}$ Division of Infectious Diseases, Western University, London, ON, Canada; ${ }^{4}$ Department of Epidemiology and Biostatistics, Western University, London, ON, Canada

Support: Supported by a resident research grant from the Department of Surgery, Western University

Introduction: Perceived and culture-positive urinary tract infections (UTIs) are a common reason for spinal cord injury (SCI) patients to use antibiotics. ${ }^{1,2}$ Antibiotic use can lead to $C$. difficile infection (CDI), which has high morbidity and mortality. ${ }^{3}$ Our objective was to characterize the use of common UTI-relevant antibiotics after a $\mathrm{SCl}$ and determine the relative risk of $\mathrm{CDI}$ from exposure to these antibiotics.

Methods: We used routinely collected data from the province of Ontario (Canada) to conduct a retrospective, cohort study. We identified people $>18$ years of age who had a traumatic, non-fatal SCl between April 1, 2003 and March 31, 2017. The primary exposure was an outpatient UTIrelevant antibiotic prescription during our observation period, and the primary outcome was evidence of a CDI. An adjusted Cox proportional hazards model was used, and antibiotic exposure was modeled as a time-varying variable.

Results: We identified 2528 patients with $\mathrm{SCl}$ that met our inclusion criteria; $1642(65 \%)$ were exposed at least once to an antibiotic of interest during followup. The most commonly prescribed UTI-related antibiotic was a fluoroquinolone (34\%). Most patients did not receive investigations for a UTI prior to use of any of the different antibiotic classes. A small number of patients $(138,5 \%)$ were started on a chronic ( $>3$ months) course of a UTI-relevant antibiotic. The overall proportion of patients diagnosed with CDI was $7.4 \%$ (188), with a rate of $9.3 / 10000$ patient days. Exposure to UTI-relevant antibiotics was associated with an adjusted hazard ratio of 2.9 (98\% confidence interval 2.0-4.3; $p<0.01)$ for CDI. Conclusions: A significant proportion of patients with $\mathrm{SCl}$ are exposed to UTI-relevant antibiotics, and most do not have UTI-related investigations carried out. The rate of $\mathrm{CDI}$ in this population is similar to that of hospitalized patients. Given the increased risk for CDI in SCI patients exposed to UTI-relevant antibiotics, efforts to reduce unnecessary UTIrelated antibiotic use should continue.

\section{References}

1. Salomon J, Denys P, Merle C, et al. Prevention of urinary tract infection in spinal cord-injured patients: Safety and efficacy of a weekly oral cyclic antibiotic (WOCA) program with a 2-year followup - an observational prospective study. J Antimicrob Chemother 2006;57:784-8. https://doi.org/10.1093/jac/dkl010

1. Evans CT, Fitzpatrick MA, Jones MM, et al. Prevalence and factors associated with multidrug-resistant gram-negative organisms in patients with spinal cord injury. Infect Control Hosp Epidemiol 2017;38:1464-71. https://doi.org/10.1017/ice.2017.238

2. Burke KE, Lamont JT. Clostridium difficile infection: A worldwide disease. Gut Liver 2014;8:1-6. https://doi.org/10.5009/gnl.2014.8.1.1

\section{MP-11.9 \\ Temporal trends and patterns in kidney transplant surgeries in Ontario}

Luke Witherspoon', Ali Dergham², Thomas A. Skinner ${ }^{3,4}$, Brian D. Blew', Jeffrey Warren ${ }^{1}$

${ }^{1}$ Division of Urology, Department of Surgery, The Ottawa Hospital and University of Ottawa, Ottawa, ON, Canada; ${ }^{2}$ School of Medicine, Faculty of Health Sciences, Queen's University, Kingston, ON, Canada; ${ }^{3}$ Department of Urology, Dalhousie University, Halifax, NS, Canada; ${ }^{4}$ Multi-Organ Transplant Program, Nova Scotia Health Authority, Halifax, NS, Canada Introduction: Studies suggest that procedures performed under fatigued conditions or on weekends may be associated with worse outcomes. Approximately 700 kidney transplants occur in Ontario per year, yet studies of their timing/trends are absent. We set out to determine these temporal trends and patterns.

Methods: Data on all deceased donor and recipient kidney transplant surgeries that occurred between April 1, 2013 and December 31, 2017 were obtained from Trillium Gift of Life Network, Ontario's organ and tissue donation agency. The rates and 95\% confidence intervals of donor and recipient transplants per unit times were calculated. To facilitate comparison, all rates were normalized to per 30 days.

Results: A total of 1116 deceased donor nephrectomies and 1858 recipient kidney transplantations were performed. Significantly more recipient surgeries were performed on Saturday than any other day. When comparing weekend and weekdays, the weekend rate is significantly greater for both donor and recipient surgeries. The rate of recipient surgeries overnight (20:00-07:00) on a weekend is greater than any other time. The 17:00-20:00 period had the highest rate of donor and recipient surgeries for donations after cardiac death. The overnight period had the greatest rate of donor surgeries for NDD donors. Similarly, the weekend overnight period had the greatest rate of recipient surgeries from NDD donors. The average cold and warm ischemia times (WIT) were 10.4 hours and 40.7 minutes, respectively. WITs were significantly longer overnight than during weekday daytime.

Conclusions: We set out to determine the temporal trends of kidney transplants across Ontario. As hypothesized, most kidney transplant surgeries are occurring at inopportune times where resources are limited and medical staff may be fatigued. This study raises questions about how to best optimize renal transplant timing, and whether the majority should be occurring at times when resources are at their minimum, and staff burnout may occur.

\section{MP-11.10}

Predictors of perioperative complications in pediatric renal transplantation: A long-term retrospective study

Cyrus Chehroudi ${ }^{1}$, Alexander Danechi ${ }^{1}$, Andrew E. MacNeily ${ }^{1}$, Kourosh Afshar ${ }^{1}$

'Department of Urologic Sciences, University of British Columbia, Vancouver, BC, Canada

Introduction: Renal transplantation is the treatment of choice for pediatric end-stage renal disease (ESRD). However, the fragility of children, size discrepancy between adult kidneys and pediatric recipients, and greater prevalence of urinary tract anomalies make pediatric renal transplantation more prone to surgical complications. Small sample sizes also make it difficult to study pediatric renal transplantation and factors associated with perioperative morbidity. Here, we report our perioperative outcomes for pediatric renal transplant over 12 years.

Methods: We conducted a retrospective chart review of renal transplant recipients from 2007-2019 at BC Children's Hospital. Perioperative complications were assessed within eight weeks of transplantation. Surgical complications were defined as vascular, urinary, or wound complications requiring intervention. Fisher's exact test and Student's t-test were used to assess statistical significance. A $p<0.05$ was taken as significant.

Results: Eighty-nine renal transplants were performed over 12 years with median age 13 and $64 \%$ in male patients. The most common causes of ESRD were posterior urethral valves $(n=13)$, focal segmental glomerulosclerosis $(n=11)$, and glomerulonephritis/vasculitis $(n=8)$. Ten patients had previous transplants. Mean vascular anastomosis time and surgical 
time were 28.3 minutes (standard deviation [SD] 9.0) and 210 minutes (SD 59.8), respectively. Only one patient required blood transfusion and median estimated blood loss was $100 \mathrm{~mL}$. Delayed graft function occurred in six cases and seven patients developed surgical complications. One patient had renal vein thrombosis managed by thrombectomy. Urine leaks were identified in two patients and managed by nephrostomy and ureteric re-implantation, respectively. Overall, six allograft nephrectomies were required for renal vein thrombosis $(n=2)$, failed embolization of arteriovenous malformation $(n=1)$, renal artery aneurysm $(n=1)$, renal pedicle torsion ( $n=1)$, and recurrent FSGS $(n=1)$. Patients who had a previous transplant, as well as those with ESRD due to obstruction or reflux were significantly more likely to experience a surgical complication ( $30 \%$ vs. $6 \%, p=0.043 ; 31 \%$ vs. $5 \%, p=0.016$, respectively). However, there was no difference between cadaveric vs. living transplants (10\% vs. $7 \%)$ or transplants performed overnight vs. daytime (14\% vs. $5.6 \%$, p $=0.39)$. Mean anastomotic time (32.3 vs. 27.9 minutes) and age (14.0 vs. 11.8 years) were similar for patients who had surgical complications compared to those who did not

Conclusions: Perioperative outcomes post-pediatric renal transplantation in our cohort are comparable to previously reported studies. Surgical complications are infrequent but cause significant morbidity. We identify previous renal transplantation and cause of ESRD as predictors for surgical complications.

\section{MP-11.11}

Patient-reported outcomes from renal transplant recipients: Initial results from a single-center study

Keesha Khehra' ${ }^{1}$, Caelie Stewart', Angela Cho ${ }^{2}$, Rohit Singla ${ }^{3,4}$, Christopher Y. Nguan ${ }^{2}$

${ }^{1} \mathrm{MD}$ Undergraduate Program, Faculty of Medicine, University of British Columbia, Vancouver, BC, Canada; ${ }^{2}$ Department of Urologic Sciences, University of British Columbia, Vancouver, BC, Canada; ${ }^{3}$ School of Biomedical Engineering, University of British Columbia, Vancouver, BC, Canada; ${ }^{4} \mathrm{MD} / \mathrm{PhD}$ Program, Faculty of Medicine, University of British Columbia, Vancouver, BC, Canada

Introduction: Patient-reported outcomes (PROs) are gaining popularity in various medical disciplines as a mechanism to improve accountability and overall quality of healthcare services. PROs can be effective tools for characterizing symptom burden and health-related quality of life. Many medical guidelines for surgical procedures do not discuss specifics of recovery or establish timelines of symptom presentation among patients. This work aims to establish a more accurate estimation of expected symptoms and report timelines for symptom recovery in renal transplant recipients.

Methods: Patients receiving a kidney transplant at Vancouver General Hospital were recruited $(n=51)$ to complete a postoperative recovery survey at a gradually reduced frequency over a six-month period. Analysis was performed to determine the number of patients that report experiencing each symptom and when the symptoms are reported to have resolved. Results: Postoperatively, common causes of concern among patients include graft rejection, sleep, pain, fluid overload, and bowel movements. The initial results give insight into the symptoms experienced by recipients including: $90 \%(n=38)$ continue to report incision site pain for up to six weeks; $57 \%(n=24)$ will have urgency while urinating, lasting up to eight weeks; and $86 \%(n=36)$ of recipients return to work within six months. Forty-three percent $(n=18)$ of patients report irritation when urinating, with this resolving within one week for $44 \%(n=18)$ of patients, within two weeks for $61 \%(n=26)$, and within one month for $78 \%(n=33)$. The average time to return to regular bowel movements is $4 \pm 2$ days and the average time of return to regular activity level is $27 \pm 13$ days.

Conclusions: Using PROs, new information can be obtained on expected symptoms and recovery, which can improve management in a patientcentric manner, as well as inform supporting research. Longer-term analysis is needed to evaluate the usability and applicability of the PRO tool in clinical practice.

\section{MP-11.12}

The role of bariatric surgery on kidney transplantation: A systematic review and meta-analysis

Yung Lee', Lucshman Raveendran², Olivia Lovrics' ${ }^{1}$, Aristithes Doumouras' ${ }^{3}$, Martin A. Koyle ${ }^{4}$, Monica Farcas ${ }^{5}$, Yonah Krawkowsky ${ }^{6}$, Dennis Hong ${ }^{3}$

${ }^{1}$ Michael G. Degroote School of Medicine, McMaster University, Hamilton, ON, Canada; ${ }^{2}$ Faculty of Medicine, University of Toronto, Toronto, ON, Canada; ${ }^{3}$ Department of Surgery, McMaster University, Hamilton, ON, Canada; ${ }^{4}$ Division of Urology, The Hospital for Sick Children, Toronto, ON, Canada; ${ }^{5}$ Division of Urology, St. Michael's Hospital, Toronto, ON, Canada; ${ }^{6}$ Department of Urology, University of Toronto, Toronto, ON, Canada

Introduction: In patients with end-stage renal disease, kidney transplantation improves survival. However, due to safety and increased rates of graft failure, severe obesity remains a relative contraindication for kidney transplantation. Kidney transplant recipients are also at a high risk for developing obesity. The aim of this systematic review and meta-analysis is to investigate the role of bariatric surgery before and after kidney transplantation.

Methods: Databases, including MEDLINE, EMBASE, CENTRAL, PubMed, and Web of Science, were searched up to March 2019, and we included studies that performed bariatric surgery on patients with severe obesity before and after kidney transplantation. Outcomes for both groups included change in body mass index (BMI) and rates of surgical mortality and complications. For studies that performed bariatric surgery before transplant, additional outcomes included the rate of patients listed for and who subsequently received renal transplantation following bariatric surgery. Pooled estimates were calculated using the random effects metaanalysis of proportions

Results: Seventeen studies were eligible for the final review. Eight studies investigated the role of bariatric surgery before kidney transplant $(n=142)$. After bariatric surgery, the 30 -day mortality rate was $0 \%$ and $84 \%$ of patients (95\% confidence interval [CI] 64-98\%) were successfully listed for transplant, $70.0 \%$ (95\% Cl 37-95\%) of whom underwent transplant. At total of $4.2 \%$ of patients experienced minor 30 -day complications, and major 30 -day complications occurred in $3.5 \%$ of patients. Nine studies investigated the role of bariatric surgery after kidney transplant $(n=274)$. After bariatric surgery, the 30 -day all-cause mortality rate was $0 \%$, with one minor $(1.54 \%)$ and one major (1.54\%) 30-day complication having occurred.

Conclusions: Bariatric surgery appears safe and effective in reducing weight to improve kidney transplant candidacy and outcomes in patients with severe obesity, as well as in patients following kidney transplantation.

\section{MP-11.13}

Sexual Health Inventory for Men (SHIM) questionnaire as a screening method for erectile dysfunction in the general urology clinic

Amjad Alwaal', Mohannad Awad', Nathan Boggs', Jake Kuzbel', Brian Snoad $^{1}$

${ }^{1}$ Urology, Marshall University, Huntington, WV, United States; ${ }^{2}$ Surgery, University of Vermont, Burlington, VT, United States

Introduction: Sexual Health Inventory for Men (SHIM) is a validated questionnaire that is widely used in urology clinics to evaluate and assess treatment efficacy for erectile dysfunction (ED). In this study, we evaluated the benefit of using SHIM questionnaire as a screening tool for ED in the general urology clinic.

Methods: All new male urology patients who are 40 years of age or older visiting the general urology clinic for non-sexual issues received SHIM questionnaire. Patients who wanted treatment for ED received a full ED evaluation and treatment.

Results: A total of 379 patients received SHIM questionnaire. Forty-eight patients $(12.7 \%)$ refused to fill the questionnaire. We excluded all patients presenting for sexual health issues (67 patients, $17.7 \%$ ). We included the remaining 264 patients (69.6\%). Mean age was 61.7 years (range $40-85$ ). Table 1 demonstrates patient characteristics. Chief complaint was not predictive of the patient's SHIM score or desire to have ED treatment. 


\begin{tabular}{|c|c|c|c|}
\hline Patient characteristic & $\begin{array}{c}\text { Number } \\
(\%)\end{array}$ & $\begin{array}{c}\text { Number } \\
\text { wanting } \\
\text { treatment (\%) }\end{array}$ & $\begin{array}{l}\text { Median SHIM } \\
\text { score (IQR) }\end{array}$ \\
\hline \multicolumn{4}{|l|}{ Age (years) } \\
\hline $40-50$ & 47 (17.8) & $11(23.4)$ & $22(16-24)$ \\
\hline $51-60$ & $75(28.4)$ & $32(42.7)$ & $17(11-24)$ \\
\hline $61-70$ & $84(31.8)$ & $38(45.2)$ & $11.5(6-20)$ \\
\hline$>70$ & $58(22.0)$ & $17(29.3)$ & $7.5(5-15)$ \\
\hline \multicolumn{4}{|l|}{ Comorbidities } \\
\hline DM & $74(28.0)$ & $38(51.4)$ & $10(6-16)$ \\
\hline Hypertension & $154(58.3)$ & $68(44.2)$ & $13(7-20)$ \\
\hline COPD & $30(11.4)$ & $15(50.0)$ & $13.5(6-17)$ \\
\hline CAD & $37(14.0)$ & $17(46.0)$ & $9(6-16)$ \\
\hline CKD & $11(4.2)$ & $4(36.4)$ & $10(7-13)$ \\
\hline \multicolumn{4}{|l|}{ Reason for visit } \\
\hline $\mathrm{BPH}$ & $135(51.1)$ & $60(44.4)$ & $13(6-19)$ \\
\hline Hematuria & $26(9.9)$ & 7 (26.9) & $19.5(8-25)$ \\
\hline Renal lesion & $2(0.8)$ & $1(50.0)$ & $9(4-14)$ \\
\hline Incontinence & $19(7.2)$ & $6(31.6)$ & $7(5-21)$ \\
\hline Nephrolithiasis & $16(6.1)$ & $5(31.3)$ & $20(15-23.5)$ \\
\hline $\begin{array}{l}\text { Orchalgia/pelvic } \\
\text { pain }\end{array}$ & $44(16.7)$ & $14(31.8)$ & $16(10-23)$ \\
\hline Vasectomy & $3(1.0)$ & $1(33.3)$ & $19(12-24)$ \\
\hline UTI & $1(0.4)$ & 0 & - \\
\hline Infertility & $1(0.4)$ & 0 & - \\
\hline Penile lesion & $4(1.5)$ & $1(25.0)$ & $10(7-18)$ \\
\hline Elevated PSA & $13(4.9)$ & $3(23.1)$ & $21(18-24)$ \\
\hline
\end{tabular}

Older patients were more likely to want ED treatment and had lower SHIM scores. However, above the age of 70 years there is a decline in the number of patients wanting treatment (Table 1). On univariate analysis, both diabetes mellitus (DM) and hypertension were significantly associated with the patient's desire to have ED treatment. On multivariate analysis, DM was associated with wanting ED treatment, but hypertension was not (Table 2). Eighty-one patients $(82.7 \%)$ were offered oral phosphodiesterase type 5 inhibitors, nine patients $(9.2 \%)$ were offered intracavernosal injection, one patient $(1.0 \%)$ was offered vacuum erection device, and seven patients $(7.1 \%)$ were offered penile prosthesis insertion.

Conclusions: SHIM questionnaire is a useful tool in the general urology clinic, as the urologist is the most capable person of treating ED. It can serve as an efficient tool to screen for and quantify ED in patients presenting for other urological issues. The maximum benefit is seen in patients between the age of 51-70 years and in diabetic patients.

\begin{tabular}{|c|c|c|c|c|}
\hline & $\begin{array}{l}\text { Unadjusted } \\
\text { OR (95\% Cl) }\end{array}$ & $\mathbf{p}$ & $\begin{array}{l}\text { Adjusted OR } \\
(95 \% \mathrm{CI})^{*}\end{array}$ & $\mathbf{p}$ \\
\hline \multicolumn{5}{|l|}{ Age } \\
\hline $40-50$ years & $0.7(0.3-1.8)$ & 0.497 & $1.1(0.4-2.8)$ & 0.853 \\
\hline $51-60$ years & $1.8(0.9-3.7)$ & 0.115 & $2(0.9-4.4)$ & 0.075 \\
\hline $61-70$ years & $2(1.0-4.1)$ & 0.057 & $2.3(1.1-4.8)$ & 0.032 \\
\hline$>70$ years & Reference & & Reference & \\
\hline DM & $2.3(1.3-4.0)$ & 0.003 & $1.9(1.0-3.4)$ & 0.040 \\
\hline Hypertension & $2.1(1.2-3.6)$ & 0.005 & $1.6(0.9-2.9)$ & 0.105 \\
\hline COPD & $1.8(0.8-3.9)$ & 0.125 & $1.8(0.8-4.1)$ & 0.152 \\
\hline CAD & $1.5(0.8-3.1)$ & 0.233 & $1.4(0.7-3.1)$ & 0.382 \\
\hline CKD & $0.9(0.3-3.4)$ & 0.950 & $0.9(0.2-3.3)$ & 0.865 \\
\hline
\end{tabular}

\section{MP-11.14}

Oral testosterone practice patterns and management: A population-based analysis of a large healthcare database Zack Li ${ }^{1}$, Jason Scovell ${ }^{2}$, Sarah McGriff', Christopher J.D. Wallis ${ }^{3}$, Yonah Krakowsky ${ }^{4}$, Premal Patel', Ranjith Ramasamy ${ }^{5}$

${ }^{1}$ Section of Urology, Department of Surgery, University of Manitoba, Winnipeg, MB, Canada; ${ }^{2}$ Scott Department of Urology, Baylor College of Medicine, Houston, TX, United States; ${ }^{3}$ Department of Urologic Surgery, Vanderbilt University, Nashville, TN, United States; ${ }^{4}$ Department of Urology, University of Toronto, Toronto, ON, Canada; ${ }^{5}$ Department of Urology, University of Miami, Miami, FL, United States

Introduction: Oral testosterone has been available in Canada since 1992. This contrasts with the U.S., where it was not approved due to its adverse effects on the liver. ${ }^{1,2}$ An oral testosterone with a different mechanism of action was approved in the U.S. in 2019. We hypothesized that prescribing and management patterns for oral testosterone differ from other routes. Methods: We performed a population-based study of men aged 66 or older that were newly treated with testosterone replacement therapy (oral, injection, gel, and patches) from 2008-2015 in Ontario using the Ontario Drug Benefit database and the Canadian Institute for Health Information, as oral testosterone was approved for use during this time period. Age, therapy type, practitioner type, and laboratory values, including liver function tests aspartate transaminase (AST) and alanine aminotransferase (ALT) were extracted. Comparisons between these values across year were evaluated. Proportional data was evaluated using a chi-squared test on PRISM8 (GraphPad Software Inc.).

Results: A total of 4187 men over the age of 65 received oral testosterone from 2008-2015, representing $27 \%$ of total testosterone prescriptions during this time period. Practitioners were less likely to check liver function tests for oral testosterone when compared to other modalities $(75 \%$ vs. $78 \% ; p<0.001)$. Oral testosterone prescriptions did not increase from 2008-2015 despite increased prescriptions for other modalities. Oral prescription used differed by practitioner type — oral ( $79 \%$ general/family practitioner [GP/FP]; $18 \%$ urology; $2 \%$ endocrine) vs other routes $(75 \%$ GP/FP; $17 \%$ urology; $8 \%$ endocrine; $\mathrm{p}<0.01$ ). There was no difference in prescription pattern by patient age or rural/urban setting $(p>0.05)$.

Conclusions: Oral testosterone utilization rates have remained low despite increasing testosterone prescription rates using other modalities. Monitoring of liver function tests is not routinely performed in men receiving oral testosterone despite its risk of liver dysfunction. 


\section{References}

1. Westaby D, Ogle SJ, Paradinas FJ, et al. Liver damage from long-term methyltestosterone. Lancet 1977;2:262-3. https://doi.org/10.1016/ S0140-6736(77)90949-7

2. Charles Osterberg E, Bernie AM, Ramasamy R. Risks of testosterone replacement therapy in men. Indian J Urol 2014;30:2-7. https://doi. org/10.4103/0970-1591.124197

\section{MP-11.15}

A significant proportion of men with a history of prostate cancer receiving testosterone therapy do not receive appropriate prostate-specific antigen monitoring

Benjamin Shiff', Jason Scovell'2, Sarah McGriff', Premal Patel', Christopher J.D. Wallis ${ }^{3}$, Yonah Krakowsky ${ }^{4}$, Ranjith Ramasamy

${ }^{1}$ Urology, University of Manitoba, Winnipeg, MB, Canada; ${ }^{2}$ Baylor College of Medicine, Houston, TX, United States; ${ }^{3}$ Urology, Vanderbilt University Medical Centre, Nashville, TN, United States; ${ }^{4}$ Urology, University of Toronto, Toronto, ON, Canada; ${ }^{5}$ Urology, University of Miami, Miami, FL, United States

Introduction: Health practitioners are hesitant to prescribe testosterone replacement therapy (TRT) for men with history of prostate cancer. Emerging data suggests that TRT is safe in men with treated prostate cancer. We characterized the prescription and laboratory monitoring patterns of patients with diagnosed prostate cancer receiving TRT in a North American healthcare system.
Methods: We performed a population-based study of men aged 66 years or older \pm a history of prostate cancer that were newly treated with TRT (oral, injection, gel, and patches) between the years 2008 and 2015 in Ontario, Canada using the Ontario Drug Benefit database and the Canadian Institute for Health Information. Patient age, type of therapy, practitioner type, and laboratory value data were extracted. Proportional data was evaluated using a chi-squared test.

Results: A total of 574 men (4\% of the cohort) with a history of prostate cancer and 14929 men without prostate cancer all over the age of 65 received TRT between 2008 and 2015. A greater proportion of men with a history of prostate cancer had their prostate-specific antigen (PSA) checked $(71 \%, \mathrm{n}=410)$ within one year of testosterone prescription than the general population $(67 \%, n=9540 ; p<0.001)$; however, this rate remained inadequately low. Men with a history of prostate cancer were more likely to be treated with TRT by a urologist compared to the general population: prostate cancer (56\% family practitioner [FP]; $37 \%$ urology; 7\% endocrinology) vs. all other patients (77\% FP; $17 \%$ urology; $6 \%$ endocrinology; $\mathrm{p}<0.01)$. Urologists were more likely to check PSA levels $(79 \%)$ in men with a history of prostate cancer on TRT than FPs and endocrinologists $(68 \%$ and $69 \% ; \mathrm{p}=0.03)$.

Conclusions: Only $70 \%$ of men who receive TRT with a history of prostate cancer have their PSA checked. Although PSA screening has declined among general practitioners, appropriate education for testing PSA among men diagnosed with prostate cancer is critical. 


\section{CUA ABSTRACTS}

\section{Moderated Poster Session 12: Pediatric Urology}

\section{MP-12.1}

Back (door) to the future: Dorsal lumbotomy for pediatric upper pole heminephrectomy

Aishwarya Roshan ${ }^{1}$, Andrew E. MacNeily $1,2,3$

${ }^{1}$ Faculty of Medicine, University of British Columbia, Vancouver, BC, Canada; '2Department of Urologic Sciences, University of British Columbia, Vancouver, BC, Canada; 'Division of Pediatric Urology, BC Children's Hospital, Vancouver, BC, Canada

Introduction: The dorsal lumbotomy approach to renal surgery has become a lost art. Upper pole heminephrectomy (UHN) is performed for two main indications: ectopic ureterocele and duplication anomalies with upper pole ectopy. Current popular techniques for conducting UHN include open flank, laparoscopic, and robotic. This study evaluates outcomes following dorsal lumbotomy (DL), an approach used historically for pyeloplasty and pyelolithotomy, and in which no clinical trials or case-series have been conducted for UHN in children. ${ }^{1,2}$

Methods: We conducted a retrospective review of $50 \mathrm{UHN}$ performed in 49 patients using the DL approach by a single surgeon from 2000-2019. Clinical variables and indicators included age, sex, weight, skin-to-skin time, tota operating room time, duration of hospital stay, postoperative complications, analgesic requirements, and postoperative ultrasound results.

Results: Twenty-three cases had a presurgical diagnosis of ectopic ureter, and 27 ureterocele. Mean weight (range) of patients was $12.61 \mathrm{~kg}$ (6.90-31.00), and mean age at surgery was 24.55 months. Mean (range) for time between skin incision and closure was 88.51 (62-132) minutes and mean (range) total operating room time was 138.46 (70-180) minutes. There were neither intraoperative complications nor transfusions. The mean (range) postoperative opioid delivered was $0.73(0.00-2.00) \mathrm{mg} / \mathrm{kg} /$ day. Mean (range) postoperative ibuprofen delivered was 5.41 (0.00-37.73) $\mathrm{mg} / \mathrm{kg} /$ day. Median length of hospital stay was two days. No patient received postoperative prescriptions for narcotics at discharge. No patient experienced wound complications. One patient had secondary atrophy of the lower pole. Secondary lower tract surgery, unrelated to surgical approach, was performed in six patients.

Conclusions: DL is a historical approach for UHN that should not be forgotten. It is safe, feasible, and produces operative outcomes and times comparable or superior to that of conventional open flank incision, laparoscopic, and robotic techniques.

\section{References}

1. Bajpai M, Kumar A, Tripathi M, et al. Dorsal lumbotomy incision in pediatric pyeloplasty. ANZ J Surg 2004;74:491-4. https://doi. org/10.1111/j.1445-1433.2004.03032.x

2. Verma A, Bajpai M, Baidya DK. Lumbotomy approach for upper urinary tract surgeries in adolescents: Feasibility and challenges. J Pediatr Urol 2014;10:1122-5. https://doi.org/10.1016/j.jpurol.2014.05.001
MP-12.2

Native nephrectomy prior to kidney transplant: A 16-year institutional experience

Lin Kyu Kim ${ }^{1,2}$, Lucshman Raveendran², Michael E Chua², Armando J. Lorenzo ${ }^{1,2}$, Walid A. Farhat', Jessica M Ming ${ }^{3}$, Martin A. Koyle ${ }^{1,2}$

'Division of Urology, Department of Surgery, University of Toronto, Toronto, ON, Canada; ${ }^{2}$ Division of Urology, Department of Surgery, Hospital for Sick Children, Toronto, ON, Canada; ${ }^{3}$ Surgery, University of New Mexico, Albuquerque, NM, United States; ${ }^{4}$ Urology, University of Wisconsin, Madison, WI, United States

Introduction: The associated risks of pre-transplant native nephrectomies in pediatric renal transplant patients remain unclear. This investigation aims to assess the clinical outcomes for pediatric renal transplant patients who underwent pre-transplant native nephrectomy.

Methods: A retrospective review of renal transplants performed at our institution from 2000-2015 was performed. Transplant recipients were divided into those who underwent native nephrectomy and those who did not. Clinical outcomes (estimated glomerular filtration rate [eGFR], ClavienDindo classification $\geq 3$ complications, graft loss, and number of readmissions) were compared. Subgroup analyses were performed for unilateral/ concurrent bilateral/staged bilateral nephrectomies.

Results: A total of 324 patients were identified. Fifty-seven patients underwent native nephrectomy (18 unilateral, 27 concurrent bilateral, 12 staged bilateral). The nephrectomy group was more likely to be younger, receiving living donor kidneys, and to have $\geq 2$ donor kidney arteries (Table 1), while also having more patients with nephrotic syndrome, nephritis, and focal sclerosing glomerulosclerosis $(p=0.002)$ (Fig. 1). Most nephrectomy indications were hypertension $(42.7 \%$ ) and proteinuria (28.1\%) (Fig. 2). In multivariate analyses (controlling for significant baseline characteristics and nephrectomy specific factors - laparoscopic, nephrectomy at time of transplant, nephrectomy prior to transplant, previous transplant nephrectomy), native nephrectomy ( $ß 1.138 ; 95 \%$ confidence interval $[\mathrm{Cl}]$ $0.137-2.138 ; \mathrm{p}=0.026)$ and bilateral nephrectomy ( $\mathrm{B} 2.733 ; 95 \% \mathrm{Cl} 1.313$ 4.152; $\mathrm{p}<0.001)$ were associated with higher readmission rates. Patients with nephrectomies were more likely to be readmitted with bacterial infections $(29.8 \%$ vs. $15.4 \%$; $\mathrm{p}=0.013$ ) (Fig. 3 ).

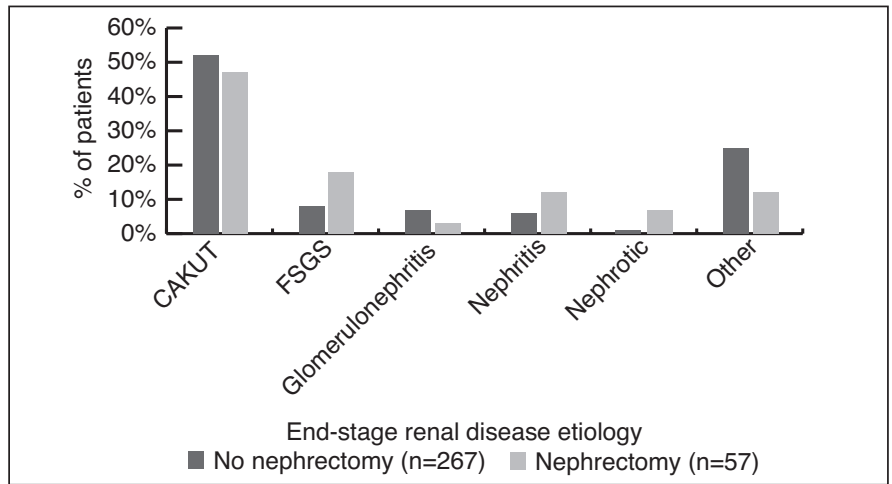

MP-12.2. Fig. 1. Comparison of end-stage renal disease etiology between two groups. 
MP-12.2. Table 1. Comparison of baseline characteristics

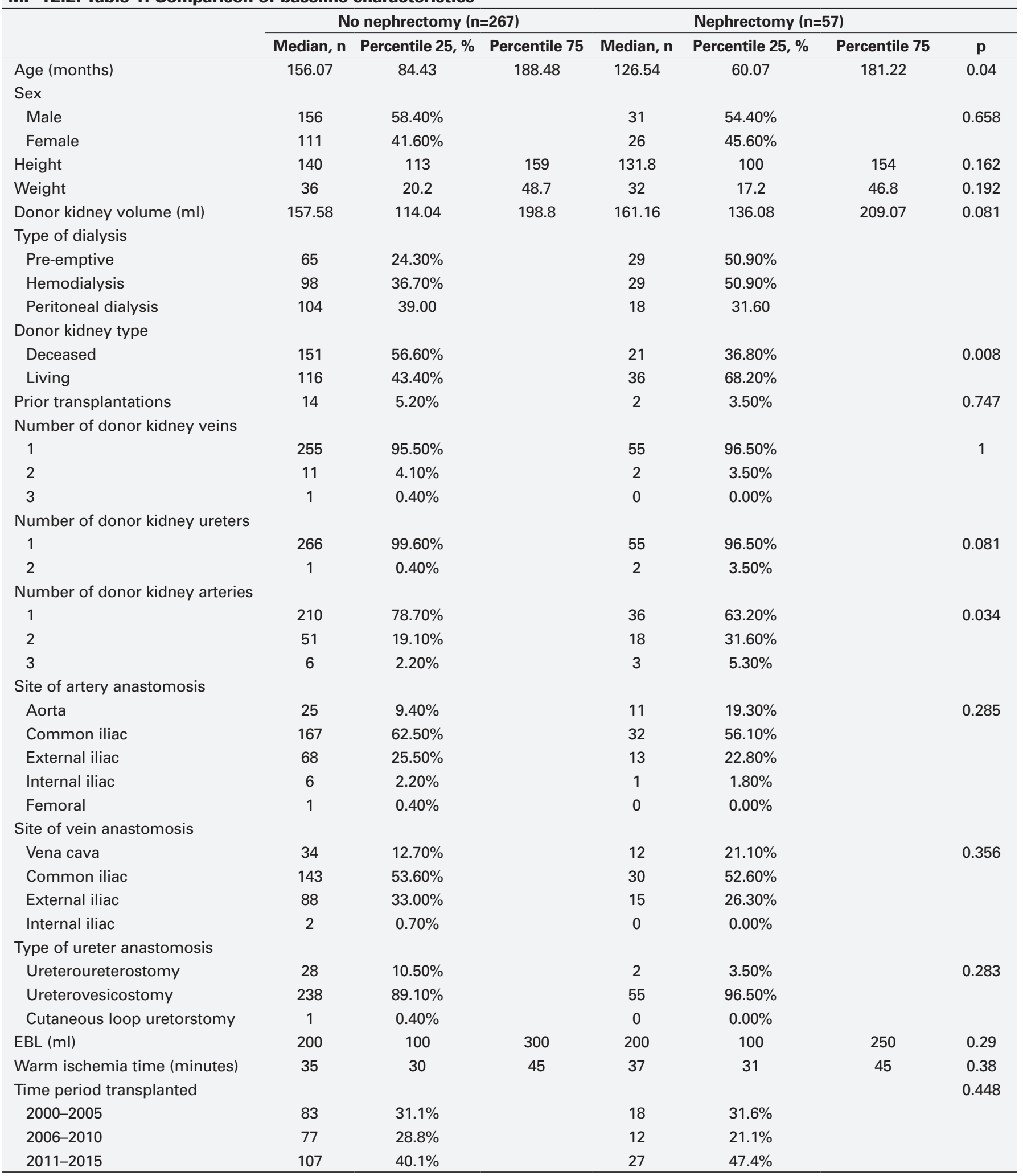




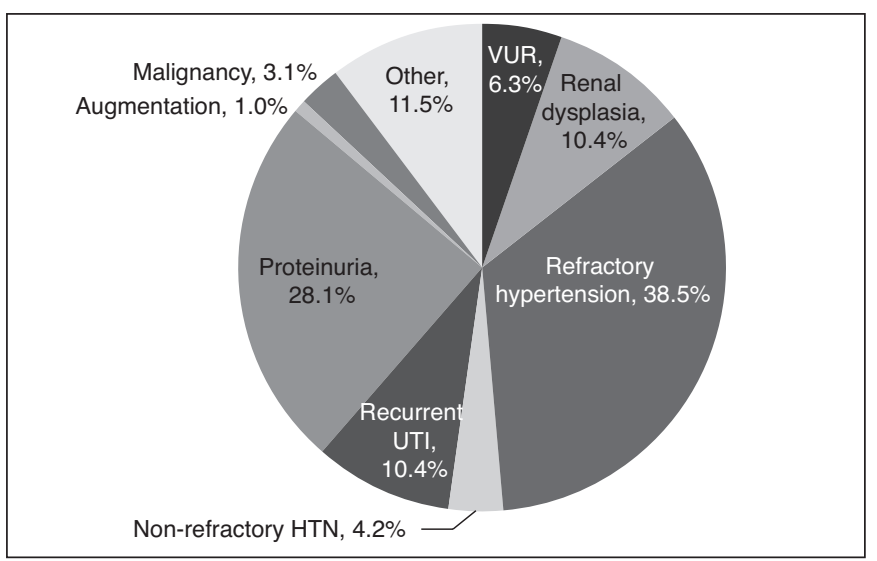

MP-12.2. Fig. 2. Indications for nephrectomy ( $n=57,96$ kidneys)

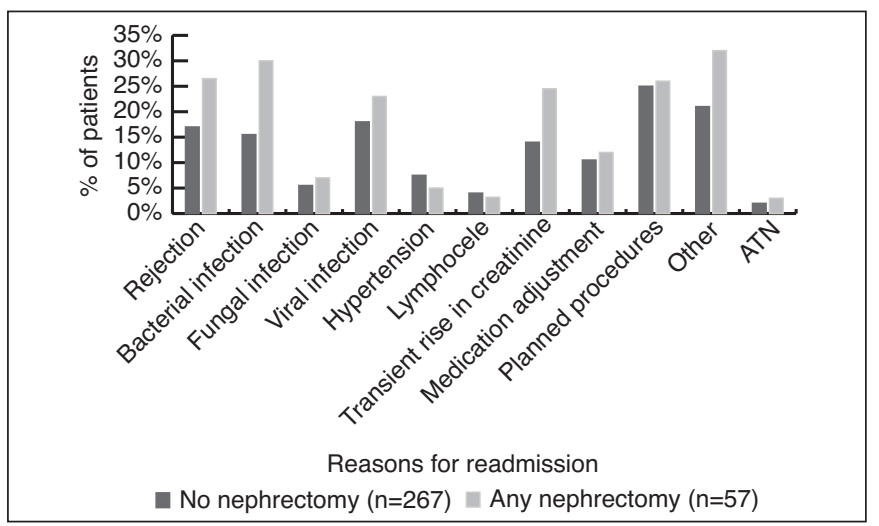

MP-12.2. Fig. 3. Reasons for readmission following kidney transplant.

Conclusions: Pre-transplant native nephrectomy may lead to worse clinical outcomes. Native nephrectomy should be reserved for select patients who will significantly benefit from them, such as those with refractory proteinuria or hypertension.

\section{MP-12.4}

How can we define ureteral dilatation according to the urinary tract dilation classification system?

Amr Hodhod $^{1}$, Iman Sadri', Francis Petrella ${ }^{1}$, J.-P. Capolicchio ${ }^{7}$, Roman Jednak', Mohamed El-Sherbiny'

${ }^{1}$ Urology, McGill University Health Centre, Montreal, QC, Canada Introduction: Ureteral dilatation in the urinary tract dilation (UTD) system is not well-defined. When a renal unit has ureteral dilatation, it is considered P2 without looking at other UTD parameters. In the literature, ureteral diameter (UD) $\leq 4 \mathrm{~mm}$ is considered normal while UD $>7 \mathrm{~mm}$ is considered megaureter. In this study, we tried to quantify the significant ureteral dilation that should be used in UTD.

Methods: We conducted a retrospective chart review of infants with postnatal hydronephrosis from 2008-2014. Reviewed data included patients' characteristics, febrile urinary tract infections (FUTIs), and surgical interventions. UD was measured retrovesically in the transverse bladder plane by a single investigator. We compared the incidence of FUTI and surgical intervention when UD was $\leq 4 \mathrm{~mm}$ and $>4 \mathrm{~mm}$, then when UD is $4.1-7$ $\mathrm{mm}$ and $>7 \mathrm{~mm}$. We studied the association between UD categories and the incidence of FUTI and surgical interventions.

Results: We included 480 patients with 604 units (Table 1). The median followup was 36.4 months; 289 units $(47.8 \%)$ were classified as P1, $27.5 \%$ (166 units) were P2, and $24.7 \%$ (149 units) were P3. Overall, units with UD $>4 \mathrm{~mm}$ had a higher incidence of FUTI and surgeries than

\begin{tabular}{|c|c|c|c|}
\hline \multicolumn{4}{|c|}{$\begin{array}{l}\text { MP-12.4. Table } 1 \text { Patients' demographics and final } \\
\text { diagnoses related to the risk group }\end{array}$} \\
\hline Parameter & UTD P1 & UTD P2 & UTD P3 \\
\hline Patients (units) ${ }^{\#}$ & $238(289)$ & $129(166)$ & 113 (149) \\
\hline $\begin{array}{l}\text { Median age at } \\
\text { presentation* (range) }\end{array}$ & $\begin{array}{c}1.07 \\
(0.06-6.8)\end{array}$ & $\begin{array}{c}0.58 \\
(0.03-12)\end{array}$ & $\begin{array}{c}0.47 \\
(0.03-11.7\end{array}$ \\
\hline \multicolumn{4}{|l|}{ Gender } \\
\hline Male (\%) & 195 (81.9) & $103(79.8)$ & 79 (69.9) \\
\hline Female (\%) & $43(18.1)$ & $26(20.2)$ & $34(30.1)$ \\
\hline \multicolumn{4}{|l|}{ Side } \\
\hline Right (\%) & $86(29.8)$ & $50(30.1)$ & $63(42.3)$ \\
\hline Left (\%) & $203(70.2)$ & $116(69.9)$ & $86(57.7)$ \\
\hline \multicolumn{4}{|l|}{ Laterality } \\
\hline Unilateral (\%) & $169(71)$ & $97(75.2)$ & $77(68.1)$ \\
\hline Bilateral $(\%)^{\#}$ & $69(29)$ & $32(24.8)$ & 36 (31.9) \\
\hline $\begin{array}{l}\text { Circumcised/non- } \\
\text { circumcised }\end{array}$ & $43 / 152$ & $29 / 74$ & $32 / 47$ \\
\hline
\end{tabular}

\begin{tabular}{|c|c|c|c|c|}
\hline \multirow[t]{2}{*}{ UTD } & \multirow[t]{2}{*}{ Parameter } & \multicolumn{2}{|c|}{ UD category } & \multirow[t]{2}{*}{$\mathbf{p}$} \\
\hline & & 0-4 mm & $>4 \mathrm{~mm}$ & \\
\hline \multirow[t]{2}{*}{ P1 } & FUTI* n (\%) & $16 / 238(6.7 \%)$ & - & - \\
\hline & Surgery\# n (\%) & $0 / 289(0)$ & - & - \\
\hline \multirow[t]{2}{*}{ P2 } & FUTI* n (\%) & $7 / 63(11.1)$ & 25/66 (40.3) & $<0.001$ \\
\hline & Surgery\# n (\%) & $14 / 75$ (18.7) & $12 / 91(14.2)$ & 0.33 \\
\hline \multirow[t]{2}{*}{ P3 } & FUTI* n (\%) & $12 / 50(24)$ & $35 / 63(55.6)$ & $<0.001$ \\
\hline & Surgery\# n (\%) & $45 / 68(66.8)$ & 63/81 (77.8) & 0.11 \\
\hline \multirow[t]{2}{*}{ Total } & FUTI* n (\%) & $35 / 351(10)$ & $60 / 129(46.5)$ & $<0.001$ \\
\hline & Surgery\# n (\%) & $59 / 432(13.7)$ & $75 / 172$ (43.6) & $<0.001$ \\
\hline UTD & Parameter & $4.1-7 \mathrm{~mm}$ & $>7 \mathrm{~mm}$ & $\mathbf{p}$ \\
\hline \multirow[t]{2}{*}{ P1 } & FUTI* n (\%) & - & - & - \\
\hline & Surgery\# n (\%) & - & - & - \\
\hline \multirow[t]{2}{*}{ P2 } & FUTI* n (\%) & $14 / 40$ (35) & $11 / 26(42.3)$ & 0.55 \\
\hline & Surgery\# n (\%) & $7 / 58(12.1)$ & $5 / 33(15.2)$ & 0.68 \\
\hline \multirow[t]{2}{*}{ P3 } & FUTI* n (\%) & $4 / 8(50)$ & $31 / 55(56.4)$ & 0.74 \\
\hline & Surgery\# n (\%) & $8 / 14(57.1)$ & $55 / 67$ (79.3) & 0.04 \\
\hline \multirow[t]{2}{*}{ Total } & FUTI* n (\%) & $18 / 48(37.5)$ & 42/81 (51.9) & 0.11 \\
\hline & Surgery\# n (\%) & $15 / 72(20.8)$ & $60 / 100(60)$ & $<0.001$ \\
\hline
\end{tabular}

those with UD $\leq 4 \mathrm{~mm}(\mathrm{p}<0.001)$ (Table 2). When UD was $>4 \mathrm{~mm}, 46.5 \%$ $(60 / 129)$ of patients had FUTI and 76 units $(44.2 \%)$ had surgeries $(p<0.001$ for both). In P2 and P3 groups, there were no differences between the UD categories $(0-4 \mathrm{~mm}$ and $>4 \mathrm{~mm})$ in terms of surgery $(p=0.33$ and 0.11 , respectively). In P2 (Table 2), there was no difference between 4.1-7 $\mathrm{mm}$ and $>7 \mathrm{~mm}$ UD categories in terms of FUTI and surgery $(\mathrm{p}=0.55$ and 0.68 , respectively), while in $\mathrm{P} 3,79.3 \%$ of units had surgeries with UD $4.1-7 \mathrm{~mm}$ in comparison to $57.1 \%$ when UD was $>7 \mathrm{~mm}(\mathrm{p}=0.04)$. In P3, $50 \%$ of patients with UD $4.1-7 \mathrm{~mm}$ and $56.4 \%$ with UD $>7 \mathrm{~mm}$ experienced FUTI $(p=0.74)$. 
Conclusions: UD $>4 \mathrm{~mm}$ is associated with a higher incidence of FUTI and surgeries when compared to UD $\leq 4 \mathrm{~mm}$. Within P2, there is no significant difference in surgical intervention or FUTI when comparing UD 4.1-7 $\mathrm{mm}$ and $>7 \mathrm{~mm}$ This leads us to believe that UD $>4 \mathrm{~mm}$ is a reliable cutoff to define ureteral dilation.

\section{MP-12.5}

Validation of modified diuretic drainage times cutoffs in antenatal hydronephrosis

Amr Hodhod ${ }^{1}$, Francis Petrella' ${ }^{1}$, Mohamed El-Sherbiny', Iman Sadri', Roman Jednak', J.-P. Capolicchio ${ }^{7}$

'Urology, McGill University Health Centre, Montreal, QC, Canada Introduction: The value of diuretic renography drainage times in congenital hydronephrosis (AHN) decision-making is controversial. Recently, the Washington group suggested a modification to the classically described diuretic drainage time cutoffs. They found that a drainage half-time (T1/2) $<5$ minutes is normal whereas a T1/2 exceeded 75 minutes predicts pyeloplasty. We sought to evaluate these criteria, and in addition, we investigated the use of global tracer washout (GW) as a predictor of pyeloplasty. Methods: We retrospectively reviewed consecutive pyeloplasties from 2004-2018 for AHN. Patients who underwent pyeloplasty due to low differential renal function (DRF) $<30 \%$ or infection were excluded. The control group comprised grade 3 and 4 AHN managed conservatively. The initial renal ultrasound and MAG 3 Lasix renogram were reviewed for SFU grade, DRF, type of renogram curve, $\mathrm{T} 1 / 2$, and GW. A ROC curve was used to evaluate the T1/2 and GW cutoff points that can predict pyeloplasty, using a $\mathrm{p}<0.05$.

Results: The pyeloplasty group consisted of 84 patients (88 renal units) and the control group consisted of 58 patients (59 renal units). The median age at pyeloplasty was 4.5 months $(0.5-26)$, while the median followup of the control group was 19 months $(9-43)$. One renal unit $(1.1 \%)$ in the pyeloplasty group had a T1/2 $<5$ minutes, whereas $26 \%(23 / 88)$ had T $1 / 2$ $>75$ minutes $(p<0.001)$ (Table 1$)$. In the control group, $48 \%(28 / 59)$ of units had T $1 / 2<5$ minutes and none had T1/2 $>75$ minutes $(\mathrm{p}<0.001)$. The ROC curve for $\mathrm{T} 1 / 2<5$ minutes demonstrated $98 \%$ sensitivity and $51 \%$ specificity, whereas a T1/2>75 minutes demonstrated $100 \%$ specificity and $26 \%$ sensitivity. Analysis of the GW using a ROC curve revealed that a cutoff of $50 \%$ GW has $100 \%$ specificity and $55 \%$ sensitivity for pyeloplasty. Conclusions: The modified T1/2 criteria are valid predictors of pyeloplasty for AHN. Unfortunately, these criteria exist in only $25 \%$ of patients. In the remaining $75 \%$ of cases, those with indeterminant $\mathrm{T} 1 / 2$, the GW $<50 \%$ was helpful in identifying a further $30 \%$ of cases requiring pyeloplasty.

\section{MP-12.6}

Outcomes of Deflux ${ }^{\circledR}$ treatment for vesicoureteral reflux following pediatric transplant: A systematic review

Karla Rebullar', Fardod O'Kelly', Martin A. Koyle', Andrew I Kirsch ${ }^{2}$, Armando J. Lorenzo ${ }^{1}$, Rusul Al-Kutbi ${ }^{1}$, Fadi Zu'bi

'Division of Urology, The Hospital for Sick Children, Toronto, ON, Canada; ${ }^{2}$ Department of Pediatric Urology, Children's Healthcare of Atlanta, Atlanta, GA, United States

Introduction: Vesicoureteral reflux (VUR) after renal transplant in the pediatric population may be associated with an increased incidence of urinary tract infection (UTIs) leading to increased morbidity. ${ }^{1}$ The nonorthotopic location of the transplanted ureter may pose challenges in correcting the VUR using endoscopic injection techniques. Herein, we report the results of a systematic review evaluating the outcomes of endoscopic treatment of VUR using Deflux in this population.

Methods: PubMed and Embase databases were searched from October 2001 to April 2019. Full-text English articles involving patients less than 18 years old at the time of transplant, with a diagnosis of VUR posttransplantation, who underwent Deflux treatment were included.

Results: We found six eligible studies describing Deflux treatment outcomes in 67 pediatric patients with post-transplant VUR where voiding cystourethrogram (VCUG) confirmed the diagnosis and resolution of VUR. Mean success rate was $36.8 \%$. Ureteral obstruction occurred in seven

MP-12.5. Table 1. Patients' demographics and initial renogram data

\begin{tabular}{|c|c|c|c|c|}
\hline & & Pyeloplasty group & Control group & $\mathbf{p}$ \\
\hline \multicolumn{2}{|c|}{ Patients number (units) } & $84(88)$ & $58(59)$ & \\
\hline \multicolumn{2}{|c|}{ Age at presentation median (range) } & 0.37 mon $(0.03-10.9)$ & 0.83 mon $(0.07-9.5)$ & 0.002 \\
\hline Gender & Female n (\%) & $21(25)$ & $13(22.4)$ & \\
\hline \multirow[t]{2}{*}{ Side } & Male n (\%) & $63(75)$ & $45(77.6)$ & 0.25 \\
\hline & Female n (\%) & $21(25)$ & $13(22.4)$ & \\
\hline Laterality & Bilateral n (\%) & $4(4.8)$ & $1(1.7)$ & \\
\hline \multirow[t]{2}{*}{ SFU at presentation } & Grade 3 n (\%) & $25(28.4)$ & $42(71.2)$ & $<0.001$ \\
\hline & Grade 4 n (\%) & $63(71.6)$ & $17(28.8)$ & \\
\hline \multicolumn{2}{|c|}{ Age at renogram median (range) } & 1.7 mon $(0.3-11)$ & 2.8 mon $(0.8-25.2)$ & 0.001 \\
\hline \multicolumn{2}{|l|}{ DRF median (range) } & $46 \%(30-100)$ & $51 \%(34-65)$ & 0.001 \\
\hline \multicolumn{2}{|l|}{ 5-75 minutes $\mathrm{n}(\%)$} & $64(72.7)$ & $31(52.5)$ & \\
\hline \multicolumn{2}{|l|}{$>75$ minutes $\mathrm{n}(\%)$} & $23(26.2)$ & 0 & \\
\hline \multicolumn{5}{|c|}{ Global isotope washout } \\
\hline \multicolumn{2}{|l|}{$<50 \%, \mathrm{n}(\%)$} & $47(53.4)$ & 0 & $<0.001$ \\
\hline \multicolumn{2}{|l|}{$50-90 \%, \mathrm{n}(\%)$} & $40(45.5)$ & $39(66.1)$ & \\
\hline \multicolumn{2}{|l|}{$>90 \%, \mathrm{n}(\%)$} & $1(1.1)$ & $20(33.9)$ & \\
\hline
\end{tabular}


cases $(10.4 \%)$. Ureteric stenting was the initial management in all cases of obstruction but was only successful in one patient (14\%). Open ureteroneocystostomy (UNC) was performed in four cases $(57 \%)$, while two were managed expectantly (29\%, unknown outcomes). Persistent VUR with urinary tract infection despite Deflux were reported in 20 cases. Of these, seven (35\%) were managed with prophylactic antibiotics and $13(65 \%)$ with UNC. Success rates were consistently low for UNC after failed Deflux (40-50\%) in comparison to redo UNC in transplant ureters without prior injection (70-80\%). $)^{2,3}$

Conclusions: Low success rates are seen following injection techniques for VUR after pediatric renal transplant. Although an appealing option, Deflux may prove counterintuitive due to the high rate of obstruction and suboptimal results if open reimplantation is required. A multi-institutional, prospective study with a larger population size may further elucidate these results.

References

1. Ranchin B, Chapuis F, Dawhara M, et al. Vesicoureteral reflux after kidney transplantation in children. Nephrol Dial Transplant 2000;15:1852e8. https://doi.org/10.1093/ndt/15.11.1852

2. Krishnan A, Swana H, Mathias R, et al. Redo ureteoneocystostomy using an extravesical approach in pediatric renal transplant patients with reflux: A retrospective analysis and description of technique. $/$ Urol 2006;176:1582-7. https://doi.org/10.1016/j.juro.2006.06.033

3. Barrero R, Fijo J, Fernandez-Hurtado M, et al. Vesicoureteral reflux after kidney transplantation in children. Pediatr Transplant 2007;11:498-503. https://doi.org/10.1111/j.13993046.2006.00668.x

\section{MP-12.7}

Early postoperative outcomes of open vs. minimally invasive surgery for vesicoureteral reflux: Analysis of the National Surgical Quality Improvement Program (NSQIP) pediatric database 2015-2017

Youshan Ding ${ }^{1}$, Jacob Davidson ${ }^{1,2}$, Leandra Stringer ${ }^{1,3}$, Zhan Tao (Peter) Wang ${ }^{1,4}$, Sumit Dave ${ }^{1,4}$

'Department of Surgery, Schulich School of Medicine \& Dentistry, Western University, London, ON, Canada; ${ }^{2}$ Division of Pediatric Surgery, Schulich School of Medicine \& Dentistry, Western University, London, ON, Canada; ${ }^{3}$ Division of Urology, Schulich School of Medicine \& Dentistry, Western University, London, ON, Canada; ${ }^{4}$ Division of Pediatric Urology, Schulich School of Medicine \& Dentistry, Western University, London, ON, Canada

Introduction: Open ureteric reimplantation (OUR) is the gold standard treatment for vesicoureteric reflux (VUR) compared to endoscopic injection (EI) and minimally invasive surgery (MIS), which includes laparoscopic or robot-assisted uretic reimplantation. As the optimal management of VUR remain controversial, information on early surgical outcomes may help with improved patient-centered decision-making. ${ }^{1,2}$

Methods: This study examined the trends in VUR surgical techniques from 2015-2017 and assessed early (30-day) outcomes for each procedure. A post-hoc analysis of data from the American College of Surgeons (ACS) National Surgical Quality Improvement Program (NSQIP) pediatric version was performed. The ACS NSQIP pediatric datasets are validated and riskadjusted and include 109 institutions across Canada and the U.S. to date. Patients undergoing VUR surgery were identified using the International Statistical Classification of Diseases and Related Health Problems (ICD) codes and by the Current Procedures Terminology (CPT) codes.

Results: Overall, 5137 pediatric VUR procedures were performed: OUR $(62.1 \%)$, EI $(30.7 \%)$, and MIS $(7.2 \%)$. Rates of each technique remained stable from 2015-2017. Median age at surgery was 4.8 years (interquartile range $2.3-7.7$ ) and $72.9 \%$ were female. OUR procedures were performed more often in younger patients $(p<0.0001)$. Median length of hospital stay was two days for OUR vs. one day for MIS. Overall, unplanned hospital readmission within 30 -days of surgery was $4.1 \%$. The most common complication was urinary tract infections (UTI), with an overall incidence of $2.9 \%$; all other complications were $<1 \%$ and non-significant across the treatment options. There was no significant difference in UTI risk between MIS and OUR, but El procedures were $60 \%$ less likely (rela- tive risk $[R R] 0.4 ; 95 \%$ confidence interval $[\mathrm{Cl}]$ 0.25-0.62) to develop UTIs compared to OUR. There was no significant difference in the risk of an unplanned hospital admission between OUR and MIS. Again, EI procedures were associated with a $79 \%$ (RR $0.21 ; 95 \% \mathrm{Cl} 0.13-0.34$ ) risk-reduction compared to OUR

Conclusions: Children undergoing OUR and MIS VUR surgery have comparable early outcomes but shorter hospital stays for the MIS group. EI procedures, as expected, have lower postoperative complications and UTI risk compared to OUR. Future prospective studies are needed to investigate the relationship between early surgical outcomes and long-term resolution of VUR in pediatric patients, while adjusting for VUR grade.

\section{References}

1. Blais AS, Bolduc S, Moore K. Vesicoureteral reflux: From prophylaxis to surgery. Can Urol Assoc / 2017;11:S13-8. https://doi.org/10.5489/ cuaj.4342

2. Boysen WR, Ellison JS, Kim C, et al. Multi-institutional review of outcomes and complications of robot-assisted laparoscopic extravesical ureteral reimplantation for treatment of primary vesicoureteral reflux in children. J Urol 2017;197:1555-61. https://doi.org/10.1016/j. juro.2017.01.062

\section{MP-12.8}

Time-driven, activity-based cost analysis of management pathways for vesicoureteral reflux

Lucshman Raveendran', Robert DiCesare', Kornelia Twardowski², David During ${ }^{2}$, Darius J. Bagli ${ }^{3}$, Myla Moretti ${ }^{2}$, Martin A. Koyle ${ }^{3}$

${ }^{1}$ Faculty of Medicine, University of Toronto, Toronto, ON, Canada; ${ }^{2}$ The Hospital for Sick Children, Toronto, ON, Canada; ${ }^{3}$ Division of Urology, Hospital for Sick Children, Toronto, ON, Canada

Support: This project was supported by the AUA Medical Student Fellowship Introduction: The approach to managing vesicoureteral reflux (VUR) remains highly variable despite being a common pediatric diagnosis, which makes costing unpredictable. Time-driven activity-based costing (TDABC) is a novel costing methodology that is purported to provide a rapid and more accurate calculation of total costs vs. traditional costing methods. The aim of the study is to employ TDABC to characterize institutional costs for three management pathways for VUR.

Methods: We developed detailed process maps for VUR management based on practice guidelines applicable to a hypothetical female VUR index patient with grade 3 unilateral reflux without bowel-bladder dysfunction at our institution. The costs of three management pathways were described: observation with continuous antibiotic prophylaxis (OCAP); minimally invasive endoscopic surgery (EDP) with dextranomer/hyaluronic acid; and open re-implantation surgery (ORP). Costs for each pathway were calculated using the capacity cost rate $(\$ /$ minute) for institutional resources and time estimates of resource use captured through direct observation and electronic medical records data.

Results: A substantial range of total costs was observed for all pathways: OCAP (\$1683.58-\$2041.12), EDP (\$2262.37-\$4012.89), and ORP (\$3317.76-\$3924.82). On average, variation in diagnostic imaging accounted for an average of $\$ 558.81$ to the cost of management pathways. Total costs for a single DMSA scan accounted for $8-15 \%$ of any pathway's overall costs. Material costs for VCUG imaging and endoscopic surgery were high at $59 \%$ and $64-76 \%$ of their individual total costs, respectively. For ORP, high costs were attributable to the longer use of operating room space (vs. EDP) and inpatient postoperative stay.

Conclusions: To our knowledge, this is the first application of TDABC to describe the cost of services delivered in pediatric urology. TDABC demonstrates significant cost variability in VUR treatment modalities and identified local cost drivers to target. 


\section{MP-12.9 \\ Younger children undergoing ureteroscopic stone management at higher risk of unplanned postoperative readmissions: An analysis of the pediatric National Surgical Quality Improvement Program database}

Youshan Ding ${ }^{1}$, Jacob Davidson 1,2, Ernest Chan', Jennifer Bjazevic 1,3, Guido Filler $^{4}$, Sumit Dave ${ }^{1,5}$, Zhan Tao (Peter) Wang ${ }^{1,5}$

${ }^{1}$ Department of Surgery, Schulich School of Medicine \& Dentistry, Western University, London, ON, Canada; ${ }^{2}$ Division of Pediatric Surgery, Schulich School of Medicine \& Dentistry, Western University, London, ON, Canada; ${ }^{3}$ Division of Urology, Schulich School of Medicine \& Dentistry, Western University, London, ON, Canada; ${ }^{4}$ Division of Pediatric Nephrology, Schulich School of Medicine \& Dentistry, Western University, London, ON, Canada; ${ }^{5}$ Division of Pediatric Urology, Schulich School of Medicine \& Dentistry, Western University, London, ON, Canada

Introduction: The incidence of pediatric urolithiasis has increased over the last two decades. Ureteroscopy with laser lithotripsy (URS) is recommended for the treatment of mid to distal ureteral calculi. However, there is currently no consensus regarding whether URS is appropriate for all pediatric patients. ${ }^{1,2}$

Methods: A post-hoc analysis of data collected from the American College of Surgeons National Surgical Quality Improvement Program (ACS NSQIP) pediatric version was performed. The ACS NSQIP pediatric datasets are validated and risk-adjusted and include 109 institutions across Canada and the U.S. to date. Outcomes of 299865 pediatric patients between 2015 and 2017 were examined. These children were identified using the International Statistical Classification of Diseases and Related Health Problems (ICD) codes and by the Current Procedures Terminology (CPT) codes.

Results: A total of 1122 pediatric URS cases were identified. The median age was 14.4 years and $55.4 \%$ of these cases were female. Overall, $8.9 \%$ of URS cases required a readmission to hospital within 30 days of the index procedure, and of these, $71 \%$ were unplanned readmissions. The two most common reasons for unplanned readmissions were postoperative urinary tract infections (UTIs, $26.4 \%$ ) and persistent ureteral calculus $(21.8 \%)$. On univariate analysis, children with unplanned readmissions were significantly younger in age (median 11.3 years) compared to those without unplanned readmissions (median 14.5 years). The relative risk (RR) of an unplanned readmission was 2.4 times higher for children aged $6-11$ years $(95 \%$ confidence interval $[\mathrm{Cl}] 1.51-3.93)$, and 4.5 times $(95 \%$ Cl 1.80-11.50) for children under two years old when compared to children $12-18$ years old.

Conclusions: This study found that younger children undergoing URS are at a higher risk of unplanned readmissions secondary to postoperative UTI and persistent calculi. As such, age must be taken into account when deciding on the optimal surgical modality for children with urolithiasis. Further prospective studies are needed to better elucidate the indications of URS for pediatric urolithiasis.

\section{References}

1. Tan AH, Al-Omar M, Denstedt JD, et al. Ureteroscopy for pediatric urolithiasis: An evolving first-line therapy. Urology 2005;65:153-6. https://doi.org/10.1016/j.urology.2004.08.032

2. Bjazevic J, Razvi H. Stones in pregnancy and pediatrics. Asian J Urol 2018;5:223-34. https://doi.org/10.1016/j.ajur.2018.05.006

\section{MP-12.10}

Single-use flexible digital ureteroscopes: Cost-effective in lowvolume pediatric centers?

Catherine McMartin ${ }^{1,2}$, Jonathan Cloutier ${ }^{1,2}$, Stéphane J. Bolduc'2,3,4

${ }^{1}$ Urology, CHU de Québec-Université Laval, Quebec City, QC Canada; '2Département de chirurgie, Université Laval, Quebec City, QC, Canada; ${ }^{3}$ Pediatric Urology, CHU de Québec-Université Laval, Quebec City, QC, Canada; ${ }^{4}$ Axe Médecine Régénératrice, Centre de rechercheCHU de Québec-Université Laval, Quebec City, QC, Canada

Introduction: Ureteroscopes (URS), especially digital flexible URS (fURS), are very fragile devices associated with high rate of breakage due to transport, handling, reprocessing, or use in clinical situations requiring high deflexion. Recently, single-use fURS have been commercialized and could represent an alternative to reusable fURS. This systematic review aimed to assess the clinical and economic relevance of single-use fURS, specifically looking at low-volume use.

Methods: A literature search was performed until September 2018 in Medline (PubMmed), Embase, Cochrane Library, Centre for Reviews and Dissemination, as well as in the grey literature, in order to retrieve studies on clinical efficacy safety and cost of single-use fURS. The US Food and Drug Administration's (FDA) Manufacturer and User Facility Device Experience (MAUDE) database was reviewed until September 20, 2018 to supplement search on safety issue. Two reviewers independently performed article selection, quality assessment, and data extraction. Disagreements were resolved with a third reviewer to achieve a consensus. The volume of fURS performed during the financial period between May 28, 2017 to May 27, 2018 was estimated from hospital databases. Reported accidents were also retrieved from hospital database during this period.

Results: Results from our review and two prospective studies suggested that efficacy of single-use fURS for urinary lithiasis in adult population was similar to reusable fURS, without any published pediatric trial identified. Two non-randomized prospective trials, two observational studies, and 13 incident reports in adults were included. Most complications associated with single-use fURS $(6-17 \%)$ were similar to those known with reusable fURS $(<15 \%)$. Five published economic studies were included using a cost-minimization approach. Global cost of single-use fURS was higher than reusable fURS considering all aspects (purchase, repair, reprocessing, and sterilization). For the 328 adult interventions using fURS registered in 2017-2018 in our tertiary center, the breakage rate of our fURS was $6.4 \%$, fixing the mean cost per intervention at $\$ 353$ CAD based on purchase of five reusable fURS amortized over six years, repair, reprocessing, and sterilization costs. Results from our cost analysis according to breakage avoidance scenario suggested that less than $10 \%$ of our ureteroscopy should be performed with single-use fURS in our hospital to stay costeffective. It also confirmed that a low-volume center (pediatric) using less than 11 single-use fURS per year was cost-effective.

Conclusions: This systematic review and economic analysis confirm that single-use fURS is as effective as reusable fURS but should not be used for every case in a large-volume center. Targeting its use for complex cases at higher risk of breakage, and in the setting of a low-volume center like our pediatric center is cost-effective. Further studies should be undertaken to assess their effectiveness and cost-effectiveness during clinical intervention in pediatric population.

References

1. de la Rosette J, et al. The clinical research office of the endourological society ureteroscopy global study: Indications, complications, and outcomes in 11885 patients. J Endourol 2014;28:131-9. https:// doi.org/10.1089/end.2013.0436

2. Lechevallier E, Saussine C, Traxer O. [Ureteroscopy for upper urinary tract stones]. Prog Urol 2008;18:912-6. https://doi.org/10.1016/j. purol.2008.09.022

3. Duty BD, Conlin MJ. Basic of urologic surgery, principes of urologic endoscopy, in Urology, Campbell-Walsh, Editor. 2016, Elservier. p. 20-22.

4. Skolarikos AA, et al. Current status of ureteroscopic treatment for urolithiasis. Int J Urol 2009; 16:713-7. https://doi.org/10.1111/ j.1442-2042.2009.02364.x

5. Doizi S, Traxer O. Flexible ureteroscopy: Technique, tips, and tricks. Urolithiasis 2018;46:47-58. https://doi.org/10.1007/s00240-0171030-x

6. Rajamahanty S, Grasso M. Flexible ureteroscopy update: indications, instrumentation and technical advances. Indian J Urol 2008;24:5327. https://doi.org/10.4103/0970-1591.44263

7. Reis Santos JM. Ureteroscopy from the recent past to the near future. Urolithiasis 2018;46:31-7. https://doi.org/10.1007/s00240017-1016-8

8. Association canadienne d'urologie : Santé urologique, l'urétéroscopie. Available at: https://www.cua.org/themes/web/assets/files/patient_ info/fr/43f-Iroscopie_s.pdf. Accessed April 27, 2020.

9. Association française d'urologie, urétéroscopie pour calcul du haut de I'appareil urinaire. (2008) Available at: https://www.urofrance.org/nc/ science-et-recherche/base-bibliographique/article/html/ureteroscopiepour- calcul-du-haut-appareil-urinaire.html. Accessed April 27, 2020. 
10. Carpentier X, Amiel J. Chirurgie endoscopique des calculs de I'uretère. EMC (Elsevier Masson SAS, Paris), Techniques chirurgicales. Urologie 2010;41-147.

11. Doizi S, Traxer O. Comment entretenir son urétéroscope souple? Prog Urol 2014;24:F95-F102. https://doi.org/10.1016/j.fpurol.2014.09.002

12. Doizi S, et al. First clinical evaluation of a new single-use flexible ureteroscope (LithoVue): A European prospective multicentric feasibility study. World J Urol 2017;35:809-18. https://doi.org/10.1007/ s00345-016-1936-x

13. Lasselin J, et al. [Flexible ureteroscope damages. Evaluation of university hospital service equipment]. Prog Urol 2015;25:265-73. https://doi.org/10.1016/j.purol.2015.01.010

14. Emiliani $\mathrm{E}$, et al. First clinical evaluation of the new single-use flexible and semirigid Pusen ureteroscopes. Cent European J Urol 2018;71:208-13.

15. Dale J, et al. Evaluation of a novel Single-use flexible ureteroscope. J Endourol 2017 March 2. [Epub ahead of print]

16. Marchini GS, et al. In vitro evaluation of single-use digital flexible ureteroscopes: A practical comparison for a patient-centered approach. J Endourol 2018;32:184-91. https://doi.org/10.1089/end.2017.0785

17. Proietti $\mathrm{S}$, et al. Comparison of new single-use digital flexible ureteroscope vs. non-disposable fiber optic and digital ureteroscope in a cadaveric model. J Endourol 2016;30:655-9. https://doi.org/10.1089/ end.2016.0051

18. Talso M, et al. Comparison of flexible ureterorenoscope quality of vision: An in vitro study. J Endourol 2018;32:523-8. https://doi. org/10.1089/end.2017.0838

19. Tom WR, et al. Next-generation single-use ureteroscopes: An in vitro comparison. J Endourol 2017;31:1301-6. https://doi.org/10.1089/ end.2017.0447

20. Wang $\mathrm{F}$, et al. The application of a single-use fiberoptic flexible ureteroscope for the management of upper urinary calculi. Int Urol Nephrol 2018;50:1235-41. https://doi.org/10.1007/s11255-018-1895-9

21. Unité d'évaluation des technologies et des modes d'intervention en santé. Guide méthodologique: démarche d'évaluation et étapes de réalisation d'un projet d'ETMIS, UETMIS du CHU de QuébecUniversité Laval, novembre 2015, 26 pages.

22. Kung J, et al. From systematic reviews to clinical recommendations for evidence-based health care: Validation of revised assessment of multiple systematic reviews (R-AMSTAR) for grading of clinical relevance. Open Dent / 2010;4:84-91. https://doi. org/10.2174/1874210601004010084

23. Brouwers MC, et al. AGREE II: Advancing guideline development, reporting and evaluation in health care. CMAJ 2010182:E839-42. https://doi.org/10.1503/cmaj.090449

24. Davis NF, et al. Single-use flexible ureteropyeloscopy: A systematic review. World J Urol 2018; 36:529-36. https://doi.org/10.1503/ cmaj.090449

25. Mager R, et al. Clinical outcomes and costs of reusable and single-use flexible ureterorenoscopes: A prospective cohort study. Urolithiasis 2018;46:587-93. https://doi.org/10.1007/s00240-018-1042-1

26. Usawachintachit $M$, et al. A prospective case-control study comparing LithoVue, a single-use, flexible disposable ureteroscope, with flexible, reusable fiber-optic ureteroscopes. J Endourol 2017;31:46875. https://doi.org/10.1089/end.2017.0027

27. Wiseman $\mathrm{O}$, et al. Comparison of a new single-use digital flexible ureteroscope (LithoVue) to a non-disposable fibre-optic flexible ureteroscope in a live porcine model. / Urol 2016;195:e682. https:// doi.org/10.1016/j.juro.2016.02.458

28. Johnson MT, Khemees TA, Knudsen BE. Resilience of disposable endoscope optical fiber properties after repeat sterilization. J Endourol 2013;27:71-4. https://doi.org/10.1089/end.2012.0323

29. Boylu U, et al. In vitro comparison of a disposable flexible ureteroscope and conventional flexible ureteroscopes. J Urol 2009;182:2347-51. https://doi.org/10.1016/j.juro.2009.07.031

30. Bader MJ, et al. The PolyScope: A modular design, semidisposable flexible ureterorenoscope system. J Endourol 2010;24:1061-6. https://doi.org/10.1089/end.2010.0077
31. Bansal $\mathrm{H}$, et al. Polyscope: A new era in flexible ureterorenoscopy. J Endourol 2011;25:317-21. https://doi.org/10.1089/end.2009.0584

32. Ding J, et al. Comparing the efficacy of a multimodular flexible ureteroscope with its conventional counterpart in the management of renal stones. Urology 2015;86:224-9. https://doi.org/10.1016/j. urology.2015.04.018

33. Gu SP, et al. Clinical effectiveness of the PolyScope endoscope system combined with holmium laser lithotripsy in the treatment of upper urinary calculi with a diameter of less than $2 \mathrm{~cm}$. Exp Ther Med 2013;6:591-5. https://doi.org/10.3892/etm.2013.1184

34. Cho SY, et al. Evaluation of performance parameters of the disposable flexible ureterorenoscope (LITHOVUE) in patients with renal stones: A prospective, observational, single-arm, multicenter study. Sci Rep 2018;8:9795. https://doi.org/10.1038/s41598-01828247-7

35. MAUDE Adverse Event Report 3005099803-2017-02868: Boston Scientific - Marlborough Lithovue single-use digital flexible ureteroscope and accessories, flexible/rigid. 2017.

36. Available at: https://www.accessdata.fda.gov/scripts/cdrh/cfdocs/ cfMAUDE/Detail.CFM?MDRFOI_ID $=6887535 \& p c=F G$ B. Accessed April 27, 2020

37. MAUDE Adverse Event Report 3005099803-2017-00406: Boston Scientific - Marlborough Lithovue single-use digital flexible ureteroscope and accessories, flexible/rigid. 2017. Available at: https://www.accessdata.fda.gov/scripts/cdrh/cfdocs/cfMAUDE/ Detail.CFM?MDRFOI_ID $=6341378 \&$ pc=FG B. Accessed April 27, 2020.

38. MAUDE Adverse Event Report \# 3005099803-2018-60107: Boston Scientific - Spencer Lithovue single-use digital flexible ureteroscope and accessories, Flexible/Rigid. (2018) Available at: https:// www.accessdata.fda.gov/scripts/cdrh/cfdocs/cfMAUDE/Detail. CFM?MDRFOI_ID=7801298\&pc=FG B. Accessed April 27, 2020.

39. MAUDE Adverse Event Report \# 3005099803-2018-02330: Boston Scientific - Spencer Lithovue single-use digital flexible ureteroscope and accessories, Flexible/Rigid. (2018) Available at: https:// www.accessdata.fda.gov/scripts/cdrh/cfdocs/cfMAUDE/Detail. CFM?MDRFOI_ID=7695886\&pc=FG B. Accessed April 27, 2020.

40. MAUDE Adverse Event Report \# 3005099803-2018-01133: Boston Scientific - Spencer Lithovue single-use digital flexible ureteroscope and accessories, Flexible/Rigid. (2018) Available at https:// www.accessdata.fda.gov/scripts/cdrh/cfdocs/cfMAUDE/Detail. CFM?MDRFOI_ID=7408278\&pc=FG B. Accessed April 27, 2020.

41. MAUDE Adverse Event Report \# 3005099803-2017-02222: Boston Scientific - Spencer Lithovue single-use digital flexible ureteroscope and accessories, Flexible/Rigid. (2017) Available at: https:// www.accessdata.fda.gov/scripts/cdrh/cfdocs/cfMAUDE/Detail. CFM?MDRFOI_ID=6740301\&pc=FG B. Accessed April 27, 2020.

42. MAUDE Adverse Event Report \# 3005099803-2017-03202: Boston Scientific - Spencer Lithovue single-use digital flexible ureteroscope and accessories, Flexible/Rigid. (2017) 62. Available at: https://www.accessdata.fda.gov/scripts/cdrh/cfdocs/cfMAUDE/ Detail.CFM?MDRFOI_ID $=6740301 \& p c=F G B$. Accessed April 27, 2020.

43. MAUDE Adverse Event Report \# 3005099803-2016-03931: Boston Scientific - Spencer Lithovue single-use digital flexible ureteroscope and sccessories, Flexible/Rigid. (2016). Available at: https:// www.accessdata.fda.gov/scripts/cdrh/cfdocs/cfMAUDE/Detail. CFM?MDRFOI_ID=6205008\&pc=FG B. Accessed April 27, 2020.

44. MAUDE Adverse Event Report \# 3005099803-2018-02093: Boston Scientific - Spencer Lithovue single-use digital flexible ureteroscope and accessories, Flexible/Rigid. (2018). Available a: https://www.accessdata.fda.gov/scripts/cdrh/cfdocs/cfMAUDE/ Detail.CFM?MDRFOI_ID=7633938\&pc=FG B. Accessed April 27, 2020.

45. MAUDE Adverse Event Report \# 7629126: Boston Scientific Spencer Lithovue single-use digital flexible ureteroscope and sccessories, Flexible/Rigid. (2018). Available at: (ttps://www.accessdata. fda.gov/scripts/cdrh/cfdocs/cfMAUDE/Detail.CFM?MDRFOI_ ID=7629126\&pc=FG B. Accessed April 27, 2020. 
46. MAUDE Adverse Event Report \# 3005099803-2018-01870: Boston Scientific - Spencer Lithovue single-use digital flexible ureteroscope and accessories, Flexible/Rigid. (2018) Available at: https://www.accessdata.fda.gov/scripts/cdrh/cfdocs/cfMAUDE/ Detail.CFM?MDRFOI_ID $=7592382 \& p c=F G$ B. Accessed April 27, 2020.

47. MAUDE Adverse Event Report \# 3005099803-2016-03209: Boston Scientific - Spencer Lithovue single-use digital flexible ureteroscope and accessories, Flexible/Rigid. (2016). Available at: https:// www.accessdata.fda.gov/scripts/cdrh/cfdocs/cfMAUDE/Detail. CFM?MDRFOI_ID=6047459\&pc=FG B. Accessed April 27, 2020.

48. MAUDE Adverse Event Report \# 3005099803-2016-01244: Boston Scientific - Spencer Lithovue single-use digital flexible ureteroscope and accessories, Flexible/Rigid. (2016). Available at: https:// www.accessdata.fda.gov/scripts/cdrh/cfdocs/cfMAUDE/Detail. CFM?MDRFOI_ID=5628325\&pc=FG B. Accessed April 27, 2020

49. Martin $\mathrm{C}$, et al. The economic implications of a reusable flexible digital ureteroscope: A cost-benefit analysis. J Urol 2017;197:730-5. https://doi.org/10.1016/j.juro.2016.09.085

50. Hennessey DB, et al. Single-use disposable digital flexible ureteroscopes: An ex vivo assessment and cost analysis. BJU Int 2018;121:55-61. https://doi.org/10.1111/bju.14235

51. Taguchi K, et al. Micro-costing analysis demonstrates comparable costs for LithoVue compared to reusable flexible fiberoptic ureteroscopes. J Endourol 2018;32:267-73. https://doi.org/10.1089/ end.2017.0523

52. Ozimek T, et al. Retrospective cost analysis of a single-center reusable flexible ureterorenoscopy program: A comparative cost simulation of disposable fURS as an alternative. / Endourol 2017; 31:122630. https://doi.org/10.1089/end.2017.0427

53. Geavlete P. Ureteroscopy complications, in Smith's Textbook of Endourology, Third Edition, G.H.B. Arthur D. Smith, Glenn M. Preminger, Louis R. Kavoussi., Editor. 2012, Blackwell Publishing Ltd. https://doi.org/10.1002/9781444345148.ch47

54. Binbay $M$, et al. Is there a difference in outcomes between digital and fiberoptic flexible ureterorenoscopy procedures? I Endouro 2010;24:1929-34. https://doi.org/10.1089/end.2010.0211

55. Somani BK, et al. Outcomes of flexible ureterorenoscopy and laser fragmentation for renal stones: comparison between digital and conventional ureteroscope. Urology 2013;82:1017-9. https://doi. org/10.1016/j.urology.2013.07.017

56. Legemate JD, et al. Durability of flexible ureteroscopes: A prospective evaluation of longevity, the factors that affect it, and damage mechanisms. Eur Urol Focus, 2019;5:1105-11. https://doi. org/10.1016/j.euf.2018.03.001

\section{MP-12.11}

Surgical outcomes in patients undergoing ileovesicostomy in Edmonton over the past 12 years

Steven Tong', David W. Chapman', Peter D. Metcalfe

${ }^{1}$ Urology, University of Alberta, Edmonton, AB, Canada

Introduction: Children born with spina bifida and other congenital abnormalities are suspect to significant urological complications, such as kidney injury, recurrent urinary tract infection (UTI), and incontinence. Management of these patients is challenging, and surgical diversion offers a possible solution. Our objective was to assess overall short- and medium-term outcomes of an incontinent ileovesicostomy in this complex pediatric and adult patient population.

Methods: We reviewed postoperative outcomes of all patients who underwent an ileovesicostomy from 2006-2018. The primary outcome was preservation of kidney function based on pre- and postoperative creatinine. The change in creatinine was evaluated with repeated-measures ANOVA. Secondary outcomes included change in hydronephrosis, recurrent UTIs, incontinence, and development of bladder stones.

Results: A total of 18 patients underwent an ileovesicostomy through the specified time frame by a single surgeon. The median age at surgery was 18 (range 8-42) and the median duration of followup was 27 months (range 0-80). Spina bifida was the most common underlying diagnosis at
$78 \%$ and high bladder pressures (dLPP $>30 \mathrm{mmH}_{2} \mathrm{O}$ ) was the most common indication for surgery at $56 \%$. Mean preoperative creatinine was 54 umol/L. Mean short-term postoperative creatinine was $55 \mathrm{umol} / \mathrm{L}(\mathrm{p}=0.66)$ and mean medium-term postoperative creatinine was $52 \mathrm{umol} / \mathrm{L}(\mathrm{p}=0.64)$, neither of which were statistically significantly elevated from preoperative creatinine. The portion of patients with hydronephrosis, recurrent UTIs, and incontinence all decreased postoperatively. The portion of patients of bladder stones increased postoperatively.

Conclusions: Ileovesicostomy is an effective tool in the pediatric surgical armamentarium in preserving renal function. It is safe procedure and can have excellent short- and medium-term urological outcomes.

\section{MP-12.12}

A consecutive, single-center, retrospective series of one-stage, vessel-preserving laparoscopic orchidopexies: Postoperative testicular atrophy rates and position

Adam Forster ${ }^{1}$, Zhan Tao (Peter) Wang ${ }^{1,2}$, Sumit Dave ${ }^{1,2}$

${ }^{1}$ Urology, Western University, London, ON, Canada; ${ }^{2}$ Pediatrics, Western University, London, ON, Canada

Introduction: Single-stage laparoscopic orchidopexy (SSO) and FowlerStephens orchidopexy (FSO) are accepted standards of care for the treatment of impalpable undescended testis (IUDT). ${ }^{1}$ There is paucity of data to suggest superiority of one approach. ${ }^{2}$ No defined intraoperative variables that favor proceeding with one approach exist. ${ }^{3}$ In this retrospective, consecutive case series, we present our results of SSO in patients with IUDT, regardless of location, treated at a single institution by one surgeon over a 10-year period.

Methods: Boys with IUDT underwent SSO from 2008-2018. SSO was performed by testicular vessel mobilization and translocation to the scrotum medial to the medial umbilical ligament. The peritoneal reflection between the gonadal vessels and vas was divided after mobilization to allow for extra mobility. Outcomes assessed included testicular atrophy defined as lack of Doppler flow on scrotal ultrasound and postoperative testicular location defined by examination at least six months postoperatively. Descriptive statistics summarize demographic variables and postoperative outcomes.

Results: Thirty-seven boys underwent surgery for IUDT (mean age $2.58 \pm 1.72$ years) with $47 \mathrm{SSO}$ procedures. Mean followup duration was $2.89 \pm 2.32$ years. Forty-six of $47(97.8 \%)$ SSO testes showed Doppler flow and were palpable on followup six months post SSO, confirming lack of testicular atrophy. Forty-five of 47 (95.4\%) were palpable in the scrotum; two were located outside the scrotum at the level of the pubic tubercle.

Conclusions: In this series, we demonstrate that a majority of boys with IUDT can undergo SSO without FSO; our data demonstrate a low atrophy rate $(2.2 \%)$ and minority of extra-scrotal testes in SSO $(4.6 \%)$. A limitation of our study design is that variables predictive of achieving an ideal scrotal location in SSO could not be elucidated. These hypothesisgenerating findings indicate a need for prospective studies to investigate the intraoperative indications for SSO vs. FSO.

\section{References}

1. Braga LH, Lorenzo AJ, Romao RL. Canadian Urological AssociationPediatric Urologists of Canada (CUA-PUC) guideline for the diagnosis, management, and followup of cryptorchidism. Can Urol Assoc J 2017;11:E251-60. https://doi.org/10.5489/cuaj.4585

2. Esposito C, Vallone G, Savanelli A, et al. Long-term outcome of laparoscopic Fowler-Stephens orchiopexy in boys with intraabdominal testis. J Urol 2009;181:1851-6. https://doi.org/10.1016/j. juro.2008.12.003

3. Penson D, Krishnaswami S, Jules A, et al. Effectiveness of hormonal and surgical therapies for cryptorchidism: A systematic review. Pediatrics 2013;131:e1897-907. https://doi.org/10.1542/peds.20130072 


\section{MP-12.13}

Randomized controlled trial of scrotal vs. inguinal orchidopexy on postoperative pain: The EXPRESSO trial

Melissa Mcgrath ${ }^{1,2,3}$, John Kim ${ }^{1}$, Luis H. Braga ${ }^{1,2,3}$

'Department of Surgery, Pediatric Urology, McMaster University, Hamilton, ON, Canada; ${ }^{2}$ McMaster Pediatric Surgery Research Collaborative, McMaster University, Hamilton, ON, Canada; ${ }^{3}$ Clinical Urology Research Enterprise (CURE), McMaster University, Hamilton, ON, Canada Introduction: We aimed to compare the impact of orchidopexy approach (scrotal $[\mathrm{SO}]$ vs. inguinal $[\mathrm{IO}]$ ) on analgesic requirements, postoperative (PO) pain, and complication rates.

Methods: A superiority randomized control trial (RCT) including boys 10-95 months of age at sx, diagnosed with palpable undescended testicles (UDT) was conducted. Patients with non-palpable or bilateral previous orchidopexies and concurrent procedures were excluded. Block randomization with 1:1 allocation ratio was employed, as was standardized anesthesia protocol with peri/postoperative analgesia. The primary outcome was $\mathrm{PO}$ pain and analgesic use in hospital and at home using validated pain scales (FLACC, CHEOPS, PPPM, TPPPS). FLACC and CHEOPS were used by two independent nurses to assess pain in the PACU and SDSU. TPPPS and PPPM were used by parents to assess pain at home within the first 24 hours postoperatively. Secondary outcomes included operative time (OpT), conversion and success rates, and complications at 6-8weeks. An intention-to-treat protocol (ITT)was followed.

Results: Of 1093 patients, 173 (15.8\%) were considered eligible and enrolled, with 14 patients withdrawing or being lost to followup. Of the159 patients who completed follow up, 81 had IO and 78 had SO (Fig. 1). No significant difference in mean operative time, pain score immediately following the surgery, and in-home administration of analgesic. PACU ibuprofen administration $(p<0.05)$ and SDSU administration of both morphine and Tylenol $(p<0.05)$ and TPPPS scores were significantly higher in patients who received inguinal orchidopexy $(p<0.05)$. The conversion rate was $29.5 \%$, with 18 of 79 SO requiring an additional incision. Of those converted SO, 13 of $18(72 \%)$ occurred in canalicular testes. The overall complication rate was $3.14 \%$, with one testicular atrophy, three re-ascents, and two wound infections.

Conclusions: $\mathrm{SO}$ is superior to $\mathrm{IO}$ on $\mathrm{PO}$ pain and analgesic consumption during the immediate recovery period but not in terms of OpT (ITT) or complications. However, SO may not be a suitable approach for all patients with canalicular testes.

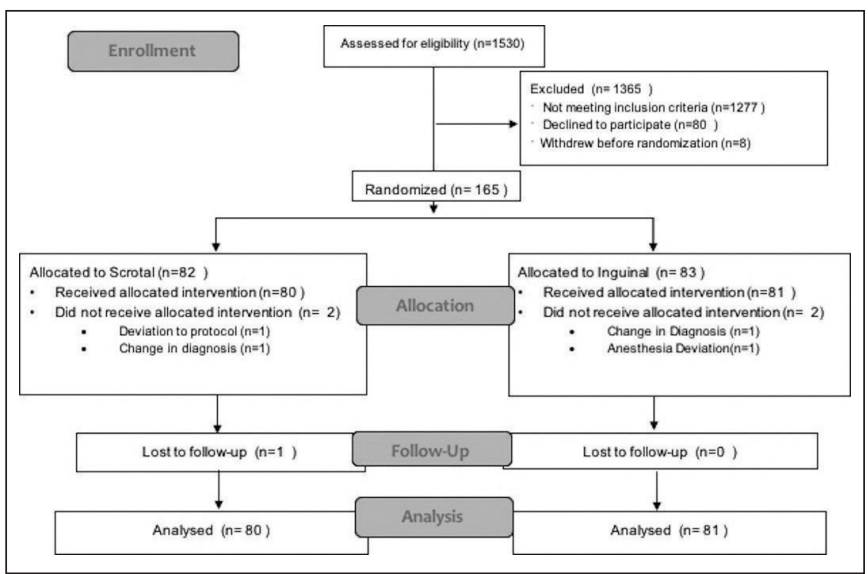

MP-12.13. Fig. 1. Eligibility and enrollment.

\section{MP-12.14}

Demystifying the diagnosis and treatment of vaginal yolk sac tumors in children: A comprehensive review of 127 cases

Bruce $\mathrm{Li}^{1}$, Adib Shamsuddin', Catherine Anne Lovatt ${ }^{2}$, Luis H. Bragat, ${ }^{1,2,3}$

${ }^{1}$ Michael G. DeGroote School of Medicine, McMaster University, Hamilton, ON, Canada; ${ }^{2}$ Division of Urology, McMaster University, Hamilton, ON, Canada; ${ }^{3}$ McMaster Pediatric Surgical Research Collaborative, McMaster University, Hamilton, ON, Canada

Support: McMaster Surgical Associates Grant. McMaster Children's Hospital Foundation

Introduction: Vaginal yolk sac tumors (vYSTs) are rare malignant germ cell tumors of infancy. While current literature is comprised of several

MP-12.14. Table 1. Summary of vaginal yolk sac tumors in published literature

\section{Characteristics}

Total number of cases reported

Presentation

Age of presentation (months)

Mean

Standard deviation

Presenting complaint, ${ }^{1} \mathrm{n}(\%)$

Vaginal bleeding

Passage of tissue

Fever

Urinary symptoms

Not stated

Diagnosis

AFP $(n g / m L)$

Standard deviation

10040.41

Not stated

18715.93

Diagnostic methods, n (\%)

Biopsy

CT

MRI

Tumor characteristics

Primary tumor location, $\mathrm{n}(\%)$

Distal third of vagina

Middle third of vagina

Proximal third of vagina

Two thirds of vagina or greater

Other ${ }^{2}$

Not stated

Tumor length $(\mathrm{cm})$

Mean

Standard deviation

Extra-vaginal spread, ${ }^{1} \mathrm{n}(\%)$

Pelvic spread

Lymphatic involvement

Distal metastasis

None

'Some cases presented with multiple features described. ${ }^{2}$ Includes anterior or posterior wall of the vagina. 


\begin{tabular}{|c|c|}
\hline \multicolumn{2}{|c|}{$\begin{array}{l}\text { MP-12.14. Table } 1 \text { (cont'd). Summary of vaginal yolk sac } \\
\text { tumors in published literature }\end{array}$} \\
\hline \multicolumn{2}{|l|}{ Treatment regimen } \\
\hline \multicolumn{2}{|l|}{ Non-surgical treatments, $\mathrm{n}(\%)$} \\
\hline Standalone chemotherapy & $44(34.7)$ \\
\hline Chemotherapy and radiation & $2(1.6)$ \\
\hline \multicolumn{2}{|l|}{ Treatments with partial vaginectomy, n (\%) } \\
\hline Partial vaginectomy with chemotherapy & $39(30.7)$ \\
\hline $\begin{array}{l}\text { Partial vaginectomy with chemotherapy and } \\
\text { radiation }\end{array}$ & $2(1.6)$ \\
\hline Standalone partial vaginectomy & $1(0.8)$ \\
\hline \multicolumn{2}{|l|}{ Other surgical treatments, $\mathrm{n}(\%)$} \\
\hline Radical surgery & $37(29.1)$ \\
\hline Unspecified surgery & $2(1.6)$ \\
\hline \multicolumn{2}{|l|}{ Total number of chemotherapy cycles } \\
\hline Study mean & 5.62 \\
\hline Study standard deviation & 3.15 \\
\hline \multicolumn{2}{|l|}{ Followup period } \\
\hline \multicolumn{2}{|l|}{ Number of months disease-free } \\
\hline Study mean & 50.41 \\
\hline Study standard deviation & 55.47 \\
\hline \multicolumn{2}{|l|}{ Mortality secondary to disease or intervention } \\
\hline \multicolumn{2}{|l|}{ Treatment group, n (\%) } \\
\hline Standalone chemotherapy & $1(0.8)$ \\
\hline Partial vaginectomy with chemotherapy & $1(0.8)$ \\
\hline Radical surgery & $7(5.5)$ \\
\hline
\end{tabular}

case reports, no studies to date have aggregated and summarized all these cases as a series. Herein, we reviewed the literature and compiled the characteristics of vYSTs.

Methods: A comprehensive literature review through MEDLINE (1946present), Embase (1974-present), and grey literature identified all case reports of vYSTs. Papers were reviewed and any cases misdiagnosed as other neoplasms were excluded. Univariate analysis compared baseline characteristics of treatment subgroups with respect to tumor size, age, extravaginal spread, alpha-fetoprotein (AFP), and cisplatin, etoposide and bleomycin (PEB) chemotherapy cycles. Multivariate linear regression was conducted to estimate change in tumor size using patient age and AFP. Multivariate Poisson regression modelled the number PEB cycles from age and tumor size. Multivariate logistic regression was conducted to predict mortality and recurrence using age, AFP, tumor size, and extravaginal spread.

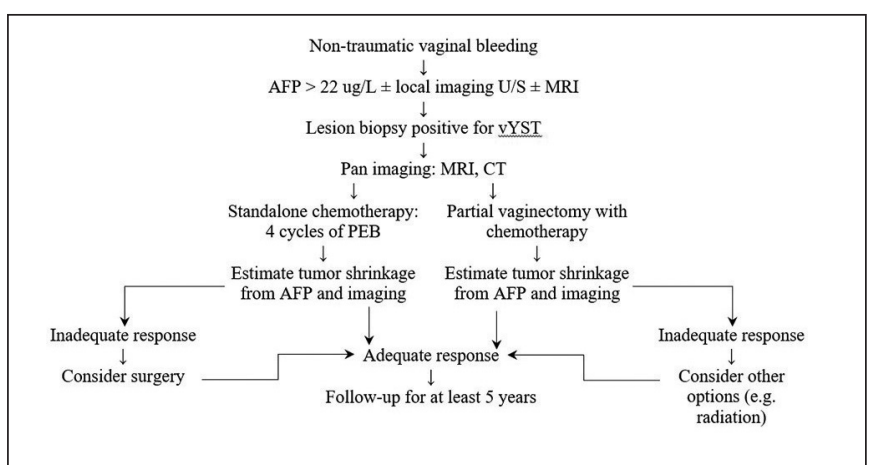

MP-12.14. Fig. 1. Tumor length as a function of AFP levels.
Results: A total of 127 cases were included (Table 1). Vaginal bleeding was the most common symptom presentation (95\%), at a mean age of presentation of 12.3 months ( \pm 6.4 months). Diagnosis was confirmed by biopsy in 125 cases $(98 \%)$ and magnetic resonance imaging (MRI) in 35 cases $(28 \%)$. Tumors were most frequently located in the proximal third of the vagina $(13 \%)$. The most frequent treatment was standalone chemotherapy (SC) (35\%), followed by partial vaginectomy with chemotherapy (PVC) (31\%). The rest had radical surgery performed prior to the 21 st century. Baseline characteristics between SC and PVC groups were similar in terms of age $(p=0.09)$, tumor size $(p=0.43)$, extravaginal spread $(p=0.99)$, and AFP $(p=0.89)$. The PVC group had 1.39 more cycles of PEB than the SC group $(p<0.05)$. AFP was found to be an independent predictor of tumor size $(p<0.001)$, adjusted for age such that a $10000 \mathrm{ng} / \mathrm{mL}$ increase in AFP predicted a $0.42 \mathrm{~cm}$ increase in tumor length. The likelihood of recurrence was independent of treatment type, age, AFP, tumor size, and presence of metastasis $(p>0.05)$. The likelihood of mortality was independent of treatment type, age, AFP, tumor size, presence of metastasis at diagnosis, and disease recurrence $(p>0.05)$. Fig. 1 is a flowchart that summarizes recommendations for clinicians.

Conclusions: Minimally invasive treatment in the form of SC or PVC have been the most common regimens since the 2000s. Since the baseline characteristics between SC and PVC were similar, treatment choice and recurrence were not affected by these characteristics. The likelihood of recurrence was independent of treatment, suggesting that either SC or PVC could be undertaken to maximize survival, albeit PVC patients received more chemotherapy than SC patients. AFP could be used to plan treatment strategy preoperatively in conjunction with $\mathrm{MRI}$, considering its predictive relationship to tumor size, and postoperatively to estimate tumor size change and relapse surveillance, reducing exposure to radiation and/or anesthesia for vaginoscopy.

\section{MP-12.15}

Initial experience of a two-attending surgeon model in the management of severe hypospadias

Loshua White ${ }^{1}$, Dawn L. MacLellan' ${ }^{1}$, Rodrigo Romao

'Urology, Dalhousie University, Halifax, NS, Canada

Introduction: Proximal hypospadias repair is associated with a high complication rate..$^{1-4}$ Two-surgeon models have been used in an attempt to improve outcomes..$^{5-7}$ Since 2013, our group has adopted a two-attending model to treat complex hypospadias cases. Our goal is to describe the outcomes of this two-surgeon approach.

Methods: A total of 42 patients (31 new cases) who underwent a twoattending surgeon hypospadias repair since 2013 were identified. The primary outcome of interest was postoperative complications requiring intervention. These were further divided into major (fistula repair/redo surgery) and minor (meatal/urethral dilatation). Perioperative data collected included: age, use of testosterone, degree of ventral curvature, location of meatus, and procedure. Postoperative outcomes assessed were: fistula, meatal stenosis, stricture, recurrent curvature, buried penis, and glans dehiscence. Three patients were excluded due to lack of followup.

Results: A total of 39 patients underwent 54 surgeries; 29 new cases and 10 re-dos. The complication rate requiring surgery was $30.7 \%$, with seven requiring major and five requiring minor additional procedures. The most common complications were: fistula $(n=10)$, glans dehiscence $(n=8)$, and meatal stenosis $(n=6)$. The 29 new cases of hypospadias were managed with 39 surgical procedures; 19 patients underwent a one-stage long-TIP repair (no to mild ventral curvature after degloving) and 10 with more severe curvature had a two-stage preputial graft repair. Complication rate for new cases was $27.5 \%$ (five major, three minor). A chi-squared test was performed comparing one and two-stage repairs. X2 $(1, n=39)=0.440$ $(p=0.507)$. We found no difference in complication rates between the one- and two-stage groups.

Conclusions: Our initial experience with limited numbers demonstrates favorable surgical outcomes for the two-attending model of hypospadias repair compared to published complication rates. Complication rates were comparable for one- vs. two-stage repairs for new cases. 


\begin{tabular}{lc}
\hline MP-12.15. Table 1. Postoperative outcomes & \\
\hline Number of cases (n=39) & $\mathbf{n}(\%)$ \\
\hline Meatal position & $0(0)$ \\
Perineal & $0(0)$ \\
Scrotal & $2(5.1)$ \\
Proximal & $0(0)$ \\
Midshaft & $7(17.9)$ \\
Coronal & $30(76.9)$ \\
Glanular & \\
Cosmesis & $7(17.9)$ \\
Poor & $5(12.8)$ \\
Acceptable & $20(51.3)$ \\
Good & $7(17.9)$ \\
Excellent & $6(15.4)$ \\
Recurrent curvature & $10(25.6)$ \\
Fistula & $5(12.8)$ \\
Meatal stenosis & $4(10.2)$ \\
Stricture & $1(2.5)$ \\
Repair breakdown & $3(7.7)$ \\
Buried penis & $8(20.5)$ \\
Glans dehiscence & \\
\hline
\end{tabular}

\section{References}

1. Long CJ, Canning DA. Proximal hypospadias: We aren't always keeping our promises. F1000Research, 2016:2379. https://doi. org/10.12688/f1000research.9230.1

2. MCNamara ER, Schaeffer AJ, Logvinenko T, et al. Management of proximal hypospadias with 2-stage repair: 20-year experience. J Urol 2015;194:1080-5. https://doi.org/10.1016/j.juro.2015.04.105

3. Long CJ, Chu DI, Tenney RW, et al. Intermediate-term followup of proximal hypospadias repair reveals high complication rate. J Urol 2016;197:852-8. https://doi.org/10.1016/j.juro.2016.11.054

4. Pippi Salle JL, Sayed S, Salle A, Bagli D, et al. Proximal hypospadias: A persistent challenge. Single institution outcome analysis of three surgical techniques over a 10-year period. J Ped Urol 2016;12:28. e1-28.e7. https://doi.org/10.1016/j.jpurol.2015.06.011

5. Snodgrass W, Bush N. Lessons learned from 20 years of TIP repair. Dialogues in Peds Urol 2014;3:3-9.

6. Snodgrass W, Bush N. Staged tubularized autograft repair for primary proximal hypospadias with 30-degree or greater ventral curvature. J Urol 2017;198:680-6. https://doi.org/10.1016/j.juro.2017.04.019

7. Ludwig AT, Inampudi L, O'Donnell MA, et al. Two-surgeon vs. single-surgeon radical cystectomy and urinary diversion: Impact on patient outcomes and costs. J Urol 2005;65:488-92. https://doi. org/10.1016/j.urology.2004.10.012 


\section{CUA ABSTRACTS}

\section{Author Index}

A

Aaron, Lorne E.

Abara, O. Emmanuel

Abara, Ogechi

Abbas, Loay

Abdul-Muhsin, Haidar

Adili, Anthony

Aditya, Ishan

Afshar, Kourosh

Ahmed, Ahmed S. Zakaria

Ajib, Khaled

Akatsuka, Jun

Al-Harbi, Badr

Al-Kutbi, Rusul

Al, Kait

Al, Kaitlin

Alaref, Amer

Albers, Patrick

Ali, Amna

Ali, Sohrab Naushad

Alibhai, Shabbir

Alinezhad, Saied

Allahdadian, Sepideh

Alsaikhan, Bader

Alwaal, Amjad

Amakawa, Ryo

Aminoltejari, Khatereh

Anderson, Kate

Anderson, Michelle

Anderson, Paul

Andkhoie, Mustafa

Andonian, Sero

Andrew, Macnab

Andrews, J. Matthew

Anscher, Mitch

Anson-Cartwight, Lynn

Antonelli, Jodi

Aprikian, Armen-G.

Aronson, Melyssa

Assmus, Mark

Atenafu, Eshetu

Aube-Peterkin, Mélanie

Avallone, Michael

Averch, Timothy

Awad, Mohannad

\section{B}

Bach, Thorsten

Bader, Gary

Bagley, Demetrius

Bagli, Darius J.

Bailly, Gregory G.

Bain, Alexandra

Bairati, Isabelle

Bakas, Vasiliki
MP-10.11

UP-3.19, UP-3.2

UP-3.2

MP-1.4, MP-1.5, MP-7.12, MP-7.8, MP-7.9

MP-7.13, MP-9.10

UP-1.21

MP-5.1

MP-11.10

MP-10.5, MP-4.13

MP-5.1, MP-8.6, UP-3.5

MP-5.10

MP-9.12

MP-12.6

MP-9.11

POD-1.6

MP-1.4

MP-9.7

MP-4.11, UP-1.20

MP-7.15

POD-4.5, UP-2.19, UP-2.20, UP-3.8

MP-8.15

MP-11.6

MP-6.1, MP-6.2, MP-9.14

MP-11.13

MP-5.10

MP-1.9

MP-2.11

MP-4.5, MP-4.6

MP-7.5

UP-1.28

MP-6.7, POD-1.4, POD-1.5

MP-11.7

MP-4.5, MP-4.6

MP-10.8

MP-3.13, MP-3.15

POD-1.5

MP-4.1, MP-7.10, UP-2.22, UP-2.23 Bilenky, Misha

MP-8.1

POD-4.1, POD-4.2

UP-1.18

MP-2.6, MP-5.12, MP-6.7, MP-7.10

MP-7.11

UP-2.5

POD-1.4, POD-1.5

$M P-11.13$

MP-7.14, MP-7.5

MP-10.12

MP-5.3

MP-12.8

MP-8.11, UP-2.15, UP-2.16

UP-1.9

UP-1.19, UP-1.22

MP-5.8
Baker, Amy

Bansal, Rahul K.

Bapat, Bharati

Barr, Richard E.

Barrett-Harlow, Brittani

Barton, Carly

Basappa, Naveen

Bauman, Glenn

Baverstock, Richard J.

Baxter, Nancy

Bayley, Conrad

Beaudry, Brianna

Beaudry, Marie-Michele

Becher, Edgardo

Bechis, Seth

Bedard, Philippe

Beech, Benjamin

Beiko, Darren T.

Bekkema, Jordan

Belanger, Eric

Bell, David

Bender, Jackie

Bergeron, Alain

Bergeron, Michelle

Berglund, Anders

Berjaoui, Mohamad Baker

Berlin, Alejandro

Bernacci, Carol

Bettan, Laurence

Beveridge, Tyler

Bhalla, Girima

Bhimani, Alisha

Bhindi, Bimal

Bhojani, Naeem

Bird, Vincent

Birk, Manjot

Bjazevic, Jennifer

Blachman-Braun, Ruben

Black, Peter C.

Blackhouse, Gord

Blanchard, Pierre

Blew, Brian D.

Bley, Derek

Bobrowski, Adam

Bochinski, Dariusz (Derek)

Boggs, Nathan

Bolduc, Stéphane J.

Bolton, Damien

Bonekamp, David

Bonneville, Scott

Booth, Christopher

Borelli-Bovo, Tiago
UP-3.10

MP-10.7, MP-4.9, UP-3.12

MP-10.12

MP-1.3

POD-3.1

MP-10.6

MP-10.14

POD-2.3

MP-10.6, MP-2.9

MP-8.2, MP-8.3

MP-9.1

MP-1.4, MP-1.5, MP-7.12, MP-7.8, MP-7.9

POD-3.3

MP-7.7

POD-1.4, POD-1.5

MP-3.13, MP-3.15, POD-4.6, UP-3.14

MP-5.13, UP-3.15

POD-1.3

MP-2.4, MP-2.5, POD-5.1

$\mathrm{MP}-3.12$

MP-8.11, UP-2.15, UP-2.16

UP-3.14

UP-2.27

$\mathrm{MP}-11.3$

POD-4.3

MP-5.1, MP-8.6, UP-2.24

MP-5.8, MP-8.6, MP-8.7, POD-2.4, POD -4.5

MP-6.4

MP-4.1

UP-3.13

MP-8.3

UP-2.24

MP-10.7, POD-2.6, POD-4.4

MP-6.5, MP-7.14, MP-7.3, MP-7.4, MP-7.5, MP-7.6, POD-1.1, POD-1.4, POD-1.5, POD-1.5, UP-1.1, UP-1.2

MP-10.12

POD-1.5

$\mathrm{MP}-4.2$

MP-1.11, MP-12.9, MP-3.6, MP-9.11, POD1.2, POD-1.6

MP-1.13, UP-2.9, UP-3.22

POD-4.1, POD-4.2

UP-1.21

MP-10.8

MP-11.9, MP-7.15

MP-1.3

MP-3.13, POD-2.4, UP-3.14, UP-3.18

MP-9.6

MP-11.13

MP-11.3, MP-12.10

MP-1.6, UP-3.10

UP-1.24

MP-1.4, MP-1.5

MP-8.5, POD-1.3

MP-7.7 
Boyle, Shawna L. Bracken, R. Bruce Braga, Luis $\mathrm{H}$.

Breau, Rodney $\mathrm{H}$.

Bremner, Karen

Brisson, Hervé

Brotherhood, Hilary L.

Buckley, Roger J.

Bui, Quang Loc

Burden, Liz

Burtnyk, Mathieu

Burton, Jeremy

Buteau, François-Alexandre

\section{C}

Cadogan, Kianna

Cafazzo, Joseph

Cagiannos, Ilias

Cambronero Santos, Javier

Camille-Ariane, Philie

Campeau, Lysanne

Canil, Thomas

Canvasser, Noah

Capolicchio, J.-P.

Carillo, Brian

Carlson, Kevin V.

Carmel, Michel

Carr, Lesley

Carrera, Raphael

Carrier, Marc

Carrier, Serge

Carrillo, Brian

Carroll, Peter

Casey, Richard W.

Cash, Hannes

Catton, Charles

Caumartin, Yves

Cavey, Colin

Cepero, Virna

Chadwick, Dianne

Chan, Ernest

Chan, Justin

Chapman, David W.

Charbonneau, Camille

Charles, David

Chehroudi, Cyrus

Chen, Eric

Cheney, Scott

Cheon, Paul

Chetner, Michael P.

Cheung, Douglas C.

Cheung, Patrick

Chew, Ben H.

Chi, Thomas

Chin, L.K. Joseph

Chiura, Allen

Cho, Angela

Choiniere, Roselyne

Choudhury, Nurul

Chu, Will

Chua, Michael
MP-4.7, UP-1.11, UP-1.14, UP-1.17

MP-8.8

MP-12.13, MP-12.14

MP-10.3, MP-5.6, MP-5.9, MP-8.9,

POD-4.4, UP-1.15, UP-2.18, UP-2.21,

UP-3.11, UP-3.9

UP-2.20

MP-4.8

MP-10.6

MP-4.3, MP-4.4

MP-3.1

MP-5.5, MP-5.7

UP-1.24

MP-9.11, POD-1.6

MP-4.8

MP-6.6

MP-5.8

MP-5.6, MP-5.9, MP-8.9, UP-1.15,

UP-2.21

POD-3.5

POD-3.3

POD-3.2

MP-6.10

POD-1.4, POD-1.5

MP-12.4, MP-12.5, UP-3.17

MP-6. 1

MP-10.6, MP-2.9, POD-3.2

MP-4. 1

$\mathrm{MP}-2.12$

MP-7.13

MP-5.9

MP-5.12, MP-6.7, MP-7.10, MP-7.11

MP-6.1, MP-6.2

POD-2.2

UP-2.6

MP-7.7

MP-10.8, MP-8.6, MP-8.7

UP-1.19

UP-3.20

MP-10.11

MP-10.1

MP-12.9

MP-1.12, MP-5.3

MP-1.10, MP-12.11, MP-2.5, UP-3.15,

UP-3.16

MP-10.6

POD-3.6, UP-2.5

MP-11.10

MP-10.1

MP-7.13

UP-1.21

MP-10.14

MP-8.12, MP-8.7, UP-2.19, UP-2.20,

UP-3.8

UP-1.13

MP-1.12, MP-1.2, MP-1.9, MP-5.3, POD-

1.4, POD-1.5

POD-1.4, POD-1.5

MP-10.10, MP-4.12, POD-2.3, UP-1.24,

UP-1.26

MP-5.3

MP-11.11, MP-5.5, MP-5.7

POD-3.3

POD-3.5

UP-1.13

MP -12.2
Chughtai, Bilal

Chun, Stacia

Chung, Hans

Chung, Peter

Ciezki, Jay

Cindolo, Luca

Clark, Roderick

Clarke, Hance

Cloutier, Jonathan

Cohen, Zane

Coles, Jenna

Comperat, Eva

Coriaty, Rhea

Couture, Félix

Cox, Ashley R.

Cox, Lindsey

Cozma, Adrian

Crook, Juanita

Crump, Trafford

Cussenot, Olivier

D

Dadoun, Simon

Dahm, Philipp

Dalla Nora, Sergio

Danechi, Alexander

Danielson, Brita

Dave, Sumit

Davidson, Jacob

Dawidek, Mark

De los Reyes, Thomas J.

De, Shubhadip (Shubha) K.

Dean, Brad

Dean, Nick

Decotiis, Keara

DeLong, Jessica

Demitor, Devin

Dere, Patricia

Dergham, Ali

Desai, Mihir

DeZeeuw, Jasmine

Dharma, Christopher

Dhillon, Jasreman

Di Lena, Michael A.

Di Lena, Richard D.

Di Meo, Amelia

Diabaté, Lamoussa

DiCesare, Robert

DiFede, Darcy

Ding, Maylynn

Ding, Youshan

Dinney, Colin

Diong, Christina

Doiron, R. Christopher

Donovan, James

Doumouras, Aristithes

Dozar, Alaina

Drachenberg, Darrel E.

Dragomir, Alice

Dubrowski, Adam

Dujardin, Thierry

Duplisea, Jon
MP-7.5

POD-2.1

UP-1.13

MP-3.13, MP-3.15, MP-8.6, MP-8.7 POD-2.4, POD-4.5, POD-4.6, UP-3.14 MP-10.8

MP-7.7

POD-5.5, MP-3.14

UP-3.8

MP-12.10

MP-8.1

UP-2.10

MP-3.1

UP-1.2

MP-4.13, MP-7.7, MP-7.2, POD-2.5,

UP-3.11, UP-1.2

MP-2.11, MP-8.11, UP-2.10

POD-3.1

MP-4.11, UP-1.20

MP-10.8

MP-10.6

MP-3.1

UP-3.22

MP-6.12

MP-10.11

MP-11.10

MP-10.14

MP-12.12, MP-12.7, MP-12.9, POD-1.2, POD-5.4

MP-12.7, MP-12.9, POD-5.4

MP-5.4

UP-2.6, UP-2.8

MP-1.10, MP-5.11, MP-9.1, MP-9.13, MP-9.5, MP-9.6, MP-9.7, MP-9.9,

UP-1.10

MP-2.13

MP-9.7, UP-1.10, UP-3.3

UP-3.18

MP-2.6

MP-1.10

MP-1.3

MP-11.9

MP-7.4, MP-7.5, POD-1.1

UP-3.25

UP-2.19

POD-4.3

POD-1.3

MP-9.12

MP-5.8

MP-4.1

MP-12.8

UP-3.22

MP-6.12

MP-12.7, MP-12.9

$\mathrm{MP}-8.14$

UP-3.8

MP-11.4, MP-2.13, POD-3.2, POD-5.2

MP-4.10

MP-11.12

UP-3.1

MP-10.7, MP-4.9, MP-6.6, UP-3.12, UP-3.9

UP-1.23, UP-2.22, UP-2.23

UP-3.25

UP-1.19, UP-1.22

MP-8.11 
During, David

Duschesne, Thierry

Dushinski, John W.

Dutka, Christopher

Dwyer, Neil T.

\section{E}

Edison, Eric

El Hajj, Albert

El-Hakim, Assaad

El-Haouly, Abir

El-Rami, Hares

El-Sherbiny, Mohamed

Elliott, Sean

Elmansy, Hazem

Elterman, Dean S

Endo, Yuki

Eng, Amanda

Erlich, Annette

Espino, Gustavo

Espinosa, Victor

Estrella, Rafael

Evans, Howard J.

Everett, Ross

\section{F}

Fairey, Adrian

Fallah-Rad, Nazanin

Farcas, Monica

Farhat, Walid A.

Fast, Andrew

Feifer, Andrew $\mathrm{H}$.

Ferguson, Thomas

Fergusson, Dean

Fernando, Sean

Ferrara, Sarah R.

Ferrari, Giovanni

Fersovich, Jordana

Fervaha, Gagan

Feuerstein, Michael

Filler, Guido

Finelli, Antonio

Flaman, Ana

Flax, Stanley

Fleshner, Neil E.

Fodness, Stephanie

Fok, Kai

Foley, Steve

Follett, Graeme

Follett, Graham

Forster, Adam

Foss, Halle

Fradet, Samuel

Fradet, Vincent

Fradet, Yves
MP-12.8

UP-1.19

MP-1.3

MP-6.6

MP-10.11, POD-3.2

POD-4.1, POD-4.2

MP-7.5

MP-10.5, MP-4.13, POD-2.5

UP-1.23

UP-1.23

MP-12.4, MP-12.5, UP-3.17

MP-2.6

MP-1.4, MP-1.5, MP-5.12, MP-7.12, MP-7.8, MP-7.9, UP-2.3

MP-6.5, MP-7.14, MP-7.3, MP-7.4, MP-7.5, MP-7.6, POD-1.1, POD-3.2,

UP-1.1, UP-1.2,

UP-2.6

MP-5.10

MP-1.13, UP-1.5, UP-2.9

MP-8.6

MP-2.6

MP-4.2

MP-2.6

MP-5.13

POD-3.6, UP-2.5

MP-10.14, POD-4.4, UP-3.11, UP-3.3, UP-3.9

MP-8.12

MP-11.12, MP-6.1, MP-6.10, MP-6.2,

MP-6.3

MP-12.2

MP-6.6

MP-4.11, UP-1.20

MP-10.7, MP-4.9

MP-5.9, UP-2.18

MP-6.6

MP-2.1, MP-2.10, MP-2.12, MP-2.8,

POD-3.1, POD-5.3, UP-2.4

MP-7.7

MP-2.2

UP-3.5

MP-1.14

MP-12.9, POD-1.2

MP-5.1, MP-5.8, POD-2.3, POD-2.4

POD-4.4, UP-1.18, UP-2.19, UP-2.20,

UP-3.11, UP-3.8, UP-3.9

UP-2.18

MP-4.3, MP-4.4

MP-10.1, MP-10.11, MP-10.12, MP-5.1, MP-8.12, MP-8.6, MP-8.7, POD-2.4 UP-1.18,

UP-2.24, UP-3.5

MP-9.5

MP-6.2

POD-3.5

MP-10.14

UP-3.3

MP-12.12

UP-2.5

UP-1.22

MP-4.1, UP-1.19, UP-1.22, UP-2.27

MP-4.1, UP-1.19, UP-1.22, UP-2.27
Frank, Steven

MP-10.8

French, Christopher G. $\quad$ UP-2.1

G

Gabara, Adam

Gabrigna Berto, Fernanda

Gaitonde, Krishnanath

Gallagher, Kevin

Gallinger, Steven

Gan, Ailsa

Gandhi, Karan

Gandhi, Shreyas

Gao, Bruce

Gao, Chuanyu

Garg, Saurabh

Gettman, Matthew

Ghai, Sangeet

Ghiculete, Daniela

Gilbert, Scott

Gilling, Peter

Gillis, Charlie

Glase, Zoe

Gobert, Aurelien

Golda, Nicole

Goldenberg, S. Larry

Gomes, Tara

Gorin, Michael

Goudie, Christine

Govindasamy, Natasha

Guan, Tianyuan

Guertin, Marie-Hélène

Guo, Yanbo

Guralnick, Michael

Guy, David

Guyatt, Gordon

H

Haan, Michael

Hafron, Jason

Hagerty, Marlon

Hahn, Ezra

Haider, Masoom

Hairston, John

Haj-Hamed, Monzer

Hajek, David

Hajiran, Ali

Hamilton, Robert J.

Hanna, Kevin

Hanna, Wael

Hansen, Aaron

Harasemiw, Oksana

Hare, Joshua

Harper, Jonathan

Harriman, David I.

Harvey, David R.

Hasegawa, Yuya

Hatiboglu, Gencay

Hayashi, Tatsuro

Hayashi, Tetsutaro

Hearn, Jason

Hemminki, Otto

Heng, Daniel

Hengel, Alexander

Hermanns, Thomas

Herrera Caceres, Jaime
MP-3.8, MP-6.9

POD-1.2

MP-4.10

POD-4.1, POD-4.2

MP-8.1

MP-5.6

POD-5.4

MP-3.3

UP-3.18

POD-4.1, POD-4.2

MP-11.6

UP-2.12

MP-10.1, MP-5.8

MP-9.14

POD-4.3

POD-1.1

MP-4.5, MP-4.6, UP-2.1, UP-2.2

UP-2.24

MP-3.1

MP-4.3, MP-4.4

MP-7.4, POD-1.1

UP-3.8

POD-2.2

UP-3.25

MP-3.9

MP-4.10, UP-1.16

UP-1.19

MP-5.2

POD-3.6, UP-2.5

$\mathrm{MP}-4.12$

POD-3.3

MP-3.14, MP-3.4

UP-1.24

POD-2.3

MP-3.15, POD-4.6, UP-3.14

MP-5.8

POD-3.5

MP-4.10

$\mathrm{MP}-4.4$

POD-4.3

MP-10.1, MP-3.13, MP-3.15, MP-5.1, POD-2.4, POD-4.6, UP-1.18, UP-3.14 MP-4.7, UP-1.14, UP-1.17

UP-1.21

MP-10.12, MP-3.13, MP-3.15, POD-4.6,

UP-3.14

MP-10.7, MP-4.9, UP-3.12

UP-3.22

POD-1.4, POD-1.5

UP-3.20

UP-2.1

MP -5.10

UP-1.24

MP-5.10

MP-3.10

UP-2.1, UP-2.2

MP-8.1

UP-3.11

MP-9.5

MP-8.2, MP-8.3, MP-8.4, POD-4.5

MP-5.1, MP-8.6, UP-3.5 
Herschorn, Sender

Hersey, Karen

Hetou, Khalil

Hewitt, Richard

Hickling, Duane R.

Hird, Amanda

Hirst, Martin

Ho, Brian

Ho, Louisa

Hoag, Nathan

Hoare, Dylan

Hobart, Michael G.

Hodhod, Amr

Hodotsuka, Naoto

Hoge, Connor

Holloway, Michael

Honey, R.J.

Hong, Dennis

Hoogenes, Jen

Horrigan, Michael

Hosier, Gregory

Hovington, Hélène

Hoy, Nathan

$\mathrm{Hu}$, Jason

Humphreys, Mitchell

Hung, Pui Long Wilson

Hurwitz, Barry

Hylton, Daniell

\section{I}

Ibrahim, Ahmed

Ikeda, Kenichiro

Ilie, Gabriela

Ischia, Joseph

Isserlin, Ruth

\section{J}

Jack, Greg

Jacobs, Lourens

Jacobsen, Niels-E. B.

Jamal, Munir A.

Javidan, Ashkan

Jebanesan, Aravinth

Jednak, Roman

Jefferies, Matthew

Jenkins, Danielle

Jewett, Michael

Jia, Ray

Jiang, Jinfeng

Jiang, Jing

Jivraj, Naheed

Johnstone, Jeannette

Jonat, Lee $M$.

Jordan, Gerald

Joshi, Kush

Joshua, Anthony

Julien, Pierre

Jung, Jae

Juvet, Tristan
MP-11.2, MP-2.1, MP-2.10, MP-2.12, MP-2.7, MP-2.8, POD-3.1, POD-3.2, POD-3.5,

POD-5.3, UP-2.4

MP-10.1, UP-2.24

MP-3.14

MP-4.5, MP-4.6

MP-8.9

MP-8.12, MP-8.7, UP-1.3, UP-2.20,

UP-2.4

MP-10.12

POD-2.1

MP-5.4, UP-2.7

MP-4.2

MP-2.3, MP-2.4

UP-1.9

MP-1.4, MP-1.5, MP-12.4, MP-12.5, MP-7.12, MP-7.8, MP-7.9, POD-2.5,

UP-3.17

MP-5.10

MP-4.10, UP-1.16

UP-2.6

MP-9.8

MP-11.12

MP-6.4, MP-9.12, POD-5.5, UP-1.21,

UP-1.8

UP-1.15

POD-1.3

MP-4.1, MP-4.8, UP-1.19, UP-2.27

MP-2.3, MP-5.11, UP-3.16

UP-2.22, UP-2.23

MP-7.13, MP-9.10

POD-2.1

UP-3.22

UP-1.21

MP-5.12, MP-6.7, MP-7.10, MP-7.11

MP-3.10

UP-2.13, UP-2.15, UP-2.14, UP-2.16

UP-3.10

MP-10.12

MP-1.6

UP-3.12

UP-3.3

MP-4.11, UP-1.20

MP-4.11, UP-1.20

UP-3.5

MP-12.4, MP-12.5, UP-3.17

POD-4.1, POD-4.2

MP-2.11

POD-4.6. MP-3.13, MP-3.15, UP-3.14

MP-4.12

UP-1.11

MP-10.6

UP-3.8

MP-6.9

MP-10.11

MP-2.6

MP-4.12

MP-10.12

MP-4.1, UP-1.19

MP-6.12

UP-2.12

\section{K}

Kadam, Rutuja

Kaler, Kamaljot (Kam) S.

Kaplan, Steven

Kapoor, Anil

Karakiewicz, Pierre

Kasivisvananthan, Veeru

Kassam, Zahra

Kasson, Matt

Kavoussi, Louis

Kelly, Kerri-Lynn

Kenk, Miran

Kenna, Tony

Keyes, Mira

Khadhouri, Sinan

Khalil, Ahmed

Khan, Asher

Khehra, Keesha

Khurram, Najia

Kiddoo, Darcie A.

Kim, Jin Kyu (Justin)

Kim, John

Kim, Kevin

Kimura, Go

Kinskey, Jacob

Kirkland, Susan

Kirsch, Andrew

Kirubarajan, Abirami

Klaassen, Zachary W.

Klausner, A

Klotz, Laurence $\mathrm{H}$.

Knee, Christopher

Kobatake, Kohei

Kodama, Ronald T.

Kokorovic, Andrea

Kolinsky, Michael

Komisarenko, Maria

Kondo, Yukihiro

Koo, Sylvia

Koschel, Samantha

Kotb, Ahmed

Koyle, Martin A.

Kozel, Zachary

Koziarz, Alex

Krahn, Murray

Krakowsky, Yonah

Kristy, Rita

Kubay, Cole

Kuhathaas, Kopika

Kuk, Cynthia

Kulendran, Saranya

Kulkarni, Girish S.

Kumar, Ravi

Kushnir, Igal

Kuzbel, Jake

Kwong, Jethro

Kwong, Justin S.

L

Labib, Yasser

Lacasse, Anaïs

Lachance, Gabriel
POD-3.4

MP-1.3

MP-7.4, POD-1.1, POD-3.5

MP-10.11, MP-5.2, POD-2.3, POD-4.4,

UP-3.9

MP-10.5, MP-4.13, POD-2.5

POD-4.1, POD-4.2

MP-3.6, UP-1.24

UP-1.16

MP-1.14

MP-2.13

MP-10.11, UP-2.24

MP-8.15

MP-10.8

POD-4.1, POD-4.2

MP-3.1

MP-10.6

MP-11.11, MP-5.5, MP-5.7

MP-10.1

UP-3.16

MP-12.2, UP-1.18

MP-12.13

POD-5.5

MP-5.10

POD-4.3

UP-2.13

MP-12.6

MP-4.3, MP-4.4

POD-2.6

MP-11.7

POD -2.3

UP-2.21

MP-3.10

MP-2.1, UP-2.4

MP-8.14

MP-10.14

UP-2.19, UP-2.20, UP-3.8

MP-5.10

MP-6.1, MP-6.2

UP-1.29

MP-1.4, MP-1.5, MP-7.12, MP-7.8, MP-7.9, UP-2.3

MP-11.12, MP-12.2, MP-12.6, MP-12.8, UP-3.18

POD-5.6, MP-1.14

MP-3.3, MP-9.14

UP-2.20

MP-11.12, MP-11.14, MP-11.15

POD-3.5

MP-6.6

MP-3.15, POD-2.4, POD-4.6, UP-3.14

MP-8.1

UP-2.24

MP-5.1, MP-8.12, MP-8.2, MP-8.3, MP-8.4, MP-8.6, MP-8.7, POD-2.4, POD-4.5, UP-1.18, UP-2.19, UP-2.20, UP-3.5, UP-3.8

MP-10.3

UP-2.21

MP-11.13

MP-4.11, UP-1.20

MP-9.8

MP-1.4, MP-1.5

UP-1.23

UP-2.27 
Lacombe, Louis

LaGrange, Chad

Lai, Enoch

Lam, Wayne

Lamarche, Benoit

Lamontagne, Valerie

Landman, Jaime

Landoni, Lauren

Landowski, Truman

Lange, Dirk

Langille, Gavin

Lantz Powers, Andrea G.

Lattouf, Jean-Baptiste

Lavallée, Luke T.

Lavi, Arnon

Laviana, Aaron

Lavoie, Callum

Law, Kyle

Lawen, Joseph

Lawen, Tarek

Lawrentschuk, Nathan L.

Lee, Jason

Lee, Lui Shiong

Lee, Taeweon

Lee, Yung

Legault, Benjamin

Léger, Caroline

Levasseur-Fortin, Patrice

Levy, Maurice

Lewis, John

Li, Bruce

Li, Kathy

Li, Roger

Li, Zack

Liandier, Frédéric

Lim, Amy

Lim, Caitlin

Lim, Jonathan

Lingeman, James

Liodakis, Peter

Liu, Bonnie

Liu, Ning

Liu, Stanley

Liu, Wei

Loblaw, Andrew

Locke, Jennifer A.

Lodde, Michele

Longo, Joseph

Lorenzo, Armando J.

Lovas, Mike

Lovatt, Catherine Anne (Kate)

Lovrics, Olivia

Luckenbaugh, Amy

Lundeen, Colin

Luo, Jack

Luong, Kathy

Lusty, Avril J.

Luu Ly, Tina

Lyman, Katie

Lynch, Mary
UP-1.19, UP-1.22

UP-1.11

MP-4.4

POD-2.1

MP-4. 1

UP-3.2

POD-1.4, POD-1.5

POD-4.6, MP-3.13, UP-3.14

POD-3.6

MP-1.9

UP-3.22

UP-1.7

UP-3.9

MP-10.3, MP-5.6, MP-5.9, MP-8.9,

POD-2.3, POD-4.4, UP-1.15, UP-2.18,

UP-2.21, UP-3.11, UP-3.9

MP-10.10, MP-3.14, MP-3.4, MP-4.12,

UP-1.26, UP-3.9

POD-2.6

MP-9.13, MP-9.9

MP-4.13, MP-6.5, MP-7.7, MP-10.5,

POD-2.5, UP-1.2

UP-2.15, UP-2.16

UP-2.16

UP-3.10

MP-3.3, MP-5.1, MP-6.3, MP-9.8

MP-10.2

POD-4.1, POD-4.2

MP-11.12

POD-3.3

UP-1.19

MP-6.5

UP-3.19

MP-3.9

MP-12.14

MP-8.2, MP-8.3, MP-8.4, POD-4.5

POD-4.3

MP-1.13, MP-11.14

UP-1.23

MP-8.14

POD-3.1

POD-1.4, POD-1.5

MP-7.14

UP-2.11

MP-11.8

MP-8.2, MP-8.3, MP-8.4, POD-4.5

UP-1.13

POD-2.3

MP-4.3, MP-4.4, UP-1.13

MP-11.6, MP-11.7, MP-2.1, MP-2.10,

MP-2.12, MP-2.8, POD-3.1, POD-3.2,

POD-5.3,

UP-2.4

UP-1.19, UP-1.22

MP-10.1

MP-12.2, MP-12.6

MP-5.8

MP-6.4, MP-12.14, POD-5.5

MP-11.12

POD-2.6

MP-1.2

POD-1.5

UP-3.1

MP-10.3, MP-7.1, MP-8.5

MP-3.14, MP-3.4

UP-3.20

MP-2.11
M

MacDonald, Morgan

Maciejewski, Conrad

MacKenzie, Kenneth

MacLellan, Dawn L.

Macnab, Andrew

MacNeily, Andrew E.

Magee, Diana E.

Major, Nicholas

Mak, Victor

Mallick, Ranjeeta

Maloni, Ranjena

Mamdani, Muhammad

Manley, Brandon

Mann, Uday

Mannas, Miles

Marien, Arnaud

Marshall, Kaitlin

Martell, Kevin

Martin, Lisa

Mason, Ross

Masoomian, Mehd

Matsubara, Akio

Matsumoto, Edward D.

Matthew, Andrew

Maynor, Sean

McAlpine, Kristen

McArthur, Eric

McGirr, Samantha

McGrath, John

McGrath, Melissa

McGregor, Thomas

McGriff, Sarah

McKeown, Martin

McLarty, Ryan

McLeod, Deborah

McMahon, Amber

McMartin, Catherine

McVary, Kevin

Merrick, Gregory

Meskawi, Malek

Metcalfe, Charles B.

Metcalfe, Peter D.

Metro, Mike

Metser, Ur

Mikami, Hikaru

Mikhail, David M.

Millar, Jeremy

Minaguchi, Shigehito

Ming, Jessica

Minotti, Simona

Misraï, Vincent

Moalwi, Adel

Mohamedali, Zeid

Mokkapati, Sharada

Moodie, Louise

Moon, Danie

Moore, Courtney

Moore, Jonathan

Moore, Sacha

Moralis Frost, Sophia

Morash, Christopher G.

Moretti, Myla
MP-8.11, UP-1.7

POD-3.2, UP-3.7

POD-4.1, POD-4.2

MP-12.15

MP-11.6

MP-11.10, MP-12.1

MP-8.12, MP-8.7, UP-1.3, UP-2.20

POD-3.1

MP-10.11

MP-5.6, MP-8.9, POD-4.4, UP-2.18,

UP-3.9

UP-3.9

MP-8.2, MP-8.3

POD-4.3

MP-1.13, MP-6.8, UP-2.9

POD-4.1, POD-4.2

UP-1.19

UP-3.13

UP-1.13

UP-2.19, UP-2.20, UP-3.8

MP-8.11, UP-1.7, UP-2.10, UP-2.15,

UP-2.16

MP-10.1

MP-3.10

MP-5.2, MP-6.4, MP-9.12, POD-5.5, UP-1.21, UP-1.8

UP-2.17

MP-4.10, UP-1.25

MP-5.9, MP-7.15

MP-11.5

UP-1.11

POD-4.1, POD-4.2

MP-12.13

POD-1.3

MP-11.14, MP-11.15

MP-11.6

MP-5.13, MP-9.7, MP-9.9

UP-2.17

MP-4.7, UP-1.14, UP-1.17

MP-12.10

POD-3.5

MP-10.8

MP-10.5, UP-2.12

MP-1.3

MP-12.11, POD-3.4, UP-3.16

POD-3.1

POD-2.3, POD-2.4

MP-5.10

MP-1.14, POD-5.6

MP-10.8

MP-5.10

MP-12.2

MP-4.11, UP-1.20

MP-6.5, MP-7.5, MP-7.7, MP-7.14, UP-1.2

UP-1.8

MP-10.11

MP-8.14

UP-2.13

UP-1.29

POD-5.5, UP-1.8

MP-7.13, MP-9.10

POD-4.1, POD-4.2

$M P-4.12$

MP-5.6, MP-5.9, MP-8.9, UP-1.15, UP-2.21

MP-12.8 
Morin, Melanie

Morris, Michael

Morton, Gerard

Mourtzinos, Arthur

Moussa, Hanane

Moussaoui, Ghizlane

Moynihan, Matthew

Mueller-Wolf, Maya

Mukherjee, Rupayan

Mule, James

Mullen, Peter

Murphy, Declan

\section{$\mathbf{N}$}

Nadeau, Geneviève

Naffouje, Rand

Nair, Shiva

Nakada, Stephen

Nambiar, Arjun

Narayan, Vikram

Nason, Gregory

Nassau, Daniel

Nathens, Avery

Navai, Neema

Navaratnam, Roshan

Nayak, Ameeta

Nayak, Jasmir (Jay) G.

Nayan, Madhur

Neff, Donald

Negrean, Cristina

Network, BIOCaPPE

Neveu, Bertrand

Nevo Pima, Amihay

$\mathrm{Ng}$, Ada

Nguan, Christopher Y.

Nguile-Makao, Molière

Nguyen, David-Dan

Nguyen, Susan

Nichols, Paige

Nickel, J. Curtis

Nielson, Matthew

Nigro, Mark K.

North, Scott

Noureldin, Yasser

Nourieh, Maya

Nozaki, Shuhei

O

O'Connor, Ellen

$\mathrm{O}^{\prime}$ Connor, R. Corey

O'Kelly, Fardod

O'Malley, Martin

Oake, Justin

Oquendo, Fabiola

Ordon, Michael

Organ, Michael K.

Ory, Jesse

Ouellette, Paul Jr.

Ouizzane, Soukaina
POD-3.3

POD-2.2

UP-1.13

POD-3.1

MP-4. 1

MP-7.2

POD-3.1

UP-1.24

MP-4.3, MP-4.4

POD-4.3

MP-10.1

UP-1.29

MP-11.3, MP-2.7, POD-3.2

MP-4.10, UP-1.16

MP-10.10, MP-3.14, MP-3.4, MP-4.12,

UP-1.24, UP-1.26, UP-3.13

POD-1.4, POD-1.5

POD-4.1, POD-4.2

MP-8.14

MP-3.15, MP-8.6, UP-1.18

MP-1.14

MP-2.1

MP-8.14

MP-2.1, MP-2.10

POD-4.4, UP-2.18

MP-10.7, MP-4.9, MP-6.8, UP-3.12

UP-3.8

MP-7.13

MP-4.13, MP-7.2, MP-10.5, POD-2.5

MP-4.1

MP-4.8

MP-7.13, MP-9.10

POD-2.1

MP-11.11, MP-5.4, MP-5.5, MP-5.7,

UP-3.20

MP-4.1, UP-1.19

MP-10.5, MP-4.13, MP-6.5, MP-7.14, MP-7.2, MP-7.6, MP-7.7, POD-1.5,

POD-2.5, UP-1.2

UP-2.24

UP-2.12

MP-2.13, MP-7.1

POD-4.1, POD-4.2

MP-11.6

MP-10.14

MP-6.7

MP-3.1

MP-5.10

UP-1.29, UP-3.10

POD-3.6, UP-2.5

MP-12.6, UP-3.18

MP-3.15, MP-3.13

MP-10.7, MP-4.9

MP-1.4, MP-1.5, MP-7.12, MP-7.8, MP-7.9

MP-9.14, MP-9.8

UP-2.1, UP-2.2, UP-3.25

UP-3.22

MP-10.11

UP-2.23
$\mathbf{P}$

Pace, Kenneth T.

MP-9.14

UP-1.24

POD-1.5

MP-5.8

MP-4.11, UP-1.20

UP-1.21

UP-1.7

MP-1.13, MP-11.14, MP-11.15, MP-6.8,

UP-1.5, UP-2.9, UP-3.23

MP-7.15

MP-7.4, POD-1.1

MP-4.10

POD-2.2

MP-3.6

UP-1.19, UP-1.22

MP-4.4

MP-10.1

POD-1.4, POD-1.5

UP-1.19

MP-5.1, MP-5.8, POD-2.4, UP-1.18

MP-10.12

MP-11.2

MP-12.4, MP-12.5, UP-3.17

MP-8.2, MP-8.3, MP-8.4, POD-4.5

MP-2.1

MP-2.6

POD-2.2

MP-9.11

POD-4.3

MP-8.14

MP-4.8, POD-2.2, POD-4.4, UP-1.19, UP-1.22, UP-3.9

POD-4.3

MP-3.14, MP-3.4, UP-3.13, UP-3.9

UP-2.1, UP-2.2

POD -3.6

UP-3.22

POD-2.2

POD-2.2

MP-1.4, MP-1.5, MP-7.12, MP-7.8, MP-7.9, UP-2.3

MP-8.2, MP-8.3, MP-8.4, POD-4.5

MP-5.1, UP-2.24

POD-3.1

UP-1.19

UP-1.3, UP-2.8

MP-11.14, MP-11.15, UP-3.22

POD-3.1

POD-5.5

UP-2.12

MP-3.9

MP-11.12, MP-12.2, MP-12.8

MP-10.14

MP-10.8

MP-1.11, MP-9.11, POD-1.2, POD-1.6

MP-12.6, MP-8.1

MP-11.4, MP-2.3, POD-3.2

POD-3.3

MP-11.8

MP-7.7

UP-1.24

UP-2.4 
Ren, Runhan

Rendon, Ricardo

Reynolds, Luke Richard, Patrick Richstone, Lee

Rijo, Enrique

Rijo, Esteban

Ringuette Goulet, Cassandra

Rittenberg, Lauren

Robinson, John

Robitaille, Karine

Rodrigues, Craig

Rodrigues, George

Rodriguez Lay, Ramón

Roehrborn, Roehrborn

Roethke, Matthias

Rolef, Jennifer

Romao, Rodrigo

Romsa, Jonathan

Roshan, Aishwarya

Ross, James

Roth, Beat

Rourke, Keith F.

Rovner, Eric

Rowe, Neal E.

Rowe, Stephen

Rudzinski, Jan K.

Rutledge, Rob

\section{$\mathbf{S}$}

Saad, Fred

Saadat, Paria

Saarela, Olli

Saavedra, Alvaro

Sadri, Iman

Saini, Jaskirat

Saldana, Carolina

Saltel, M. Eric

Saltzman, Russell

Samarasinghe, Natasha

Sanders, Jeremiah

Sano, Yujiro

Saperstein, Lawrence

Saranchuk, Jeffery W.

Sarcona, Joseph

Sarlieve, Philippe

Sathianathen, Niranjan

Savard, Josée

Sbrocca, Rheanne

Schlemmer, Heinz-Peter

Schuler, Trevor D.

Schwartz, Russell

Scotland, Kymora

Scovell, Jason

Sears, Carly

Semotiuk, Kara

Seo, Nuley

Sexton, Wade

Shah, Taimur

Shahine, Hanna

Shahrour, Walid
MP-9.6

MP-8.11, POD-4.4, UP-2.15, UP-3.11,

UP-2.16, UP-3.9

MP-6.3

POD-3.3, UP-3.11

MP-1.14, POD-5.6

MP-6.5, MP-7.5

UP-1.2

MP-4.8

POD-3.1

UP-2.17

UP-1.12

MP-10.10

MP-2.6

MP-7.4, POD-1.1

UP-1.24

POD-3.1

MP-12.15

MP-3.6

MP-12.1

MP-8.9

MP-8.14

MP-11.4, MP-2.2, MP-2.3, MP-2.4, MP-2.5, POD-3.2, POD-5.1, POD-5.2,

UP-3.15

POD-3.1

MP-3.12, MP-7.15

POD-2.2

MP-3.9, MP-5.13, UP-3.3

UP-2.13, UP-2.14, UP-2.15, UP-2.16

MP-4.1

MP-8.15

UP-3.8

MP-2.2

MP-12.4, MP-12.5, MP-7.14, UP-1.2,

UP-3.17

UP-1.8

MP-3.1

UP-1.5

UP-3.22

UP-3.19

MP-10.8

MP-3.4

POD-2.2

MP-10.7, MP-4.9, UP-3.12

MP-1.14, POD-5.6

UP-3.16

UP-1.29

UP-1.19, UP-1.22

MP-9.14

UP-1.24

MP-5.11, MP-9.1, MP-9.13, MP-9.5,

MP-9.6, MP-9.9

POD-2.5

MP-1.12, MP-1.2, MP-5.3, POD-1.4 POD-1.5

MP-11.14, MP-11.15

UP-2.17

MP-8.1

MP-6.1, MP-6.2

POD-4.3

POD-4.1, POD-4.2

POD-2.5, MP-10.5, MP-4.13

MP-1.4, MP-1.5, MP-7.12, MP-7.8,

MP-7.9, UP-2.3
Shamout, Samer

Shamsuddin, Adib

Shayegan, Bobby

Shiau, Gillian

Shibasaki, Mikio

Shiff, Benjamin

Shivaram Alva, Ajjai

Short, Thomas

Sidana, Abhinav

Siegel, Barry

Siemens, D. Robert

Silverman, Michael

Singbo, Narcisse

Singla, Rohit

Sioufi, Richard

Sistani, Golmehr

Sivalingam, Sri

Skeldon, Sean

Skinner, Thomas A.

Skogberg, Jill

Sleightholm, Richard

Snoad, Brian

So, Alan I.

Soderberg, Leah

Southall, Thomas

Speich, I

Spevack, Les

Spierling, Anna-Lena

Spiess, Philippe

Spodek, Jeffrey $M$.

Spooner, Jesse

Spooner, Lucie

Sridhar, Srikala

Srigley, John

St Martin, Blair A

Starcevic, Danielle

Staruch, Robert

Steele, Stephen S.

Stefanova, Veselina

Stern, Karen

Stern, Noah

Stewart, Caelie

Stock, Richard

Stoletov, Konstantin

Streeper, Necole

Stothers, Lynn

Stringer, Leandra

Struve, Timothy

Suarez, George

Sullivan, Frank

Sun, Ryan

Sundi, Debasish

Sur, Roger

Sutradhar, Rinku

Sweeney, Ellen

Sweet, Joan

Syeda, Ayesha

Syvestre, Nickolas

Szafron, Michael

Szumacher, Ewa

\section{$T$}

Tabibzadeh, Aaron

Takeda, Hayato

Talarek, Jeffrey

Tan, Guan Hee

Tang, Chad
MP-2.9

MP-12.14

MP-10.11, POD-5.5, UP-1.21, UP-1.8

MP-9.7, UP-1.10

MP-5.10

MP-10.7, MP-11.15, UP-3.12, UP-3.23

POD-2.2

MP-4.11, UP-1.20

MP-4.10, UP-1.16, UP-1.25

POD-2.2

MP-7.1, MP-8.5, POD-1.3, UP-1.12

$\mathrm{MP}-11.8$

UP-1.22

MP-11.11, MP-5.4, MP-5.5, MP-5.7,

UP-3.20

MP-10.11

MP-3.6

POD-1.4, POD-1.5

MP-8.1

MP-11.9

MP-7.12

MP-4.7, UP-1.14

MP-11.13

MP-7.4, POD-1.1, POD-4.4, UP-3.9

MP-6.12

MP-4.5, MP-4.6

$\mathrm{MP}-11.7$

MP-4.3, MP-4.4

MP-9.11

POD-4.3

MP-10.11

MP-4.2

MP-5.4, MP-5.7

MP-8.12, MP-8.6, MP-8.7, POD-4.5

$M P-4.11, U P-1.20$

UP-1.9

$\mathrm{MP}-4.12$

UP-1.24

POD-3.2

MP-4.3, MP-4.4

MP-9.10

MP-1.11

MP-11.11

MP-10.8

MP-3.9

POD-1.5

MP-11.6, MP-11.7, UP-2.7

$\mathrm{MP}-12.7$

UP-1.16

MP-2.6

MP-10.8

MP-6.6, UP-3.12

MP-8.14

POD-1.4, POD-1.5

MP-8.2, MP-8.3

UP-2.14

MP-3.13, MP-3.15

UP-2.20

UP-3.2

UP-1.28

UP-1.13

MP-1.14, POD-5.6

MP-5.10

UP-1.25

MP-8.6

MP-10.8 
Tangri, Navdeep

Tanguay, Simon

Tarride, Jean-Eric

Tasian, Gregory

Tawfiek, Ehab

Teishima, Jun

Tennankore, Karthik

Thames, Howard

Thangasamy, Isaac

Tholomier, Côme

Thomas, Patrick

Tiguert, Rabi

Tilley, Derek

Timm, Brennan

Tiwari, Raj

Tjong, Michael

Tobler, Juliana

Todd, Gerry

Tohidi, Mina

Tong, Steven

Toren, Paul

Touma, Naji J.

Traboulsi, Samer

Trinh, Hong Son

Trudel, Dominique

Trudel, Julie

Truong, Tran

Tsang, Chiu Fung

Tseng, Eric

Tsu, Hok Leung

Tu, Le Mai

Tunis, Adam

Twardowski, Kornelia

U

Uy, Michael John

V

Valley, Zachary

Van der Kwast, Theodorus

Van Leeuwen, Bryant

Vanhuyse, Marie

Vasquez-Lastra, Carlos

Veinberg, Natan

Vela, Ian

Veneziano, Domenico

Vera Badillo, Francisco

Verma, Sadhna

Vesprini, Danny

Vigil, Humberto R.

Violette, Philippe D.

Viprakasit, Davis

Virasoro, Ramón

Visram, Kashif

Vrabec, George

\section{W}

Wagner, Heidi

Walker, Lauren

Wallis, Christopher J.D.

Wang, Betty
MP-10.7, MP-4.9, UP-3.12

MP-7.10, POD-4.4, UP-3.11, UP-3.9

UP-1.21

POD-1.3

MP-5.3

MP-3.10

UP-2.10

MP-10.8

UP-1.29

MP-4.13, MP-7.2, MP-7.7, POD-2.5,

UP-1.2

MP-8.15

UP-1.19, UP-1.22

MP-10.14, UP-3.3

MP-1.6, UP-2.11, UP-3.10

MP-10.2

MP-8.6

UP-1.25

MP-10.14

MP-7.1

MP-12.11, UP-3.16

UP-1.19, UP-1.22

MP-3.8, MP-6.9

MP-10.5

MP-3.1

UP-1.19

UP-3.7

POD-4.6, UP-3.14

POD-2.1

UP-1.13

POD-2.1

POD-3.2, POD-3.3

MP-4.4

MP-12.8

MP-6.4, MP-9.12

MP-1.3

MP-10.1, MP-8.2, MP-8.3, MP-8.4, POD-4.5

MP-4.7, UP-1.11, UP-1.14, UP-1.17

UP-2.22, UP-2.23

MP-7.7

MP-4.12

MP-8.15

POD-5.6

MP-3.8

MP-4.10, UP-1.16, UP-1.25

UP-1.13

POD-5.3

POD-3.3

POD-1.5

MP-2.6

MP-3.8, UP-1.12

MP-10.11

UP-2.24

UP-2.17

MP-11.14, MP-11.15, POD-2.6

MP-5.11
Wang, Luke

Wang, Ming-Hsien

Wang, Wendy

Wang, Yuding

Wang, Zhan Tao (Peter)

Warde, Padraig

Warner, Andrew

Warren, Jeffrey

Wassersug, Richard

Webster, George

Welk, Blayne K.

Werlang, Paulo

Westney, O. Lenaine

Wettstein, Marian

Whalen, Stewart

Whelan, Emily

White, Joshua

Whitehead, Marlo

Wiafe, Bridget

Wibowo, Erik

Wijeysundera, Duminda

Wiljer, David

Williams, Elizabeth

Willmore, Katherine

Witherspoon, Luke

Wollin, Tim

Wong, Julie

Wong, Rache

Wong, Victor

Wood, Lori A.

Woodward, Aiden

Wszolek, Matthew

$X$

Xie, Feng

$\mathbf{Z}$

Zakaria, Ahmed

Zemp, Logan W.

Zhang, Tinghua

Zheng, Yu

Zimmerman, Kate

Zimmermann, Eleanor

Zlotta, Alexandre

Zorn, Kevin C.

Zu'bi, Fadi

Zukotynski, Katherine
MP-5.10

MP-10.6

MP-3.12

MP-6.7

UP-2.21

MP-4.5, MP-4.6, UP-2.2

UP-3.1

MP-6.6

POD-5.5

POD-5.4

MP-3.13, MP-3.15, MP-8.7, POD-4.6,

UP-3.14

$\mathrm{MP}-11.9$

UP-2.17

UP-1.15, UP-2.2

POD-3.1

-8.2, MP-8.3, MP-8.4, POD-4.5

UP-1.3

MP-12.15, UP-2.10, UP-2.15

UP-2.17

MP-8.15

UP-3.13

PP-11.9, MP-3.12, MP-5.6 MP-9.7, MP-9.9, POD-4.1

MP-1.12, MP-1.2, MP-5.3

POD -4.4

MP-9.12

MP-8.14

MP-6.5, MP-7.10, MP-7.2, MP-7.7, POD2.5, UP-1.2

POD-4.3

MP-7.15

POD-3.1

$\mathrm{MP}-8.15$

POD-4.1, POD-4.2

MP-5.1, MP-8.1, MP-8.6, MP-8.7, UP-3.5 MP-10.5, MP-4.13, MP-6.5, MP-7.14, MP-7.2, MP-7.3, MP-7.4, MP-7.5, MP-7.6, MP-7.7, POD-1.1, POD-2.5, UP-1.1, UP-1.2

MP-12.6

POD-2.3 